\title{
A CONSPECTUS OF THE MOSSES OF ANTARCTICA, SOUTH GEORGIA, THE FALKLAND ISLANDS AND SOUTHERN SOUTH AMERICA
}

By

DOROTHY M. GREENE

Institute of Terrestrial Ecology

Natural Environment Research Council

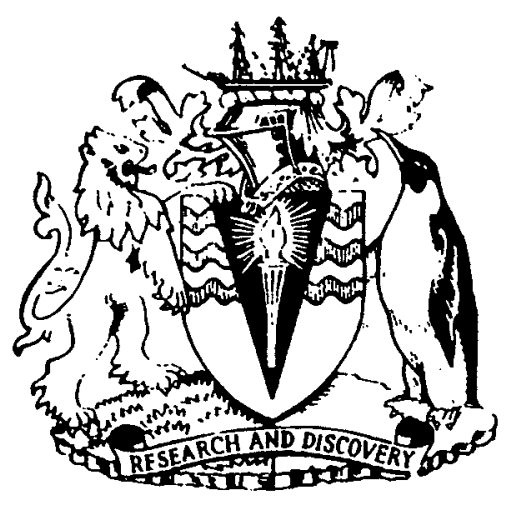

LIBRARY

BRITISH ANTARCTIC SURVEY

MADINGLEY ROAD

CAMBRIDGE CB3 OET 
Front Cover: Tortula robusta, Cumberland Bay, South Georgia. Photo: I Everson

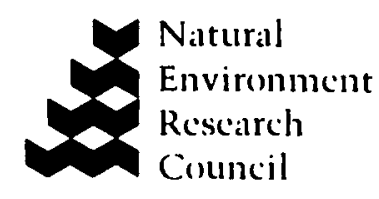

\section{$£ 7.00$}

Authors address:

Copies available from:

Institute of Terrestrial Ecology

The Distribution Centre

Monkswood Experimental Station

Blackhorse Road

Abbots Ripton

Letchworth

Huntingdon PE17 2LS

Herts SG6 IHN

United Kingdom

United Kingdom

Telephone (04873 381/8 


\title{
A CONSPECTUS OF THE MOSSES OF ANTARCTICA, SOUTH GEORGIA, THE FALKLAND ISLANDS AND SOUTHERN SOUTH AMERICA
}

By

\author{
DOROTHY M. GREENE
}

Institute of Terrestrial Ecology

Natural Environment Research Council

\begin{abstract}
A sURVEY is presented of the taxa of mosses which have been reported from Antarctica, the islands of the Scotia Ridge, South Georgia, the Falkland Islands and southem South America, i.e.

Argentina, Chile and Uruguay, including Juan Femandez, from earliest times up to the end of 1977. The results show that 172.7 species and 254 subspecies or varieties are currently accepted for the area; of these 254 species and 40 subspecies or varieties are believed to be endemic.

Nomenclature is based on that in Index muscorum (van der Wijk and others, 1959, 1962, 1964, 1967,1969 ) or in later treatments where appropriate but in four cases, viz. Racomitrium crispulum, the Polytrichaceae, Bryum antarcticum and Sphagnum, reasons are given for adopting a different approach. The list of synonyms includes the basionym and those epithets which have been used for material from the area but is not nomenclaturally exhaustive. Some 60 nomina nuda are also listed since specimens bearing these names are frequently encountered in herbaria.

Distribution follows the worldwide scheme adopted in Index muscorum grouped here under six headings, viz. Juan Fernandez, southern South America, the Falkland Islands, South Georgia, Antarctic Peninsula region and Antarctic cortinental region, the records for the South Orkney Islands being included with the peninsula region. It was found that 41 of the taxa listed in Index muscorum as occurring in the area could not be substantiated from any other source and so have been deleted from the list. As genera and species are revised, further reductions in the totals of currently accepted taxa can be expected, although further field work may add others.

The list of references is extensive and includes about 900 entries, all of which have been examined by the author. Included in the list is the literature relating to the basionyms. Where appropriate, both bibliographic and effective dates have been given to facilitate literature retrieval and taxonomic requirements.
\end{abstract}

\section{INTRODUCTION}

FACILITIES provided during the International Geophysical Year (1957-58) offered bryologists their first opportunities to work in Antarctica. As a result of visits by S. W. Greene in 1960-61, R. E. Longton in 1963-65, R. I. L. Smith in 1964-67, 1969-71, the author in 1967-68, G. C. S. Clarke in $1967-68$ and B. G. Bell in 1971-72, substantial collections were assembled in the British Antarctic Survey Herbarium (Greene, 1972) to be used for revisionary work on Antarctic bryophytes. Most pre-IGY collections had been made from ship-borne expeditions by collectors who were almost entirely non-botanists and who sent their material to European specialists for examination. As a consequence there developed a very scattered literature published in a variety of journals and expedition reports. The first attempt to draw this together was made by Steere $(1961 a, b)$, who produced check lists for Antarctica and South Georgia, respectively, which were later updated by Greene (1968a).

As the collections made by the above-mentioned bryologists contained so much that was new for the region, it soon became clear that the work of identification and preparation of taxonomic 
revisions could not proceed satisfactorily without detailed knowledge of the taxa, and records of their distribution, in neighbouring areas. This applied in particular to southern South America and New Zealand, since it appeared that the Antarctic Peninsula and continental Antarctic moss floras were most closely related to the flora of Magellanic and Nova Zealandic regions. For the latter region Sainsbury's (1955) A handbook of the New Zealand mosses and Dixon's earlier Studies in the bryology of New Zealand with special reference to the herbarium of Robert Brown, Christchurch, New Zealand (Dixon, 1913, 1914, 1923, 1926, 1927, 1929a) provided a reasonable background. Kühnemann (1938) had compiled from the literature a check list of mosses for Argentina, which included the Falkland Islands and later he gave a list of genera for the neighbourhood of Buenos Aires (Kühnemann, 1944). Looser (1952) reviewed the contribution made by Thériot to knowledge of the Chilean moss flora, while Herter $(1927-28,1953)$ listed many relevant works for Uruguay. For the Magellanic region, where the first moss recorded as having been collected was obtained in 1690 (Middleton, 1909; Gunckel, 1971), Cardot (1908a) in La flore bryologique des terres Magellaniques, de la Géorgie du Sud et de l'Antarctide drew together the work of the early expeditions. But all these accounts remained unsynthesized, a different nomenclature was used in each and much of relevance had been published subsequently.

So, in response to the needs of the British Antarctic Survey's bryologists, a synthesis of the literature was undertaken to update Cardot (1908a) and to amalgamate into a single consolidated account the reports and records of mosses from southern South American and Antarctic regions based on the nomenclature of van der Wijk and others $(1959,1962,1964,1967,1969)$ in Index muscorum. This synthesis is provided here in the form of a conspectus of taxa for almost the whole of the areas referred to in Index muscorum as Antarctica (Ant.) and America area No. 6 (Am. 6), i.e.:
Antarctica
Antarctic Peninsula and continental region together with all off-shore
islands.

America 6

Chile, Argentina, Uruguay, Juan Fernandez and Easter Island, Falkland Islands, South Georgia, South Sandwich Islands.

The one exception is the records for the Easter Island flora which have been excluded since they are not believed to have any real affinity with the flora of Antarctica. It should also be appreciated that the moss floras of the sub-Antarctic Marion and Prince Edward Islands, îles Crozet, îles Kerguelen, Heard and Macdonald Islands and Macquarie Island, as well as oceanic island groups to the north of these, e.g. the islands of the Tristan-Gough group in the South Atlantic, New Amsterdam and St. Paul Islands in the South Indian Ocean and the islands normally referred to as the New Zealand Shelf islands, are not treated as they form parts of other geographical areas,
sensu Index muscorum.

The present work lists 1959 currently accepted taxa, 1728 species and 232 subspecies or varieties, of which 254 species and 40 subspecies or varieties are, according to Index muscorum, endemic; in addition 60 nomina nuda are included. It covers the period from the earliest records up to 1977 , although a few papers from 1978 and 1979 have been included. Purely ecological papers have been excluded except where these works contain lists of species from an area which
is not covered in any taxonomic work.

Nomenclature follows that in Index muscorum, Crosby (1977a), or the most recent treatment, in which case the authority is given. Exceptions are the treatment of Racomitrium crispulum by Clifford (1955) and the Polytrichaceae by Smith (1971), because it was found that contemporary opinion did not accept the conclusions in these works. Another exception is the list of synonyms given for Bryum antarcticum by Dixon and Watts (1918). In recent years considerable doubt has been cast on the validity of these reductions by Horikawa and Ando (1967), Greene (1968a), and others. In view of this, and the fact that doubt exists as to whether $B$. antarcticum really belongs to the genus Bryum, it was decided to list all the species involved under their pre-Dixon and Watts (1918) nomenclature. The taxonomic status of Bryum inconnexum Card. is also in considerable doubt in spite of a recent paper by Ochi (1976) in Japanese.

The treatment of the genus Sphagnum has been presented in a simplified form. It proved too time-consuming and beyond the competence of the author to treat the nomenclature for Sphagnum in the same way as has been done for other genera. On the advice of Mr A. Eddy, of the British Museum (Nat. Hist.), the Sphagnum taxa which have been reported for the region are listed with their geographical distribution but no attempt has been made to quote basionyms or any other synonyms, although Index muscorum has been followed as to status. This is indicated in the same way as in the rest of the text.

Apart from the foregoing cases, the author has not made any taxonomic decisions, so obvious anomalies or mistakes remain, although some attempt has been made to draw attention to them.

The list is arranged alphabetically in genera and species and, as in Index muscorum, nothing under varietal rank has been given, although forms are sometimes mentioned in the distribution. Validly published, currently accepted names are given in bold italics, synonyms in italics, nomina illegitima, homonyma illegitima and nomina nuda are in Roman type (Table I) with the status indicated in each case. Synonyms are cross-referenced to their final names. The list of synonyms is not exhaustive but always includes the basionym and those names which have been used for the geographical regions covered by the list, as well as those which were considered necessary to make the nomenclature clear. 
TABLE I

ABBREVIATIONS AND CONVENTIONS

\title{
Abbreviations
}

comb. inval.

distr.

err.

f.

fide

forma

hom. illeg.

nom. illeg.

nom. ival.

nom. nud.

nom. rejic.

non

pro parte

sensu

ssp.

syn.

synon. excl.

var.

\author{
invalid combination \\ distribution \\ in error \\ filius or son \\ according to \\ lowest-ranked infraspecific category \\ later and thus illegitimate homonym \\ illegitimate name \\ invalidly published name \\ name lacking a description or reference to a description \\ name rejected in favour of a later conserved name \\ not \\ in part \\ as understood by \\ subspecies or highest-ranked infraspecific category \\ synonym \\ excluding synonyms in original publication \\ variety or intermediate-ranked infraspecific category
}

The foregoing terms are to be understood in accordance with usage in botanical nomenclature, as explained in the introductory matter to volume one of Index muscorum (van der Wijk and others, 1959).

\section{Conventions}

Names in bold italics

Names in italics

Names in Roman type (e.g. Achrophyllum dentatum) are those currently accepted.

(e.g. Hookeria dentata) are synonyms.

(e.g. Hookeria denticulata) are those which, for one reason or another contravene the rules of The international code of botanical nomenclature (Stafleu, 1978).

Although nomina nuda have no nomenclatural significance, they have been treated in the same way as valid species because both Mueller and Dusén created a significant number and, in the case of Dusén, specimens bearing these names are frequently encountered in world herbaria. It will be noted that on a number of occasions a name has been used by an author prior to its valid publication by the authority cited. For example, Paris frequently published names attributed to Dusén a year before Dusén himself published his descriptions. Camp $(1915,1921)$ and Costes (1921) both cited Theriot names before the latter validly published them. In those cases where it has been possible to be sure that the record pre-dating the valid publication of the taxon is based on the same type as the later valid publication, it has been cited without further comment in the distribution, although technically these names are all nomina nuda. Where the literature has revealed a misidentification, this has been shown by giving the reference followed by fide and a reference to the appropriate source. Orthographical errors not listed in Index muscorum are mentioned in the distribution lists after a reference as orthographical variants.

In a number of cases an author has used a specific epithet which is not given in Index muscorum or elsewhiere and although an authority was cited it has proved impossible to trace the original publication. These names are included in the list as nomina nuda. One other situation deserves comment. A number of authors have reported Hypnum liliputeanum from Chile but, as Thériot (1916) pointed out, Montagne (1842) described the taxon on material from Cuba. Mitten (1869) erroneously listed it for Chile and this error has been faithfully copied by later authors.

The distribution lists are an amalgamation of all the papers in which a taxon or its synonyms have been reported as occurring in the area. This extended method of citation has been adopted as an aid to specimen recovery for the taxonomic work. Despite what appears to be an extensive literature, the specimen base is surprisingly small, the same specimen, quite often the type, being cited by several authors as the only one available.

All taxa reported in Index muscorum as occurring in America 6 or Antarctica have been abstracted, but not all of these appear in the list of taxa. In most cases, this is due to the fact that synonyms irrelevant to the region have been deleted. There remain the 41 taxa cited in Table II where Index muscorum appears to be in error as the author has been unable to find any other reference to the occurrence of any of these taxa in the region.

The abbreviations for the author citations follow those used in Index muscorum; in the case of more recent authors, not cited in that work, the full name is used. The abbreviations of standard nomenclatural terms used in the text are summarized in Table I. 
TABLE II

TAXA REPORTED ERRONEOUSLY FROM SOUTHERN SOUTH AMERICA OR ANTARCTICA IN Index muscorum

\section{Southern South America}

Aulacomnium androgynum (Hedw.) Schwaegr.

Bartramia micrangia Herz.

Breutelia karsteniana (C. Muell.) Jaeg.

Breutelia wainioi Broth.

Bryum cirrhatum Hoppe et Hornsch. hom. illeg.

Bryum incacorralis Herz.

Bryum incrasso-limbatum Card.

Bryum micro-laevigatum C. Muell.

Bryum pungens Tayl.

Bryum urbanskyi Broth.

Campylopus acervatus Mitt.

Campylopus bogotensis Thér.

Coscinodon trinervis (Williams) Broth.

Cryphaea patens Hornsch. ex C. Muell.

Cryphaea patens Hornsch. ex C. Muell. var. dilatata Herz.

Fissidens brevifolius Hook. f. et Wils.

Fissidens mollis Mitt.

Fissidens subcrispus Besch.

Fissidens taylorii C. Muell.

Funaria megalostoma Mitt.

Leptodontium subacutifolium Besch. nom. nud.

Meesia uliginosa Hedw.

Microdus densus (Hook.) Besch.

Mielichhoferia campylocarpa (Hook. et Arnott) Mitt.

Octoblepharum ampullaceum Mitt.

Orthotrichum albidum Hedw.

Papillaria pseudo-funalis C. Muell.

Philonotis hastata (Dub.) Wijk et Marg.

Pilotrichum fendleri C. Muell.

Polytrichum alpinum var. polare (C. Muell.) Hag.

Pseudocrossidium apiculatum Williams

Ptychomitrium serratum B.S.G.

Racopilum cuspidigerum (Schwaegr.) Aongstr.

Schlotheimia rugifolia (Hook.) Schwaegr.

Sematophyllum contiguum (Mitt.) Mitt.

Tortula austro-ruralis (C. Muell.) Broth.

Verrucidens macrosporus Reim.

\section{Antarctica}

Brachythecium pallidoflavens Card.

Isopterygium ambiguum Card.

Isopterygium brownii Card.

Rhynchostegium pseudo-murale (Hamp. ) Jaeg.

The list of references has been made as complete as possible and is arranged under the bibliographic (i.e. title page) date. In taxonomic work it is the effective date, i.e. the date of issue or availability, that is important. In Index muscorum only the effective dates are cited, which presents difficulty in locating many works since card indices and other retrieval systems in libraries are based exclusively on bibliographic dates. To facilitate retrieval, the references are arranged in the bibliography according to bibliographic dates with the effective date given within square brackets where it differs. The same policy has been adopted for author citations but elsewhere in the text only the bibliographic date is used. If the evidence for an effective date is available internally in a publication, e.g. in those volumes of the Revue Bryologique et Lichénologique, where the effective date is given on the cover, the date itself is cited without a source; in all other cases the authority for the effective date is given.

Extensive use has been made of the following works when determining effective dates of publication:

van Steenis-Krauseman and Stearn (1954),

Sayre $(1959,1969,1971,1975)$,

Stafleu (1967) and

Stafleu and Cowan $(1976,1979)$. 
Considerable difficulty was experienced in finding effective dates of publication for the first edition of Index bryologicus (Paris, 1894, 1895, 1896, 1897, 1898), Index muscorum being found to be inconsistent in the dates quoted for the different parts. The dates used here are those given by Paris (1900b), but the complicated inter-relationships between the dates of publication of the fascicules and the order in which pages appeared in Actes de la Société linnéenne de Bordeaux have been dealt with by Stafleu and Cowan (1983).

Every reference has been checked personally by the author. This includes the literature of the basionyms, since many of these works are often cited inaccurately and a lot of them are rare and not readily available outside of large institutional libraries. It is hoped that the trouble taken to trace these works will facilitate their recovery by working taxonomists.

Inevitably a number of papers came to hand too late to be incorporated into the body of the text. Grassi's (1975a, b, 1976) introduction to Argentine bryophytes gives lists of species for the north-west of the country, while Mahu's (1979) paper presents a list of genera of Chilean mosses based on Index muscorum. The existence of an early paper by Porter (1900), dealing with Chilean mosses which is, as its author clearly states, a résumé of the appropriate parts of Cardot's (1900) preliminary account of the mosses collected by the Belgian Antarctic Expedition, has only recently come to the author's attention. Zander (1978) has proposed a number of new combinations in Didymodon and the relevant nomenclatural changes involving taxa treated here in Barbula and Trichostomopsis have been shown as footnotes in the text. This has also been done for Ochi (1977) and for his provisional revision of the genus Bryum in Antarctica (Ochi, 1979) in which some new synonyms are proposed. However, there is, as yet, no answer to the vexed question of the identity and synonymy of Bryum antarcticum Hook.f. et Wils. 
Acanthocladium hom. illeg.

A. breviflagellosum Broth. (Brotherus, 1908) nom. nud.

Syn. Heterophyllum breviflagellosum C. Muell. (Mueller in Brotherus, 1908) nom. nud.

Distr. Southern South America (Hosseus, 1940).

\section{Acaulon}

A. lorentzii C. Muell.

Astomum lorentzii

A. vesiculosum C. Muell. (Mueller, 1888).

Syn. Sphaerangium vesiculosum (C. Muell.) Kindb. (Kindberg, 1889).

Distr. Southern South America (Mueller, 1888; Paris, 1905b; Roth, 1911; Brotherus, 1924a; Kühnemann, 1938).

\section{Achrohypnella}

A. subnervis Herz.

Vesiculariopsis spirifolium

\section{Achrophyllum}

A. anomalum (Schwaegr.) H. Robinson (Robinson, 1974).

Syn. Racopilum anomalum Schwaegr. (Schwaegrichen, 1830). Hookeria anomala (Schwaegr.) C. Muell. (Mueller, 1851a). Pterygophyllum anomalum (Schwaegr.) Mitt. (Mitten, 1869).

Distr. Juan Fernandez (Brotherus, 1924b, 1925; Espinosa B., 1941; Bartram, 1959; Robinson, 1974, 1975).

Southem South America (Mitten, 1869; Mueller, 1885; Paris, 1905b; Cardot, 1905a, 1908a; Brotherus, 1925; Kühnemann, 1938; Welch, 1969).

A. dentatum (Hook. f. et Wils.) Vitt et Crosby (Vitt and Crosby, 1972).

Syn. Hookeria dentata Hook. f. et Wils. (Hooker and Wilson, 1844). Pterygophyllum dentatum (Hook. f. et Wils.) Dix. (Dixon, 1912).

Hookeria denticulata Hook. f. et Wils. (Hooker and Wilson in Wilson and Hooker, 1845) hom. illeg.

Pterygophyllum denticulatum Mitt. (Mitten, 1869) nom. illeg.

Hookeria nigella Hook. f. et Wils. (Hooker and Wilson in Wilson, 1854).

Pterygophyllum nigellum (Hook. f. et Wils.) Mitt. (Mitten, 1856).

Distr. Juan Fernandez (Mitten. 1885; Johow, 1896; Paris, 1905b; Brotherus, 1924b, 1925; Bartram, 1959; Robinson, 1975).

Southern South America (Wilson and Hooker, 1847; Mueller, 1851a, 1885; Mitten, 1869 Jaeger, 1877; Paris, 1905b; Cardot, 1908a; Thériot, 1917b; Cardot and Brotherus, 1923; Brotherus, 1925; Kühnemann, 1938; Herzog, 1939, 1954).

Falkland Islands Wilson and Hooker, 1847; Mueller, 1851a, 1885; Jaeger, 1877; Paris, 1905b; Cardot, 1908a; Cardot and Brotherus, 1923; Brotherus, 1925; Kühnemann, 1938; Welch, 1969).

A. tenuinerve (Broth.) H. Robinson (Robinson, 1974).

Syn. Pterygophyllum tenuinerve Broth. (Brotherus, 1924b).

Distr. Juan Fernandez (Brotherus, 1924b, 1925; Espinosa B., 1941; Bartram, 1959; Welch, 1969; Matteri, 1972; Robinson, 1974, 1975).

Southern South America (Matteri, 1972).

\section{Acidodontium}

A. kunthii Schwaegr.

Acidodontium megalocarpum

A. macropoma (C. Muell.) Broth. (Brotherus, 1903).

Syn. Bryum macropoma C. Muell. (Mueller, 1879a). Brachymenium macropoma (C. Muell.) Kindb. (Kindberg, 1889).

Distr. Southern South America (Mueller, 1879a; Paris, 1894, 1903; Brotherus, 1903, 1924a; Kühnemann, 1938). 
A. megalocarpum (Hook.) Ren. et Card. (Renauld and Cardot, 1892 [1893]).

Syn. Bryum megalocarpum Hook. (Hooker in Kunth, 1822).

Acidodontium kunthii Schwaegr. (Schwaegrichen, 1827a) nom. illeg.

Distr. Southern South America (Hornschuch, 1840; Herter, 1928, 1933a).

Acroceratium nom. nud.

A. auriculatum (Mont.) Mitt.

Acrocladium auriculatum

A. politum (Hook. f. et Wils.) Mitt.

Catagonium politum

A. sarmentosum (Wahlenb.) Mitt.

Calliergon sarmentosum

\section{Acrocladiopsis}

A. complanata Card.

Catagonium politum

A. myura Card.

Catagonium myurum

A. nitida (Hook. f. et Wils.) Card.

Catagonium nitidum

\section{Acrocladium}

A. auriculatum (Mont.) Mitt. (Mitten, 1869).

Syn. Hypnum auriculatum Mont. (Montagne, 1843).

Stereodon auriculatus (Mont.) Mitt. (Mitten, 1859).

Coelidium auriculatum (Mont.) Jaeg. (Jaeger, 1878).

Acroceratium auriculatum (Mont.) Mitt. (Mitten, 1883).

Lembophyllum auriculatum (Mont.) Par. (Paris, 1896).

Hypnum chlamydophyllum Hook. f. et Wils. (Hooker, J. D. and Wilson, 1844).

Acrocladium chlamydophyllum (Hook. f. et Wils.) C. Muell. et Broth. (Mueller and Brotherus, 1900).

Hypnum encalyptratum Schimp. (Schimper in Ångström, 1872) hom. illeg.

Eurhynchium encalyptratum Schimp. (Schimper in Jaeger, 1878) nom. nud.

Eurhynchium eucalyptratum Schimp. ex Par. (Schimper ex Paris, 1895) nom. nud.

Hypnum eucalyptratum Lindb. ex Card. (Lindberg ex Cardot, 1908a) err.

Plagiothecium magellanicum Schimp. (Schimper in Jaeger, 1878) nom. nud.

Distr. Juan Fernandez (Robinson, 1975).

Southem South America (Montagne, 1843, 1845b, 1850, 1856; Wilson and Hooker, 1847 (and as Hypnum chlamydophyllum fide Karczmarz, 1966); Mueller, 1851a, 1885, 1889; Sullivant, 1859 (as Hypnum chlamydophyllum fide Karczmarz, 1966); Mitten, 1869; Ångström, 1872; Jaeger, 1878; Bescherelle, 1889; Paris, 1896, 1905a; Neger, 1899; Cardot, 1900, 1901, 1905a, 1908a (and as A. chlamydophyllum), 1913a;

Dusén, 1903a, 1905d; Spegazzini, 1922; Cardot and Brotherus, 1923; Herzog, 1923, 1939, 1940, 1954, 1957, 1960; Brotherus, 1925; Roivainen, 1934; Thériot, 1934b; Kühnemann, 1938; Andrews, 1949a; Bartram, 1952; Karczmarz, 1966; Seki, 1974).

A. chlamydophyllum (Hook. f. et Wils.) C. Muell. et Broth.

Acrocladium auriculatum

A. cuspidatum (Hedw.) Lindb.

Calliergonella cuspidata

A. morenoi (C. Muell.) Dus.

Pleurozium schreberi

A. phyllogonium Jaeg.

Catagonium politum var. phyllogonium

A. politum (Hook. f. et Wils.) Mitt.

Catagonium politum

A. sarmentosum (Wahlenb.) Richs. et Wall.

Calliergon sarmentosum

A. stramineum (Brid.) Richs. et Wall.

Calliergon stramineum

Acrocryphaea nom. illeg.

A. julacea (Mitt.) Jaeg. (Jaeger, 1876).

Syn. Cryphaea julacea Mitt. (Mitten, 1869).

Grimmia julacea Hornsch. (Hornschuch, 1840) hom. illeg.

Distr. Southem South America (Brotherus, 1925; Kühnemann, 1938; Herzog, 1952a).

\section{Acroschisma}

A. wilsonii (Hook. f.) Jaeg.

Andreaea wilsonii 


\section{Aloina}

A. catillum (C. Muell.) Broth. (Brotherus, 1902).

Syn. Barbula catillum C. Muell. (Mueller, 1879a).

Distr. Southern South America (Mueller, 1879a; Brotherus, 1902, 1924a; Paris, 1903; Kühnemann, 1938; Delgadillo, 1975).

A. cucullatifolia (C. Muell.) Broth.

Aloinella cucullatifolia

A. galeata (C. Muell.) Broth.

Aloinella galeata

A. recurvipatula (C. Muell.) Broth. (Brotherus, 1902).

Syn. Barbula recurvipatula C. Muell. (Mueller, 1882).

Distr. Southern South America (Mueller, 1882; Brotherus, 1902, 1924a; Paris, 1903; Hosseus, 1935b, 1937; Kühnemann, 1938).

A. roseae (Williams) Delgadillo (Delgadillo, 1973).

Syn. Pterygoneurum roseae Williams (Williams, 1915).

Crossidium roseae (Williams) Bartr. (Bartram, 1942).

Distr. Southern South America (Bartram, 1942, 1943, 1952).

A. sedifolia (C. Muell.) Broth. (Brotherus, 1902).

Syn. Barbula sedifolia C. Muell. (Mueller, 1879a).

Distr. Southern South America (Mueller, 1879a; Brotherus, 1902, 1924a; Paris, 1903; Kurtz, 1904; Hosseus, 1935b, 1937; Kühnemann, 1938).

\section{Aloinella}

A. cucullatifolia (C. Muell.) Broth. (Brotherus, 1924a).

Syn. Barbula cucullatifolia C. Muell. (Mueller, 1879a).

Aloina cucullatifolia (C. Muell.) Broth. (Brotherus, 1902).

Distr. Southern South America (Mueller, 1879a; Brotherus, 1902, 1924a; Paris, 1903; Kühnemann, 1938).

A. galeata (C. Muell.) Broth. (Brotherus, 1924a).

Syn. Barbula galeata C. Muell. (Mueller, 1879a).

Aloina galeata (C. Muell.) Broth. (Brotherus, 1902).

Distr. Southern South America (Mueller, 1879a; Brotherus, 1902, 1924a; Paris, 1903; Kühnemann, 1938).

Amblystegiella nom. illeg.

A. densissima (Card.) Broth. (Brotherus, 1908).

Syn. Amblystegium densissimum Card. (Cardot, 1900).

Distr. Antarctic, peninsula region (Cardot, 1900, 1901, 1908a; Paris, 1903; Brotherus, 1908 , 1925; Robinson, 1972 (as Platydictya densissima)).

A. tenuifolia (Hedw.) Fleisch.

Rhynchostegium tenuifolium

\section{Amblystegium}

A. aduncum (Hedw.) De Not.

Drepanocladus aduncus

ssp. orthothecioides (Lindb.) Lindb.

Drepanocladus uncinatus var. subjulaceus

var. plumosum (Schimp.) Hult

Drepanocladus uncinatus

A. austro-fluviatile (C. Muell.) Kindb.

Hygroamblystegium austro-fluviatile

A. austro-riparium Broth. (Brotherus in Kurtz, 1904) nom. nud.

Distr. Southern South America (Kurtz, 1904; Hosseus, 1936; Kühnemann, 1938).

A. austro-serpens (C. Muell.) Kindb.

Haplocladium microphyllum

A. brachiatum (Mitt.) Broth.

Drepanocladus brachiatus 
A. brachypelmatum $\mathrm{C}$. Muell.

A. campicola (C. Muell.) Kindb.

A. chilense Lor.

A. Clarazii (Dub.) Jaeg.

A. densissimum Card.

A. dusenii Broth. (Brotherus in Paris, 1906) nom. nud.

Distr. Southern South America (Paris, 1906).

A. exannulatum (B.S.G.) De Not.

A. excurrens Card. et Broth.

A. fluitans (Hedw.) De Not.

ssp. exannulatum (B.S.G.) Arn. et C. Jens.

var. exannulatum (B.S.G.) Lindb.

var. falcifolium (Ren.) Arn. et C. Jens.

A. fuegianum Mitt.

A. intermedium Lindb. var. revolvens (Sw.) Vent. et Bott.

A. jaffuelii Thér.

A. kneiffii B.S.G. var. polycarpum (Voit) Braithw.

A. kochii B.S.G.

A. longifolium Mitt.

A. lucidum (Hook. f. et Wils.) Jaeg.

A. megachaete Dus. (Dusén, 1903a) nom. nud.

Distr. Southern South America (Dusén, 1903a).

A. orthocarpus Broth. (Brotherus in Kurtz, 1904) nom. nud.

Distr. Southern South America (Kurtz, 1904; Hosseus, 1936, 1937; Kühnemann, 1938).

A. orthothecioides (Lindb.) Lindb. ex Par.

Drepanocladus uncinatus var. subjulaceus

A. philonotis Dus. (Dusén in Paris, 1906) nom. nud.

Distr. Southern America (Paris, 1906; Dixon, 1929b).

A. polycarpum (Voit) Vent. et Bott.

A. polygamum B.S.G.

A. pulchellum C. Muell.

A. radicale ( $P$. Beauv.) Mitt.

A. revolvens (Sw.) De Not.

A. riparium (Hedw.) B.S.G. ssp. kochii (B.S.G.) Ren. et Card.

var. kochii (B.S.G.) Buyss.

A. rivulare (C. Muell.) Broth. (Brotherus, 1908) hom. illeg.

Syn. Dimerodontium rivulare C. Muell. (Mueller, 1897a).

Distr. Southern South America (Mueller, 1897a; Paris, 1900a, 1904b; Brotherus, 1908, 1925;

Fleischer, 1920; Kühnemann, 1938).

A. sarmentosum (Wahlenb.) De Not.

Calliergon sarmentosum

A. serpens (Hedw.) B.S.G. (Bruch and others, 1853).

Syn. Hypnum serpens Hedw. (Hedwig, 1801).

r.ypnum oligorrhizon Guemb. (Guembel in Austin, 1877) nom. nud.

Distr. Juan Fernandez (Robinson, 1975).

Southern South America (Gibert, 1873; Roivainen, 1934; Piovano, 1954).

Falkland Islands (Cardot and Brotherus, 1923; Kühnemann, 1938). 
A. sparsifolium (Hamp.) Jaeg. (Jaeger in Jaeger and Sauerbeck, 1879a).

Syn. Hypnum sparsifolium Hamp. (Hampe, 1870).

Distr. Southern South America (Felippone, 1928; Herter, 1933a).

A. stramineum (Brid.) De Not.

Calliergon stramineum

A. subvarium Broth. (Brotherus, 1906a).

Distr. Antarctic, peninsula region (Darbishire, 1923; Greene, 1968a).

A. tenellum Card. et Broth.

Hygroamblystegium tenellum

A. trichopodium (Schultz) Hartm. ssp. kochii (B.S.G.) Loesk.

Leptodictyum kochii

var. Kochii (B.S.G.) Lindb.

Leptodictyum kochii

A. uncinatum (Hedw.) De Not.

Drepanocladus uncinatus

var. orthothecioides (Lindb.) C. Jens.

Drepanocladus uncinatus var. subjulaceus

var. plumosum (Schimp.) Moell.

Drepanocladus uncinatus

var. subjulaceum (B.S.G.) C. Jens.

Drepanocladus uncinatus var. subjulaceus

var. symmetricum (Ren. et Card.) Dus.

Drepanocladus uncinatus var. symmetricus

A. varium (Hedw.) Lindb. (Lindberg, 1879).

Syn. Leskea varia Hedw. (Hedwig, 1801).

Hypnum varium (Hedw.) P. Beauv. (Palisot de Beauvois, 1805a).

Stereodon varius (Hedw.) Mitt. (Mitten, 1864).

Rigodium varium (Hedw.) Kindb. (Kindberg, 1883).

Amblystegium pulchellum C. Muell. (Mueller in Ule, 1899) nom. nud.

Hygroamblystegium varium (Hedw.) Moenk. (Mönkemeyer, 1911).

Distr. Southern South America (Williams, 1930).

var. patagonicum Card.

Drepanocladus aduncus var. polycarpus

\section{Amphidium}

A. cyathicarpum (Mont.) Broth.

Amphidium tortuosum

A. tortuosum (Hornsch.) H. Robinson (Robinson, 1975).

Syn. Syrrhopodon tortuosus Hornsch. (Hornschuch, 1841).

Zygodon cyathicarpus Mont. (Montagne, 1845a).

Didymodon cyathicarpus (Mont.) Mitt. (Mitten, 1860).

Rhabdoweisia cyathicarpa (Mont.) Mitt. (Mitten, 1869).

Amphoridium cyathicarpum (Mont.) Jaeg. (Jaeger, 1874).

Oncophorus cyathicarpus (Mont.) Mitt. (Mitten, 1885).

Amphidium cyathicarpum (Mont.) Broth. (Brotherus, 1902).

Weisia sphaerothecia C. Muell. (Mueller, 1882).

Rhabdoweisia sphaerothecia (C. Muell.) Kindb. (Kindberg, 1889).

Distr. Juan Fernandez (Mitten, 1885; Johow, 1896; Paris, 1900a, 1903; Skottsberg, 1914; Espinosa B., 1941; Bartram, 1959; Robinson, 1975).

Southern South America (Montagne, 1845a, 1850, 1856; Mueller, 1849, 1882; Jaeger, 1874: Brotherus, 1902, 1924a; Dusén, 1903a; Paris, 1903; Cardot, 1908a; Thériot, 1917b, 1921a; Costes, 1921; Kühnemann, 1938; Herzog, 1939; Lawton, 1961; Seki, 1974).

South Georgia (Cardot and Brotherus, 1923; Steere, 1961b).

Amphoridium hom. illeg.

A. cyathicarpum (Mont.) Jaeg.

Amphidium tortuosum

\section{Amphoritheca}

A. bonplandii (Hook.) Jaeg.

Funaria bonplandii

A. Clavellata (Mitt.) Jaeg.

Funaria clavellata

A. papillosa (C. Muell.) Kindb.

Funaria papillosa 


\section{Anacalypta}

A. brunnea (C. Muell.) Kindb.

Didymodon brunneus

A. glauco-viridis C. Muell.

Didymodon glauco-viridis

A. gracillima (C. Muell.) C. Muell.

Trichostomum gracillimum

A. gymna (C. Muell.) Kindb.

Didymodon gymnus

A. imperfecta (C. Muell.) Kindb.

Barbula imperfecta

A. microthecium C. Muell.

Didymodon microthecius

A. nuda (C. Muell.) Kindb.

Didymodon brunneus

A. spathulato-linearis (C. Muell.) Kindb.

Didymodon spathulato-linearis

\section{Anacolia}

A. aurea Dus. (Dusén, 1903a) nom. nud.

Distr. Southem South America (Dusén, 1903a).

A. auricolor Dus.

Breutelia integrifolia

A. laevisphaera (Tayl.) Flow. (Flowers, 1935).

Syn. Glyphocarpa laevisphaera Tayl. (Taylor, 1846).

Bartramia laevisphaera (Tayl.) C. Muell. (Mueller, 1849).

Glyphocarpus laevisphaerus (Tayl.) Jaeg. (Jaeger, 1875).

Bartramia subsessilis Tayl. (Taylor, 1847).

Anacolia subsessilis (Tayl.) Broth. (Brotherus, 1904).

Anacolia subsessilis (Tayl.) Broth. var. brevifolia Broth. (Brotherus, 1924b).

Distr. Juan Fernandez (Brotherus, 1924b; Espinosa B., 1941; Flowers, 1952; Bartram, 1959; Robinson, 1975).

Southem South America (Thériot, 1936; Hosseus, 1938c, d. 1939; Flowers, 1952; Herzog, 1960).

A. subsessilis (Tayl.) Broth.

Anacolia laevisphaera

var. brevifolia Broth.

Anacolia laevisphaera

\section{Ancistrodes}

A. genuflexa (C. Muell.) Crosby (Crosby, 1976).

Syn. Pilotrichum genuflexum C. Muell. (Mueller, 1844b [1845]).

Meteorium genuflexum (C. Muell.) Mitt. (Mitten, 1869).

Duseniella genuflexa (C. Muell.) Broth. (Brotherus, 1906b).

Bryodusenia genuflexa (C. Muell.) H. Robinson (Robinson, 1974).

Hookeria ancistrodes Mont. (Montagne, 1845a).

Pilotrichum ancistrodes (Mont.) Lor. (Lorentz, 1866).

Meteorium ancistrodes (Mont.) Par. (Paris, 1896) hom. illeg.

Distr. Southern South America (Mueller, 1844b, 1850; Montagne, 1845a, 1850, 1856; Lorentz, 1866; Mitten, 1869; Jaeger, 1877; Paris, 1897, 1900a, 1905a; Brotherus, 1906b, 1925; Cardot, 1908a; Herzog, 1923, 1939, 1954, 1960; Reimers, 1926; Thériot, 1934b; Kühnemann, 1938; Seki, 1974).

\section{Andreaea}

A. acuminata Mitt. (Mitten in Wilson, 1859).

Syn. Andreaea opaca Card. ex Roth (Cardot ex Roth, 1911) fide Greene, $1968 \mathrm{~b}$.

Distr. South Georgia (Roth, 1911, 1913; Cardot and Brotherus, 1923; Brotherus, 1924a; Steere, 1961b; Greene, 1968b).

A. acutifolia Hook. f. et Wils. (Hooker, J.D. and Wilson, 1844).

Distr. Southern South America (Hooker, J.D. and Wilson, 1844; Wilson and Hooker, 1847; Mueller, 1848a, 1885; Mitten, 1869; Jaeger, 1870; Bescherelle, 1889; Dusén, 1903a; Paris, 1903; Cardot, 1905a, 1908a; Roth, 1910; Cardot and Brotherus, 1923; Brotherus, 1924a; Roivainen and Bartram, 1937; Kühnemann, 1938; Seki, 1974). 
Falkland Islands (Hooker, J.D. and Wilson, 1844; Wilson and Hooker, 1847; Mueller, 1848a; Jaeger, 1870; Paris, 1903; Cardot, 1908a; Roth, 1910; Cardot and Brotherus, 1923; Brotherus, 1924a; Kühnemann, 1938).

var. rufescens Hook. f. et Wils. (Hooker and Wilson in Wilson and Hooker, 1847).

Distr. Southern South America (Wilson and Hooker, 1847).

A. alpina Hedw. (Hedwig, 1801).

Syn. Andreaea squamata C. Muell. (Mueller, 1884 [1883]) fide Greene, $1968 a$.

Andreaea loricata Dus. (Dusén, 1903b).

Andreaea brevifolia Dus. (Dusén, 1903b) fide Roivainen and Bartram, 1937.

Andreaea parallela C. Muell. var. brevifolia (Dus.) Card. (Cardot, 1908a) fide Roivainen and Bartram, 1937.

Andreaea lanceolata Dus. ex Roth. (Roth, 1910) hom. illeg.

Andreaea ruficaulis Dus. (Dusén in Roivainen and Bartram, 1937) nom. nud.

Distr. Juan Femandez (Robinson, 1975).

Southern South America (Wilson and Hooker, 1847; Sullivant, 1859; Mitten, 1969; Jaeger, 1870; Dusén, 1903a, b; Paris, 1906; Cardot, 1908a; Roth, 1910; Cardot and Brotherus, 1923; Brotherus, 1924a; Roivainen and Bartram, 1937; Kühnemann, 1938; Greene, 1968b; Seki, 1974).

Falkland Islands (Cardot and Brotherus, 1923; Roivainen and Bartram, 1937; Kühnemann, 1938; Greene, 1968b).

South Georgia (Cardot, 1906a, 1908a; Roth, 1910; Steere, 1961b (and as A. regularis var. brevifolia); Greene, 1968a, b).

A. appendiculata B.S.G. (Bruch and others, 1855).

Distr. Southern South America (Wilson and Hooker, 1847 (as A. rupestris L.); Bruch and others, 1855; Mueller, 1864, 1885; Mitten, 1869; Jaeger, 1870; Dusén, 1903a; Paris, 1903; Cardot, 1908a; Roth, 1910; Cardot and Brotherus, 1923; Brotherus, 1924a; Kühnemann, 1938).

A. arachnoidea C. Muell. (Mueller, 1879a).

Distr. Southem South America (Mueller, 1879a; Paris, 1903; Roth, 1910; Brotherus, 1924a; Kühnemann, 1938).

A. brevifolia Dus.

Andreaea alpina

A. depressinervis Card. (Cardot, 1900).

Syn. Andreaea depressinervis Card. var. compacta Card. (Cardot, 1901) fide Greene, $1968 b$.

Distr. South Georgia (Greene, 1968b; Schultze-Motel, 1970).

Antarctic, peninsula region (Cardot, 1900, 1901, 1906a, 1907c, 1908a, 1911b, c, d, 1912b, 1913a; Paris, 1903; Wright, 1905 (as Andreaea sp.); Roth, 1910; Dixon, 1935; Steere, 1961a; Greene, 1968b; Greene and others, 1970; Robinson, 1972).

var. compacta Card.

Andreaea depressinervis

A. flabellata C. Muell. (Mueller, 1884 [1883]).

Syn. Andreaea grimmioides Dus. (Dusén, 1903b) fide Greene, $1968 b$.

Andreaea pumila Card. (Cardot, 1906a) fide Greene, 1968b.

Distr. Southern South America (Dusén, 1903b; Cardot, 1905a, 1908a; Roth, 1910; Brotherus, 1924a; Roivainen and Bartram, 1937; Kühnemann, 1938; Herzog, 1954; Greene,
1968b).

Falkland Islands (Cardot and Brotherus, 1923; Kühnemann. 1938; Greene, 1968b).

South Georgia (Cardot, 1906a, 1908a; Roth, 1910; Brotherus, 1924a; Steere, 1961b; Greene, 1968b)

A. fragilis C. Muell. (Mueller, 1879a).

Distr. Southern South America (Mueller, 1879a; Paris, 1903; Roth, 1910; Kühnemann, 1938). 
A. fuegiana (Card.) S.W. Greene (Greene, 1972 [1973]).

Syn. Neuroloma fuegiana Card. (Cardot, 1911a) hom. illeg. non Neuroloma Cand.

Distr. Southern South America (Cardot, 1911a; Roth, 1913; Cardot and Brotherus, 1923, Brotherus, 1924a; Roivainen and Bartram, 1937; Kühnemann, 1938; Seki, 1974). South Georgia (Greene, 1968b, 1972).

A. gainii Card. (Cardot, 1911b).

Distr. South Georgia (Greene, 1968b).

Antarctic, peninsula region (Cardot, 1911b, d, 1913a; Roth, 1913; Brothenus, 1924a; Bartram, 1957 (as A. rupestris forma fide Greene, 1968b); Steere, 1961a; Greene, 1968b; Greene and others, 1970; Robinson, 1972).

var. parallela (C. Muell.) S.W. Greene

Andreaea parallela

A. grimmioides Dus.

Andreaea flabellata

A. heterophylla Card.

Andreaea regularis

A. hookeri Schimp. (Schimper in Bruch and others, 1855).

Distr. Southern South America (Bruch and others, 1855).

A. lanceolata Dus. ex Roth.

Andreaea alpina

A. latinervis Bartr. (Bartram in Roivainen and Bartram, 1937).

Distr. Southern South America (Roivainen and Bartram, 1937).

A. laxifolia Hook. f. et Wils. (Hooker, J.D. and Wilson, 1844).

Distr. Southern South America (Hooker, J.D. and Wilson, 1844; Wilson and Hooker, 1847; Mueller, 1848a, 1885; Mitten, 1869; Jaeger, 1870; Dusén, 1903a; Paris, 1903; Cardot, 1908a; Roth, 1910; Brotherus, 1924a).

var. minor Hook. f. et Wils. (Hooker, J.D. and Wilson, 1844).

Distr. Southem South America (Hooker, J.D. and Wilson, 1844; Wilson and Hooker, 1847; Paris, 1903; Cardot, 1908a).

A. leiophylla Card. ex Roth (Cardot ex Roth, 1913).

Distr. Southern South America (Roth, 1913; Cardot and Brotherus, 1923; Brotherus, 1924a; Roivainen and Bartram, 1937; Kühnemann, 1938).

A. liliputana C. Muell. (Mueller, 1901a [1900]) nom. nud.

Distr. Southern South America (Mueller, 1901a).

A. Iorentziana C. Muell. (Mueller, 1879a).

Syn. Andreaea lorentzii C. Muell. ex Kindb. (Kindberg, 1889) nom. illeg.

Distr. Southem South America (Mueller, 1879a; Paris, 1903; Roth, 1910; Brotherus, 1924a; Kühnemann, 1938).

A. Iorentzii C. Muell. ex Kindb.

Andreaea lorentziana

A. loricata Dus.

Andreaea alpina

A. marginata Hook. f. et Wils. (Hooker, J.D. and Wilson, 1844).

Distr. Southern South America (Hooker, J.D. and Wilson, 1844; Wilson and Hooker, 1847; Mueller, 1848a, 1885; Mitten, 1869; Jaeger, 1870; Dusén, 1903a, b; Paris, 1903; Cardot, 1908a; Roth, 1910; Brotherus, 1924a; Kühnemann, 1938; Herzog, 1954; Seki, 1974).

A. mutabilis Hook. f. et Wils.

Andreaea rupestris

var. microphylla Hook. f. et Wils. (Hooker, J.D. and Wilson, 1844).

Distr. Southern South America (Cardot and Brotherus, 1923; Kühnemann, 1938).

var. subsecunda Hook. f. et Wils. (Hooker, J.D. and Wilson, 1844).

Distr. Falkland Islands (Hooker, J.D. and Wilson, 1844; Wilson and Hooker, 1847; Mueller, 1848a; Paris, 1903; Cardot, 1908a; Kühnemann, 1938). 
var. uncinata Hook, f. et Wils. (Hooker, J.D. and Wilson, 1844).

Distr. Falkland Islands (Hooker, J.D. and Wilson, 1844; Wilson and Hooker, 1847; Mueller, 1848a; Paris, 1903; Cardot, 1908a; Kühnemann, 1938).

A. opaca Card. ex Roth

Andreaea acuminata

A. pachyphylla (C. Muell.) Broth. (Brotherus, 1901).

Syn. Grimmia pachyphylla C. Muell. (Mueller, 1885).

Andreaea pachyphylla (C. Muell.) Broth. var. acutifolia Dus. (Dusén, 1903b) fide Schultze-Motel, 1970.

Distr. Southern South America (Mueller, 1885; Brotherus, 1901, 1924a; Dusén, 1903a, b; Paris, 1903; Cardot, 1908a; Roth, 1910; Roivainen and Bartram, 1937; Kühnemann, 1938; Herzog, 1954; Schultze-Motel, 1970; Seki, 1974).

var. acutifolia Dus.

Andreaea pachyphylla

A. parallela C. Muell. (Mueller, 1884 [1883]).

Syn. Andreaea gainii Card. var. parallela (C. Muell.) S.W. Greene (Greene, 1968b) hom. illeg.

Distr. South Georgia (Cardot, 1906a, 1908a; Roth, 1910; Steere, 1961b; Greene, 1968b). Antarctic, peninsula region (Greene and others, 1970).

var. brevifolia (Dus.) Card. (Cardot, 1908a).

Andreaea alpina

A. patagonica Dus. (Dusén, 1903b).

Andreaea alpina

Distr. Southern South America (Dusén, 1903b; Cardot, 1908a; Roth, 1910; Cardot and Brotherus, 1923; Brotherus, 1924a; Kühnemann, 1938).

A. perichaetialis (Hook. f. et Wils.) Jaeg.

Andreaea subulata

A. petrophila Fuernr.

Andreaea rupestris

A. pilifera Herz. et Thér. (Herzog and Thériot in Herzog, 1937).

Distr. Southern South America (Herzog, 1937).

A. pseudo-alpina C. Muell. (Mueller, 1859).

Distr. Southern South America (Mueller, 1859, 1885; Jaeger, 1870; Dusén, 1903a, b; Paris, 1903; Cardot, 1908a; Roth, 1910; Brotherus, 1924a).

A. pseudomutabilis Dus. (Dusén, 1903b).

Distr. Southern South America (Dusén, 1903b; Cardot, 1908a; Roth, 1910; Brotherus, 1924a).

A. pseudo-subulata C. Muell.

Andreaea subulata

A. pulvinatula Dus. (Dusén, 1903a) nom. nud.

Distr. Southern South America (Dusén, 1903a).

A. pumila Card.

Andreaea flabellata

A. purpurascens (Dus.) Roth (Roth, 1910).

Syn. Andreaea remotifolia Dus. var. purpurascens Dus. (Dusén, 1903b).

Distr. Southern South America (Dusén, 1903a, b; Cardot, 1908a; Roth, 1910; Brotherus, 1924a).

A. pycnotyla Card.

Andreaea regularis

A. pygmaea Card.

Andreaea regularis

A. regularis C. Muell. (Mueller, 1890a).

Syn. Andreaea pycnotyla Card. (Cardot, 1900) fide Greene, $1968 \mathrm{~b}$.

Andreaea regularis C. Muell. var. pycnotyla (Card.) Card. (Cardot, 1908a) fide Greene, $1968 b$.

Andreaea heterophylla Card. (Cardot, 1906a) fide Greene, 1968 b.

Andreaea pygmaea Card. (Cardot, 1900) fide Greene, 1968b.

Andreaea verruculosa Card. (Cardot, 1905a) fide Greene, $1968 \mathrm{~b}$. 
Andreaea viridis C. Muell. (Mueller, 1890a) fide Greene, $1968 b$.

Andreaea willii C. Muell. (Mueller, 1890a) fide Greene, 1968b.

Distr. Southern South America (Cardot, 1905a, 1908a (including A. mutabilis fide Greene, 1968b); Roth, 1910; Cardot and Brotherus, 1923; Brotherus, 1924a; Roivainen and Bartram, 1937 (including A. mutabilis p.p. fide Greene, 1968b); Kühnemann, 1938; Bartram, 1952; Greene, 1968b; Seki, 1974).

Falkland Islands (Cardot and Brotherus, 1923 (as A. mutabilis fide Greene, 1968b); Greene, 1968b).

South Georgia (Mueller, 1890a; Paris, 1903; Cardot, 1906a, 1908a; Roth, 1910; Cardot and Brotherus, 1923; Brotherus, 1924a; Steere, 1961b; Greene, 1968b).

Antarctic, peninsula region (Cardot, 1900, 1901, 1906a, b, 1907a, C, 1908a, 1911b, d, 1913a; Paris, 1903; Roth, 1910; Brotherus, 1924a; Bartram, 1957 (as A. rupestris fide Greene, 1968b); Steere, 1961 a (and as A. rupestris fide Greene, 1968b); Greene, 1968b; Greene and others, 1970; Robinson, 1972).

var. pycnotyla (Card.) Card.

Andreaea regularis

A. remotifolia Dus. (Dusén, 1903b).

Distr. Southern South America (Dusén, 1903b; Cardot, 1908a; Roth, 1910; Brotherus, 1924a). var. purpurascens Dus.

Andreaea purpurascens

A. rothii Web. et Mohr (Weber et Mohr, 1807).

Syn. Andreaea rupestris A. Roth (Roth, 1802) hom. illeg.

Distr. Falkland Islands (Cardot and Brotherus, 1923; Kühnemann, 1938).

A. ruficaulis Dus.

Andreaea alpina

A. rupestris Hedw. (Hedwig, 1801).

Syn. Andreaea mutabilis Hook. f. et Wils. (Hooker, J.D. and Wilson, 1844). Andreaea petrophila Fuernr. (Fürnrohr, 1827).

Distr. Southern South America (Mueller, 1851a; Jaeger, 1870; Dusén, 1903a; Paris, 1903; Cardot, 1905a, 1908a; Roth, 1910; Cardot and Brotherus, 1923; Brotherus, 1924a; Roivainen and Bartram, 1937; Kühnemann, 1938; Bartram, 1952).

Falkland Islands (Jaeger, 1870; Dusén, 1903a; Paris, 1903; Roth, 1910; Cardot and Brotherus, 1923; Brotherus, 1924a; Kühnemann, 1938).

A. rupestris A. Roth

Andreaea rothii

A. semi-squarrosa C. Muell. (Mueller, 1879a).

Distr. Southern South America (Mueller, 1879a; Paris, 1903; Roth, 1910; Brotherus, 1924a; Kühnemann, 1938).

A. squamata C. Muell.

Andreaea alpina

A. squarrosa Mitt. (Mitten, 1869).

Distr. Southern South America Wilson and Hooker, 1847 (as A. alpina Hedw. var. 1 fide Mitten, 1879); Donat, 1936a; Herzog, 1954; Seki, 1974).

A. subremotifolia Dix. (Dixon, 1920).

Distr. South Georgia (Dixon, 1920; Greene, 1968b).

A. subulata Harv. (Harvey in Hooker, 1839).

Syn. Andreaea subulata Harv. var. perichaetialis Hook. f. et Wils. (Hooker, J.D. and Wilson, 1844).

Andreaea subulata Harv. var. rigida Hook. f. et Wils. (Hooker, J.D. and Wilson, 1844).

Andreaea pseudo-subulata C. Muell. (Mueller, 1864).

Andreaea perichaetialis (Hook. f. et Wils.) Jaeger (Jaeger, 1870) hom. illeg.

Distr. Southern South America (Hooker, J.D. and Wilson, 1844; Wilson and Hooker, 1847; Mueller, 1848a, 1864, 1885; Mitten, 1869; Jaeger, 1870; Dusén, 1903a, b; Paris, 1903; Cardot, 1908a; Roth, 1910; Cardot and Brotherus, 1923; Roivainen and Bartram, 1937; Kühnemann, 1938; Seki, 1974).

Falkland Islands (Hooker, J.D. and Wilson, 1844; Wilson and Hooker, 1847; Mueller. 1848a; Jaeger, 1870; Dusén, 1903a; Paris, 1903; Cardot, 1908a; Roth, 1910; Cardot and Brotherus, 1923; Kühnemann, 1938). 
var. perichaetialis Hook. f. et Wils.

Andreaea subulata

var. rigida Hook. f. et Wils.

Andreaea subulata

A. vaginalis Herz. (Herzog in Donat, 1936a).

Distr. Southern South America (Donat, 1936a, b; Seki, 1974).

A. vermicularis Dus. (Dusén, 1903a) nom. nud.

Distr. Southern South America (Dusén, 1903a).

A. verruculosa Card.

Andreaea regularis

A. viridis C. Muell.

Andreaea regularis

A. willii C. Muell.

Andreaea regularis

A. wilsonii Hook. f. (Hooker in Hooker, J.D. and Wilson, 1844).

Syn. Acroschisma wilsonii (Hook. f.) Jaeg. (Jaeger, 1870).

Distr. Southern South America (Hooker, J.D. and Wilson, 1844; Wilson and Hooker, 1847;

Mueller, 1851a, 1885; Mitten, 1869; Jaeger, 1870; Dusén, 1903a, b; Paris, 1903;

Cardot, 1908a; Roth, 1910; Cardot and Brotherus, 1923; Brotherus, 1924a; Roivainen and Bartram, 1937; Kühnemann, 1938; Bartram, 1952; Herzog, 1954; Seki, 1974).

Anictangium nom. rejic.

A. ciliatum Hedw.

Hedwigia ciliata

A. coronatum Hook. et Wils.

Erpodium coronatum

A. humboldtii Hook.

A. imberbe (Sm.) Hook. et Tayl.

Rhacocarpus purpurascens

Hedwigidium integrifolium

A. orthotrichoides Gill. ex Grev. (Gillies ex Greville, 1830).

Distr. Southern South:America (Greville, 1830).

A. subsessile (Brid.) Grev. et Arnott

Pterygoneurum subsessile

\section{Anisothecium}

A. capituligerum (C. Muell.) Thér. (Thériot, 1934b [1935]).

Syn. Aongstroemia capituligera C. Muell. (Mueller, 1879a). Dicranella capituligera (C. Muell.) Kindb. (Kindberg, 1889).

Dichodontium capituligerum (C. Muell.) Par. (Paris, 1900a).

Distr. Southern South America (Mueller, 1879a; Paris, 1895, 1900a, 1903; Brotherus, 1901. 1924a; Kurtz, 1904; Hosseus, 1935b, 1937; Kühnemann, 1938).

A. elegans (Dub.) Thér. (Thériot, 1934b [1935]).

Syn. Dicranum elegans Dub. (Duby, 1870).

Dichodontium elegans (Dub.) Jaeg. (Jaeger in Jaeger and Sauerbeck, 1879b).

Aongstroemia elegans (Dub.) Broth. (Brotherus, 1901).

Distr. Southern South America (Duby, 1870; Jaeger and Sauerbeck, 1879b; Brotherus, 1901, 1924a; Paris, 1903; Herzog, 1923, 1938; Thériot, 1934b).

A. flexisetum Dus. (Dusén in Herzog, 1938) nom. nud.

Distr. Southern South America (Herzog, 1938).

A. hookeri (C. Muell.) Broth.

Dicranella hookeri

var. minus Card. et Thér. (Cardot and Thériot in Thériot, 1929c [1930]).

Distr. Southern South America (Thériot, 1929c).

A. jamesonii Mitt.

Anisothecium vaginatum

A. krausei (Lor.) Herz. (Herzog, 1939).

Syn. Dicranella krausei Lor. (Lorentz, 1866).

Dichodontium krausei (Lor.) Jaeg. (Jaeger, 1872). 
Distr. Southern South America (Lorentz, 1866; Paris, 1904b; Herzog, 1939, 1954; Seki, 1974).

A. Iorentzii (C. Muell.) Broth. (Brotherus, 1924a).

Syn. Aongstroemia lorentzii C. Muell. (Mueller, 1882).

Dicranella lorentzii (C. Muell.) Kindb. (Kindberg, 1889).

Dichodontium lorentzii (C. Muell.) Par. (Paris, 1894).

Distr. Southern South America (Mueller, 1882; Paris, 1894, 1904b; Brotherus, 1901, 1924a; Kühnemann, 1938).

A. paludella (Besch.) Broth. (Brotherus, 1924a).

Syn. Dichodontium paludella Besch. (Bescherelle, 1885a).

Aongstroemia paludella (Besch.) C. Muell. (Mueller, 1901a [1900]).

Dicranella paludella (Besch.) Dus. (Dusén, 1905a).

Distr. Southern South America (Bescherelle, 1885a, 1889; Mueller, 1901a; Paris, 1904b;

Dusén, 1905a; Cardot, 1908a; Cardot and Brotherus, 1923; Brotherus, 1924a;

Roivainen and Bartram, 1937; Kühnemann, 1938; Herzog, 1954; Seki, 1974).

A. perpusillum Dus.

Dicranella hookeri

A. persquarrosum (Dus.) Broth.

Dicranella cardotii

var. falklandicum (Card.) Wijk et Marg. (van der Wijk and Margadant, 1960b).

Syn. Dichodontium dicranelloides Card. var. falklandicum Card. (Cardot, 1905a).

Dichodontium persquarrosum (Dus.) Card. var. falklandicum (Card.) Card. (Cardot, 1908a).

Distr. Falkland Islands (Cardot, 1905a, 1908a).

A. pseudorufescens (Card. et Broth.) Broth. (Brotherus, 1924a).

Syn. Dicranella pseudorufescens Card. et Broth. (Cardot and Brotherus, 1923).

Distr. Southem South America (Cardot and Brotherus, 1923; Brotherus, 1924a; Kühnemann, 1938).

var. leptocladus (Card. et Broth.) Kühn. (Kühnemann, 1938).

Syn. Dicranella pseudorufescens Card. et Broth. var. leptoclada Card. et Broth. (Cardot and Brotherus, 1923).

Distr. Southern South America (Cardot and Brotherus, 1923; Kühnemann, 1938).

A. rufipes (C. Muell.) Dus. (Dusén, 1903a).

Syn. Aongstroemia rufipes C. Muell. (Mueller, 1882).

Dicranella rufipes (C. Muell.) Kindb. (Kindberg, 1889).

Dichodontium rufipes (C. Muell.) Par. (Paris, 1894).

Distr. Southem South America (Mueller, 1882; Paris, 1894, 1904b); Brotherus, 1901, 1924a; Dusén, 1903a; Kühnemann, 1938).

A. skottsbergii (Card. et Broth.) Broth. (Brotherus, 1924a).

Syn. Dicranella skottsbergii Card. et Broth. (Cardot and Brotherus, 1923).

Distr. Southem South America (Cardot and Brotherus, 1923; Brotherus, 1924a; Kühnemann, 1938; Herzog, 1954).

A. vaginatum (Hook.) Mitt. (Mitten, 1869).

Syn. Dicranum vaginatum Hook. (Hooker, 1816).

Aongstroemia vaginata (Hook.) C. Muell. (Mueller, 1851a).

Dichodontium vaginatum (Hook.) Jaeg. (Jaeger, 1872).

Dicranella vaginata (Hook.) Card. (Cardot, 1908a).

Dicranum jamesonii Tayl. (Taylor, 1847) hom. illeg.

Leptotrichum jamesonii Mitt. (Mitten, 1859).

Anisothecium jamesonii Mitt. (Mitten, 1869).

Dichodontium jamesonii (Mitt.) Jaeg. (Jaeger, 1872).

Aongstroemia jamesonii (Mitt.) C. Muell. (Mueller, 1901a [1900]).

Dicranella jamesonii (Mitt.) Broth. (Brotherus, 1901). 
Distr. Southem South America (Montagne, $1839 b$ (as Dicranum vaginatum Hook.?); Wilson and Hooker, 1847; Mitten, 1869; Jaeger, 1872; Brotherus, 1901, 1924a; Paris, 1904b; Dusén, 1905a; Thériot, 1934b; Hosseus, 1938c, $d$, 1939; Herzog, 1938, 1939, 1940. 1954, 1960 (as Anisodontium jamesonii Tayl.)).

\section{Anoectangium}

A. acaule (Web. et Mohr) Roehl.

Pterygoneurum subsessile

A. ciliatum (Hedw.) Roehl.

Hedwigia ciliata

A. euchloron (Schwaegr.) Mitt.

Gymnostomum aestivum

A. excelsum (C. Muell.) Kindb.

Molendoa sendtneriana

A. fernandezianum Card. (Cardot in Skottsberg, 1914) nom. nud.

Distr. Juan Fernandeż (Skottsberg, 1914).

A. humboldtii (Hook.) Brid.

Rhacocarpus purpurascens

var. australe Hook. f. et Wils.

A. integrifolium (P. Beauv.) Schwaegr.

Rhacocarpus purpurascens

A. laxifolium Par.

Hedwigidium integrifolium

A. lechlerianum Mitt.

Gymnostomum tenerrimum

Molendoa sendtneriana

var. limbatulum Bartr. (Bartram, 1936).

Distr. Southem South America (Bartram, 1936; Hosseus, 1938c, 1939).

A. lineare (C. Muell.) Kindb. (Kindberg, 1889).

Syn. Zygodon linearis C. Muell. (Mueller, 1879a). Anoectangium mandonianum (C. Muell.) Par. (Paris, 1894) nom. inval. Zygodon mandonianus Schimp. ex C. Muell. (Schimper ex Mueller, 1897b).

Distr. Southern South America (Mueller, 1879a; Paris, 1894, 1903; Brotherus, 1924a; Hosseus, 1938d; Kühnemann, 1938).

A. mandonianum (C. Muell.) Par.

Anoectangium lineare

A. patagonicum Card. et Broth. (Cardot and Brotherus, 1923).

Distr. Southem South America (Cardot and Brotherus, 1923).

A. sendtnerianum B.S.G.

Molendoa sendtneriana

A. subsessile (Brid.) Spreng.

Pterygoneurum subsessile

A. tenerrimum C. Muell.

Gymnostomum tenerrimum

\section{Anomobryum}

A. brachymeniopsis (C. Muell.) Broth. (Brotherus, 1903).

Syn. Bryum brachymeniopsis C. Muell. (Mueller, 1879a).

Distr. Southern South America (Mueller, 1879a; Paris, 1903; Brotherus, 1903, 1924a; Kühnemann, 1938).

A. conicum (Hornsch.) Broth. (Brotherus, 1903).

Syn. Bryum conicum Hornsch. (Hornschuch, 1840).

Distr. Southern South America (Felippone, 1909; Herter, 1933a).

A. cygnicollum (C. Muell.) Jaeg. (Jaeger, 1875).

Syn. Bryum cygnicollum C. Muell. (Mueller, 1858).

Mielichhoferia cygnicolla Hamp. (Hampe in Mueller, 1858) nom. nud.

Climacodontium cygnicollum (C. Muell.) Hamp. (Hampe in Jaeger and Sauerbeck, 1879b) comb. inval.

Bryum climacodontium Par. (Paris, 1894) nom. illeg.

Distr. Southern South America (Mueller, 1858; Paris, 1894, 1904a). 
A. felipponei Thér. (Thériot in Felippone, 1929 [1930]).

Distr. Southern South America (Felippone, 1929; Herter, 1933a).

A. filiforme (Dicks.) Husn. (Husnot, 1888) hom. illeg.

Syn. Bryum filiforme Dicks. (Dickson, 1801).

Pohlia filiformis (Dicks.) Andrews (Andrews, 1935).

Bryum julaceum Schrad. ex Gaertn., Meyer et Scherb. (Schrader in Gärtner and others, 1802).

Distr. Southern South America (Montagne, 1850; Mitten, 1869; Gibert, 1873; Paris, 1903; Campo, 1921; Cardot and Brotherus, 1923; Brotherus, 1924c; Herter, 1928, 1933a, b, 1943; Kühnemann, 1938; Herzog, 1938, 1939, 1954; Espinsoa B., 1941; Seki, 1974).

A. laxirete Card. et Broth. (Cardot et Brotherus, 1923).

Distr. Southern South America (Cardot and Brotherus, 1923; Kühnemann, 1938).

A. semireticulatum (C. Muell.) Broth. (Brotherus, 1903).

Syn. Bryum semi-reticulatum C. Muell. (Mueller, 1879a).

Distr. Southern South America (Mueller, 1879a, 1882; Paris, 1903; Brotherus, 1903, 1924a; Hosseus, 1938c, d, 1939; Kühnemann, 1938).

A. terminale (C. Muell.) Broth. (Brotherus, 1903).

Syn. Bryum terminale C. Muell. (Mueller, 1879a).

Distr. Southem South America (Mueller, 1879a; Paris, 1903; Brotherus, 1903, 1924a; Hosseus, 1938c, d, 1939; Kühnemann, 1938).

\section{Anomodon}

A. curtipendulus (Hedw.) Hook. et Tayl.

Antitrichia curtipendula

A. pellicola C. Muell.

Leskea pellicula

A. pellicula C. Muell.

Leskea pellicula

A. toccoae Sull. et Lesq.

Herpetineuron toccoae

\section{Antitrichia}

A. curtipendula (Hedw.) Brid. (Bridel, 1819).

Syn. Neckera curtipendula Hedw. (Hedwig, 1801).

Hypnum curtipendulum (Hedw.) L. ex With. (Linnaeus ex Withering, 1801).

Anomodon curtipendulus (Hedw.) Hook. et Tayl. (Hooker and Taylor, 1818).

Cyrtopus curtipendulus Hedw. Spruc. (Spruce, 1849).

Haptymenium curtipendulum (Hedw.) Fuernr. (Fürnrohr, 1827).

Leucodon curtipendulus (Hedw.).T. Jens. (Jensen, 1856).

Distr. Southem South America (Bridel, 1801b, 1817, 1827b; Paris, 1903).

\section{Aongstroemia}

A. argentinica C. Muell.

Dicranella argentinica

A. aulacocarpa (Mont.) C. Muell.

Dicranella aulacocarpa

A. capituligera C. Muell.

Anisothecium capituligerum

A. elegans (Dub.) Broth.

Anisothecium elegans

A. euchlora (Mont.) C. Muell.

Microdus euchlorus

A. gayana (Mont.) C. Muell. (Mueller, 1848a).

Syn. Dicranum gayanum Mont. (Montagne, 1845a).

Distr. Southern South America (Montagne, 1845a, 1850, 1856; Mueller, 1848a; Lorentz, 1866; Mitten, 1869; Jaeger, 1872; Brotherus, 1901, 1924a; Paris, 1903; Dusén, 1905a;

Campo, 1921; Costes, 1921; Thériot, 1921a; Herzog, 1938, 1939, 1954; Seki, 1974).

A. hilariana (Mont.) C. Muell.

Dicranella hilariana 
A. hookeri C. Muell.

Dicranella hookeri

A. jamesonii (Mitt.) C. Muell.

Anisothecium vaginatum

A. leiocarpa C. Muell. (Mueller, 1901a [1900]) nom. nud.

Syn. Cynodontium leiocarpum Lor. (Lorentz in Mueller, 1901a [1900]) nom. nud.

Distr. Southern South America (Mueller, 1901a).

A. lorentzii C. Muell.

Anisothecium lorentzii

A. paludella (Besch.) C. Muell.

A. patagonica C. Muell.

A. persquarrosa Dus.

Anisothecium paludella

A. rufipes C. Muell.

A. subclathrata (Lor.) C. Muell.

A. tenuirostris (Schwaegr.) C. Muell.

A. vaginata (Hook.) C. Muell.

Dicranella patagonica

Dicranella cardotii

Anisothecium rufipes

Dicranella hookeri

Dicranella hilariana

Anisothecium vaginatum

\section{Apalodium}

A. australe (Hook. f. et Wils.) Mitt.

A. confine (Hamp.) Mitt.

A. lineare (Schwaegr.) Mitt.

A. pellucens (Hook.) Mitt.

A. tenue Mitt.

Orthodontium lineare

Orthodontium pellucens

Orthodontium lineare

Orthodontium pellucens

Pohlia humilis

\section{Aptychella}

A. chilensis Herz. (Herzog, 1954).

Distr. Southern South America (Herzog, 1954; Seki, 1974; Tixier, 1977).

\section{Aptychus}

A. ampullatus C. Muell. ex Par.

A. ampullulatus C. Muell.

A. aureo-viridis C. Muell.

A. catilliformis C. Muell.

A. cochleatulus C. Muell.

A. condensatulus $C$. Muell.

A. diaphanodictyus C. Muell.

A. grandicellulosus C. Muell.

A. laxo-alaris C. Muell.

A. macrocytus C. Muell.

A. micrangius $C$. Muell.

A. micropyxis C. Muell.

A. nano-cephalus C. Muell. var. subglauculus C. Muell.

A. serifolius C. Muell.

A. temperatus C. Muell.

A. tenerifolius C. Muell.

Sematophyllum ampullulatum

Sematophyllum ampullulatum

Sematophyllum aureo-viride

Sematophyllum bracteatum

Rhaphidostegium cochleatulum

Rhapidostegium condensatulum

Rhaphidostegium diaphanodictyon

Sematophyllum grandicellulosum

Entodon laxo-alaris

Sematophyllum macrocytus

Sematophyllum micrangium

Trichosteleum micropyxidium

Sematophyllum nanocephalum

Sematophyllum nanocephalum var. subglauculum

Sematophyllum serifolium Sematophyllum temperatum Sematophyllum tenerifolium

\section{Archidium}

A. amplexicaule C. Muell. (Mueller, 1882). 
Distr. Southern South America (Mueller, 1882; Paris, 1903; Roth, 1911; Brotherus, 1924a; Herter, 1933a; Kühnemann, 1938; Hosseus, 1939).

var. brevi-solonaceum C. Muell. (Mueller, 1882).

Distr. Southern South America (Mueller, 1882; Paris, 1903; Roth, 1911).

A. arechavaletae C. Muell. (Mueller, 1888).

Distr. Southern South America (Mueller, 1888; Paris, 1903; Roth, 1911; Felippone, 1912; Brotherus, 1924a; Herter, 1933a).

A. ephemeroides C. Muell. (Mueller, 1882).

Distr. Southern South America (Mueller, 1882; Paris, 1903; Roth, 1911; Brotherus, 1924a; Herter, 1933a; Kühnemann, 1938; Hosseus, 1939).

A. gibertii Mitt. (Mitten, 1886).

Distr. Southern South America (Mitten, 1886; Paris, 1903; Roth, 1910; Brotherus, 1924a; Herter, 1933a).

A. julaceum C. Muell. (Mueller, 1882).

Distr. Southern South America (Mueller, 1882; Paris, 1903; Roth, 1911; Thériot, 1918, 1924 : Campo, 1921; Brotherus, 1924a; Herter, 1933a; Kühnemann, 1938; Hosseus, 1939).

A. Iorentzii C. Muell. (Mueller, 1882).

Distr. Southern South America (Mueller, 1882; Paris, 1903; Roth, 1911; Brotherus, 1924a; Herter, 1933a; Kühnemann, 1938; Hosseus, 1939; Herzog, 1952a).

Aschistodon nom. rejic.

A. conicus Mont.

Ditrichum conicum

Asteriscium hom. illeg.

A. australasiae (Hook. et Grev.) Hilp.

A. curvipes (C. Muell.) Hilp.

A. deciduum Hilp.

A. flavisetum Herz.

A. geniculatum (Mont.) Herz.

A. graminicolor (C. Muell.) Hilp.

A. poeppigianum (C. Muell.) Hilp.

A. riparium (C. Muell.) Hilp.

A. subtophaceum (Williams) Hilp.

A. umbrosum (C. Muell.) Herz.

Trichostomopsis australasiae

Trichostomopsis curvipes

Barbula uruguensis

Trichostomopsis umbrosa

Barbula geniculata

Barbula graminicolor

Trichostomopsis australasiae

Barbula riparia

Trichostomopsis australasiae

Trichostomopsis umbrosa

\section{Astomiopsis}

A. amblyocalyx C. Muell. (Mueller, 1882).

Syn. Astomiopsis amblyocarpa C. Muell. ex Par. (Mueller ex Paris, 1894) nom. inval. Pleuridiopsis amblyocalyx (C. Muell.) Par. (Paris, 1897) comb. inval.

Distr. Southern South America (Mueller, 1882; Paris, 1897, 1905b; Brotherus, 1924a; Kühnemann, 1938).

A. amblyocarpa C. Muell. ex Par.

Astomiopsis amblyocalyx

A. pacifica Buck et Landrum (Buck and Landrum, 1977).

Distr. Juan Fernandez (Buck and Landrum, 1977).

A. subulata C. Muell. (Mueller, 1882).

Syn. Pleuridiopsis subulata (C. Muell.) Par. (Paris, 1897) comb. inval. 
Distr. Southern South America (Mueller, 1882; Paris, 1897, 1905b; Brotherus, 1924a; Kühnemann, 1938).

\section{Astomum}

A. chilense Williams

Tetrapterum recurvirostre

A. fruchartii (C. Muell.) Broth. (Brotherus, 1900).

Syn. Phascum fruchartii C. Muell. (Mueller, 1888).

Systegium fruchartii (C. Muell.) Kindb. (Kindberg, 1889).

Distr. Southern South America (Mueller, 1888; Paris, 1905b; Roth, 1911; Felippone, 1912; Brotherus, 1924a; Herter, 1933a, 1943).

A. Iorentzii (C. Muell.) Broth. (Brotherus, 1901).

Syn. Acaulon lorentzii C. Muell. (Mueller, 1882).

Sphaerangium lorentzii (C. Muell.) Par. (Paris, 1897).

Distr. Southern South America (Mueller. 1882; Paris, 1897, 1905b; Brotherus. 1901, 1924a;

Roth, 1911, 1914; Herter, 1933a; Kühnemann, 1938; Hosseus, 1939).

A. nervosum (Hook.) C. Muell.

A. recurvirostre (C. Muell.) Roth

A. robinsonii (Mont.) C. Muell.

Pleuridium nervosum

A. subnervosum C. Muell.

Tetrapterum recurvirostre

Pleuridium robinsonii

Pleuridium subnervosum

\section{Atractylocarpus}

A. patagonicus Herz. et Thér. (Herzog and Thériot in Herzog and others, 1933).

Distr. Southern South America (Herzog and others, 1933).

\section{Atrichopsis}

A. compressa (Hook. f. et Wils.) G.L. Smith (Smith, 1969a).

Syn. Polytrichum compressum Hook. f. et Wils. (Hooker, J.D. and Wilson, 1844).

Catharinea compressa (Hook. f. et Wils.) C. Muell. (Mueller, 1848a).

Psilopilum compressum (Hook. f. et Wils.) Mitt. (Mitten, 1869).

Oligotrichum compressum (Hook. f. et Wils.) Kindb. (Kindberg, 1888).

Atrichopsis magellanica Card. (Cardot, 1912a) fide Smith, 1969a.

Distr. Southern South America (Hooker, J.D. and Wilson, 1844; Wilson and Hooker, 1847;

Mueller, 1848a, 1885; Mitten, 1869; Jaeger, 1875; Paris, 1905b; Cardot, 1908a,

1912a; Cardot and Brotherus, 1923; Brotherus, 1925; Kühnemann, 1938; Herzog,
1939; Smith, 1969a).

Falkland Islands (Cardot, 1912a; Cardot and Brotherus, 1923; Brotherus, 1925;
$\quad$ Kühnemann, 1938; Smith, 1969a).

A. magellanica Card.

Atrichopsis compressa

\section{Atrichum}

A. planifolium (C. Muell.) Jaeg.

Atrichum polycarpum

A. polycarpum (C. Muell.) Mitt. (Mitten, 1869).

Syn. Catharinea polycarpa C. Muell. (Mueller, 1851a).

Catharinea planifolia C. Muell. (Mueller in Lorentz, 1868).

Atrichum planifolium (C. Muell.) Jaeg. (Jaeger, 1875).

Distr. Southern South America (Lorentz, 1868; Jaeger, 1875; Frye and Duckering, 1948).

A. rigidum Lor.

Oligotrichum rigidum

\section{Aulacomnium}

A. chilense C. Muell.

Rhizogonium mnioides 
A. gaudichaudii (Schwaegr.) Mitt.

Leptotheca gaudichaudii

A. palustre (Hedw.) Schwaegr. (Schwaegrichen, 1827b).

Syn. Mnium palustre Hedw. (Hedwig, 1801).

Bryum palustre (Hedw.) Gaertn., Meyer et Scherb. (Gärtner and others, 1802) hom. illeg.

Hypnum palustre (Hedw.) Web. et Mohr (Weber and Mohr, 1803) hom. illeg.

Bartramia palustris (Hedw.) P. Beauv. (Palisot de Beauvois, 1805b).

Orthopyxis palustris (Hedw.) P. Beauv. (Palisot de Beauvois, 1805a).

Gymnocybe palustris (Hedw.) Fries (Fries, 1825).

Limnobryum palustre (Hedw.) Rabenh. (Rabenhorst, 1863).

Sphaerocephalus palustris (Hedw.) Lindb. (Lindberg, 1879).

Distr. Southem South America (Dusén, 1903a; Cardot, 1908a; Cardot and Brotherus, 1923; Kühnemann, 1938; Herzog, 1957).

A. pentastichum Mont.

Zygodon pentastichus

\section{Aulacopilum}

A. glaucum Wils. Wilson, 1848).

Syn. Aulacopilum intermedium Broth. (Brotherus, 1918) fide Crum, 1972.

Distr. Southem South America (Brotherus, 1918, 1925; Kühnemann, 1938; Crum, 1972).

A. intermedium Broth.

Aulacopilum glaucum

\section{Barbula}

B. aculeonervis C. Muell.

Tortula aculeonervis

B. aestiva (Hedw.) Schultz

Tortula muralis var. aestiva

B. alpicola C. Muell. (Mueller, 1901a [1900]).

Distr. Southern South America (Mueller, 1901a).

B. amblyophylla (Hook.) Jaeg.

B. amphidiifolia C. Muell.

Didymodon amblyophyllus

B. anacamptophylla C. Muell.

Tortula amphidiifolia

Tortula lepto-syntrichia

B. anastomosans C. Muell. (Mueller, 1879a).

Syn. Barbula anostomosans Par. (Paris, 1894) err.

Distr. Southem South America (Mueller, 1879a; Paris, 1894, 1904a; Brotherus, 1918, 1924a; Kühnemann, 1938).

B. anderssonii (Aongstr.) Jaeg.

Tortula anderssonii

$B$. annullus $\mathrm{C}$. Muell.

Tortula muralis

B. anostomosans Par.

Barbula anastomosans

B. antarctica Hamp.

Tortula princeps

B. apoclada Par.

Barbula perrevoluta

B. appressa (Mitt.) Jaeg.

Tortula appressa

B. arechavaleta C. Muell. (Mueller, 1901a [1900]) nom. nud.

Distr. Southem South America (Mueller, 1901a).

B. arenae Besch.

Tortula arenae

B. arenicola Dus. (Dusén, 1906).

Distr. Southern South America (Dusén, 1906; Brotherus, 1924a; Kühnemann, 1938).

B. argentinica Par.

Didymodon argentinicus

B. atrovirens (Sm.) Schimp.

Desmatodon convolutus

B. australasiae (Hook. et Grev.) Brid.

Trichostomopsis australasiae

B. australis Par. 
B. austrogracilis Dus. (Dusén, 1906).

Distr. Southern South America (Dusén, 1906; Brotherus, 1924a).

B. berteroana C. Muell.

Tortula muralis

B. berteroi C. Muell. ex Kindb.

Tortula muralis

B. brachychaete Dus.

B. breviseta (Mont.) C. Muell.

Tortula brachychaete

B. brunnea (C. Muell.) Broth.

Tortula breviseta

B. byrdii Bartr. (Bartram, 1938).

Distr. Antarctic, continental region (Bartram, 1938, 1957; Steere, 1961a).

B. caespitosa Schwaegr.

Tortella humilis

B. calycina Schwaegr.

Tortella calycina

B. calyculosa (Mitt.) Jaeg. (Jaeger, 1873).

Syn. Tortula calyculosa Mitt. (Mitten, 1869).

Distr. Southem South America (Herzog, 1954).

B. cardotii Dus. (Dusén, 1905b).

Distr. Southem South America (Dusén, 1905b, 1906; Brotherus, 1924a; Kühnemann, 1938).

B. catillum C. Muell.

B. cavifolia Schimp.

Aloina catillum

B. characodonta C. Muell.

B. chilensipilifera C. Muell. (Mueller, 1901a [1900]) nom. nud.

Pterygoneurum ovatum

Tortula characodonta

Distr. Southem South: America (Mueller, 1901a).

B. chrysopila C. Muell.

B. conotricha C. Muell. var. fagicola C. Muell.

B. contorta Hamp. ex C. Muell.

Tortula anderssonii var. fagicola

B. costessii Thér. (Thériot, 1921a).

Tortula contorta

Distr. Southem South America (Thériot, 1921a; Costes, 1921; Brotherus, 1924a).

B. crinita Schultz

B. crispatula C. Muell. ex Par.

B. cucullatifolia C. Muell.

B. curvipes C. Muell.

B. curvirostris Lindb.

B. decidua C. Muell.

Tortula pilifera

Calyptopogon mnioides

Aloinella cucullatifolia

Trichostomopsis curvipes

Hymenostylium recurvirostre

B. declivium C. Muell. (Mueller, 1882).

Barbula uruguensis

Distr. Southem South America (Mueller, 1882; Paris, 1904a; Brotherus, 1924a; Kühnemann,
1938).

B. densifolia Hook. f. et Wils.

B. depressa Sull.

Tortula densifolia

Tortula pilifera

var. denticulata (Dus.) Thér.

var. gracilis (Dus.) Thér.

var. oliviensis (Card.) Thér.

B. dioica (R. Brown ter.) Par.

Tortula pilifera var. denticulata

Tortula pilifera var. gracilis

Tortula pilifera var. oliviensis

Tortula robusta 
B. filaris C. Muell.

Tortula filaris

B. flagellaris Schimp.

Tortula flagellaris

var. denticulata Dus.

Tortula pilifera var. denticulata

var. gracilis Dus.

Tortula pilifera var. gracilis

B. flavido-pilosa Dus.

Tortula flavido-pilosa

B. flaviseta (Herz.) Wijk et Marg.

Trichostomopsis umbrosa

B. fontana C. Muell.

Tortula fontana

B. fuegiana (Mitt.) Jaeg.

Tortula fuegiana

B. fusca C. Muell. (Mueller, 1849).

Syn. Tortula fusca (C. Muell.) Mont. (Montagne, 1850).

Distr. Southem South America (Mueller, 1849; Montagne, 1850; Mitten, 1869; Jaeger, 1873; Paris. 1904a; Dusén, 1906; Cardot, 1908a; Thériot, 1918, 1921a, 1928; Campo, 1921; Costes, 1921; Cardot and Brotherus, 1923; Brotherus, 1924a; Kühnemann, 1938; Herzog, 1938; Bartram, 1952; Seki, 1974).

B. fuscinervia (Mitt.) Jaeg. (Jaeger, 1873).

Syn. Tortula fuscinervia Mitt. (Mitten, 1869).

Distr. Southem South America (Mitten, 1869; Jaeger, 1873; Paris, 1904a; Brotherus, 1924a, c, Skottsberg, 1950; Bartram, 1952; Herzog, 1954, 1960; Seki, 1974).

B. fusco-viridis Broth. ex Thér. (Brotherus ex Thériot, 1921a).

Distr. Southem South America (Paris, 1906; Campo, 1921; Thériot, 1921a).

B. fuscula C. Muell.

Erythrophyllopsis fuscula

B. galeata C. Muell.

Aloinella galeata

B. geniculata (Mont.) C. Muell. (Mueller, 1849).

Syn. Tortula geniculata Mont. (Montagne, 1845a). Asteriscium geniculatum (Mont.) Herz. (Herzog, 1938).

Distr. Southem South America (Montagne, 1845a, 1850, 1856: Mueller, 1849; Mitten, 1869; Jaeger, 1873; Paris, 1904a; Brothenus, 1924a; Herzog, 1938. 1954).

B. glacialis Kunz. ex C. Muell.

Tortula glacialis

B. glaucula C. Muell. (Mueller, 1882).

Distr. Southem South America (Mueller, 1882).

B. graminicolor C. Muell. (Mueller, 1849).

Syn. Tortula graminicolor (C. Muell.) Mont. (Montagne, 1850). Asteriscium graminicolor (C. Muell.) Hilp. (Hilpert, 1933).

Distr. Southem South America (Mueller, 1849; Montagne, 1850: Mitten, 1869; Jaeger, 1873; Paris, 1904a; Thériot, 1921b; Brotherus, 1924a).

ssp. subgraminicolor Thér. (Thériot, 1917b).

Syn. Barbula subgraminicolor Thér. (Thériot, 1917b) nom. inval.

Distr. Southem South America (Thériot, 1917b: Costes, 1921).

B. grimmiacea C. Muell. (Mueller, 1882).

Distr. Southem South America (Mueller, 1882; Paris, 1904a; Brotherus, 1924a; Hilpert, 1933; Kühnemann, 1938).

B. hosseusii Thér. (Thériot, 1935b).

Distr. Southem South America (Bauer, 1934; Thériot, 1935b; Hosseus, 1936, 1937, 1938a, b, c, d, 1939; Kühnemann, 1938). 
B. humilis Hedw.

Tortella humilis

B. hyperborea Mont. ex Par.

Tortula mucronifolia var. arctica

B. imperfecta (C. Muell.) Broth. (Brotherus, 1902).

Syn. Trichostomum imperfectum C. Muell. (Mueller, 1879a).

Anacalypta imperfecta (C. Muell.) Kindb. (Kindberg, 1889).

Pottia imperfecta (C. Muell.) Par. (Paris, 1898).

Distr. Southern South America (Mueller, 1879a; Paris, 1898; Brotherus, 1902, 1924a; Warnstorf, 1916; Kühnemann, 1938).

B. inundata (Mitt.) Jaeg.

Didymodon inundatus

B. juniperoidea C. Muell. (Mueller, 1882). Distr. Southern South America (Mueller, 1882; Paris, 1904a; Brotherus, 1924a; Kühnemann,
1938).

B. kunzeana C. Muell.

B. kunzei C. Muell. ex Kindb.

Tortula kunzeana

B. laevigata* (Mitt.) Jaeg. (Jaeger, 1873).

Syn. Tortula laevigata Mitt. (Mitten, 1869).

Distr. Southern South America (Dusén, 1907; Cardot, 1908a: Seki, 1974).

B. laevipila (Brid.) Garov.

Tortula laevipila

B. lamprocalyx C. Muell. (Mueller, 1849).

Syn. Tortula lamprocalyx (C. Muell.) Mont. (Montagne, 1850).

Distr. Southern South America (Mueller, 1849; Montagne, 1850; Jaeger, 1873; Paris, 1904a;
Brotherus, 1924a).

B. lechleri C. Muell.

Tortula lechleri

B. lepto-syntrichia C. Muell.

B. leucocalyx (Mont.) C. Muell.

Tortula lepto-syntrichia

B. Ionchodonta C. Muell. (Mueller, 1879a).

Distr. Southern South America (Mueller, 1879a; Kurtz, 1904; Paris, 1904a; Brotherus, 1924a; Hosseus, 1935a, 1937; Kühnemann, 1938).

B. lorentzii C. Muell.

Tortula lorentzii

B. Iurida Hornsch. (Hornschuch, 1840).

Distr. Southern South America (Hosseus, 1938C).

B. magellanica (Mont.) C. Muell.

B. magellanica C. Muell.

B. marginato-serrata Dus.

Tortula magellanica

Tortula fuegiana

B. mendozensis (Mitt.) Jaeg. (Jaeger, 1873).

Syn. Tortula mendozensis Mitt. (Mitten, 1869).

Distr. Southern South America (Mitten, 1869; Jaeger, 1873; Paris, 1904a; Brotherus, 1924a).

B. micro-runcinata Dus.

B. minutirosula C. Muell.

Leptodontium longicaule var. microruncinatum

B. mniadelphus C. Muell.

Tortula minutirosula

B. mnioides Schwaegr.

Tortula mniadelphus

Calyptopogon mnioides

* = Didymodon laevigatus (Mitt.) Zander fide Zander (1978). 
var. prostrata (Mont.) C. Muell.

Tortula prostrata

B. muelleri B.S.G.

Tortula princeps

B. muelleri Kindb.

Barbula perrevoluta

B. muralis (Hedw.) Crom.

Tortula muralis

ssp. aestiva (Hedw.) Kindb.

Tortula muralis var. aestiva

var. aestiva (Hedw.) Roehl.

Tortula muralis var. aestiva

var. australis Hamp.

Tortula muralis

var. rupestris Schultz

Tortula muralis

B. muricola C. Muell.

Tortula muralis

B. nanophylla Dus. (Dusén, 1903a) nom. nud.

Distr. Southern South America (Dusén, 1903a).

B. oliviensis Card.

B. ovata (Hedw.) Schimp.

Tortula pilifera var. oliviensis

B. pachyneura Dus.

B. patagonica (Mitt.) Jaeg.

B. patagonica C. Muell. Pterygoneurum ovatum

Pseudocrossidium leucocalyx Didymodon patagonicus

Tortula fuegiana

B. perangusta Dus. (Dusén, 1903a) nom. nud.

Distr. Southern South America (Dusén, 1903a).

B. percarnosa C. Muell.

Tortula percarnosa

B. perfirma Dus. (Dusén in Paris, 1906) nom. nud.

Distr. Southern South America (Paris, 1906; Roivainen, 1934).

B. perflaccida Broth.

Tortula flagellaris

B. pernana C. Muell. (Mueller, 1879a).

Distr. Southern South America (Mueller, 1879a; Paris, 1904a; Brotherus, 1924a; Kühnemann, 1938).

B. perpusilla C. Muell.

Tortula perpusilla

B. perrevoluta C. Muell. (Mueller, 1882).

Syn. Barbula subrevoluta C. Muell. (Mueller, 1879a) hom. illeg.

Barbula muelleri Kindb. (Kindberg, 1889) hom. illeg.

Barbula apoclada Par. (Paris, 1900a) nom. illeg.

Distr. Southern South America (Mueller, 1879a, 1882; Paris, 1900a, 1904a; Kurtz, 1904; Brotherus, 1924a; Hosseus, 1935a, b, 1937; Kühnemann, 1938).

var. acutifolia (C. Muell.) Par. (Paris, 1894).

Syn. Barbula subrevoluta C. Muell. var. acutifolia C. Muell. (Mueller, 1879a).

Distr. Southern South America (Mueller, 1879a; Paris, 1894, 1904a; Hosseus, 1935b, 1937; Kühnemann, 1938).

var. linearifolia (C. Muell.) Par. (Paris, 1894).

Syn. Barbula subrevoluta C. Muell. var. linearifolia C. Muell. (Mueller, 1879a).

Distr. Southern South America (Mueller, 1879a; Paris, 1894, 1904a; Hosseus, 1935a, 1937; Kühnemann, 1938).

B. perrubiginosa Dus.

Tortula anderssonii

B. perrufula C. Muell.

Tortella perrufula

B. pertorquescens Broth. (Brotherus, 1918).

Distr. Southern South America (Brotherus, 1918, 1924a; Kühnemann, 1938).

B. pilifera (Hook.) Brid.

Tortula pilifera 
B. plebeja C. Muell. (Mueller, 1882).

Distr. Southern South America (Mueller, 1882; Paris, 1904a; Brotherus, 1924a; Kühnemann, 1938).

B. podocarpi C. Muell.

Tortula podocarpi

B. poeppigiana C. Muell. Trichostomopsis australisiae

B. princeps (De Not.) C. Muell. Tortula princeps

B. prostrata (Mont.) Jaeg. Tortula prostrata

B. pseudo-caespitosa C. Muell. Tortella pseudo-caespitosa var. brachybasis C. Muell. var. pungens $\mathrm{C}$. Muell. Tortella pseudo-caespitosa var. brachybasis Tortella pseudo-caespitosa var. pungens

B. punctulata (Brid.) Jaeg.

Tortula robusta

B. purpurascens Dus. (Dusén, 1906).

Distr. Southem South America (Dusén, 1906; Cardot, 1908a; Brotherus, 1924a; Kühnemann, 1938; Herzog, 1938, 1960; Seki, 1974).

B. pusilla Jaeg.

Tortula brachypelma

B. pycnophylla Card. (Cardot, 1906a).

Distr. Southern South America (Dusén, 1906; Cardot, 1908a; Brotherus, 1924a; Bartram, 1952; Seki, 1974).

South Georgia (Cardot, 1906a, 1908a; Brotherus, 1924a; Steere, 1961b).

B. recurvipatula C. Muell.

Aloina recurvipatula

B. replicata Tayl. (Taylor, 1846).

Syn. Tortula replicata (Tayl.) Wils. (Wilson, 1846).

Distr. Southern South America (Bartram, 1942, 1943, 1952).

B. rigidula (Hedw.) Mitt.

Didymodon rigidulus

B. riparia C. Muell. (Mueller, 1882).

Syn. Asteriscium riparium (C. Muell.) Hilp. (Hilpert, 1933).

Distr. Southern South America (Mueller, 1882; Paris, 1904a; Brotherus, 1924a; Hosseus, 1938a, d; Kühnemann, 1938).

B. ripicola Card.

B. robusta (Hook. et Grev.) Brid.

Hymenostylium recurvirostre

B. rubiginosa C. Muell. Tortula robusta

B. rubra (Mitt.) Jaeg. Didymodon argentinicus

B. rubricaulis Kindb. (Kindberg, 1891) nom. nud. Tortula robusta

Distr. Southern South America (Mueller, 1901a).

B. rubriseta Bartr. (Bartram, 1936).

Distr. Southern South America (Bartram, 1936; Hosseus, 1938c, d, 1939).

B. runcinata C. Muell.

Tortula robusta var. runcinata

B. ruralis Hedw.

Tortula ruralis

B. santessonii Bartr. (Bartram, 1952).

Distr. Southern South America (Bartram, 1952; Seki, 1974).

B. santiagensis Broth. (Brotherus in Dusén, 1907).

Distr. Southern South America (Dusén, 1907; Brotherus, 1924a; Thériot, 1934b; Herzog, 1954). 
var. piligera Broth. (Brotherus in Dusén, 1907).

Distr. Southern South America (Dusén, 1907).

B. scabrella Dus.

Tortula scabrella

B. scabrinervis C. Muell.

Tortula scabrinervis

B. schimperi (Mont.) Mitt.

Didymodon schimperi

B. schnyderiC. Muell.

Tortula schnyderi

B. sedifolia C. Muell.

Aloina sedifolia

B. serripungens Lor. et C. Muell.

Tortula serripungens

var. exesa C. Muell.

Tortula serripungens var. exesa

B. serrulata (Hook. et Grev.) Brid.

Tortula robusta

B. speciosa Hook. f. et Wils.

Tortula robusta

B. spiralis Schimp. (Schimper in Mueller, 1849).

Distr. Southern South America (Hosseus, 1938a, d).

B. streptostega C. Muell. (Mueller, 1901a [1900]) nom. nud.

Distr. Southern South America (Mueller, 1901a).

B. subdecidua Kindb.

Barbula uruguensis

B. subgraminicolor Thér.

Barbula graminicolor ssp. subgraminicolor

B. subgrimmiacea Thér. (Thériot in Felippone, 1929 [1930]).

Distr. Southem South America (Felippone, 1929; Herter, 1933a).

B. subrevoluta C. Muell.

Barbula perrevoluta

var. acutifolia C. Muell.

Barbula perrevoluta var. acutifolia

var. linearifolia C. Muell.

B. subrubiginosa Kindb.

Barbula perrevoluta var. linearifolia

Didymodon argentinicus

B. subruncinata C. Muell. (Mueller, 1901a [1900]).

Distr. Southern South America (Mueller, 1901a).

B. tenella (Broth.) Par.

Tortula tenella

B. tessellata C. Muell. (Mueller, 1901a [1900]) nom. nud.

Distr. Am. 6 in Index muscorum, 1, 156, but not found in Mueller (1901a).

B. tortelloides C. Muell. (Mueller, 1879a).

Distr. Southern South America (Mueller, 1879a; Paris, 1904a; Brotherus, 1924a; Kühnemann, 1938).

B. tortuosa (Hedw.) Web. et Mohr

Tortella tortuosa

B. umbrosa C. Muell.

Trichostomopsis umbrosa

B. uncinicoma C. Muell. (Mueller, 1879a).

Distr. Southern South America (Mueller, 1879a; Kurtz, 1904; Paris, 1904a; Brotherus, 1924a; Williams, 1930; Hosseus, 1935a, 1937; Kühnemann, 1938).

B. unguiculata Hedw. (Hedwig, 1801).

Syn. Bryum unguiculatum (Hedw.) With. (Withering, 1801).

Tortula unguiculata (Hedw.) A. Roth exP. Beauv. (A. Roth ex Palisot de Beauvois, 1805a) hom. illeg.

Streblotrichum unguiculatum (Hedw.) Loesk. (Loeske, 1910).

Distr. Southern South America (Felippone, 1917; Herter, 1933a).

var. patagonica C. Muell. (Mueller, 1843a).

Syn. Syntrichia ericetorum (With.) Brid. (Bridel, 1826). 
Distr. Southem South America (Bridel, 1826; Mueller, 1843a, 1849; Paris, 1904a).

B. unguiculatula C. Muell. (Mueller, 1879a). Distr. Southem South America (Mueller, 1879a; Paris, 1904a; Brotherus, 1924a; Hosseus,
1938c, $d$; Kühnemann, 1938).

B. uruguayensis Broth. (Brotherus, 1900). Distr. Southern South America (Brotherus, 1900; Paris, 1904a; Felippone, 1912; Herter,
1933a; Hosseus, 1938d).

B. uruguensis Par. (Paris, 1894).

Syn. Barbula decidua C. Muell. (Mueller, 1882) hom. illeg. Barbula subdecidua Kindb. (Kindberg, 1889) nom nud. Asteriscium deciduum Hilp. (Hilpert, 1933).

Distr. Southem South America (Mueller, 1882; Paris, 1894, 1904a; Felippone, 1912 Brotherus, 1924a; Herter, 1933a; Hosseus, 1938a, c; Herzog, 1938; Kühnemann.

B. vaginata Warnst. (Warnstorf, 1915).

Distr. Southern South America (Warnstorf, 1915).

B. vahliana Schultz

B. ventanae C. Muell. ex Par.

Tortula vahliana

B. ventanae C. Muell.

\section{Barbula ventanica}

Tortula pseudo-robusta

B. ventanica C. Muell. (Mueller, 1897a).

Syn. Barbula ventanae C. Muell. ex Par. (Mueller ex Paris, 1900a) err.

Distr. Southern South America (Mueller, 1897a; Paris, 1900a; Kühnemann, 1938).

B. vinealis*Bid. var. flaccida B.S.G. (Bruch and others, 1842).

Syn. Tortula vinealis (Brid.) Spruc. var. flaccida (B.S.G.) Spruc. (Spruce, 1845).

Distr. Southern South America (Bruch and others, 1842; Montagne, 1850).

\section{Bartramia}

B. abbreviata Card. et Broth. (Cardot and Brotherus, 1923).

Distr. Southern South America (Cardot and Brotherus, 1923; Kühnemann, 1938).

B. acicularis $\mathrm{C}$. Muell.

Philonotis acicularis

B. acutissima C. Muell.

Philonotis acutissima

B. ambigua Mont. (Montagne, 1845a).

Distr. Southem South America (Montagne, 1845a, 1850, 1856; Mueller, 1849; Mitten, 1869; Jaeger, 1875; Paris, 1904a; Theriot, 1915, 1917b, 1921b; Campo, 1921; Costes, 1921: Cardot and Brotherus, 1923; Brotherus, 1924a, $c$; Reimers, 1926; Roivainen, 1934; Herzog, 1938, 1939, 1940, 1954, 1957, 1960; Kühnemann, 1938; Espinosa B. 1941; Bart

var. horrida Card. et Broth. (Cardot and Brotherus, 1923).

Distr. Southem South America (Cardot and Brotherus, 1923; Kühnemann, 1938).

var. penicillatula (C. Muell.) Herz. (Herzog, 1920).

Syn. Bartramia penicillatula C. Muell. (Mueller, 1882).

Distr. Southem South America (Mueller, 1882; Paris, 1904a: Brotherus, 1924a; Kühnemann 1938).

B. aristata Schimp. ex C. Muell. (Schimper ex Mueller, 1862).

Syn. Bartramia lechleri Schimp. (Schimper in Mueller, 1901a [1900]) nom. illeg.

= Didymodon vinealis (Brid.) Zander var. flaccida (B.S.G.) Zander fide Zander (1978).
Distr. Juan Femandez (Brotherus, 1924b; Bartram, 1959; Robinson, 1975)

Southem South America (Lechler, 1857; Mueller, 1862; Lorentz, 1866; Jaeger, 1874

Mueller, 1901a; Dusen, 1903a; Paris, 1904a; Cardot, 1905a, 1908a; Theriot, 1918, 1934b; Campo, 1921; Brotherus, 1924a; Reimers, 1926; Herzog, 1938, 1957; Kühnemann, 1938)

B. aureola Besch. ex C. Muell.

Breutelia aureola

B. australis Mitt.

B. austro-arcuata C. Muell.

B. austro-georgica Par.

Conostomum pentastichum

Breutelia austro-arcuata

Bartramia patens

B. austro-stricta C. Muell. (Mueller, 1901a [1900]) nom. nud.

Distr. Southern South America (Mueller, 1901a).

B. carinata Mitt.

B. chilensis Lor.

B. chrysura C. Muell.

B. comiramea C. Muell.

B. comosa Mitt.

B. crenatula C. Muell.

B. crispa Brid. var. pomiformis (Hedw.) Lindb.

B. curvata Hamp.

B. cuspidatissima C. Muell.

B. cygnea Mont.

B. declivium C. Muell.

B. defecta C. Muell.

B. delicatula C. Muell. (Mueller, 1901a [1900]) nom. nud.

Breutelia plicata

Breutelia dumosa

Breutelia integrifolia

see Breutelia dumosa

see Breutelia dumosa

Philonotis crenatula

Bartramia pomiformis

Philonotis curvata

Breutelia cuspidatissima

Bartramidula cygnea

Philonotis pomangium

Philonotis glaucescens

B. diminutiva C. Muell. (Mueller, 1884 [1883]).

Distr. South Georgia (Cardot, 1906a, 1908a; Dixon, 1920; Steere, 1961b). Antarctic, peninsula region (Cardot, 1907c, 1908a, 1911b, $d, 1913 a$; Steere, 1961a).

B. dimorpha Schimp.

B. dumosa (Mitt.) Mitt.

B. elegantula Tayl.

B. elegantula Par.

B. elongatula C. Muell.

Philonotis vagans

Breutelia dumosa

Philonotis rufiflora

Philonotis elongatula

var. tenella C. Muell. (Mueller, 1882) nom. nud.

Philonotis elongatula

Distr. Southern South America (Mueller, 1882).

B. exigua Sull.

Philonotis scabrifolia

B. fernandeziana Card. (Cardot in Thériot, 1921b).

Distr. Juan Fernandez (Thériot, 1921b; Brotherus, 1924a, b).

B. flaviuscula C. Muell. (Mueller, 1901a [1900]) nom. nud.

Distr. Southern South America (Mueller, 1901a).

B. flexipes (C. Muell.) C. Muell.

Philonotis flexipes

B. fontana (Hedw.) Turn.

var. magellanica (Brid.) P. Beauv.

B. fontanoides Gill. et Grev. 
B. fragilifolia C. Muell. (Mueller, 1897b).

Distr. Southem South America (Bartram, 1965).

B. glaucescens Hornsch.

B. graminicola C. Muell.
Philonotis glaucescens Breutelia integrifolia

\section{B. halleriana Hedw. (Hedwig, 1801).}

Distr. Southem South America (Mitten, 1869; Bartram, 1952; Seki, 1974).

var. brachydonta Kab. (Kabiersch, 1937).

Syn. Bartramia mossmaniana C. Muell. (Mueller, 1851b).

Bartramia mossmanii C. Muell. ex Kindb. (Mueller ex Kindberg, 1888) nom. illeg.

Distr. Southem South America (Ångström, 1872; Jaeger, 1875; Mueller, 1885; Neger, 1899; Dusén, 1903a, 1905d; Paris, 1904a; Cardot, 1905a, 1908a, 1913a; Cardot and 1934; Theriot, 1935a; Kabiersch, 1937; Kühnemann, 1938).

B. hariotiana Besch. ex C. Muell.

Breutelia plicata B. integrifolia Tayl.

Breutelia integrifolia

B. ithyphylla Brid. (Bridel, 1803).

Syn. Plagiopus ithyphyllus (Brid.) Mach. (Machado, 1919).

Distr. Southern South America (Montagne, 1850; Campo, 1921 (as Bartramia ithyla)).

var. arenae Besch. (Bescherelle, 1889). Distr. Southem South America (Bescherelle, 1889; Dusén, 1903a; Paris, 1904a; Cardot,
1908a; Kühnemann, 1938).

B. ithyphylloides Schimp. ex C. Muell. (Schimper ex Mueller, 1859).

Distr. Southern South America (Lechler, 1857; Mueller, 1859; Jaeger, 1875; Neger, 1899; Paris, 1904a; Brotherus, 1924a; Roivainen, 1934; Thériot, 1934b; Herzog, 1939,
1954; Seki, 1974).

B. jacquenotiana Schimp. (Schimper in Paris, 1904a) nom. nud.

Distr. Southern South America (Paris, 1904a).

B. joannae C. Muell. (Mueller, 1901a [1900]) nom. nud.

Distr. Southem South America (Mueller, 1901a).

B. krauseiC. Muell.

B. laevisphaera (Tayl.) C. Muell.

Philonotis krausei

B. lechleri Schimp.

Anacolia laevisphaera

Bartramia aristata

B. leptophylla Card. et Broth. (Cardot and Brotherus, 1923).

Distr. Southem South America (Cardot and Brotherus, 1923; Brotherus, 1924a).

B. leptophylla Card. (Cardot in Skottsberg, 1914) nom. nud. fide (Brotherus, 1924b)

Distr. Juan Femandez (Skottsberg, 1914).

B. leucocolea Card.

Bartramia patens

var. brevifolia Broth. et Card.

Bartramia patens

var. glaucoflava Broth. et Card. (Brotherus and Cardot in Cardot and Brotherus, 1923).

Distr. Southern South America (Cardot and Brotherus, 1923; Kühnemann, 1938).

B. leucolomacea C. Muell.

B. lorentzii C. Muell.

Bartramia patens

B. magellanica (Sull.) Mitt.

Breutelia lorentzii

B. magellanica Aongstr.

B. microbasis C. Muell. (Mueller, 1882).

Distr. Southem South America (Mueller, 1882; Paris, 1904a; Brotherus, 1924a; Hosseus, 1935b, 1937; Kühnemann, 1938; Bizot, 1967).

B. mossmaniana C. Muell.

B. mossmanii C. Muell. ex Kindb.

Bartramia halleriana var. brachydonta

B. nevadensis C. Muell. (Mueller, 1882).

Distr. Southem South America (Mueller, 1882; Brotherus, 1924a; Hosseus, 1938c, d, 1939; Kühnemann, 1938).

B. oreadella C. Muell.

var. microphylla Card.

Bartramia patens

B. palustris (Hedw.) P. Beauv.

Bartramia patens

B. patens Brid. (Bridel, 1803).

Syn. Bartramia reticulata P. Beauv. (Palisot de Beauvois, 1805a).

Bartramia magellanica Brid. (Bridel in Bescherelle, 1889) nom nud.

Bartramia leucolomacea C. Muell. (Mueller, 1890a) fide Newton, 1974a

Bartramia oreadella C. Muell. (Mueller, 1890a) fide Newton, 1974a.

Bartramia oreadella C. Muell. var. microphylla Card. (Cardot, 1905a) fide Newton,

1974a.

Bartramia pycnocolea C. Muell. (Mueller, 1890a) fide Newton, 1974a.

Bartramia subpatens C. Muell. (Mueller, 1890a) hom. illeg.

Bartramia austro-georgica Par. (Paris, 1894).

Bartramia leucocolea Card. (Cardot, 1905a) fide Newton, 1974a.

Card

Distr. Juan Femandez (Brotherus, 1924b; Robinson, 1975).

Southem South America (Bridel, 1803, 1827b; Palisot de Beauvois, 1805a;

Schwaegrichen, 1816; Wilson and Hooker, 1847; Mueller, 1849, 1885, 1889;

Montagne, 1850; Sullivant, 1859; Mitten, 1869; Jaeger, 1875; Bescherelle, 1889; 1923. Herzog, 1923, 1954, 1957 1960. Brotherus, 1924a; Roivainen, 1934: 1923, Herog, 1923, 1954,1 Card, 190 , Bat 1908 ,

Falkand lsands (Wilson and Hooker, 1847 ; 174 ).

(1875; Mueller, 1885; Bescherelle, 1889; Dusén, 1903a; Paris,

South Georgia (Mueller, 1890a; Paris, 1894, 1904a; Cardot, 1906a, 1908a; Dixon, 1920 1934; Cardot and Brotherus, 1923; Brotherus, 1924a; Steere, 1961b; Newton, 1974a; Bell, 1977b). Antarctic, peninsula region (Cardot, 1906a, 1908a, 1911b, d, 1913a; Bartram, 1957;
Steere, 1961a; Greene, 1968a; Robinson, 1972).

var. arenae Card. (Cardot in Cardot and Brotherus, 1923) nom. nud.

Distr. Southern South America (Cardot and Brotherus, 1923; Kühnemann, 1938).

Falkland Islands (Cardot and Brotherus, 1923).

var. intermedia Hook. f. et Wils. (Hooker and Wilson in Wilson and Hooker, 1847).

Distr. Falkland Islands (Wilson and Hooker, 1847; Kühnemann, 1938).

var. minor Dus. (Dusén, 1903a).

Distr. Southern South America (Dusén, 1903a; Kühnemann, 1938).

B. pendula (Sm.) Hook.

B. penicillatula C. Muell.

see Breutelia dumosa

B. pentasticha Brid.

Bartramia ambigua var. penicillatula

B. perpumila C. Muell. (Mueller, 1882).

Distr. Southern South America (Mueller, 1882; Paris, 1904a; Kühnemann, 1938; Brotherus 1924a; Bartram, 1965). 
B. plicata (Mitt.) Mitt.

B. pomangium C. Muell.

B. pomiformis Hedw. (Hedwig, 1801).

Syn. Bryum pomiforme (Hedw.) With. (Withering, 1801). (1872) nom. illeg. Plagiopus pomiformis (Hedw.) Mach. (Machado, 1919).

Distr. Southem South America (Bridel, 1803, 1827b; Montagne, 1845b, 1850; Mitten, 1869; Mueller, 1885; Dusén, 1903a; Paris, 1904a; Cardot, 1908a).

var. crispa (Brid.) B.S.G.

Bartramia pomiformis var. elongata

var. elongata Turn. (Turner, 1805).

Syn. Bartramia pomiformis Hedw. var. crispa (Brid.) B.S.G. (Bruch and others, 1842). nom. illeg.

Distr. Southern South America (Wilson and Hooker, 1847; Rowlee, 1916).

B. potosica Mont. (Montagne, 1838).

Distr. Southern South America (Mitten, 1869; Jaeger, 1875; Paris, 1904a; Brotherus, 1924a).

B. pseudo-cryptopodium C. Muell. (Mueller, 1882).

Distr. Southem South America (Mueller, 1882; Paris, 1904a; Brotherus, 1924a; Kühnemann, 1938).

B. pusilla Sull.

B. pycnocolea C. Muell.

B. reticulata P. Beauv.

B. robusta Hook. f. et Wils. (Hooker and Wilson in Wilson and Hooker, 1845)

Syn. Breutelia robusta (Hook. f. et Wils.) Jaeg. (Jaeger, 1875).

Distr. Southem South America (Sullivant, 1859; Jaeger, 1875; Mueller, 1885; Dusén, 1903a Paris, 1904a; Cardot, 1908a; Brotherus, 1924a).

B. rufiflora Hornsch.

Philonotis rufiflora

B. rupestris Mitt.

Breutelia plicata

B. rupta C. Muell. (Mueller, 1882).

Distr. Southem South America (Mueller, 1882; Paris, 1904a; Brotherus, 1924a ; Hosseus, 1935b, 1937, 1939; Kühnemann, 1938).

B. scaberrima Card. et Broth. (Cardot and Brotherus, 1923).

Distr. Southern South America (Cardot and Brotherus, 1923; Brotherus, 1924a; Kühnemann, 1938).

B. scabrifolia (Hook. f. et Wils.) C. Muell.

Philonotis scabrifolia

B. simplicissima C. Muell.

Philonotis simplicissima

B. striata C. Muell.

Philonotis striata

B. stricta Brid. (Bridel, 1803).

Syn. Plagiopus strictus (Brid.) Mach. (Machado, 1919).

Distr. Juan Fernandez (Montagne, 1835; Johow, 1896).

Southem South America (Bridel, 1803, 1827b; Schwaegrichen, 1816; Montagne, 1850; Paris, 1904a).

B. strictiuscula (C. Muell.) C. Muell.

B. subpatens C. Muell.

Philonotis strictiuscula

Bartramia patens

B. subsecunda C. Muell. (Mueller, 1901a [1900]) nom. nud.

Distr. Southem South America (Mueller, 1901a).
B. subsymmetrica Card. (Cardot, 1906a).

Syn. Exodokidium subsymmetricum (Card.) Card. (Cardot, 1908a) fide Newton, 1974a.

Distr. South Georgia (Cardot, 1906a, 1908a; Brotherus, 1924a; Newton, 1974a; Bell, 1977b).

B. tenella (Brid.) C. Muell.

Philonotis glaucescens

B. thrausta Schimp. ex Britt. (Schimper ex Britton, 1896).

Distr. Southem South America (Paris, 1904a).

B. vagans (Hook. f. et Wils.) Mitt.

B. ventanae $C$. Muell.

Philonotis vagans

B. wilkesiana Par. (Paris, 1894) nom. nud.

Distr. Southem South America (Paris, 1894, 1904a).

B. willii C. Muell.

Philonotis scabrifolia

\section{Bartramidula}

B. cygnea (Mont.) Par. (Paris, 1894).

Syn. Bartramia cygnea Mont. (Montagne, 1845a). Glyphocarpus cygneus (Mont.) Jaeg. (Jaeger, 1875).

Distr. Southern South America (Montagne, 1845a, 1850, 1856; Mueller, 1848a; Lorentz, 1866 Mitten, 1869; Jaeger, 1875; Paris, 1894, 1904a; Herzog, 1923; Brotherus, 1924
Seki, 1974).

B. defecta (C. Muell.) Broth.

Philonotis glaucescens

B. exigua (Sull.) Par.

Philonotis scabrifolia

B. krausei (C. Muell.) Par.

Philonotis krause

Blindia

B. acuta (Hedw.) B.S.G. (Bruch and others, 1846).

Syn. Weisia acuta Hedw. (Hedwig, 1801).

Distr. Southem South America (Wilson and Hooker, 1847; Mueller, 1848a; Paris, 1904a). Falkland Islands (Wilson and Hooker, 1847; Kühnemann, 1938).

B. antarctica C. Muell.

B. arcuata Mitt.

B. auriculata C. Muell.

B. austro-crispula C. Muell.

Dicranoweisia antarctica

Blindia magellanica

Holodontium inerme

B. brevipes C. Muell.

Dicranoweisia austro-crispula

Dicranoweisia brevipes

B. capillifolia Card. (Cardot, 1906a).

Distr. South Georgia (Cardot, 1906a, 1908a; Brotherus, 1924a; Steere, 1961b).

B. churuccana Besch.

Holodontium pumilum

B. consimilis Card.

Blindia magellanica

B. contecta (Hook. f. et Wils.) C. Muell. (Mueller, 1848a).

Syn. Weisia contecta Hook. f. et Wils. (Hooker, J. D. and Wilson, 1844). Dicranoweisia contecta (Hook. f. et Wils.) Par. (Paris, 1896)

Distr. Southern South America (Ångström, 1872; Paris, 1896; Dusén, 1903a; Paris, 1904a; Cardot, 1908a; Brotherus, 1924a; Kühnemann, 1938; Seki, 1974).

B. curviseta Mitt. (Mitten, 1869).

Syn. Dicranum gracilescens Dus. (Dusén in Paris, 1904b) nom. nud. Blindia praticola Card. et Broth. (Cardot and Brotherus, 1923). 
Distr. Southem South America (Mitten, 1869; Mueller, 1885; Paris, 1900a, 1904a, b; Dusén, 1903a; Cardot, 1908a; Cardot and Brotherus, 1923; Brotherus, 1924a; Roivainen, 1934; Roivainen and Bartram, 1937; Kühnemann, 1938; Herzog, 1939). Falkland Islands (Dusén, 1903a; Paris, 1904a; Cardot, 1908a.

South Georgia (Cardot and Brotherus, 1923).

B. dicranellacea C. Muell. (Mueller, 1890a).

Distr. South Georgia (Mueller, 1890a; Paris, 1904a; Cardot, 1906a, 1908a; Cardot and Brothenus, 1923; Brotherus, 1924a; Steere, 1961b).

B. globularis Dus.

B. grimmiacea C. Muell.

B. humilis $\mathrm{C}$. Muell.

B. inundata (Card.) Card.

B. leptotrichacea C. Muell. ex. Par.

B. leptotrichocarpa C. Muell.

var. strictiuscula C. Muell.

\section{Blindia magellanica \\ Dicranoweisia grimmiacea \\ Holodontium pumilum \\ Blindia magellanica var. inundata \\ Ditrichum hookeri \\ Ditrichum hookeri \\ Ditrichum hookerivar strictiusculum}

B. Iygodipoda C. Muell. (Mueller, 1885)

Syn. Blindia lygopodina C. Muell. (Mueller in Paris, 1894) nom. illeg.

Distr. Southern South America (Mueller, 1885; Paris, 1894, 1904a; Dusén, 1903a; Cardot 1908a; Brotherus, 1924a; Kühnemann, 1938).

B. lygopodina C. Muell.

Blindia Iygodipoda

B. magellanica Schimp. (Schimper in Mueller, 1862).

Syn. Blindia arcuata Mitt. (Mitten, 1869).

Blindia consimilis Card. (Cardot, 1905a).

(1937) nom. nud.

Distr. Southern South America (Mueller, 1862, 1885; Mitten, 1869; Jaeger, 1873; Dusén, 1903a, 1905a, Paris, 1904a, 1906, Cardot, 1908a; Campo, 1921, Theriot, 1921 Cardot and Brotherus, 1923; Brotherus, 1924a; Roivan Herzog, 1938, 1939; Kunnem, Broterus, 1924a, Roivaine.

Falkland Islands (Cardot, 1905a, 1908a: Cardot and Brotherus, 1923: Kühnemann,

var. inundata (Card.) Herz. (Herzog, 1939)

Syn. Ditrichum inundatum Card. (Cardot, 1905a). Blindia inundata (Card.) Card. (Cardot, 1908a).

Distr. Southem South America (Cardot, 1905a, 1908a; Brotherus, 1924a; Roivainen and Bartram, 1937; Herzog, 1939, 1940, 1960)

\section{B. microdicranum Dus.}

Blindia magellanica

\section{B. pallidifolia C. Muell.}

Dicranoweisia subinclinata

B. praticola Card. et Broth.

Blindia curviseta

B. pseudolygodipoda Card. (Cardot, 1905a).

Distr. Southem South America (Cardot, 1905a, 1908a; Cardot and Brotherus, 1923;

Brotherus, 1924a, Kunnemann, 1938).

B. pseudorobusta Dus.

Blindia robusta

B. robusta Hamp. (Hampe, 1860)

Syn. Dicranum tenuifolium Hook. f. et Wils. (Hooker, J. D. and Wilson, 1844) hom. illeg

Blindia tenuifolia Mitt. (Mitten, 1869 ).
Distr. Southern South America (Hooker, J. D. and Wilson, 1844; Wilson and Hooker, 1847; Mueller, 1848a, 1885; Sullivant, 1859; Mitten, 1969; Jaeger, 1872, 1873; Dusén, 1903a, 1905a; Paris, 1904a; Cardot, 1908a; Cardot and Brotherus, 1923: Brotherus, 1924a; Roivainen and Bartram, 1937; Herzog, 1938, 1954; Kühnemann, 1938; Seki, 1974).

B. savatieri C. Muell.

Holodontium sp.

B. savatieriana C. Muell. Holodontium sp.

B. skottsbergii Card. (Cardot, 1906a).

Distr. South Georgia (Cardot, 1906a, 1908a; Dixon, 1920; Brotherus, 1924a; Steere, 1961b) Antarctic, peninsula region (Wright, 1905 (as Campylopus vesticauls), Cardot, 1906a, 1908a; 1911c, 1912b; Brotherus, 1924a; Steere, 1961a; Greene, 1968a; Robinson 1972)

B. subinclinata C. Muell.

Dicranoweisia subinclinata

B. sulphurea Dus.

Dicranoweisia austro-crispula

B. tenuifolia Mitt.

Blindia robusta

B. torrentium Card. et Broth. (Cardot and Brotherus, 1923).

Distr. Falkland Islands (Cardot and Brotherus, 1923; Brotherus, 1924a; Kühnemann, 1938).

B. turpis Card.

Verrucidens turpis

\section{Brachymenium}

B. ellipticum Jaeg.

Brachymenium meyenianum var. ellipticum

B. erubescens (C. Muell.) Kindb. (Kindberg, 1889).

Syn. Bryum erubescens C. Muell. (Mueller, 1882).

Distr. Southern South America (Mueller, 1882; Paris, 1904a; Brotherus, 1924a; Kühnemann 938).

B. fabroniellum Par. (Paris, 1906) nom. nud

Syn. Dicranobryum fabroniellum Dus. (Dusén in Paris, 1906) nom. nud.

Distr. Southem South America (Paris, 1906).

B. fabronioides (C. Muell.) Kindb. (Kindberg, 1889)

Syn. Bryum fabronioides C. Muell. (Mueller, 1879a).

Distr. Southem South America (Mueller, 1879a; Paris, 1904a; Brotherus, 1924a; Hosseus 1935b; Kühnemann, 1938).

var. densirete Thér. (Thériot, 1935b).

Distr. Southern South America (Thériot, 1935b; Hosseus, 1938c, d).

B. gilliesii (Hook.) Jaeg.

Bryum gilliesii

B. hyalinum Thér. (Thériot in Felippone, 1929 [1930]).

Distr. Southem South America (Felippone, 1929; Herter, 1933a).

B. indicum (Doz. et Molk.) Bosch et Lac. (van den Bosch and van der Sande Lacoste, 1860).

Syn. Bryum indicum Doz. et Molk. (Dozy and Molkenboer, 1845).

Peromnion magellanicum Sull. (Sullivant, 1850).

Bryum magellanicum (Sull.) C. Muell. (Mueller, 1851a).
Brachymenium magellanicum (Sull.) Par. (Paris, 1894).

Distr. Southern South America (Sullivant, 1850, 1859; Mueller, 1851a, 1885; Mitten, 1869 1938).

B. macropoma (C. Muell.) Kindb.

B. magellanicum (Sull.) Par.

Acidodontium macropoma

Brachymenium indicum 
B. malachiticum (C. Muell.) Kindb. (Kindberg, 1889).

Syn. Bryum malachiticum C. Muell. (Mueller, 1882). Distr. Southem South America (Mueller, 1882; Paris, 1904a; Brotherus, 1924a; Kühnemann,

B. meyenianum (C. Muell.) Jaeg. (Jaeger, 1875)

Syn. Webera meyeniana Hamp. (Hampe, 1837c).

Bryum meyenianum (Hamp.) C. Muell. (Mueller, 1848a).

Brachymenium meyenii Hamp. ex Kindb. (Hampe ex Kindberg, 1888) nom. illeg.

Dicranobryum subfabroniellum Dus. (Dusen in Paris, 1906) nom. nud.

Distr. Southern South America (Hampe, 1837c; Mueller, 1848a; Montagne, 1850; Mitten, 1869; Jaeger, 1875; Paris, 1904a, 1906; Brotherus, 1924a; Thériot, 1928; Hosseus,
1936, 1937; Kühnemann, 1938; Herzog, 1939, 1954).

var. ellipticum (Jaeg.) Thér. (Thériot, 1928).

Syn. Bryum ellipticum Mitt. (Mitten, 1869) hom. illeg.
Brachymenium ellipticum Jaeg. (Jaeger, 1875).

Distr. Southern South America (Mitten, 1869; Jaeger, 1875; Paris, 1904a; Costes, 1921; Thériot, 1921b, 1928; Brotherus, 1924a).

B. meyenii Hamp. ex Kindb.

B. ovatum Hook. f. et Wils.

B. papillosum Jaeg.

Brachymenium meyenianum Leptotheca gaudichaudii Pohlia papillosa

B. porteri Thér. (Thériot, 1925a).

Distr. Southern South America (Thériot, 1925a, 1926).

B. robertii Broth. (Brotherus, 1918).

Distr. Southern South America (Brotherus, 1918, 1924a; Kühnemann, 1938).

B. subfabroniellum Par.

B. wilsonii (Mitt.) Jaeg.

\section{Brachypodium hom. illeg.}

B. crispatum (Hedw.) Brid.

Brachysteleum nom. rejic.

B. brevifolium C. Muell.

B. chimborazense (Mitt.) Thér. var. obtusifolium Thér.

Ptychomitrium chimborazense var. obtusifolium

B. crispatum (Hedw.) Hornsch.

B. cummingii (Dub.) Hamp.

B. deltorii Thér.

B. emersum C. Muell.

B. ligulatum (Mitt.) C. Muell.

B. sellowianum C. Muell.

B. sellowii Aongstr.

B. uruguense C. Muell.

B. vernicosum C. Muell.

\section{Brachythecium}

B. alari-decurrens Dus.
Brachymenium meyenianum Leptobryum wilsonii

Ptychomitrium crispatum

Ptychomitrium balansae

Ptychomitrium crispatum Ptychomitrium fernandesianum

Ptychomitrium deltori

Ptychomitrium emersum

Ptychomitrium ligulatum

Ptychomitrium sellowianum

Ptychomitrium sellowianum

Ptychomitrium balansae

Ptychomitrium vernicosum

Brachythecium subplicatum
B. antarcticum Card.

Brachythecium austro-salebrosum Brachythecium austro-salebrosum

var. cavifolium Card.

B. arenarium Card. et Broth. (Cardot and Brotheru's, 1923).

Distr. Southern South America (Cardot and Brotherus, 1923; Brotherus, 1925; Kühnemann 1938).

B. argyrocaulon Dus. (Dusén in Paris, 1906) nom. nud.

Distr. Southern South America (Paris, 1906).

B. austro-glareosum (C. Muell.) Kindb. (Kindberg, 1891).

Syn. Hypnum austro-glareosum C. Muell. (Mueller, 1884 [1883]). Brachythecium austro-glareosum (C. Muell.) Kindb. var. diffusum Card. (Cardot, 1911b) fide Newton, $1979 a$.

Distr. South Georgia (Newton, 1979a, b).

Antarctic, peninsula region (Cardot, 1906b, 1907c, 1908a, 1911b, d, 1913a; Brotherus 1924a; Steere, 1961a; Robinson, 1972; Newton, 1979a).

var. diffusum Card.

Brachythecium austro-glareosum

B. austro-salebrosum (C. Muell.) Kindb. (Kindberg, 1889).

Syn. Hypnum austro-salebrosum C. Muell. (Mueller, 1884 [1883]).

1890a) fide Newton 1979 a Hypnum georgico-glareosum C. Muell. (Muell.) Kindb. (Kindberg, 1891) fide Newton, Brachythe.

Brachythecium antarcticum Card. (Cardot, 1900) fide Newton, 1979a. Brachythecium antarcticum Card. var. cavifolium Card. (Cardot, 1900) fide Newton, $1979 a$.

Brachythecium skottsbergii Card. (Cardot, 1906a) fide Newton, 1979a.

Distr. Southern South America (Cardot, 1905a, 1908a; Cardot and Brotherus, 1923: Falkland South Georgia (Mueller, 1890a; Paris, 1894, 1904a; Cardot, 1906a, 1908a; Cardot and Brotherus, 1923; Brotherus, 1925; Dixon, 1932; Steere. 1961b; Newton 1979a, b). Antarctic, peninsula region (Cardot, 1900, 1901, 1906a, b, 1907c. 1908a, 191 1 b, $d$,
1913a; Paris, 1904a; Dixon, 1920, 1935; Brotherus, 1925; Bartram, 1957; Steere 1961a; Robinson, 1972; Newton, 1979a).

B. calerae (C. Muell.) Kindb. (Kindberg, 1888).

Syn. Hypnum calerae C. Muell. (Mueller, 1879a).

Distr. Southern South America (Mueller, 1879a; Paris, 1894, 1904a; Kurtz, 1904; Brotherus,

Distr. Southern South America (Mueller, 1879a; Paris, 1894).
1925; Hosseus, 1935b, 1937; Kühnemann, 1938).

B. clinocarpum (Tayl.) Jaeg.

B. confluens (C. Muell.) Kindb.

Oxyrrhynchium clinocarpum Sciaromium confluens

B. conostomum (Tayl.) Jaeg. (Jaeger, 1878).

Syn. Hypnum conostomum Tayl. (Taylor, 1848a). Hypnum taylorii Mitt. (Mitten, 1869) nom. illeg.

Distr. Southern South America (Reimers, 1926; Herzog, 1938).

B. cuspidarioides Dus.

Brachythecium subplicatum

B. fasciculato-caudatum C. Muell. (Mueller, 1897a).

Distr. Southern South America (Mueller, 1897a; Paris, 1900a, 1904a; Brotherus, 1925; Kühnemann, 1938).

B. filirameum C. Muell. (Mueller, 1897a).

Distr. Southern South America (Mueller, 1897a; Paris, 1900a, 1904a; Brotherus, 1925; Kühnemann, 1938). 
B. filirepens Dus. (Dusén, 1905b). Distr. Southern South America (Dusén, 1905b; Paris, 1906; Cardot, 1908a; Brotherus, 1925
Kühnemann, 1938: Seki, 1974).

B. flagellare (Hedw.) Jenn

Brachythecium plumosum

B. fuegianum Broth. (Brotherus, 1908).

Syn. Stereophyllum fuegianum Besch. (Bescherelle, 1885a syn. excl.) nom. nud. Stereophyllum fuegianum Besch. (Bescherelle, 1889) hom. illeg.

Distr. Southern South America (Bescherele, 1885a, 1889; Boschers, 1908, 1925). nud.

georgico-garesum (C. Muerica (Bescherelle, 1885a, 1889; Brotherus, 1908, 1925).

(C. Muell.) Kindb.

Brachythecium austro-salebrosum

B. glaciale B.S.G. (Bruch and others, 1853).

Distr. Southern South America (Newton, 1979a, b).
South Georgia (Newton, 1979a, b).

B. grandirete C. Muell. (Mueller, 1897b).

Syn. Brachythecium pumilum Dus. (Dusén, 1905b).

Distr. Southern South America (Dusén, 1905b; Brotherus, 1925).

B. lepidopiloides (C. Muell.) Kindb.

Stereophyllum lepidopiloides

B. longidecurrens Bartr. (Bartram, 1946).

Distr. Southern South America (Bartram, 1946).

B. longidens (C. Muell.) Kindb. (Kindberg, 1888).

Syn. Hypnum longidens C. Muell. (Mueller, 1885).

Distr. Southern South America (Mueller, 1885; Dusén, 1903a; Paris, 1904a, 1905b; Cardot. 1908a; Kühnemann, 1938; Bartram, 1946).

B. macroconum (C. Muell.) Kindb.

var. robustius (C. Muell.) Par. (Paris, 1894).

Syn. Hypnum macroconum C. Muell. var. robustius C. Muell. (Mueller, 1879a).

Distr. Southern South America (Mueller, 1879a; Paris, 1894, 1904a).

B. macrogynum Card. (Cardot, 1905a).

Distr. Southem South America (Cardot, 1905a, 1908a; Brotherus, 1925; Kühnemann, 1938; Seki, 1974).

B. majusculum M. Newton (Newton, 1974d).

Syn. Brachythecium majusculum Dus. (Dusén, 1903a) nom. nud.

Distr. Southem South America (Dusén, 1903a; Newton, 1974d, 1979a, b). South Georgia (Newton, 1974d, 1979a, b).

B. majusculum Dus.

Brachythecium majusculum

B. microcollinum Bartr. (Bartram, 1946).

Distr. Southern South America (Roivainen, 1934; Bartram, 1946).

B. minusculifolium C. Muell. (Mueller, 1897a).

Distr. Southern South America (Mueller, 1897a ; Paris, 1900a, 1904a; Brotherus, 1925;
Kühnemann, 1938). Kühnemann, 1938).

B. mollirameum C. Muell. (Mueller, 1897a).

Distr. Southern South America (Mueller, 1897a; Paris, 1900a, 1904a; Kühnemann, 1938). B. morenoi C. Muell.

Brachythecium sericeo-virens
B. nematogonium (C. Muell.) Kindb. (Kindberg, 1888).

Syn. Hypnum nematogonium C. Muell. (Mueller, 1879a).

Distr. Southern South America (Mueller, 1879a; Kurtz, 1904; Paris, 1904a; Brotherus, 1925; Hosseus, 1935b, 1937; Kühnemann, 1938).

B. paradoxum (Hook. f. et Wils.) Jaeg. (Jaeger, 1878). Syn. Hypnum paradoxum Hook. f. et Wils. (Hooker, J. D. and Wilson, 1844).
Rhynchostegium mollissimum Dus. (Dusén 1903a) nom. nud.

Distr. Southern South America (Hooker, J. D. and Wilson, 1844; Wilson and Hooker, 1847; Mueller, 1851a, 1885; Mitten, 1869; Jaeger, 1878; Bescherelle, 1889; Cardot, 1901, 1905a, 1908a; Dusén, 1903a; Paris, 1904a, 1905b; Cardot and Brotherus, 1923;
Brotherus, 1925; Roivainen, 1934; Kühnemann, 1938; Herzog, 1954; Seki, 1974).

B. parvulum Dus. (Dusén in Paris, 1904a) nom. nud. Distr. Southern South America (Paris, 1904a).

B. patagonicum Card. et Broth. (Cardot and Brotherus, 1923).

Distr. Southern South America (Cardot and Brotherus, 1923; Brotherus, 1925; Kühnemann, 1938).

B. plumosum (Hedw.) B.S.G. (Bruch and others, 1853).

Syn. Hypnum plumosum Hedw. (Hedwig, 1801).

Hypnum macroconum C. Muell. (Mueller, 1879a).

Brachythecium macroconum (C. Muell.) Kindb. (Kindberg, 1888).

Hypnum flagellare Hedw. (Hedwig, 1801).

Brachythecium flagellare (Hedw.) Jenn. (Jennings, 1913) nom. illeg. Distr. Southern South America (Mueller, 1879a; Paris, 1904a; Brotherus, 1925; Roivainen,
1934; Kühnemann, 1938).

B. pumilum Dus.

Brachythecium grandirete

B. rivulare B.S.G. (Bruch and others, 1853).

Distr. Southem South America (Thériot, 1934b).

B. rivularioides (C. Muell.) Par. (Paris, 1894).

Syn. Hypnum rivularioides C. Muell. (Mueller, 1879a).

Distr. Southern South America (Mueller, 1879a; Paris, 1894, 1904a; Kurtz, 1904; Brotherus, 1925; Hosseus, 1935a, 1937; Kühnemann, 1938).

B. rutabulum (Hedw.) B.S.G. (Bruch and others, 1853).

Syn. Hypnum rutabulum Hedw. (Hedwig, 1801).

Southem South America Wilson and Hooker, 1847: Cardot, 1905a, 1908a; Cardot and

Distr. Southern South America Wilson and Hooker, 1847; Cardot, 1905a, 1908
Brotherus, 1923; Brotherus, 1925; Reimers, 1926; Kühnemann, 1938).

B. sericeo-virens (C. Muell.) Kindb. (Kindberg, 1888).

Syn. Hypnum sericeo-virens C. Muell. (Mueller, 1885).

Brachythecium morenoi C. Muell. (Mueller, 1879a) fide Seki, 1974.

Distr. Southern South America (Mueller, 1885, 1897a; Paris, 1900a, 1904a; Dusén, 1903a; Cardot, 1905a, 1908a; Brotherus, 1925; Kühnemann, 1938; Seki, 1974).

B. serribracteatum Dix.

Brachythecium subpilosum

B. skottsbergii Card.

Brachythecium austro-salebrosum

B. spegazzinii (C. Muell.) Kindb.

Hypnum spegazzini

B. spurio-albicans C. Muell. (Mueller, 1897a).

Distr. Southern South America (Mueller, 1897a; Paris, 1900a. 1904a; Dusén, 1903a; Cardot, 1908a; Brotherus, 1925; Kühnemann, 1938). 
B. squalidissimum (C. Muell.) Kindb. (Kindberg, 1888).

Syn. Hypnum squalidissimum C. Muell. (Mueller, 1879a).

Distr. Southern South America (Mueller, 1879a; Kurtz, 1904; Paris, 1904a; Brotherus, 1925; Hosseus, 1935b, 1937; Kühnemann, 1938).

B. stereophyllum C. Muell.

Brachythecium fuegianum

B. subpilosum (Hook. f. et Wils.) Jaeg. (Jaeger, 1878).

Syn. Hypnum subpilosum Hook. f. et Wils. (Hooker, J. D. and Wilson, 1844). Brachythecium serribracteatum Dix. (Dixon in Newton, 1979a) nom. nud.

Distr. Southern South America (Hooker, J. D. and Wilson, 1844; Wilson and Hooker, 1847; Mueller, 1851a, 1885; Mitten, 1869; Jaeger, 1878; Paris, 1904a; Cardot, 1905a, 1908a; Cardot and Brotherus, 1923; Herzog, 1923; Brotherus, 1925; Kühnemann, 1938; Newton, 1979a).

Falkland Islands (Dusén, 1903a; Paris, 1904a; Cardot, 1905a, 1908a; Cardot and Brotherus, 1923; Brotherus, 1925; Kühnemann, 1938).

South Georgia (Cardot, 1906a, 1908a; Dixon, 1920; Cardot and Brotherus, 1923;

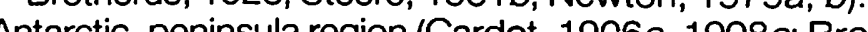

B. subplicatum (Hamp.) Jaeg. (Jaeger, 1878).

Syn. Hypnum subplicatum Hamp. (Hampe in Mueller, 1851a).

Hypnum rutabulum Hedw. var. 5 (in Wilson and Hooker, 1847) fide Mueller, $1851 a$ Brachythecium cuspidarioides Dus. (Dusén, 1905b) fide Cardot, $1908 a$. Brachythecium alari-decurrens Dus. (Dusén in Newton, 1979a)

Distr. Southern South America (Mueller, 1851a, 1885; Dusén, 1903a, 1905b; Paris, 1906; Cardot, 1908a; Herzog, 1923, 1939, 1954; Brotherus, 1925; Thériot, 1929c; Roivainen, 1934, Kuhnemann, 1938; Bartram, 1952; Seki, 1974; Newton, 1979a). Falkland Islands Wison and Hooker, 1847; Mueller, 1851 a; Jaeger, 1878; Cardot.

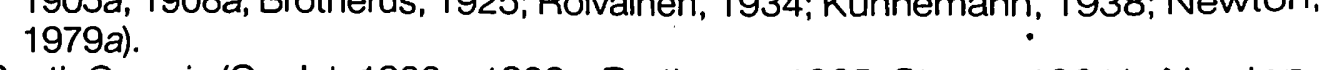

South Georgia (Cardot, 1906a, 1908a; Brotherus, 1925; Steere, 1961b; Newton, 1979a,
b). b).

var. dilaceratum Card. (Cardot, 1905a).

Distr. Southern South America (Cardot, 1905a, 1908a; Kühnemann, 1938).

var. minus Card. et Broth. (Cardot and Brotherus, 1923)

Distr. Falkland Islands (Cardot and Brotherus, 1923; Kühnemann, 1938).

B. subtilicaule Dus. (Dusén, 1903a) nom. nud.

Distr. Southern South America (Dusén, 1903a; Paris, 1904a).

B. sulphureum (Geh. et Hamp.) Par. (Paris, 1894).

Syn. Hypnum sulphureum Geh. et Hamp. (Geheeb and Hampe in Hampe and Geheeb, 1881).

Distr. Southern South America (Paris, 1894; Brotherus, 1918, 1925; Hosseus, 1938a (as Brachystegium), b, c, d, 1939. Kühnemann, 1938).

B. tenui-prostratum C. Muell. (Mueller, 1897a).

Distr. Southern South America (Mueller, 1897a; Paris, 1900a, 1904a; Brotherus, 1925; Kühnemann, 1938).

B. trachychaete Dus. (Dusén, 1905b).

Distr. Southern South America (Dusén, 1905b; Cardot, 1908a; Brotherus, 1925; Newton, 1979a).

B. turgens Dus.

Brachythecium subplicatum
B. turquetii Card. (Cardot, 1906b).

Distr. Antarctic, peninsula region (Cardot, 1906b, 1907c, 1908a; Brotherus, 1925; Steere,
1961a).

Braunia

B. argentinica C. Muell.

B. canescens Schimp. ex Britt.

B. chilensis Broth.

Braunia cirrhifolia

Braunia cirrhifolia

B. cirrhifolia (Mitt.) Wils. ex Jaeg. (Wilson ex Jaeger, 1876).

Syn. Harrisonia secunda (Hook.) Spreng. var. cirrhifolia Mitt. (Mitten, 1851) nom. nud. Braunia incana C. Muell. (Mueller, 1879a).

Braunia argentinica C. Muell. (Mueller, 1882).

Braunia canescens Schimp. ex Britt. (Schimper ex Britton, 1896).

Distr. Southern South America (Mueller, 1879a, 1882; Kurtz, 1904; Paris, 1904a; Brotherus.

1918, 1925; Hosseus, 1936, 1937, 1938c, d, 1939; Kühnemann, 1938).

B. cochlearifolia C. Muell. (Mueller, 1897a).

Distr. Southem South America (Mueller, 1897a; Paris, 1900a, 1904a; Brotherus, 1925

Hosseus, 1936, 1937, 1938c, $d$, 1939; Kühnemann, 1938)

B. exserta C. Muell. (Mueller, 1879a). Distr. Southern South America (Mueller, 1879a; Paris, 1904a; Brotherus, 1925; Kühnemann.
1938: Bartram, 1965).

var. reflexifolia C. Muell.

B. gibertii Besch.

B. humboldtii (Hook.) Hook. f.

Braunia reflexifolia

Hedwigidium gibertii

B. incana C. Muell.

B. patagonica C. Muell.

Braunia cirrhifolia

B. reflexifolia (C. Muell.) Bartr. (Bartram, 1965).

Syn. Braunia exserta C. Muell. var reflexifolia C. Muell. (Mueller, 1879a).

Distr. Southern South America (Mueller, 1879a; Paris, 1904a; Bartram, 1965).

B. rhabdocarpa (Hamp.) C. Muell.

Hedwigidium integrifolia

Braunia Hamp. hom. illeg.

B. cuspidata (Sull.) Hamp.

Dendrocryphaea cuspidata

\section{Breutelia}

B. angustiretis Bartr. (Bartram, 1946).

Distr. Southern South America (Bartram, 1946; Matteri, 1973a; Seki, 1974).

B. areola Besch.

Breutelia aureola

B. aureola (C. Muell.) Besch. (Bescherelle, 1885a).

Syn. Bartramia aureola Besch. ex C. Muell. (Bescherelle ex Mueller, 1885). Breutelia areola Besch. (Bescherelle in Cardot and Brotherus, 1923) err. Breutelia rupestris (Mitt.) Jaeg. var. gracilior Card. (Cardot, 1908a) fide Matteri,

Distr. Southern South America (Mueller, 1885; Bescherelle, 1885a, 1889; Dusén, 1903a (and as B. elongata fide Matteri, 1973a); Paris, 1904a; Cardot. 1905a, 1908a (and as B. Kühnemann, 1938; Herzog, 1954: Matteri, 1973a: Seki. 1974).

B. auricolor Herz.

Breutelia integrifolia 
B. austro-arcuata (C. Muell.) Kindb. (Kindberg, 1889).

Syn. Bartramia austro-arcuata C. Muell. (Mueller, 1882).

Distr. Southern South America (Mueller, 1882; Paris, 1894, 1904a; Brotherus, $1924 a$$$
\text { Kühnemann, 1938). }
$$

B. brachycoma Besch.

B. carinata (Mitt.) Jaeg.

B. chilensis (Lor.) Par.

B. chrysura (C. Muell.) Broth.

B. comosa (Mitt.) Mitt.

B. comosa Besch.

\section{Breutelia dumosa \\ Breutelia plicata \\ Breutelia dumosa \\ Breutelia integrifolia \\ see Breutelia dumosa \\ Breutelia dumosa}

B. cuspidatissima (C. Muell.) Kindb. (Kindberg, 1889).

Syn. Bartramia cuspidatissima C. Muell. (Mueller, 1882)

Distr. Southern South America (Mueller, 1882; Paris, 1894, 1904a; Brotherus, 1924a; Thériot. 1934b; Kühnemann, 1938).

\section{B. dumosa Mitt. (Mitten, 1860).}

Syn. Bartramia dumosa (Mitt.) Mitt. (Mitten, 1869).

Bartramia chilensis Lor. (Lorentz, 1866) fide Matteri, 1973a.

Breutelia chilensis (Lor.) Par. (Paris, 1904a) fide Matteri, 1973a.

Breutelia brachycoma Besch. (Bescherelle, 1885a) fide Matteri, 1973a.

Breutelia comosa Besch. (Bescherelle, 1885a) nom. nud.
Breutelia longipes Schimp. (Schimper in Paris, 1904a) nom. nud. pro parte.

Distr. Southem South America (Wilson and Hooker, 1847 (as Bartramia pendula vars. 1 and 2 fide Matteri, 1973a); Mueller, 1849 (as Bartramia pendula fide Matteri, 1973a), 1885 (and as Bartramia comosa fide Matteri, 1973a). 1901a (as Bartramia comiramea fide Matteri, 1973a); Sullivant, 1859; Mitten 1860, 1869; Lorentz, 1866; Jaeger, 1875 (and as B. pendula fide Matteri, 1973a); Bescherelle, 1885a, 1889; Dusén, 1903a (and as B. elongata fide Matteri, 1973a); Paris, 1904a; Cardot, $1908 a$ (and as B. elongata an 1954:Brotherus, 1924a; Roivand 1954; Brotherus, 1924a; Roivainen, 1934; Kühnemann, 1938; Matteri, 1973a; Seki,
B. elongata (Hook. f. et Wils.) Mitt.

B. glabrifolia Dus.

B. graminicola Broth.

B. hariotii Besch. ex Kindb.

see Breutelia aureola, B. dumosa and B. plicata Breutelia integrifolia

Breutelia integrifolia

Breutelia plicata

Breutelia plicata

B. integrifolia (Tayl.) Jaeg. (Jaeger, 1875).

Syn. Bartramia integrifolia Tayl. (Taylor, 1846).

Breutelia chrysura (C. Muell.) Broth. (Brotherus, 1904).

Bartramia graminicola C. Muell. (Mueller, 1884 [1883]) hom illeg. fide Matteri, 1973a.

Breutelia graminicola Broth. (Brotherus, 1904) nom. illeg. fide Matteri, 1973a.

Philonotis kerguelensis Par. (Paris, 1897) fide Matteri, 1973a. Matteri, 1973a.

Breutelia skottsbergii Card. var. horrida Card. et Broth. (Cardot and Brotherus, 1923) Matteri, 1973a.

Breutelia integrifolia (Tayl.) Jaeg. var. patagonica Card. et Broth. (Cardot and

Anacolia auricolor Dus. (Dusén in Paris, 1906) nom. nud. fide Matteri, 1973a. Breutelia auricolor Herz. (Herzog, 1909) nom. nud. fide Matteri 1973a.

Breutelia thysanomitrioides Dus. (Dusén in Paris, 1904a) nom. nud. fide Matteri.
1973a.
B. hariotiana (C. Muell.) Besch.

Bartramia chrysura C. Muell. (Mueller, 1884 [1883]).

Breutelia kerguelensis (Par.) Wijk et Marg. (van der Wijk and Margadant, 1958) fide

elia skottsbergii Card. (Cardot, 1905a) fide Matteri, 1973a. Brotherus, 1923) fide Matteri, 1973a.
Distr. Southem South America (Sullivant, 1859; Neger, 1899; Dusén, 1903a, 1905b; Paris, 1904a, 1906; Cardot, 1905a, 1908a; Campo, 1921; Cardot and Brotherus, 1923; Brotherus, 1924a, 1925: Thériot, 1924, 1925a; Roivainen, 1934: Kühnemann, 1938; Herzog, 1940, 1954, 1957; Bartram, 1952; Matteri, 1973a; Seki, 1974).

Falkland Islands (Cardot and Brotherus, 1923; Kühnemann, 1938; Matteri, 1973a).

var. patagonica Card. et Broth.

Breutelia integrifolia

B. kerguelensis (Par.) Wijk et Marg.

Breutelia integrifolia

B. longipes Schimp. Breutelia dumosa, Breutelia plicata and Breutelia subplicata

B. lorentzii (C. Muell.) Kindb. (Kindberg, 1889).

Syn. Bartramia lorentzii C. Muell. (Mueller, 1882).

Distr. Southern South America (Mueller, 1882; Paris, 1904a; Brotherus, 1924a; Kühnemann, 1938).

B. masafuerae Broth. (Brotherus, 1924b).

Syn. Breutelia subplicata Broth. var. masafuerae (Broth.) Matteri (Matteri, 1973a) fide Robinson, 1975.

Distr. Juan Femandez (Brotherus, 1924b; Bartram, 1959; Matteri, 1973a; Robinson, 1975). B. pendula (Sm.) Mitt.

B. persquarrosa Dus.
B. perruncinata Dus.

Breutelia subplicata

Syn. Bartramia plicata (Mitt.) Mitt. (Mitten, 1869).

ex Mueller, 1885)

Breutelia hariotiana (C. Muell.) Besch. (Bescherelle, 1885a).

Breutelia hariotii Besch. ex Kindb. (Bescherelle ex Kindberg, 1888) hom. illeg.

Bartramia carinata Mitt. (Mitten, 1869) fide Matteri, 1973a.

Breutelia carinata (Mitt.) Jaeg. (Jaeger, 1875).

Bartratma rupestris (Mit) Jateg. (Jager, 1875) 1973a.

Bartamia upestis Mitt. (Mithen. 1869) fide Matteri, 1973a.

Breutia $1973 a$.

Breutelia speciosa Card. (Cardot in Herzog, 1954) nom. nud. fide Seki, 1974.

Distr. Southern South America (Mitten, 1860, 1869; Lorentz, 1866; Jaeger, 1875; Mueller, 1885; Bescherelle, 1885a, 1889; Neger, 1899; Dusén, 1903a (and as B. elongata fide Matteri, 1973a): Cardot and Brotherus, 1923: Brotherus, 19242: Reimers, 1926: Kühnemann, 1938; Herzog, 1939, 1954; Matteri, 1973a; Seki, 1974).

B. robusta (Hook. f. et Wils.) Jaeg.

B. rupestris (Mitt.) Jaeg.

var. gracilior Card.

Bartramia robusta

B. scabrifolia Dus. (Dusén in Paris, 1904a) nom. nud.

Distr. Southern South America (Paris, 1904a).

B. skottsbergii Card.

var. horrida Card. et Broth.

B. speciosa Card.

B. subelongata Broth.

Breutelia integrifolia

Breutelia plicata

Breutelia plicata
Breutelia plicata

Breutelia aureola South Georgia (Cardot, 1906a, 1908a; Cardot and Brotherus, 1923; Steere, 1961b; Matteri, 1973a; Newton, 1974a).

B. plicata Mitt. (Mitten, 1860).

B. subplicata Broth. (Brotherus in Thériot, 1911).

Syn. Breutelia persquarrosa Dus. (Dusén in Paris, 1904a) nom. nud. 
Breutelia perruncinata Dus. (Dusén in Paris, 1904a) nom. nud. fide Matteri, 1973a. ( Matteri, 1973 .

Distr. Juan Fernandez (Matteri, 1973a: Robinson, 1975).

Southem South America (Paris, 1904a; Thériot, 1911, 1934b, 1935a; Brotherus, 1924a; Roivainen, 1934; Herzog, 1938, 1939, 1940, 1954, 1960; Bartram, 1952; Matteri, 1973a; Seki, 1974)

var. masafuerae (Broth.) Matteri

Breutelia masafuerae

B. thysanomitrioides Dus.

\section{B. ulicina Herz. (Herzog, 1927).}

Distr. Southern South America (Herzog, 1927).

\section{Bruchia}

B. brevipes Harv. ex Hook. (Harvey ex Hooker, 1840).

Syn. Phascum brevipes (Hook.) Schwaegr. (Schwaegrichen, 1842).

Distr. Southem South America (Montagne, 1850).

B. hampeana C. Muell. (Mueller, 1848a).

Syn. Sporledera hampeana (C. Muell.) Mitt. (Mitten, 1869).

Sporledera schwaegrichenii Hamp. (Hampe in Jaeger, 1869) nom. nud.

Distr. Southem South America (Mueller, 1848a; Jaeger, 1869, 1873; Mitten, 1869; Paris.

$$
\text { 1904a; Roth, 1911; Brotherus, 1924a). }
$$

B. schwaegrichenii Jaeg.

Bruchia hampeana

B. uleana C. Muell. ex Roth (Mueller ex Roth, 1911).

Distr. Southem South America (Hosseus, 1940).

B. uruguensis C. Muell. (Mueller, 1879a).

Distr. Southem South America (Mueller, 1879a; Jaeger and Sauerbeck, 1879b; Paris, 1904a; Roth, 1911; Brotherus, 1924a; Herter, 1933a; Kühnemann, 1938; Hosseus, 1939).

\section{Bryodusenia}

B. genuflexa (C. Muell.) H. Robinson

Ancistrodes genuflexa

\section{Bryoerythrophyllum}

B. campylocarpum (Ç. Muell.) Crum (Crum, 1957).

Syn. Trichostomum campylocarpum C. Muell. (Mueller, 1851a). Didymodon calymperidictyon Broth. (Brotherus, 1924b).
Didymodon linearis Broth. (Brotherus, 1924b) hom. illeg.

Distr. Juan Fernandez (Brotherus, 1924a, b; Bartram, 1959; Robinson, 1975).

B. recurvirostre (Hedw.) Chen (Chen, 1941).

Syn. Weisia recurvirostris Hedw. (Hedwig, 1801).

Bryum lacustre Brid. (Bridel, 1803).

Dermatodon rubellus Genth (Genth, 1835).

Didymodon rubellus B.S.G. (Bruch and others, 1846) nom. illeg

Distr. Southern South America (Bruch and others, 1839; Wilson and Hooker, 1847; Cardot, 1905a, 1908a; Kühnemann, 1938).

var. antarctica Savicz et Z. Smirn. (Savich-Ljubitskaya and Smirnova, 1963b) Distr. Antarctica, continental region (Savich-Ljubitskaya and Smirnova, 1963b; Greene, 1968a;
Savich-Ljubitskaya, 1978).

\section{Bryoporteria}

B. chilensis Thér.
Camptodontium cryptodon
Bryum

B. aequabile C. Muell. (Mueller, 1882).

Distr. Southem South America (Mueller, 1882; Paris, 1904a; Brotherus, 1924a; Hosseus, 1936, 1937, 1938b, d; Kühnemann, 1938)

B. aerugo Dus. (Dusén, 1903a) nom. nud.

Distr. Southern South America (Dusén, 1903a; Paris, 1904a).

B. albicans Roehl.

B. albidum (Hedw.) P. Beauv.

Pohlia wahlenbergii Octoblepharum albidum

B. algens Card. (Cardot, 1907a).

Syn. Bryum atgens Card. (Cardot in Holzinger, 1911) err.

Distr. Antarctic, peninsula region (Cardot, 1911b, d, 1913a; Dixon, 1920; Steere, 1961a). Antarctic, continental region (Cardot, 1907a, 1908a; Brotherus, 1924a; Steere, 1961a;
Savich-Ljubitskaya and Smimova, 1972; Savich-Ljubitskaya, 1978).

B. alticaule C. Muell.

Pohlia wahlenbergii var. glacialis

var. robustius C. Muell.

Mniobryum alticaule var. robustius

B. amblyodon C. Muell. (Mueller, 1879a).

Distr. Southern South America (Mueller, 1879a; Paris, 1904a; Kühnemann, 1938).

B. amblyolepis ${ }^{\star}$ Card. (Cardot, 1900)

Distr. Southern South America (Costes, 1921).

Antarctic, peninsula region (Cardot, 1900, 1901, 1908a; Paris, 1904a; Brotherus, 1924a; Antarctic, continenter

B. amplirete C. Muell.

Pohlia wahlenbergii var. glacialis

B. andicola Hook. (Hooker in Kunth, 1822) see Ochi, 1972.

Syn. Rhodobryum andicola (Hook.) Par. (Paris, 1897). Bryum lechleri C. Muell. (Mueller, 1856) see Ochi, 1972 Rhodobryum lechleri (C. Muell.) Par. (Paris, 1897)

Distr. Juan Fernandez (Brotherus, 1924b; Robinson, 1975). Southern South America (Mueller, 1856; Lorentz, 1866; Mitten, 1869; Jaeger, 1875 Paris, 1897, 1904a; Theriot, 1915, 1918, 1935a; Campo, 1921; Cardot and Brotherus, 1923; Herzog, 1923, 1938, 1939; Brotherus, 1924a, c; Reimers, 1926; Kühnemann, 1938; Espinosa B., 1941; Skottsberg, 1950)
Falkland Islands (Cardot and Brotherus, 1923).

B. angulosum Menz.

Conostomum pentastichum

B. anisodontacum Dus. (Dusén, 1903a) nom. nud.

Distr. Southern South America (Dusén, 1903a; Paris, 1904a).

B. anomobryoides Card. et Broth. (Cardot and Brotherus, 1923) Distr. Southern South America (Cardot and Brotherus, 1923; Brotherus, 1924a; Kühnemann,
1938).

B. antarcticum Hook. f. et Wils. (Hooker and Wilson in Wilson and Hooker, 1847)

Syn. Webera antarctica (Hook. f. et Wils.) Jaeg. (Jaeger, 1875).

Distr. Antarctic, peninsula region (Wilson and Hooker, 1847; Mueller, 1851a; Jaeger, 1875 Cardot, 1906a, 1908a; Paris, 1906; Darbishire, 1923;

Antarctic, continental region (Cardot, 1907a, 1908a, 1910; Dixon and Watts, 1918; Brotherus, 1924a; Bartram, 1938, 1957; Rayner, 1940; Clifford, 1957; Steere, 1961 a (as B. algens see Clifford, 1957); Filson, 1966; Robinson. 1972; Savich-Ljubitskaya and Smirnova, 1972).

= Bryum argenteum Hedw. fide Ochi (1979). 
B. apiculatum Schwaegr. (Schwaegrichen, 1816).

Distr. Southem South America (Hornschuch, 1840; Mueller, 1848a; Mitten, 1869; Gibert 1873; Jaeger, 1875; Paris, 1904a; Felippone, 1912; Herter, 1933a)

B. apocarpum (Hedw.) L. ex With.

Schistidium apocarpum

var. ciliatum (Hedw.) With.

Hedwigia ciliato

B. arechavaletae Broth. (Brotherus in Krieger, 1904) nom. nud.

Distr. Southem South America (Krieger, 1904; Herter, 1939b).

B. arenae C. Muell.

Bryum argenteum

B. argenteum Hedw. (Hedwig, 1801)

Syn. Bryum argyreum C. Muell. (Mueller, 1882).

Bryum atro-sanguineum C. Muell. (Mueller, 1882)

Bryum capillisetum C. Muell. (Mueller, 1882).

Bryum compactulum C. Muell. (Mueller, 1882)

Bryum griseum Dus. ex Thér. (Dusén ex Thériot, 1917b) fide Ochi, 1969.

Bryum siplei Bartr. (Bartram, 1938) fide Greene, 1967.

Distr. Juan Fernandez (Robinson, 1975).

Southem South America (Montagne, 1850; Lorentz, 1866; Ångström, 1872; Mueller.

1917b. Campo, 1921: C3a, Pars, 1904a, Cardot, 1908a, Felippone, 1912, The 1024, Herter 1933a, 1939b; Hoses, $1935 a, b, 1937,1940$; Herzog, 1938, 1939, 1957:

Falkland Islands Wilson and Hooker, 1847; Cardot, 1905a, 1908a; Cardot and Brotherus, 1923; Kühnemann, 1938).

Antarctic, peninsula region (Wilson and Hooker, 1847; Paris, 1904a; Cardot, 1906a, 1908a, 1911b, d, 1913a; Steere, 1961a; Robinson, 1972).

Antarctic, continental region (Gepp, 1902; Cardot, 1907a, 1908a, 1910; Bartram, 1938. 1957; Horikawa, 1961; Horikawa and Ando, 1961, 1967; Steere, 1961a; SavichLjubitskaya and Smirnova, 1964b; Greene, 1967; Robinson, 1972).

var. lanatum (P. Beauv.) Hamp. (Hampe, 1839).

Syn. Mnium lanatum P. Beauv. (Palisot de Beauvois, 1805a)

Distr. Southem South America (Hosseus, 1938a, b, c, d)

var. obtusifolium Broth. (Brotherus, 1906a).

Distr. Antarctic, continental region (Savich-Ljubitskaya, 1978).

var. subamblyolepis Card. et Broth. (Cardot and Brotherus, 1923).

Distr. Falkland Islands (Cardot and Brotherus, 1923; Kühnemann, 1938).

B. argentinense Thér. (Thériot in Herzog, 1957) nom. nud.

Distr. Southem South America (Herzog, 1957)

B. argyreum C. Muell.

Bryum argenteum

B. aspillagae Thér. (Thériot, 1917b).

Distr. Southem South America (Thériot, 1917b; Costes, 1921; Brotherus, 1924a).

B. atgens Card. in Holz.

B. atro-sanguineum C. Muell.

Bryum algens

B. aubertii (Schwaegr.) Brid.

Bryum argenteum

B. austro-affine Broth. (Brotherus, 1916).

Distr. Southem South America (Felippone, 1928; Herter, 1933a). B. austro-albicans C. Muell.

Pohlia wahlenbergii
B. austro-polare* Card. (Cardot, 1900).

Distr. Southern South America (Looser, 1932; Thériot, 1934a).

Antarctic, peninsula region (Cardot, 1900, 1901, 1906b, 1907c, 1908a; Paris, 1904a Brotherus, 1924a; Steere, 1961a (as B. antarcticum); ? Filson, 1966 (as B. Benticum): Ochi 1970 (as B. pseudotiquerum for Racovitza, 234b)).

B. austro-turbinatum C. Muell. (Mueller, 1882). Distr. Southern South America (Mueller, 1882; Paris, 1904a; Brotherus, 1924a; Hosseus,
1935a, 1937; Kühnemann, 1938).

B. badium (Brid.) Schimp. (Schimper, 1876).

Syn. Bryum caespiticium L. ex Hedw. var. badium Bruch ex Brid. (Bridel, 1827a). Bryum caespiticium L. ex Hedw. ssp. badium (Brid.) Lindb. (Lindberg, 1879).
Bryum caespiticium L. ex Hedw. var. gracilescens B.S.G. (Bruch and others, 1839) nom. illeg.

Distr. Falklands Islands (Wilson and Hooker, 1847; Kühnemann, 1938).

B. bartramiopsis C. Muell. (Mueller, 1882).

Distr. Southern South America (Mueller, 1882; Paris, 1904a; Brotherus, 1924a; Kühnemann 1938)

B. beyrichiae Hornsch. ex Kindb.

B. beyrichianum (Hornsch.) C. Muell.

Rhodobryum beyrichianum

B. beyrichii Aongstr.

Rhodobryum beyrichianum

Rhodobryum beyrichianum

B. bicolor Dicks. (Dickson, 1801).

Syn. Bryum dichotomum auct. non Hedwig fide Ochi, 1972.

um Mont. (Montagne, 1841).

Bnym ferriviae C. Muell. (Mueller in Ule, 1899) nom. nud. Distr. Southerm South America (Mitten, 1869; Gibert, 1873; Jaeger, 1875; Paris, 1904a

B. billardieri Schwaegr. (Schwaegrichen, 1816)

Distr. Falkland Islands (Wilson and Hooker, 1847; Kühnemann, 1938)

B. bimum (Scherb.) Turn.

ssp. pseudotriquetrum (Hedw.) Dix.

Bryum pseudotriquetrum ssp. bimum

Bryum pseudotriquetrum

B. brachycarpum Bartr. (Bartram, 1946) hom. illeg

Distr. Southem South America (Bartram, 1946)

B. brachychaete Card. et Broth. (Cardot and Brotherus, 1923).

Distr. Southern South America (Cardot and Brotherus, 1923; Brotherus, 1924a).

B. brachymeniopsis $\mathrm{C}$. Muell.

Anomobryum brachymeniopsis

B. brevigemmatum Dus. (Dusén, 1903a) nom. nud.

Distr. Southem South America (Dusén, 1903a; Paris, 1904a; Seki, 1974).

B. bulbillinum C. Muell. (Mueller, 1882)

Distr. Southern South America (Mueller, 1882; Paris, 1904a; Brotherus, 1924a; Kühnemann, 1938).

B. bulbillosum Mont.

Bryum bicolor

B. caespiticium L. ex Hedw. (Hedwig, 1801).

Syn. Mnium caespiticium (Hedw.) With. (Withering, 1801).

Hypnum caespiticium (Hedw.) Web. et Mohr (Weber and Mohr, 1803).

* = Bryum pseudotriquetrum (Hedw.) Gaertn., Meyer et Scherb. fide Ochi $(1970,1979)$

Distr. South South America (Roivainen, 1934; Bartram, 1946). 
Distr. Juan Femandez (Montagne, 1835, 1845b). Southem South America (Wilson and Hooker, 1847; Kühnemann, 1938; Seki, 1974). Falkland Islands (Wilson and Hooker, 1847). ssp. badium (Brid.) Lindb.

var. badium Bruch ex Brid.

var. gracilescens B.S.G.

B. caespitosum (Hopp. et Hornsch.) Brid.

B. calopyxis C. Muell.

Bryum badium

Bryum badium

Bryum badium

Pohlia nutans

Pohlia calopyxis

B. campoanum Thér. (Thériot, 1926).

Distr. Southem South America (Campo, 1921; Thériot, 1926)

B. campylothecium Tayl. (Taylor, 1846).

Syn. Bryum jaffuelii Thér. (Thériot, 1926) fide Ochi, 1977.

Distr. Southem South America (Ochi, 1977).

B. campylotrichum C. Muell. (Mueller, 1901a [1900]) nom. nud. Distr. Southem South America (Mueller, 1901a).

B. canariense Brid. (Bridel, 1817).

Syn. Bryum jaffuelii Thér. (Thériot, 1926).

Distr. Southem South America (Montagne, 1850; Thériot, 1926).

B. candicans Tayl. (Taylor in Mitten, 1869).

Distr. Southem South America (Thériot, 1918; Campo, 1921).

B. canescens Schimp. (Schimper in Paris, 1904a) nom. nud.

Distr. Southern South America (Lechler, 1857; Paris, 1904a).

B. capillaceum (Hedw.) Dicks. ex With.

Distichium capillaceum

B. capillare Hedw. (Hedwig, 1801).

Syn. Bryum philippianum C. Muell. (Mueller, 1844b [1845]) fide Ochi, 1969. Bryum chilense Reichdt. (Reichard, 1868 ) Thde Ochi, 1969.

Bryum nanocoma C. Muell. (Mueller, 1882) fide Ochi, 1969.

Distr. Southern South America (Mueller, 1844b, 1848a, 1882; Montagne, 1850; Lorentz, 1866: Reichardt, 1868, 1870; Mitten, 1869; Jaeger, 1875; Neger, 1899; Paris, 1904a; 1974).

ssp. torquescens (B.S.G.) Kindb. (Kindberg, 1897).

Syn. Bryum torquescens B.S.G. (Bruch and others, 1839).

Distr. Southern South America (Bruch and others, 1839; Mueller, 1848a; Montagne, 1850; Mitten, 1869; Gibert, 1873; Paris, 1904a; Felippone, 1912; Thériot, 1928; Herter, 1928, 1933a, 1939b)

var. cymbifolium Hook. f. et Wils.

Bryum cavum

var. flaccidum (Brid.) B.S.G. (Bruch and others, 1839).

Syn. Bryum flaccidum Brid. (Bridel, 1826).

Distr. Southern South America (Bruch and others, 1839).

\section{B. capillisetum C. Muell.}

Bryum argenteum

B. capituliforme Warnst. (Warnstorf, 1915).

Distr. Southern South America (Warnstorf, 1915).
Bryum philippii C. Muell. ex Kindb. (Mueller ex Kindberg, 1888) nom. illeg.

Syn. Bryum capillare Hedw. var. cymbifolium Hook. f. et Wils. (Hooker, J. D. and Wilson, 1844).

Distr. Southern South America (Mitten, 1869; Gibert, 1873; Paris, 1904a; Herter, 1928, 1933a, b).

B. cephalozioides Card. (Cardot, 1906a)

Distr. Antarctic, peninsula region (Cardot, 1906a, 1908a: Steere, 1961a).

B. chilense Reichdt.

Bryum capillare

B. chiloense Thér. (Thériot, 1926).

Distr. Southern South America (Thériot, 1926).

B. chorizodontum Card. et Broth. (Cardot and Brotherus, 1923).

Distr. Southern South America (Cardot and Brotherus, 1923; Brotherus, 1924a).

B. chryseum Mitt. (Mitten, 1869).

Distr. Juan Femandez (Bartram, 1959; Robinson, 1975).

B. ciliatum (Hedw.) Dicks.

B. cirrhatum Hopp. et Hornsch. var. australe Card. (Cardot, 1905a).

Hedwigia ciliata

Distr. Southem South America (Cardot, 1905a, 1908a).

B. clavatum (Schimp.) C. Muell. (Mueller, 1848a).

Syn. Pohlia clavata Schimp. (Schimper, 1836).

Distr. Southern South America (Schimper, 1836; Mueller, 1848a; Montagne, 1850; Mitten, 1869; Paris, 1904a; Brotherus, 1924a).

B. climacodontium Par.

Anomobryum cygnicollum

B. cochlearifolium Card. et Broth. (Cardot and Brotherus, 1923) hom. illeg.

Distr. Southern South America (Donat, 1936a; Herzog, 1960; Seki, 1974).

South Georgia (Cardot and Brotherus, 1923; Brotherus, 1924a; Steere, 1961b).

B. coelophyllum Eaton (Eaton, 1892).

Distr. Southern South America (Eaton, 1892).

B. commutatum (Schimp.) Boul.

B. compactulum C. Muell.

Pohlia drummondii

Bryum argenteum

B. concavifolium Thér. (Thériot, 1926).

Distr. Southern South America (Costes, 1921 (as B. concarifolium); Thériot, 1926; Herzog, 1939).

B. conicum Hornsch.

B. controversum (Hedw.) P. Beauv.

Anomobryum conicum

B. coronatum Schwaegr. (Schwaegrichen, 1816).

Distr. Southern South America (Mueller, 1848a; Montagne, 1850; Jaeger, 1875; Seki, 1974).

B. corralense Herz. et Thér. (Herzog and Thériot in Herzog, 1938).

Distr. Southern South America (Herzog, 1938).

B. crassicaule Dus.

Bryum laevigatum

B. crassinervium Lor. (Lorentz, 1866).

Distr. Southern South America (Lorentz, 1866; Jaeger, 1875; Paris. 1904a; Costes, 1921;

B. cavum C. Muell. (Mueller, 1844a).
Distr. Southern South America
Brotherus, 1924a). 
B. crateris* Dix. (Dixon, 1920).

Distr. Antarctic, peninsula region (Dixon, 1920; Brotherus, 1924a; Steere, 1961a).

B. crispatum Dicks. ex With.

Rhabdoweisia crispata

Pohlia cruda

B. cruegeri Hamp. (Hampe in Mueller, 1848a).

Syn. Pohlia cruegeri (Hamp.) Andrews (Andrews, 1935).

Distr. Southern South America (Kühnemann, 1938).

B. cygnicollum C. Muell.

Anomobryum cygnicollum

B. decurrentinervium C. Muell. (Mueller, 1897a).

Distr. Southem South America (Mueller, 1897a; Paris, 1900a, 1904a; Brotherus, 1918, 1924a; Kühnemann, 1938).

B. delitescens Card. (Cardot, 1905a).

Distr. Southem South America (Cardot, 1905a, 1908a; Cardot and Brotherus, 1923; Brotherus, 1924a; Kühnemann, 1938).

B. demissum Dus. (Dusén, 1903a) nom. nud.

Distr. Southern South America (Dusén, 1903a).

B. densifolium Brid. (Bridel, 1827a).

Syn. Rhodobryum gracilescens $C$. Muell. (Mueller in Hampe, 1874).

Distr. Southern South America (Herter, 1933a; Hosseus, 1940; Herzog, 1952a).

B. denticulatinervium Ochi (Ochi, 1977).

Distr. Southern South America (Ochi, 1977).

B. diaphanum C. Muell. (Mueller, 1897a).

Distr. Southern South America (Mueller, 1897a, 1901a; Paris, 1904a).

B. dicarpum Bartr. (Bartram, 1946).

Distr. Southem South America (Bartram, 1946).

B. dichotomum auct. non Hedwig

Bryum bicolor

B. dissolutinerve C. Muell. (Mueller, 1882).

Distr. Southem South America (Mueller, 1882; Paris, 1904a; Kühnemann, 1938).

B. donati Herz. et Thér.

Bryum redboonii

B. donatii Thér. (Thériot, 1934b [1935]).

Distr. Southem South America (Thériot, 1934b; Herzog, 1957).

B. drummondii C. Muell.

Pohlia drummondii

B. elegantulum Lor. (Lorentz, 1864).

Distr. Southern South America (Lorentz, 1864, 1866; Jaeger, 1875; Paris, 1904a; Brotherus,
1924a, C; Espinosa B., 1941).

B. ellipsifolium C. Muell. (Mueller, 1890b).

Syn. Bryum myurella Dus. (Dusén, 1905b) hom. illeg. fide Ochi, 1972.

Distr. Southern South America (Dusén, 1903a, 1905b; Cardot, 1908a; Brotherus, 1924a; Kühnemann, 1938; Ochi, 1972; Seki, 1974).

B. ellipticum Mitt.

B. emergens C. Muell.

Brachymenium meyenianum var. ellipticum

Mielichhoferia emergens

* = Bryum pseudotriquetrum (Hedw.) Gaertn., Meyer et Scherb. fide Ochi (1979).
B. encalyptaceum C. Muell. (Mueller, 1882). Distr. Southern South America (Mueller, 1882; Paris, 1904a; Brotherus, 1924a; Kühnemann,
1938).

B. erubescens $\mathrm{C}$. Muell.

Brachymenium erubescens

B. erythrocarpoides C. Muell. et Hamp. (Mueller and Hampe, 1855).

Syn. Bryum reichertii Herz. (Herzog, 1926) fide Seki, 1974.

Distr. Southem South America (Herzog, 1926; Seki, 1974).

B. fabronia C. Muell. (Mueller, 1882).

Syn. Bryum fabroniae C. Muell. ex Kindb. (Mueller ex Kindberg, 1889) nom. inval.

Distr. Southern South America (Mueller, 1882; Paris, 1904a; Brotherus, 1924a; Kühnemann,
1938).

B. fabroniae C. Muell. ex Kindb.

B. fabronioides C. Muell.

Brachymenium fabronioides

B. felipponei Thér. (Thériot in Felippone, 1929 [1930]).

Distr. Southem South America (Felippone, 1929; Herter, 1933a).

B. fernandezianum Broth. (Brotherus, 1924b). Distr. Juan Fernandez (Skottsberg, 1914; Brotherus, 1924b; Espinosa B., 1941; Bartram,
1959; Robinson, 1975).

B. ferriviae C. Muell.

Bryum bicolor

B. filicaule Broth. (Brotherus, 1906a). Distr. Antarctic, continental region (Cardot, 1908a; Brotherus, 1924a; Steere, 1961a (as B.
antarcticum); Filson, 1966 (as B. antarcticum)).

B. filiforme Dicks.

Anomobryum filiforme

B. flaccidum Brid.

Bryum capillare var. flaccidum

B. flagellans C. Muell. (Mueller, 1901a [1900]).

Distr. Southern South America (Mueller, 1901a).

B. flagellicoma C. Muell. (Mueller, 1882).

Distr. Southern South America (Mueller, 1882; Paris, 1904a; Kühnemann, 1938).

B. flavo-pallidum Dus. (Dusén, 1903a).

Distr. Southern South America (Dusén, 1903a).

B. flexisetum Mitt. (Mitten, 1869).

Distr. Southern South America (Bartram, 1942, 1943).

B. fontanum (Hedw.) Gaertn., Meyer et Scherb.

Philonotis fontana

B. fuegianum Bartr. (Bartram, 1946).

Distr. Southern South America (Bartram, 1946).

B. fusco-mucronatum C. Muell. (Mueller, 1897a).

Distr. Southern South America (Mueller, 1897a; Paris, 1901a; Brotherus, 1924a; Herter,
1933a; Kühnemann, 1938).

B. ganophyllum C. Muell. (Mueller, 1882).

Distr. Southern South America (Mueller, 1882; Brotherus, 1904, 1924a; Kühnemann, 1938)

B. gaudichaudii (Schwaegr.) Spreng.

Leptotheca gaudichaudii 
B. gayanum Mont. (Montagne in Mueller, 1848a).

Syn. Bryum gayi Mont. ex Kindb. (Montagne ex Kindberg, 1888) nom. illeg.

Distr. Southem South America (Mueller, 1848a; Montagne, 1850, 1856; Mitten, 1869; Jaeger,

$$
\text { 1875; Paris, 1904a; Brotherus, 1924a). }
$$

B. gayi Mont. ex Kindb.

Bryum gayanum

B. gemmaceum Besch. (Bescherelle in Mueller, 1901a [1900] nom. nud.

Distr. Southem South America (Mueller, 1901a).

\section{B. gemmatum C. Muell. (Mueller, 1885).}

Distr. Southern South America (Mueller, 1885; Paris, 1904a; Cardot, 1905a, 1908a; Cardot and Brotherus, 1923; Brotherus, 1924a; Kühnemann, 1938; Seki, 1974).

B. gerlachei* Card. ex Broth. (Cardot ex Brotherus, 1904).

Syn. Webera gerlachei* Card. (Cardot, 1900).

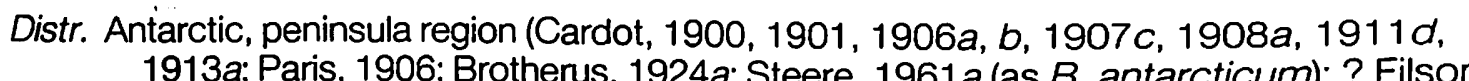
1913a; Paris, 1906; Brotherus, 1924a; Steere, 1961 (as B. antarcticum): ? Filson,
1966 (as B. antarcticum); Ochi, 1970 (as B. pseudotriquetrum for Racovitza 229a)).

B. gibertii C. Muell. (Mueller, 1901a [1900]).

Distr. Southem South America (Mueller, 1901a).

B. gilliesii Hook. (Hooker, 1829).

Syn. Pohlia gilliesii (Hook.) Mont. (Montagne, 1839b).

Brachymenium gilliesii (Hook.) Jaeg. (Jaeger, 1875).

Distr. Southem South America (Hooker, 1829; Montagne, 1839b; Mueller, 1848a, 1879a; Mitten, 1869; Jaeger, 1875; Kurtz, 1904; Paris, 1904a; Brotherus, 1924a; Hosseus, 1935a, 1937; Kühnemann, 1938; Herzog, 1957).

B. glaciale Brid.

Pohlia wahlenbergii var. glacialis

B. glauco-viride C. Muell. (Mueller, 1882).

Distr. Southem South America (Mueller, 1882; Paris, 1904a; Brotherus, 1924a; Kühnemann,
1938).

B. gracilisetum Hornsch. (Hornschuch, 1840).

Distr. Southem South America (Krieger, 1904; Herter, 1943).

B. gracili-torquescens C. Muell. (Mueller in Kindberg, 1891) nom. nud.

Distr. Southem South America (Paris, 1900a; Thériot, 1917b, 1918, 1921a; Campo, 1921;
Costes, 1921).

B. gracillimum Broth. (Brotherus in Felippone, 1909).

Distr. Southem South America (Felippone, 1909; Brotherus, 1924a; Herter, 1933a).

B. griffithianum Dicks.

B. griseum Dus. ex Thér.

Oedipodium griffithianum

Bryum argenteum

B. hallei Card.

B. hamatum* Dus. (Dusén, 1903a) nom. nud.

Bryum schleicheri var. patagonicum

Bryum perlimbatum pro parte

\section{B. hamipilum C. Muell. (Mueller, 1882)} Distr. Southem South America (Mueller, 1882; Paris, 1904a; Brotherus, 1918; Kühnemann.
1938).

B. hatcheri Dus. (Dusén, 1903a).

Distr. Southern South America (Dusén, 1903a; Cardot, 1908a; Kühnemann, 1938).

* = Bryum pseudotriquetrum (Hedw.) Gaertn., Meyer et Scherb. fide Ochi (1979).

$*$ * Bryum viridescens Welw. et Dub. fide Ochi (1977).
B. hauthalli C. Muell. (Mueller, 1897a).

Distr. Southern South America (Mueller, 1897a; Paris, 1900a, 1904a; Brotherus, 1924a; Kühnemann, 1938).

B. hedwigii Brotero

B. heimii (Hedw.) Dicks. ex With.

Hedwigia ciliata

Pottia heimii

B. heteroblepharum Card. et Broth. (Cardot and Brotherus, 1923).

Distr. Falkland Islands (Cardot and Brotherus, 1923; Brotherus, 1924a).

B. heteromallum (Hedw.) P. Beauv.

Ditrichum heteromallum

B. heterophyllum Card. et Broth. (Cardot and Brotherus, 1923) hom. illeg.

Distr. Southern South America (Cardot and Brotherus, 1923; Kühnemann, 1938).

B. heterostichum (Hedw.) Dicks.

B. hickenii Herz.

B. hieronymi C. Muell.

B. humile Mont.

Racomitrium heterostichum

Bryum pseudotriquetrum

Rhodobryum hieronym

Pohlia humilis

B. hypnoides L. ex With.

Racomitrium lanuginosum

B. imperfectum Card. (Cardot, 1900).

Distr. Southern South America (Herzog, 1957). Antarctic, peninsula region (Cardot, 1900, 1901, 1908a, 1911b, d, 1913a; Paris, $1904 a$ Brotherus, 1924a; Steere, 1961a; Robinson, 1972).

B. inclinatum (Brid.) Bland. (Blandow, 1809) hom. illeg.

Syn. Pohlia inclinata Sw. ex Brid. (Swartz ex Bridel, 1803)

Leskea inclinata (Brid.) Web. et Mohr (Weber and Mohr, 1803).

Distr. Southern South America (Montagne, 1850).

var. magellanicum Card. (Cardot, 1900).

Distr. Southern South America (Paris, 1904a; Cardot, 1905a, 1908a; Cardot and Brotherus 1923; Herzog, 1923; Brotherus, 1924a; Kühnemann, 1938).
Antarctic, peninsula region (Cardot, 1900, 1901; Bartram, 1957; Steere, 1961a).

B. inconnexum ${ }^{*}$ Card. (Cardot, 1900).

Distr. Antarctic, peninsula region (Cardot, 1900, 1901, 1906b, 1907c, 1908a; Paris, 1904a Brotherus, 1924a; Steere, 1961a (as B. antarcticum); ? Filson, 1966 (as $B$.

Antarctic, continental region (Greene, 1968a; Kuc, 1969; Robinson, 1972; Ochi, 1976).

var. fragile Hor. et Ando (Horikawa and Ando, 1961).

Distr. Antarctic, continental region (Horikawa and Ando, 1961)

var. tomentosum Card. (Cardot, 1906b).

Distr. Antarctic, peninsula region (Cardot, 1906b, 1907c, 1908a; Greene, 1968a). B. indicum Doz. et Molk.

Brachymenium indicum

B. inflexum C. Muell.

Pohlia inflexa

B. jaffuelii Thér.

see Bryum canariense and Bryum campylothecium

B. julaceum Schrad. ex Gaertn., Meyer et Scherb.

Anomobryum filiforme

B. kermesinum Herz. et Thér. (Herzog and Thériot in Herzog, 1939)

Distr. Southern South America (Herzog, 1939).

B. Klinggraeffi Schimp. (Schimper in Klinggraeff, 1858).

Distr. Southern South America (Crundwell and Nyholm, 1964).

* = Bryum pseudotriquetrum (Hedw.) Gaertn., Meyer et Scherb. fide Ochi (1979). 
B. korothkevieziae Sav. et Smirn. (Savich-Ljubitskaya and Smirnova, 1959).

Distr. Antarctic, continental region (Savich-Ljubitskaya and Smirnova, 1959; SavichLjubitskaya, 1978).

var. hollerbachii Sav. et Smirn. (Savich-Ljubitskaya and Smirnova, 1960).

Distr. Antarctic, continental region (Savich-Ljubitskaya and Smirnova, 1960; Greene, 1968a). B. lacustre Brid.

Bryoerythrophyllum recurvirostre

B. laetevirens Card. et Broth. (Cardot and Brotherus, 1923).

Distr. Southem South America (Cardot and Brotherus, 1923; Brotherus, 1924a; Herzog, 1954; Seki, 1974).

B. laevigatum Hook. f. et Wils. (Hooker, J. D. and Wilson, 1844).

Syn. Bryum levigatum Hook. f. et Wils. (Hooker and Wilson in Mitten, 1860) err. Bryum crassicaule Dus. (Dusén in Paris, 1904a) nom. nud.

Distr. Southem South America (Hooker, J. D. and Wilson, 1844; Wilson and Hooker, 1847; Mueller, 1848a, 1885; Sullivant, 1859; Jaeger, 1875; Bescherelle, 1889; Dusén, 1903a; Paris, 1904a; Cardot, 1905a, 1908a; Campo, 1921; Cardot and Brotherus, 1923; Brotherus, 1924a; Thériot, 1934b, 1935a; Kühnemann, 1938; Herzog, 1938 1939, 1954; Ochi, 1970; Seki, 1974).

Falkland Islands (Hooker, J. D. and Wilson, 1844; Wilson and Hooker, 1847; Mueller Brotherus, 1924a; Kühnemann, 1938).

B. lamprocarpum C. Muell. (Mueller, 1890a).

Syn. Zieria lamprocarpa (C. Muell.) Kindb. (Kindberg, 1891).

Plagiobryum lamprocarpum (C. Muell.) Par. (Paris, 1897)

Distr. Falkland Islands (Kühnemann, 1938).

South Georgia (Mueller, 1890a; Paris, 1897, 1905b; Cardot, 1906a, 1908a; Cardot and Brotherus, 1923; Brotherus, 1924a; Steere, 1961 b).

\section{B. lamprochaete Dus. (Dusén, 1903a).}

Distr. Southem South America (Dusén, 1903a; Cardot, 1908a; Cardot and Brotherus, 1923; Brotherus, 1924a; Kühnemann, 1938; Herzog, 1957).

\section{B. lamprocomum C. Muell. (Mueller, 1879a).}

Distr. Southem South America (Mueller, 1879a; Paris, 1904a; Brotherus, 1924a; Kühnemann, 1938).

B. lanuginosum (Hedw.) Brotero

Racomitrium lanuginosum

B. laticeps C. Muell. (Mueller, 1879a).

Distr. Southern South America (Mueller, 1879a; Kurtz, 1904; Paris, 1904a; Brotherus, $1924 a$ Hosseus, 1937; Kühnemann, 1938).

\section{B. lechleri C. Muell.}

Bryum andicola

B. leptotrichum C. Muell. (Mueller, 1897a).

Distr. Southern South America (Mueller, 1897a; Paris, 1900a, 1904a).

B. leuco-aristatum Dus. (Dusén, 1903a) nom. nud.

Distr. Southern South America (Dusén, 1903a).

B. levigatum Hook. f. et Wils.

Bryum laevigatum

B. liliputanum Dus. (Dusén, 1903a) nom. nud.

Distr. Southern South America (Dusén, 1903a).

B. linearifolium C. Muell. (Mueller, 1882).

Distr. Southern South America (Mueller, 1882; Paris, 1904a; Brotherus, 1924a).
B. litoris Card. (Cardot in Cardot and Brotherus, 1923).

Distr. Falkland Islands (Cardot and Brotherus, 1923; Brotherus, 1924a; Kühnemann, 1938).

B. lonchochaete Dus. (Dusén, 1903a) nom. nud.

Distr. Southern South America (Dusén, 1903a).

B. longidens Thér. (Thériot, 1926).

Distr. Southern South America (Campo, 1921; Thériot, 1926).

B. longirostre Brid.

B. Iorentzianum C. Muell.

Mnium rostratum

B. maceratum C. Muell. (Mueller, 1882).

Distr. Southem South America (Mueller, 1882; Paris, 1904a; Brotherus, 1918, 1924a; Kühnemann, 1938).

B. macro-carneum C. Muell. (Mueller, 1901a [1900]) nom. nud.

Distr. Southem South America (Mueller, 1901a).

B. macrochaete Card. (Cardot, 1905a).

Distr. Southern South America (Cardot, 1905a, 1908a; Cardot and Brotherus, 1923; Brotherus, 1924a; Donat, 1936a; Kühnemann, 1938; Herzog, 1954).

B. macropelma C. Muell. (Mueller, 1848a). Distr. Southem South America (Mueller, 1848a; Montagne, 1850; Mitten, 1869; Jaeger, 1875;
Paris, 1904a; Brotherus, 1924a).

B. macrophyllum Card. et Broth. (Cardot and Brotherus, 1923).

Syn. Bryum macrophyllum Card. et Broth. var. latifolium Broth. (Brotherus in Cardot and Brotherus, 1923) fide Ochi, 1967.

Bryum megalophyllum Card. (Cardot in Cardot and Brotherus, 1923) nom. nud. Distr. Falkland Islands (Cardot and Brotherus, 1923; Brotherus, 1924a; Kühnemann, 1938;
Ochi, 1967).

var. latifolium Broth.

B. macropoma C. Muell.

Bryum macrophyllum

B. macrosporum Bartr. (Bartram, 1946).

Distr. Southern South America (Bartram, 1946).

B. magellanicum (Sull.) C. Muell.

B. malachiticum C. Muell.

Brachymenium indicum

B. malacophyllum Broth. (Brotherus in Herzog, 1916a).

Distr. Southern South America (Cardot and Brotherus, 1923; Kühnemann, 1938; Herzog, 1954).

B. mancum C. Muell.

B. megalocarpum Hook.

Mielichhoferia manca

B. megalophyllum Card. Acidodontium megalocarpum

Bryum macrophyllum

B. megalothecium Thér. (Thériot, 1926).

Distr. Southern South America (Thériot, 1926; Bartram, 1942, 1943; Herzog, 1957).

B. meyenianum (Hamp.) C. Muell.

Brachymenium meyenianum

B. microglossum C. Muell. (Mueller, 1897a).

Distr. Southern South America (Mueller, 1897a; Paris, 1900a, 1904a; Brotherus, 1924a;
Kühnemann, 1938). 
B. micron C. Muell. (Mueller, 1882).

Distr. Southem South America (Mueller, 1882; Paris, 1904a; Brotherus, 1924a; Hosseus, 1935b, 1937; Kühnemann, 1938)

B. micro-pendulum C. Muell.

Bryum nano-pendulum

B. microphyllum Card. et Broth. (Cardot and Brotherus, 1923).

Distr. Southern South America (Cardot and Brotherus, 1923; Brotherus, 1924a; Kühnemann, 1938; Seki, 1974).

B. minusculum C. Muell. (Mueller, 1885).

Distr. Southem South America (Mueller, 1885; Dusén, 1903a, 1905d; Paris, 1904a; Cardot, 1908a; Brotherus, 1924a).

B. miserum Card. (Cardot, 1905a).

Distr. Falkland Islands (Cardot, 1905a, 1908a; Cardot and Brotherus, 1923; Brotherus, 1924a; Kühnemann, 1938; Herzog, 1939).

B. montevidense Broth. (Brotherus in Felippone, 1928 [1929]).

Distr. Southem South America (Felippone, 1928; Herter, 1933a).

B. montevidense Thér.

B. mucronatum C. Muell. (Mueller, 1901a [1900]) nom. nud.

Distr. Southem South America (Mueller, 1901a).

B. murale (Hedw.) L. ex With.

B. myurella Dus.

B. nanocoma C. Muell.

Tortula muralis

Bryum ellipsifolium

Bryum capillare

\section{B. nano-pendulum C. Muell. (Mueller, 1882).}

Syn. Bryum micro-pendulum C. Muell. (Mueller, 1879b) hom. illeg. Distr. Southem South America (Mueller, 1879a, 1882; Paris, 1904a; Brotherus, 1924a;
Kühnemann, 1938).

B. nevadense C. Muell.

Pohlia nevadensis

\section{B. nivale C. Muell. (Mueller, 1848a).}

Distr. Southem South America (Mueller, 1848a; Montagne, 1850; Sullivant, 1859; Mitten 1869; Jaeger, 1875; Paris, 1904a; Brotherus, 1924a).

B. nutans (Hedw.) Turn.

var. minus Hook.

Pohlia nutans

B. obliquatulum Dus. (Dusén in Paris, 1904a) nom. nud.

Distr. Southem South America (Paris, 1904a).

B. obliquum C. Muell. (Mueller, 1890a).

Distr. South Georgia (Mueller, 1890a; Paris, 1904a; Cardot, 1908a; Steere, 1961b).

B. obscurum Card. et Broth. (Cardot and Brotherus, 1923) hom. illeg. Distr. Southem South America (Cardot and Brotherus, 1923; Brotherus, 1924a; Kühnemann,
1938)

B. oediloma C. Muell. (Mueller in Brotherus, 1895) Distr. Southern South America (Bartram, 1965).

B. ongulense* Hor. et Ando (Horikawa and Ando, 1961).

Distr. Antarctic, continental region (Horikawa and Ando, 1961; Savich-Ljubitskaya and Z.
Smirnova, 1972).

* = Bryum pseudotriquetrum (Hedw.) Gaertn., Meyer et Scherb. fide Ochi (1979).
B. orbiculatifolium Card. et Broth. (Cardot and Brotherus, 1923).

Distr. Southern South America (Cardot and Brotherus, 1923; Brotherus, 1924a; Herzog, 1954; Seki, 1974).

B. orthodontioides C. Muell. (Mueller, 1848a). Distr. Southern South America (Herter, 1943).

B. orthothecium Card. et Broth. (Cardot and Brotherus, 1923).

Distr. Southern South America (Cardot and Brotherus, 1923; Brotherus, 1924a).

B. pachyneuron Dus. (Dusén in Paris, 1904a) nom. nud. Distr. Southern South America (Paris, 1904a).

B. pallescens Schleich. ex Schwaegr. (Schleicher ex Schwaegrichen, 1816). Syn. Bryum pseudotriquetrum (Hedw.) Gaertn., Meyer et Scherb. ssp. pallescens (Schwaegr.) Dix. (Dixon, 1924b).

Distr. Southern South America (Wilson and Hooker, 1847).

Falkland Islands (Wilson and Hooker, 1847: Kühnemann, 1938).

B. pallidipes C. Muell. (Mueller, 1897a). Distr. Southern South America (Mueller, 1897a; Paris, 1900a, 1904a; Brotherus, 1924a;
Kühnemann, 1938).

B. pallido-viride Card. (Cardot, 1905a).

Distr. Southern South America (Cardot, 1905a, 1908a; Kühnemann, 1938; Herzog, 1957).

B. palustre (Hedw.) Gaertn., Meyer et Scherb.

B. papillosum C. Muell.

Aulacomnium palustre

B. parvirete Dus. (Dusén in Paris, 1904a) nom. nud.

Pohlia papillosa

Distr. Southern South America (Paris, 1904a).

B. parvulum Card. (Cardot, 1906a) hom. illeg.

Distr. Southern South America (Cardot, 1908a; Cardot and Brotherus, 1923; Brotherus,

1924a; Kunhnemann, 1938).
South Georgia (Cardot, 1906a, 1908a; Brotherus, 1924a; Steere, 1961b).

B. parvulum Broth. (Brotherus in Felippone, 1928 [1929]) hom. illeg.

Distr. Southern South America (Felippone, 1928; Herter, 1933a).

B. patens Hook. f. et Wils.

Byum rigidum

B. pauperculum Bartr. (Bartram, 1946).

Distr. Southern South America (Bartram, 1946).

B. pellucens Hook.

Orthodontium pellucens

B. perangustidens ${ }^{*}$ Card. (Cardot, $\left.1911 b, d\right)$.

Distr. Antarctic, peninsula region (Cardot, 1911b, d, 1913a; Brotherus, 1924a; Steere, 1961a).

B. perlimbatum Card. (Cardot, 1905a).

Syn. Bryum hamatum Dus. (Dusén, 1903a) nom. nud. pro parte fide Seki, 1974.

Distr. Southern South America (Dusén, 1903a; Paris, 1906; Cardot and Brotherus, 1923; Herzog, 1923, 1954; Seki, 1974).

Falkland Islands (Cardot, 1905a, 1908a; Cardot and Brotherus, 1923; Brotherus, 1924a Kühnemann, 1938: Ochi, 1970, 1972).

* = Bryum pseudotriquetrum (Hedw.) Gaertn., Meyer et Scherb. fide Ochi (1979). 
B. pertenerumt Thér. (Thériot, 1926).

Distr. Southem South America (Campo, 1921; Thériot, 1926).

B. pertomentosum Dus. (Dusén in Paris, 1904a) nom. nud.

Distr. Southem South America (Paris, 1904a).

B. pertriste C. Muell. (Mueller, 1882).

Syn. Bryum triste C. Muell. (Mueller, 1879a) hom. illeg.

Distr. Southem South America (Mueller, 1879a, 1882; Paris, 1904a; Brotherus, 1924a).

B. phallus C. Muell. (Mueller, 1875)

Distr. Southem South America (Mueller, 1875; Jaeger and Sauerbeck, 1879b; Paris, 1904a). B. philippianum C. Muell.

B. philippii C. Muell. ex Kindb.

Byum capillare

B. philonoteum C. Muell.

Bryum capillare

Mniobryum philonoteum

B. philonotoides C. Muell. (Mueller, 1901a [1900]).

Distr. Southern South America (Mueller, 1901a).

B. philonotula C. Muell. (Mueller, 1901a [1900]) nom. nud.

Distr. Southem South America (Mueller, 1901a).

B. piriforme Par.

B. platense C. Muell.

Leptobryum pyriforme

Rhodobryum platense

B. platyphylloides C. Muell. (Mueller, 1879a). Distr. Southem South America (Mueller, 1879a; Kurtz, 1904; Paris, 1904a; Brotherus, 1924a;
Hosseus, 1935b, 1937; Kühnemann, 1938).

B. platyphyllum (Schwaegr.) C. Muell. (Mueller, 1848a).

Syn. Pohlia platyphylla Schwaegr. (Schwaegrichen, 1842).

Distr. Southem South America (Schwaegrichen, 1842; Mueller, 1848a; Montagne, 1850 Mitten, 1869; Jaeger, 1875; Paris, 1904a; Brotherus, 1924a).

B. pluricolor Dus. ex Thér. (Dusén ex Thériot, 1917b).

Distr. Southern South America (Thériot, 1917b).

B. polycarpum Mitt.

B. pomiforme (Hedw.) With.

Pohlia polycarpa

B. porteri Thér. (Thériot, 1926).

Distr. Southern South America (Thériot, 1926).

B. posthumum C. Muell. (Mueller, 1897a).

Syn. Bryum pseudogemmatum Card. (Cardot in Cardot and Brotherus, 1923) nom. nud.

Distr. Southem South America (Mueller, 1897a; Paris, 1900a, 1904a; Brotherus, 1924a;

Kühnemann, 1938).
Falkland Islands (Cardot and Brotherus, 1923; Kühnemann, 1938).

B. pseudo-acidodontium C. Muell. (Mueller, 1882).

Distr. Southem South America (Mueller, 1882; Paris, 1904a; Brotherus, 1924a; Kühnemann,

B. pseudo-fallax Card. et Broth. (Cardot and Brotherus, 1923).

Distr. Falkland Islands (Cardot and Brotherus, 1923; Brotherus, 1924a; Kühnemann, 1938). B. pseudogemmatum Card.

Bryum posthumum
B. pseudo-micron C. Muell. (Mueller, 1882).

Distr. Southern South America (Mueller, 1882; Paris, 1904a; Hosseus, 1935b, 1937

Kühnemann, 1938)

B. pseudothyridium Bartr. (Bartram, 1946).

Distr. Southern South America (Bartram, 1946)

B. pseudotriquetrum (Hedw.) Gaertn., Meyer et Scherb. (Gärtner and others, 1802).

Syn. Mnium pseudo-triquetrum Hedw. (Hedwig, 1801).

Hypnum pseudotriquetrum (Hedw.) Web. et Mohr (Weber and Mohr, 1807) Bryum ventricosum Relh. var. pseudotriquetrum (Hedw.) Hamp. (Hampe, 1837a) nom. illeg.

Hypnum ventricosum Web. et Mohr (Weber and Mohr, 1803) nom. illeg.

Bryum bimum (Scherb.) Turn. ssp. pseudotriquetrum (Hedw.) Dix. (Dixon, 1896) hom.$$
\text { Billeg. }
$$

Bryurn yendangaianum Card. (Cardot in Cardot and Brotherus, 1923) nom. nud. Bryum hickenii Herz. (Herzog, 1926) fide Seki, 1974.

Distr. Southern South America (Cardot and Brotherus, 1923; Herzog, 1926; Hosseus, 1938c d; Kühnemann, 1938; Bartram, 1952; Ochi, 1970; Seki, 1974).

ssp. bimum (Schreb.) Hartm. (Hartman, 1849).

Syn. Mnium bimum Schreb. (Schrebius, 1802).

Bryum bimum (Schreb.) Turn. (Turner, 1804a).

By ventricosum Relh. var. bimum (Schreb.) Hamp. (Hampe, 1837a).

Distr. Southern South America (Dusén, 1903a; Cardot, 1908a; Kühnemann, 1938).

ssp. pallescens (Schwaegr.) Dix.

Bryum pallescens

B. puconense Herz. et Thér. (Herzog and Thériot in Herzog, 1938).

Distr. Southern South America (Herzog, 1938).

B. pulchellum Hedw.

B. pulvinatum C. Muell.

Mniobryum pulchellum

Pohlia nutans

B. pumilum C. Muell. (Mueller in Brotherus, 1918) nom. nud.

Distr. Southem South America (Brotherus, 1918; Kühnemann, 1938).

B. purpuratum C. Muell. (Mueller, 1882).

Distr. Southern South America (Mueller, 1882; Paris, 1904a; Brotherus, 1924a; Kühnemann, 1938).

B. pycnodictyum C. Muell. (Mueller, 1901a [1900]).

Distr. Southern South America (Mueller, 1901a).

B. pycnotylum Card. et Broth. (Cardot and Brotherus, 1923).

Distr. Southern South America (Cardot and Brotherus, 1923; Brotherus, 1924a).

B. pyriforme (Hedw.) Gaertn., Meyer et Scherb.

var. antarcticum C. Muell.

Leptobryum pyriforme

var. fuegianum C. Muell.

Leptobryum pyriforme var. antarcticum

Leptobnyum pyriforme var. fuegianum

B. pyrrhothrix C. Muell. (Mueller, 1882). Distr. Southern South America (Mueller, 1882; Paris, 1904a; Brotherus, 1924a; Kühnemann,
1938).

B. raminerve C. Muell. (Mueller, 1901a [1900]) nom. nud.

Distr. Southern South America (Mueller, 1901a).

$\dagger=$ Bryum funkii Schwaegr. fide Ochi (1977). 
B. redboonii Donat (Donat, 1936a).

Syn. Bryum donati Herz. et Thér. (Herzog and Thériot in Donat, 1936b) hom. illeg.

Distr. Southem South America (Donat, 1936a, b; Kühnemann, 1938).

B. reichertii Herz.

Bryum erythrocarpoides

B. revolutum C. Muell. (Mueller, 1879a).

Distr. Southem South America (Mueller, 1879a; Kurtz, 1904; Paris, 1904a; Brotherus, 1924a; Hosseus, 1935b, 1937; Kühnemann, 1938).

B. rhizoblastum Card. et Broth. (Cardot and Brotherus, 1923).

Distr. Falkland Islands (Cardot and Brotherus, 1923; Brotherus, 1924a; Kühnemann, 1938).

B. rigidulum (Hedw.) Dicks.

Didymodon rigidulus

B. rigidum (Hornsch.) C. Muell. (Mueller, 1848a) hom. illeg.

Syn. Mnium rigidum Hornsch. (Hornșchuch, 1840).

Rhodobryum rigidum (Hornsch.) Par. (Paris, 1897).

Bryum patens Hook. f. et Wils. (Hooker, J. D. and Wilson, 1844) hom. illeg.

Distr. Southem South America (Homschuch, 1840; Mueller, 1848a; Mitten, 1869; Gibert, 1873; Jaeger, 1875; Hampe, 1879; Paris, 1897, 1904a; Herter, 1928, 1933a).

B. rigochaete Dus. (Dusén, 1903a).

Distr. Southem South America (Dusén, 1903a; Cardot, 1908a; Cardot and Brotherus, 1923; Brotherus, 1924a; Kühnemann, 1938).

B. rimicola Card. et Broth. (Cardot and Brotherus, 1923).

Distr. Falkland Islands (Cardot and Brotherus, 1923; Brotherus, 1924a; Kühnemann, 1938).

B. rivale C. Muell. (Mueller, 1879a).

Distr. Southem South America (Mueller, 1879a, 1882; Paris, 1904a; Kühnemann, 1938).

B. roivainenii Bartr. (Bartram, 1946).

Distr. Southern South America (Bartram, 1946).

B. roseolum C. Muell. (Mueller, 1879a).

Syn. Rhodobryum roseolum (C. Muell.) Par. (Paris, 1897).

Distr. Southem South America (Mueller, 1879a; Paris, 1897, 1904a; Brotherus, 1924a; Kühnemann, 1938).

B. rostratum (Schrad.) Sm.

Mnium rostratum

B. rosulans C. Muell. (Mueller, 1897a).

Distr. Southern South America (Mueller, 1897a; Paris, 1900a, 1904a; Brotherus, 1924a).

B. rubellicaule C. Muell. or Dus. (Mueller or Dusén in Neger, 1899) nom. nud.

Distr. Southem South America (Neger, 1899).

B. rubinerve Card. et Broth. (Cardot and Brotherus, 1923).

Distr. Falkland Islands (Cardot and Brotherus, 1923; Brotherus, 1924a; Kühnemann, 1938).

B. rurale (Hedw.) L. ex With.

Tortula ruralis

B. sabuletorum Broth. et Card. (Brotherus and Cardot in Cardot and Brotherus, 1923).

Distr. Southem South America (Cardot and Brotherus, 1923; Brotherus, 1924a; Kühnemann, 1938).

B. schilleri Herz. (Herzog, 1916b).

Distr. Southem South America (Herzog, 1916b). 
B. schleicheri Cand. (de Candolle, 1815).

Syn. Mnium schleicheri (Cand.) Brid. (Bridel, 1817).

Bryum turbinatum (Hedw.) Turn. ssp. schleicheri (Cand.) Kindb. (Kindberg, 1883).

Bryum turbinatum (Hedw.) Turn. var. schleicheri (Cand.) Fuernr. (Fürnrohr, 1829).

Distr. Southern South America (Amann, 1924; Thériot, 1926).

var. patagonicum Broth. (Brotherus in Cardot and Brotherus, 1923).

Syn. Bryum hallei Card. (Cardot in Cardot and Brotherus, 1923) nom. nud.

Distr. Southern South America (Cardot and Brotherus, 1923; Kühnemann, 1938).

B. schnyderi C. Muell. (Mueller, 1882).

Distr. Southern South America (Mueller, 1882; Paris, 1904a; Kühnemann, 1938).

B. semi-reticulatum C. Muell.

Anomobryum semireticulatum

B. senopyxis C. Muell. (Mueller, 1882).

Distr. Southern South America (Mueller, 1882; Brotherus, 1924a (as B. stenopyxis); Kühnemann, 1938).

B. senopyxoides C. Muell. (Mueller, 1901a [1900]) nom. nud.

Distr. Southem South America (Mueller, 1901a).

B. siplei Bartr.

Bryum argenteum

B. skottsbergii Card. et Broth. (Cardot and Brotherus, 1923).

Distr. Southern South America (Cardot and Brotherus, 1923; Brotherus, 1924a; Kühnemann, 1938).

B. spegazzinii C. Muell. (Mueller, 1885).

Distr. Southern South America (Mueller, 1885; Dusén, 1903a; Paris, 1904a; Cardot, 1908a; Cardot and Brotherus, 1923; Brotherus, 1924a; Kühnemann, 1938).

Falkland Islands (Cardot and Brotherus, 1923; Kühnemann, 1938).

B. sphagnadelphus $\mathrm{C}$. Muell.

Pohlia nutans

B. splachnobryoides C. Muell. (Mueller, 1882).

Distr. Southern South America (Mueller, 1882; Paris, 1904a; Brotherus, 1924a; Hosseus, 1935b, 1937; Kühnemann, 1938).

B. steffenii Dus. ex Herz. (Dusén ex Herzog, 1926).

Distr. Southem South America (Paris, 1904a; Herzog, 1926; Seki, 1974).

B. subclavatum Thér. (Thériot, 1926).

Distr. Southern South America (Campo, 1921; Thériot, 1926).

B. subgracillimum Thér. (Thériot, 1926).

Distr. Southern South America (Thériot, 1926).

B. sublamprocarpum Card. et Broth. (Cardot and Brotherus, 1923).

Distr. Southern South America (Cardot and Brotherus, 1923; Brotherus, 1924a).

B. submicrolaevigatum Card. et Broth. (Cardot and Brotherus, 1923).

Distr. Southern South America (Cardot and Brotherus, 1923; Brotherus, 1924a; Kühnemann, 1938).

B. subpercurrente Thér. (Thériot in Felippone, 1930).

Syn. Bryum montevidense Thér. (Thériot in Felippone, 1928 [1929]) hom. illeg.

Distr. Southern South America (Felippone, 1928, 1930; Herter, 1933a).

B. synoico-crudum C. Muell.

Pohlia cruda 
B. tenuicaule Mont. (Montagne, 1845a).

Distr. Southem South America (Montagne, 1845a, 1846, 1850, 1856; Mueller, 1848a; Mitten, 1869; Jaeger, 1875; Paris, 1904a; Costes, 1921; Brotherus, 1924a).

B. tenuirete Dus. ex Card. (Dusén ex Cardot, 1905a).

Distr. Southern South America (Dusén, 1903a; Paris, 1904a; Cardot, 1905a, 1908a; Brotherus, 1924a).

B. terminale C. Muell.

Anomobryum terminale

B. torquescens B.S.G.

Bryum capillare ssp. torquescens

B. tortuosum (Hedw.) L. ex With.

Tortella tortuosa

B. triste C. Muell.

Bryum pertriste

B. truncatulum L. ex With.

Pottia truncata

B. truncatum (Hedw.) Brotero

Pottia truncata

B. truncorum (Brid.) Brid. (Bridel, 1819).

Syn. Mnium truncorum Brid. (Bridel, 1817).

Distr. Southern South America (Kühnemann, 1938).

Falkland Islands (Wilson and Hooker, 1847).

B. turbinatum (Hedw.) Turn. (Turner, 1804a).

Syn. Mnium turbinatum Hedw. (Hedwig, 1801).

Hypnum turbinatum (Hedw.) Web. et Mohr (Weber and Mohr, 1803).

Mnium bimum Schreb. var. turbinatum (Hedw.) Wahlenb. (Wahlenberg, 1826).

Distr. Southem South America (Herzog, 1923; Seki, 1974).

ssp. schleicheri (Cand.) Kindb.

Bryum schleicheri

var. patagonicum Broth. (Brotherus in Herzog, 1923) nom. nud.

Distr. Southem South America (Herzog, 1923).

var. schleicheri (Cand.) Fuernr.

Bryum schleicheri

B. uliginosum (Brid.) B.S.G. (Bruch and others, 1839).

Syn. Cladodium uliginosum Brid. (Bridel, 1827a).

Distr. Southern South America (Ochi, 1967).

B. unguiculatum (Hedw.) With.

Barbula unguiculata

B. uruguense C. Muell. (Mueller, 1901a [1900]) nom. nud.

Distr. Southem South America (Mueller, 1901a).

B. uspallatense Herz. (Herzog, 1916b).

Distr. Southern South America (Herzog, 1916b).

var. turgidum Herz. (Herzog, 1916b).

Distr. Southern South America (Herzog, 1916b).

B. uvidum Card. et Broth. (Cardot and Brotherus, 1923).

Distr. Falkland Islands (Cardot and Brotherus, 1923; Brotherus, 1924a; Kühnemann, 1938).

B. vagans Hook. f. et Wils.

Philonotis vagans

B. valdiviae Lor. (Lorentz, 1864).

Distr. Southem South America (Lorentz, 1864, 1866; Jaeger, 1875; Brotherus, 1924a).

B. valparaisense Thér. (Thériot, 1917b).

Distr. Southem South America (Thériot, 1917b; Costes, 1921; Brotherus, 1924a; Herzog, 1938). 
B. ventricosum Dicks. ex Relh.

Bryum pseudotriquetrum

var. bimum (Schreb.) Hamp.

Bryum pseudotriquetrum ssp. bimum

var. pseudotriquetrum (Hedw.) Hamp.

Bryum pseudotriquetrum

B. vernicosum Dus. (Dusén, 1903a) hom. illeg.

Distr. Southern South America (Dusén, 1903a; Cardot, 1908a; Kühnemann, 1938; Herzog, 1957).

B. violaceum Crundw. et Nyh. (Crundwell and Nyholm, 1963).

Distr. Southern South America (Crundwell and Nyholm, 1963, 1964).

B. viridatum C. Muell.

Pohlia cruda

B. vulcanicum Dus. (Dusén in Paris, 1904a) nom. nud.

Distr. Southern South America (Paris, 1904a).

B. wahlenbergii (Web. et Mohr) Schwaegr.

Pohlia wahlenbergii

B. weberoides Card. et Broth. (Cardot and Brotherus, 1923).

Distr. Southern South America (Cardot and Brotherus, 1923; Brotherus, 1924a; Kühnemann, 1938).

B. weisii Dicks. ex With.

Ditrichum heteromallum

B. wilsonii Mitt.

Leptobryum wilsonii

B. yendangaianum Card.

Bryum pseudotriquetrum

B. zeballosicum Card. et Broth. (Cardot and Brotherus, 1923).

Distr. Southern South America (Cardot and Brotherus, 1923; Brotherus, 1924a; Kühnemann, 1938).

\section{Bryum sp.}

Syn. Hypnum aquicola C. Muell. (Mueller, 1879a).

Sematophyllum aquicola (C. Muell.) Kindb. (Kindberg, 1888).

Distr. Southern South America (Mueller, 1879a; Paris, 1900a, 1904a; Kurtz, 1904; Hosseus, 1935b; Kühnemann, 1938).

Bucklandia hom. illeg.

B. bartramii Roiv.

Racomitrium bartramii

\section{Bucklandiella}

B. bartramii Roiv.

Racomitrium bartramii

\section{Callicostella*}

C. incurva (Hornsch.) Aongstr.

Hookeriopsis incurva

C. scabriuscula (C. Muell.) Jaeg. (Jaeger, 1877).

Syn. Hookeria scabriuscula C. Muell. (Mueller, 1848b).

Distr. Southern South America (Mueller, 1848b, 1851a; Mitten, 1869; Jaeger, 1877; Paris, 1904b; Brotherus, 1925).

\section{Calliergidium}

C. austro-stramineum (C. Muell.) Bartr. (Bartram, 1946).

Syn. Hypnum austro-stramineum C. Muell. (Mueller, 1890a).

Drepanocladus austro-stramineus (C. Muell.) Broth. ex Par. (Brotherus ex Paris, 1909).

Distr. Southern South America (Bartram, 1946; Seki, 1974).

South Georgia (Mueller, 1890a; Paris, 1904c; Cardot, 1908a; Brotherus, 1925; Dixon, 1932; Steere, 1961b).

* According to Crosby (1975) the generic name should be Schizomitrium B.S.G. 
Antarctic, peninsula region (Cardot, 1900, 1906b; Robinson, 1972).

var. gracillimum (C. Muell.) Wijk et Marg. (van der Wijk and Margadant, 1965).

Syn. Hypnum austro-stramineum C. Muell. var. gracillimum C. Muell. (Mueller, 1890a). Drepanocladus austro-stramineus (C. Muell.) Broth. ex Par. var. gracillimus (C. Mueil.) Card. et Broth. (Cardot and Brotherus, 1923).

Distr. South Georgia (Mueller, 1890a; Cardot, 1908a; Dixon, 1932; Steere, 1961b). Antarctic, peninsula region (Cardot, 1901, 1908a; Steere, 1961a).

var. minus (Card.) Wijk et Marg. (van der Wijk and Margadant, 1965).

Syn. Hypnum austro-stramineum C. Muell. var. minus Card. (Cardot, 1906b).

Drepanocladus austro-stramineus (C. Muell.) Broth- ex Par. var. minus (Card.) Steere (Steere, 1961a).

Distr. Antarctic, peninsula region (Çardot, 1906b, 1907c, 1908a; Steere, 1961a).

var. subfluitans (C. Muell.) Wijk et Marg. (van der Wijk and Margadant, 1965).

Syn. Hypnum austro-stramineum C. Muell. var. subfluitans C. Muell. (Mueller, 1890a).

Drepanocladus austro-stramineus (C. Muell.) Broth. ex Par. var. subfluitans (C. Muell.) Steere (Steere, 1961b).

Distr. South Georgia (Mueller, 1890a; Cardot, 1906a, 1908a; Steere, 1961b).

\section{Calliergon}

C. cuspidatum (Hedw.) Kindb.

Calliergonella cuspidata

C. sarmentosum (Wahlenb.) Kindb. (Kindberg, 1894).

Syn. Hypnum sarmentosum Wahlenb. Wahlenberg, 1812).

Amblystegium sarmentosum (Wahlenb.) De Not. (De Notaris, 1867).

Acroceratium sarmentosum (Wahlenb.) Mitt. (Mitten in Reid and Ridley, 1888).

Acrocladium sarmentosum (Wahlenb.) Richs. et Wall. (Richards and Wallace, 1950).

Distr. Southem South America (Cardot and Brotherus, 1923; Brotherus, 1925; Kühnemann, 1938; Bartram, 1946).

South Georgia (Cardot, 1906a, 1908a; Brotherus, 1925; Dixon, 1932; Steere, 1961b; Newton, 1979b).

Antarctic, peninsula region (Cardot, 1906a, 1908a; Brotherus, 1925; Steere, 1961a; Robinson, 1972).

C. stramineum (Brid.) Kindb. (Kindberg, 1894).

Syn. Hypnum stramineum Dicks. ex Brid. (Dickson ex Bridel, 1801b).

Amblystegium stramineum (Brid.) De Not. (De Notaris, 1867).

Acrocladium stramineum (Brid.) Richs. et Wall. (Richards and Wallace, 1950).

Distr. Southem South America (Donat, 1936a; Seki, 1974).

\section{Calliergonella}

C. cuspidata (Hedw.) Loesk. (Loeske, 1911).

Syn. Hypnum cuspidatum Hedw. (Hedwig, 1801).

Acrocladium cuspidatum (Hedw.) Lindb. (Lindberg, 1879).

Calliergon cuspidatum (Hedw.) Kindb. (Kindberg, 1894).

Cuspidaria fulvo-acuta C. Muell. (Mueller, 1897a).

Hypnum fulvo-acutum (C. Muell.) Par. (Paris, 1900a).

Distr. Southem South America (Mueller, 1897a; Paris, 1900a, 1904C; Brotherus, 1925; Kühnemann, 1938).

Calliergonella hom. illeg.

C. complanata Card. et Broth.

C. nitida (Hook. f. et Wils.) Card.

\section{Calymperes}

C. fasciculatum (Hook. et Grev.) Mitt. 
C. glaziovii Hamp. (Hampe, 1878).

Distr. Southern South America (Hosseus, 1940).

\section{Calyptopogon}

C. crispatulus (C. Muell.) Broth.

Calyptopogon mnioides

C. mnioides (Schwaegr.) Broth. (Brotherus, 1902).

Syn. Barbula mnioides Schwaegr. (Schwaegrichen, 1842).

Tortula mnioides (Schwaegr.) Mont. (Montagne, 1850).

Streptopogon mnioides (Schwaegr.) Mitt. (Mitten, 1860).

Syntrichia crispatula C. Muell. (Mueller, 1897a).

Streptopogon crispatulus (C. Muell.) Par. (Paris, 1898).

Barbula crispatula C. Muell. ex Par. (Mueller ex Paris, 1900a) nom. inval.

Calyptopogon crispatulus (C. Muell.) Broth. (Brotherus, 1902).

Platylomidium crispatula C. Muell. (Mueller in Salmon, 1903) nom. nud.

Distr. Southern South America (Schwaegrichen, 1842; Montagne, 1850; Mitten, 1860, 1869; Lorentz, 1866; Jaeger, 1873; Mueller, 1897a; Paris, 1898, 1904a; Brotherus, 1902, 1924a; Salmon, 1903; Dusén, 1906; Cardot, 1908a; Thériot, 1921a; Cardot and Brotherus, 1923; Herzog, 1923, 1938, 1939, 1954, 1960; Reimers, 1926;

Kühnemann, 1938; Bartram, 1952; Seki, 1974).

var. prostratus (Mont.) Par.

Tortula prostrata

\section{Camptochaete}

C. arbusculans Broth. ex Bartr.

Camptochaete orbiculata

C. gracilis (Hook. f. et Wils.) Par. (Paris, 1894).

Syn. Hypnum gracile Hook. f. et Wils. (Hooker, J. D. and Wilson, 1844) hom. illeg. Isothecium gracile Hook. f. et Wils. (Hooker and Wilson in Wilson, 1854).

Stereodon gracilis (Hook. f. et Wils.) Mitt. (Mitten, 1860).

Microthamnium gracile (Hook. f. et Wils.) Jaeg. (Jaeger, 1878).

Porotrichum gracile (Hook. f. et Wils.) Mitt. (Mitten, 1883).

Hypnum gracilescens C. Muell. (Mueller, 1851a) hom. illeg.

Hypnum micans Wils. var. laxum Hook. f. (Wilson and Hooker, 1847) fide Mueller, $1851 a$.

Distr. Southern South America Wilson and Hooker, 1847; Paris, 1904a; Brotherus, 1907, 1925).

C. orbiculata (Thér.) Robins. (Robinson, 1970b).

Syn. Weymouthia orbiculata Thér. (Thériot, 1934b [1935]).

Camptochaete arbusculans Broth. ex Bartr. (Brotherus ex Bartram, 1946) fide Robinson, 1970b.

Distr. Southern South America (Paris, 1904a; Brotherus, 1925; Roivainen, 1934; Thériot, 1934b; Herzog, 1938, 1940, 1957, 1960; Bartram, 1946; Robinson, 1970b).

\section{Camptodontium}

C. brotheri Dus.

Camptodontium cryptodon

var. grandirete Dus. (Dusén, 1907).

Distr. Southern South America (Dusén, 1907; Cardot, 1908a).

C. cryptodon (Mont.) Reim. (Reimers, 1936).

Syn. Weisia cryptodon Mont. (Montagne, 1845a).

Dicranum cryptodon (Mont.) Mitt. (Mitten, 1869).

Dicranoweisia cryptodon (Mont.) Par. (Paris, 1895).

Grimmia cryptodon (Mont.) Hamp. (Hampe in Reimers, 1936) nom. inval.

Dicranoweisia tenella Dus. (Dusén, 1903a) nom. nud.

Camptodontium brotheri Dus. (Dusén, 1905b).

Bryoporteria chilensis Thér. (Thériot, 1933).

Distr. Southern South America (Montagne, 1845a, 1850; Mueller, 1849; Mitten, 1869; Jaeger, 
1870; Paris, 1895, 1904a, b; Neger, 1899; Dusén, 1903a, 1905b, 1907; Cardot, 1908a; Campo, 1921; Brotherus, 1924a; Reimers, 1926, 1936; Thériot, 1933;

Roivainen, 1934; Herzog, 1938, 1954, 1957, 1960; Seki, 1974).

\section{Campylium}

C. polygamum (B.S.G.) C. Jens. (Jensen in Lange and Jensen, 1887).

Syn. Amblystegium polygamum B.S.G. (Bruch and others, 1853).

Hypnum polygamum (B.S.G.) Wils. (Wilson, 1855).

Chrysohypnum polygamum (B.S.G.) Loesk. (Loeske, 1903).

Distr. Southem South America (Cardot and Brotherus, 1923; Kühnemann, 1938; Seki, 1974).

C. radicale (P. Beauv.) Grout (Grout, 19.29).

Syn. Hypnum radicale P. Beauv. (Palisot de Beauvois, 1805a).

Amblystegium radicale (P. Beauv.) Mitt. (Mitten, 1869) hom. illeg.

Distr. Southem South America (Bizot and Piovano, 1953; Piovano, 1954).

C. squarroso-byssoides C. Muell. (Mueller, 1897a).

Syn. Hypnum squarroso-byssoides (C. Muell.) Par. (Paris, 1900a).

Distr. Southem South America (Mueller, 1897a; Paris, 1900a, 1905a; Brotherus, 1925; Kühnemann, 1938).

\section{Campylopodium}

C. flaccidum Herz. (Herzog, 1926).

Distr. Southem South America (Herzog, 1926; Seki, 1974).

\section{Campylopus}

C. aberrans Broth. (Brotherus, 1924b).

Distr. Juan Fernandez (Brotherus, 1924b, 1925; Espinosa B., 1941; Bartram, 1959; Frahm, 1975a, 1976a).

var. viridis Broth. (Brotherus, 1924b).

Distr. Juan Fernandez (Brotherus, 1924b; Frahm, 1975a).

C. acuminatus Mitt. (Mitten, 1869).

Syn. Dicranum acuminatum (Mitt.) C. Muell. (Mueller, 1885).

Distr. Southem South America (Mitten, 1869; Jaeger, 1872; Mueller, 1885; Paris, 1904a; Cardot, 1908a; Frahm, 1975a, 1976a).

C. alopecurus (C. Muell.) Kindb. (Kindberg, 1889).

Syn. Dicranum alopecurus C. Muell. (Mueller, 1882).

Distr. Southem South America (Mueller, 1882; Paris, 1904a; Brotherus, 1924a; Kühnemann, 1938; Frahm, 1975a).

C. amabills (C. Muell.) Kindb. (Kindberg, 1888).

Syn. Dicranum amabile C. Muell. (Mueller, 1882).

Distr. Southem South America (Mueller, 1882; Paris, 1904a; Brotherus, 1924a; Hosseus, 1938c, d; Kühnemann, 1938; Frahm, 1975a).

C. arctocarpus (Hornsch.) Mitt. (Mitten, 1869).

Syn. Dicranum arctocarpum Hornsch. (Hornshuch, 1840). Thysanomitrion scabrisetum Hamp. (Hampe, 1844).

Distr. Southem South America (Hornschuch, 1840; Hampe, 1844; Mueller, 1848a; Mitten, 1869; Jaeger, 1872; Gibert, 1873; Paris, 1904a; Brotherus, 1924a; Herter, 1933a; Frahm, 1975a). 
C. arenicola (C. Muell.) Mitt.

C. areodictyon (C. Muell.) Mitt. (Mitten, 1869).

Syn. Dicranum areodictyon C. Muell. (Mueller, 1848a).

Distr. Juan Fernandez (Brotherus, 1924b; Espinosa, B., 1941; Frahm, 1975a, 1976a).

C. asperifolius Mitt.

Dicranodontium asperifolium

C. atratus Schimp.

Campylopus patagonicus

C. aurificus (C. Muell.) Kindb.

Campylopus introflexus

var. flavescens (C. Muell.) Par. (Paris, 1894).

Syn. Dicranum aurificum C. Muell. var. flavescens C. Muell. (Mueller, 1882).

Distr. Southern South America (Mueller, 1882; Paris, 1894).

C. austro-alpinus (C. Muell.) Kindb. (Kindberg, 1889).

Syn. Dicranum austro-alpinum C. Muell. (Mueller, 1882).

Distr. Southern South America (Mueller, 1882; Paris, 1904a; Kühnemann, 1938; Frahm, 1975a).

C. berteroanus Dub.

Campylopus introflexus

C. berteroi Dub. ex Kindb.

Campylopus introflexus

C. birgeri Card. (Cardot, 1905a).

Distr. Falkland Islands (Cardot, 1905a, 1908a; Cardot and Brothenus, 1923; Brotherus, 1924a; Kühnemann, 1938; Frahm, 1975a).

C. blindiaceus C. Muell. (Mueller, 1901a [1900]) nom. nud.

Distr. Southern South America (Mueller, 1901a).

C. blindioides Broth. (Brotherus, 1924b).

Distr. Juan Fernandez (Brotherus, 1924b, 1925; Frahm, 1975a, 1976a; Robinson, 1975).

C. brachythysanos (C. Muell.) Par. (Paris, 1900a).

Syn. Dicranum brachythysanos C. Muell. (Mueller, 1897a).

Distr. Southern South America (Mueller, 1897a; Paris, 1900a; Frahm, 1975a).

C. cacti (C. Muell.) Kindb. (Kindberg, 1889).

Syn. Dicranum cacti C. Muell. (Mueller, 1882).

Distr. Southern South America (Mueller, 1882; Paris, 1904a; Kühnemann, 1938; Frahm, 1975a).

C. campoanus Thér. (Thériot, 1918).

Distr. Southern South America (Thériot, 1918; Campo, 1921; Brotherus, 1924a; Herzog, 1938 , 1939; Frahm, 1975a, 1976a).

C. canescens (C. Muell.) Schimp. (Schimper in Jaeger, 1872).

Syn. Dicranum canescens C. Muell. (Mueller, 1859).

Dicranum perincanum C. Muell. (Mueller, 1885) fide Frahm, 1975a.

Campylopus perincanus (C. Muell.) Kindb. (Kindberg, 1888) fide Frahm, 1975a.

Distr. Southern South America (Mueller, 1885; Paris, 1904a; Cardot, 1905a, 1908a; Kühnemann, 1938; Frahm, 1975a, 1976a).

Falkland Islands (Mueller, 1859; Jaeger, 1872; Paris, 1904a; Cardot, 1905a, 1908a; Cardot and Brotherus, 1923; Kühnemann, 1938; Frahm, 1975a, 1976a).

C. carbonicolus Thér. (Thériot, 1918).

Distr. Southern South America (Thériot, 1918; Campo, 1921; Frahm, 1975a, 1976a). 
C. carinatus.C. Muell. (Mueller, 1901a [1900]) nom. nud.

Distr. Southern South America (Mueller, 1901a; Brotherus, 1924a; Kühnemann, 1938; Frahm, 1975a).

C. chalarobasis C. Muell. ex Thér. (Mueller ex Thériot, 1934b [1935]).

Distr. Southern South America (Thériot, 1934b; Frahm, 1975a, 1976a).

C. chilensis De Not. (De Notaris, 1859).

Distr. Southern South America (De Notaris, 1859; Mitten, 1869; Jaeger, 1872; Paris, 1904a; Brotherus, 1924a; Frahm, 1975a).

C. chrismarii (C. Muell.) Mitt. (Mitten, 1869).

Syn. Dicranum chrismarii C. Muell. (Mueller, 1855).

Distr. Juan Femandez (Frahm, 1975a).

C. clavatus (R. Brown) Wils. (Wilson, 1854).

Syn. Dicranum clavatum R. Brown (R. Brown in Schwaegrichen, 1829).

Campylopus leptodus Mont. (Montagne, 1845a).

Trichostomum leptodum (Mont.) Mitt. (Mitten in Hooker, 1867).

Pilopogon leptodus (Mont.) Broth. (Brotherus, 1901).

Thysanomitrion leptodus (Mont.) Broth. (Brotherus in Skottsberg, 1914).

Campylopus corralensis Broth. (Brotherus in Paris, 1904a) nom. nud.

Campylopus montagnei De Not. (De Notaris, 1859) fide Frahm, $1975 a$.

Distr. Juan Femandez (Mitten, 1885; Paris, 1900a, 1905a; Skottsberg, 1914; Brotherus, 1924b; Bartram, 1959; Frahm, 1975a, 1976a; Robinson, 1975).

Southern South America (Montagne, 1845a, 1850, 1856; De Notaris, 1859; Mitten, 1869; Jaeger, 1872; Brotherus, 1901, 1924a; Paris, 1904a, 1905a; Dusén, 1905a; Cardot, 1908a; Herzog, 1923, 1938; Thériot, 1925a, 1935a; Bartram, 1952; Frahm, $1975 a, 1976 a, b)$.

C. concolor (Hook.) Brid. (Bridel, 1826).

Syn. Dicranum concolor Hook. (Hooker, 1816).

Distr. Southem South America (Bartram, 1965; Frahm, 1975a).

C. corralensis Broth.

Campylopus clavatus

C. crassissimus Besch. (Bescherelle, 1885a).

Syn. Campylopus sordido-nigritus Dus. (Dusén, 1903a) nom. nud.

Distr. Southem South America (Bescherelle, 1885a, 1889; Dusén, 1903a, 1905c; Paris, 1904a; Cardot, 1908a; Brotherus, 1924a; Roivainen, 1934; Frahm, 1975a, 1976a).

C. cummingii Dub. (Duby, 1870).

Distr. Southern South America (Duby, 1870; Jaeger and Sauerbeck, 1879b; Paris, 1904a).

C. curvatifolius Card. (Cardot, 1905a).

Distr. Falkland Islands (Cardot, 1905a, 1908a; Cardot and Brotherus, 1923; Brotherus, 1924a; Kühnemann, 1938; Frahm, 1975a).

C. fernandezianus Card. (Cardot in Skottsberg, 1914) nom. nud.

Distr. Southem South America (Skottsberg, 1914).

C. fernandezianus Bartr. (Bartram, 1959).

Distr. Juan Fernandez (Bartram, 1959; Frahm, 1975a, 1976a).

C. fibrobasis Dus.

Campylopus torquatus

C. flavissimus (C. Muell.) Besch. (Bescherelle, 1885a).

Syn. Dicranum flavissimus C. Muell. (Mueller, 1885). 
Distr. Southern South America (Bescherelle, 1885a, 1889; Mueller, 1885; Cardot, 1901, 1908a; Paris, 1904a; Dusén, 1905c; Brotherus, 1924a; Frahm, 1975a, 1976a).

C. flavo-nigritus Dus. (Dusén, 1905c).

Distr. Southern South America (Dusén, 1903a, 1905c; Paris, 1904a; Cardot, 1908a; Cardot and Brotherus, 1923; Brotherus, 1924a; Frahm, 1975a, 1976a).

C. flavo-viridis Dus. (Dusén, 1905c).

Distr. Southern South America (Paris, 1904a; Dusén, 1905c; Cardot, 1908a; Seki, 1974; Frahm, 1975a, 1976a).

C. flexuosus (Hedw.) Brid. (Bridel, 1819).

Syn. Dicranum flexuosum Hedw. (Hedwig, 1801).

Dicranum flexuosum Hedw. var. minus Schwaegr. (Schwaegrichen in Gaudichaud, 1826 [1828]) nom. nud.

Distr. Southern South America (Montagne, 1850).

Falkland Islands (Dumont D'Unille, 1825; Gaudichaud, 1825, 1826; Bridel, 1827b).

C. fuegianus Dus. (Dusén, 1905c).

Distr. Southern South America (Dusén, 1905c; Cardot, 1908a; Cardot and Brotherus, 1923; Brotherus, 1924a; Frahm, 1975a, 1976a).

C. fuscatus Besch. (Bescherelle, 1891).

Distr. Southern South America (Bescherelle, 1891; Paris, 1904a; Felippone, 1912; Herter, 1933a; Frahm, 1975a).

C. fusco-luteus Card. (Cardot in Skottsberg, 1914) nom. nud.

Distr. Juan Fernandez (Skottsberg, 1914).

C. gaudichaudii Besch. (Bescherelle, 1891).

Distr. Southern South America (Bescherelle, 1891; Paris, 1904a; Frahm, 1975a).

C. grimmioides (C. Muell.) Kindb. (Kindberg, 1889).

Syn. Dicranum grimmioides C. Muell. (Mueller, 1882).

Distr. Southern South America (Mueller, 1882; Paris, 1904a; Brotherus, 1924a; Hosseus, 1935b, 1937; Kühnemann, 1938; Frahm, 1975a).

C. griseus (Hornsch.) Jaeg. (Jaeger, 1872).

Syn. Thysanomitrion griseum Hornsch. (Hornschuch, 1840). Dicranum griseum (Hornsch.) C. Muell. (Mueller, 1848a).

Dicranum recurvipilum C. Muell. (Mueller, 1900).

Campylopus recurvipilus (C. Muell.) Par. (Paris, 1904a).

Distr. Southern South America (Hornschuch, 1840; Mueller, 1848a; Jaeger, 1872; Paris, 1904a; Felippone, 1912; Herter, 1933a, 1943; Frahm, 1975a).

C. guaitecae Dus. (Dusén, 1905c).

Distr. Juan Fernandez (Frahm, 1976a).

Southern South America (Paris, 1904a; Dusén, 1905c; Cardot, 1908a; Cardot and Brothenus, 1923; Brotherus, 1924a; Thériot, 1934b; Roivainen and Bartram, 1937; Kühnemann, 1938; Frahm, 1975a, 1976a).

C. hamatus Bartr. (Bartram in Roivainen and Bartram, 1937).

Distr. Southern South America (Roivainen and Bartram, 1937; Frahm, 1975a, 1976a).

C. humifugus (C. Muell.) Kindb. (Kindberg, 1889).

Syn. Dicranum humifugum C. Muell. (Mueller, 1882).

Distr. Southern South America (Mueller, 1882; Paris, 1904a; Brotherus, 1924a; Kühnemann, 1938; Frahm, 1975a). 
C. incrassatus C. Muell. (Mueller, 1844b [1845]).

Syn. Dicranum incrassatum (C. Muell.) C. Muell. (Mueller, 1848a).

Distr. Southem South America (Mueller, 1844b, 1847c, 1848a; Montagne, 1850; Mitten, 1869; Jaeger, 1872; Paris, 1904a; Dusén, 1905c; Thériot, 1917b; Costes, 1921; Brotherus, 1924a; Espinosa B., 1924; Herzog, 1938, 1960; Frahm, 1975a, 1976a).

C. introflexus (Hedw.) Brid. (Bridel, 1819).

Syn. Dicranum introflexum Hedw. (Hedwig, 1801).

Thysanomitrion introflexum (Hedw.) Arnott (Arnott, 1827).

Campylopus introflexus (Hedw.) Brid. var. genuinus Giac. (Giacomini, 1955) hom. illeg.

Campylops xanthophyllus Mont. (Montagne, 1845a).

Dicranum xanthophyllum Mont. ex Par. (Montagne ex Paris, 1895).

Dicranum aurificum C. Muell. (Mueller, 1882) fide Frahm, $1975 a$.

Campylopus aurificus (C. Muell.) Kindb. (Kindberg, 1889) fide Frahm, $1975 a$.

Campylopus lechleri Schimp. (Schimper in Lorentz, 1866) nom. nud. fide Frahm, 1975a.

Campylopus berteroanus Dub. (Duby, 1875b).

Campylopus berteroi Dub. ex Kindb. (Duby ex Kindberg, 1888) hom. illeg.

Campylopus rectipilus C. Muell. (Mueller in Kindberg, 1891) nom. nud.

Campylopus nodicaulis C. Muell. (Mueller in Frahm, 1975a) nom. nud.

Campylopus latipilis C. Muell. (Mueller in Kindberg, 1891) nom. nud. fide Frahm, 1975a.

Distr. Juan Fernandez (Montagne, 1835; Duby, 1875a, $b$; Jaeger and Sauerbeck, 1879b; Johow, 1896 (as C. leptodus fide Brotherus, 1924b); Paris, 1904a; Skottsberg, 1914; . Brotherus, 1924b; Thériot, 1927b; Espinosa B., 1941; Bartram, 1959; Frahm, 1974, 1975a; Robinson, 1975).

Southem South America (Hornschuch, 1840; Mueller, 1844b; Montagne, 1845a, 1846. 1850, 1856; Lorentz, 1866; Mitten, 1869; Jaeger, 1872; Gibert, 1873; Paris, 1895, 1904a; Dusén, 1905c; Cardot 1908a; Campo, 1915, 1921; Thériot, 1918; Brotherus, 1924a, $C$; Reimers, 1926; Herter, 1928, 1933a, b, 1939b; Roivainen and Bartram, 1937; Hosseus, 1938c, d; Herzog, 1938, 1939, 1954, 1960; Espinosa B., 1941; Skottsberg, 1950; Bartram, 1952; Giacomini, 1955; Bizot, 1967; Seki, 1974; Frahm, 1974, 1975a, 1976a, b).

Falkland Islands (Wilson and Hooker, 1847; Mueller, 1848a; Jaeger, 1872; Cardot, 1905a; 1908a; Cardot and Brotherus, 1923; Kühnemann, 1938; Giacomini, 1955; Frahm, 1974, 1975a).

var. brachycarpus Giac. (Giacomini, 1955).

Distr. Southem South America (Giacomini, 1955; Frahm, 1975a).

var. cordobaensis Thér.

Campylopus pilifer

var. genuinus Giac.

Campylopus introflexus var. introflexus

C. jamesonil (Hook.) Jaeg. (Jaeger, 1872).

Syn. Dicranum jamesonii Hook. (Hooker, 1837).

Distr. Southern South America (Frahm, 1975a).

C. jugorum Herz. (Herzog, 1909).

Distr. Southern South America (Hosseus, 1938c, d, 1939; Frahm, 1975a).

C. kunkelii Bartr. (Bartram, 1959).

Distr. Juan Femandez (Bartram, 1959; Frahm, 1975a, 1976a; Robinson, 1975).

C. laetivirens (Broth.) Frahm (Frahm, 1975a) hom. illeg.

Syn. Thysanomitrion laetevirens Broth. (Brotherus, 1924C) fide Frahm, 1975a.

Distr. Southern South America (Frahm, 1975a, b, 1976a).

C. laniger (C. Muell.) Besch.

C. latipilus $\mathrm{C}$. Muell.

Chorisodontium lanigerum

C. laxiretis Bartr. 
C. lechleri Schimp.

C. leptodus Mont.

C. Iorentzii C. Muell. ex Par.

Conostomum lorentzii

C. maulensis Broth. (Brotherus in Espinosa B., 1941) nom. nud.

Distr. Southern South America (Espinosa B., 1941).

C. masafuerae Frahm (Frahm, 1975a).

Syn. Campylopus laxiretis Bartr. (Bartram, 1959) hom. illeg. fide Frahm, $1975 a$.

Distr. Juan Fernandez (Bartram, 1959; Frahm, 1975a, 1976a).

C. modestus Card. (Cardot, 1905a).

Distr. Southem South America (Herzog, 1938, 1939; Frahm, 1975a, 1976a).

Falkland Islands (Cardot, 1905a, 1908a; Cardot and Brotherus, 1923; Brotherus, 1924a; Kühnemann, 1938; Frahm, 1975a, 1976a).

var. deciduus Card. et Broth. (Cardot and Brotherus, 1923).

Distr. Falkland Islands (Cardot and Brotherus, 1923; Kühnemann, 1938; Frahm, 1975a).

C. montagnei De Not.

Campylopus clavatus

C. morenoi (C. Muell.) Par. (Paris, 1900a).

Syn. Dicranum morenoi C. Muell. (Mueller, 1897a).

Distr. Southem South America (Mueller, 1897a; Paris, 1900a, 1904a; Cardot, 1908a; Frahm, 1975a).

C. mucorinus C. Muell. (Mueller, 1901a [1900]) nom. nud.

Distr. Southern South America (Mueller, 1901a).

C. muricatus Dix.

Chorisodontium aciphyllum

C. nanocaudatus C. Muell.

Chorisodontium aciphyllum

C. nanotrichus C. Muell. (Mueller, 1901a [1900]) nom. nud.

Distr. Southern South America (Mueller, 1901a).

C. nitidus Thér.

C. nodicaulis C. Muell.

Campylopus urugensis

Campylopus introflexus

C. occultoviridis Dus. (Dusén, 1903a) nom. nud.

Distr. Southern South America (Dusén, 1903a).

C. orthocomus (C. Muell.) Besch.

Chorisodontium aciphyllum

C. patagonicus Broth. ex Dus. (Brotherus in Dusén, 1905c).

Syn. Campylopus atratus Schimp. (Schimper in Mueller, 1901a [1900]) nom. nud. fide Frahm, $1975 a$.

Distr. Southern South America (Dusén, 1903a, 1905c; Paris, 1904a; Cardot, 1908a; Brotherus, 1924a; Kühnemann, 1938; Seki, 1974; Frahm, 1975a, 1976a).

Falkland Islands (Mueller, 1901a).

C. perhorridus Dus. (Dusén, 1905c).

Syn. Dicranum perhorridum (Dus.) Card. (Cardot, 1908a).

Distr. Southem South America (Dusén, 1905c; Cardot, 1908a; Cardot and Brotherus, 1923; Brotherus, 1924a; Roivainen and Bartram, 1937; Herzog, 1938; Kühnemann, 1938; Frahm, 1975a, 1976a).

C. perincanus (C. Muell.) Kindb.

Campylopus canescens

C. permollis Thér. (Thériot, 1926).

Distr. Southern South America (Thériot, 1926, 1934b; Frahm, 1975a, 1976a). 
C. pilifer Brid. (Bridel, 1819).

Syn. Campylopus polytrichoides De Not. (De Notaris, 1838b) fide Gradstein and Sipman, 1978.

Campylopus introflexus (Hedw.) Brid. var. cordobaensis Thér. (Thériot in Bauer, 1934) fide Gradstein and Sipman, 1978.

Distr. Juan Femandez (Brotherus, 1924b).

Southern South America (Bauer, 1934; Hosseus, 1936, 1937, 1938c, d, 1939;

Kühnemann, 1938; Espinosa B., 1941; Frahm, 1975a; Gradstein and Sipman, 1978).

C. pilopogon C. Muell. (Mueller, 1901a [1900]) nom. nud.

Distr. Southern South America (Mueller, 1901a).

C. polytrichoides De Not.

Campylopus pilifer

C. purpureocaulis Dus. (Dusén, 1905c).

Distr. Southem South America (Dusén, 1903a, 1905c; Cardot, 1908a; Cardot and Brotherus, 1923; Brotherus, 1924a; Herzog, 1954; Seki, 1974; Frahm, 1975a, 1976a).

C. rectipilus C. Muell.

Campylopus introflexus

C. recurvifolius Dus. (Dusén, 1905c).

Distr. Southem South America (Dusén, 1905c; Brotherus, 1924a; Herzog, 1938, 1960; Frahm, 1975a, 1976a).

C. recurvipilus (C. Muell.) Par.

Campylopus griseus

C. richardii Brid. (Bridel, 1819).

Syn. Thysanomitrion richardii (Brid.) Schwaegr. (Schwaegrichen, 1823).

Weisia richardii (Brid.) Schwaegr. (Schwaegrichen in Bridel, 1826) nom. inval.

Grimmia richardii (Brid.) Spreng. (Sprengel, 1827).

Dicranum richardii (Brid.) C. Muell. (Mueller, 1848a).

Pilopogon richardii (Brid.) Broth. (Brotherus, 1901).

Distr. Juan Fernandeż (Brotherus, 1924b, 1925; Espinosa B., 1941; Frahm, 1975a, 1976a).

Southem South America (Mueller, 1848a; Paris, 1905a).

C. rigescens Kindb. (Kindberg, 1889) nom. nud.

Distr. No locality given; see Index muscorum, 1, p. 453.

C. saddleanus (C. Muell.) Besch.

Campylopus vesticaulis

C. sancti-caroli Besch. (Bescherelle, 1891).

Distr. Southem South America (Bescherelle, 1891; Paris, 1904a; Frahm, 1975a).

C. scaberrimus Broth.

Chorisodontium aciphyllum

C. scabrophyllus (C. Muell.) Par. (Paris, 1900a).

Syn. Dicranum scabrophyllum C. Muell. (Mueller, 1897a).

Distr. Southem South America (Mueller, 1897a; Paris, 1900a, 1904a; Felippone, 1912; Herter, 1933a; Frahm, 1975a).

C. senilis Lor. (Lorentz in Mueller, 1901 a [1900]) nom. nud.

Distr. Southern South America (Mueller, 1901a).

C. sordido-nigritus Dus.

C. spegazzinii (C. Muell.) Kindb.

Campylopus crassissimus

C. spiralis Dus. (Dusén, 1905c).

Distr. Southern South America (Paris, 1904a; Dusén, 1905c; Cardot, 1908a; Thériot, 1934b, 1935a; Herzog, 1939; Frahm, 1975a, 1976a).

C. stenocarpus (Hamp.) Jaeg. (Jaeger, 1872).

Syn. Thysanomitrion stenocarpum Hamp. (Hampe, 1844).

Dicranum stenocarpum (Hamp.) C. Muell. (Mueller, 1851a).

Dicranum stenopus C. Muell. (Mueller, 1848a) nom. illeg. 
Distr. Southern South America (Hampe, 1844; Mueller, 1848a; Jaeger, 1872; Paris, 1904a; Felippone, 1912; Herter, 1933a; Frahm, 1975a).

C. subareodictyon Broth. (Brotherus, 1924b).

Distr. Juan Fernandez (Brotherus, 1924b, 1925; Frahm, 1975a, 1976a).

C. subexalaris C. Muell. (Mueller, 1901 a [1900]) nom. nud.

Distr. Southern South America (Mueller, 1901a).

C. substygius C. Muell. (Mueller in Kindberg, 1889) nom. nud.

Distr. No locality given, see Index muscorum, 1, 458.

C. sulfureo-nigritus Dus. (Dusén, 1905c).

Distr. Southern South America (Dusén, 1903a, 1905c; Paris, 1904a; Cardot, 1908a; Brotherus, 1924a; Frahm, 1975a, 1976a).

C. surinamensis C. Muell. (Mueller, 1848b).

Syn. Dicranum arenicola C. Muell. (Mueller, 1855).

Campylopus arenicola (C. Muell.) Mitt. (Mitten, 1869).

Distr. Southern South America (Herter, 1939b; Frahm, 1975a).

C. torquatus Mitt. (Mitten in Wilson, 1859).

Syn. Campylopus fibrobasis Dus. (Dusén, 1905c) fide Frahm, 1975a.

Distr. Southern South America (Paris, 1904a; Dusén, 1905c; Cardot, 1908a; Brotherus, 1924a; Thériot, 1934b; Herzog, 1938, 1954; Kühnemann, 1938; Frahm, 1975a, 1976a).

C. truncatus C. Muell. (Mueller, 1844b [1845]).

Syn. Dicranum truncatum (C. Muell.) C. Muell. (Mueller, 1848a).

Distr. Juan Fernandez (Skottsberg, 1914; Thériot, 1921b; Brotherus, 1924b; Frahm, 1976a). Southern South America (Mueller, 1844b, 1848a; Jaeger, 1872; Paris, 1904a; Reimers, 1926; Thériot, 1934b; Herzog, 1938; Frahm, 1975a, 1976a).

C. urugensis Frahm (Frahm, 1975a).

Syn. Campylopus nitidus Thér. (Thériot in Felippone, 1929 [1930]) hom. illeg. fide Frahm, $1975 a$.

Distr. Southern South America (Felippone, 1929; Herter, 1933a; Frahm, 1975a).

C. ventanus C. Muell. (Mueller, 1901a [1900]) nom. nud.

Distr. Southern South America (Mueller, 1901a).

C. verticillatus (Hamp.) Kindb. (Kindberg, 1889).

Syn. Dicranum verticillatum Hamp. (Hampe, 1879).

Distr. Southern South America (Hampe, 1879; Paris, 1894, 1904a; Frahm, 1975a).

C. vesticaulis Mitt. (Mitten, 1875).

Syn. Dicranum saddleanum Besch. ex C. Muell. (Mueller, 1885).

Campylopus saddleanus (C. Muell.) Besch. (Bescherelle, 1885a).

Distr. Southern South America (Bescherelle, 1885a, 1889; Mueller, 1885; Paris, 1904a; Cardot, 1908a; Brotherus, 1924a; Roivainen and Bartram, 1937; Frahm, 1975a, 1976a).

Falkland Islands (Cardot, 1905a, 1908a; Cardot and Brotherus, 1923; Brotherus, 1924a; Kühnemann, 1938; Frahm, 1975a, 1976a).

C. xanthophyllus Mont. (Montagne, 1845a).

Campylopus introflexus

\section{Catagoniopsis}

C. berteroana (Mont.) Broth. (Brotherus, 1909). 
Syn. Hypnum berteroanum Mont. (Montagne, 1845a).

Rhynchostegium berteroanum (Mont.) Jaeg. (Jaeger, 1878).

Rhynchostegium berteroi Kindb. (Kindberg, 1888) nom. illeg.

Distr. Juan Femandez (Mitten, 1885; Johow, 1896; Paris, 1905b; Brotherus, 1924b, 1925; Robinson, 1975).

Southem South America (Montagne, 1845a, 1850, 1856; Mueller, 1851 a; Sullivant, 1859; Mitten, 1869; Jaeger, 1878; Paris, 1905b; Brotherus, 1909, 1924c, 1925; Thériot, 1917b, 1918, 1928; Campo, 1921; Costes, 1921; Herzog, 1938, 1954; Espinosa B., 1941; Skottsberg, 1950; Bartram, 1952).

\section{Catagonium}

C. callichroum (Mont.) Neger

Catagonium politum var. phyllogonium

C. complanatum (Card. et Broth.) Broth. Catagonium politum

C. myurum Card. et Thér. (Cardot and Thériot in Thériot, 1929c [1930]).

Syn. Acrocladiopsis myura Card. (Cardot, 1914a) nom. nud.

Distr. Southern South America (Cardot, 1914a; Thériot, 1929c; Herzog, 1957).

C. nitidum (Hook. f. et Wils.) Dus. ex Broth. (Dusén ex Brotherus, 1908).

Syn. Leskea nitida Hook. f. et Wils. (Hooker, J. D. and Wilson, 1844).

Hypnum nitidum (Hook. f. et Wils.) C. Muell. (Mueller, 1851a).

Stereodon nitidus (Hook. f. et Wils.) Mitt. (Mitten, 1869).

Acrocladiopsis nitida (Hook. f. et Wils.) Card. (Cardot, 1914a).

Calliergonella nitida (Hook. f. et Wils.) Card. (Cardot in Cardot and Brotherus, 1923).

Plagiothecium nitidum (Hook. f. et Wils.) Bartr. (Bartram, 1946) hom. illeg.

Hypnum hookeri Lindb. (Lindberg, 1868a) hom. illeg.

Distr. Southern South America (Hooker, J. D. and Wilson, 1844; Wilson and Hooker, 1847; Mueller, 1851a, 1885; Mitten, 1869; Jaeger and Sauerbeck, 1879a; Bescherelle, 1889; Paris, 1904C; Brotherus, 1908, 1925; Cardot, 1908a, 1914a; Cardot and Brotherus, 1923; Roivainen, 1934; Kühnemann, 1938; Bartram, 1946; Seki, 1974).

C. politum (Hook. f. et Wils.) Dus. ex Broth. (Dusén ex Brotherus, 1908).

Syn. Hypnum politum Hook. f. et Wils. (Hooker, J. D. and Wilson, 1844).

Acrocladium politum (Hook. f. et Wils.) Mitt. (Mitten, 1869).

Acroceratium politum (Hook. f. et Wils.) Mitt. (Mitten, 1883).

Eucatagonium politum (Hook. f. et Wils.) Broth. (Brotherus, 1925).

Acrocladiopsis complanata Card. (Cardot, 1914a) nom. nud.

Calliergonella complanata Card. et Broth. (Cardot and Brotherus, 1923).

Catagonium complanatum (Card. et Broth.) Broth. (Brotherus, 1925).

Orthorrhynchium chilense Williams (Williams, 1924 [1925]).

Distr. Juan Fernandez (Robinson, 1975).

Southem South America (Hooker, J. D. and Wilson, 1844; Wilson and Hooker, 1847;

Sullivant, 1859; Mitten, 1869; Jaeger, 1878; Mueller, 1885; Bescherelle, 1889; Neger,

1899; Dusén, 1903a; Paris, 1903; Brotherus, 1908, 1925; Cardot, 1908a, 1914a;

Cardot and Brotherus, 1923; Herzog, 1923, 1938, 1939, 1954, 1957, 1960; Williams, 1924; Reimers, 1926; Kühnemann, 1938; Bartram, 1946; Seki, 1974).

South Georgia (Newton, 1977a).

var. filescens Card. et Broth. (Cardot and Brotherus, 1923).

Syn. Eucatagonium politum (Hook. f. et Wils.) Broth. var. filescens (Card. et Broth.) Herz. (Herzog, 1954).

Distr. Southem South America (Cardot and Brotherus, 1923; Herzog, 1954).

var. phyllogonium Card. (Cardot in Cardot and Brotherus, 1923).

Syn. Phyllogonium callichroum Mont. (Montagne, 1845a).

Hypnum callichroum (Mont.) C. Muell. (Mueller, 1851a) hom. illeg.

Catagonium callichroum (Mont.) Neger (Neger, 1899).

Hypnum phyllogonium C. Muell. (Mueller, 1851a) hom. illeg.

Acrocladium phyllogonium Jaeg. (Jaeger, 1878) hom. illeg.

Distr. Southem South America (Montagne, 1845a, 1850; Mueller, 1851a; Jaeger, 1878; Neger, 1899; Cardot and Brotherus, 1923). 
Catharinea nom. rejic.

C. antarctica C. Muell.

Psilopilum trichodon

C. canaliculata (Hook. et Arnott) C. Muell.

Oligotrichum canaliculatum var. major Lor.

C. compressa (Hook. f. et Wils.) C. Muell.

Oligotrichum canaliculatum var. majus

C. dendroides (Hedw.) Hamp.

Atrichopsis compressa

C. gymnostoma C. Muell. ex Par.

Dendroligotrichum dendroides

C. gymnostomula C. Muell.

Psilopilum gymnostomulum

C. magellanica (Hedw.) Brid.

Psilopilum gymnostomulum

C. molinae (Mont.) C. Muell.

Polytrichadelphus magellanicus

C. planifolia C. Muell.

Oligotrichum canaliculatum var. molinae

C. polycarpa C. Muell.

Atrichum polycarpum

C. rigida (Lor.) C. Muell.

Atrichum polycarpum

C. simplex C. Muell. (Mueller, 1901a [1900]) nom. nud.

Oligotrichum rigidum

Distr. Southern South America (Mueller, 1901a).

C. squamosa (Hook. f. et Wils.) C. Muell.

Dendroligotrichum squamosum

C. tapes C. Muell.

Psilopilum tapes

Cecalyphum nom. illeg.

C. dichotomum P. Beauv.

Dicranoloma billardieri

C. semi-completum (Hedw.) P. Beauv.

Fissidens semicompletus

Cephalotrichum hom. illeg.

C. oligodus B.S.G.

Pogonatum oligodus

\section{Ceratodon}

C. amblyocalyx C. Muell. exÅngstr.

Ceratodon purpureus var. amblyocalyx

C. antarcticus Card. (Cardot, 1900).

Distr. Antarctic, peninsula region (Cardot, 1900, 1901, 1908a; Paris, 1904a; Dixon, 1935; Horikawa and Ando, 1961, 1963; Steere, 1961a).

C. chilensis (Mont.) Par.

Cheilothela chilensis

C. convolutus Reichdt.

Ceratodon purpureus

C. corralensis (Lor.) Jaeg.

Zygodon corralensis

C. crassinervis Lor. (Lorentz, 1866).

Distr. Southern South America (Lorentz, 1866; Paris, 1904a: Thériot, 1918, 1921a, 1935a;

Campo, 1921; Reimers, 1926; Herzog, 1938).

C. dubius Dus.

Cheilothela chilensis

C. grossiretis Card. (Cardot, 1906a).

Distr. Southern South America (Thériot, 1926).

South Georgia (Dixon, 1920; Steere, 1961b; Horikawa and Ando, 1963).

Antarctic, peninsula region (Cardot, 1906a, 1908a, 1911b, $d, 1913 a$; Horikawa and Ando, 1961, 1963; Steere, 1961a; Robinson, 1972).

Antarctic, continental region (Bartram, 1957; Steere, 1961a; Horikawa and Ando, 1963).

var. validus Card.

Ceratodon validus

C. minutifolius Card. (Cardot, 1911b, d).

Distr. Antarctic, peninsula region (Cardot, 1911b, $d, 1913 a$; Steere, 1961a; Horikawa and Ando, 1963). 
C. purpureus (Hedw.) Brid. (Bridel, 1826).

Syn. Dicranum purpureum Hedw. (Hedwig. 1801).

Ceratodon purpureus (Hedw.) Brid. var. xanthopus Sull. (Sullivant in Sullivant and Lesquereux, 1865).

Ceratodon purpureus (Hedw.) Brid. var. fastigiatus Warnst. (Warnstorf, 1904).

Ceratodon convolutus Reichdt. (Reichardt, 1868).

Distr. Juan Fernandez (Brotherus, 1924b; Espinosa B., 1941; Robinson, 1975).

Southem South America (Montagne, 1845b, 1850; Wilson and Hooker, 1847; Lorentz, 1866; Mitten, 1869; Cardot, 1900, 1905a, 1908a; Krieger, 1904; Dusén, 1905a; Thériot, 1918, 1935a; Campo, 1921; Cardot and Brotherus, 1923; Herzog, 1923. 1938, 1939, 1954, 1957; Roivainen, 1934; Roivainen and Bartram 1937; Hosseus, 1938c, d; Kühnemann, 1938; Bartram, 1952; Seki, 1974).

Falkland Islands (Dumont d'Unille, 1825; Gaudichaud, 1825, 1826; Bridel, 1827a, Wilson and Hooker, 1847; Cardot, 1905a, 1908a; Cardot and Brotherus, 1923; Kühnemann, 1938).

Antarctic, peninsula region (Cardot, 1900, 1901, 1906a, 1908a, 1911 b, d, 1913a; Paris, 1904a; Dixon and Watts, 1918; Bartram, 1957; Steere, 1961a; Robinson, 1972).

Antarctic, continental region (Cardot, 1907a, 1908a; Steere, 1961a; Horikawa, 1961; Horikawa and Ando, 1961, 1967; Kuc, 1969; Robinson, 1972).

var. amblyocalyx (Aongstr.) C. Muell. (Mueller, 1885).

Syn. Ceratodon amblyocalyx C. Muell. ex Aongstr. (Mueller ex Ångström, 1876a).

Distr. Juan Fernandez (Skottsberg, 1914).

Southem South America (Ângström, 1872 (as C. purpureus), 1876a; Jaeger and Sauerbeck, 1879b; Mueller, 1885; Bescherelle, 1889; Cardot, 1900, 1901, 1905a, 1908a; Paris, 1904a; Dusén, 1905a, d; Kühnemann, 1938).

Falkland Islands (Cardot, 1905a, 1908a; Kühnemann, 1938).

var. falklandicus Card. et Broth. (Cardot and Brotherus, 1923).

Distr. Falkland Islands (Cardot and Brotherus, 1923; Kühnemann, 1938).

var. fastigiatus Warnst. :

Ceratodon purpureus

var. xanthopus Sull.

Ceratodon purpureus

C. valdiviae C. Muell. (Mueller, 1901a [1900]).

Distr. Southem South America (Mueller, 1901a).

C. validus (Card.) Hor. et Ando (Horikawa and Ando, 1963).

Syn. Ceratodon grossiretis Card. var. validus Card. (Cardot, 1906a) fide Horikawa and Ando, 1963.

Distr. Antarctic, peninsula region (Cardot, 1906a, 1908a; Horikawa and Ando, 1961, 1963; Greene, 1968a).

Chaetephora hom. illeg.

C. cristata (Hedw.) Brid.

Eriopus cristatus

C. incurva Hornsch.

Hookeriopsis incurva

C. orthotrichoides (Raddi) Brid.

Macrocoma orthotrichoides

\section{Cheilothela}

C. chilensis (Mont.) Broth. (Brotherus, 1901).

Syn. Trichostomum chilense Mont. (Montagne, 1845a).

Ceratodon chilensis (Mont.) Par. (Paris, 1904a).

Chrysoblastella chilensis (Mont.) Reim. (Reimers, 1926).

Ceratodon dubius Dus. (Dusén in Paris, 1904a) nom. nud.

Cheilothela dubia Dus. (Dusén, 1905a) fide Newton, $1977 a$.

Chrysoblastella dubia (Dus.) Reim. (Reimers, 1926).

Chrysoblastella revoluta Herz. (Herzog, 1916a) fide Newton, $1977 a$.

Cheilothela revoluta (Herz.) Thér. (Thériot, 1934b [1935]).

Distr. Southem South America (Montagne, 1845a, 1850, 1856; Mueller, 1849; Jaeger, 1873; Brotherus, 1901, 1924a; Paris, 1904a; Dusén, 1905a; Reimers, 1926; Hilpert, 1933; 
Thériot, 1934b; Herzog, 1938, 1954; Newton, 1974b; Seki, 1974).

South Georgia (Newton, 1977a).

C. dubia Dus.

Cheilothela chilensis

C. revoluta (Herz.) Thér.

Cheilothela chilensis

\section{Chorisodontium}

C. aciphyllum (Hook. f. et Wils.) Broth. (Brotherus, 1924a).

Syn. Dicranum aciphyllum Hook. f. et Wils. (Hooker, J. D. and Wilson, 1844).

Dicranum rigens Besch. (Bescherelle, 1885a).

Dicranoloma rigens (Besch.) Par. (Paris, 1904b).

Dicranum leucopterum C. Muell. (Mueller, 1885).

Chorisodontium leucopterum (C. Muell.) Roiv. (Roivainen in Roivainen and Bartram, 1937).

- Dicranum orthocomus Besch. ex C. Muell. (Bescherelle ex Mueller, 1885).

Campylopus orthocomum (C. Muell.) Besch. (Bescherelle, 1885a).

Chorisodontium orthocomum (C. Muell.) Roiv. (Roivainen in Roivainen and Bartram, 1937).

Dicranum tenuicuspitatum C. Muell. (Mueller, 1890a).

Campylopus nanocaudatus C. Muell. (Mueller, 1901a [1900]) nom. nud. fide Bell, $1977 a$.

Dicranum scaberrimum Dus. (Dusén, 1903a).

Campylopus scaberrimus Broth. (Brotherus in Dusén, 1903a) nom. nud.

Dicranum nordenskioeldii Card. (Cardot, 1906a) fide Bell, $1973 a$.

Chorisodontium nordenskioeldii (Card.) Roiv. (Roivainen in Roivainen and Bartram, 1937) fide Bell, 1973a.

Campylopus muricatus Dix. (Dixon, 1932) fide Dixon, 1934 and Bell, $1977 a$.

Dicranum atro-viride Card. (Cardot in Roivainen and Bartram, 1937).

Dicranum hatscheri Dus. (Dusén in Roivainen and Bartram, 1937) nom. nud.

Distr. Juan Fernandez (Bartram, 1959; Robinson, 1975).

Southern South America (Hooker, J. D. and Wilson, 1844; Wilson and Hooker, 1847; Mueller, 1848a, 1885; Sullivant, 1859; Mitten, 1869; Jaeger, 1872; Bescherelle, 1885a, 1889; Cardot, 1900, 1901, 1905a, 1908a, 1913a; Dusén, 1903a, 1905a, d; Paris, 1904a, b; Spegazzini, 1922; Cardot and Brotherus, 1923; Brotherus, 1924a; Roivainen and Bartram, 1937; Kühnemann, 1938; Bartram, 1952; Seki, 1974).

Falkland Islands (Hooker, J. D. and Wilson, 1844; Wilson and Hooker, 1847; Mueller, 1848a, 1885; Paris, 1904b; Cardot, 1905a, 1908a; Cardot and Brotherus, 1923; Brotherus, 1924a; Kühnemann, 1938).

South Georgia (Mueller, 1890a, 1901a; Paris, 1904b; Cardot, 1906a, 1908a; Cardot and Brotherus, 1923; Brotherus, 1924a; Dixon, 1932; Steere, 1961b; Bell, 1973b; Frahm, 1975a).

Antarctic, peninsula region (Wright, 1905 (as Campylopus introflexus fide Cardot, 1908a); Cardot, 1906a, 1908a, 1911b, $c, d, 1912 b, 1913 a$; Brotherus, 1924a; Dixon, 1935; Steere, 1961a; Greene, 1968a; Robinson, 1972 (as C. nordenskioeldi)).

C. dicranellatum (Dus.) Roiv. (Roivainen in Roivainen and Bartram, 1937).

Syn. Dicranum dicranellatum Dus. (Dusén, 1903a).

Distr. Southern South America (Dusén, 1903a, 1905a (and as Campylopus perhorridus pro parte fide Roivainen and Bartram, 1937); Cardot, 1908a; Cardot and Brotherus, 1923; Roivainen and Bartram, 1937; Kühnemann, 1938; Seki, 1974).

C. lanigerum (C. Muell.) Broth. (Brotherus, 1924a).

Syn. Dicranum lanigerum C. Muell. (Mueller, 1885).

Campylopus laniger (C. Muell.) Besch. (Bescherelle, 1885a).

Distr. Southern South America (Bescherelle, 1885a, 1889; Mueller, 1885; Dusén, 1903a, 1905a; Paris, 1904a; Cardot, 1908a, 1912a; Cardot and Brotherus, 1923; Brotherus, 1924a; Roivainen and Bartram, 1937; Kühnemann, 1938; Bartram, 1952; Seki, 1974).

C. leucopterum (C. Muell.) Roiv.

Chorisodontium aciphyllum

C. Iuridum Bartr. (Bartram in Roivainen and Bartram, 1937).

Distr. Southern South America (Roivainen and Bartram, 1937). 
C. macropus (C. Muell.) Broth. (Brotherus, 1924a).

Syn. Dicranum macropus Kunz. ex C. Muell. (Kunze ex Mueller, 1844b [1845]).

Distr. Southern South America (Mueller, 1844b, 1848a; Montagne, 1850; Mitten, 1869; Jaeger, 1872; Paris, 1904b; Cardot and Brotherus, 1923; Brotherus, 1924a; Roivainen and Bartram, 1937; Seki, 1974).

C. magellanicum (Card.) Bartr. (Bartram in Roivainen and Bartram, 1937).

Syn. Dicranum magellanicum Card. (Cardot, 1901).

Dicranum percompactum Dus. (Dusén, 1903a) nom. nud.

Distr. Southern South America (Cardot, 1901, 1905a, 1908a; Dusén, 1903a, 1905a; Cardot and Brotherus, 1923; Roivainen and Bartram, 1937; Kühnemann, 1938).

C. microdus (Lor.) Broth. (Brotherus, 1924a).

Syn. Dicranum microdus Lor. (Lorentz, 1866).

Distr. Southern South America (Lorentz, 1866; Jaeger, 1872; Paris, 1904b; Brotherus, 1924a).

C. nordenskioeldii (Card.) Roiv.

C. orthocomum (C. Muell.) Roiv.

Chorisodontium aciphyllum

C. sericeum Bartr. (Bartram, 1946).

Chorisodontium aciphyllum

Distr. Southem South America (Bartram, 1946).

C. spegazzinii (C. Muell.) Roiv. (Roivainen in Roivainen and Bartram, 1937).

Syn. Dicranum spegazzinii C. Muell. (Mueller, 1885).

Campylopus spegazzinii (C. Muell.) Kindb. (Kindberg, 1888).

Dicranum blindiaceum C. Muell. (Mueller in Kindberg, 1889) nom. nud.

Distr. Southern South America (Mueller, 1885; Paris, 1894, 1904a; Cardot, 1900, 1901, 1908a; Cardot and Brotherus, 1923; Brotherus, 1924a; Roivainen and Bartram, 1937; Kühnemann, 1938).

C. sphagneticola Roiv. (Roivainen in Roivainen and Bartram, 1937).

Distr. Southern South America (Roivainen and Bartram, 1937; Kühnemann, 1938).

\section{Chrysoblastella}

C. chilensis (Mont.) Reim.

Cheilothela chilensis

C. dubia (Dus.) Reim. Cheilothela chilensis

C. revoluta (Herz.) Thér.

Cheilothela chilensis

Chrysohypnum hom. illeg.

C. polygamum (B.S.G.) Loesk.

Campylium polygamum

\section{Cinclidium}

c.

C. stygium Sw. (Swartz, 1803).

Syn. Meesia stygia (Sw.) Brid. (Bridel, 1803).

Mnium stygium (Sw.) B.S.G. (Bruch and others, 1838).

Mnium subpunctatum Card. et Broth. (Cardot and Brotherus, 1923) fide Koponen, 1969.

Distr. Southern South America (Cardot, 1905a, 1908a; Cardot and Brotherus, 1923; Kühnemann, 1938; Koponen, 1969).

\section{Cladodium}

C. uliginosum Brid.

Bryum uliginosum

\section{Cladomnion}

C. crenato-obtusum Dus.

C. gracile (Hamp.) Mitt. 
C. membranosum Dus.

Juratzkaea membranosa

C. montevidense C. Muell.

Felipponea montevidensis

C. poeppigianum C. Muell.

Glyphothecium gracile

C. valdiviae C. Muell. (Mueller, 1897a).

Distr. Southern South America (Mueller, 1897a; Paris, 1904a).

\section{Cladomniopsis}

C. crenato-obtusa Fleisch. (Fleischer, 1906 [1908]).

Syn. Cladomnion crenato-obtusum Dus. (Dusén, 1903a) nom. nud.

Distr. Southern South America (Dusén, 1903a; Paris, 1904a; Fleischer, 1906; Brotherus, 1925; Matteri, 1973d; Seki, 1974; Kühnemann and Gonçalves, 1975).

Climacodontium nom. nud.

C. cygnicollum (C. Muell.) Hamp.

Anomobryum cygnicollum

\section{Codonoblepharon}

C. menziesii Schwaegr.

Zygodon menziesii

C. papillatum (Mont.) Jaeg.

Zygodon papillatus

C. uncinatum (Mitt.) Jaeg.

Zygodon pentastichus

Coelidium hom. illeg.

C. auriculatum (Mont.) Jaeg.

Acrocladium auriculatum

C. cochlearifolium (Schwaegr.) Reichdt.

Weymouthia cochlearifolia

Conomitrium nom. illeg.

C. atratum C. Muell.

Fissidens atratus

C. berterii Mont.

Fissidens fontanus

C. berteroi Mont. ex Kindb.

Fissidens fontanus

C. dillenii (Pyl.) Mont.

Fissidens fontanus

C. hedwigii Mont.

Fissidens semicompletus

C. julianum (Cand.) Mont.

Fissidens fontanus

C. Iorentziae C. Muell.

Fissidens lorentziae

C. molle C. Muell.

Fissidens permollis

C. nigritellum C. Muell.

Fissidens nigritellus

C. nigritum C. Muell. (Mueller, 1901a [1900]) nom. nud.

Distr. Southern South America (Mueller, 1901a).

C. osmundoides (Hedw.) C. Muell.

Fissidens osmundoides

C. smaragdinum Lor. et $\mathrm{C}$. Muell.

Fissidens smaragdinus

\section{Conostomum}

C. australe Sw.

Conostomum pentastichum

var. microphyllum Card. et Broth. (Cardot and Brotherus, 1923).

Distr. Southern South America (Cardot and Brotherus, 1923; Kühnemann, 1938).

Falkland Islands (Cardot and Brotherus, 1923).

var. piliferum Card. et Broth. (Cardot and Brotherus, 1923).

Distr. Southern South America (Cardot and Brotherus, 1923; Kühnemann, 1938).

C. Iorentzii C. Muell. (Mueller, 1882).

Syn. Campylopus lorentzii C. Muell. ex Par. (Mueller ex Paris, 1894) err. 
Distr. Southern South America (Mueller, 1882; Paris, 1894, 1900a, 1904a; Brotherus, 1924a; Kühnemann, 1938).

C. magellanicum Sull. (Sullivant, 1850).

Syn. Bartramia magellanica (Sull.) Mitt. (Mitten, 1869).

Conostomum perangulatum Card. (Cardot, 1905a) fide Bell, 1973a.

Distr. Southem South America (Sullivant, 1850, 1859; Mueller, 1851a, 1885; Mitten, 1869; Jaeger, 1875; Paris, 1904a; Cardot, 1905a, 1908a; Brotherus, 1924a; Kühnemann, 1938).

South Georgia (Bell, 1973b).

Antarctic, peninsula region (Bell, 1973a).

C. pentastichum (Brid.) Lindb. (Lindberg, 1863).

Syn. Bartramia pentasticha Brid: (Bridel, 1803).

Weisia pentasticha (Brid.) Hedw. (Hedwig in Bridel, 1826) nom. inval.

Conostomum australe Sw. (Swartz, 1806a) nom. illeg.

Philonotis australis Mitt. (Mitten, 1860).

Bartramia australis Mitt. (Mitten, 1869) hom. illeg.

Bryum angulosum Menz. (Menzies in Bridel, 1826) nom. nud.

Conostomum rhynchostegium C. Muell. (Mueller, 1890a).

Distr. Southern South America (Bridel, 1803, 1819, 1826; Swartz, 1806a; Schwaegrichen, 1811, 1824; Wilson and Hooker, 1847; Mueller, 1848a, 1885; Montagne, 1850;

Sullivant, 1859; Mitten, 1869; Jaeger, 1875; Bescherelle, 1889; Paris, 1904a; Cardot, 1905a, 1908a, 1913a; Cardot and Brotherus, 1923; Brotherus, 1924a; Kühnemann, 1938; Bartram, 1952; Seki, 1974).

Falkland Islands Wilson and Hooker, 1847; Jaeger, 1875; Paris, 1904a; Cardot, 1908a; Cardot and Brotherus, 1923; Brotherus, 1924a; Kühnemann, 1938).

South Georgia (Mueller, 1890a; Paris, 1904a; Cardot, 1906a, 1908a; Dixon, 1920, 1934; Cardot and Brotherus, 1923; Brotherus, 1924a; Steere, 1961b; Bell, 1973b, 1977b).

Antarctic, peninsula region (Bell, 1973a).

C. perangulatum Card.

Conostomum magellanicum

var. majus Card. et Broth. (Cardot and Brotherus, 1923).

Distr. Southern South America (Cardot and Brotherus, 1923; Kühnemann, 1938).

C. perpusillum Card. et Broth. (Cardot and Brotherus, 1923).

Distr. Southern South America (Cardot and Brotherus, 1923).

C. rhynchostegium C. Muell.

Conostomum pentastichum

\section{Costesia}

C. spongiosa Thér. (Thériot, 1917b).

Distr. Southem South America (Thériot, 1917b, 1921a, 1930; Costes, 1921; Brotherus,
1924a).

\section{Cratoneuron}

C. filicinum (Hedw.) Spruc. (Spruce, 1867).

Syn. Hypnum filicinum Hedw. (Hedwig, 1801).

Distr. Southem South America (Robinson, 1964).

C. mendozense Herz. (Herzog, 1916b).

Distr. Southern South America (Herzog, 1916b).

C. perplicatum (Dus.) Broth. (Brotherus, 1909).

Syn. Hypnum perplicatum Dus. (Dusén, 1903a).

Drepanocladus perplicatus (Dus.) Roth (Roth, 1908).

Distr. Southern South America (Dusén, 1903a; Cardot, 1908a; Roth, 1908; Brotherus, 1909, 1925; Kühnemann, 1938). 


\section{Crossidium}

C. roseae (Williams) Bartr.

Aloina roseae

\section{Crossomitrium}

C. patrisiae (Brid.) C. Muell. (Mueller, 1874).

Syn. Hypnum patrisiae Brid. (Bridel, 1827b).

Hookeria patrisiae (Brid.) Hamp. (Hampe, 1844).

Lepidopilum patrisiae (Brid.) Mitt. (Mitten, 1869).

Distr. Southern South America (Hampe, 1844; Mueller, 1851a; Mitten, 1869; Gibert, 1873; Herter, 1933a; Welch, 1969).

\section{Cryphaea}

C. aurantiorum C. Muell.

Cryphidium leucocoleum

C. caldensis Aongstr. (Ångström, 1876a).

Distr. Southern South America (Herzog, 1952a).

C. consimilis Mont.

Cryphaea tenella

C. cuspidata Sull.

Dendrocryphaea cuspidata

C. furcinervis C. Muell. (Mueller, 1882).

Distr. Southern South America (Mueller, 1882; Paris, 1904a; Brotherus, 1925; Kühnemann, 1938).

C. gorveana Mont.

Dendrocryphaea gorveana

C. julacea Mitt.

Acrocryphaea julacea

C. lechleri C. Muell.

Dendrocryphaea lechleri

C. Iorentziana C. Muell. (Mueller, 1882).

Syn. Cryphaea lorentzii C. Muell. ex Kindb. (Mueller ex Kindberg, 1891) nom. illeg.

Distr. Southern South America (Mueller, 1882; Paris, 1904a; Brotherus, 1925; Kühnemann, 1938).

C. lorentzii C. Muell. ex Kindb.

Cryphaea lorentziana

C. mollis Dus.

Cryphaeophilum molle

C. orbifolia Besch.

Cryphidium leucocoleum

C. pulchella Par. et Schimp.

C. ramosissima Schimp.

Dendrocryphaea ramosissima

C. ramosissima Par.

Dendrocryphaea lechleri

C. rhacomitrioides C. Muell. (Mueller, 1879a).

Distr. Southern South America (Mueller, 1879a, 1882; Paris, 1904a; Brotherus, 1925; Kühnemann, 1938; Herzog, 1952a).

var. gracilis C. Muell. (Mueller, 1882).

Distr. Southern South America (Mueller, 1882; Kühnemann, 1938).

C. tenella (Schwaegr.) Hornsch. ex C. Muell. (Hornschuch ex Mueller, 1844b [1845]).

Syn. Neckera tenella Schwaegr. (Schwaegrichen, 1827a).

Pilotrichum tenellum (Schwaegr.) C. Muell. (Mueller, 1851a).

Cryphaea consimilis Mont. (Montagne, 1845a).

Pilotrichum consimile (Mont.) C. Muell. (Mueller, 1851a).

Distr. Southern South America (Montagne, 1845a, 1850, 1856; Mueller, 1851a; Mitten, 1869; Jaeger, 1876; Paris, 1904a; Cardot, 1908a; Herzog, 1923; 1938; Brotherus, 1925; Reimers, 1926; Kühnemann, 1938; Seki, 1974).

C. tenella Mitt.

Dendrocryphaea ramosissima 


\section{Cryphaeophilum}

C. molle (Dus.) Fleisch. (Fleischer, 1914).

Syn. Cryphaea mollis Dus. (Dusén, 1905b).

Distr. Southern South America (Dusén, 1903a, 1905b; Paris, 1904a; Cardot, 1908a; Fleischer, 1914; Brotherus, 1925; Kühnemann, 1938; Herzog, 1954; Seki, 1974; Kühnemann and Gonçalves, 1976).

var. enerve Herz. (Herzog, 1954).

Distr. Southem South America (Herzog, 1954; Seki, 1974).

\section{Cryphidium}

C. aurantiorum (C. Muell.) Broth.

Cryphidium leucocoleum

C. leucocoleum (Mitt.) Jaeg. (Jaeger, 1876).

Syn. Neckera leucocolea Mitt. (Mitten, 1869).

Cryphaea aurantiorum C. Muell. (Mueller, 1882).

Cryphidium aurantiorum (C. Muell.) Broth. (Brotherus, 1905b).

Cryphaea orbifolia Besch. (Bescherelle, 1891).

Cryphidium orbifolium (Besch.) Broth. (Brotherus, 1905b).

Distr. Southern South America (Mitten, 1869; Gibert, 1873; Jaeger, 1877; Mueller, 1882; Bescherelle, 1891; Paris, 1904a, 1905a; Brotherus, 1905b, 1925; Herter, 1928. 1933a, b; Kühnemann, 1938; Herzog, 1952a).

C. orbifolium (Besch.) Broth.

Cryphidium leucocoleum

Cupressina hom. illeg.

C. cupressiformis (Hedw.) C. Muell.

C. pallido-nitida C. Muell.

Hypnum cupressiforme

Cuspidaria hom. illeg.

C. fulvo-acuta C. Muell.

C. morenoi C. Muell.

Calliergonella cuspidata

C. pseudo-pura C. Muell.

Pleurozium schreberi

Cyathophorum

C. splendidissimum (Mont.) Hamp. et Lor.

Schimperobryum splendidissimum

\section{Cyclodictyon}

C. albicans (Hedw.) O. Kuntze (Kuntze, 1891).

Syn. Leskea albicans Hedw. (Hedwig, 1801).

Distr. Southern South America (Herzog, 1952a).

C. sublimbatum (C. Muell.) O. Kuntze (Kuntze, 1891).

Syn. Hookeria sublimbata C. Muell. (Mueller, 1851a).

Pterygophyllum sublimbatum (C. Muell.) Mitt. (Mitten, 1885).

Distr. Southern South America (Mueller, 1851a; Mitten, 1869, 1885; Jaeger, 1877; Paris, 1904b, 1905b; Brotherus, 1925; Welch, 1969).

\section{Cylindrothecium}

C. flavo-virens (C. Muell.) Par. var. flagellaceum (C. Muell.) Par.

C. platygyrioides (C. Muell.) Par.

C. suberythropus (C. Muell.) Par.

Pseudoscleropodium pseudopurum

Rhaphidorrhynchium pallido-nitidum

var. complanatulum (C. Muell.) Par.

Entodon flavo-virens

Entodon flavo-virens var. flagellaceus

Erythrodontium platygyrioides

Entodon suberythropus

Entodon suberythropus var. complanatulus 
var. ellipticum (C. Muell.) Par.

var. flaccidum (C. Muell.) Par.

var. gracillimum (C. Muell.) Par.

var. Iutescens (C. Muell.) Par.

var. microtheca (C. Muell.) Par.

var. myosuroides (C. Muell.) Par.

var. rufulum (C. Muell.) Par.

C. truncatum (C. Muell.) Par.
Entodon suberythropus var. ellipticus

Entodon suberythropus var. flaccidus

Entodon suberythropus var. gracillimus

Entodon suberythropus var. lutescens

Entodon suberythropus var. microtheca

Entodon suberythropus var. myosuroides

Entodon suberythropus var. rufulus

Entodon truncatus

\section{Cynodontium}

C. capillaceum (Hedw.) Brid.

C. conicum (Mont.) Mitt.

Distichium capillaceum

C. fuegianum (Card.) Card.

C. hookeri (C. Muell.) Mitt.

C. leiocarpum Lor.

Ditrichum conicum

Oncophorus fuegianus

Ditrichum hookeri

Aongstroemia leiocarpa

Cynontodium nom. rejic.

C. affine (C. Muell.) Mitt.

Ditrichum difficile

C. australe Mitt.

Ditrichum strictum

C. capillaceum Hedw.

Distichium capillaceum

C. elongatum (Hook. f. et Wils.) Mitt.

Ditrichum cylindricarpum

C. hookeri (C. Muell.) Mitt.

Ditrichum hookeri

C. hyalinum (Mitt.) Mitt.

Ditrichum hyalinum

\section{Cyptodon}

C. crassinervis Broth. (Brotherus, 1924b).

Distr. Juan Fernandez (Brothenus, 1924b, 1925; Robinson, 1975).

\section{Cyrto-Hypnum}

C. filarium (Mitt.) Hamp.

Thuidiopsis furfurosa

\section{Cyrtopus}

C. curtipendulus (Hedw.) Spruc.

Antitrichia curtipendula

Daltonia hom. illeg.

D. ariquensis Schimp.

Daltonia gracilis

D. aristata Geh. et Hamp. (Geheeb and Hampe in Hampe, 1879).

Syn. Daltonia uleana C. Muell. (Mueller, 1898c).

Distr. Southern South America (Reimers, 1926).

D. gracilis Mitt. (Mitten, 1869).

Syn. Daltonia krauseana C. Muell. ex Dus. (Mueller ex Dusén, 1905b).

Daltonia ariquensis Schimp. (Schimper in Bartram, 1931) nom. nud.

Distr. Juan Femandez (Robinson, 1975).

Southern South America (Dusén, 1905b; Brotherus, 1925; Bartram, 1931, 1952;

Herzog, 1954; Welch, 1969; Seki, 1974; Matteri, 1975b).

D. krauseana C. Muell. ex Dus.

Daltonia gracilis

D. patagonica Dus. (Dusén, 1903a) nom. nud.

Distr. Southern South America (Dusén, 1903a). 
D. polytrichoides (Hedw.) Arnott

Lepidopilum polytrichoides

D. trachydontia Mitt. ex Bartr.

Daltonia trachyodonta

D. trachyodonta Mitt. (Mitten, 1869).

Syn. Daltonia trachydontia Mitt. ex Bartr. (Mitten ex Bartram, 1931) err.

Daltonia valdiviae Herz. (Herzog, 1923).

Distr. Southem South America (Herzog, 1923; Brotherus, 1925; Bartram, 1931; Welch, 1969; Seki, 1974; Matteri, 1975b).

D. uleana C. Muell.

Daltonia aristata

D. valdiviae Herz.

Daltonia trachyodonta

Decodon hom. illeg.

D. demissus C. Muell.

Rhachithecium perpusillum

\section{Dendrocryphaea}

D. cuspidata (Sull.) Broth. ex Par. (Brotherus ex Paris, 1909).

Syn. Cryphaea cuspidata Sull. (Sullivant, 1857 [1854]).

Braunia cuspidata (Sull.) Hamp. (Hampe, 1867a).

Distr. Juan Femandez (Skottsberg, 1914; Brotherus, 1924b).

Southem South America (Sullivant, 1857, 1859; Mitten, 1869; Paris, 1904a, 1909;

Brotherus, 1905b, 1925; Thériot, 1924, 1934b, 1935a; Herzog, 1954, 1960).

D. gorveana (Mont.) Par. et Schimp. (Paris and Schimper in Brotherus, 1905b).

Syn. Cryphaea gorveana Mont. (Montagne, 1845a).

Dendropogon gorveanus (Mont.) B.S.G. (Bruch and others, 1850).

Pilotrichum gorveanum (Mont.) C. Muell. (Mueller, 1851a).

Distr. Southem South America (Montagne, 1845a, 1850, 1856; Mueller, 1851a; Lorentz, 1866; Mitten, 1869; Jaeger, 1876; Neger, 1899; Paris, 1904a; Brotherus, 1905b; Cardot. 1908a; Herzog, 1923; Kühnemann, 1938; Seki, 1974).

var. lechleri Fleisch.

Dendrocryphaea lechleri

D. lechleri (Fleisch.) Par. et Schimp. ex Thér. (Paris and Schimper ex Thériot, 1934b [1935]).

Syn. Cryphaea lechleri C. Muell. (Mueller in Lorentz, 1866) nom. nud.

Dendropogon lechleri C. Muell. (Mueller in Fleischer, 1914) nom. nud.

Dendrocryphaea gorveana (Mont.) Par. et Schimp. var. lechleri Fleisch. (Fleischer, 1914).

Cryphaea ramosissima Schimp. (Schimper in Bescherelle, 1876) nom. nud.

Dendrocryphaea ramosissima Par. et Schimp. (Paris and Schimper in Paris, 1894) nom. nud.

Distr. Southern South America (Lorentz, 1866; Paris, 1894, 1904a; Fleischer, 1914; Thériot, 1934b).

D. pulchella (Par. et Schimp.) Fleisch.

Dendrocryphaea ramosissima

D. ramosissima Par. et Schimp.

Dendrocryphaea lechleri

D. ramosissima (Par.) Wijk et Marg. (van der Wijk and Margadant, 1959).

Syn. Cryphaea ramosissima Par. (Paris in Jaeger, 1876).

Cryphaea tenella Mitt. (Mitten, 1869) hom. illeg.

Dendrocryphaea tenella Thér. (Thériot, 1934b [1935]) nom. illeg.

Cryphaea pulchella Par. et Schimp. (Paris and Schimper in Paris, 1894).

Dendrocryphaea pulchella (Par. et Schimp.) Fleisch. (Fleischer, 1914).

Distr. Southern South America (Mitten, 1869; Jaeger, 1876; Paris, 1894, 1904a; Cardot, 1908a; Brotherus, 1925; Thériot, 1934b; Kühnemann, 1938).

D. tenella Thér.

Dendrocryphaea ramosissima

\section{Dendro-Hypnum}

D. fasciculatum (Hedw.) Hamp.

Porothamnium fasciculatum 


\section{Dendroligotrichum}

D. dendroides (Hedw.) Broth. (Brotherus, 1905a).

Syn. Polytrichum dendroides Brid. ex Hedw. (Bridel ex Hedwig, 1801).

Pogonatum dendroides (Hedw.) Brid. (Bridel, 1827b).

Catharinea dendroides (Hedw.) Hamp. (Hampe, 1847).

Polytrichadelphus dendroides (Hedw.) Mitt. (Mitten, 1869).

Oligotrichum dendroides (Hedw.) Kindb. (Kindberg, 1888).

Gymnopilum dendroides (Hedw.) Schimp. (Schimper in Paris, 1895) nom. inval.

Distr. Juan Fernandez (Skottsberg, 1914; Brotherus, 1924b, 1925; Espinosa B., 1941; Bartram, 1959; Robinson, 1975).

Southern South America (Hedwig, 1801; Bridel, 1803, 1806, 1827b; Palisot de Beauvois, 1805a; Schwaegrichen, 1816, 1826; Bridel, 1827b; Montagne, 1845b, 1850; Wilson and Hooker, 1847; Mueller, 1848a, 1851a, 1885; Sullivant, 1859; Lorentz, 1866; Mitten, 1869; Jaeger, 1875; Bescherelle, 1889; Hariot, 1891; Neger, 1899; Cardot, 1900, 1901, 1908a, 1913a; Paris, 1905b; Thériot, 1918; Campo, 1921; Cardot and Brotherus, 1923; Herzog, 1923, 1938, 1939, 1940, 1954, 1957, 1960; Brotherus, 1925; Reimers, 1926; Kühnemann, 1938; Bartram, 1952; Seki, 1974).

D. squamosum (Hook. f. et Wils.) Broth. (Cardot, 1908a).

Syn. Polytrichum squamosum Hook. f. et Wils. (Hooker, J. D. and Wilson, 1844). Catharinea squamosa (Hook. f. et Wils.) C. Muell. (Mueller, 1848a).

Polytrichadelphus squamosus (Hook. f. et Wils.) Mitt. (Mitten, 1869).

Oligotrichum squamosum (Hook. f. et Wils.) Kindb. (Kindberg, 1888).

Distr. Southern South America (Hooker, J. D. and Wilson, 1844; Wilson and Hooker, 1847; Mueller, 1848a, 1885; Sullivant, 1859; Mitten, 1869; Ångström, 1872; Jaeger, 1875; Cardot, 1905a, 1908a, 1913a; Paris, 1905b; Cardot and Brotherus, 1923; Brotherus, 1925; Bartram, 1946, 1952; Herzog, 1954; Seki, 1974).

Falkland Islands (Paris, 1905b; Cardot, 1908a; Cardot and Brotherus, 1923; Brotherus, 1925).

South Georgia (Greene, 1973).

\section{Dendropogon}

D. gorveanus (Mont.) B.S.G.

Dendrocryphaea gorveana

D. lechleri C. Muell.

Dendrocryphaea lechleri

\section{Dermatodon}

D. rubellus Genth

Bryoerythrophyllum recurvirostre

\section{Desmatodon}

D. amblyophyllus Mont.

Tortula platyphylla

D. argentinicus Broth. (Brotherus, 1918).

Distr. Southern South America (Brotherus, 1918, 1924a; Kühnemann, 1938).

D. atro-virens (Sm.) Jur.

Desmatodon convolutus

D. cavifolius Mitt.

Pterygoneurum ovatum

D. convolutus (Brid.) Grout (Grout, 1939).

Syn. Trichostomum convolutum Brid. (Bridel, 1806).

Grimmia atro-virens Sm. (Smith, 1809).

Tortula atro-virens (Sm.) Lindb. (Lindberg, 1864a).

Barbula atrovirens (Sm.) Schimp. (Schimper, 1876).

Desmatodon atro-virens (Sm.) Jur. (Juratzka, 1882).

Distr. Southern South America (Mitten, 1869; Thériot, 1921a, b; Brotherus, 1924a; Bartram, 1952; Herzog, 1954).

D. heimii (Hedw.) Mitt.

Pottia heimii

D. rigidulus Hassk.

Didymodon rigidulus

D. ruber (Mitt.) Hamp.

Tortula robusta 
D. schimperi (Mont.) Jaeg.

Didymodon schimperi

D. serrulatus (Hook. et Grev.) Mitt.

Tortula robusta

D. subtophaceus (Williams) Williams

Trichostomopsis australasiae

\section{Dichodontium}

D. brotheri Dix.

Oncophorus fuegianus

D. capituligerum (C. Muell.) Par.

Anisothecium capituligerum

D. dicranelloides Card.

Dicranella cardotii

var. falklandicum Card.

D. elegans (Dub.) Jaeg.

Anisothecium persquarrosum var. falklandicum

D. jamesonii (Mitt.) Jaeg.

Anisothecium elegans

D. krausei (Lor.) Jaeg.

Anisothecium vaginatum

D. lorentzii (C. Muell.) Par.

Anisothecium krausei

D. paludella Besch.

Anisothecium lorentzii

D. patagonicum (C. Muell.) Par.

Anisothecium paludella

D. persquarrosum (Dus.) Card.

Dicranella patagonica

var. falklandicum (Card.) Card.

Dicranella cardotii

D. rufipes (C. Muell.) Par.

Anisothecium persquarrosum var. falklandicum

D. subclathratum (Lor.) Par.

Anisothecium rufipes

D. vaginatum (Hook.) Jaeg.

Dicranella hookeri

Anisothecium vaginatum

\section{Dicranella}

D. argentinica (C. Muell.) Kindb. (Kindberg, 1889).

Syn. Aongstroemia argentinica C. Muell. (Mueller, 1879a).

Distr. Southern South America (Mueller, 1879a; Kurtz, 1904; Paris, 1904b; Hosseus, 1935b; Kühnemann, 1938).

D. aulacocarpa (Mont.) Mitt. (Mitten, 1869).

Syn. Dicranum aulacocarpum Mont. (Montagne, 1845a).

Aongstroemia aulacocarpa (Mont.) C. Muell. (Mueller, 1848a).

Distr. Southem South America (Montagne, 1845a, 1850, 1856; Mueller, 1848a; Mitten, 1869; Jaeger, 1872; Paris, 1904b).

D. capituligera (C. Muell.) Kindb.

Anisothecium capituligerum

D. cardotii (R. Brown ter.) Dix. (Dixon, 1923).

Syn. Dicranum cardotii R. Brown ter. (Brown, 1902 [1903]).

Aongstroemia persquarrosa Dus. (Dusén, 1905a) fide Newton, 1977 a.

Dichodontium persquarrosum (Dus.) Card. (Cardot, 1908a).

Anisothecium persquarrosum (Dus.) Broth. (Brotherus, 1924a).

Dichodontium dicranelloides Card. (Cardot, 1905a).

Distr. Southern South America (Dusén, 1903a, 1905a; Cardot, 1905a, 1908a; Cardot and Brotherus, 1923; Brotherus, 1924a; Roivainen, 1934; Roivainen and Bartram, 1937; Kühnemann, 1938; Bartram, 1952; Herzog, 1954; Seki, 1974).

Falkland Islands (Cardot and Brotherus, 1923).

South Georgia (Newton, 1977a).

D. circinata Herz. (Herzog, 1954).

Distr. Southern South America (Herzog, 1954).

D. costata Broth. (Brotherus, 1924b).

Distr. Juan Fernandez (Brotherus, 1924a, b; Robinson, 1975).

D. euchlora (Mont.) Lor. 
D. flexipes Card. et Broth. (Cardot and Brotherus, 1923).

Distr. Southern South America (Cardot and Brotherus, 1923; Brotherus, 1924a; Kühnemann, 1938; Seki, 1974).

D. flexiseta Dus. (Dusén in Paris, 1904b) nom. nud.

Distr. Southern South America (Paris, 1904b).

D. fuegiana Card. et Broth. (Cardot and Brotherus, 1923).

Distr. Southern South America (Cardot and Brotherus, 1923; Brotherus, 1924a; Kühnemann. 1938).

D. guilleminiana (Mont.) Mitt. (Mitten, 1869).

Syn. Dicranum guilleminianum Mont. (Montagne, 1841).

Distr. Southern South America (Krieger, 1904).

D. hilariana (Mont.) Mitt. (Mitten, 1869).

Syn. Dicranum hilarianum Mont. (Montagne, 1839a).

Aongstroemia hilariana (Mont.) C. Muell. (Mueller, 1848a).

Microdus hilarianus (Mont.) Besch. (Bescherelle in Paris, 1896).

Dicranum tenuirostre Kunz. ex Schwaegr. (Kunze ex Schwaegrichen, 1842).

Aongstroemia tenuirostris (Schwaegr.) C. Muell. (Mueller, 1848a).

Dicranella tenuirostris (Schwaegr.) Mitt. (Mitten, 1869).

Microdus tenuirostris (Schwaegr.) Besch. (Bescherelle in Paris, 1896).

Dicranum sellowii Hornsch. (Hornschuch, 1840).

Distr. Southern South America (Hornschuch, 1840; Mueller, 1844b, 1848a; Montagne, 1850 , 1856; Jaeger, 1872; Gibert, 1873; Paris, 1896, 1905a; Herter, 1933a).

D. hookeri (C. Muell.) Card. (Cardot, 1906a) fide Newton, 1977 a.

Syn. Aongstromia hookeri C. Muell. (Mueller, 1851a).

Anisothecium hookeri (C. Muell.) Broth. (Brotherus, 1924a).

Dicranum vaginatum Hook. f. et Wils. (Hooker and Wilson in Mueller, 1851a) nom. nud.

Dicranella subclathrata Lor. (Lorentz, 1864).

Dichodontium subclathratum (Lor.) Par. (Paris, 1894).

Aongstroemia subclathrata (Lor.) C. Muell. (Mueller, 1901a [1900]).

Anisothecium perpusillum Dus. (Dusén, 1903a) fide Newton, 1977 a.

Dicranella perpusilla (Dus.) Card. (Cardot, 1908a).

Distr. Southern South America (Wilson and Hooker, 1847; Mueller, 1851a, 1885, 1901a: Lorentz, 1864, 1866; Jaeger, 1872; Paris, 1894, 1904b; Dusén, 1903a; Cardot. 1908a; Cardot and Brotherus, 1923; Thériot, 1923b, 1924; Brotherus. 1924a: Roivainen and Bartram, 1937; Herzog, 1938, 1954; Kühnemann, 1938; Bartram. 1952; Seki, 1974).

South Georgia (Cardot, 1906a, 1908a; Brotherus, 1924a; Steere, 1961b; Newton. 1977a).

Antarctic, continental region (Cardot, 1910; Brotherus, 1924a; Greene, 1968a; Newton. 1977b).

var. flexisubulata Card. et Broth. (Cardot and Brotherus, 1923).

Distr. Southern South America (Cardot and Brotherus, 1923; Seki, 1974).

D. jamesonii (Mitt.) Broth.

Anisothecium vaginatum

D. Krausei Lor.

Anisothecium krausei

D. kunzeana (C. Muell.) Mitt.

Microdus kunzeanus

D. lorentzii (C. Muell.) Kindb.

Anisothecium lorentzii

D. paludella (Besch.) Dus.

Anisothecium paludella

D. patagonica (C. Muell.) Broth. (Brotherus, 1901).

Syn. Aongstroemia patagonica C. Muell. (Mueller, 1897a).

Dichodontium patagonicum (C. Muell.) Par. (Paris, 1900a).

Distr. Southern South America (Mueller, 1897a; Paris, 1900a, 1904b; Brotherus, 1901: Cardot, 1908a; Herzog, 1923). 
D. perpusilla (Dus.) Card.

Dicranella hookeri

D. pseudorufescens Card. et Broth. Anisothecium pseudorufescens var. leptoclada Card. et Broth.

D. rufipes (C. Muell.) Kindb.

Anisothecium pseudorufescens var. leptocladus

D. skottsbergii Card. et Broth.

D. subclathrata Lor.

Anisothecium rufipes

D. tenuirostris (Schwaegr.) Mitt.

D. vaginata (Hook.) Card.

Anisothecium skottsbergii

Dicranella hookeri

Dicranella hilariana

Anisothecium vaginatum

Dicranobryum nom. nud.

D. fabroniellum Dus.

Brachymenium fabroniellum

D. subfabroniellum Dus.

Brachymenium meyenianum

Dicranodon nom. illeg.

D. adianthoides (Hedw.) Behéré

D. bryoides (Hedw.) Behéré

Fissidens adianthoides

Fissidens bryoides

\section{Dicranodontium}

D. asperifolium (Mitt.) Broth. (Brotherus, 1924a).

Syn. Campylopus asperifolius Mitt. (Mitten, 1869).

Distr. Southern South America (Mitten, 1869; Jaeger, 1872; Paris, 1904a).

D. schwabei Herz. et Thér. (Herzog and Thériot in Herzog, 1939).

Distr. Southern South America (Herzog, 1939).

\section{Dicranoloma}

D. australe (C. Muell.) Par. (Paris, 1904b).

Syn. Dicranum australe Besch. ex C. Muell. (Bescherelle ex Mueller, 1885).

Leucoloma australe (Besch.) Broth. (Brotherus, 1901).

Dicranum pseudo-australe C. Muell. (Mueller in Kindberg, 1891) nom. nud.

Dicranoloma pseudo-australe Ren. (Renauld, 1909) nom. nud.

Leucoloma robustum Hook. f. et Wils. var. flexuosum Dus. (Dusén, 1905b) fide Cardot, 1908a.

Distr. Southem South America (Bescherelle, 1885a, 1889; Mueller, 1885, 1889; Hariot, 1891; Dusén, 1903a, $1905 b$ (and as Leucoloma setosum fide Cardot, 1908a), 1905d; Paris, 1904b; Cardot, 1905a, 1908a; Renauld, 1909; Cardot and Brotherus, 1923; Brotherus, 1924a; Roivainen and Bartram, 1937; Seki, 1974).

Falkland Islands (Cardot and Brotherus, 1923).

var. condensatum Broth. (Brotherus in Cardot and Brotherus, 1923).

Distr. Falkland Islands (Cardot and Brotherus, 1923; Kühnemann, 1938).

var. schwabei Herz. et Thér. (Herzog and Thériot in Herzog, 1939).

Distr. Southern South America (Herzog, 1939).

D. austro-georgicum Broth.

Dicranoloma hariotii

D. billardieri (Brid.) Par. (Paris, 1904b).

Syn. Dicranum billardieri Brid. (Bridel, 1802).

Oncophorus billardieri (Brid.) Brid. (Bridel, 1826).

Leucoloma.billardieri (Brid.) Broth. (Brotherus, 1901).

Dicranum boryanum Schwaegr. (Schwaegrichen, 1823).

Trematodon boryanus (Schwaegr.) Spreng. (Sprengel, 1827) hom. illeg.

Cecalyphum dichotomum P. Beauv. (Palisot de Beauvois, 1805a).

Dicranum dichotomum (P. Beauv.) Brid. (Bridel, 1806) hom. illeg.

Oncophorus dichotomus (P. Beauv.) Brid. (Bridel, 1826).

Dicranoloma dichotomum (P. Beauv.) Ren. (Renauld, 1901). 
Dicranum callocaulon C. Muell. (Mueller in Kindberg, 1889) nom. nud.

Dicranum helvolum C. Muell. (Mueller in Kindberg, 1891) nom. nud.

Dicranoloma fernandezianum Broth. (Brotherus, 1924b) fide Robinson, 1975.

Distr. Juan Fernandez (Montagne, 1835; Mitten, 1869, 1885; Jaeger, 1872; Johow, 1896; Paris, 1904b; Skottsberg, 1914; Brotherus, 1924a, b, 1925; Espinosa B., 1941; Bartram, 1959; Robinson, 1975).

Southern South America (Montagne, 1850; Mitten, 1869; Jaeger, 1872; Brotherus, 1901, 1924a; Dusén, 1903a, 1905a; Paris, 1904b; Cardot, 1905a, 1908a; Thériot, 1918, 1935a; Campo, 1921; Spegazzini, 1922; Cardot and Brotherus, 1923; Herzog, 1923, 1938, 1939, 1954, 1960; Roivainen, 1934; Roivainen and Bartram, 1937; Kühnemann, 1938; Bartram, 1952; Bizot, 1967; Seki, 1974).

Falkland Islands, Wilson and Hooker, 1847; Mueller, 1848a; Jaeger, 1872; Brotherus, 1901, 1924a; Paris, 1904b; Cardot, 1908a; Kühnemann, 1938).

var. compactum (Card.) Card. et Broth. (Cardot and Brotherus, 1923).

Syn. Dicranum billardieri Brid. var. compactum Card. (Cardot, 1905a).

Distr. Southern South America (Cardot and Brotherus, 1923).

Falkland islands (Cardot, 1905a, 1908a).

var. moelleri Bauer (Bauer, 1934).

Distr. Southern South America (Bauer, 1934).

D. capillare (Dus.) Broth. (Brotherus, 1909).

Syn. Leucoloma capillare Dus. (Dusén, 1905a).

Dicranum capillare (Dus.) Card. (Cardot, 1908a).

Leucoloma nigricaule (Aongstr.) Par. var. gracile Dus. (Dusén, 1905a).

Leucoloma peruncinatum Dus. var. gracile Dus. (Dusén in Roivainen and Bartram, 1937) nom. nud.

Distr. Southern South America (Dusén, 1905a; Cardot, 1908a; Brotherus, 1909, 1924a; Roivainen and Bartram, 1937; Kühnemann, 1938; Herzog, 1939, 1957; Seki, 1974).

D. capillifolioides Broth. (Brotherus, 1924b).

Distr. Juan Fernandez (Brotherus, 1924b; Bartram, 1959; Robinson, 1975).

D. capillifolium (Card.) Broth.

Dicranoloma menziesii

D. dichotomum (P. Beauv.) Ren.

Dicranoloma billardieri

D. dusenii (Broth.) Broth. (Brotherus, 1909).

Syn. Leucoloma dusenii Broth. (Brotherus in Dusén, 1905a).

Distr. Southern South America (Dusén, 1905a; Paris, 1905a; Brotherus, 1909, 1924a; Herzog, 1954).

D. falklandicum (Card.) Broth. (Brotherus, 1909).

Syn. Dicranum falklandicum Card. (Cardot, 1905a).

Distr. Southern South America (Cardot and Brotherus, 1923).

Falkland Islands (Cardot, 1905a, 1908a; Brotherus, 1909, 1924a; Kühnemann, 1938).

D. fernandezianum Broth.

Dicranoloma billardieri

D. fuegianum (Dus.) Broth.

Dicranoloma hariotii

D. hariotii (C. Muell.) Par. (Paris, 1904b).

Syn. Dicranum hariotii C. Muell. (Mueller, 1885).

Leucoloma hariotii (C. Muell.) Broth. (Brotherus, 1901).

Leucoloma fuegianum Dus. (Dusén, 1905a).

Dicranum fuegianum (Dus.) Card. (Cardot, 1908a).

Dicranoloma fuegianum (Dus.) Broth. (Brotherus, 1909).

Leucoloma fuegianum Dus. var. laxum Dus. (Dusén, 1905a).

Dicranum fuegianum (Dus.) Card. var. laxum (Dus.) Card. (Cardot, 1908a).

Dicranoloma hariotii (C. Muell.) Par. var. laxum (Dus.) Card. et Broth. (Cardot and Brotherus, 1923).

Dicranum austro-georgicum C. Muell. (Mueller, 1890a). 
Dicranoloma austro-georgicum Broth. (Brotherus in Roivainen and Bartram, 1937) nom. nud.

Leucoloma alboalare Dus. (Dusén in Cardot, 1908a) nom. nud.

Distr. Southern South America (Bescherelle, 1885a, 1889; Mueller, 1885; Cardot, 1900, 1901, 1908a; Brotherus, 1901, 1909, 1924a; Dusén, 1904, 1905a; Paris, 1904b; Cardot and Brotherus, 1923; Roivainen and Bartram, 1937; Kühnemann, 1938; Herzog, 1954; Seki, 1974).

Falkland Islands (Cardot and Brotherus, 1923; Kühnemann, 1938).

South Georgia (Mueller, 1890a; Paris, 1904b; Cardot, 1906a, 1908a; Dixon, 1920; Steere, 1961b; Bell, 1973b, 1977b).

var. laxum (Dus.) Card. et Broth.

Dicranoloma hariotii

D. hollermayeri Thér. (Thériot, 1935a).

Distr. Southern South America (Thëriot, 1935a).

D. imponens (Mont.) Ren. (Renauld, 1909).

Syn. Dicranum imponens Mont. (Montagne, 1843).

Leucoloma imponens (Mont.) Dus. (Dusén, 1905a).

Dicranum involutifolium Sull. (Sullivant, 1850).

Leucoloma robustum (Hook. f. et Wils.) Broth. var. lagunicola Dus. (Dusén, 1905a).

Dicranum robustum Hook. f. et Wils. var. lagunicola (Dus.) Card. (Cardot, 1908a).

Distr. Southern South America (Montagne, 1843, 1845b, 1850, 1856; Wilson and Hooker, 1847; Mueller, 1848a, 1851a, 1885; Sullivant, 1850, 1859; De Notaris, 1859; Mitten, 1869; Jaeger, 1872; Bescherelle, 1889; Eaton, 1892; Paris, 1904b; Cardot, 1905a, 1908a; Dusén, 1905a; Renauld, 1909; Thériot, 1921a, 1934b; Cardot and Brotherus, 1923; Brotherus, 1924a; Roivainen, 1934; Roivainen and Bartram, 1937; Kühnemann, 1938; Herzog, 1939; Seki, 1974).

D. kunkelii H. Robins. (Robinson, 1974).

Distr. Juan Fernandez (Robinson, 1974, 1975).

D. menziesii (Hook. f. et Wils.) Par. (Paris, 1904b).

Syn. Dicranum menziesii Hook. f. et Wils. (Hooker, J. D. and Wilson, 1844).

Leucoloma menziesii (Hook. f. et Wils.) Broth. (Brotherus, 1901).

Dicranum suberectum Hamp. (Hampe, 1860).

Leucoloma suberectum (Hamp.) Broth. (Brotherus, 1901).

Dicranoloma suberectum (Hamp.) Par. (Paris, 1904b).

Leucoloma capillifolium Broth. (Brotherus in Dusén, 1905a) hom. illeg. fide Robinson, 1975.

Dicranum capillifolium Card. (Cardot, 1908a).

Dicranoloma capillifolium (Card.) Broth. (Brotherus, 1909).

Distr. Juan Fernandez (Brotherus, 1924b; Espinosa B., 1941; Bartram, 1959; Robinson, 1975).

Southern South America (Dusén, 1903a, 1905a; Paris, 1904b; Cardot, 1908a; Brotherus, 1909, 1924a; Campo, 1915, 1921; Thériot, 1918; Cardot and Brotherus, 1923; Herzog, 1938, 1954, 1960; Kühnemann, 1938).

var. chilense Thér. (Thériot, 1924).

Distr. Southern South America (Thériot, 1924).

var. fernandezianum (Thér.) Broth. (Brotherus, 1924b).

Syn. Dicranum menziesii Hook f. et Wils. var. fernandezianum Card. ex Thér. (Cardot ex Thériot, 1921b).

Distr. Juan Fernandez (Skottsberg, 1914; Thériot, 1921b; Brotherus, 1924b).

D. muelleri Dus.

Dicranoloma robustum var. setosum

D. muelleri (Dus.) Roiv. (Roivainen in Roivainen and Bartram, 1937).

Syn. Leucoloma muelleri Dus. (Dusén, 1905a).

Distr. Southern South America (Dusén, 1905a; Paris, 1905a; Cardot and Brotherus, 1923 (as D. capillifolium fide Roivainen and Bartram, 1937); Roivainen and Bartram, 1937;

Kühnemann, 1938; Herzog, 1954; Seki, 1974). 
var. strictifolium (Dus.) Herz.

Dicranoloma robustum var. strictifolium

D. nigricaule (Aongstr.) Par. (Paris, 1904b).

Dicranoloma robustum var. strictifolium

Syn. Dicranum nigricaule Aongstr. (Ångström, 1872).

Leucoloma nigricaule (Aongstr.) Broth. (Brotherus, 1901).

Dicranum racovitzae Card. (Cardot, 1900).

Dicranum chilense De Not. (De Notaris, 1859).

Leucoloma peruncinatum Dus. (Dusén, 1905a).

Dicranum peruncinatum (Dus.) Card. (Cardot, 1908a).

Dicranoloma peruncinatum (Dus.) Broth. (Brotherus, 1909).

Distr. Juan Fernandez (Brotherus, 1924b; Robinson, 1975).

Southem South America (De Notaris, 1859; Ångström, 1872; Jaeger and Sauerbeck, 1879b; Mueller, 1885; Bescherelle, 1889; Cardot, 1900, 1901, 1905a, 1908a, 1913a; Brotherus, 1901, 1909, 1924a; Dusén, 1903a, 1905a; Paris, 1904b; Cardot and Brotherus, 1923; Roivainen and Bartram, 1937; Kühnemann, 1938; Bartram, 1952; Seki, 1974).

Falkland Islands (Cardot and Brotherus, 1923; Kühnemann, 1938).

var. flexuosulum (Dus.) Bartr. (Bartram in Roivainen and Bartram, 1937).

Syn. Leucoloma nigricaule (Aongstr.) Broth. var. flexuosulum Dus. (Dusén, 1905a).

Dicranum nigricaule Aongstr. var. flexuosulum (Dus.) Card. (Cardot, 1908a).

Distr. Southern South America (Dusén, 1905a; Cardot, 1908a; Roivainen and Bartram, 1937).

D. perremotifolium (Dus.) Broth. (Brotherus, 1924a).

Syn. Leucoloma perremotifolium Dus. (Dusen, 1905a).

Dicranum perremotifolium (Dus.) Card. (Cardot, 1908a).

Distr. Southern South America (Paris, 1904a, 1905a; Dusén, 1905a; Cardot, 1908a; Brotherus, 1924a; Roivainen, 1934; Kühnemann, 1938; Herzog, 1954, 1960; Seki, 1974).

var. fragile (Dus.) Thér. (Thériot, 1935a).

Syn. Leucoloma perremotifolium Dus. var. fragile Dus. (Dusén, 1905a).

Distr. Southem South America (Dusén, 1905a; Thériot, 1935a).

D. peruncinatum (Dus.) Broth.

Dicranoloma nigricaule

D. pseudo-australe Ren.

Dicranoloma australe

D. pungens (Hook. f. et Wils.) Par.

Dicranoloma robustum

D. rigens (Besch.) Par.

Chorisodontium aciphyllum

D. robustum (Hook. f. et Wils.) Par. (Paris, 1904b).

Syn. Dicranum robustum Hook. f. et Wils. (Hooker, J. D. and Wilson, 1844).

Leucoloma robustum (Hook. f. et Wils.) Broth. (Brotherus, 1901).

Dicranum grandialare Dus. (Dusén, 1903a) nom. nud.

Leucoloma grandialare Dus. (Dusén, 1905a).

Dicranum pungens Hook. f. et Wils. (Hooker, J. D. and Wilson, 1844).

Leucoloma pungens (Hook. f. et Wils.) Broth. (Brotherus, 1901).

Dicranoloma pungens (Hook. f. et Wils.) Par. (Paris, 1904b).

Dicranum robustum Hook. f. et Wils. var. pungens (Hook. f. et Wils.) Hook. f. (Hooker, 1867).

Dicranum hookeri Schimp. (Schimper in Paris, 1895) nom. nud.

Distr. Southern South America (Hooker, J. D. and Wilson, 1844; Wilson and Hooker, 1847; Mueller, 1848a, 1885; Sullivant, 1859; Mitten, 1869; Angström, 1872; Jaeger 1872; Eaton, 1892; Cardot, 1900, 1908a, 1913a; Brotherus, 1901, 1924a; Dusén, 1903a, 1905a, d; Paris, 1904b; Cardot and Brotherus, 1923; Herzog, 1923, 1938, 1939. 1957; Reimers, 1926; Thériot, 1934b, 1935a; Kühnemann, 1938; Bartram, 1952; Seki, 1974).

Falkland Islands (Mueller, 1848a, 1885; Jaeger, 1872; Cardot, 1908a).

var. longisetaceum Herz. (Herzog, 1938).

Distr. Southern South America (Herzog, 1938). 
var. setosum (Hook. f. et Wils.) Sains. (Sainsbury, 1931 [1932]).

Syn. Dicranum setosum Hook. f. et Wils. (Hooker, J. D. and Wilson, 1844).

Leucoloma setosum (Hook. f. et Wils.) Broth. (Brotherus, 1901).

Dicranoloma setosum (Hook. f. et Wils.) Par. (Paris, 1904b).

Dicranoloma muelleri Dus. (Dusén in Paris, 1904b) nom. nud.

Distr. Southern South America (Ångström, 1872; Paris, 1904b; Dusén, 1905a; Cardot, 1908a; Herzog, 1923, 1954; Brotherus, 1924a; Roivainen, 1934; Kühnemann, 1938; Bartram, 1952; Bizot, 1967; Seki, 1974).

var. strictifolium (Dus.) Wijk et Marg. (van der Wijk and Margadant, 1960a).

Syn. Leucoloma muelleri Dus. var strictifolium Dus. (Dusén, 1905a).

Dicranoloma muelleri (Dus.) Roiv. var. strictifolium (Dus.) Herz. (Herzog, 1940).

Dicranum setosum Hook. f. et Wils. var. strictifolium (Dus.) Card. (Cardot, 1908a).

Dicranoloma setosum (Hook. f. et Wils.) Par. var. strictifolium (Dus.) Kühnemann

(Kühnemann, 1938).

Distr. Southern South America (Dusén, 1905a; Cardot, 1908a; Kühnemann, 1938; Herzog, 1940).

D. setosum (Hook. f. et Wils.) Par.

Dicranoloma robustum var. setosum

var. strictifolium (Dus.) Kühnemann

Dicranoloma robustum var. strictifolium

D. skottsbergii (Card.) Broth. (Brotherus, 1909).

Syn. Dicranum skottsbergii Card. (Cardot, 1905a).

Distr. Southem South America (Cardot, 1905a, 1908a; Brotherus, 1909, 1924a; Kühnemann, 1938).

D. suberectum (Hamp.) Par.

Dicranoloma menziesii

D. subimponens (Card.) Broth. (Brotherus, 1909).

Syn. Dicranum subimponens Card. (Cardot, 1905a).

Dicranum turgidissimum Dus. (Dusén in Roivainen and Bartram, 1937) nom. nud.

Distr. Southern South America (Cardot, 1905a, 1908a; Brotherus, 1909, 1924a; Cardot and Brotherus, 1923; Roivainen and Bartram, 1937; Kühnemann, 1938; Bartram, 1952; Seki, 1974).

South Georgia (Bell, 1973b).

D. subnigricaule Dus. (Dusén in Paris, 1904b) nom. nud.

Distr. Southem South America (Paris, 1904b).

D. truncorum (C. Muell.) Par. (Paris, 1904b).

Syn. Dicranum truncorum Schimp. ex C. Muell. (Schimper ex Mueller, 1859).

Distr. Southem South America (Lechler, 1857; Mueller, 1859; Lorentz, 1866; Jaeger, 1872; Paris, 1904b).

\section{Dicranoweisia}

D. antarctica (C. Muell.) Kindb. (Kindberg, 1888).

Syn. Blindia antarctica C. Muell. (Mueller, 1848a).

Dicranum antarcticum (C. Muell.) Mitt. (Mitten, 1869).

Weisia antarctica (C. Muell.) Wils. (Wilson in Jaeger, 1870).

Distr. Southem South America (Wilson and Hooker, 1847 (as Weissia crispula fide Mueller, 1848a); Mueller, 1848a, 1879a, 1885; Mitten, 1869; Jaeger, 1870; Paris, 1904b; Cardot, 1908a; Cardot and Brotherus, 1923; Brotherus, 1924a; Roivainen and Bartram, 1937; Kühnemann, 1938; Herzog, 1939, 1954; Bartram, 1952; Seki, 1974).

Antarctic, peninsula region (Bell, 1976)

var. nigricans Card. et Broth. (Cardot and Brotherus, 1923).

Distr. Southern South America (Cardot and Brotherus, 1923; Kühnemann, 1938).

D. auriculata (C. Muell.) Broth.

Holodontium inerme 
D. austro-crispula (C. Muell.) Par. (Paris, 1895).

Syn. Blindia austro-crispula C. Muell. (Mueller, 1885). Dicranoweisia perpulvinata Dus. (Dusén, 1903a). Blindia sulphurea Dus. (Dusén, 1903a) nom. nud. Dicranoweisia sulfurea Dus. (Dusén in Paris, 1904b) nom. nud. Hymenoloma nordenskjoeldii Dus. (Dusén, 1905a). Dicranoweisia nordenskjoeldii (Dus.) Reimd. (Reimers, 1936) hom. illeg.

Distr. Southern South America (Mueller, 1885; Paris, 1895, 1904b; Dusén, 1903a, 1905a; Cardot, 1905a, 1908a; Cardot and Brotherus, 1923; Brotherus, 1924a; Donat, 1936a; Roivainen and Bartram, 1937; Kühnemann, 1938; Bartram, 1952; Seki, 1974).

D. brevipes (C. Muell.) Card. (Cardot, 1905a).

Syn. Blindia brevipes C. Muell. (Mueller, 1890a).

Distr. South Georgia (Mueller, 1890a; Paris, 1904a; Cardot, 1906a, 1908a; Brotherus, 1924a; Steere, 1961b).

D. breviseta Card. (Cardot, 1905a).

Syn. Dicranoweisia breviseta Card. var. atrata Card. et Broth. (Cardot and Brotherus, 1923).

Distr. Southem South America (Cardot, 1905a, 1908a; Cardot and Brothenus, 1923; Roivainen and Bartram, 1937; Kühnemann, 1938; Seki, 1974).

var. atrata Card. et Broth.

Dicranoweisia breviseta

D. contecta (Hook. f. et Wils.) Par.

Blindia contecta

D. cryptodon (Mont.) Par.

Camptodontium cryptodon

D. funiculipes Card. et Broth. (Cardot and Brotherus, 1923).

Distr. Southern South America (Cardot and Brotherus, 1923; Brotherus, 1924a; Kühnemann, 1938).

D. grimmiacea (C. Muell.) Broth. (Brotherus, 1901).

Syn. Blindia grimmiacea C. Muell. (Mueller, 1890a).

Distr. South Georgia (Mueller, 1890a; Brotherus, 1901, 1924a; Paris, 1904b; Cardot, 1906a, 1908a; Cardot and Brotherus, 1923; Dixon, 1934; Steere, 1961b).

Antarctic, peninsula region (Cardot, 1906a, 1908a, 1911c, 1912b; Brotherus, 1924a; Bartram, 1957; Steere, 1961a; Greene, 1968a; Robinson, 1972; Bell, 1976).

D. humilis (C. Muell.) Broth.

Holodontium pumilum

D. inermis (Mitt.) Par.

Holodontium inerme

D. jugellifera Dus. (Dusén, 1905a).

Distr. Southern South America (Dusén, 1905a; Cardot, 1908a).

D. nordenskjoeldii (Dus.) Reim.

Dicranoweisia austro-crispula

D. pallidifolia (C. Muell.) Par.

Dicranoweisia subinclinata

D. perpulvinata Dus.

Dicranoweisia austro-crispula

D. subinclinata (C. Muell.) Broth. (Brotherus, 1901).

Syn. Blindia subinclinata C. Muell. (Mueller, 1890a).

Blindia pallidifolia C. Muell. (Mueller, 1890a).

Dicranoweisia pallidifolia (C. Muell.) Par. (Paris, 1895).

Distr. Southern South America (Cardot, 1905a, 1908a; Cardot and Brotherus, 1923; Brotherus, 1924a; Kühnemann, 1938).

Falkland Islands (Cardot and Brotherus, 1923).

South Georgia (Mueller, 1890a; Paris, 1895, 1904b; Brotherus, 1901, 1924a; Cardot, 1906a, 1908a; Cardot and Brotherus, 1923; Dixon, 1934; Steere, 1961b).

Antarctic, peninsula region (Cardot, 1911b, $d, 1913 a$; Steere, 1961a; Robinson, 1972).

D. sulfurea Dus.

Dicranoweisia austro-crispula

D. tenella Dus. 


\section{Dicranum}

D. aciphyllum Hook. f. et Wils.

Chorisodontium aciphyllum

D. acuminatum (Mitt.) C. Muell.

Campylopus acuminatus

D. adianthoides (Hedw.) Web. et Mohr

Fissidens adianthoides

D. albo-alare C. Muell. (Mueller in Kindberg, 1889) nom. nud.

Distr. No locality given; see Index muscorum, 2, p. 56.

$D$. alopecurus $\mathrm{C}$. Muell.

D. amabile C. Muell.

D. antarcticum (C. Muell.) Mitt.

D. arctocarpum Hornsch.

D. arenicola C. Muell.

$D$. areodictyon C. Muell.

D. asplenioides (Hedw.) Web. et Mohr

D. atro-viride Card.

D. aulacocarpum Mont.

D. aurificum C. Muell.

var. flavescens $\mathrm{C}$. Muell.

D. australe Besch. ex C. Muell.

$D$. austro-alpinum C. Muell.

$D$. austro-georgicum C. Muell.

Campylopus alopecurus

Campylopus amabilis

Dicranoweisia antarctica

Campylopus arctocarpus

Campylopus surinamensis

Campylopus areodictyon

Fissidens asplenioides

Chorisodontium aciphyllum

Dicranella aulacocarpa

Campylopus introflexus

D. berteroanum Brid.

$D$. billardieri Brid.

var. compactum Card.

var. flavicans Card. (Cardot, 1908a).

Campylopus aurificus var. flavescens

Dicranoloma australe

Campylopus austro-alpinus

Dicranoloma hariotii

Syrrhopodon incompletus var. berteroanus

Dicranoloma billardieri

Distr. Southern South America (Bescherelle, 1889 (as D. imponens for île Wellington, leg. Dr. Contraud fide Cardot, 1908a); Cardot, 1908a).

D. blindiaceum C. Muell.

D. boryanum Schwaegr.

$D$. brachythysanos C. Muell.

D. bryoides (Hedw.) Sw.

D. cactiC. Muell.

D. callocaulon C. Muell.

D. canescens C. Muell.

D. capillare (Dus.) Card.

D. capillifolium Card.

D. cardotii R. Brown ter.

D. chilense De Not.

D. chiloense C. Muell. (Mueller in Kindberg, 1889) nom. nud.

Distr. No locality given; see Index muscorum, 2, p. 63.

D. chrismarii C. Muell.

D. cirrhifolium Schimp. ex Dus.

D. clavatum R. Brown

D. concolor Hook.

D. cryptodon (Mont.) Mitt.

D. dichotomum (P. Beauv.) Brid.

Chorisodontium spegazzinii

Dicranoloma billardieri

Campylopus brachythysanos

Fissidens bryoides

Campylopus cacti

Dicranoloma billardieri

Campylopus canescens

Dicranoloma capillare

Dicranoloma menziesii

Dicranella cardotii

Dicranoloma nigricaule

Campylopus chrismarii

Platyneurum laticostatum

Campylopus clavatus

Campylopus concolor

Camptodontium cyrptodon

Dicranoloma billardieri 
D. dicranellatum Dus.

D. elegans Dub.

D. euchlorum Mont.

D. falklandicum Card.

D. flavissimus C. Muell.

D. flexifolium Hook.

D. flexuosum Hedw. var. minus Schwaegr.

D. fuegianum (Dus.) Card. var. laxum (Dus.) Card.

$D$. gayanum Mont.

D. gracilescens Dus.

D. grandialare Dus.

D. grimmioides C. Muell.

D. griseum (Hornsch.) C. Muell.

D. guilleminianum Mont.

D. hariotii C. Muell.

D. hatscheri Dus.

D. helvolum C. Muell.

D. hilarianum Mont.

D. hookeri Schimp.

$D$. humifugum $\mathrm{C}$. Muell.

D. imponens Mont.

D. incrassatum (C. Muell.) C. Muell.

$D$. inerme Mitt.

D. introflexum Hedw.

$D$. involutifolium Sull.

D. jamesonii Hook.

D. jamesonii Tayl.

D. lanigerum C. Muell.

D. laticostatum Card.
Chorisodontium dicranellatum

Anisothecium elegans Microdus euchlorus

Dicranoloma falklandicum

Campylopus flavissimus

Ditrichum difficile

Campylopus flexuosus

Campylopus flexuosus

Dicranoloma hariotii

Dicranoloma hariotii

Aongstroemia gayana

Blindia curviseta

Dicranoloma robustum

Campylopus grimmioides

Campylopus griseus

Dicranella guilleminiana

Dicranoloma hariotii

Chorisodontium aciphyllum

Dicranoloma billardieri

Dicranella hilariana

Dicranoloma robustum

Campylopus humifugus

Dicranoloma imponens

Campylopus incrassatus

Holodontium inerme

Campylopus introflexus

Dicranoloma imponens

Campylopus jamesonii

Anisothecium vaginatum

Chorisodontium lanigerum

Platyneurum laticostatum

D. leucobryoides Besch. ex C. Muell. (Bescherelle ex Mueller, 1901a [1900]).

Distr. Southern South America (Mueller, 1901a).

D. leucopterum C. Muell.

Chorisodontium aciphyllum var. oncophoriopsis C. Muell. (Mueller, 1885).

Distr. Southern South America (Mueller, 1885; Paris, 1904b; Cardot, 1908a).

D. Ionchotrix C. Muell. (Mueller, 1901a [1900]) nom. nud.

Distr. Southern South America (Mueller, 1901a).

D. macropus Kunz. ex C. Muell.

Chorisodontium macropus

D. magellanicum Card.

Chorisodontium magellanicum

D. menziesii Hook. f. et Wils.

Dicranoloma menziesii

var. fernandezianum Card. ex Thér.

D. microdus Lor.

Dicranoloma menziesii var. fernandezianum

D. morenoi C. Muell.

Chorisodontium microdus

Campylopus morenoi 
D. nano-cespitosum C. Muell. (Mueller in Kindberg, 1891) nom. nud.

Distr. No locality given; see Index muscorum, 2, p. 86.

D. nigricaule Aongstr.

Dicranoloma nigricaule

var. flexuosulum (Dus.) Card.

Dicranoloma nigricaule var. flexuosulum

D. nordenskioeldii Card.

Chorisodontium aciphyllum

D. oleodictyon Dix. (Dixon, 1934).

Distr. South Georgia (Dixon, 1934; Steere, 1961b; Bell, 1973b).

Antartic, peninsula regionN (Bell, 1973a).

$D$. orthocomum Besch. ex C. Muell.

Chorisodontium aciphyllum

D. osmundoides (Hedw.) Turn.

Fissidens osmundoides

D. percompactum Dus.

D. perhorridum (Dus.) Card.

Chorisodontium magellanicum

D. perincanum C. Muell.

Campylopus perhorridus

D. perremotifolium (Dus.) Card.

Campylopus canescens

D. peruncinatum (Dus.) Card.

Dicranoloma perromotifolium

D. pseudo-australe C. Muell.

Dicranoloma nigricaule

D. pseudo-leucoloma C. Muell. (Mueller, 1882).

Dicranoloma australe

Distr. Southem South America (Muelle,r 1882; Paris, 1904b; Kühnemann, 1938).

D. pseudo-truncorum C. Muell. (Mueller in Kindberg, 1889) nom. nud.

Distr. No locality given; see Index muscorum, 2, p. 93.

D. pumilum (Mitt.) Mitt.

Holodontium pumilum

D. pungens Hook. f. et Wils.

Dicranoloma robustum

D. purpureum Hedw.

Ceratodon purpureus

D. racovitzae Card.

Dicranoloma nigricaule

D. ramulosum Mitt.

D. recurvipilum C. Muell.

D. richardii (Brid.) C. Muell.

Holodontium ramulosum

$D$. rigens Besch.

Campylopus griseus

D. robustum Hook. f. et Wils.

Campylopus richardii

Chorisodontium aciphyllum

Dicranoloma robustum

Card. (Cardot, 1908a).

Distr. Southem South America (Cardot, 1901 (as D. robustum fide Cardot, 1908a); Dusén, $1903 a$ (p. 68 as D. australe fide Cardot, 1908a); Cardot, 1908a).

var. lagunicola (Dus.) Card.

var. pungens (Hook. f. et Wils.) Hook. f.

$D$. saddleanum Besch. ex $\mathrm{C}$. Muell.

$D$. scaberrimum Dus.

D. scabrophyllum C. Muell.

D. scoparium Hedw. var. reflectens Brid. (Bridel, 1826).

Distr. Southern South America (Montagne, 1845b, 1850; Kühnemann, 1938).

D. sellowii Hornsch.

Dicranella hilariana

D. semicompletum (Hedw.) Mohr

$D$. setosum Hook. f. et Wils.

var. strictifolium (Dus.) Card.

D. skottsbergii Card.
Dicranoloma imponens

Dicranoloma robustum

Campylopus vesticaulis

Chorisodontium aciphyllum

Campylopus scabrophyllus
Fissidens semicompletus

Dicranoloma robustum var. setosum

Dicranoloma robustum var. strictifolium

Dicranoloma skottsbergii 
D. spegazzinii C. Muell.

Chorisodontium spegazzinii

D. starkei Web. et Mohr

Kiaeria starkei

D. stenocarpum (Hamp.) C. Muell.

Campylopus stenocarpus

D. stenopus C. Muell.

Campylopus stenocarpus

D. strictum Schwaegr.

Pilopogon gracilis

D. suberectum Hamp.

Dicranoloma menziesii

D. subimponens Card.

Dicranoloma subimponens

D. syrrhopodontoides C. Muell. (Mueller, 1897a).

Syn. Leucoloma syrrhodictyon (C. Muell.) Broth. (Brotherus, 1901).

Distr. Southern South America (Mueller, 1897a; Paris, 1900a, 1904b; Brotherus, 1901).

D. taxifolium (Hedw.) Web. et Mohr

Fissidens taxifolius

D. tenuicuspidatum C. Muell.

Chorisodontium aciphyllum

D. tenuifolium Hook. f. et Wils.

Blindia robusta

D. tenuirostre Kunz. ex Schwaegr.

Dicranella hilariana

D. toninii C. Muell. (Mueller, 1897a).

Distr. Southem South America (Mueller, 1897a; Paris, 1900a, 1904b; Cardot, 1908a).

D. truncatum (C. Muell.) C. Muell.

Campylopus truncatus

D. truncorum Schimp. ex C. Muell.

Dicranoloma truncorum

D. turgidissimum Dus.

D. vaginatum Hook.

Dicranoloma subimponens

D. vaginatum Hook. f. et Wils.

Anisothecium vaginatum

D. verticillatum Hamp.

Dicranella hookeri

D. xanthopyllum Mont. ex Par.

Campylopus verticillatus

Campylopus introflexus

\section{Didymodon}

D. amblyophyllus (Hook.) Broth. (Brotherus, 1902).

Syn. Gymnostomum amblyophyllum Hook. (Hooker, 1830).

Pottia amblyophylla (Hook.) C. Muell. (Mueller, 1849).

Tortula amblyophylla (Hook.) Mitt. (Mitten, 1869).

Barbula amblyophylla (Hook.) Jaeg. (Jaeger, 1873).

Distr. Southern South America (Hooker, 1830; Mueller, 1849; Mitten, 1869; Jaeger, 1873;

Paris, 1904b; Warnstorf, 1916; Brotherus, 1924a; Kühnemann, 1938).

D. ampliretis Bartr. (Bartram, 1946).

Distr. Southern South America (Bartram, 1946).

D. andreaeoides Card. et Broth. (Cardot and Brotherus, 1923).

Distr. Southern South America (Cardot and Brotherus, 1923; Kühnemann, 1938).

D. angustifolius Herz. (Herzog, 1960) hom. illeg.

Distr. Southern South America (Bartram, 1965 (as D. angustatus Herz.)).

D. argentinicus (Par.) Par. (Paris, 1904b).

Syn. Barbula argentinica Par. (Paris, 1894).

Barbula rubiginosa C. Muell. (Mueller, 1882) hom. illeg.

Didymodon rubiginosus Broth. (Brotherus, 1902) hom. illeg.

Barbula subrubiginosa Kindb. (Kindberg, 1889) nom. nud.

Distr. Southern South America (Mueller, 1882; Paris, 1894, 1904b; Brotherus, 1902, 1924a; Kühnemann, 1938).

D. argentinicus Warnst. ex Broth.

Didymodon argentiniensis 
D. argentiniensis Warnst. (Warnstorf, 1916).

Syn. Didymodon argentinicus Warnst. ex Broth. (Warnstorf ex Brotherus, 1924a) hom. illeg.

Distr. Southern South America (Warnstorf, 1916; Brotherus, 1924a; Kühnemann, 1938).

D. berthoanus Thér. (Thériot, 1926).

Distr. Southern South America (Thériot, 1926).

D. brachydontius (Bruch) Wils.

Trichostomum brachydontium

D. brunneus (C. Muell.) Warnst. (Warnstorf, 1916).

Syn. Trichostomum brunneum C. Muell. (Mueller, 1879a).

Anacalypta brunnea (C. Muell.) Kindb. (Kindberg, 1889).

Pottia brunnea (C. Muell.) Par. (Paris, 1897).

Barbula brunnea (C. Muell.) Broth. (Brotherus, 1902) hom. illeg.

Trichostomum nudum C. Muell. (Mueller, 1882).

Anacalypta nuda (C. Muell.) Kindb. (Kindberg, 1889).

Pottia nuda (C. Muell.) Par. (Paris, 1897).

Distr. Southern South America (Mueller, 1879a, 1882; Paris, 1897, 1905b; Brotherus, 1902 , 1924a; Warnstorf, 1916; Hosseus, 1935b, 1937; Kühnemann, 1938).

D. calymperidictyon Broth.

D. capillaceus (Hedw.) Web. et Mohr

Bryoerythrophyllum campylocarpum

var. compactus Hueb.

D. crassinervis Thér. (Thériot in Costes, 1921) nom. nud.

Distichium capillaceum

Distr. Southern South America (Costes, 1921 (as Didymodon)).

D. cyathicarpus (Mont.) Mitt.

D. dusenii Card. (Cardot in Cardot and Brotherus, 1923) nom. nud.

Amphidium tortuosum

Distr. Southern South America (Cardot and Brotherus, 1923; Kühnemann, 1938).

D. gelidus Card. (Cardot, 1907a).

Distr. Antarctic, peninsula region (Dixon, 1920).

Antarctic, continental region (Cardot, 1907a, 1908a; Brotherus, 1924a; Steere, 1961a).

D. glacialis Hook. f. et Wils.

D. glauco-viridis (C. Muell.) Broth. (Brotherus, 1902).

Sarconeurum glaciale

Syn. Trichostomum glauco-viride C. Muell. (Mueller, 1882).

Anacalypta glauco-viridis C. Muell. (Mueller, 1882) nom. nud.

Pottia glauco-viridis (C. Muell.) Par. (Paris, 1897).

Distr. Southem South America (Mueller, 1882; Paris, 1897, 1904b; Brotherus, 1902, 1924a;

Warnstorf, 1916; Hosseus, 1938c; Kühnemann, 1938).

D. gracilescens Bartr. (Bartram, 1936) hom. illeg.

Distr. Southern South America (Bartram, 1936; Hosseus, 1938c, d, 1939).

D. gracilis Hook.

Pilopogon gracilis

var. robustum Hook.

Pilopogon gracilis

D. gymnus (C. Muell.) Broth. (Brotherus, 1902).

Syn. Trichostomum gymnum C. Muell. (Mueller, 1879a).

Anacalypta gymna (C. Muell.) Kindb. (Kindberg, 1889).

Pottia gymna (C. Muell.) Par. (Paris, 1897).

Distr. Southern South America (Mueller, 1879a; Paris, 1897, 1904b; Warnstorf, 1916; Brotherus, 1902, 1918, 1924a; Kühnemann, 1938).

D. heimii (Hedw.) Kindb.

Pottia heimii

D. heteromallus (Hedw.) Hook. et Tayl.

Ditrichum heteromallum

D. homomallus Hedw. 
D. inundatus (Mitt.) Broth. (Brotherus, 1902).

Syn. Tortula inundata Mitt. (Mitten, 1869).

Barbula inundata (Mitt.) Jaeg. (Jaeger, 1873).

Distr. Southem South America (Mitten, 1869; Gibert, 1873; Jaeger, 1873; Brotherus, 1902, 1924a; Paris, 1904b; Herter, 1928, 1933a; Hosseus, 1938d).

D. linearis Broth.

Bryoerythrophyllum campylocarpum

D. longifolius (Brid.) Hook. f. et Wils.

Leucoloma longifolium

var. curvifolius Hook. f. et Wils. (Hooker and Wilson in Wilson and Hooker, 1847).

Distr. Southern South America (Wilson and Hooker, 1847; Kühnemann, 1938).

var. penicillatus Hook. f. et Wils.

Ditrichum strictum

var. tenuifolius Hook. f. et Wils.

Ditrichum hyalinum

D. Iorentzianus (C. Muell.) Broth. (Brotherus, 1902).

Syn. Pottia lorentziana C. Muell. (Mueller, 1879a).

Distr. Southern South America (Mueller, 1879a; Brotherus, 1902, 1924a; Kurtz, 1904; Paris, 1904b; Warnstorf, 1916; Hosseus, 1935b, 1937; Kühnemann, 1938).

D. microthecius (C. Muell.) Broth. (Brotherus, 1902).

Syn. Trichostomum microthecium C. Muell. (Mueller, 1882).

Anacalypta microthecium C. Muell. (Mueller, 1882) nom. nud.

Pottia microthecia (C. Muell.) Par. (Paris, 1897).

Distr. Southern South America (Mueller, 1882; Paris, 1897, 1904b; Brotherus, 1902, 1924a; Warnstorf, 1916; Kühnemann, 1938).

D. montevidensis Broth. (Brotherus in Felippone, 1917).

Distr. Southern South America (Felippone, 1917; Brotherus, 1924a; herter, 1933a).

D. oligondontus Card. (Cardot in Skottsberg, 1914) nom. nud.

Distr. Juan Fernandez (Skottsberg, 1914; Brotherus, 1924b).

D. orbignyanus (C. Muell.) Broth. (Brotherus, 1902).

Syn. Pottia orbignyana C. Muell. (Mueller, 1879a).

Distr. Southern South America (Mueller, 1879a; Brotherus, 1902, 1924a, c; Paris, 1904b; Warnstorf, 1916; Espinosa B., 1941).

D. patagonicus (Mitt.) Broth. (Brotherus, 1924a).

Syn. Tortula patagonica Mitt. (Mitten, 1869).

Barbula patagonica (Mitt.) Jaeg. (Jaeger, 1873).

Distr. Southern South America (Mitten, 1869; Jaeger, 1873; Paris, 1904a; Cardot, 1908a; Brotherus, 1924a; Kühnemann, 1938).

D. pichinchensis Tayl.

Zygodon pichinchensis

D. polycephalus Mont. (Montagne, 1845a).

Distr. Southern South America (Montagne, 1845a, 1856; Mueller, 1848a; Jaeger, 1873).

D. pungens Mitt.

Leptodontium pungens

D. reflexus Thér. (Thériot, 1924).

Distr. Southern South America (Thériot, 1924; Brotherus, 1925; Hosseus, 1938d).

D. reticulatus Gillies ex Grev. (Gillies ex Greville, 1830).

Distr. Southern South America (Greville, 1830).

D. rigidulus Hedw. (Hedwig, 1801). 
Syn. Bryum rigidulum (Hedw.) Dicks. (Dickson, 1801).

Trichostomum rigidulum (Hedw.) Turn. (Turner, 1804a).

Desmatodon rigidulus Hassk. (Hasskarl, 1851) err.

Barbula rigidula (Hedw.) Mitt. (Mitten, 1864).

Tortula rigidula (Hedw.) Lindb. (Lindberg, 1864a).

Distr. Southern South America (Roivainen, 1934).

Falkland Islands (Cardot and Brotherus, 1923; Kühnemann, 1938).

D. rubellus B.S.G.

D. rubiginosus Broth.

Bryoerythrophyllum recurvirostre

Didymodon argentinicus

D. schilleri Herz. et Thér. (Herzog and Thériot in Herzog and others, 1933). Distr. Southern South America (Herzog and others, 1933; Herzog, 1938, 1957; Kühnemann,
1938).

D. schimperi (Mont.) Broth. (Brotherus, 1902).

Syn. Trichostomum schimperi Mont. (Montagne, 1845a).

Barbula schimperi (Mont.) Mitt. (Mitten, 1859).

Desmatodon schimperi (Mont.) Jaeg. (Jaeger, 1873).

Distr. Southern South America (Montagne, 1845a, 1850, 1856; Mueller, 1849; Jaeger, 1873;

Brotherus, 1902, 1924a; Paris, 1904b; Williams, 1930).

D. spathulato-linearis (C. Muell.) Broth. (Brotherus, 1902).

Syn. Trichostomum spathulato-lineare C. Muell. (Mueller, 1879a).

Anacalypta spathulato-linearis (C. Muell.) Kindb. (Kindberg, 1889).

Pottia spathulato-linearis (C. Muell.) Par. (Paris, 1897).

Distr. Southern South America (Mueller, 1879a; Paris, 1897, 1904b; Brotherus, 1902; Warnstorf, 1916; Kühnemann, 1938).

D. subtophaceus Williams

Trichostomopsis australasiae

\section{Dimerodontium}

D. acuminatum C. Muell. (Mueller, 1879a).

Distr. Southern South America (Mueller, 1879a; Brotherus, 1925; Herter, 1933a; Kühnemann, 1938; Hosseus, 1939; Herzog, 1952a).

D. aurescens C. Muell. (Mueller, 1879a).

Distr. Southern South America (Mueller, 1879a; Paris, 1904b; Brotherus, 1925; Kühnemann, 1938; Hosseus, 1939).

D. chlorophyllosum C. Muell. (Mueller, 1879a).

Distr. Southern South America (Mueller, 1879a; Paris, 1904b; Brotherus, 1925; Herter, 1933a; Kühnemann, 1938; Hosseus, 1939; Herzog, 1952a).

D. mendozense Mitt. (Mitten, 1869).

Distr. Southern South America (Mitten, 1869; Gibert, 1873; Jaeger, 1878; Mueller, 1879a; Paris, 1904b; Krieger, 1904; Kurtz, 1904; Brotherus, 1925; Herter, 1928, 1933a, 1939a, $b$; Hosseus, 1935a, $b, 1936,1937,1938 a, b, c, d$; Kühnemann, 1938; Herzog, 1952a).

D. ovatifolium Herz. (Herzog, 1916b).

Distr. Southern South America (Herzog, 1916b).

D. pellucidum (Schwaegr.) Mitt. (Mitten, 1869).

Syn. Sclerodontium pellucidum Schwaegr. (Schwaegrichen, 1830).

Leskea pellucida Hook. (Hooker in Schwaegrichen, 1830) nom. nud.

Hypnum sclerodontium C. Muell. (Mueller, 1851a).

Fabriona longidens Dub. (Duby, 1868).

Distr. Southern South America (Duby, 1868; Mitten, 1869; Jaeger, 1878; Paris, 1904b; Thériot, 1919; Brotherus, 1925; Kühnemann, 1938). 
D. rivulare C. Muell.

D. schnyderi C. Muell. (Mueller, 1882).

Distr. Southern South America (Mueller, 1882; Paris, 1904b; Brotherus, 1925; Kühnemann, 1938).

\section{Diplostichum}

D. longirostre (Brid.) Mont. (Montagne, 1845a).

Syn. Pterigynandrum longirostre Brid. (Bridel, 1819). Eustichia longirostris (Brid.) Brid. (Bridel, 1827b).

Distr. Juan Fernandez (Robinson, 1975).

Southern South America (Montagne, 1845a, 1850, 1856; Mueller, 1848a; Lorentz, 1866; Jaeger, 1873; Paris, 1904b; Seki, 1974).

D. Iorentzii C. Muell. (Mueller, 1897a).

Syn. Eustichia lorentzii (C. Muell.) Par. (Paris, 1900a).

Distr. Southern South America (Mueller, 1897a; Paris, 1900a; Kurtz, 1904; Brotherus, 1924a; Hosseus, 1936, 1937; Kühnemann, 1938).

D. poeppigii C. Muell. ex Card. ex Thér. (Mueller ex Cardot ex Thériot, 1921a).

Syn. Eustichia poeppigii Par. (Paris, 1900a) nom. nud.

Distr. Juan Fernandez (Skottsberg, 1914; Brotherus, 1924b; Espinosa B., 1941; Bartram, 1959).

Southern South America (Paris, 1900a; Cardot, 1908a; Campo, 1921; Thériot, 1921a; Cardot and Brotherus, 1923; Brotherus, 1924a: Reimers, 1926; Roivainen, 1934; Kühnemann, 1938; Herzog, 1939, 1940, 1954, 1960; Bartram, 1952).

var. laetevirens Card. (Cardot in Skottsberg, 1914) nom. nud.

Distr. Juan Fernandez (Skottsberg, 1914).

\section{Discophyllum}

D. dicksonii (Hook. et Grev.) Mitt.

Distichophyllum dicksonii

\section{Dissodon}

D. dubyanus Besch.

D. magellanicus (Brid.) Hamp.

D. mirabilis Card.

D. plagiopus (Mont.) C. Muell.

\section{Distichium}

D. austro-georgicum C. Muell. (Mueller, 1890a).

Distr. South Georgia (Mueller, 1890a; Paris, 1904b; Cardot, 1908a; Dixon, 1932; Steere, 1961b).

D. austro-inclinatum C. Muell. (Mueller, 1882).

Distr. Southern South America (Mueller, 1882; Paris, 1904b; Brotherus, 1924a; Kühnemann, 1938).

D. capillaceum (Hedw.) B.S.G. (Bruch and others, 1846).

Syn. Cynontodium capillaceum Hedw. (Hedwig, 1801).

Bryum capillaceum (Hedw.) Dicks. ex With. (Dickson ex Withering, 1801).

Didymodon capillaceus (Hedw.) Web. et Mohr (Weber and Mohr, 1803).

Trichostomum capillaceum (Hedw.) Sm. (Smith, 1803).

Cynodontium capillaceum (Hedw.) Brid. (Bridel, 1806).

Leptotrichum capillaceum (Hedw.) Mitt. (Mitten, 1860).

Ditrichum capillaceum (Hedw.) Watts et Whitel. Watts and Whitelegge, 1902). 
Distr. Southern South America (Wilson and Hooker, 1847; Dusén, 1903a, 1905a; Cardot, 1905a, 1908a; Cardot and Brotherus, 1923; Brotherus, 1924a; Roivainen and Bartram, 1937; Kühnemann, 1938; Bartram, 1952).

South Georgia (Brotherus, 1924a; Newton, 1977a).

Antarctic, peninsula region (Cardot, 1900, 1901, 1908a, 1911b, d, 1913a; Paris, 1904b; Steere, $1961 a$ (all these references as $D$. capillaceum var. brevifolium fide Newton, 1977b); Newton 1977b).

Antarctic, continental region (the record for Cardot, 1907a given by Steere, 1961a and mentioned by Newton, $1977 b$ is erroneous as is the Cardot, $1908 a$ record for a Skottsberg specimen from Moss Island, see original Cardot papers).

var. brevifolium B.S.G.

Distichium capillaceum var. compactum

var. compactum (Hueb.) Torre et Sarnth. (Dalla Torre and Sarnthein, 1904).

Syn. Didymodon capillaceus (Hedw.) Web. et Mohr var. compactus Hueb. (Hübener, 1833).

Distichium capillaceum (Hedw.) B.S.G. var. brevifolium B.S.G. (Bruch and others, 1846) nom. illeg.

Distr. Southern South America (Roivainen and Bartram, 1937).

Falkland Islands (Cardot and Brotherus, 1923).

D. Iorentzii C. Muell. (Mueller, 1882).

Distr. Southern South America (Mueller, 1882; Krieger, 1904; Paris, 1904b; Kühnemann, 1938).

\section{Distichophyllum}

D. apiculatum (Hook. f. et Wils.) Mitt.

Eriopus apiculatus

D. assimile Broth. (Brotherus, 1924b).

Syn. Mniadelphus assimilis Broth. (Brotherus in Fleischer, 1922) nom. nud.

Distr. Juan Fernandez (Brotherus, 1924b; Bartram, 1959; Welch, 1969; Matteri, 1975b; Robinson, 1975).

D. cavifolium (Card.) Card.

var. montanum Card.

Distichophyllum eremitae

D. crispatissimum Dus.

Distichophyllum eremitae

D. dicksonii (Hook. et Grev.) Mitt. (Mitten, 1869).

Syn. Hookeria dicksonii Hook. et Grev. (Hooker and Greville, 1825a).

Mniadelphus dicksonii (Hook. et Grev.) Hamp. (Hampe in Mueller, 1850).

Discophyllum dicksonii (Hook. et Grev.) Mitt. (Mitten, 1868).

Distr. Southern South America (Hooker and Greville, 1825a; Hooker, J. D. and Wilson, 1844; Wilson and Hooker, 1847; Mueller, 1850, 1885; Sullivant, 1859; Mitten, 1869; Jaeger, 1877; Bescherelle, 1889; Paris, 1904b; Cardot, 1908a; Brotherus, 1925; Herzog, 1938, 1954; Kühnemann, 1938; Welch, 1969; Seki, 1974; Matteri, 1975a, b).

Falkland Islands (Hooker, J. D. and Wilson, 1844; Wilson and Hooker, 1847; Paris, 1904b; Cardot, 1908a; Cardot and Brotherus, 1923; Brotherus, 1925; Kühnemann, 1938; Matteri, 1975b).

D. ellipticum Herz. (Herzog, 1954).

Syn. Distichophyllum crispatissimum Dus. (Dusén, 1903a) nom. nud. fide Matteri, $1975 a$.

Distr. Southern South America (Dusén, 1903a; Paris, 1904b; Herzog, 1939, 1954; Welch, 1969; Seki, 1974; Matteri, 1975a, b).

D. eremitae (Jaeg.) Par. (Paris, 1895).

Syn. Mniadelphus eremitae Jaeg. (Jaeger, 1877).

Distichophyllum procumbens Mitt. (Mitten, 1869) hom. illeg .

Mniadelphus procumbens C. Muell: (Mueller, 1885) hom. illeg.

Mniadelphus cavifolius Card. (Cardot, 1905a) fide Matteri, $1975 a$.

Distichophyllum cavifolium (Card.) Card. (Cardot, 1908a) fide Matteri, 1975a.

Distichophyllum cavifolium (Card.) Card. var. montanum Card. (Cardot in Cardot and

Brotherus, 1923) nom. nud. fide Matteri, 1975a. 
Distr. Southern South America (Mitten, 1869; Jaeger, 1877; Mueller, 1885, 1889; Paris, 1895 , 1904b; Cardot, 1905a, 1908a; Cardot and Brotherus, 1923; Brotherus, 1925; Kühnemann, 1938; Welch, 1969; Seki, 1974; Matteri, 1975a, b).

Falkland Islands (Matteri, 1975b).

D. fasciculatum Mitt. (Mitten, 1876).

Distr. Southern South America (Herzog, 1960).

D. fernandezianum Broth. (Brotherus, 1924b).

Distr. Juan Fernandez (Brotherus, 1924a, b; Welch, 1969; Matteri, 1975b; Robinson, 1975 (as synonym of $D$. assimile)).

D. flaccidum (Hook. f. et Wils.) Mitt. (Mitten, 1869).

Syn. Hookeria flaccida Hook. f. et Wils. (Hooker, J. D. and Wilson, 1844).

Mniadelphus flaccidus (Hook. f. et Wils.) Hamp. (Hampe in Mueller, 1850).

Distr. Southern South America (Hooker, J. D. and Wilson, 1844; Wilson and Hooker, 1847; Mueller, 1850, 1885; Mitten, 1869; Jaeger, 1877; Paris, 1905a; Cardot, 1908a; Brotherus, 1925; Welch, 1969; Matteri, 1975a, b).

D. hahnianum Fleisch.

Eriopus apiculatus

D. fuegianum Matteri (Matteri, 1975a).

Distr. Southern South America (Matteri, 1975a, b).

D. grandifolium Matteri (Matteri, 1975b).

Distr. Southern South America (Matteri, 1975b).

D. kraussei (Lor.) Mitt. (Mitten, 1885).

Syn. Mniadelphus kraussei Lor. (Lorentz, 1864).

Mniadelphus krausei Lor. (Lorentz in Jaeger, 1877) err.

Mniadelphus nigricans C. Muell. (Mueller in Bescherelle, 1889) nom. nud. fide Matteri, $1975 a$.

Distichophyllum nigricans Besch. (Bescherelle, 1889) fide Matteri, 1975a.

Distichophyllum latilimbatum Card. (Cardot in Matteri, 1975a) nom. nud. fide Matteri, $1975 a$.

Distr. Southern South America (Lorentz, 1864, 1866; Jaeger, 1877; Bescherelle, 1889; Neger, 1899; Paris, 1904b, 1905a; Cardot, 1908a; Brotherus, 1925; Kühnemann, 1938; Welch, 1969; Seki, 1974; Matteri, 1975a, b).

D. Iatilimbatum Card.

Distichophyllum kraussei

D. molle Besch.

Eriopus bescherellei

D. nanospathulatum Herz. (Herzog. 1954).

Distr. Southern South America (Herzog, 1954; Welch, 1969; Matteri, 1975b).

D. nigricans Besch.

Distichophyllum kraussei

D. patagonicum Besch. (Bescherelle, 1889).

Distr. Southern South America (Bescherelle, 1889; Paris, 1904b; Cardot, 1908a; Brotherus, 1925; Welch, 1969).

D. procumbens Mitt.

Distichophyllum eremitae

D. rotundifolium (Hook. f. et Wils.) C. Muell. et Broth. (Mueller and Brotherus, 1900).

Syn. Hookeria rotundifolia Hook. f. et Wils. (Hooker, J. D. and Wilson, 1844).

Mniadelphus rotundifolius (Hook. f. et Wils.) C. Muell. (Mueller, 1851a).

Distr. Southern South America (Cardot and Brotherus, 1923; Kühnemann, 1938; Herzog, 1960; Welch, 1969; Seki, 1974; Matteri, 1975b).

D. subelimbatum Broth. (Brotherus, 1924b).

Distr. Juan Fernandez (Brotherus, 1924b, 1925; Espinosa B., 1941; Bartram, 1959; Welch, 1969; Matteri, 1975b; Robinson, 1975). 
D. submersum Matteri (Matteri, 1975a).

Distr. Southern South America (Matteri, 1975a, b).

D. theriotianum Matteri (Matteri, 1975b).

Distr. Southem South America (Matteri, 1975b).

\section{Ditrichum}

D. affine (C. Muell.) Hamp.

Ditrichum difficile

D. australe (Mitt.) Mitt.

Ditrichum strictum

D. capillaceum (Hedw.) Watts et Whitel.

Distichium capillaceum

D. conicum(Mont.) Mitt. (Mitten, 1879 ).

Syn. Aschistodon conicus Mont. (Montagne, 1845a).

Cynontodium conicum (Mont.) Mitt. (Mitten, 1869).

Leptotrichum montagnei C. Muell. (Mueller, 1848a) nom. illeg.

Distr. Southem South America (Montagne, 1845a, 1850, 1856; Mueller, 1848a; Lorentz, 1866; Mitten, 1869; Jaeger, 1873; Paris, 1904b; Dusén, 1905a; Cardot, 1908a; Cardot and

Brotherus, 1923; Brotherus, 1924a; Thériot, 1935a; Roivainen and Bartram, 1937;

Herzog, 1938, 1939, 1957; Kühnemann, 1938; Bartram, 1952; Seki, 1974).

var. glaciale Card. et Broth. (Cardot and Brotherus, 1923).

Distr. Southern South America (Cardot and Brotherus, 1923; Roivainen and Bartram, 1937).

D. cylindricarpum (C. Muell.) Hamp. (Hampe, 1880c).

Syn. Leptotrichum cylindricarpum C. Muell. (Mueller, 1851b).

Trichostomum cylindricarpum (C. Muell.) Wils. (Wilson in Wilson, 1859).

Trichostomum elongatum Hook. f. et Wils. (Hooker and Wilson in Wilson, 1854).

Cynontodium elongatum (Hook. f. et Wils.) Mitt. (Mitten, 1869).

Leptotrichum élongatum (Hook. f. et Wils.) Jaeg. (Jaeger, 1873).

Ditrichum elongatum (Hook. f. et Wils.) Mitt. (Mitten, 1883).

Distr. Southem South America (Mitten, 1869; Jaeger, 1873; Paris, 1904b; Dusén, 1905a; Cardot, 1908a; Herzog, 1923, 1957; Brotherus, 1924a; Kühnemann, 1938; Seki, 1974).

D. difficile (Dub.) Fleisch. (Fleischer, 1904).

Syn. Trichostomum difficile Dub. (Duby in Moritzi, 1846).

Leptotrichum difficile (Dub.) C. Muell. (Mueller, 1901a [1900]).

Leptotrichum affine C. Muell. (Mueller, 1847c).

Trichostomum affine (C. Muell.) Mont. (Montagne, 1850) hom. illeg.

Ditrichum affine (C. Muell.) Hamp. (Hampe, 1867b).

Cynontodium affine (C. Muell.) Mitt. (Mitten, 1869).

Dicranum flexifolium Hook. (Hooker, 1819) hom. illeg.

Leptotrichum flexifolium Mitt. (Mitten, 1856).

Ditrichum flexifolium (Mitt.) Hamp. (Hampe, 1867b).

Trichostomum laxifolium Hook. f. et Wils. (Hooker and Wilson in Wilson, 1854).

Ditrichum laxifolium (Hook. f. et Wils.) Mitt. (Mitten, 1883).

Leptotrichum laxifolium (Hook. f. et Wils.) C. Muell. (Mueller, 1901a [1900]).

Distr. Juan Fernandez (Mitten, 1869; Jaeger, 1873; Paris, 1904b; Skottsberg, 1914; Brotherus, 1924b; Espinosa B., 1941; Bartram, 1959; Robinson, 1975).

Southern South America (Mueller, 1847c, 1848a; Montagne, 1850; Lorentz, 1866; Jaeger, 1873; Paris, 1904b; Dusén, 1905a; Cardot, 1908a; Thériot, 1915, 1921a; Herzog, 1923, 1938, 1954; Brotherus, 1924a; Kühnemann, 1938; Seki, 1974).

D. elongatum (Hook. f. et Wils.) Mitt.

Ditrichum cylindricarpum

D. flexifolium (Mitt.) Hamp.

Ditrichum difficile

D. fontanum Herz. (Herzog, 1954).

Distr. Southern South America (Herzog, 1954).

"See also footnote to entries for Pseudodistichium atlanticum Dix. and $P$. taitaoense Froehl. 
D. hallei Card. et Broth. (Cardot and Brotherus, 1923).

Distr. Southern South America (Cardot and Brotherus, 1923; Brotherus, 1924a; Roivainen, 1934; Roivainen and Bartram, 1937; Kühnemann, 1938).

D. heteromallum (Hedw.) Britt. (Britton, 1913).

Syn. Weisia heteromalla Hedw. (Hedwig, 1801).

Grimmia heteromalla (Hedw.) Web. et Mohr (Weber and Mohr, 1803).

Bryum heteromallum (Hedw.) P. Beauv. (Palisot de Beauvois, 1822) hom. illeg.

Didymodon heteromallus (Hedw.) Hook. et Tayl. (Hooker and Taylor, 1818).

Didymodon homomallus Hedw. (Hedwig, 1801).

Grimmia homomalla (Hedw.) Sm. (Smith, 1804b).

Leptotrichum homomallum (Hedw.) Hamp. (Hampe in Mueller, 1848a).

Ditrichum homomallum (Hedw.) Hamp. (Hampe, 1867b).

Bryum weisii Dicks. ex With. (Dickson ex Withering, 1801).

Distr. Southern South America (Cardot and Brotherus, 1923; Brotherus, 1924a; Kühnemann, 1938).

D. homomallum (Hedw.) Hamp.

Ditrichum heteromallum

var. leptocladum Card. et Broth. (Cardot and Brotherus, 1923).

Distr. Southem South America (Cardot and Brotherus, 1923; Kühnemann, 1938).

D. hookeri (C. Muell.) Hamp. (Hampe, 1867b).

Syn. Leptotrichum hookeri C. Muell. (Mueller, 1848a).

Cynontodium hookeri (C. Muell.) Mitt. (Mitten, 1869).

Cynodontium hookeri (C. Muell.) Mitt. (Mitten, 1876).

Blindia leptotrichocarpa C. Muell. (Mueller, 1885).

Blindia leptotrichacea C. Muell. ex Par. (Mueller ex Paris, 1894) err.

Distr. Southem South America (Mueller, 1848a, 1885; Mitten, 1869; Jaeger, 1873;

Bescherelle, 1889; Paris, 1894, 1904a, b; Dusén, 1905a; Cardot, 1905a, 1908a,

1913a; Cardot and Brotherus, 1923; Brotherus, 1924a; Roivainen and Bartram, 1937; Kühnemann, 1938; Herzog, 1954; Seki, 1974).

Falkland Islands (Cardot and Brotherus, 1923).

var. strictiusculum (C. Muell.) Wijk et Marg. (van der Wijk and Margadant, 1959).

Syn. Blindia leptotrichocarpa C. Muell. var. strictiuscula C. Muell. (Mueller, 1885).

Distr. Southern South America (Mueller, 1885; Paris, 1904a; Cardot, 1908a; Kühnemann, 1938).

D. hyalinocuspidatum Card. (Cardot, 1906a).

Distr. Southern South America (Cardot and Brotherus, 1923; Kühnemann, 1938).

Falkland Islands (Cardot and Brotherus, 1923; Kühnemann, 1938).

South Georgia (Cardot, 1906a, 1908a; Brotherus, 1924a; Steere, 1961b).

D. hyalinum (Mitt.) O. Kuntze (Kuntze, 1891).

Syn. Leptotrichum hyalinum Mitt. (Mitten, 1860).

Cynontodium hyalinum (Mitt.) Mitt. (Mitten, 1869).

Didymodon longifolius (Brid.) Hook. f. et Wils. var. tenuifolius Hook. f. et Wils. (Hooker and Wilson in Hooker, 1847).

Distr. Southern South America (Mitten, 1860, 1869; Jaeger, 1873; Mueller, 1885; Bescherelle, 1889; Dusén, 1903a, 1905a; Paris, 1904b; Cardot, 1908a; Brotherus, 1924a; Kühnemann, 1938; Herzog, 1954; Seki, 1974).

Falkland Islands Milson and Hooker, 1847; Mitten, 1860; Paris, 1904b; Cardot, 1908a; Brotherus, 1924a; Kühnemann, 1938).

$D$. inundatum Card.

D. laxifolium (Hook. f. et Wils.) Mitt.

Blindia magellanica var. inundata

D. longisetum (Lor.) Hamp. (Hampe, 1867b).

Syn. Leptotrichum longisetum Lor. (Lorentz, 1866). 
Distr. Juan Fernandez (Brotherus, 1924b; Espinosa B., 1941; Bartram, 1959; Robinson, 1975).

Southern South America (Lorentz, 1866; Jaeger, 1873; Bescherelle, 1889; Paris, 1904b; Dusén, 1905a; Cardot, 1908a; Brotherus, 1924a; Kühnemann, 1938; Herzog, 1939; Seki, 1974).

D. perporodictyon Dix. (Dixon, 1932).

Distr. South Georgia (Dixon, 1932; Steere, 1961b).

D. praealtum (Mitt.) O. Kuntze (Kuntze, 1891).

Syn. Leptotrichum praealtum Mitt. (Mitten, 1860).

Distr. Southem South America (Mitten, 1860; Jaeger and Sauerbeck, 1879b; Mueller, 1885;

Paris, 1904b; Cardot, 1908a: Brotherus, 1924a).

D. roivanenii Bartr. et Roiv. (Bartram and Roivainen in Roivainen and Bartram, 1937).

Syn. Southern South America (Roivainen and Bartram, 1937).

D. rufescens (Hamp.) Hamp. (Hampe, 1867b).

Syn. Leptotrichum rufescens Hamp. (Hampe, 1862a).

Trichostomum strictum Web. et Mohr (Weber and Mohr, 1803).

Ditrichum strictum (Web. et Mohr) Card. (Cardot, 1905a) hom. illeg.

Ditrichum stenostomum Card. (Cardot, 1908a).

Distr. Southem South America (Jaeger, 1873; Mueller, 1879a; Cardot, 1905a, 1908a; Brotherus, 1924a; Roivainen and Bartram, 1937; Kühnemann, 1938).

Falkland Islands (Cardot, 1908a; Kühnemann, 1938).

D. stenostomum Card.

Ditrichum rufescens

D. strictum (Hook. f. et Wils.) Hamp. (Hampe, 1867b).

Syn. Lophiodon strictus Hook. f. et Wils. (Hooker, J. D. and Wilson, 1844).

Trichostomum australe Mitt. (Mitten in Wilson, 1859).

Leptotrichum australe Mitt. (Mitten, 1860) nom. illeg.

Cynontodium australe Mitt. (Mitten, 1869).

Ditrichum australe (Mitt.) Mitt. (Mitten, 1879) nom. illeg.

Didymodon longifolius (Brid.) Hook. f. et Wils. var. penicillatus Hook. f. et Wils.

(Hooker and Wilson in Wilson and Hooker, 1847).

Distr. Southern South America (Jaeger, 1873; Paris, 1905a; Brotherus, 1924a). Falkland Islands Wilson and Hooker, 1847; Mitten, 1860; Paris, 1905a; Brotherus,
1924a).

D. strictum (Web. et Mohr) Card.

Ditrichum rufescens

Dorcadion nom. illeg.

D. rupestre (Schwaegr.) Lindb.

Orthotrichum rupestre

\section{Drepanium}

D. circinale (Hook.) Roth

D. cupressiforme (Hedw.) Roth

D. revolutum (Mitt.) C. Jens.

Hypnum circinale

Hypnum cupressiforme

Hypnun revolutum

\section{Drepanocladus}

D. abbreviatus Card. et Broth.

Drepanocladus brachiatus var. abbreviatus

D. aduncus (Hedw.) Warnst. (Warnstorf, 1903).

Syn. Hypnum aduncum Hedw. (Hedwig, 1801).

Amblystegium aduncum (Hedw.) De Not. (De Notaris, 1867).

Harpidium aduncum (Hedw.) Con. (Conard, 1945) nom. inval.

Distr. Southern South America (Sullivant, 1859; Cardot, 1908a).

Falkland Islands (Dumont d'Unville, 1825; Gaudichaud, 1825, 1826; Bridel, 1827b). 
var. polycarpus (Voit) Roth (Roth, 1904).

Syn. Hypnum polycarpon Bland. ex Voit (Blandow ex Voit in Sturm, 1813).

Amblystegium polycarpum (Voit) Vent. et Bott. (Nenturi and Bottini, 1884).

Drepanocladus polycarpus (Voit) Warnst. (Warnstorf, 1903).

Harpidium polycarpum (Voit) Williams (Williams, 1903b).

Hypnum aduncum Hedw. var. polycarpum (Voit) B.S.G. (Bruch and others, 1854).

Hypnum aduncum Hedw. ssp. polycarpum (Noit) Meyl. (Meylan, 1905).

Hypnum fluitans Hedw. var. polycarpon (Voit) Fiedl. (Fiedler, 1844).

Amblystegium kneiffii B.S.G. var. polycarpum (Voit) Braithw. (Braithwaite, 1898).

Hypnum kneiffii (B.S.G.) Schimp. var. polycarpus (Voit) Fam. (Familler, 1913).

Amblystegium varium (Hedw.) Lindb. var. patagonicum Card. (Cardot in Cardot and

Brotherus, 1923) nom. nud.

Distr. Southern South America (Cardot and Brotherus, 1923).

var. plumosus (Schimp.) Warnst.

Drepanocladus uncinatus

var. subjulaceum (B.S.G.) Warnst.

Drepanocladus uncinatus var. subjulaceus

D. austro-stramineus (C. Muell.) Broth. ex Par.

Calliergidium austro-stramineum

var. gracillimus (C. Muell.) Card. et Broth.

Calliergidium austro-stramineum var. gracillimum

var. minus (Card.) Steere

Calliergidium austro-stramineum var. minus

var. subfluitans (C. Muell.) Steere

Calliergidium austro-stramineum var. subfluitans

var. tenuissimus Card. et Broth. (Cardot and Brotherus, 1923).

Distr. South Georgia (Cardot and Brotherus, 1923; Steere, 1961b).

D. brachiatus (Mitt.) Dix. (Dixon, 1912).

Syn. Hypnum brachiatum Mitt. (Mitten in Hooker, 1867).

Amblystegium brachiatum (Mitt.) Broth. (Brotherus in Mueller and Brotherus, 1900).

Amblystegium longifolium Mitt. (Mitten, 1869).

Hypnum longifolium (Mitt.) Jaeg. (Jaeger and Sauerbeck, 1879a) hom. illeg.

Drepanocladus longifolius (Mitt.) Broth. ex Par. (Brotherus ex Paris, 1909).

Distr. Southern South America (Jaeger and Sauerbeck, 1879a; Cardot, 1908a; Cardot and Brotherus, 1923; Brotherus, 1925; Dixon, 1929a, b; Roivainen, 1934; Kühnemann, 1938; Bartram, 1952; Herzog, 1957).

Falkland Islands (Mitten, 1869; Jaeger and Sauerbeck, 1879a; Paris, 1904c; Cardot, 1908a; Cardot and Brotherus, 1923; Dixon, 1929b).

South Georgia (Dixon, 1932; Steere, 1961b).

Antarctic, peninsula region (Dixon, 1929b, 1935).

var. abbreviatus (Card. et Broth.) Dix. (Dixon, 1929b).

Syn. Drepanocladus abbreviatus Card. et Broth. (Cardot and Brotherus, 1923).

Distr. Southern South America (Cardot and Brotherus, 1923; Brotherus, 1925; Dixon, 1929b).

D. exannulatus (B.S.G.) Warnst. (Warnstorf, 1903).

Syn. Hypnum exannulatum B.S.G. (Bruch and others, 1854).

Amblystegium exannulatum (B.S.G.) De Not. (De Notaris, 1869).

Amblystegium fluitans (Hedw.) De Not. var. exannulatum (B.S.G.) Lindb. (Lindberg and Arnell, 1890) nom. inval.

Amblystegium fluitans (Hedw.) De Not. ssp. exannulatum (B.S.G.) Arn. et C. Jens. (Arnell and Jensen, 1910).

Distr. Southern South America (Seki, 1974).

D. fluitans (Hedw.) Warnst. (Warnstorf, 1903).

Syn. Hypnum fluitans Hedw. (Hedwig, 1801).

Harpidium fluitans (Hedw.) Spruc. (Spruce, 1867).

Amblystegium fluitans (Hedw.) De Not. (De Notaris, 1867).

Hypnum fluitans Hedw. var. falcifolium Ren. (Renauld in Husnot, 1894).

Amblystegium fluitans (Hedw.) De Not. var. falcifolium (Ren.) Arn. et C. Jens. (Arnell and Jensen, 1910) nom. inval.

Drepanocladus fluitans (Hedw.) Warnst. var. falcifolium (Ren.) Thér. (Thériot, 1924). 
Distr. Southern South America (Montagne, 1845b, 1850; Wilson and Hooker, 1847; Sullivant, 1859; Eaton, 1892; Paris, 1904c; Cardot, 1908a; Thériot, 1924; Bartram, 1952; Seki, 1974).

var. australe (Card.) Broth. (Brotherus, 1908).

Syn. Hypnum fluitans Hedw. var. australe Card. (Cardot, 1905a).

Distr. Southern South America (Cardot, 1905a; Brotherus, 1908, 1925).

Falkland Islands (Cardot, 1905a, 1908a; Brotherus, 1908, 1925; Kühnemann, 1938).

var. falcifolius (Ren.) Thér.

Drepanocladus fluitans

D. fuegianus (Mitt.) Broth. ex Par. (Brotherus ex Paris, 1909).

Syn. Amblystegium fuegianum Mitt. (Mitten, 1869).

Hypnum fuegianum (Mitt.) C.: Muell. (Mueller, 1885).

Distr. Southern South America (Mitten, 1869; Jaeger and Sauerbeck, 1879a; Mueller, 1885;

Paris, 1904c; Cardot, 1908a; Cardot and Brotherus, 1923; Brotherus, 1925;

Kühnemann, 1938).

var. stenophyllus Card. et Broth. (Cardot and Brotherus, 1923).

Distr. Falkland Islands (Cardot and Brotherus, 1923; Kühnemann, 1938).

D. kneiffii (B.S.G.) Warnst. var. chilense Thér. (Thériot, 1924).

Distr. Southern South America (Thériot, 1924).

D. laculosus (C. Muell.) Broth. (Brotherus in Cardot and Brotherus, 1923).

Syn. Hypnum laculosum C. Muell. (Mueller, 1885).

Hypnum fluitans Hedw. var. laculosum (C. Muell.) Par. (Paris, 1896).

Distr. Southern South America (Mueller, 1885; Paris, 1896, 1904C; Cardot, 1908a; Cardot and Brotherus, 1923; Brotherus, 1925; Kühnemann, 1938).

D. longifolius (Mitt.) Broth. ex Par.

Drepanocladus brachiatus

D. orthothecioides (Lindb.) Roth

Drepanocladus uncinatus var. subjulaceus

D. perfoliatus C. Muell. or Dus. (Mueller or Dusén in Neger, 1899) nom. nud.

Distr. Southem South America (Neger, 1899).

D. perplicatus (Dus.) Roth

Cratoneuron perplicatum

D. plicatus Dix. (Dixon, 1934).

Distr. South Georgia (Dixon, 1934; Steere, 1961b).

D. polycarpus (Noit) Warnst.

Drepanocladus aduncus var. polycarpus

D. revolvens (Sw.) Warnst. (Warnstorf, 1903).

Syn. Hypnum revolvens Sw. (Swartz, 1801).

Amblystegium revolvens (Sw.) De Not. (De Notaris, 1867).

Harpidium revolvens (Sw.) C. Jens. (Jensen in Lange and Jensen, 1887).

Limprichtia revolvens (Sw.) Loesk. (Loeske, 1907).

Hypnum aduncum Hedw. var. revolvens (Sw.) Bland. (Blandow, 1809).

Hypnum intermedium Lindb. var. revolvens (Sw.) San. (Sanione, 1883) nom. illeg.

Amblystegium intermedium Lindb. var. revolvens (Sw.) Vent. et Bott. (Nenturi and

Bottini, 1884) nom. illeg.

Distr. Southern South America (Wilson and Hooker, 1847; Cardot and Brotherus, 1923;

Kühnemann, 1938).

D. secundifolius (C. Muell.) Dix. (Dixon, 1921).

Syn. Hypnum secundifolium C. Muell. (Mueller, 1851a).

Sematophyllum secundifolium (C. Muell.) Mitt. (Mitten, 1869).

Rhapidostegium secundifolium (C. Muell.) Jaeg. (Jaeger, 1878).

Hypnum lithophilum Hook. f. et Wils. (Hooker and Wilson in Wilson and Hooker, 1847) hom. illeg. fide Dixon, 1921.

Distr. Southern South America Wilson and Hooker, 1847; Mueller, 1851a, 1885; Mitten, 1869; Jaeger, 1878; Paris, 1905b; Cardot, 1908a; Dixon, 1921; Brotherus, 1925). 
D. subjulaceus (B.S.G.) Roiv.

D. symmetricus (Ren. et Card.) Card.
Drepanocladus uncinatus var. subjulaceus

Drepanocladus uncinatus var. symmetricus

D. uncinatus (Hedw.) Warnst. (Warnstorf, 1903).

Syn. Hypnum uncinatum Hedw. (Hedwig, 1801).

Amblystegium uncinatum (Hedw.) De Not. (De Notaris, 1867).

Harpidium uncinatum (Hedw.) C. Jens. (Jensen in Lange and Jensen, 1887).

Sanionia uncinata (Hedw.) Loesk. (Loeske, 1907).

Hypnum georgico-uncinatum C. Muell. (Mueller, 1890a).

Hypnum uncinatum Hedw. var. plumosum Schimp. (Schimper, 1860).

Drepanocladus uncinatus (Hedw.) Warnst. var. plumosus (Schimp.) Warnst. (Warnstorf, 1903).

Amblystegium uncinatum (Hedw.) De Not. var. plumosum (Schimp.) Moell. (Möller, 1907).

Amblystegium aduncum (Hedw.) De Not. var. plumosum (Schimp.) Hult (Hult, 1886).

Drepanocladus aduncus Warnst. var. plumosus (Schimp.) Warnst. (Warnstorf, 1906).

Distr. Southern South America (Wilson and Hooker, 1847; Ångström, 1872; Jaeger and Sauerbeck, 1879a; Dusén, 1903a; Cardot, 1905a, 1908a; Cardot and Brotherus, 1923; Brotherus, 1925; Roivainen, 1934; Kühnemann, 1938; Herzog, 1939, 1940. 1954, 1957; Seki, 1974).

Falkland Islands (Cardot, 1905a, 1908a; Cardot and Brotherus, 1923; Kühnemann, 1938).

South Georgia (Mueller, 1890a; Paris, 1904c; Cardot, 1906a, 1908a; Cardot and Brotherus, 1923; Dixon, 1932; Steere, 1961b).

Antarctic, peninsula region (Cardot, 1900, 1901, 1906a, b, 1907c, 1908a, 1911b, c, d, 1912b, 1913a; Paris, 1904c; Wright, 1905; Brotherus, 1925; Dixon, 1935; Bartram, 1957; Steere, 1961a; Greene, 1968a; Robinson, 1972).

var. orthothecioides (Lindb.) Broth.

Drepanocladus uncinatus var. subjulaceus

var. plumosus (Schimp.) Warnst.

Drepanocladus uncinatus

var. stenocarpus Card. et Broth. (Cardot and Brotherus, 1923).

Distr. Southern South America (Cardot and Brotherus, 1923).

var. subjulaceus (B.S.G.) Warnst. (Warnstorf, 1903).

Syn. Hypnum uncinatum Hedw. var. subjulaceum B.S.G. (Bruch and others, 1854).

Amblystegium uncinatum (Hedw.) De Not. var. subjulaceum (B.S.G.) C. Jens. (Jensen, 1898 [1897]).

Drepanocladus subjulaceus (B.S.G.) Roiv. (Roivainen, 1954).

Drepanocladus aduncus Warnst. var. subjulaceum (B.S.G.) Warnst. Warnstorf, 1906).

Hypnum uncinatum Hedw. var. orthothecioides (Lindb.) Zett. (Zetterstedt, 1876).

Harpidium uncinatum (Hedw.) C. Jens. var. orthothecioides (Lindb.) C. Jens. (Jensen in Lange and Jensen, 1887).

Amblystegium uncinatum (Hedw.) De Not. var. orthothecioides (Lindb.) C. Jens. (Jensen, 1898 [1897]).

Drepanocladus uncinatus (Hedw.) Warnst. var. orthothecioides (Lindb.) Broth. (Brotherus, 1910b).

Amblystegium aduncum (Hedw.) De Not. ssp. orthothecioides (Lindb.) Lindb. (Lindberg, 1879).

Amblystegium orthothecioides (Lindb.) Lindb. ex Par. (Lindberg ex Paris, 1894).

Hypnum orthothecioides (Lindb.) Par. (Paris, 1904c).

Sanionia orthothecioides (Lindb.) Loesk. (Loeske, 1907).

Drepanocladus orthothecioides (Lindb.) Roth (Roth, 1904).

Distr. Southern South America (Cardot and Brotherus, 1923; Kühnemann, 1938).

South Georgia (Cardot and Brotherus, 1923; Steere, 1961b).

Antarctic, peninsula region (Cardot, 1913a).

var. symmetricus (Ren. et Card.) Grout (Grout, 1929).

Syn. Hypnum uncinatum Hedw. ssp. symmetricum Ren. et Card. (Renauld and Cardot, 1888).

Amblystegium uncinatum (Hedw.) De Not. var. symmetricum (Ren. et Card.) Dus. (Dusén, 1903a).

Hypnum symmetricum (Ren. et Card.) Ren. et Card. (Renauld and Cardot, 1889). 
Sanionia symmetrica (Ren. et Card.) Wheld. (Wheldon, 1921).

Drepanocladus symmetricus (Ren. et Card.) Card. (Cardot in Cardot and Brotherus, 1923).

Hypnum uncinatum Hedw. var. cylindropyxis Dus. (Dusén in Paris, 1905a) nom. nud.

Distr. Southem South America (Dusén, 1903a; Paris, 1905a; Cardot, 1908a, 1913a; Cardot and Brotherus, 1923; Kühnemann, 1938).

\section{Drummondia}

D. obtusifolia C. Muell. (Mueller, 1849).

Syn. Leiotheca obtusifolia (C. Muell.) Hamp. (Hampe, 1867a).

Distr. Southern South America (Mueller, 1849; Montagne, 1850; Mitten, 1869; Jaeger, 1874; Brotherus, 1925; Herzog, 1938, 1954, 1960).

\section{Dryptodon}

D. crispulus Hook. f. et Wils.

D. donnianus (Sm.) Hartm.

Racomitrium crispulum

D. rupestris Hook. f. et. Wils.

D. trichophyllus (Grev.) Brid.

Grimmia donniana

Racomitrium crispulum var. rupestre

Grimmia trichophylla

Dusenia nom. illeg.

D. coronata (Mont.) C. Muell.

Forsstroemia coronata

\section{Duseniella}

D. genuflexa (C. Muell.) Broth.

Ancistrodes genuflexa

\section{Ectropothecium}

E. berberidis Dus.

E. cygnisetum C. Muell.

E. pallido-nitidum (C. Muell.) Par.

E. rutilans (Brid.) Mitt.

Rhaphidorrhynchium pallido-nitidum

E. spirifolium Dus.

E. sublimatum (Lor.) Par.

Vesicularia vesicularis

Vesiculariopsis spirifolium

Hypnum chrysogaster

\section{Encalypta}

E. armata Broth. ex Dus. (Brotherus ex Dusén, 1906).

Distr. Southern South America (Dusén, 1906; Brotherus, 1924a; Kühnemann, 1938).

E. asperifolia Mitt. (Mitten, 1869).

Syn. Encalypta emersa C. Muell. (Mueller, 1879a).

Distr. Southem South America (Mueller, 1879a; Paris, 1904b; Brotherus, 1924a; Kühnemann, 1938).

E. australis Mitt.

Encalypta vulgaris

E. austrociliata Broth. ex Dus. (Brotherus ex Dusén, 1906).

Distr. Southern South America (Dusén, 1906; Cardot, 1908a; Cardot and Brotherus, 1923; Brotherus, 1924a; Kühnemann, 1938; Herzog, 1957).

E. berthoana Thér. (Thériot, 1923b).

Distr. Southern South America (Thériot, 1923b; Brotherus, 1925).

E. crispata Hedw.

E. emersa C. Muell.

Ptychomitrium crispatum

Encalypta asperifolia

E. obtusata Card. et Broth. (Cardot and Brotherus, 1923).

Distr. Southem South America (Cardot and Brotherus, 1923; Brotherus, 1924a; Kühnemann. 1938). 
E. patagonica Broth. (Brotherus in Dusén, 1905b).

Distr. Southern South America (Dusén, 1903a (as Eucalypta), 1905b, 1906; Paris, 1904b; Cardot, 1905a, 1908a; Brotherus, 1924a; Kühnemann, 1938).

Antarctic, peninsula region (Newton, 1974c).

E. procera Bruch (Bruch, 1832).

Distr. South Georgia (Newton, 1974c).

Antarctic, peninsula region (Newton, 1974c).

E. vulgaris Hedw. (Hedwig, 1801).

Syn. Encalypta australis Mitt. (Mitten in Wilson, 1859).

Distr. Southem South America (Mitten, 1869; Jaeger, 1873; Paris, 1904b).

\section{Entodon}

E. argentinicus C. Muell.

Enthrodontium argentinicum

E. beyrichii (Schwaegr.) C. Muell. (Mueller, 1844b [1845]).

Syn. Neckera beyrichii Schwaegr. (Schwaegrichen, 1827a).

Distr. Southem South America (Hosseus, 1940; Herzog, 1952a).

E. flavo-virens C. Muell. (Mueller, 1879a).

Syn. Cylindrothecium flavo-virens (C. Muell.) Par. (Paris, 1894).

Distr. Southem South America (Mueller, 1879a; Paris, 1894, 1904b; Brotherus, 1925; Kühnemann, 1938).

var. flagellaceus C. Muell. (Mueller, 1879a).

Syn. Cylindrothecium flavo-virens (C. Muell.) Par. var. flagellaceum (C. Muell.) Par. (Paris, 1894).

Distr. Southern South America (Mueller, 1879a; Paris, 1894, 1904b; Kühnemann, 1938).

E. laxo-alaris (C. Muell.) Broth. ex Par. (Brotherus ex Paris, 1909).

Syn. Aptychus laxo-alaris C. Muell. (Mueller, 1897a). Rhaphidostegium laxo-alare (C. Muell.) Par. (Paris, 1897).

Distr. Southern South America (Mueller, 1897a; Paris, 1897, 1905b).

E. micans Herz. (Herzog, 1916a).

Distr. Southern South America (Hosseus, 1940).

E. platygyrioides C. Muell.

Enthrodontium platygyrioides

E. schreberi (Brid.) Moenk. Pleurozium schreberi

E. suberythropus C. Muell. (Mueller, 1879a).

Syn. Cylindrothecium suberythropus (C. Muell.) Par. (Paris, 1894).

Distr. Southern South America (Mueller, 1879a; Paris, 1894, 1904b; Kurtz, 1904; Brotherus, 1918, 1925; Hosseus, 1938b, c, d; Kühnemann, 1938; Herzog, 1952a).

var. complanatulus C. Muell. (Mueller, 1879a).

Syn. Cylindrothecium suberythropus (C. Muell.) Par. var. complanatulum (C. Muell.) Par. (Paris, 1894).

Distr. Southern South America (Mueller, 1879a; Paris, 1894, 1904b; Kühnemann, 1938).

var. ellipticus C. Muell. (Mueller, 1879a).

Syn. Cylindrothecium suberythropus (C. Muell.) Par. var. ellipticum (C. Muell.) Par. (Paris, 1894).

Distr. Southern South America (Mueller, 1879a; Paris, 1894, 1904b; Hosseus, 1935a, b, 1937. 1938b; Kühnemann, 1938). 
var. flaccidus C. Muell. (Mueller, 1879a).

Syn. Cylindrothecium suberythropus (C. Muell.) Par. var. flaccidum (C. Muell.) Par. (Paris, 1894).

Distr. Southern South America (Mueller, 1879a; Paris, 1894, 1904b; Kühnemann, 1938).

var. gracillimus C. Muell. (Mueller, 1879a).

Syn. Cylindrothecium suberythropus (C. Muell.) Par. var. gracillimum (C. Muell.) Par. (Paris, 1894).

Distr. Southem South America (Mueller, 1879a; Paris, 1894, 1904b; Kühnemann, 1938).

var. Iutescens C. Muell. (Mueller, 1879a).

Syn. Cylindrothecium suberythropus (C. Muell.) Par. var. lutescens (C. Muell.) Par. (Paris, 1894).

Distr. Southern South America (Muieller, 1879a; Paris, 1894, 1904b; Kühnemann, 1938).

var. microtheca C. Muell. (Mueller, 1879a).

Syn. Cylindrothecium suberythropus (C. Muell.) Par. var. microtheca (C. Muell.) Par. (Paris, 1894).

Distr. Southem South America (Mueller, 1879a; Paris, 1894, 1904b; Hosseus, 1935a, 1937, 1938d; Kühnemann, 1938).

var. myosuroides C. Muell. (Mueller, 1879a).

Syn. Cylindrothecium suberythropus (C. Muell.) Par. var. myosuroides (C. Muell.) Par. (Paris, 1894).

Distr. Southem South America (Mueller, 1879a; Paris, 1894, 1904b; Kühnemann, 1938). var. rufulus C. Muell. (Mueller, 1879a).

Syn. Cylindrothecium suberythropus (C. Muell.) Par. var. rufulum (C. Muell.) Par. (Paris, 1894).

Distr. Southem South America (Mueller, 1879a; Paris, 1894, 1904b; Kühnemann, 1938).

E. truncatus (C. Muell.) Mitt. (Mitten, 1869).

Syn. Neckera truncata C. Muell. (Mueller, 1850).

Cylindrothecium truncatum (C. Muell.) Par. (Paris, 1894).

Distr. Southern South America (Mueller, 1850; Mitten, 1869; Jaeger, 1878; Paris, 1894, 1904b; Brotherus, 1925).

Entopogon nom. nud.

E. cylindricus C. Muell.

Eriodon conostomus

\section{Entosthodon}

E. acutifolius Hamp.

Funaria acutifolia

E. balansae Besch.

Funaria balansae

E. bonplandii (Hook.) Mitt.

Funaria bonplandii

E. brachyodus Mont. Funaria integra

E. chiloënsis Mitt.

Funaria chiloënsis

E. clavellatus Mitt.

Funaria clavellata

E. flexisetus C. Muell.

E. mathewsii Hook. f. var. integer C. Muell.

Funaria flexiseta

var. integrifolius $P$ ar.

Funaria integra

Funaria integra

E. papillosus C. Muell.

Funaria papillosa

E. plagiothecius C. Muell.

E. rhizomaticus C. Muell.

Funaria plagiothecia

Funaria rhizomatica 


\section{Ephemerum}

E. conicum C. Muell. (Mueller, 1879a).

Distr. Southem South America (Mueller, 1879a, 1882; Roth, 1911; Kühnemann, 1938).

E. uleanum C. Muell. (Mueller, 1898c).

Distr. Southem South America (Hosseus, 1940; Herzog, 1952a).

Eremodon nom. illeg.

E. magellanicus (Brid.) Brid.

Tayloria magellanica

E. spathulatus Hook. f. et Wils.

Tayloria spathulata

\section{Eriodon}

E. conostomus Mont. (Montagne, 1845a).

Syn. Hypnum conostomum (Mont.) Mitt. (Mitten, 1869) hom. illeg.

Neckera eriodon C. Muell. (Mueller, 1850) nom. illeg.

Hookeria eriodon C. Muell. ex Card. (Mueller ex Cardot, 1908a) err.

Entopogon cylindricus C. Muell. (Mueller in Paris, 1895) nom. nud.

Distr. Southern South America (Montagne, 1845a, 1850, 1856; Mueller, 1850; Lorentz, 1866;
Mitten, 1869; Jaeger, 1878; Neger, 1899; Paris, 1904b; Cardot, 1908a; Thériot, 1918;
Campo, 1921; Herzog, 1923, 1954; Brotherus, 1925; Reimers, 1926; Kühnemann, 1938; Bartram, 1952; Gunckel, 1964; Seki, 1974).

\section{Eriopus}

E. apiculatus (Hook. f. et Wils.) Mitt. (Mitten, 1869).

Syn. Hookeria apiculata Hook. f. et Wils. (Hooker, J. D. and Wilson, 1844).

Pterygophyllum apiculatum (Hook. f. et Wils.) Jaeg. (Jaeger, 1877).

Distichophyllum apiculatum (Hook. f. et Wils.) Mitt. (Mitten, 1883).

Mniadelphus apiculatus (Hook. f. et Wils.) Mitt. (Mitten in Paris, 1896).

Mniadelphus hahnii C. Muell. (Mueller in Kindberg, 1889) nom. nud. fide Matteri, $1975 a$.

Mniadelphus hahnianus C. Muell. (Mueller in Fleischer, 1922) nom. nud. fide Matteri, $1975 a$.

Distichophyllum hahnianum Fleisch. (Fleischer, 1922) nom nud. fide Matteri, 1975a.

Eriopus apiculatus (Hook. f. et Wils.) Mitt. var. platyloma Card. et Broth. (Cardot and Brotherus, 1923) fide Matteri, $1975 a$.

Distr. Southern South America (Hooker, J. D. and Wilson, 1844; Wilson and Hooker, 1847; Mueller, 1851a, 1885; Mitten, 1869; Jaeger, 1877; Paris. 1904b; Cardot, 1908a; Fleischer, 1922; Brotherus, 1925; Bartram, 1952; Herzog, 1954; Welch, 1969; Seki, 1974; Matteri, 1975a, b).

Falkland Islands (Cardot and Brotherus, 1923; Kühnemann, 1938; Welch, 1969; Matteri, 1975b).

var. platyloma Card. et Broth.

Eriopus apiculatus

var. spathulus Matteri (Matteri, 1975b).

Distr. Southern South America (Matteri, 1975b).

E. bescherellei Thér. (Thériot, 1925a).

Syn. Distichophyllum molle Besch. (Bescherelle, 1889) nom. nud. fide Matteri, 1975a.

Mniadelphus mollis C. Muell. (Mueller in Bescherelle, 1889) nom. nud. fide Matteri, $1975 a$.

Distr. Southern South America (Bescherelle, 1889; Paris, 1904b; Cardot, 1908a; Thériot, 1917b, 1925a; Costes, 1921; Brotherus, 1925; Herzog, 1954; Seki, 1974; Matteri, $1975 a, b)$.

E. cristatus (Hedw.) Brid. (Bridel, 1827b).

Syn. Leskea cristata Hedw. (Hedwig, 1801).

Chaetophora cristata (Hedw.) Brid. (Bridel, 1819).

Mnium cristatum (Hedw.) P. Beauv. (Palisot de Beauvois, 1822).

Hookeria cristata (Hedw.) Hook. et Grev. (Hooker and Greville, 1825a). 
Hypnum cristatum Poir. (Poiret in Bridel, 1827b) nom. nud.

Lepidopilum cristatum (Hedw.) Hamp. (Hampe, 1865).

Distr. Southem South America (Wilson and Hooker, 1847; Welch, 1969).

E. flexicollis (Mitt.) Jaeg. (Jaeger, 1877).

Syn. Hookeria flexicollis Mitt. (Mitten in Hooker, 1867).

Distr. Southem South America (Cardot and Brotherus, 1923; Welch, 1969; Seki, 1974).

E. grandiretis Broth. (Brotherus, 1924b).

Distr. Juan Fernandez (Brotherus, 1924b, 1925; Bartram, 1959; Welch, 1969; Matteri, 1975b).

E. leptoloma Broth. (Brotherus, 1924b).

Distr. Juan Fernandez (Brotherus, 1924b, 1925; Welch, 1969; Matteri, 1975b).

E. odontoloma Dus. ex Matteri (Matteri, 1975b).

Distr. Southem South America (Dusén, 1903a; Paris, 1904b; Seki, 1974; Matteri, 1975b).

\section{Erpodium}

E. balensae C. Muell.

Erpodium glaziouii

E. beccarii C. Muell. ex Vent. (Mueller ex Venturi, 1872).

Syn. Erpodium lorentzianum C. Muell. (Mueller, 1879a) fide Crum, 1972.

Erpodium lorentzii C. Muell. ex Kindb. (Mueller ex Kindberg, 1888) nom. illeg.

Erpodium paraguense Besch. (Bescherelle, 1877) fide Crum, 1972.

Distr. Southem South America (Mueller, 1879a; Jaeger and Sauerbeck, 1879b; Paris, 1904b;

Brotherus, 1918, 1925; Kühnemann, 1938; Herzog, 1952a; Crum, 1972).

E. chlorophyllosum C. Muell.

Erpodium coronatum

E. coronatum (Hook. et Wils.) Mitt. (Mitten, 1869).

Syn. Anictangium coronatum Hook. et Wils. (Hooker, W. J. and Wilson, 1844).

Erpodium chlorophyllosum C. Muell. (Mueller, 1879a) fide Crum, 1972.

Distr. Southem South America (Mueller, 1879a; Paris, 1904b; Brotherus, 1925; Kühnemann, 1938; Crum, 1972).

E. glaziouii Hamp. (Hampe, 1872).

Syn. Erpodium balansae C. Muell. (Mueller, 1887b) fide Crum, 1972.

Distr. Southern South America (Brotherus, 1925; Kühnemann, 1938; Hosseus, 1940; Herzog, 1952a).

E. lorentzinum C. Muell.

Erpodium beccarii

E. lorentzii C. Muell. ex Kindb.

Erpodium beccarii

E. paraguense Besch.

Erpodium beccarii

\section{Enythrodontium}

E. argentinicum (C. Muell.) Par. (Paris, 1904b).

Syn. Entodon argentinicus C. Muell. (Mueller, 1879a).

Distr. Southern South America (Mueller, 1879a; Paris, 1904b; Brotherus, 1918, 1925; Kühnemann, 1938).

E. bicolor (Aongstr.) Broth.

Enthrodontium squarrosum

E. platygyrioides (C. Muell.) Fleisch. (Fleischer, 1922).

Syn. Entodon platygyrioides C. Muell. (Mueller, 1879a).

Cylindrothecium platygyrioides (C. Muell.) Par. (Paris, 1894).

Distr. Southern South America (Mueller, 1879a; Paris, 1894, 1904b; Fleischer, 1922;

Brotherus, 1925; Kühnemann, 1938). 
E. squarrosum (Hamp.) Par. (Paris, 1904b).

Syn. Leptohymenium squarrosum Hamp. (Hampe, 1862b). Erythrodontium bicolor (Aongstr.) Broth. (Brotherus in Ule, 1899). Pterigynandrum bicolor Lindb. ex Aongstr. (Lindberg ex Ångström, 1876a).

Distr. Southern South America (Krieger, 1904; Hosseus, 1940; Herzog, 1952a).

\section{Enythrophyllopsis}

E. boliviana Broth.

Erythrophyllopsis fuscula

E. fuscula (C. Muell.) Hilp. (Hilpert, 1933).

Syn. Barbula fuscula C. Muell. (Mueller, 1879a).

Erythrophyllopsis boliviana Broth. (Brotherus in Herzog, 1916a).

Distr. Southern South America (Mueller, 1879a; Kurtz, 1904; Paris, 1904a; Brotherus, 1924a; Hilpert, 1933; Hosseus, 1935b, 1937; Kühnemann, 1938; Bartram, 1965).

\section{Eucamptodon}

E. perichaetialis (Mont.) Mont. (Montagne, 1845a).

Syn. Weisia perichaetialis Mont. (Montagne, 1845a).

Distr. Juan Fernandez (Brotherus, 1924b; Robinson, 1975).

Southern South America (Montagne, 1845a, 1850, 1856; Mueller, 1848a; Mitten, 1869; Jaeger, 1872; Paris, 1904b; Dusén, 1905c; Cardot, 1908a; Herzog, 1923, 1938;

Brotherus, 1924a; Kühnemann, 1938; Seki, 1974).

Eucatagonium nom. illeg.

E. politum (Hook. f. et Wils.) Broth.

Catagonium politum

var. filescens (Card. et Broth.) Herz.

Catagonium politum var. filescens

\section{Eucladium}

E. calcareum (Nees et Hornsch.) C. Jens.

Gymnostomum aeruginosum

E. recurvirostre (Hedw.) C. Jens.

Hymenostylium recurvirostre

E. verticillatum (Brid.) B.S.G. (Bruch and others, 1846).

Syn. Weisia verticillata Brid. (Bridel, 1801a).

Distr. Southern South America (Roivainen, 1934).

Euglossophyllum nom. inval.

E. brevipes (C. Muell.) Hamp.

Stereophyllum brevipes

Eugrimmia nom. nud.

E. trichophylla (Grev.) Hamp.

Grimmia trichophylla

\section{Eurhynchiella}

E. acanthophylla (Mont.) Fleisch. (Fleischer, 1923).

Syn. Hypnum acanthophyllum Mont. (Montagne, 1845a).

Rhynchostegium acanthophyllum (Mont.) Jaeg. (Jaeger, 1878) nom. inval.

Rhynchostegiella acanthophylla (Mont.) Broth. (Brotherus, 1909).

Distr. Southern South America (Montagne, 1845a, 1850, 1856; Mueller, 1851a; Mitten, 1869; Jaeger, 1878; Paris, 1905b; Thériot, 1915, 1918; Campo, 1921; Costes, 1921; Herzog, 1923, 1938, 1939, 1954, 1957, 1960; Brotherus, 1924C, 1925; Espinosa B. 1941; Seki, 1974).

E. fuegiana (Card.) Fleish.

Oxyrrhynchium fuegianum

E. tenuinervis Herz. (Herzog, 1952a).

Distr. Southern South America (Herzog, 1952a). 


\section{Eurhynchium}

E. acanthophyllum (Mont.) Dus. ex Thér.

Oxyrrhynchium corralense

E. confusum Thér. Oxyrrhynchium corralense

E. corralense (Lor.) Jaeg. Oxyrrhynchium corralense

E. cuestarum C. Muell. (Mueller, 1897a).

Distr. Southern South America (Mueller, 1897a; Paris, 1900a, 1904b).

E. cygnisetum C. Muell.

Ptychomnion cygnisetum

E. dives C. Muell. (Mueller, 1897a).

Distr. Southem South America (Mueller, 1897a; Paris, 1900a, 1904b).

E. encalyptratum Schimp.

Acrocladium auriculatum

E. eucalyptratum Schimp. ex Par. Acrocladium auriculatum

E. fuegianum Card. Oxyrrhynchium fuegianum

E. pendulum (Brid.) Jaeg. Helicodontium pendulum

E. pulchellum (Hedw.) Jenn. (Jennings, 1913).

Syn. Hypnum strigosum Hoffm. ex Web. et Mohr (Hoffman ex Weber and Mohr, 1803).

Distr. Southern South America (Mueller, 1851a).

E. remotifolium (Grev.) Jaeg.

Oxyrrhynchium clinocarpum

E. riparioides (Hedw.) Richs.

E. rusciforme Mild.

E. striatellum Schimp. Platyhypnidium riparioides Platyhypnidium riparioides Rigodium toxarion

Eustichia hom. illeg.

E. longirostris (Brid.) Brid.

E. lorentzii (C. Muell.) Par.

Diplostichum longirostre

E. poeppigii Par. Diplostichum lorentzii Diplostichum poeppigii

\section{Exodokidium}

E. donatii Herz. (Herzog, 1937).

Distr. Southern South America (Herzog, 1937).

E. subsymmetricum (Card.) Card.

Bartramia subsymmetrica

\section{Fabronia}

F. affinis Thér. (Thériot in Felippone, 1929 [1930]).

Distr. Southern South America (Felippone, 1929; Herter, 1933a; Herzog, 1957).

F. andina Mitt. (Mitten, 1869).

Distr. Southern South America (Mitten, 1869; Jaeger, 1878; Paris, 1904a; Felippone, 1909; Brotherus, 1925; Herter, 1933a; Herzog, 1938, 1954).

var. jaffuelii Thér. (Thériot, 1924).

Distr. Southern South America (Thériot, 1924).

F. apophysatula C. Muell. (Mueller, 1897a).

Distr. Southern South America (Mueller, 1897a; Paris, 1900a, 1904b; Kühnemann, 1938; Herzog, 1960).

F. argentinica C. Muell. (Mueller, 1879a).

Distr. Southerm South America (Mueller, 1879a; Kurtz, 1904; Paris, 1904b; Hosseus, 1934. 1935b, 1937, 1939; Kühnemann, 1938). 
F. balansae Besch. (Bescherelle, 1891).

Syn. Fabronia balansaeana Besch. (Bescherelle, 1885b) nom. nud.

Distr. Southern South America (Herzog, 1952a).

F. balansaeana Besch.

Fabronia balansae

F. basilaris C. Muell. (Mueller, 1879a).

Distr. Southern South America (Mueller, 1879a; Paris, 1904b; Fleischer, 1920; Brotherus, 1925; Kühnemann, 1938).

F. contermina Thér. (Thériot in Felippone, 1929 [1930]).

Distr. Southern South America (Felippone, 1929; Herter, 1933a).

F. enervis Herz. (Herzog, 1960 [1961]) hom. illeg.

Distr. Southern South America (Herzog, 1960).

F. felipponei Broth. (Brotherus in Felippone, 1917).

Distr. Southern South America (Felippone, 1917; Brotherus, 1925; Herter, 1933a, 1939a).

F. filamentosa C. Muell. (Mueller, 1897a).

Distr. Southem South America (Mueller, 1897a; Paris, 1900a, 1904a; Brotherus, 1925; Kühnemann, 1938).

F. imbricata Hamp. (Hampe, 1878).

Distr. Southern South America (Thériot, 1936; Hosseus, 1938a, d, 1939; Herter, 1939b).

F. latidens Dub. (Duby, 1870).

Distr. Southern South America (Duby, 1870; Jaeger, 1878; Paris, 1904a; Brotherus, 1925; Herzog, 1960).

F. latifolia C. Muell. (Mueller, 1897a).

Distr. Southern South America (Mueller, 1897a; Paris, 1900a, 1904b; Herter, 1933a; Hosseus, 1934, 1939; Kühnemann, 1938).

F. longidens Dub.

Dimerodontium pellucidum

F. Iorentziae C. Muell. (Mueller, 1897a).

Distr. Southern South America (Mueller, 1897a; Paris, 1900a, 1904b; Brotherus, 1925; Hosseus, 1934; Kühnemann, 1938; Herzog, 1954).

F. lorentzii C. Muell. (Mueller, 1879a).

Distr. Southern South America (Mueller, 1879a; Kurtz, 1904; Paris, 1904b; Fleischer, 1920; Brotherus, 1925; Williams, 1930; Hosseus, 1935a, b, 1937; Kühnemann, 1938).

F. minutissima Dub. (Duby, 1880a).

Distr. Southern South America (Duby, 1880a, b; Paris, 1904b; Brotherus, 1925).

F. obtusatula C. Muell. (Mueller, 1897a).

Syn. Helicondontium obtusatulum (C. Muell.) Fleisch. (Fleischer, 1920).

Distr. Southern South America (Mueller, 1897a; Paris, 1900a, 1904b; Fleischer, 1920; Brotherus, 1925; Herter, 1933a; Hosseus, 1934, 1939; Kühnemann, 1938).

F. palmicola C. Muell. (Mueller, 1879a).

Distr. Southern South America (Mueller, 1879a; Paris, 1904b; Brotherus, 1925; Herter, 1933a, 1939b; Hosseus, 1934, 1939; Kühnemann, 1938).

F. physcomitriocarpa C. Muell. (Mueller, 1879a).

Distr. Southern South America (Mueller, 1879a; Paris, 1904b; Brotherus, 1925; Kühnemann, 1938). 
F. podocarpi C. Muell. (Mueller, 1879a).

Distr. Southem South America (Mueller, 1879a; Paris, 1904b; Brotherus, 1925; Kühnemann, 1938).

F. polycarpa Hook. (Hooker, 1818).

Distr. Southern.South America (Mueller, 1869; Gibert, 1873; Paris, 1904b; Herter, 1928. 1933a; Herzog, 1952a).

F. spegazzinii C. Muell. (Mueller, 1897a).

Distr. Southern South America (Mueller, 1897a; Paris, 1900a, 1904b; Brotherus, 1925; Hosseus, 1938c, d; Kühnemann, 1938).

F. tucumanensis C. Muell. (Mueller, 1897a).

Distr. Southern South America (Muèller, 1897a; Paris, 1900a, 1904b; Hosseus, 1934, 1939; Kühnemann, 1938).

F. uruguensis C. Muell. (Mueller, 1879a).

Distr. Southern South America (Mueller, 1879a; Brotherus, 1925; Herter, 1933a; Hosseus, 1934, 1939; Kühnemann, 1938).

\section{Fabroniella}

F. krausei Lor. ex C. Muell.

Juratzkaea krausei

\section{Felipponea}

F. hollermayeri Thér. (Thériot, 1935a).

Distr. Southern South America (Thériot, 1935a).

F. montevidensis (C. Muell.) Broth. (Brotherus in Felippone, 1912).

Syn. Cladomnion montevidense C. Muell. (Mueller, 1897a).

Pterogoniadelphus montevidensis (C. Muell.) Fleisch. (Fleischer, 1917).

Distr. Southern South America (Mueller, 1897a; Paris, 1900a, 1904b; Felippone, 1912;

Fleischer, 1917; Brotherus, 1925; Herter, 1933a, 1939b).

\section{Fissidens}

F. acacioides Schrad.

Fissidens osmundoides

F. adianthoides Hedw. (Hedwig, 1801).

Syn. Hypnum adianthoides (Hedw.) L. ex With. (Linnaeus ex Withering, 1801).

Dicranum adianthoides (Hedw.) Web. et Mohr (Weber and Mohr, 1803).

Skitophyllum adianthoides (Hedw.) Pyl. (De la Pylaie, 1814 [1815]).

Dicranodon adianthoides (Hedw.) Béhéré (Béhéré, 1826).

Schistophyllum adianthoides (Hedw.) Lindb. (Lindberg, 1879). Fissidens taxifolius Hedw. var. adianthoides (Hedw.) Wahlenb. ex Hueb. (Hübener,
1833) nom. inval.

Distr. Southern South America (Dusén, 1906; Cardot, 1908a).

F. anacamptodes Besch.

Fissidens hauthalii var. obtusatulus

F. angustelimbatus Mitt. (Mitten, 1869).

Distr. Southern South America (Gibert, 1873; Herter, 1928, 1933a).

F. asplenioides Hedw. (Hedwig, 1801).

Syn. Hypnum asplenioides (Hedw.) Dicks. ex With. (Dickson ex Withering, 1801).

Dicranum asplenioides (Hedw.) Web. et Mohr (Weber and Mohr, 1803).

Skitophyllum asplenioides (Hedw.) Pyl. (De la Pylaie, 1814 [1815]).

Schistophyllum asplenioides (Hedw.) Brid. (Bridel, 1827b) nom. inval.

Fissidens pycnotylus Broth. (Brotherus, 1924b) fide Robinson, 1975.

Distr. Juan Fernandez (Mitten, 1869, 1885; Johow, 1896; Paris, 1904b; Brotherus, 1924b, 1925; Robinson, 1975). 

Southern South America (Hosseus, 1938c, d, 1939, 1940; Herzog, 1938, 1939, 1954;
Bartram, 1952, 1965).

var. australis Card. (Cardot in Skottsberg, 1914) nom. nud.

Distr. Juan Fernandez (Skottsberg, 1914).

F. atratus (C. Muell.) Broth. (Brotherus, 1901).

Syn. Conomitrium atratum C. Muell. (Mueller, 1897a).

Distr. Southern South America (Mueller, 1897a; Paris, 1900a, 1904a; Brotherus, 1901, 1924a; Kühnemann, 1938).

F. berterii (Mont.) C. Muell.

Fissidens fontanus

F. brachyloma Card. (Cardot in Skottsberg, 1914) nom. nud.

Distr. Juan Fernandez (Skottsberg, 1914).

F. brevipes Besch. (Bescherelle, 1891).

Distr. Southern South America (Herzog, 1952a).

F. brotheri Dus.

Fissidens brotherianus

F. brotherianus Par. (Paris in Thériot, 1921a).

Syn. Fissidens brotheri Dus. (Dusén, 1906) hom. illeg. Fissidens valdiviensis Herz. (Herzog, 1923).

Distr. Southern South America (Dusén, 1906; Costes, 1921; Thériot, 1921a, 1924, 1930; Herzog, 1923, 1938, 1954; Brotherus, 1924a; Bartram, 1952).

var. longisetus Thér. (Thériot, 1921a).

Distr. Southern South America (Costes, 1921; Thériot, 1921a).

F. bryoides Hedw. (Hedwig, 1801).

Syn. Hypnum bryoides (Hedw.) L. ex With. (Linnaeus ex Withering, 1801).

Dicranum bryoides (Hedw.) Sw. (Swartz, 1801).

Skitophyllum bryoides (Hedw.) Pyl. (De la Pylaie, 1814 [1815]).

Dicranodon bryoides (Hedw.) Béhéré (Béhéré, 1826).

Schistophyllum bryoides (Hedw.) Lindb. (Lindberg, 1879).

Fissidens viridulus (Sw.) Wahlenb. var. bryoides (Hedw.) Roehl. (Röhling, 1813) nom. illeg.

Distr. Juan Fernandez (Montagne, 1835).

Southern South America (Montagne, 1850).

ssp. incurvus (Roehl.) Bertsch (Bertsch, 1949).

Syn. F. incurvus Stark ex Roehl. (Röhling, 1813).

Distr. Southern South America (Montagne, 1850).

var. osmundoides (Hedw.) Mathieu

Fissidens osmundoides

F. campylopus Mont. (Montagne, 1845a).

Distr. Southern South America (Montagne, 1845a, 1850, 1856; Mueller, 1848a; Mitten, 1869; Jaeger, 1876; Paris, 1904b; Dusén, 1906; Brotherus, 1924a).

F. chilensis Dus. (Dusén, 1906).

Distr. Southern South America (Paris, 1904b; Dusén, 1906; Thériot, 1921a; Brotherus, 1924a; Herzog, 1938, 1954).

F. colonialis C. Muell.

F. compactus Mitt. (Mitten in Gibert, 1873) nom. nud.

Fissidens hauthallii var. minor

Distr. Southern South America (Gibert, 1873; Herter, 1928, 1933a).

F. costesii Thèr. (Thériot, 1921a).

Distr. Southern South America (Costes, 1921; Thériot, 1921a; Brotherus, 1924a). 
F. crassicuspis Broth. (brotherus, 1924b).

Distr. Juan Femandez (Brotherus, 1924b, 1925; Robinson, 1975).

F. crispus Mont. (Montagne, 1838).

Distr. Southern South America (Montagne, 1838, 1856; Jaeger, 1876; Paris, 1904b; Brotherus, 1924a; Kühnemann, 1938).

F. curvinervis Card. (Cardot in Skottsberg, 1914) nom. nud.

Distr. Juan Fernandez (Skottsberg, 1914).

F. decursivus C. Muell. (Mueller, 1879a).

Distr. Southern South America (Mueller, 1879a; Kurtz, 1904; Paris, 1904b; Brotherus, 1924a; Hosseus, 1935b, 1937; Kühnemann, 1938).

F. dietrichiae C. Muell.

Fissidens fontanus

F. dillenii (Pyl.) Hamp.

F. distichellus Besch. (Bescherelle, 1891).

Distr. Southern South America (Bescherelle, 1891; Paris, 1904b; Brotherus, 1924a; Herter, 1933a).

F. diversiformis Broth. (Brotherus, 1924c). Distr. Southern South America (Brotherus, 1924c, 1925; Espinosa B., 1941; Skottsberg,
1950).

F. echinellus Herz. (Herzog, 1954).

Distr. Southern South America (Herzog, 1954).

F. felipponei Broth. (Brotherus in Felippone, 1909).

Distr. Southern South America (Felippone, 1909; Brotherus, 1924a; Herter, 1933a, 1939a, 1943).

F. fernandezianus Card. (Cardot in Skottsberg, 1914) nom. nud.

Distr. Juan Fernandez (Skottsberg, 1914).

F. fernandezianus Broth. (Brotherus, 1924b).

Distr. Juan Fernandez (Brotherus, 1924b, 1925; Bartram, 1959; Robinson, 1975).

F. fontanus (Pyl.) Steud. (Steudel, 1824).

Syn. Skitophyllum fontanum Pyl. (De la Pylaie, 1814 [1815]).

Octodiceras fontanum (Pyl.) Lindb. (Lindberg, 1863).

Skitophyllum dillenii Pyl. (De la Pylaie, 1814 [1815]).

Octodiceras dillenii (Pyl.) Brid. (Bridel, 1827b).

Conomitrium dillenii (Pyl.) Mont. (Montagne, 1837).

Fissidens dillenii (Pyl.) Hamp. (Hampe, 1839).

Fontinalis juliana Savi ex Cand. (Savi ex de Candolle, 1815).

Skitophyllum julianum (Cand.) Brid. (Bridel, 1827 b) nom. inval.

Octodiceras julianum (Cand.) Brid. (Bridel, 1827 b).

Conomitrium julianum (Cand.) Mont. (Montagne, 1837).

Fissidens julianus (Cand.) Schimp. (Schimper, 1838).

Schistophyllum julianum (Cand.) Lindb. (Lindberg, 1879).

Conomitrium berterii Mont. (Montagne, 1837).

Fissidens berterii (Mont.) C. Muell. (Mueller, 1848a).

Octodiceras berterii (Mont.) Jaeg. (Jaeger, 1876).

Conomitrium berteroi Mont. ex Kindb. (Montagne ex Kindberg, 1888) err.

Fissidens dietrichae C. Muell. (Mueller, 1872).

Fissidens parvus Mitt. (Mitten, 1869) hom. illeg.

Distr. Southern South America (De la Pylaie, 1814; Bridel, 1827b; Montagne, 1837, 1839c, 1850, 1856; Schwaegrichen, 1842; Mueller, 1848a; Mitten, 1869; Jaeger, 1876; Paris, 1904a; Dusén, 1906; Cardot, 1908a; Brotherus, 1924a; Kühnemann, 1938; Herzog, 1954). 
F. fossicola C. Muell. (Mueller, 1882).

Distr. Southern South America (Mueller, 1882; Paris, 1904b; Brotherus, 1924a; Kühnemann, 1938).

F. geheebii C. Muell. (Mueller, 1879a).

Distr. Southern South America (Mueller, 1879a; Paris, 1904b; Brotherus, 1924a; Kühnemann, 1938).

F. glossophyllus C. Muell. (Mueller, 1879a).

Distr. Southern South America (Mueller, 1879a; Paris, 1904b; Brotherus, 1924a; Hosseus, 1937; Kühnemann, 1938).

var. brevifolius C. Muell. (Mueller, 1879a).

Distr. Southern South America (Mueller, 1879a; Paris, 1904b; Hosseus, 1935a; Kühnemann, 1938).

F. hauthalii C. Muell. (Mueller, 1897a).

Distr. Southern South America (Mueller, 1897a; Paris, 1900a, 1904b; Felippone, 1912; Brotherus, 1924a; Herter, 1933a; Kühnemann, 1938).

var. angustissimus C. Muell. (Mueller, 1897a).

Syn. Fissidens stoloniferus C. Muell. (Mueller, 1897a) nom. nud.

Distr. Southern South America (Mueller, 1897a; Paris, 1900a, 1904b; Brotherus, 1924a; Herter, $1933 a$ (as F. stolonifer C. Muell.).

var. gracillimus C. Muell. (Mueller, 1897a).

Syn. Fissidens semilaminatus C. Muell. (Mueller, 1897a) nom. nud. Fissidens semi-lamellosus C. Muell. (Mueller, 1897a) nom. nud.

Distr. Southern South America (Mueller, 1897a; Paris, 1900a, 1904b).

var. minor C. Muell. (Mueller, 1897a).

Syn. Fissidens colonialis C. Muell. (Mueller, 1897a) nom. nud.

Distr. Southern South America (Mueller, 1897a; Paris, 1900a, 1904b).

var. obtusatulus C. Muell. (Mueller, 1897a).

Syn. Fissidens anacamptodes Besch. (Bescherelle in Mueller, 1897a) nom. nud.

Distr. Southern South America (Mueller, 1897a; Paris, 1900a, 1904b).

F. hedwigii C. Muell.

Fissidens semicompletus

F. hornschuchii Mont. (Montagne, 1840a).

Distr. Southern South America (Montagne, 1850).

F. inclinatulus C. Muell.

Fissidens platensis

F. incompletus Hedw. ex Dix.

Fissidens semicompletus

F. incurvus Stark ex Roehl.

Fissidens bryoides ssp. incurvus

F. indistinctus C. Muell. (Mueller, 1879a).

Distr. Southern South America (Mueller, 1879a; Paris, 1904b; Brotherus, 1924a; Kühnemann, 1938).

var. major C. Muell. (Mueller, 1879a).

Distr. Southern South America (Mueller, 1879a; Paris, 1904b).

F. jaffueli Thér. (Thériot in Costes, 1921) nom. nud.

Distr. Southern South America (Costes, 1921).

F. jujuiensis Broth. (Brotherus, 1918).

Distr. Southern South America (Brotherus, 1918, 1924a; Kühnemann, 1938).

F. julaceus (Hedw.) Lindb.

Leucodon julaceus 
F. julianus (Cand.) Schimp.

F. jungermanniopsis C. Muell. (Mueller, 1879a).

Distr. Southern South America (Mueller, 1879a; Paris, 1900a, 1904b; Kurtz, 1904; Brotherus, 1924a; Hosseus, 1937; Kühnemann, 1938).

F. lechleri Hamp. ex C. Muell.

Fissidens rigidulus

F. leptocaulis C. Muell. (Mueller, 1897a).

Distr. Southern South America (Mueller, 1897a; Paris, 1900a, 1904b; Felippone, 1912; Brotherus, 1924a; Herter, 1933a).

F. leptochaete Dus. (Dusén, 1906).

Distr. Juan Fernandez (Brotherus, 1924b; Robinson, 1975).

Southern South America (Dusén, 1906; Brotherus, 1924a).

F. leucodictyus C. Muell. (Mueller, 1897a).

Distr. Southem South America (Mueller, 1897a; Paris, 1904b; Brotherus, 1924a; Kühnemann, 1938).

F. Ionchothecius C. Muell. (Mueller, 1879a).

Distr. Southem South America (Mueller, 1879a; Paris, 1904b; Brothenus, 1924a; Hosseus, 1938a, $d, 1939$; Kühnemann, 1938).

F. Iorentziae (C. Muell.) Broth. (Brotherus, 1901).

Syn. Conomitrium lorentziae C. Muell. (Mueller, 1897a).

Distr. Southem South America (Mueller, 1897a; Paris, 1900a, 1904a; Brotherus, 1901, 1924a; Felippone, 1928; Herter, 1933a; Hosseus, 1936, 1937, 1938c, d, 1939; Kühnemann, 1938).

F. macro-bryoides C. Muell. (Mueller, 1879a).

Distr. Southern South America (Mueller, 1879a; Paris, 1904b; Brotherus, 1924a; Kühnemann, 1938).

F. maschalanthus Mont. (Montagne, 1845a).

Distr. Juan Fernandez (Brotherus, 1924b; Espinosa B., 1941; Bartram, 1959; Robinson, 1975).

Southern South America (Montagne, 1845a, 1850, 1856; Mueller, 1848a; Lorentz, 1866; Mitten, 1869; Jaeger, 1876; Paris, 1904b; Dusén, 1906; Cardot, 1908a; Campo, 1921; Thériot, 1921a; Brotherus, 1924a; Reimers, 1926; Herzog, 1938, 1939, 1954. 1960; Kühnemann, 1938; Seki, 1974).

var. minor Dus. ex Thér. (Dusén ex Thériot, 1918).

Distr. Juan Fernandez (Skottsberg, 1914).

Southern South America (Paris, 1906; Thériot, 1918; Campo, 1921).

F. mollis (C. Muell.) Broth.

Fissidens permollis

F. nigritellus (C. Muell.) Broth. (Brotherus, 1901).

Syn. Conomitrium nigritellum C. Muell. (Mueller, 1897a).

Distr. Southern South America (Mueller, 1897a; Brotherus, 1901, 1924a; Paris, 1904a; Kühnemann, 1938).

F. obliquifolius C. Muell. (Mueller, 1897a).

Distr. Southern South America (Mueller, 1897a; Paris, 1900a, 1904b; Brotherus, 1924a; Kühnemann, 1938).

F. odontoloma C. Muell. (Mueller, 1879a).

Distr. Southern South America (Mueller, 1879a; Paris, 1904b; Brotherus, 1924a; Kühnemann, 1938).

F. oeditrachelos C. Muell.

Fissidens vitreo-limbatus 
F. ornaticostatus Robins. (Robinson, 1974).

Distr. Juan Fernandez (Robinson, 1974, 1975).

F. osmundoides Hedw. (Hedwig, 1801).

Syn. Dicranum osmundoides (Hedw.) Turn. (Turner, 1804a).

Skitophyllum osmundoides (Hedw.) Pyl. (De la Pylaie, 1814 [1815]).

Conomitrium osmundoides (Hedw.) C. Muell. (Mueller, 1851a).

Schistophyllum osmundoides (Hedw.) Lindb. (Lindberg, 1879).

Fissidens bryoides Hedw. var. osmundoides (Hedw.) Mathieu (Mathieu, 1853).

Fissidens osmundoides Hedw. var. microcarpus B.S.G. (Bruch and others, 1843).

Schistophyllum osmundoides (Hedw.) Lindb. var. microcarpum (B.S.G.) Kindb.

(Kindberg, 1883).

Fissidens acacioides Schrad. (Schrader, 1803b).

Hypnum acacioides (Schrad.) L. ex Turn. (Linnaeus ex Turner, 1804b).

Skitophyllum acacioides (Schrad.) Pyl. (De la Pylaie, 1814 [1815]).

Schistophyllum acacioides (Schrad.) Lindb. (Lindberg, 1883).

Distr. Southern South America (Bridel, 1803, 1806, 1827b; Mueller, 1848a, $1851 a$; Jaeger, 1876; Lindberg, 1883; Paris, 1904b).

var. microcarpus B.S.G.

Fissidens osmundoides

F. pachylomatos C. Muell.

Fissidens vitreo-limbatus

F. pachylomulus C. Muell.

Fissidens vitreo-limbatus

F. palmatus Hedw. (Hedwig, 1801).

Distr. Southern South America (Montagne, 1850).

F. parvus Mitt.

Fissidens fontanus

F. permollis Thér. (Thériot, 1931b [1932]).

Syn. Conomitrium molle C. Muell. (Mueller, 1879a).

Fissidens mollis (C. Muell.) Broth. (Brotherus, 1900) hom. illeg.

Distr. Southern South America (Mueller, 1879a; Paris, 1904a; Brotherus, 1924a; Herter, 1933a, 1939b; Kühnemann, 1938).

F. plagiothecioides C. Muell. (Mueller, 1879a).

Distr. Southern South America (Mueller, 1879a; Paris, 1904b; Brotherus, 1924a; Kühnemann, 1938).

F. platensis Par. (Paris, 1900a).

Syn. Fissidens inclinatulus C. Muell. (Mueller, 1897a) hom. illeg.

Distr. Southern South America (Mueller, 1897a; Paris, 1900a, 1904b; Brotherus, 1924a; Kühnemann, 1938).

F. prionocheilos C. Muell. (Mueller, 1879a).

Distr. Southern South America (Mueller, 1879a; Paris, 1904b; Brotherus, 1924a; Kühnemann, 1938).

F. pulvinatus Timm ex Hedw.

Grimmia pulvinata

F. pycnoglossus C. Muell. (Mueller, 1879a).

Distr. Southern South America (Mueller, 1879a; Kurtz, 1904; Paris, 1904b; Brotherus, 1924a; Hosseus, 1937; Kühnemann, 1938).

F. pycnotylus Broth.

Fissidens asplenioides

F. repandus Wils. (Wilson in Mitten, 1851).

Distr. Southern South America (Gibert, 1873; Herter, 1928, 1933a; Bartram, 1965).

F. rigidulus Hook. f. et Wils. (Hooker and Wilson in Wilson, 1854).

Syn. Fissidens lechleri Hamp. ex C. Muell. (Hampe ex Mueller, 1864). 
Distr. Juan Fernandez (Brotherus, 1924b; Bartram, 1959; Robinson, 1975).

Southem South America (Lechler, 1857; Mueller, 1864; Mitten, 1869; Jaeger, 1876; Neger, 1899; Paris, 1904b; Dusén, 1906; Cardot, 1908a; Campo, 1921; Thériot, 1921a, 1925a; Brotherus, 1924a; Reimers, 1926; Roivainen, 1934; Roivainen and Bartram, 1937; Kühnemann, 1938; Herzog, 1939, 1954; Espinosa B., 1941; Seki, 1974).

F. scalaris Mitt. (Mitten, 1869).

Distr. Southem South America (Mitten, 1869; Gibert, 1873; Jaeger, 1876; Paris, 1904b; Campo, 1921; Thériot, 1921a; Brotherus, 1924c; Herter, 1928, 1933a; Herzog, 1938. 1954, 1960; Kühnemann, 1938; Espinosa B., 1941; Bartram, 1952).

F. semicompletus Hedw. (Hedwig, 1801).

Syn. Cecalyphum semi-completum (Hedw.) P. Beauv. (Palisot de Beauvois, 1805a).

Dicranum semicompletum (Hedw.) Mohr (Mohr, 1806).

Skitophyllum semi-completum (Hedw.) Pyl. (De la Pylaie, 1814 [1815]).

Schistophyllum semicompletum (Hedw.) Lindb. (Lindberg, 1883).

Fissidens incompletus Hedw. ex Dix. (Dixon, 1933 [1934]) err.

Conomitrium hedwigii Mont. (Montagne, 1837) nom. illeg.

Fissidens hedwigii C. Muell. (Mueller, 1848a) nom. illeg.

Octodiceras hedwigii Lindb. (Lindberg, 1863) nom. illeg.

Octodiceras fissidentoides Brid. (Bridel, 1827 b) nom. illeg.

Distr. Southem South America (Hedwig, 1801; Bridel, 1803, 1806; Schwaegrichen, 1816 , 1842; Montagne, 1837, 1850; Mueller, 1848a; Mitten, 1869; Jaeger, 1876; Paris, 1904a; Brotherus, 1924a).

F. semi-lamellosus C. Muell.

Fissidens hauthalii var. gracillimus

F. semilaminatus C. Muell.

Fissidens hauthalii var. gracillimus

F. smaragdinus (Lor. et C. Muell.) Broth. (Brotherus, 1901).

Syn. Conomitrium smaragdinum Lor. et C. Muell. (Lorentz and Mueller in Mueller, 1879a).

Distr. Southern South America (Mueller, 1879a; Brotherus, 1901, 1924a; Kurtz, 1904; Paris, 1904a; Hosseus, 1935b, 1937; Kühnemann, 1938; Bruggeman-Nannenga, 1973).

F. stolonaceus C. Muell. (Mueller, 1879a).

Distr. Southem South America (Mueller, 1879a; Kurtz, 1904; Paris, 1904b; Brotherus, 1924a; Hosseus, 1935b, 1937; Kühnemann, 1938).

F. stoloniferus C. Muell.

Fissidens hauthalii var. angustissimus

F. subaloma Dus. (Dusén, 1906).

Distr. Southem South America (Paris, 1904b; Dusén, 1906; Costes, 1921; Thériot, 1921a, 1924; Brotherus, 1924a, C; Espinosa B., 1941).

F. synoicus C. Muell. (Mueller, 1879a) hom. illeg.

Distr. Southem South America (Mueller, 1879a; Paris, 1904b; Brotherus, 1924a; Kühnemann, 1938).

F. taxifolius Hedw. (Hedwig, 1801).

Syn. Hypnum taxifolium (Hedw.) L. ex With. (Linnaeus ex Withering, 1801).

Dicranum taxifolium (Hedw.) Web. et Mohr (Weber and Mohr, 1803).

Skitophyllum taxifolium (Hedw.) Pyl. (De la Pylaie, 1814 [1815]).

Schistophyllum taxifolium (Hedw.) Lindb. (Lindberg, 1879).

Distr. Southern South America (Brothenus, 1924C; Espinosa B., 1941).

var. adianthoides (Hedw.) Wahlenb. ex Hueb.

Fissidens adianthoides

F. tener Dus. (Dusén, 1906).

Distr. Southern South America (Dusén, 1906).

F. terebrifolius C. Muell. (Mueller, 1897a).

Distr. Southern South America (Mueller, 1897a; Paris, 1900a, 1904a; Brotherus, 1924a; Kühnemann, 1938). 
F. valdiviensis Herz.

F. ventanae C. Muell. (Mueller, 1897a).

Distr. Southern South America (Mueller, 1897a; Paris, 1904b; Brotherus, 1924a; Kühnemann, 1938).

F. viridulus (Sw.) Wahlenb. var. bryoides (Hedw.) Roehl.

Fissidens bryoides

F. vitreo-limbatus C. Muell. (Mueller, 1897a).

Syn. Fissidens oeditrachelos C. Muell. (Mueller, 1897a) nom. nud.

Fissidens pachylomatos C. Muell. (Mueller in Paris, 1900a) nom. nud.

Fissidens pachylomulus C. Muell. (Mueller, 1897a) nom. nud.

Distr. Southern South America (Mueller, 1897a; Paris, 1900a, 1904b; Felippone, 1917; Brotherus, 1924a; Herter, 1933a; Kühnemann, 1938).

\section{Floribundaria}

F. cardotii (Broth.) Broth. (Brotherus, 1906b).

Syn. Papillaria cardotii Broth. (Brotherus, 1895).

Distr. Southern South America (Herzog, 1952a).

F. usneoides (Broth.) Broth. (Brotherus, 1906b).

Syn. Papillaria usneoides Broth. (Brotherus, 1893).

Distr. Southern South America (Hosseus, 1940).

Florschuetzia hom. illeg.

F. pilmaiquen Crosby

Muscoflorschuetzia pilmaiquen

\section{Fontinalis}

F. juliana (Savi) Savi ex Cand.

Fissidens fontanus

\section{Forsstroemia}

F. coronata (Mont.) Par. (Paris, 1895).

Syn. Leptodon coronatus Mont. (Montagne, 1838).

Neckera coronata (Mont.) C. Muell. (Mueller, 1850).

Leucodon coronatus (Mont.) Mitt. (Mitten, 1869).

Lasia coronata (Mont.) C. Muell. (Mueller, 1879a).

Dusenia coronata (Mont.) C. Muell. (Mueller in Fleischer, 1917) nom. inval.

Distr. Southern South America (Montagne, 1838, 1839c; Mueller, 1850, 1879a, 1882; Mitten, 1869; Jaeger, 1877; Felippone, 1917; Brotherus, 1918; Herter, 1933a; Kühnemann, 1938; Herzog, 1952a).

F. felipponei Thèr. (Thériot in Felippone, 1929 [1930]).

Distr. Southern South America (Felippone, 1929; Herter, 1933a).

F. subcoronata (Besch.) Par. (Paris, 1895).

Syn. Lasia subcoronata Besch. (Bescherelle, 1877).

Distr. Southern South America (Herzog, 1952a).

\section{Funaria}

F. acutifolia (Hamp.) Broth. (Brotherus, 1903).

Syn. Entosthodon acutifolius Hamp. (Hampe in Mueller, 1859).

Distr. Southern South America (Thériot, 1927a).

F. aristatula C. Muell. (Mueller, 1879a).

Distr. Southern South America (Mueller, 1879a; Kurtz, 1904; Paris, 1904b; Brotherus, 1924a; Hosseus, 1935b, 1937; Kühnemann, 1938). 
F. balansae (Besch.) Broth. (Brotherus, 1900).

Syn. Entosthodon balansae Besch. (Bescherelle, 1877).

Distr. Southern South America (Herter, 1933a, 1939a; Herzog, 1939).

F. berteroana Hamp. ex C. Muell. (Hampe ex Mueller, 1862).

Syn. Funaria fontanesii B.S.G. non Schwaegr. (Bruch and others, 1841) pro parte fide Paris, 1904b.

Funaria berteroi Hamp. ex Kindb. (Hampe ex Kindberg, 1888) nom. illeg.

Distr. Southem South America (Mueller, 1844b, 1851a, 1862; Jaeger, 1874; Paris, 1904b;

Costes, 1921; Herzog, 1923; Brotherus, 1924a; Thériot, 1927a).

var. inclinata Thér. (Thériot, 1927a).

Distr. Southern South America (Thẹ́riot, 1927a).

F. berteroi Hamp. ex Kindb.

Funaria berteroana

F. bonplandii (Hook.) Broth. (Brotherus, 1903).

Syn. Gymnostomum bonplandii Hook. (Hooker, 1816).

Physcomitrium bonplandii (Hook.) De Not. (De Notaris, 1838b).

Entosthodon bonplandii (Hook.) Mitt. (Mitten, 1869).

Amphoritheca bonplandii (Hook.) Jaeg. (Jaeger, 1874).

Distr. Southem South America (Mueller, 1844b, 1848a; Montagne, 1850; Jaeger, 1874;

Brotherus, 1903, 1924a; Paris, 1904b; Thériot, 1927a).

F. calvescens Schwaegr.

var. crispa C. Muell.

Funaria hygrometrica var. calvescens

Funaria hygrometrica var. crispa

F. cavifolia Card. et Broth. (Cardot and Brotherus, 1923).

Distr. Southern South America (Cardot and Brotherus, 1923).

F. chilensis (Thér.) Thér. (Thériot, 1926).

Syn. Funaria clavellata (Mitt.) Broth. var. chilensis Thér. (Thériot, 1924).

Distr. Southem South America (Thériot, 1924, 1926, 1927a).

F. chiloënsis (Mitt.) Broth. (Brotherus, 1903).

Syn. Entosthodon chiloënsis Mitt. (Mitten, 1869).

Distr. Southern South America (Mitten, 1869; Jaeger, 1874; Brotherus, 1903, 1924a; Krieger, 1904; Paris, 1904b; Thériot, 1927a).

F. clavellata (Mitt.) Broth. (Brotherus, 1903).

Syn. Entosthodon clavellatus Mitt. (Mitten, 1869).

Amphoritheca clavellata (Mitt.) Jaeg. (Jaeger, 1874).

Distr. Southern South America (Mitten, 1869; Gibert, 1873; Jaeger, 1874; Brotherus, 1903. 1924a; Paris, 1904b; Felippone, 1909; Herter, 1928, 1933a).

var. chilensis Thér.

Funaria chilensis

F. commixta Thér. (Thériot, 1926).

Distr. Southem South America (Thériot, 1926, 1927a; Herzog, 1954).

F. costesii Thér. (Thériot, 1921a).

Distr. Southern South America (Thériot, 1921a, 1927a; Costes, 1921; Brotherus, 1924a).

F. erectiuscula Mitt. (Mitten, 1869). Distr. Southern South America (Mitten, 1869; Jaeger, 1874; Paris, 1904b; Brotherus, 1924a;
Thériot, 1927a).

F. flexiseta (C. Muell.) Broth. (Brotherus, 1903).

Syn. Entosthodon flexisetus C. Muell. (Mueller, 1879a). 
Distr. Southern South America (Mueller, 1879a; Brotherus, 1903, 1924a; Paris, 1904b; Kühnemann, 1938).

F. fontanesii B.S.G. non Schwaegr.

see Funaria berteroana and $\boldsymbol{F}$. integra

F. fuegiana C. Muell.

Funaria hygrometrica var. fuegiana

F. hosseusii Bartr. (Bartram, 1936).

Distr. Southern South America (Bartram, 1936; Hosseus, 1938c, $d, 1939$ ).

F. hygrometrica Hedw. (Hedwig, 1801).

Distr. Juan Fernandez (Mitten, 1885; Johow, 1896; Skottsberg, 1914; Brotherus, 1924b; Espinosa B., 1941; Robinson, 1975).

Southern South America (Schwaegrichen, 1816; Montagne, 1850; Lorentz, 1866; Ångström, 1872; Gibert, 1873; Bescherelle, 1889; Dusén, 1903a; Cardot, 1905a, 1908a; Felippone, 1912; Williams, 1915, 1930; Thériot, 1915, 1917b, 1918, 1921a, 1927a; Campo, 1921; Costes, 1921; Cardot and Brotherus, 1923; Brotherus, 1924c; Espinosa B., 1924, 1941; Reimers, 1926; Herter, 1928, 1933a, 1939a, b; Roivainen, 1934; Hosseus, 1938c, d; Kühnemann, 1938; Herzog, 1939, 1940, 1954, 1957; Bartram, 1952; Bizot and Piovano, 1953; Piovano, 1954; Seki, 1974).

Falkland Islands (Wilson and Hooker, 1847; Kühnemann, 1938).

var. calvescens (Schwaegr.) Mont. (Montagne, 1839a).

Syn. Funaria calvescens Schwaegr. (Schwaegrichen, 1816).

Distr. Southern South America (Montagne, 1846, 1850; Mitten, 1869; Mueller, 1879a; Krieger, 1904; Felippone, 1912; Thériot, 1927a; Herter, 1933a; Hosseus, 1938a, c, d; Kühnemann, 1938; Herzog, 1954).

var. crispa (C. Muell.) Par. (Paris, 1895).

Syn. Funaria calvescens Schwaegr. var. crispa C. Muell. (Mueller, 1848a).

Distr. Southern South America (Mueller, 1848a; Paris, 1895, 1904b; Thériot, 1927a).

var. fuegiana (C. Muell.) Besch. (Bescherelle, 1889).

Syn. Funaria fuegiana C. Muell. (Mueller, 1885).

Funaria magellanica C. Muell. (Mueller in Bescherelle, 1889) nom. nud.

Distr. Southern South America (Mueller, 1885; Bescherelle, 1889; Paris, 1904b; Cardot, 1908a; Brotherus, 1924a; Thériot, 1927a; Kühnemann, 1938).

F. incompleta C. Muell. (Mueller, 1879a).

Distr. Southern South America (Mueller, 1879a; Paris, 1904b; Brotherus, 1924a; Kühnemann, 1938).

F. integra (C. Muell.) Broth. (Brotherus, 1903).

Syn. Entosthodon mathewsii Hook. f. var. integer C. Muell. (Mueller, 1848a). Entosthodon mathewsii Hook. f. var. integrifolius Par. (Paris, 1895) nom. inval. Entosthodon brachyodus Mont. (Montagne in Mueller, 1851a) nom. nud.

Funaria fontanesii B.S.G. non Schwaegr. (Bruch and others, 1841) pro parte fide Paris, $1904 b$.

Distr. Southern South America (Mueller, 1844b, 1848a, 1851a; Montagne, 1850; Jaeger, 1874; P-aris, 1895, 1904b; Brotherus, 1903, 1924a, c; Thériot, 1927a; Skottsberg, 1950; Herzog, 1954).

var. serrulata Broth. (Brotherus, 1924C).

Distr. Southern South America (Brotherus, 1924C; Espinosa B., 1941).

F. jujuiensis C. Muell. (Mueller, 1879a).

Distr. Southern South America (Mueller, 1879a; Paris, 1904b; Brotherus, 1924a; Kühnemann, 1938).

F. linearidens C. Muell. (Mueller, 1879a).

Distr. Southern South America (Mueller, 1879a; Paris, 1904b; Brotherus, 1924a; Kühnemann, 1938). 
F. Iorentzii C. Muell. (Mueller, 1879a).

Distr. Southem South America (Mueller, 1879a; Kurtz, 1904; Paris, 1904b; Brotherus, 1924a; Hosseus, 1935b, 1937; Kühnemann, 1938).

F. magellanica C. Muell.

Funaria hygrometrica var. fuegiana

F. meeseacea C. Muell. (Mueller, 1882).

Distr. Southem South America (Mueller, 1882; Paris, 1904b; Brotherus, 1924a; Kühnemann, 1938).

F. papillosa (C. Muell.) Wijk. et Marg. (van der Wijk and Margadant, 1960a).

Syn. Entosthodon papillosus C. Muell. (Mueller, 1882).

Amphoritheca papillosa (C. Muell.) Kindb. (Kindberg, 1889).

Funaria tucumanica Broth. (Brotherus, 1903) nom. illeg.

Distr. Southem South America (Múeller, 1882; Brotherus, 1903, 1924a; Paris, 1904b; Kühnemann, 1938).

F. plagiothecia (C. Muell.) Broth. (Brotherus, 1903).

Syn. Entosthodon plagiothecius C. Muell. (Mueller, 1879a).

Distr. Southem South America (Mueller, 1879a; Brotherus, 1903, 1924a; Paris, 1904b; Kühnemann, 1938).

F. porteri Thér. (Thériot, 1928 [1929]).

Distr. Southern South America (Thériot, 1928).

F. pulchricolor C. Muell. (Mueller, 1879a).

Distr. Southem South America (Mueller, 1879a; Paris, 1904b; Brotherus, 1924a; Hosseus, 1938a, d; Kühnemann, 1938).

F. pungens Bartr. (Bartram, 1946).

Distr. Southern South America (Bartram, 1946).

F. rhizomatica (C. Muell.) Broth. (Brotherus, 1903).

Syn. Entosthodon rhizomaticus C. Muell. (Mueller, 1879a).

Distr. Southem South America (Mueller, 1879a; Brotherus, 1903, 1924a; Paris, 1904b; Kühnemann, 1938).

F. schnyderiC. Muell. (Mueller, 1882).

Distr. Southem South America (Mueller, 1882; Paris, 1904b; Brotherus, 1924a; Kühnemann, 1938).

F. suberecta Mitt. (Mitten, 1869).

Distr. Southern South America (Williams, 1915; Thériot, 1927a).

F. tenella C. Muell. (Mueller, 1879a).

Distr. Southern South America (Mueller, 1879a; Kurtz, 1904; Paris, 1904b; Brotherus, 1924a; Hosseus, 1935b, 1937, 1938a, d; Kühnemann, 1938).

F. tucumanica Broth.

Funaria papillosa

F. valdiviae C. Muell. (Mueller, 1901a [1900]).

Distr. Southern South America (Mueller, 1901a).

\section{Gigaspermum}

G. imbricatum (Mitt.) Kindb.

Lorentziella imbricata

Glossophyllum hom. illeg.

G. brevipes (C. Muell.) Hamp.

Stereophyllum brevipes 


\section{Glyphocarpa}

G. Iaevisphaera Tayl.

Glyphocarpus nom. illeg.

G. cygneus (Mont.) Jaeg.

Bartramidula cygnea

G. exiguus (Sull.) Jaeg.

Philonotis scabrifolia

G. krausei (C. Muell.) Jaeg.

Philonotis krausei

G. laevisphaerus (Tayl.) Jaeg.

Anacolia laevisphaera

\section{Glyphomitrium}

G. balansae (Besch.) Broth.

Ptychomitrium balansae

G. brevifolium (C. Muell.) Broth.

Ptychomitrium balansae

G. cummingii (Dub.) Broth.

Ptychomitrium fernandesianum

G. emersum (C. Muell.) Broth.

Ptychomitrium emersum

G. fernandesianum Mitt.

Ptychomitrium fernandesianum

G. hieronymi (Besch.) Broth.

Ptychomitrium hieronymi

G. kurtzii Broth. (Brotherus in Kurtz, 1904) nom. nud.

Distr. Southern South America (Kurtz, 1904; Hosseus, 1937; Kühnemann, 1938).

G. ligulatum Mitt.

Ptychomitrium ligulatum

G. sellowianum (C. Muell.) Mitt.

Ptychomitrium sellowianum

G. uruguense (C. Muell.) Broth.

Ptychomitrium balansae

G. vernicosum (C. Muell.) Broth.

Ptychomitrium vernicosum

\section{Glyphothecium}

G. gracile (Hamp.) Broth. (Brotherus in Fleischer, 1906 [1908]).

Syn. Leucodon gracilis Hamp. (Hampe, 1844).

Cladomnion gracile (Hamp.) Mitt. (Mitten, 1869).

Glyptothecium gracile (Hamp.) Broth. (Brotherus, 1905b).

Neckera poeppigiana C. Muell. (Mueller, 1850).

Cladomnion poeppigianum C. Muell. (Mueller in Fleischer, 1917) nom. nud.

Leskea distans Mont. (Montagne, 1845a).

Distr. Southern South America (Hampe, 1844; Montagne, 1845a, 1850, 1856; Mueller, 1850; Lorentz, 1866; Mitten, 1869; Jaeger, 1877; Neger, 1899; Paris, 1904a; Brotherus, 1905a, 1925; Fleischer, 1906, 1917; Cardot, 1908a; Thériot, 1915; Reimers, 1926 ;Herzog, 1938, 1954, 1960; Kühnemann, 1938; Bizot, 1967; Seki, 1974; Kühnemann and Gonçalves, 1975).

var. nanum Herz. (Herzog, 1923).

Distr. Southern South America (Herzog, 1923).

Glyptothecium nom. illeg.

G. gracile (Hamp.) Broth.

Glyphothecium gracile

\section{Goniobryum}

G. reticulatum Lindb.

Philonotis vagans

var. evanidum Herz. (Herzog in Donat, 1936a).

Distr. Southern South America (Donat, 1936a; Kühnemann, 1938).

G. subbasilare (Hook.) Lindb. (Lindberg, 1864b [1865]).

Syn. Hypnum subbasilare Hook. (Hooker, 1818).

Rhizogonium subbasilare (Hook.) Schimp. (Schimper in Mueller, 1847b).

Mnium subbasilare (Hook.) C. Muell. (Mueller, 1848a).

Trachyloma subbasilare (Hook.) Mitt. (Mitten, 1860).

Photinophyllum subbasilare (Hook.) Mitt. (Mitten, 1868).

Webera hariotiana Besch. (Bescherelle in Mueller, 1901a [1900]) nom. nud. 
Distr. Southem South America (Hooker, 1818; Bridel, 1827b; Schwaegrichen, 1829; Wilson and Hooker, 1847; Mueller, 1848a, 1851a, 1885, 1901a; Mitten, 1869; Ångström, 1872; Jaeger, 1875; Bescherelle, 1889; Dusén, 1903a; Paris, 1904b; Cardot, 1908a, 1913a; Spegazzini, 1922; Cardot and Brotherus, 1923; Brotherus, 1924a; Kühnemann, 1938).

\section{Grimmia}

G. abscondita Card. (Cardot, 1908a).

Distr. Southern South America (Cardot, 1908a; Brotherus, 1924a; Kühnemann, 1938).

G. alpicola sw. ex Hedw.

Schistidium alpicola

var. maritima (Turn.) Wahlenb.

Schistidium maritimum

var. rivularis (Brid.) Wahlenb.

Schistidium apocarpum

G. amblyophylla C. Muell. (Mueller, 1849).

Syn. Schistidium amblyophyllum (C. Muell.) Herz. (Herzog, 1960 [1961]).

Distr. Southem South America Milson and Hooker, 1847 (as G. apocarpa var. pro parte fide Mueller, 1849); Mueller, 1849, 1885; Mitten, 1869; Jaeger, 1874; Paris, 1904b; Dusén, 1907; Cardot, 1908a; Brotherus, 1924a; Kühnemann, 1938; Bartram, 1952; Herzog.
1960).

G. andreaeacea C. Muell. (Mueller, 1882).

Distr. Southem South America (Mueller, 1882; Paris, 1904b; Kühnemann, 1938).

G. antarctici Card.

var. percompacta Bartr.

Schistidium antarctici

var. pilifera Bartr.

Schistidium antarctici

G. apocarpa Hedw.

Schistidium antarctici

ssp. alpicola (Hedw.) C. Hartm.

Schistidium apocarpum

ssp. conferta (Funck) Boul.

var. conferta (Funck) Spreng.

var. rivularis (Brid.) Roehl.

G. aquatica (Schrad.) C. Muell.

G. arachnoidea Dus.

G. atro-virens Sm.

Schistidium alpicola

G. austro-georgica C. Muell. ex Par.

G. austro-leucophaea Besch.

G. austro-patens C. Muell.

Schistidium apocarpum ssp. confertum

Schistidium apocarpum ssp. confertum

Schistidium apocarpum

Racomitrium aquaticum

Grimmia orbicularis var. patagonica

Desmatodon convolutus

Racomitrium austro-georgicum

Willia austro-leucophaea

G. calotricha C. Muell. (Mueller, 1882).

Racomitrium austro-georgicum

Distr. Southern South America (Mueller, 1882; Paris, 1904b; Brotherus, 1924a; Hosseus, 1935b, 1937; Kühnemann, 1938).

G. celata Card. (Cardot, 1906a).

Distr. Southern South America (Cardot and Brotherus, 1923; Kühnemann, 1938).

South Georgia (Cardot, 1906a, 1908a; Brotherus, 1924a; Steere, 1961b)

G. chilensis Thér. (Thériot in Herzog, 1957).

Distr. Southern South America (Herzog, 1957; Seki, 1974).

G. chubutensis Card. et Broth. (Cardot and Brotherus, 1923). Distr. Southern South America (Cardot and Brotherus, 1923; Brotherus, 1924a; Kühnemann.
1938).

G. conferta Funck

Schistidium apocarpum ssp. confertum 
G. consobrina Kunz. ex C. Muell. (Kunze ex Mueller, 1849).

Distr. Southern South America (Mueller, 1849; Montagne, 1850; Mitten, 1869; Jaeger, 1874; Paris, 1904b; Dusén, 1907; Thériot, 1917b, 1921a, 1935a; Campo, 1921; Costes, 1921; Cardot and Brotherus, 1923; Brotherus, 1924a; Reimers, 1926; Roivainen, 1934 ;Herzog, 1938, 1939, 1954, 1957; Kühnemann, 1938; Bartram, 1952; Seki, 1974).

G. controversa (Hedw.) Web. et Mohr

Weisia controversa

G. convoluta (Mont.) C. Muell.

Racomitrium crispulum

G. crassiretis Card. et Broth. (Cardot and Brotherus, 1923).

Distr. Southern South America (Cardot and Brotherus, 1923; Kühnemann, 1938).

G. crispula (Hook. f. et Wils.) C. Muell.

Racomitrium crispulum

G. cryptodon (Mont.) Hamp.

Camptodontium cryptodon

G. decurrentipilis Thér. (Thériot in Felippone, 1929 [1930]).

Distr. Southern South America (Felippone, 1929; Herter, 1933a).

G. depressa C. Muell. (Mueller, 1885).

Distr. Southern South America (Mueller, 1885; Bescherelle, 1889; Paris, 1904b; Cardot, 1908a; Kühnemann, 1938).

var. terrestris C. Muell. (Mueller, 1885).

Distr. Southern South America (Mueller, 1885; Paris, 1904b; Cardot, 1908a).

G. dicksonii (Dus.) Dus.

Grimmia flexicaulis var. dicksonii

G. didyma Mont.

Racomitrium didymum

G. donatii Herz. et Thér. (Herzog and Thériot in Herzog, 1937).

Distr. Southern South America (Herzog, 1937).

G. doniana Sm. ex Spruc.

Grimmia donniana

G. donii Sm. ex Lindb.

Grimmia donniana

G. donniana Sm. (Smith, 1804a).

Syn. Weisia donniana (Sm.) Poir. (Poiret in Bridel, 1826) nom. inval.

Dryptodon donnianus (Sm.) Hartm. (Hartman, 1838).

Grimmia obtusa Schwaegr. var. donniana (Sw.) Hartm. (Hartman, 1849) nom. illeg.

Grimmia donnii Gray (Gray, 1821) nom. illeg.

Grimmia doniana Sm. ex Spruc. (Smith ex Spruce, 1847) nom. illeg.

Grimmia donii Sm. ex Lindb. (Smith ex Lindberg, 1879) nom. illeg.

Distr. Antarctic, peninsula region (Cardot, 1901, 1908a, 1911b, d, 1913a; Paris, 1904b;

Brotherus, 1924a; Bartram, 1957, Steere, 1961a; Robinson, 1972).

Antarctic, continental region (Kuc, 1969; Savich-Ljubitskaya and Smimova, 1969;

Savich-Ljubitskaya, 1978).

G. donii Gray

Grimmia donniana

G. fallax Dus. (Dusén, 1903a).

Distr. Southern South America (Dusén, 1903a, 1907; Cardot, 1908a; Brotherus, 1924a;

Thériot, 1924; Kühnemann, 1938).

G. fasciculata Dus. (Dusén, 1905b) hom. illeg.

Distr. Southern South America (Paris, 1904b; Dusén, 1905b, 1907; Cardot, 1908a; Brotherus, 1924a; Kühnemann, 1938).

G. fastigiata Card. (Cardot, 1905a).

Distr. Southern South America (Cardot, 1905a, 1908a; Cardot and Brotherus, 1923;

Brotherus, 1924a; Kühnemann, 1938).

Antarctic, continental region (Dixon and Watts, 1918; Steere, 1961a). 
G. flexicaulis C. Muell. (Mueller, 1882).

Distr. Southern South America (Mueller, 1882; Paris, 1904b; Brotherus, 1924a; Kühnemann, 1938).

var. dicksonii Dus. (Dusén, 1905b).

Syn. Grimmia dicksonii (Dus.) Dus. (Dusén, 1907) hom. illeg.

Grimmia pycnophylla Dus. (Dusén, 1903a) nom. nud.

Grimmia pachyphylla Dus. (Dusén, 1905b) hom. illeg.

Distr. Southem South America (Dusén, 1903a, 1905b, 1907; Paris, 1904b; Cardot, 1908a; Brotherus, 1924a; Kühnemann, 1938; Seki, 1974).

G. flexiseta Dus.

Grimmia reflexidens

G. gibertii Mitt. (Mitten, 1869).

Distr. Southern South America (Mitten, 1869; Gibert, 1873; Jaeger, 1874; Krieger, 1904; Paris, 1904b; Felippone, 1909; Brotherus, 1924a; Herter, 1928, 1933a, 1939a, b, 1943).

G. glacialis C. Muell.

Racomitrium lanuginosum

G. grisea Card. (Cardot, 1906a).

Distr. Southern South America (Cardot and Brotherus, 1923; Thériot, 1934b; Kühnemann, 1938).

South Georgia (Cardot, 1906a, 1908a; Cardot and Brotherus, 1923; Dixon, 1932).

G. gusindei Froehl. (Froehlich, 1953).

Distr. Southern South America (Froehlich, 1953).

G. heteromalla (Hedw.) Web. et Mohr

Ditrichum heteromallum

G. heterosticha (Hedw.) C. Muell.

G. homomalla (Hedw.) Sm.

Racomitrium heterostichum

Ditrichum heteromallum

G. humilis Mitt. (Mitten, 1869).

Syn. Grimmia macropulvinata Dus. (Dusén, 1903a) fide Cardot, $1908 a$.

Distr. Southem South America (Mitten, 1869; Jaeger, 1874; Mueller, 1885; Dusén, 1903a, 1907; Paris, 1904b; Cardot, 1908a; Cardot and Brotherus, 1923; Brotherus, 1924a; Espinosa B., 1924; Kühnemann, 1938; Bartram, 1952; Herzog, 1957; Seki, 1974).

G. hyalino-cuspidata C. Muell. (Mueller, 1890a).

Syn. Racomitrium hyalino-cuspidatum (C. Muell.) Kindb. (Kindberg, 1891).

Distr. Southern South America (Cardot and Brotherus, 1923; Brotherus, 1924a; Kühnemann, 1938).

South Georgia (Mueller, 1890a; Paris, 1905b; Cardot, 1908a; Brotherus, 1924a; Steere, 1961b).

var. mutica Card. et Broth. (Cardot and Brotherus, 1923).

Distr. South Georgia (Cardot and Brotherus, 1923; Steere, 1961 b).

G. imberbis Kunz. ex C. Muell. (Kunze ex Mueller, 1849).

Distr. Southern South America (Mueller, 1849; Montagne, 1850; Mitten, 1869; Jaeger, 1874;

Paris, 1904b; Dusén, 1907; Brotherus, 1924a; Herzog, 1938, 1957, 1960).

G. immerso-leucophaea (C. Muell.) Kindb. (Kindberg, 1891).

Syn. Guembelia immerso-leucophaea C. Muell. (Mueller, 1890a).

Distr. South Georgia (Mueller, 1890a; Paris, 1904b; Cardot, 1908a; Brotherus, 1924a; Steere, 1961b).

G. integridens C. Muell.

Grimmia subovata

G. julacea (Hedw.) Web. et Mohr

Leucodon julaceus

G. julacea Hornsch.

Acrocryphaea julacea

G. laevigata Mitt.

Racomitrium laevigatum 
G. lamprocarpa C. Muell.

G. lanuginosa (Hedw.) C. Muell.

G. lawiana J. H. Willis (Willis in Filson, 1966).

Distr. Antarctic, continental region (Filson, 1966; Greene, 1968a).

G. leucophaeola C. Muell. (Mueller, 1882).

Distr. Southern South America (Mueller, 1882; Paris, 1904b; Brotherus, 1924a; Kühnemann, 1938).

G. Iorentzii C. Muell. ex Kindb.

Grimmia lorentziana

G. lorentziana (C. Muell.) Par. (Paris, 1895).

Syn. Guembelia lorentziana C. Muell. (Mueller, 1882).

Grimmia lorentzii C. Muell. ex Kindb. (Mueller ex Kindberg, 1889) nom. illeg.

Distr. Southern South America (Mueller, 1882; Paris, 1895, 1904b; Brotherus, 1924a; Hosseus, 1936; Kühnemann, 1938).

G. macropulvinata Dus.

Grimmia humilis

G. macrothylla Card. et Broth.

Grimmia macrotyla

G. macrotyla Card. et Broth. (Cardot and Brotherus, 1923).

Syn. Grimmia macrothylla Card. et Broth. in Broth. (Cardot and Brotherus in Brotherus, 1924a) err.

Distr. Southern South America (Cardot and Brotherus, 1923; Brotherus, 1924a; Kühnemann, 1938).

G. maritima Turn.

Schistidium maritimum

G. montevidensis Thér. (Thériot in Felippone, 1929 [1930]).

Distr. Southern South America (Felippone, 1929; Herter, 1933a).

G. murina C. Muell.

Grimmia reflexidens

G. nigrita C. Muell.

Racomitrium crispulum

G. nivea Dus. (Dusén, 1905b).

Distr. Southern South America (Dusén, 1905b, 1907; Cardot, 1908a; Brotherus, 1924a; Kühnemann, 1938; Seki, 1974).

G. nordenskioldii Card. (Cardot, 1906a).

Distr. Southern South America (Cardot and Brotherus, 1923).

South Georgia (Cardot, 1906a, 1908a; Cardot and Brotherus, 1923; Brotherus, 1924a; Steere, 1961b).

G. obtusa Schwaegr. var. donniana (Sm.) Hartm.

Grimmia donniana

G. occulta C. Muell. (Mueller, 1890a).

Distr. Southern South America (Dusén, 1907; Cardot, 1908a; Cardot and Brotherus, 1923; Kühnemann, 1938).

South Georgia (Mueller, 1890a; Paris, 1904b; Cardot, 1906a, 1908a; Cardot and Brotherus, 1923; Brotherus, 1924a; Dixon, 1935; Steere, 1961b).

G. orbicularis Bruch (Bruch in Wilson, 1844).

Syn. Guembelia orbicularis (Bruch) Hamp. (Hampe in Mueller, 1849).

Distr. Southern South America (Cardot and Brotherus, 1923; Brotherus, 1924a; Kühnemann, 1938 (as G. onvicularis); Seki, 1974).

var. patagonica Card. (Cardot, 1908a).

Syn. Grimmia arachnoidea Dus. (Dusén, 1903a) fide Cardot, 1908a, p. 284.

Distr. Southern South America (Dusén, 1903a, 1905b (as G. orbicularis fide Cardot, 1908a), 1907 (as G. orbicularis fide Cardot, 1908a); Cardot, 1908a (and as G. orbicularis)). 
G. pachyloma (Mont.) Mitt.

Sciaromium pachyloma

G. pachyphylla C. Muell.

Andreaea pachyphylla

G. pachyphylla Dus.

Grimmia flexicaulis var. dicksonii

G. patagonica Mitt.

Scouleria patagonica

G. perplexa Thér. (Thériot, 1923a).

Distr. Southern South America (Thériot, 1923a, 1934b; Brotherus, 1925).

G. phyllorhizans Broth. (Brotherus, 1924b).

Distr. Juan Fernandez (Brotherus, 1924b, 1925; Espinosa B., 1941; Robinson, 1975).

G. plagiopodia Hedw. (Hedwig, 1801).

Syn. Weisia plagiopodi (Hedw.) Poir. (Poiret, 1808).

Schistidium plagiopodium (Hedw.) Loesk. (Loeske, 1913).

Distr. Antarctic, peninsula region (Bartram, 1957; Steere, 1961a).

Antarctic, continental region (Bartram, 1957; Steere, 1961a).

var. antarctica Bartr. (Bartram, 1957).

Distr. Antarctic, continental region (Bartram, 1957; Steere, 1961a; Savich-Ljubitskaya and Smimova, 1970; Savich-Ljubitskaya, 1978).

G. praemorsa (C. Muell.) Kindb. (Kindberg, 1889).

Syn. Guembelia praemorsa C. Muell. (Mueller, 1882).

Schistidium praemorsum (C. Muell.) Herz. (Herzog, 1916a).

Distr. Southern South America (Mueller, 1882; Paris, 1904b; Brotherus, 1924a; Hosseus, $1938 c$, d; Kühnemann, 1938).

G. protensum (Braun) Mitt.

Racomitrium aquaticum

G. ptychophylla Mitt.

Racomitrium ptychophyllum

G. pulvinata (Hedw.) Sm. (Smith, 1807).

Syn. Fissidens pulvinatus Timm ex Hedw. (Hedwig, 1801).

Distr. Southern South America (Montagne, 1850).

G. pycnophylla Dus.

Grimmia flexicaulis var. dicksonii

G. quadricruris C. Muell. (Mueller, 1882).

Distr. Southern South America (Mueller, 1882; Paris, 1904b; Brotherus, 1924a; Kühnemann, 1938 (as quatricruris C. Muell.); Bartram, 1965).

G. raphidostega C. Muell. ex Kindb.

Grimmia rhaphidostega

G. reflexidens C. Muell. (Mueller, 1849).

Syn. Grimmia murina C. Muell. (Mueller, 1897a).

Grimmia flexiseta Dus. (Dusén, 1903a) nom. nud.

Distr. Southem South America (Mueller, 1849, 1897a; Montagne, 1850; Mitten, 1869; Jaeger, 1874; Paris, 1900a, 1904b; Dusén, 1903a, 1905b, 1907; Brotherus, 1924a; Kühnemann, 1938).

G. rhaphidostega C. Muell. (Mueller, 1882).

Syn. Grimmia raphidostega C. Muell. ex Kindb. (Mueller ex Kindberg, 1889) err.

Distr. Southern South America (Mueller, 1882; Paris, 1904b; Brotherus, 1924a; Kühnemann, 1938).

G. richardii (Brid.) Spreng.

Campylopus richardii

G. rivularis Brid.

Schistidium apocarpum

G. rupestris (Hook. f. et Wils.) C. Muell.

Racomitrium crispulum var. rupestre 
G. scabripes Bartr. (Bartram, 1946).

Distr. Southern South America (Roivainen, 1934; Bartram, 1946).

G. senilis Besch.

Willia austro-leucophaea

G. serripila Dus. (Dusén in Paris, 1904b) nom. nud.

Distr. Southern South America (Paris, 1904b; Thériot, 1923b).

G. stolonifera C. Muell. (Mueller, 1884 [1883]).

Distr. Antarctic, continental region (Dixon and Watts, 1918; Steere, 1961a).

G. striata Schrad.

Rhabdoweisia fugax

G. subcrispipila C. Muell.

Racomitrium subcrispipilum

G. sublamprocarpa C. Muell.

Racomitrium sublamprocarpum

G. subnigrita C. Muell.

Racomitrium subnigritum

G. subovata Schimp. (Schimper in Mueller, 1882).

Syn. Grimmia integridens C. Muell. (Mueller, 1882).

Distr. Southem South America (Mueller, 1882; Kurtz, 1904; Paris, 1904b; Brotherus, 1924a; Hosseus, 1936, 1937; Kühnemann, 1938).

G. symphyodon Mitt.

Racomitrium symphyodon

G. symphyodonta C. Muell.

Racomitrium symphyodontum

G. syntrichiacea C. Muell. (Mueller, 1890a).

Distr. Southern South America (Cardot and Brotherus, 1923; Kühnemann, 1938).

South Georgia (Mueller, 1890a; Paris, 1904b; Cardot, 1908a; Cardot and Brotherus, 1923; Brotherus, 1924a; Steere, 1961b).

G. tenuicaulis Hamp. (Hampe in Jaeger, 1874) nom. nud.

Distr. Southern South America (Jaeger, 1874).

G. tortuosa Hook. f. et Wils. (Hooker, J. D. and Wilson, 1844).

Distr. Falkland Islands (Hooker, J. D. and Wilson, 1844; Wilson and Hooker, 1847; Mueller, 1849; Jaeger, 1874; Paris, 1904b; Cardot, 1908a; Brotherus, 1924a; Kühnemann, 1938).

G. trichophylla Grev. (Greville, 1824).

Syn. Dryptodon trichophyllus (Grev.) Brid. (Bridel, 1827a).

Eugrimmia trichophylla (Grev.) Hamp. (Hampe, 1880c) comb. inval.

Distr. Southern South America (Montagne, 1850).

G. unicruris C. Muell. (Mueller, 1882).

Distr. Southern South America (Mueller, 1882; Paris, 1904b; Brotherus, 1924a; Kühnemann, 1938; Herzog, 1957).

G. urnulacea C. Muell. (Mueller, 1890a).

Distr. South Georgia (Mueller, 1890a; Paris, 1904b; Cardot, 1906a, 1908a; Brotherus, 1924a; Steere, 1961b).

G. vernicosula C. Muell. (Mueller, 1882).

Distr. Southern South America (Mueller, 1882; Paris, 1904b; Brotherus, 1924a; Kühnemann, 1938).

G. willii C. Muell.

Racomitrium willii

\section{Groutiella}

G. apiculata (Hook.) Crum et Steere (Crum and Steere, 1950).

Syn. Orthotrichum apiculatum Hook. (Hooker, 1818).

Macromitrium apiculatum (Hook.) Schwaegr. (Schwaegrichen, 1826). 
Distr. Southern South America (Krieger, 1904).

G. tomentosa (Hornsch.) Wijk et Marg. (van der Wijk and Margadant, 1960a).

Syn. Macromitrium tomentosum Hornsch. (Hornschuch, 1840). Micromitrium tomentosum (Hornsch.) Par. (Paris, 1905a).

Distr. Southem South America (Hornschuch, 1840; Mueller, 1849; Gibert, 1873; Jaeger, 1874; Paris, 1905a; Brotherus, 1925; Herter, 1933a).

\section{Guembelia}

G. austro-leucophaea C. Muell.

Willia austro-leucophaea

G. immerso-leucophaea C. Muell.

G. Iorentziana C. Muell.

Grimmia immerso-leucophaea

G. orbicularis (Bruch) Hamp.

Grimmia lorentziana

G. praemorsa C. Muell.

Grimmia orbicularis

Grimmia praemorsa

Gymnocybe nom. rejic.

G. palustris (Hedw.) Fries

Aulacomnium palustre

Gymnopilum nom. nud.

G. dendroides (Hedw.) Schimp.

Dendroligotrichum dendroides

G. magellanicum (Hedw.) Schimp.

Polytrichadelphus magellanicus

\section{Gymnostomum}

G. aeruginosum Sm. (Smith, 1804b).

Syn. Gymnostomum calcareum Nees et Hornsch. (Nees and Hornschuch in Nees von Esenbeck and others, 1823) fide Zander, 1977.

Weisia calcarea (Nees et Hornsch.) C. Muell. (Mueller, 1849) hom. illeg. fide Zander, 1977.

Hymenostylium calcareum (Nees et Hornsch.) Mitt. (Mitten, 1859) fide Zander, 1977.

Trichostomum calcareum (Nees et Hornsch.) Lindb. (Lindberg, 1864a) fide Zander, 1977.

Eucladium calcareum (Nees et Hornsch.) C. Jens. (Jensen, 1923).

Gymnostomum calcareum Nees et Hornsch. var. tenellum B.S.G. (Bruch and others, 1846).

Distr. Juan Fernandez (Brotherus, 1924b; Espinosa B., 1941; Robinson, 1975).

Southern South America (Montagne, 1850; Mitten, 1869; Paris, 1904b; Dusén, 1906; Thériot, 1921a; Hosseus, 1938c, d; Herzog, 1938, 1940, 1954; Bizot and Piovano, 1953; Zander, 1977).

G. aestivum Hedw. (Hedwig, 1801).

Syn. Gymnostomum euchloron Schwaegr. (Schwaegrichen, 1827a) fide Zander, 1977. Anoectangium euchloron (Schwaegr.) Mitt. (Mitten, 1869) fide Zander, 1977.

Distr. Southern South America (Thériot, 1936; Hosseus, 1938c, d, 1939; Bartram, 1965; Zander, 1977).

G. amblyophyllum Hook.

Didymodon amblyophyllus

G. antarcticum Aongstr.

Pottia antarctica

G. bonplandii Hook.

Funaria bonplandii

G. calcareum Nees et Hornsch.

var. tenellum B.S.G.

G. curvirostre Hedw. ex Brid.

G. euchloron Schwaegr.

G. griffithianum (Dicks.) Sm.

G. heimii Hedw.

G. imberbe Sm.

Gymnostomum aeruginosum

Gymnostomum aeruginosum

Hymenostylium recurvirostre

Gymnostomum aestivum

Oedipodium griffithianum

Pottia heimii

Hedwigidium integrifolium 
G. kunzeanum (C. Muell.) Par.

Hymenostylium kunzeanum

G. laxifolium Kunz. ex Par. Gymnostomum tenerrimum

G. longirostre Kunz. ex C. Muell.

Hymenostylium kunzeanum

G. magellanicum Aongstr. ex Par.

Pottia antarctica

G. marginatum Kunz. ex Mont.

Hymenostomum kunzeanum

G. menziesii (R. Brown) Hook.

Leptostomum menziesii

G. orbignyanum Mont.

G. ovatum Hedw.

Physcomitrium orbignyanum

G. pachyloma Mont.

Pterygoneurum ovatum

G. pyriforme Hedw.

Sciaromium pachyloma

G. recurvirostre Hedw.

Physcomitrium pyriforme

G. subsessile Brid.

Hymenostylium recurvirostre

Pterygoneurum subsessile

G. tenerrimum (C. Muell.) Wijk et Marg. (van der Wijk and Margadant, 1959).

Syn. Anoectangium tenerrimum C. Muell. (Mueller, 1847c).

Zygodon tenerrimus (C. Muell.) C. Muell. (Mueller, 1849).

Anoectangium laxifolium Par. (Paris, 1894) hom. illeg.

Gymnostomum laxifolium Kunz. ex Par. (Kunze ex Paris, 1904b) nom. illeg.

Distr. Southern South America (Mueller, 1847c, 1849; Lorentz, 1866; Mitten, 1869; Jaeger, 1870; Paris, 1894, 1904b; Brotherus, 1924a).

G. tortula Schwaegr.

Hyophila tortula

G. truncatum Hedw.

Pottia truncata

\section{Gyroweisia}

G. detersifolia (Hamp.) Broth. (In Piovano, 1954) nom. nud.

Distr. Southern South America (Piovano, 1954).

\section{Haplocladium}

H. amblyostomum (C. Muell.) Broth.

Haplocladium microphyllum

H. austro-serpens (C. Muell.) Broth.

Haplocladium microphyllum

H. laplatae (C. Muell.) Broth.

Haplocladium microphyllum

H. laterculi (C. Muell.) Broth.

Haplocladium microphyllum

H. malachiticum C. Muell. (Mueller in Reimers, 1937) nom. nud.

Distr. Southern South America (Reimers, 1937).

H. microphyllum (Hedw.) Broth. (Brotherus, 1907).

Syn. Hypnum microphyllum Hedw. (Hedwig, 1801).

Leskea microphylla (Hedw.) Sw. (Swartz in Mitten, 1859).

Thuidium microphyllum (Hedw.) Jaeg. (Jaeger, 1878).

Pseudoleskea microphylla (Hedw.) Sauerb. (Sauerbeck in Jaeger and Sauerbeck. $1879 b)$.

Hypnum austro-serpens C. Muell. (Mueller, 1879a).

Amblystegium austro-serpens (C. Muell.) Kindb. (Kindberg, 1891).

Thuidium austro-serpens (C. Muell.) Kindb. (Kindberg, 1891).

Haplocladium austro-serpens (C. Muell.) Broth. (Brotherus, 1907).

Hypnum laterculi C. Muell. (Mueller, 1879a).

Thuidium laterculi (C. Muell.) Kindb. (Kindberg, 1891).

Thuidium laterale Par. (Paris, 1898) err.

Haplocladium laterculi (C. Muell.) Broth. (Brotherus, 1907).

Hypnum attenuatifolium C. Muell. (Mueller, 1882) nom. nud.

Hypnum paludicola C. Muell. (Mueller, 1882) nom. nud.

Hypnum amblyostomum C. Muell. (Mueller, 1882).

Thuidium amblyostomum (C. Muell.) Kindb. (Kindberg, 1891).

Haplocladium amblyostomum (C. Muell.) Broth. (Brotherus, 1907). 
Hypnum amblyostomum C. Muell. var. attenuatifolium C. Muell. (Mueller, 1882).

Thuidium amblyostomum (C. Muell.) Kindb. var. attenuatifolium (C. Muell.) Par. (Paris, 1898).

Hypnum amblyostomum C. Muell. var. paludicola C. Muell. (Mueller, 1882).

Thuidium amblyostomum (C. Muell.) Kindb. var. paludicola (C. Muell.) Par. (Paris, 1898).

Haplocladium pseudo-gracile C. Muell. (Mueller, 1897a).

Pseudoleskea pseudogracilis (C. Muell.) Par. (Paris, 1896).

Thuidium pseudo-gracile (C. Muell.) Par. (Paris, 1906) hom. illeg.

Haplodontium pseudo-gracile C. Muell. ex Par. (Mueller ex Paris, 1906) err.

Pseudoleskea uruguensis C. Muell. (Mueller, 1897a).

Haplocladium uruguense (C. Muell.) Broth. (Brotherus, 1907).

Thuidium semi-lunare C. Muell. (Mueller, 1897a).

Haplocladium semilunare (C. Muell.) Broth. (Brotherus, 1907).

Hypnum semilunare C. Mue!l. (Mueller in Reimers, 1937) nom. nud.

Thuidium torskii Kiaer ex C. Muell. (Kiaer ex Mueller, 1897a).

Haplocladium torskii (C. Muell.) Broth. (Brotherus, 1907).

Pseudoleskea laplatae C. Muell. (Mueller, 1897a).

Haplocladium laplatae (C. Muell.) Broth. (Brotherus, 1907).

Distr. Southem South America (Montagne, 1839c; Mueller, 1879a, 1882, 1897a; Paris. 1896. 1898, 1905b, 1906; Brotherus, 1907, 1918, 1925; Herter, 1933a, 1943; Kühnemann, 1938; Hosseus, 1939, 1940; Herzog, 1952a).

H. persistens (C. Muell.) Broth. (Brotherus, 1907).

Syn. Hypnum persistens C. Muell. (Mueller, 1882).

Thuidium persistens (C. Muell.) Kindb. (Kindberg, 1891).

Distr. Southern South America (Mueller, 1882; Paris, 1906; Brotherus, 1907, 1925; Kühnemann, 1938).

H. pseudo-gracile C. Muell.

H. semilunare (C. Muell.) Broth.

H. torskii (C. Muell.) Broth.

$H$. uruguense (C. Muell.) Broth.

Haplocladium microphyllum

Haplocladium microphyllum

Haplocladium microphyllum

Haplocladium microphyllum

\section{Haplodontium}

H. brachycladum Broth. (Brotherus in Felippone, 1909).

Syn. Haplodontium brachycladulum Broth. (Brotherus, 1924a) err.

Distr. Southern South America (Felippone, 1909; Brotherus, 1924a; Herter, 1933a).

H. brachycladulum Broth.

Haplodontium brachycladum

H. humipetens C. Muell. (Mueller, 1879a).

Syn. Mielichhoferia humipetens (C. Muell.) Par. (Paris, 1896).

Distr. Southern South America (Mueller, 1879a; Paris, 1896, 1904b; Brotherus, 1924a; Kühnemann, 1938).

H. pernanum C. Muell.

Haplodontium sanguinolentum

var. robustior C. Muell. (Mueller, 1879a).

Syn. Mielichhoferia pernana (C. Muell.) Par. var. robustior (C. Muell.) Par. (Paris, 1896).

Distr. Southern South America (Mueller, 1879a; Paris, 1896, 1904b; Kühnemann, 1938).

H. pseudo-gracile C. Muell. ex Par.

Haplocladium microphyllum

H. sanguinolentum C. Muell. (Mueller, 1879a).

Syn. Mielichhoferia sanguinolenta (C. Muell.) Par. (Paris, 1896).

Haplodontium pernanum C. Muell. (Mueller, 1879a).

Mielichhoferia pernana (C. Muell.) Par. (Paris, 1896).

Distr. Southern South America (Mueller, 1879a; Paris, 1896, 1904b; Brotherus, 1924a; Hosseus, 1936, 1937; Kühnemann, 1938; Bartram, 1965). 
Haptymenium nom. illeg.

H. curtipendulum (Hedw.) Fuernr.

Antitrichia curtipendula

Harpidium hom. illeg.

$\mathrm{H}$. aduncum (Hedw.) Con.

Drepanocladus aduncus

H. fluitans (Hedw.) Spruc.

Drepanocladus fluitans

H. polycarpum (Voit) Williams

$H$. revolvens (Sw.) C. Jens.

Drepanocladus aduncus var. polycarpus

H. uncinatum (Hedw.) C. Jens.

Drepanocladus revolvens

var. orthothecioides (Lindb.) C. Jens.

Drepanocladus uncinatus

Drepanocladus uncinatus var. subjulaceus

Harrisonia hom. illeg.

H. australis (Hook. f. et Wils.) Hamp.

Rhacocarpus purpurascens

$H$. crasso-limbata C. Muell.

Rhacocarpus purpurascens

H. humboldtii (Hook.) Spreng.

Rhacocarpus purpurascens

var. argentinica C. Muell.

Rhacocarpus purpurascens var. argentinicus

H. orbiculata (Mitt.) Jaeg.

Rhacocarpus orbiculatus

H. purpurascens (Brid.) C. Muell.

Rhacocarpus purpurascens

H. rhabdocarpa Hamp.

Hedwigidium integrifolium

H. secunda (Hook.) Spreng. var. cirrhifolia Mitt.

Braunia cirrhifolia

\section{Hedwigia}

$H$. albicans Lindb.

Hedwigia ciliata

$H$. argentinica C. Muell.

Hedwigia ciliata

H. ciliata (Hedw.) P. Beauv. (Palisot de Beauvois, 1805a).

Syn. Anictangium ciliatum Hedw. (Hedwig, 1801).

Bryum ciliatum (Hedw.) Dicks. (Dickson, 1801).

Anoectangium ciliatum (Hedw.) Roehl. (Röhling, 1809).

Perisiphorus ciliatus (Hedw.) P. Beauv. (Palisot de Beauvois, 1822).

Hedwigidium ciliatum (Hedw.) Hartm. (Hartman, 1849).

Pilotrichum ciliatum (Hedw.) C. Muell. (Mueller, 1851a).

Bryum apocarpum (Hedw.) L. ex With. var. ciliatum (Hedw.) With. (Withering, 1801).

Bryum hedwigii Brotero (Brotero, 1804).

Hedwigia albicans Lindb. (Lindberg, 1864b) nom. illeg.

Hedwigia argentinica C. Muell. (Mueller in Fleischer, 1920) nom. nud.

Distr. Southern South America (Jaeger, 1876; Fleischer, 1920).

H. cirrhifolia Mitt.

Braunia cirrhifolia

H. humboldtii (Hook.) Hook.

var. australis (Hook. f. et Wils.) Wils.

Rhacocarpus purpurascens

H. imberbis (Sm.) Spruc.

Rhacocarpus purpurascens

H. integrifolia P. Beauv.

Hedwigidium integrifolium

H. orbiculata Mitt.

Hedwigidium integrifolium

Rhacocarpus orbiculatus

H. patagonica C. Muell. (Mueller in Kindberg, 1891) nom. nud.

Distr. No locality given, see Index muscorum, 5, p. 674.

\section{Hedwigidium}

H. chilense Fleisch. (Fleischer, 1920) nom. nud.

Syn. Braunia chilensis Broth. (Brotherus in Fleischer, 1920) nom. nud.

Distr. Southern South America (Fleischer, 1920).

H. ciliatum (Hedw.) Hartm.

Hedwigia ciliata 
H. gibertii Fleisch. (Fleischer, 1920) nom. nud.

Syn. Braunia gibertii Besch. (Bescherelle in Fleischer, 1920) nom. nud.

Distr. Southem South America (Fleischer, 1920).

H. imberbe (Sm.) B.S.G.

Hedwigidium integrifoliuma

H. integrifolium (P. Beauv.) Dix. (Dixon in Jensen, 1939).

Syn. Hedwigia integrifolia P. Beauv. (Palisot de Beauvois, 1805a).

Anoectangium integrifolium (P. Beauv.) Schwaegr. (Schwaegrichen, 1811).

Gymnostomum imberbe Sm. (Smith, 1811).

Anictangium imberbe (Sm.) Hook. et Tayl. (Hooker and Taylor, 1818).

Schistidium imberbe ( $\mathrm{Sm}$.) Nees et Hornsch. (Nees von Esenbeck and others, 1823).

Hedwigidium imberbe (Sm.) B.S.G. (Bruch and others, 1846).

Hedwigia imberbis (Sm.) Spruc. (Spruce, 1847).

Neckera imberbis (Sm.) C. Muell. (Mueller, 1850).

Schistidium ciliatum (Hedw.) Brid. var. imberbe (Sm.) Hueb. (Hübener, 1833).

Harrisonia rhabdocarpa Hamp. (Hampe, 1863).

Braunia rhabdocarpa (Hamp.) C. Muell. (Mueller, 1879a).

Hedwigidium rhabdocarpum (Hamp.) Jaeg. (Jaeger in Jaeger and Sauerbeck, 1879b).

Distr. Southern South America (Mueller, 1879a; Paris, 1904b; Brotherus, 1905a, 1925;

Reimers, 1926; Herzog, 1938; Kühnemann, 1938; Bartram, 1952).

H. patagonicum Fleisch. (Fleischer, 1920) nom. nud.

Syn. Braunia patagonica C. Muell. (Mueller in Fleischer, 1920) nom. nud.

Distr. Southem South America (Fleischer, 1920).

H. rhabdocarpum (Hamp.) Jaeg.

Hedwigidium integrifolium

\section{Helicodontium}

H. acuminatum C. Muell. (Mueller; 1897a).

Distr. Southem South America (Mueller, 1897a; Paris, 1900a, 1904b: Brotherus, 1925; Kühnemann, 1938).

H. chloronema C. Muell. (Mueller, 1897a).

Syn. Helicodontium montevidense C. Muell. (Mueller in Fleischer, 1920) nom. nud.

Distr. Southern South America (Mueller, 1897a; Paris, 1900a, 1904b; Fleischer, 1920; Brotherus, 1925; Hosseus, 1934, 1939; Kühnemann, 1938).

H. clarazii (Dub.) Par. (Paris, 1895).

Syn. Hypnum clarazii Dub. (Duby, 1868).

Rudia clarazii (Dub.) Jaeg. (Jaeger, 1878) comb. inval.

Amblystegium clarazii (Dub.) Jaeg. (Jaeger in Jaeger and Sauerbeck, 1879a) nom. inval.

Distr. Southern South America (Duby, 1868; Jaeger, 1878; Paris, 1895, 1904b).

H. complanatum Broth. (Brotherus, 1895).

Distr. Southern South America (Herzog, 1952a).

H. montevidense C. Muell.

Helicodontium chloronema

H. obtusatulum (C. Muell.) Fleisch.

Fabronia obtusatula

H. pendulum (Brid.) Lindb. (Lindberg, 1883).

Syn. Isothecium pendulum Brid. (Bridel, 1827b).

Hypnum pendulum (Brid.) C. Muell. (Mueller, 1851a) hom. illeg.

Eurhynchium pendulum (Brid.) Jaeg. (Jaeger, 1878).

Rhynchostegium pendulum (Brid.) Jaeg. (Jaeger, 1878).

Distr. Southern South America (Bridel, 1827b; Mueller, 1851a; Mitten, 1869; Gibert, 1873; Jaeger, 1878; Lindberg, 1883; Paris, 1904b; Herter, 1928, 1933b). 
H. pervirens (C. Muell.) Broth. (Brotherus, 1895).

Syn. Hypnum pervirens C. Muell. (Mueller, 1879a).

Distr. Southern South America (Mueller, 1879a; Paris, 1904b; Brothenus, 1925; Herter, 1933a, 1943; Hosseus, 1938; Kühnemann, 1938; Herzog, 1952a).

H. pseudo-limnobium (C. Muell.) Kindb. (Kindberg, 1891).

Syn. Hypnum pseudo-limnobium C. Muell. (Mueller, 1882).

Distr. Southern South America (Mueller, 1882; Paris, 1904b; Brotherus, 1925; Kühnemann, 1938; Herzog, 1952a).

H. rhyparobolax C. Muell. (Mueller, 1897a).

Distr. Southern South America (Mueller, 1897a; Paris, 1900a, 1904b; Brotherus, 1925; Hosseus, 1934, 1939; Kühnemann, 1938).

H. seminerve (Schwaegr.) Schwaegr.

Juratzkaea seminervis

H. siambonense C. Muell. (Mueller, 1897a).

Syn. Helicondontium siambonicum C. Muell. (Mueller in Fleischer, 1920) nom. nud.

Distr. Southern South America (Mueller, 1897a; Paris, 1900a, 1904b; Fleischer, 1920; Brotherus, 1925; Kühnemann, 1938).

H. siambonicum C. Muell.

Helicodontium siambonense

H. tenuifolium (Hedw.) C. Muell.

Rhynchostegium tenuifolium

H. tenuirostre Schwaegr. (Schwaegrichen, 1830).

Syn. Hypnum tenuirostre (Schwaegr.) C. Muell. (Mueller, 1851a) hom. illeg.

Distr. Southern South America (Jaeger, 1878; Paris, 1904b; Brotherus, 1925).

Hepaticina nom. inval.

H. lamellata Fleisch.

Pterygophyllum lamellatum

H. peruncinata Dus.

Pterygophyllum peruncinatum

\section{Herpetineuron}

H. toccoae (Sull. et Lesq.) Card. (Cardot, 1905c).

Syn. Anomodon toccoae Sull. et Lesq. (Sullivant and Lesquereux, 1856).

Distr. Southern South America (Hosseus, 1940; Herzog, 1952a).

\section{Heterocladium}

H. austro-alpinum (C. Muell.) Broth.

Leptopterigynandrum austro-alpinum

H. implexum (Schwaegr.) Lor.

Rigodium implexum

H. lechleri Schimp.

Rigodium arborescens and $\boldsymbol{R}$. toxarion

H. prolixum Schimp.

Rigodium arborescens and $R$. toxarion

\section{Heterophyllum}

H. breviflagellosum C. Muell.

Acanthocladium breviflagellosum

\section{Holodontium}

H. inerme (Mitt.) Broth. (Brotherus, 1924a).

Syn. Dicranum inerme Mitt. (Mitten, 1869).

Dicranoweisia inermis (Mitt.) Par. (Paris, 1895).

Blindia auriculata C. Muell. (Mueller, 1885).

Dicranoweisia auriculata (C. Muell.) Broth. (Brotherus, 1901).

Distr. Southern South America (Mueller, 1885; Paris, 1895, 1904a; Brotherus, 1901, 1924a; Dusén, 1905a; Cardot, 1905a, 1908a, 1913a; Cardot and Brotherus, 1923; Roivainen, 1934; Roivainen and Bartram, 1937; Kühnemann, 1938; Herzog, 1939).

South Georgia (Bell, 1973b, p. 33). 
H. pumilum (Mitt.) Broth. (Brotherus, 1924a).

Syn. Symblepharis pumila Mitt. (Mitten in Hooker, 1867).

Dicranum pumilum (Mitt.) Mitt. (Mitten, 1869) hom. illeg.

Holomitrium pumilum (Mitt.) Kindb. (Kindberg, 1888).

Blindia humilis C. Muell. (Mueller, 1885).

Dicranoweisia humilis (C. Muell.) Broth. (Brotherus, 1901).

Blindia churuccana Besch. (Bescherelle, 1885a).

Distr. Southern South America (Mitten, 1869; Mueller, 1885; Bescherelle, 1885a, 1889; Cardot, 1900, 1901, 1905a, 1908a; Brotherus, 1901, 1924a; Paris, 1904a, b; Dusén, 1905a; Cardot and Brotherus, 1923; Roivainen and Bartram, 1937; Kühnemann, 1938; Herzog, 1954; Seki, 1974).

Falkland Islands (Cardot and Brotherus, 1923).

H. ramulosum (Mitt.) Broth. (Brotherus, 1924a).

Syn. Dicranum ramulosum Mitt. (Mitten, 1869).

Distr. Southern South America (Mitten, 1869; Mueller, 1885; Paris, 1904b; Cardot, 1908a; Brotherus, $1924 a$ (as $H$. ramosum)).

\section{Holodontium sp.}

Syn. Blindia savatieriana C. Muell. (Mueller in Bescherelle, 1889).

Blindia savatieri C. Muell. (Mueller, 1901a [1900]) nom. illeg.

Distr. Southem South America (Bescherelle, 1889; Mueller, 1901a; Paris, 1904a; Cardot, 1908a; Brotherus, 1924a).

\section{Holomitrium}

H. pumilum (Mitt.) Kindb.

Holodontium pumilum

\section{Homalia}

H. glabella (Hedw.) B.S.G. (Bruch and others, 1850).

Syn. Leskea glabella Hedw. (Hedwig, 1801).

Neckera glabella (Hedw.) Web. et Mohr (Weber and Mohr, 1803).

Neckera patagonica Brid. (Bridel, 1801b).

Pilotrichum patagonicum (Brid.) P. Beauv. (Palisot de Beauvois, 1805a).

Hypnum patagonicum Brid. (Bridel in Steudel, 1824) nom. nud.

Neckera dillenii Lindb. (Lindberg, 1883) nom. illeg.

Distr. Southern South America (Bridel, 1801b, 1827b; Schwaegrichen, 1816; Lindberg, 1883).

\section{Hookeria}

H. ancistrodes Mont.

Ancistrodes genuflexa

H. anomala (Schwaegr.) C. Muell.

Achrophyllum anomalum

H. apiculata Hook. f. et Wils.

Eriopus apiculatus

H. cirrhifolia Arnott

H. concinna (Hook.) Hook. et Grev.

Sematophyllum cirrhifolium

H. cristata (Hedw.) Hook. et Grev.

Lopidium concinnum

H. dentata Hook. f. et Wils.

Eriopus cristatus

H. denticulata Hook. f. et Wils.

Achrophyllum dentatum

H. dicksonii Hook. et Grev.

Achrophyllum dentatum

H. eriodon C. Muell. ex Card.

Distichophyllum dicksonii

$H$. erectiuscula Tayl.

Eriodon conostomus

H. flaccida Hook. f. et Wils.

Lepidopilum erectiusculum

H. flexicollis Mitt.

Distichophyllum flaccidum

Eriopus flexicollis

H. incurva (Hornsch.) Hook. et Grev.

Hookeriopsis incurva

H. Iorentzii C. Muell. (Mueller, 1882).

Distr. Southern South America (Mueller, 1882; Paris, 1904b; Brotherus, 1925; Kühnemann, 1938; Welch, 1969). 
H. magellanica (P. Beauv.) Arnott (Arnott, 1827).

Syn. Hypnum magellanicum P. Beauv. (Palisot de Beauvois, 1805a).

Distr. Southern South America (Palisot de Beauvois, 1805a; Bridel, 1817, 1827b; Wilson and Hooker, 1847; Mueller, 1850; Paris, 1904c; Kühnemann, 1938; Welch, 1969).

H. nigella Hook. f. et Wils.

Achrophyllum dentatum

H. obscura Mont.

H. patrisiae (Brid.) Hamp.

Pterygophyllum crassirete

H. polytrichoides (Hedw.) Spreng.

Crossomitrium patrisiae

H. rigida (Schwaegr.) Arnott

Lepidopilum polytrichoides

$H$. rotundifolia Hook. f. et Wils.

Lepidopilum polytrichoides

H. scabriuscula C. Muell.

Distichophyllum rotundifolium

H. sellovii Hornsch.

Callicostella scabriuscula

H. speciosissima Mont. ex Schwaegr.

Mittenothamnium sellovii

H. splendidissima Mont.

Schimperobryum splendidissimum

Schimperobryum splendidissimum

H. sublimbata C. Muell.

Cyclodictyon sublimbatum

$H$. tenella Hook. f. et Wils.

Sauloma tenella

H. uliginosa C. Muell. (Mueller, 1882).

Distr. Southern South America (Mueller, 1882; Paris, 1904b; Brotherus, 1925; Kühnemann, 1938; Welch, 1969).

\section{Hookeriopsis}

H. incurva (Hornsch.) Broth. (Brotherus, 1907).

Syn. Chaetephora incurva Hornsch. (Hornschuch, 1820a).

Hookeria incurva (Hornsch.) Hook. et Grev. (Hooker and Greville, 1825a).

Lepidopilum incurvum (Hornsch.) C. Muell. (Mueller, 1848b).

Callicostella incurva (Hornsch.) Aongstr. (Ångström, 1876a).

Distr. Southern South America (Hornschuch, 1820a, b; Hooker and Greville, 1825a; Bridel, $1827 b$; Schwaegrichen, 1829; Montagne, 1850; Mueller, 1851a; Jaeger, 1877; Paris, 1904b; Brotherus, 1907, 1925; Welch, 1969; Matteri. 1972).

\section{Husnotiella}

H. baueri Bartr. (Bartram, 1936).

Distr. Southern South America (Bartram, 1936; Hosseus, 1938c, d, 1939).

H. glossophylla Herz. (Herzog, 1916a).

Distr. Southern South America (Hosseus, 1936, 1937 (both as H. glaucophylla Herz.); Kühnemann, 1938).

\section{Hygroamblystegium}

H. antarcticum (Card.) Reim. (Reimers, 1926).

Syn. Pseudoleskea antarctica Card. (Cardot, 1900).

Distr. Southern South America (Reimers, 1926).

South Georgia (Cardot, 1906a, 1908a; Brotherus, 1925; Reimers, 1926; Steere, 1961b).

Antarctic, peninsula region (Cardot, 1900, 1901, 1908a; Paris, 1905b; Brotherus, 1925;

Steere, 1961a).

H. austro-fluviatile (C. Muell.) Broth. (Brotherus, 1908).

Syn. Hypnum austro-fluviatile C. Muell. (Mueller, 1890a).

Amblystegium austro-fluviatile (C. Muell.) Kindb. (Kindberg, 1891).

Distr. Southern South America (Thériot, 1924; Bartram, 1942, 1943).

South Georgia (Mueller, 1890a; Paris, 1903; Cardot, 1906a, 1908a; Brotherus, 1908 ,

1925: Reimers, 1926; Steere, 1961b). 
H. calochroum (Card.) Reim. (Reimers, 1926).

Syn. Pseudoleskea calochroa Card. (Cardot, 1906a).

Distr. Southern South America (Cardot and Brotherus, 1923; Brotherus, 1925; Kühnemann, 1938).

Falkland Islands (Cardot and Brotherus, 1923; Brotherus, 1925; Kühnemann, 1938).

South Georgia (Cardot, 1906a, 1908a; Brotherus, 1925; Reimers, 1926; Steere, 1961b).

Antarctic, peninsula region (Cardot, 1911b, $d, 1913 a$; Steere, 1961a).

H. chilense (Lor.) Reim. (Reimers, 1926).

Syn. Amblystegium chilense Lor. (Lorentz, 1866).

Orthotheciella austro-chilense C. Muell. (Mueller in Reimers, 1926) nom. nud.

Distr. Southern South America (Lorentz, 1866; Jaeger and Sauerbeck, 1879a; Paris, 1903;

Reimers, 1926; Herzog, 1939, 1940, 1954; Seki, 1974).

H. crassicostatum Bartr. (Bartram, 1942) hom. illeg.

Distr. Southern South America (Bartram, 1942, 1943).

H. excurrens (Card. et Broth.) Reim.

Hygroamblystegium fuegianum var. excurrens

H. filum (C. Muell.) Reim. (Reimers, 1926).

Syn. Hypnum filum C. Muell. (Mueller, 1884 [1883]).

Orthothecium filum (C. Muell.) Kindb. (Kindberg, 1891).

Pseudoleskea filum (C. Muell.) Par. (Paris, 1897).

Distr. Southern South America (Cardot, 1905a, 1908a; Thériot, 1918; Campo, 1921; Herzog, 1923, 1938, 1939; Brotherus, 1925; Reimers, 1926; Roivainen, 1934; Kühnemann, 1938; Bartram, 1946, 1952).

var. compactum Bartr. (Bartram, 1946).

Distr. Southem South America (Bartram, 1946).

H. fuegianum (Besch.) Reim. (Reimers, 1926).

Syn. Leskea fuegiana Besch. (Bescherelle, 1889).

Pseudoleskea fuegiana (Besch.) Broth. (Brotherus in Cardot, 1905a).

Distr. Southern South America (Bescherelle, 1889; Dusén, 1903a; Cardot, 1905a, 1908a; Paris 1905a; Thériot, 1915, 1917b; Campo, 1921; Costes, 1921; Cardot and Brotherus, 1923; Herzog, 1923, 1938, 1960; Reimers, 1926; Looser, 1932; Roivainen, 1934; Kühnemann, 1938; Bartram, 1946; Seki, 1974).

Falkland Islands (Cardot, 1905a, 1908a; Cardot and Brotherus, 1923; Kühnemann, 1938).

var. excurrens (Card. et Broth.) Bartr. (Bartram, 1946).

Syn. Amblystegium excurrens Card. et Broth. (Cardot and Brotherus, 1923). Hygroamblystegium excurrens (Card. et Broth.) Reim. (Reimers, 1926).

Distr. Southem South America (Cardot and Brotherus, 1923; Brotherus, 1925; Reimers, 1926; Bartram, 1946, 1952).

var. gracilis (Card. et Broth.) Bartr. (Bartram, 1946).

Syn. Pseudoleskea fuegiana (Besch.) Broth. var. gracilis Card. et Broth. (Cardot and Brotherus, 1923).

Distr. Southern South America (Cardot and Brotherus, 1923; Bartram, 1946).

var. secundum Bartr. (Bartram, 1946).

Distr. Southern South America (Bartram, 1946).

var. skottsbergii (Card.) Bartr. (Bartram in Roivainen, 1934).

Syn. Pseudoleskea fuegiana (Besch.) Broth. var. skottsbergii Card. (Cardot, 1905a).

Distr. Southern South America (Cardot, 1905a, 1908a; Cardot and Brotherus, 1923;

Roivainen, 1934; Kühnemann, 1938; Bartram, 1946).

Falkland Islands (Cardot and Brotherus, 1923). 
H. Iuridum (Card.) Reim. (Reimers, 1926).

Syn. Pseudoleskea lurida Card. (Cardot, 1905a).

Distr. Southern South America (Cardot, 1905a, 1908a; Cardot and Brotherus, 1923; Herzog, 1923; Brotherus, 1925; Reimers, 1926; Kühnemann, 1938).

H. platyphyllum (Card.) Reim. (Reimers, 1926).

Syn. Pseudoleskea platyphylla Card. (Cardot, 1906a).

Distr. South Georgia (Cardot, 1906a, 1908a; Brotherus, 1925; Reimers, 1926; Steere, 1961b).

H. sordidoviride (Card. et Broth.) Reim. (Reimers, 1926).

Syn. Pseudoleskea sordidoviridis Card. et Broth. (Cardot and Brotherus, 1923).

Distr. Falkland Islands (Cardot and Brotherus, 1923; Brotherus, 1925; Reimers, 1926; Kühnemann, 1938).

H. strictulum (Card.) Reim. (Reimers, 1926).

Syn. Pseudoleskea strictula Card. (Cardot, 1906a).

Distr. South Georgia (Cardot, 1906a, 1908a; Brotherus, 1925; Reimers, 1926; Steere, 1961b).

H. tenellum (Card. et Broth.) Reim. (Reimers, 1926).

Syn. Amblystegium tenellum Card. et Broth. (Cardot and Brotherus, 1923).

Distr. Southern South America (Cardot and Brotherus, 1923; Brotherus, 1925; Reimers, 1926; Kühnemann, 1938; Herzog, 1954).

H. varium (Hedw.) Moenk.

Amblystegium varium

\section{Hygrodicranum}

H. falklandicum Card. (Cardot, 1911a).

Distr. Falkland Islands (Cardot, 1911a; Cardot and Brotherus, 1923; Brotherus, 1924a; Kühnemann, 1938).

\section{Hygrohypnum}

H. aureum Herz. (Herzog, 1916a).

Distr. Southern South America (Bartram, 1965).

H. falcifolium Bartr. (Bartram, 1965).

Distr. Southern South America (Bartram, 1965).

H. peruviense Williams (Williams, 1916).

Distr. Southern South America (Bartram, 1942, 1943).

\section{Hylocomium}

H. lagurus (Hook.) Kindb.

Lepyrodon lagurus

H. schreberi (Brid.) De Not.

Pleurozium schreberi

H. splendidissimum De Not.

Ptychomnion cygnisetum

\section{Hymenocleiston}

H. magellanicum Dub.

Tayloria dubyi

var. edenense Besch.

Tayloria dubyi var. edenensis

\section{Hymenodon}

H. ovatus (Hook. f. et Wils.) C. Muell.

Leptotheca gaudichaudii

\section{Hymenoloma}

H. intermedium (Dix.) Broth.

Verrucidens intermedius

$H$. nordenskjoeldii Dus. 
H. subglobosum Herz. (Herzog, 1937).

Distr. Southern South America (Herzog, 1937).

H. turpe (Card.) Card. et Broth.

Verrucidens turpis

\section{Hymenostomum}

H. argentinicum (C. Muell.) Kindb. (Kindberg, 1891).

Syn. Weisia argentinica C. Muell. (Mueller, 1879a).

Distr. Southern South America (Mueller, 1879a; Kurtz, 1904; Paris, 1904b; Brotherus, 1924a; Hosseus, 1935b, 1938c, d; Kühnemann, 1938).

H. balansae Besch. ex Kindb.

Hymenostomum balansaeanum

H. balansaeanum Besch. (Bescherelle, 1877). Syn. Hymenostomum balansae Besch. ex Kindb. (Bescherelle ex Kindberg, 1888) nom.
illeg.

Weisia balansaeana (Besch.) C. Muell. (Mueller, 1882).

Distr. Southern South America (Mueller, 1882; Felippone, 1909; Brotherus, 1924a; Herter, 1933a, 1943).

var. densirete Thér. (Thériot, 1935b).

Distr. Southern South America (Thériot, 1935b; Hosseus, 1938a, d).

H. griffithianum (Dicks.) Spreng.

Oedipodium griffithianum

H. kunzeanum (C. Muell.) Broth. (Brotherus, 1902).

Syn. Weisia kunzeana C. Muell. (Mueller, 1849).

Gymnostomum marginatum Kunz. ex Mont. (Kunze ex Montagne, 1850) nom. illeg.

Hymenostomum marginatum Par. (Paris, 1904b) nom. illeg.

Distr. Juan Fernandez (Brotherus, 1924b; Robinson, 1975).

Southern South America (Mueller, 1849; Montagne, 1850; Mitten, 1869; Jaeger, 1870;

Brotherus, 1902, 1924a; Paris, 1904b; Thériot, 1935a; Herzog, 1939, 1954).

H. marginatum Par.

Hymenostomum kunzeanum

H. semi-involutum (C. Muell.) Kindb. (Kindberg, 1889).

Syn. Weisia semi-involuta C. Muell. (Mueller, 1882). Distr. Southern South America (Mueller, 1882; Paris, 1904b; Brotherus, 1924a; Kühnemann,
1938).

\section{Hymenostylium}

H. calcareum (Nees et Hornsch.) Mitt.

Gymnostomum aeruginosum

H. curvirostre Mitt.

Hymenostylium recurvirostre

H. kunzeanum (C. Muell.) C. Muell. (Mueller, 1901a [1900]).

Syn. Pottia kunzeana C. Muell. (Mueller in Montagne, 1850).

Gymnostomum kunzeanum (C. Muell.) Par. (Paris, 1895) nom. illeg.

Gymnostomum longirostris Kunz. ex C. Muell. (Mueller, 1847c) hom. illeg.

Pottia longirostris C. Muell. (Mueller, 1849) hom. illeg.

Weisia longirostris Mitt. (Mitten, 1869) hom. illeg.

Hymenostylium longirostre Broth. (Brotherus, 1902) hom. illeg.

Distr. Southern South America (Mueller, 1847c, 1849, 1901a; Montagne, 1850; Mitten, 1869; Jaeger, 1870; Paris, 1895, 1904b; Brotherus, 1902, 1924a; Warnstorf, 1916).

$\mathrm{H}$. longirostre Broth.

Hymenostylium kunzeanum

H. longo-pulvinatum Dus.

Hymenostylium recurvirostre

H. recurvirostre (Hedw.) Dix. (Dixon, 1933 [1934]).

Syn. Gymnostomum recurvirostre Hedw. (Hedwig, 1801).

Pottia recurvirostra Ehrh. ex Hedw. (Hedwig, 1801) nom. inval.

Weisia recurvirostris (Hedw.) Dix. (Dixon, 1902) hom. illeg. 
Eucladium recurvirostre (Hedw.) C. Jens. (Jensen, 1939).

Gymnostomum curvirostre Hedw. ex Brid. (Hedwig ex Bridel, 1801a) nom. illeg.

Weisia curvirostris C. Muell. (Mueller, 1849) hom. illeg.

Hymenostylium curvirostre Mitt. (Mitten, 1859) nom. illeg.

Barbula curvirostris Lindb. (Lindberg, 1879) hom. illeg.

Hymenostylium longo-pulvinatum Dus. (Dusén, 1906).

Barbula ripicola Card. (Cardot in Cardot and Brotherus, 1923) nom. nud.

Distr. Southern South America (Paris, 1904b; Dusén, 1906; Cardot, 1908a; Cardot and Brotherus, 1923; Brotherus, 1924a; Kühnemann, 1938; Seki, 1974).

\section{Hyophila}

H. argentinica Thér. (Thériot, 1935b).

Distr. Southern South America (Bauer, 1934; Thériot, 1935b; Hosseus, 1936, 1937, 1938c, d, 1939; Kühnemann, 1938).

H. macrocarpa (Schimp.) Kindb.

Pottia macrocarpa

H. poeppigiana (C. Muell.) Jaeg.

Trichostomopsis australasiae

H. poeppigii C. Muell. ex Kindb.

Trichostomopsis australasiae

H. tortula (Schwaegr.) Hamp. (Hampe, 1846).

Syn. Gymnostomum tortula Schwaegr. (Schwaegrichen, 1826).

Distr. Southern South America (Herzog, 1952a).

\section{Hypnodendron}

H. arcuatum (Hedw.) Lindb. (Lindberg, 1861).

Syn. Hypnum arcuatum Hedw. (Hedwig, 1801).

Pterygophyllum arcuatum (Hedw.) Brid. (Bridel, 1827b).

Hypopterygium arcuatum (Hedw.) C. Muell. (Mueller, 1850).

Racopilum arcuatum (Hedw.) Jaeg. (Jaeger, 1876).

Hypnum spininervium Hook. (Hooker, 1818).

Rhizogonium spininervium (Hook.) Schimp. (Schimper in Mueller, 1847c).

Isothecium spininervium (Hook.) Wils. (Wilson, 1854).

Hypnodendron spininervium (Hook.) Reichdt. (Reichardt, 1870).

Isothecium spininervium (Hook.) Wils. var. arcuatum (Hook.) Wils. (Wilson, 1854).

Hypnodendron spininervium (Hook.) Reichdt. var. arcuatum (Hook.) Jaeg. (Jaeger and Sauerbeck, 1879a) nom. illeg.

Distr. Southern South America (Lorentz, 1866).

H. Krausei (C..Muell.) Jaeg.

Hypnodendron microstictum

H. microstictum Mitt. (Mitten, 1869).

Syn. Hypnum krausei C. Muell. (Mueller, 1874).

Hypnodendron krausii (C. Muell.) Jaeg. (Jaeger and Sauerbeck, 1879a).

Distr. Juan Fernandez (Brotherus, 1924b; Bartram, 1959; Touw. 1971; Robinson, 1975).

Southern South America (Mitten, 1869; Mueller, 1874a; Jaeger and Sauerbeck, 1879̈a;

Neger, 1899; Paris, 1904b; Cardot, 1908a; Herzog. 1923, 1938, 1939; 1954, 1960;

Brotherus, 1924a; Reimers, 1926; Kühnemann, 1938: Bizot, 1967; Touw, 1971; Seki. 1974).

H. naumannii (C. Muell.) Kindb.

Porothamnium arbusculans

H. spininervium (Hook.) Reichdt.

Hypnodendron arcuatum

var. arcuatum (Hedw.) Jaeg.

Hypnodendron arcuatum

\section{Hypnodon}

H. demissus C. Muell.

Rhachithecium perpusillum

H. perpusillus (Thwait. et Mitt.) C. Muell.

Rhachithecium perpusillum

\section{Hypnopsis}

H. schreberi (Brid.) Podp.

Pleurozium schreberi 


\section{Hypnum}

H. acacioides (Schrad.) L. ex Turn.

Fissidens osmundoides

H. acanthophyllum Mont.

H. aciculare Brid.

Eurhynchiella acanthophylla

H. adianthoides (Hedw.) L. ex With.

Ptychomnion aciculare

H. aduncum Hedw.

Fissidens adianthoides

ssp. polycarpum (Noit) Meyl.

var. polycarpum (Noit) B.S.G.

Drepanocladus aduncus

var. revolvens (Sw.) Bland.

Drepanocladus aduncus var. polycarpus

Drepanocladus aduncus var. polycarpus

H. albicans Web. et Mohr

Drepanocladus revolvens

H. alopecurum L. ex Hedw. var. flagelliferum Mont. (Montagne, 1835).

Distr. Juan Fernandez (Montagne, 1835).

H. altisetum (C. Muell.) Broth.

H. amblyostomum C. Muell.

Oxyrrhynchium altisetum

var. attenuatifolium C. Muell.

var. paludicola C. Muell.

Pohlia wahlenbergii

H. amoenum Hedw.

H. aquicola C. Muell.

Rhaphidorrhynchium amoenum

$H$. arborescens C. Muell.

Bryum sp.

H. arbuscula P. Beauv.

Rigodium arborescens

$H$. arbusculans C. Muell.

Hypopterygium arbuscula

H. arcuatum Hedw.

$H$. argentinicum C. Muell.

H. asplenioides (Hedw.) Dicks. ex With.

Haplocladium microphyllum

Haplocladium microphyllum

Haplocladium microphyllum

$H$. attenuatifolium C. Muell.

H. auriculatum Mont.

H. austro-fluviatile C. Muell.

$H$. austro-glareosum C. Muell.

H. austro-salebrosum C. Muell.

$H$. austro-serpens C. Muell.

H. austro-stramineum C. Muell. Porothamnium arbusculans

Hypnodendron arcuatum

Rigodium argentinicum

Fissidens asplenioides

Haplocladium microphyllum

Acrocladium auriculatum

Hygroamblystegium austro-fluviatile

Brachythecium austro-glareosum

Brachythecium austro-salebrosum

Haplocladium microphyllum

var. gracillimum C. Muell.

var. minus Card.

var. subfluitans $\mathrm{C}$. Muell.

H. berteroanum Mont.

$H$. beskeanum C. Muell.

Calliergidium austro-stramineum

Calliergidium austro-stramineum var. gracillimum

Calliergidium austro-stramineum var. minus

Calliergidium austro-stramineum var. subfluitans

Catagoniopsis berteroana

H. bimum (Schreb.) Web. et Mohr

H. brachiatum Mitt.

Rhynchostegium beskeanum

H. brachypelma C. Muell.

$H$. brachypodium C. Muell.

H. brachypus Hamp.

H. bracteatum C. Muell.

$H$. brevipes C. Muell.

H. bryoides (Hedw.) L. ex With.

Bryum pseudotriquetrum ssp. bimum

Drepanocladus brachiatus

Rigodium brachypodium

Rigodium brachypodium

Rhaphidorrhynchium brachypus

Sematophyllum bracteatum

Stereophyllum brevipes

Fissidens bryoides 
H. cacti C. Muell.

Isopterygium cacti

H. cacticola C. Muell.

Rhynchostegium cacticola

H. caespiticium (Hedw.) Web. et Mohr

Bryum caespiticium

H. calerae C. Muell.

Brachythecium calerae

H. callichroum C. Muell.

Catagonium politum var. phyllogonium

H. callidum Mont.

Rhaphidorrhynchium callidum

H. campicola C. Muell.

Leptodictyum campicola

H. campoanum (Thér.) Broth. (Brotherus, 1925).

Syn. Stereodon campoanus Thér. (Thériot, 1918).

Distr. Southern South America (Thériot, 1918; Campo, 1921; Brotherus, 1925; Herzog, 1938. 1954, 1960).

H. camptorrhynchium Hamp.

Mittenothamnium camptorrhynchium

H. chlamydophyllum Hook. f. et Wils.

Acrocladium auriculatum

H. chrysogaster C. Muell. (Mueller, 1851a).

Syn. Hypnum cupressiforme L. ex Hedw. var. chrysogaster (C. Muell.) Sains. (Sainsbury, 1952).

Hypnum lechleri C. Muell. (Mueller, 1856) fide Ando, 1972.

Stereodon lechleri (C. Muell.) Mitt. (Mitten, 1869) fide Ando, 1972.

Hypnum lechleri C. Muell. var. fernandezianum Card. ex Thèr. (Cardot ex Thériot, 1921b) fide Ando, 1976.

Hypnum sublimatum Lor. (Lorentz, 1866) fide Ando, 1976.

Ectropothecium sublimatum (Lor.) Par. (Paris, 1895) fide Ando, 1976.

Hypnum cupressoides Schimp. (Schimper in Lechler, 1857) nom. nud. fide Ando, 1976.

Distr. Juan Fernandez (Jaeger and Sauerbeck, 1879a; Johow, 1896; Paris, 1904c; Skottsberg. 1914; Thériot, 1921b; Brotherus, 1924b, 1925; Espinosa B., 1941; Bartram, 1959; Robinson, 1975; Ando, 1976).

Southern South America (Mueller, 1856; Lechler, 1857; Lorentz, 1866; Mitten, 1869; Jaeger and Sauerbeck, 1879a; Paris, 1895, 1904b, c; Cardot, 1908a; Campo, 1915; Cardot and Brotherus, 1923; Herzog, 1923, 1938, 1939, 1954, 1960; Brotherus, 1925: Roivainen, 1934; Kühnemann, 1938; Seki, 1974: Ando, 1976).

H. circinale Hook. (Hooker, 1819).

Syn. Hypnum circinnale Hook. ex Macoun et Kindb. (Macoun and Kindberg, 1892).err Drepanium circinale (Hook.) Roth (Roth, 1904).

Distr. Juan Fernandez (Montagne, 1835; Johow, 1896; Paris. 1904C).

Southern South America (Montagne, 1850; Mueller, 1851a).

H. circinnale Hook. ex Macoun et Kindb.

Hypnum circinale

$H$. cirrifolium (Arnott) Schwaegr.

H. clarazii Dub.

Sematophyllum cirrifolium

H. clinocarpum Tayl. Helicodontium clarazii

H. cochlearifolium Schwaegr. Oxyrrhynchium clinocarpum

H. complanum Mitt.

Weymouthia cochlearifolia

H. confertum Dicks.

Rhynchostegium complanum

$H$. confluens $\mathrm{C}$. Muell.

Rhynchostegium confertum

H. conostomum Tayl.

Sciaromium confluens

H. conostomum (Mont.) Mitt.

Brachythecium conostomum

$H$. conspissatum Hook. f. et Wils.

Eriodon conostomus

$H$. corralense Lor.

Sciaromium conspissatum

H. crassiusculum Brid.

Oxyrrhynchium corralense

H. crinitifolium C. Muell.

Sematophyllum crassiusculum

Leucomium crinitifolium 
H. cristatum Poir.

Eriopus cristatus

H. crudum (Hedw.) Web. et Mohr

Pohlia cruda

H. cucullifolium P. Beauv.

Ptychomnion aciculare

H. cupressiforme L. ex Hedw. (Hedwig, 1801).

Syn. Stereodon cupressiformis (Hedw.) Brid. ex Mitt. (Bridel ex Mitten, 1859).

Cupressina cupressiformis (Hedw.) C. Muell. (Mueller, 1896a).

Drepanium cupressiforme (Hedw.) Roth (Roth, 1904).

Hypnum cupressiforme L. ex Hedw. var. tectorum Brid. (Bridel, 1827b).

Distr. Southern South America (Campo, 1915; Cardot and Brotherus, 1923; Brotherus, 1925;

Reimers, 1926; Herzog, 1938, 1940; Kühnemann, 1938; Bartram, 1952; Seki, 1974).

var. chrysogaster (C. Muell.) Sains.

var. tectorum Brid.

Hypnum chrysogaster

Hypnum cupressiforme

H. cupressoides Schimp.

H. curtipendulum (Hedw.) L. ex With.

Hypnum chrysogaster

H. cuspidatum Hedw.

H. cygnisetum C. Muell.

H. delicatulum Hedw.

H. densifolium Brid.

H. denticulatum Hedw.

Antitrichia curtipendula

Calliergonella cuspidata

Ptychomnion cygnisetum

var. obtusifolium Turn.

H. diminutivum Hamp.

H. dolomiticum Mild.

H. donnianum Sm.

H. drepanocladioides C. Muell.

H. encalyptratum Schimp.

H. eucalyptratum Lindb. ex Card.

H. exannulatum B.S.G.

H. falcatum Brid.

H. fasciculatum Hedw.

H. filarium (Mitt.) Hamp.

H. filicinum Hedw.

$H$. filum C. Muell.

H. fissidens C. Muell.

H. flagellare Hedw.

H. fluitans Hedw.

var. australe Card.

var. falcifolium Ren.

var. laculosum (C. Muell.) Par.

var. polycarpon (Voit) Fiedl.

H. fuegianum (Mitt.) C. Muell.

H. fulvo-acutum (C. Muell.) Par.

H. furfurosum Hook. f. et Wils.

H. gayanum (Mont.) Lor.

Thuidium recognitum var. delicatulum

Ptychomnion densifolium

Plagiothecium denticulatum

Plagiothecium denticulatum var. obtusifolium

Mittenothamnium diminutivum

Hypnum revolutum var. dolomiticum

Plagiothecium denticulatum var. obtusifolium

Rhynchostegium drepanocladioides

Acrocladium auriculatum

Acrocladium auriculatum

Drepanocladus exannulatus

see Hypnum longifolium

Porothamnium fasciculatum

Thuidiopsis furfurosa

Cratoneuron filicinum

Hygroamblystegium filum

Rhynchostegium fissidens

Brachythecium plumosum

Drepanocladus fluitans

Drepanocladus fluitans var. australe

Drepanocladus fluitans

Drepanocladus laculosus

Drepanocladus aduncus var. polycarpus

Drepanocladus fuegianus

Calliergonella cuspidata

Thuidiopsis furfurosa

Ptychomnion ptychocarpum

H. georgico-antarcticum C. Muell.

Plagiothecium georgico-antarcticum 
H. georgico-glareosum C. Muell.

H. georgico-uncinatum C. Muell.

H. glauco-virescens C. Muell.

H. globipyxis C. Muell.

H. gracile Hook. $f$ et Wils.

H. gracile Par. (Paris, 1904C) nom. nud.

Syn. Stereodon gracilis Dus. (Dusén in Paris, 1904C) nom. nud.

Distr. Southern South America (Paris, 1904c).

$H$. gracilescens $C$. Muell.

$H$. harrisona Poir.

H. heufleri Jur.

H. homaliocaulon C. Muell.

$H$. hookeri Lindb.

H. implexum (Schwaegr.) C. Muell.

$H$. intermedium Lindb. var. revolvens (Sw.) San.

H. julaceum (Hedw.) Schleich.

H. kneiffii (B.S.G.) Schimp. var. polycarpus (Voit) Fam.

Drepanocladus aduncus var. polycarpus

H. krausei C. Muell.

H. laculosum C. Muell.

$H$. lagurum Menz.

H. laterculi C. Muell.

H. lechleri C. Muell. var. fernandezianum Card. ex Thér.

H. lepidopiloides C. Muell.

H. leptorhynchum Brid.

H. leucocytus C. Muell.

H. leucophyllum Hamp.

H. leucostegum (Brid.) C. Muell.

H. lithophilum Hook. f. et Wils.

$H$. longidens C. Muell.

H. longifolium Wils. (Wilson, 1855) hom. illeg.

Distr. Falkland Islands (Wilson and Hooker, 1847 (as H. falcatum Brid. fide Wilson, 1855); Wilson, 1855; Kühnemann, 1938 (as H. falcatum Brid. fide Wilson, 1855)).

$H$. longifolium (Mitt.) Jaeg.

H. loxense Hook.

H. Iucidulum Hook. f. et Wils.

H. lucidum Hook. f. et Wils.

H. macroconum C. Muell. var. robustius C. Muell.

H. magellanicum P. Beauv.

H. magellanicum C. Muell.

H. masafuerae Bartr. (Bartram, 1959).
Brachythecium austro-salebrosum

Drepanocladus uncinatus

Rhynchostegium glauco-virescens

Rhynchostegium globipyxis

Camptochaete gracilis
Camptochaete gracilis

Neckeropsis undulata

Hypnum revolutum

Rhynchostegium homaliocaulon

Catagonium nitidum

Rigodium implexum

Drepanocladus revolvens

Leucodon julaceus

Hypnodendron microstictum

Drepanocladus laculosus

Lepyrodon lagurus

Haplocladium microphyllum

Hypnum chrysogaster

Hypnum chrysogaster

Stereophyllum lepidopiloides

Rhaphidorrhynchium amoenum

Sematophyllum leucocytus Isoptenggium leucophyllum

Stereophyllum leucostegum

Drepanocladus secundifolius

Brachythecium longidens
Sematophyllum caespitosum

Plagiothecium lucidulum

Plagiothecium lucidum

Brachythecium plumosum

Brachythecium macroconum var. robustius

Hookeria magellanica

Isopterygium fuegianum

Distr. Juan Fernandez (Bartram, 1959). 
H. micans Wils. var. laxum Hook. $f$.

Campochaete gracilis

$H$. microcarpoides C. Muell.

H. microphyllum Hedw.

Sematophyllum microcarpoides

H. micropyxis C. Muell.

Haplocladium microphyllum

H. mnioides Hook.

Trichosteleum sp.

H. morenoi (C. Muell.) Par.

Rhizogonium mnioides

H. nanum C. Muell.

Pleurozium schreberi

H. naumanii C. Muell.

Rhaphidostegium nanum

H. neckera Schimp.

Porothamnium arbusculans

H. neckeraeforme Hamp.

Porothamnium valdiviae

H. neckeroides Hook.

Porothamnium neckeraeforme

H. neei Mohr

Thamnium neckeroides

Rigodium implexum

H. nematogonium C. Muell.

Brachythecium nematogonium

H. nigrescens Hedw.

Papillaria nigrescens

H. nitidum (Hook. f. et Wils.) C. Muell.

Catagonium nitidum

H. obtusifolium (Turn.) Brid.

H. oligorrhizon Guemb.

Plagiothecium denticulatum var. obtusifolium

H. ornithopodioides Brid.

Amblystegium serpens

$\mathrm{H}$. orthocarpon Hornsch. ex C. Muell.

Racopilum tomentosum

H. orthothecioides (Lindb.) Par.

H. pachyloma (Mont.) C. Muell.

H. pallens Schimp. ex Card.

H. paludicola C. Muell.

H. palustre (Hedw.) Web. et Mohr

Haplocladium microphyllum

Aulacomnium palustre

H. pampae C. Muell.

Rhynchostegium pampae

H. panduraefolium C. Muell.

H. paradoxum Hook. f. et Wils.

var. leptocladum C. Muell. (Mueller, 1889).

Distr. Southem South America (Mueller, 1889).

$H$. parietinum Wahlenb.

Pleurozium schreberi

H. patagonicum Brid.

Homalia glabella

H. patrisiae Brid.

Crossomitrium patrisiae

H. pendulum (Brid.) C. Muell.

Helicodontium pendulum

H. perplicatum Dus.

Cratoneuron perplicatum

H. persistens C. Muell.

Haplocladium persistens

H. pervirens C. Muell.

Helicodontium pervirens

H. phyllogonium C. Muell.

Catagonium politum var. phyllogonium

H. pinnicaule C. Muell.

Rhynchostegium pinnicaule

H. piriforme Web. et Mohr ex Par.

Leptobryum pyriforme

H. plicatile (Mitt.) Lesq. et Jam. ssp. revolutum (Mitt.) Kindb.

Hypnum revolutum

H. plinthophilum C. Muell.

H. plumosum Hedw.

Mittenothamnium plinthophilum

H. politum Hook. f. et Wils.

Brachythecium plumosum

H. polycarpon Bland. ex Voit

Catagonium politum

Drepanocladus aduncus var. polycarpus 
H. polygamum (B.S.G.) Wils.

Campylium polygamum

H. polytrichoides Hedw.

Lepidopilum polytrichoides

H. pseudo-limnobium C. Muell.

Helicodontium pseudo-limnobium

H. pseudo-pallens Dus. (Dusén in Paris, 1905a) nom. nud.

Distr. Southern South America (Paris, 1905a).

var. gracile Dus. (Dusén in Paris, 1905a) nom. nud.

Distr. Southern South America (Paris, 1905a).

H. pseudo-purum (C. Muell.) Par.

Pseudoscleropodium pseudopurum

H. pseudo-triquetrum (Hedw.) Web. et Mohr

Bryum pseudotriquetrum

H. ptychocarpum Schwaegr.

Ptychomnion ptychocarpum

H. purpurascens Brid.

H. pyriforme (Hedw.) Web. et Mohr

Rhacocarpus purpurascens

$H$. radicale $P$. Beauv.

Leptobryum pyriforme

H. raphidostegum C. Muell.

Campylium radicale

$H$. remotifolium Grev.

Thuidium raphidostegum

H. reticulatum Hook. f. et Wils.

Oxyrrhynchium clinocarpum

Philonotis vagans

H. revolutum (Mitt.) Lindb. (Lindberg, 1866 [1867]).

Syn. Stereodon revolutus Mitt. (Mitten, 1859).

Drepanium revolutum (Mitt.) C. Jens. (Jensen in Lange and Jensen, 1887).

Hypnum plicatile (Mitt.) Lesq. et Jam. ssp. revolutum (Mitt.) Kindb. (Kindberg, 1897) nom. illeg.

Hypnum heufleri Jur. (Juratzka, 1861).

Distr. Antarctic, peninsula region (Cardot, 1900, 1901, 1908a; Paris, 1905a; Steere, 1961a; Robinson, 1972; Ando, 1973).

Antarctic, continental region (Bartram, 1957; Steere, 1961a).

var. dolomiticum (Mild.) Moenk. (Mönkemeyer, 1927).

Syn. Hypnum dolomiticum Mild. (Milde, 1864a).

Distr. Antarctic, peninsula region (Ando, 1973).

H. revolvens Sw.

Drepanocladus revolvens

H. rhaphidostegum C. Muell. ex Par.

Thuidium raphidostegum

H. rigidum Schwaegr.

Lepidopilum polytrichoides

H. riparioides Hedw.

H. riparium L. ex Hedw. ssp. kochii (B.S.G.) Boul.

$H$. rivularioides $\mathrm{C}$. Muell.

H. rostratum (Schrad.) Web. et Mohr

Platyhypnidium riparioides

Leptodictyum kochii

H. iusciforme Brid.

H. rutabulum Hedw.

$H$. sarmentosum Wahlenb.

H. scabrifolium Hook. f. et Wils.

Brachythecium rivularioides

Mnium rostratum

Platyhypnidium riparioides

Brachythecium rutabulum

Calliergon sarmentosum

Philonotis scabrifolia

H. schreberi Brid.

Pleurozium schreberi

$H$. sclerodontium C. Muell.

Dimerodontium pellucidum

H. scorpiurus Mont.

$H$. secundifolium C. Muell.

Rhaphidorrhynchium scorpiurus

$H$. sellowianum C. Muell.

Drepanocladus secundifolius

$H$. sellowii Hornsch.

Rhynchostegium sellowii

$H$. semilunare C. Muell.

Rhynchostegium sellowii

Haplocladium microphyllum 
H. semi-nerve (Schwaegr.) C. Muell.

Juratzkaea seminervis

H. semi-tortulum C. Muell. Rhynchostegium semi-tortulum

H. sericeo-virens C. Muell.

Brachythecium sericeo-virens

H. serpens Hedw.

Amblystegium serpens

H. serrulatum Hedw.

Rhynchostegium serrulatum

H. simorhynchum Hamp.

Mittenothamnium simorhynchum

H. simplex Brid.

Sematophyllum subsimplex

H. skottsbergii Ando (Ando, 1972) nom nud.

Syn. Rhaphidostegium pallens Dus. (Dusén, 1903a) nom. nud.

Stereodon pallens Broth. (Brotherus, 1908) nom. nud.

Hypnum pallens Schimp. ex Card. (Schimper ex Cardot, 1908a) hom. illeg.

Distr. Southem South America (Mueller, 1885; Dusén, 1903a; Paris, 1904c; Cardot, 1905a, 1908a; Brotherus, 1908, 1925; Cardot and Brotherus, 1923; Herzog, 1923, 1938. 1939, 1940, 1954, 1957, 1960; Thériot, 1934b; Kühnemann, 1938; Seki, 1974).

H. smithii Hedw.

Leptodon smithii

H. sparsifolium Hamp.

Amblystegium sparsifolium

H. speciosum C. Muell.

Hypopterygium arbuscula

H. spegazzinii C. Muell. (Mueller, 1885).

Syn. Brachythecium spegazzinii (C. Muell.) Kindb. (Kindberg, 1888). Stereodon spegazzinii (C. Muell.) Broth. (Brotherus, 1908).

Distr. Southem South America (Mueller, 1885; Paris, 1905a; Brotherus, 1908; Cardot, 1908a).

$H$. spiniforme Hedw.

H. spininervium Hook.

$H$. squalidissimum C. Muell.

H. squarroso-byssoides (C. Muell.) Par.

$H$. stenopyxidium C. Muell.

H. stramineum Dicks. ex Brid.

H. strigosum Hoffm. ex Web. et Mohr

H. subbasilare Hook.

H. sublimatum Lor.

H. subpilosum Hook. f. et Wils.

H. subplicatum Hamp.

H. subsimplex Hedw.

$H$. sulphureum Geh. et Hamp.

H. symmetricum (Ren. et Card.) Ren. et Card.

H. tamariscinum Hedw.

var. delicatulum (Hedw.) Brid.

H. taxarion Schwaegr. ex Mont.

H. taxifolium (Hedw.) L. ex With.

H. taylorii Mitt.

H. tenerum Sw.

H. tenuifolium Hedw.

H. tenuirostre (Schwaegr.) C. Muell.

H. thelistegum C. Muell.

$H$. thieleanum C. Muell.
Rhizogonium spiniforme

Hypnodendron arcuatum

Brachythecium squalidissimum

Campylium squarroso-byssoides

Sematophyllum stenopyxidium

Calliergon stramineum

Eurhynchium pulchellum

Goniobryum subbasilare

Hypnum chrysogaster

Brachythecium subpilosum

Brachythecium subplicatum

Sematophyllum subsimplex

Brachythecium sulphureum

Drepanocladus uncinatus var. symmetricus

Thuidium tamariscinum

Thuidium recognitum var. delicatulum

Rigodium toxarion

Fissidens taxifolius

Brachythecium conostomum

Isopterygium tenerum

Rhynchostegium tenuifolium

Helicodontium tenuirostre

Mittenothamnium diminutivum

Pinnatella thieleana 
H. thouinii Schwaegr.

Hypopterygium arbuscula

H. tomentosum Hedw.

Racopilum tomentosum

H. torquatum Hedw.

Schlotheimia torquata

$H$. toxarion Schwaegr.

Rigodium toxarion

H. trichopodium Schultz var. kochii Lindb. ex Par.

Leptodictyum kochii

H. turbinatum (Hedw.) Web. et Mohr

Bryum turbinatum

H. uncinatum Hedw.

Drepanocladus uncinatus

ssp. symmetricum Ren. et Card.

Drepanocladus uncinatus var. symmetricus

var. cylindropyxis Dus.

var. orthothecioides (Lindb.) Zett.

Drepanocladus uncinatus var. symmetricus

var. plumosum Schimp.

Drepanocladus uncinatus var. subjulaceus

var. subjulaceum B.S.G.

Drepanocladus uncinatus

H. unguiculatum Hook. f. et Wils.

Drepanocladus uncinatus var. subjulaceus

Thuidiopsis furfurosa

$H$. valdiviae C. Muell.

Porothamnium valdiviae

var. pallidum Lor.

H. varium (Hedw.) P. Beauv.

Porothamnium valdiviae var. pallidum

$H$. ventricosum Web. et Mohr

Amblystegium varium

H. vesiculare Schwaegr.

Bryum pseudotriquetrum

H. wahlenbergii Web. et Mohr

Vesicularia vesicularis

Pohlia wahlenbergii

\section{Hypoptenygium}

H. arbuscula Brid. (Bridel, 1827b).

Syn. Hypnum arbuscula P. Beauv. (Palisot de Beauvois, 1805a) hom. illeg.

Hypnum speciosum C. Muell. (Mueller, i 844b [1845]) hom. illeg.

Hypopterygium speciosum C. Muell. (Mueller, 1844b [1845]).

Hypnum thounii Schwaegr. (Schwagrichen, 1830).

Hypopterygium thounii (Schwaegr.) Mont. (Montagne, 1845a).

Distr. Juan Fernandez (Brotherus, 1924a, b; Espinosa B., 1941; Bartram, 1959; Matteri, 1973b; Robinson, 1975).

Southern South America (Palisot de Beauvois, 1805a; Bridel, 1827b; Schwaegrichen, 1830; Mueller, 1844b, 1850, 1885; Montagne, 1845a, 1850, 1856; Wilson and Hooker, 1847; Lorentz, 1866; Mitten, 1869; Jaeger, 1876; Bescherelle, 1885a; Hariot, 1891; Eaton, 1892; Neger. 1899; Paris, 1905a; Cardot, 1908a; Campo, 1915, 1921; Thériot, 1915, 1918; Cardot and Brotherus, 1923; Herzog, 1923, 1938, 1939, 1940, 1954, 1957, 1960; Brotherus, 1924a; Reimers, 1926; Roivainen, 1934; Kühnemann, 1938; Bartram, 1952; Bizot, 1967; Matteri, 1973b. 1975a; Seki, 1974).

H. arcuatum (Hedw.) C. Muell.

Hypnodendron arcuatum

H. argentinicum Lor. ex C. Muell. (Lorentz ex Mueller, 1879a).

Syn. Hypopterygium tamarisci (Sw.) Brid. ex C. Muell. ssp. argentinicum (C. Muell.) Kindb. (Kindberg, 1901).

Distr. Southern South America (Mueller, 1879a; Kindberg, 1901; Paris, 1905a; Brotherus, 1925; Herter, 1933a, 1939a; Kühnemann, 1938; Herzog, 1952a).

H. concinnum (Hook.) Brid.

Lopidium concinnum

H. didictyon C. Muell. (Mueller, 1850).

Distr. Southern South America Milson and Hooker, 1847 (as H. laricinum fide Mueller, 1850); Mueller, 1850. 1885; Sullivant, 1859; Mitten, 1869; Jaeger, 1876; Bescherelle, 1889; Hariot, 1891; Neger, 1899; Kindberg, 1901; Cardot. 1905a, 1908a; Paris, 1905a; Cardot and Brotherus, 1923; Herzog, 1923, 1938, 1939, 1940, 1954. 1960; Thériot. 1924, 1934b; Brotherus, 1925; Kühnemann, 1938; Bartram, 1952; Matteri, 1973b. 1975a; Seki, 1974). 
H. flavescens Hamp. (Hampe, 1847).

Distr. Southern South America (Herzog, 1952a).

H. flexisetum Hamp.

Lopidium concinnum

H. incrassato-limbatum C. Muell.

H. krauseanum C. Muell.

Hypopterygium laricinum ssp. incrassato-limbatum

Hypopterygium novae-seelandiae var. chilensis

H. laricinum (Hook.) Brid. ssp. incrassato-limbatum (C. Muell.) Kindb. (Kindberg, 1901).

Syn. Hypopterygium incrassato-limbatum C. Muell. (Mueller, 1850).

Distr. Southem South America (Krieger, 1904).

H. novae-seelandiae C. Muell. var. chilènsis Lor. (Lorentz, 1866).

Syn. Hypopterygium krauseanum C. Muell. (Mueller in Kindberg, 1901).

Distr. Southem South America (Lorentz, 1866; Kindberg, 1901; Paris, 1905a).

H. pallens (Hook. f. et Wils.) Mitt.

Lopidium concinnum

ssp. plumarium (Mitt.) Kindb.

Lopidium concinnum

H. plumarium Mitt.

Lopidium concinnum

H. speciosum C. Muell. Hypopterygium arbuscula

H. squarrulosum C. Muell. (Mueller, 1897a).

Distr. Southern South America (Mueller, 1897a; Paris, 1900a, 1905a; Brotherus, 1925; Herter. 1933a, 1943; Kühnemann, 1938 (as H. squamulosum C. Muell.); Hosseus, 1939).

H. tamarisci (Sw.) Brid. ex C. Muell. ssp. argentinicum (C. Muell.) Kindb.

H. thouinii (Schwaegr.) Mont.

Hypopterygium argentinicum

H. tomentosum (Hedw.) C. Muell.

Hypopterygium arbuscula

H. uliginosum C. Muell. (Mueller, 1882).

Distr. Southern South America (Mueller, 1882; Paris, 1905a; Brotherus, 1925; Kühnemann,
1938).

H. wolffhuegelii Herz. (Herzog, 1923).

Distr. Southern South America (Herzog, 1923; Brotherus, 1925).

\section{Isopterygium}

I. adflatum (C. Muell.) Par. (Paris, 1900a).

Syn. Taxicaulis adflatus C. Muell. (Mueller, 1897a).

Distr. Southern South America (Mueller, 1897a; Paris, 1900a, 1905a; Brotherus, 1925;
Kühnemann, 1938).

I. arechavaletae Broth. (Brotherus in Krieger, 1904) nom. nud.

Distr. Southem South America (Krieger, 1904).

I. byssobolax (C. Muell.) Par. (Paris, 1900a).

Syn. Taxicaulis byssobolax C. Muell. (Mueller, 1897a).

Distr. Southern South America (Mueller, 1897a; Paris, 1900a, 1905a; Brotherus, 1918, 1925; Kühnemann, 1938).

I. cacti (C. Muell.) Kindb. (Kindberg, 1888).

Syn. Hypnum cacti C. Muell. (Mueller, 1879a).

Plagiothecium cacti (C. Muell.) Kindb. (Kindberg, 1891).

Distr. Southern Suuth America (Mueller, 1879a; Paris, 1905a; Brotherus, 1925; Herter, 1933a; Kühnemann, 1938; Hosseus, 1939). 
I. elegans (Brid.) Lindb. var. nanum (Jur.) Jaeg. (Jaeger, 1878).

Syn. Plagiothecium nanum Jur. (Juratzka in Milde, 1864b). Isopterygium nanum (Jur.) Loesk. (Loeske, 1910) nom. inval.

Plagiothecium schimperi Jur. et Mild. var. nanum (Jur.) Walth. et Mol. (Walther and Molendo, 1868).

Plagiothecium elegans (Brid.) Sull. var. nanum (Jur.) Mol. (Molendo, 1875).

Distr. Falkland Islands (Cardot and Brotherus, 1923).

I. fernandezianum Broth.

Isoptengium tenerum

var. longisetum Broth. (Brotherus, 1924b).

Distr. Juan Fernandez (Brotherus, 1924b; Espinosa B., 1941; Bartram, 1959).

I. fuegianum Besch. (Bescherelle, 1889).

Syn. Plagiothecium fuegianum (Besch.) Dus. (Dusén, 1903a).

Hypnum magellanicum C. Muell. (Mueller, 1885) hom. illeg.

Plagiothecium magellanicum Par. (Paris, 1896) nom. illeg.

Distr. Southern South America (Mueller, 1885; Bescherelle, 1889; Paris, 1896, 1905b; Dusen, 1903a: Cardot, 1908a; Cardot and Brotherus, 1923; Brotherus, 1925; Kühnemann, 1938).

I. leptoplumosum (Dus.) Card. (Cardot, 1908a).

Syn. Plagiothecium leptop/umosum Dus. (Dusén, 1903a).

Distr. Southern South America (Dusén, 1903a; Cardot, 1908a; Kühnemann, 1938; Seki, 1974).

I. leucophyllum (Hamp.) Mitt. (Mitten, 1869).

Syn. Hypnum leucophyllum Hamp. (Hampe in Mueller, 1851a).

Plagiothecium leucophyllum Hamp. (Hampe in Mueller, 1851a).

Distr. Southern South America (Mueller, 1851a; Mitten, 1869; Jaeger, 1878).

I. nanum (Jur.) Loesk.

Isopterygium elegans var. nanum

I. pyrrhopus (C. Muell.) Par. (Paris, 1900a).

Syn. Taxicaulis pyrrhopus C. Muell. (Mueller, 1897a).

Distr. Southern South America (Mueller, 1897a; Paris, 1900a, 1905a; Brotherus, 1925; Kühnemann, 1938).

I. saprophilum (C. Muell.) Par. (Paris, 1900a).

Syn. Taxicaulis saprophilus C. Muell. (Mueller, 1897a).

Distr. Southern South America (Mueller, 1897a; Paris, 1900a, 1905a; Brotherus, 1925; Kühnemann, 1938).

I. tenerum (Sw.) Mitt. (Mitten, 1869).

Syn. Hypnum tenerum Sw. (Swartz, 1806b).

Isopterygium fernandezianum Broth. (Brotherus, 1924b) fide Robinson, 1975.

Distr. Juan Fernandez (Skottsberg. 1914; Brotheus, 1924b, 1925; Bartram, 1959; Robinson, 1975).

\section{Isothecium}

1. amoenum (Hedw.) Brid.

I. cochlearifolium (Schwaegr.) Mitt.

Rhaphidorrhynchium amoenum

I. gracile Hook. f. et Wils.

Weymouthia cochlearifolia

I. molle (Hedw.) Mitt.

Camptochaete gracilis

1. neckeroides (Hook.) Brid.

Weymouthia mollis

I. orthocarpum Brid.

Thamnium neckeroides

I. pendulum Brid.

I. pumilum Hook. f. et Wils. 
I. serpens Dus. (Dusén, 1903a) nom. nud.

Distr. Southern South America (Neger, 1899; Dusén, 1903a; Paris, 1905a; Seki, 1974).

1. spininervium (Hook.) Wils.

Hypnodendron arcuatum

var. arcuatum (Hedw.) Wils.

Hypnodendron arcuatum

I. subsimplex (Hedw.) Brid.

Sematophyllum subsimplex

I. tenuifolium (Hedw.) Brid.

Rhynchostegium tenuifolium

\section{Juratzkaea}

J. krausei (C. Muell.) Fleisch. (Fleischer, 1923).

Syn. Fabroniella krausei Lor. ex C. Muell. (Lorentz ex Mueller, 1875). Stereophyllum krausei (C. Muell.) Broth. (Brotherus, 1907).

Distr. Southern South America (Mueller, 1875; Jaeger, 1878; Paris, 1904b; Brotherus, 1907, 1925; Campo, 1921 (as Atereophyllum krausei Lor.); Fleischer, 1923; Thériot, 1935).

J. membranosa Fleisch. (Fleischer, 1917) nom. nud.

Syn. Cladomnion membranosum Dus. (Dusén in Fleischer, 1917) nom. nud.

Distr. Southern South America (Fleischer, 1917).

J. seminervis (Schwaegr.) Lor. (Lorentz, 1866).

Syn. Leskea seminervis Kunz. ex Schwaegr. (Kunze ex Schwaegrichen, 1829).

Helicodontium seminerve (Schwaegr.) Schwaegr. (Schwaegrichen, 1830) nom. inval.

Hypnum semi-nerve (Schwaegr.) C. Muell. (Mueller, 1851a).

Stereophyllum seminerve (Schwaegr.) Mitt. (Mitten, 1869).

Distr. Southern South America (Schwaegrichen, 1829; Mueller, 1851a; Sullivant, 1859 (as Pylaisea seminervis Kunz.); Lorentz, 1866; Mitten, 1869; Reichardt, 1870; Jaeger, 1878; Paris, 1904b; Thériot, 1917b, 1918, 1921b, 1928; Campo, 1921; Costes, 1921; Herzog, 1923, 1938, 1952b, 1954; Brotherus, 1924a, c; Hosseus, 1938b; Espinosa B., 1941; Bartram, 1942, 1943, 1952).

var. arenicola Thér. (Thériot, 1926).

Distr. Southern South America (Thériot, 1926).

var. argentinica Thér. (Thériot in Bauer, 1931).

Distr. Southem South America (Bauer, 1931; Thériot, 1935b; Hosseus, 1936, 1937, 1938b, c, d, 1939; Kühnemann, 1938; Herzog, 1952a).

var. pilifera Thér. (Thériot in Verdoorn, 1938).

Distr. Southern South America Nerdoorn, 1938).

\section{Kiaeria}

K. starkei (Web. et Mohr) Hag. (Hagen, 1915).

Syn. Dicranum starkei Web. et Mohr (Weber and Mohr, 1807).

Oncophorus starkei (Web. et Mohr) Brid. (Bridel, 1826).

Distr. Southem South America (Wilson and Hooker, 1847 (as vars 1 and 2)).

Lamprophyllum hom. illeg.

L. crudum (Hedw.) Lindb.

Pohlia cruda

L. nutans (Hedw.) Lindb.

Pohlia nutans

Lamprophyllum hom. illeg.

L. splendidissimum (Mont.) Broth.

Schimperobryum splendidissimum

Lasia hom. illeg.

L. coronata (Mont.) C. Muell.

Forsstroemia coronata

L. orthotrichoides Raddi

Macrocoma orthotrichoides

L. subcoronata Besch. 
Leiotheca nom. rejic

L. filiformis Brid.

Macrocoma orthotrichoides

L. microstoma (Hook. et Grev.) Brid.

Macromitrium microstomum

L. obtusifolia (C. Muell.) Hamp.

Drummondia obtusifolia

L. urceolata (Hook.) Brid.

Macromitrium urceolatum

\section{Lembophyllum}

L. auriculatum (Mont.) Par.

Acrocladium auriculatum

L. cochlearifolium (Schwaegr.) Lindb.

Weymouthia cochlearifolia

\section{Lepidopilum}

L. aurescens C. Muell. (Mueller, 1879a).

Distr. Southern South America (Mueller, 1879a; Paris, 1905a; Brotherus, 1925; Kühnemann, 1938; Welch, 1969).

L. cristatum (Hedw.) Hamp.

Eriopus cristatus

L. erectiusculum (Tayl.) Mitt. (Mitten, 1869).

Syn. Hookeria erectiuscula Tayl. (Taylor, 1846).

Distr. Southern South America (Mitten, 1869; Jaeger, 1877; Paris, 1905a; Brotherus, 1925; Welch, 1969).

L. incurvum (Hornsch.) C. Muell.

Hookeriopsis incurva

L. patrisiae (Brid.) Mitt.

Crossomitrium patrisiae

L. plebejum C. Muell. (Mueller, 1900).

Distr. Southern South America (Welch, 1969).

L. polytrichoides (Hedw.) Brid. (Bridel, 1827b).

Syn. Hypnum polytrichoides Hedw. (Hedwig, 1801).

Orthotrichum polytrichoides (Hedw.) Brid. (Bridel, 1801a).

Neckera polytrichoides (Hedw.) Web. et Mohr (Weber and Mohr, 1803).

Pilotrichum polytrichoides (Hedw.) Brid. (Bridel, 1819).

Daltonia polytrichoides (Hedw.) Arnott (Arnott, 1827).

Hookeria polytrichoides (Hedw.) Spreng. (Sprengel, 1827).

Hypnum rigidum Schwaegr. (Schwaegrichen, 1816).

Pterygophyllum rigidum (Schwaegr.) Brid. (Bridel, 1819).

Hookeria rigida (Schwaegr.) Arnott (Arnott, 1827).

Distr. Southern South America (Paris, 1905a; Brotherus, 1925; Welch, 1969).

L. scabrisetum (Schwaegr.) Steere (Steere, 1948).

Syn. Neckera scabriseta Schwaegr. (Schwaegrichen, 1816).

Pilotrichum scabrisetum (Schwaegr.) Brid. (Bridel, 1819).

Lepidopilum subenerve Brid. (Bridel, 1827b) hom. illeg.

Distr. Southern South America (Welch, 1969).

L. splendidissimum (Mont.) Mitt.

Schimperobryum spendidissimum

L. subenerve Brid.

Lepidopilum scabrisetum

L. uruguense Broth. (Brotherus in Krieger, 1904) nom. nud.

Distr. Southern South America (Krieger, 1904).

Leptangium hom. illeg.

L. imbricatum Mitt.

Lorentziella imbricata

\section{Leptobryum}

L. pottiaceum Dus. (Dusén, 1903a).

Syn. Wollnya pottiacea (Dus.) Herz. (Herzog, 1954). 
Distr. Southem South America (Dusén, 1903a, 1905d; Cardot, 1908a; Brotherus, 1924a; Kühnemann, 1938; Herzog, 1954).

L. pyriforme (Hedw.) Wils. (Wilson, 1855).

Syn. Webera pyriformis Hedw. (Hedwig, 1801).

Bryum pyriforme (Hedw.) Gaertn., Meyer and Scherb. (Gärtner and others, 1802) hom. illeg.

Bryum piriforme Par. (Paris, 1894) err.

Hypnum pyriforme (Hedw.) Web. et Mohr (Weber and Mohr, 1803).

Hypnum piriforme Web. et Mohr ex Par. (Weber and Mohr ex Paris, 1896) err.

Distr. Southern South America (Bescherelle, 1889; Dusén, 1903a, 1905d; Krieger, 1904; Cardot, 1908a; Cardot and Brotherus, 1923; Brotherus, 1924a; Kühnemann, 1938 (as L. pysiforme Dus.); Seki, 1974).

var. antarcticum Besch. (Bescherelle, 1889) nom. nud.

Syn. Bryum pyriforme (Hedw.) Gaertn., Meyer and Scherb. var. antarcticum C. Muell. (Mueller, 1885) nom. nud.

Distr. Southem South America (Mueller, 1885; Paris, 1905a; Cardot, 1908a; Kühnemann, 1938).

var. fuegianum Par. (Paris, 1896) nom. nud.

Syn. Bryum pyriforme (Hedw.) Gaertn., Meyer and Scherb. var. fuegianum C. Muell. (Mueller, 1889) nom. nud.

Distr. Southem South America (Mueller, 1889; Paris, 1905a; Cardot, 1908a).

L. wilsonii (Mitt.) Broth. (Brotherus, 1903).

Syn. Bryum wilsonii Mitt. (Mitten, 1851).

Webera wilsonii (Mitt.) Jaeg. (Jaeger, 1875).

Brachymenium wilsonii (Mitt.) Jaeg. (Jaeger, 1875).

Wollnya wilsonii (Mitt.) Herz. (Herzog, 1911).

Distr. Southern South America (Bartram, 1952; Herzog, 1954).

Leptochlaena hom. illeg.

L. chilensis Mont.

Mielichhoferia chilensis

\section{Leptodictyum}

L. brachypelmatum (C. Muell.) Broth. (Brotherus, 1925).

Syn. Amblystegium brachypelmatum C. Muell. (Mueller, 1897a).

Distr. Southem South America (Mueller, 1897a; Paris, 1900a, 1903; Brotherus, 1925; Hosseus, 1938c, d; Kühnemann, 1938).

L. campicola (C. Muell.) Broth. (Brotherus, 1925).

Syn. Hypnum campicola C. Muell. (Mueller, 1879a).

Amblystegium campicola (C. Muell.) Kindb. (Kindberg, 1888).

Distr. Southern South America (Mueller, 1879a; Paris, 1903; Kurtz, 1904; Brotherus, 1925; Herter, 1933a; Hosseus, 1935a, 1936, 1937; Kühnemann, 1938).

L. kochii (B.S.G.) Warnst. (Warnstorf, 1906).

Syn. Amblystegium kochii B.S.G. (Bruch and others, 1853).

Amblystegium riparium (Hedw.) B.S.G. var. kochii (B.S.G.) Buyss. (Du Buysson, 1883).

Amblystegium riparium (Hedw.) B.S.G. ssp. kochii (B.S.G.) Ren. et Card. (Renauld and Cardot, 1893a).

Hypnum riparium L. ex Hedw. ssp. kochii (B.S.G.) Boul. (Boulay, 1884).

Amblystegium trichopodium (Schultz) Hartm. var. kochii (B.S.G.) Lindb. (Lindberg, 1872).

Amblystegium trichopodium (Schultz) Hartm. ssp. kochii (B.S.G.) Loesk. (Loeske, 1903).

Hypnum trichopodium Schultz var. kochii Lindb. ex Par. (Lindberg ex Paris, 1896) err.

Leptodictyum trichopodium (Schultz) Warnst. var. kochii (B.S.G.) Broth. (Brotherus, 1925). 
Distr. Southern South America (Cardot and Brotherus, 1923).

L. riparium (Hedw.) Warnst. (Warnstorf, 1906).

var. jaffuelii (Thér.) Thér. (Thériot, 1934b [1935]).

Syn. Amblystegium jaffuelii Thér. (Thériot, 1924).

Distr. Southern South America (Thériot, 1924; Brotherus, 1925).

var. looseri Thér. (Thériot, 1934b [1935]).

Distr. Southern South America (Thériot, 1934b).

L. trichopodium (Schultz) Warnst. var. kochii (B.S.G.) Broth.

Leptodictyum kochii

\section{Leptodon}

L. coronatus Mont.

Forsstroemia coronata

L. dillenii Lindb. (Lindberg, 1883).

Distr. Southern South America (Lindberg, 1883).

L. patagonicus C. Muell.

Leptodon smithii

L. smithii (Hedw.) Web. et Mohr (Weber and Mohr, 1803).

Syn. Hypnum smithii Hedw. (Hedwig, 1801).

Orthotrichum smithii (Hedw.) Brid. (Bridel, 1801a).

Pilotrichum smithii (Dicks.) P. Beauv. (Palisot de Beauvois, 1805a).

Pterigynandrum smithii (Hedw.) Lam. et Cand. (Lamark and de Candolle, 1805).

Maschalocarpus smithii (Hedw.) Spreng. (Sprengel, 1827).

Neckera smithii (Hedw.) C. Muell. (Mueller, 1850).

Leptodon patagonicus C. Muell. (Mueller in Paris, 1905a) nom. nud. fide Thériot, $1929 c$.

Distr. Juan Fernandez (Johow, 1896; Paris, 1905a; Brotherus, 1924b; Bartram, 1959; Robinson, 1975).

Southern South America (Mitten, 1869; Paris, 1905a; Cardot, 1908a; Fleischer, 1917; Cardot and Brotherus, 1923; Brotherus, 1924a; Thériot. 1924; Roivainen, 1934;

Herzog, 1938, 1954, 1960) Kühnemann, 1938;

var. longirepens Card. et Thér. (Cardot and Thériot in Thériot, 1924).

Distr. Juan Fernandez (Thériot, 1924).

\section{Leptodontium}

L. arachnoideum C. Muell.

Leptodontium capituligerum

L. braunioides C. Muell.

Leptodontium pungens

L. bullockii Thèr.

Leptostomum bullockii

L. capituligerum C. Muell. (Mueller, 1879a).

Syn. Leptodontium arachnoideum C. Muell. (Mueller, 1879a) fide Zander, 1972.

Leptodontium felipponei Broth. (Brotherus in Felippone, 1917) fide Zander, 1972.

Leptodontium gracile C. Muell. ex Britt. var. gemmascens Bartr. (Bartram, 1936) fide Zander, 1972.

Distr. Southern South America (Mueller, 1879a; Kurtz, 1904; Paris, 1905a; Felippone, 1917; Brotherus, 1924a; Herter, 1933a; Hosseus, 1935b, 1937, 1938c, d, 1939; Bartram, 1936; Kühnemann, 1938; Zander, 1972; Seki, 1974).

L. chiloense C. Muell. (Mueller in Kindberg, 1889) nom. nud.

Distr. No locality given, see Index muscorum, 3, p. 233.

L. felipponei Broth.

L. fernandezianum Broth.

Leptodontium longicaule var. microruncinatum

L. filicaule Dus. (Dusén in Paris, 1905a) nom. nud.

Distr. Southern South America (Paris, 1905a). 
L. filicola Herz. (Herzog, 1916a).

Distr. Southern South America (Zander, 1972).

L. gracile C. Muell. ex Britt. var. gemmascens Bartr.

L. gracile C. Muell. ex Britt. var. gracilescens Bartr. nom. nud.

Distr. Southern South America (Bartram, 1936; Hosseus, 1938c, 1939).

L. kurtzii Broth. (Brotherus in Kurtz, 1904) nom. nud.

Distr. Southern South America (Kurtz, 1904; Hosseus, 1936, 1937; Kühnemann, 1938).

L. longicaule Mitt. var. microruncinatum (Dus.) Zander (Zander, 1972).

Syn. Barbula micro-runcinata Dus. (Dusén, 1903a) nom. nud. fide Zander, 1972.

Leptodontium microruncinatum Dus. (Dusén, 1906) fide Zander, 1972.

Syntrichia micro-runcinata Dus. (Dusén in Paris, 1906) nom. nud.

Tortula micro-runcinata Par. (Paris, 1906) nom. nud.

Leptodontium fernandezianum Broth. (Brotherus, 1924b) fide Zander, 1972.

Distr. Juan Femandez (Brotherus, 1924b, 1925; Espinosa B., 1941; Bartram, 1959; Zander, 1972; Robinson, 1975).

Southem South America (Dusén, 1903a, 1906; Paris, 1906; Cardot, 1908a; Brotherus, 1924a; Thériot, 1925a; Herzog, 1938, 1954; Zander, 1972).

L. menziesii R. Brown ex Thér.

Leptostomum menziesii

L. microruncinatum Dus.

Leptodontium longicaule var. microruncinatum

L. orthotrichoides (C. Muell.) Par. (Paris, 1900a).

Syn. Trichostomum orthotrichoides C. Muell. (Mueller, 1874).

Zygodon orthotrichoides (C. Muell.) Broth. (Brotherus, 1918).

Zygodon palmarum C. Muell. (Mueller, 1882).

Distr. Southern South America (Mueller, 1882; Paris, 1906; Brotherus, 1918, 1925; Kühnemann, 1938).

L. pungens (Mitt.) Kindb. (Kindberg, 1888).

Syn. Didymodon pungens Mitt. (Mitten, 1863).

Trichostomum pungens (Mitt.) Par. (Paris, 1898).

Leptodontium braunioides C. Muell. (Mueller, 1879a) fide Zander, 1972.

Leptodontium skottsbergii Bartr. (Bartram, 1959) fide Zander, 1972.

Distr. Juan Fernandez (Bartram, 1959; Zander, 1972; Robinson, 1975).

Southem South America (Mueller, 1879a; Paris, 1905a; Brotherus, 1924a; Kühnemann, 1938; Zander, 1972).

L. quennoae C. Muell.

L. racomitrioides Lor. et C. Muell.

L. rhacomitrioides Lor. et C. Muell.

L. skottsbergii Bartr.

L. splachnoides (Hook. et Arnott) Thér.
Leptodontium viticulosoides var. viticulosoides Leptodontium viticulosoides var. viticulosoides Leptodontium viticulosoides var. viticulosoides

Leptodontium pungens Leptostomum splachnoides

L. viticulosoides (P. Beauv.) Wijk et Marg. (van der Wijk and Margadant, 1960a). var. viticulosoides

Syn. Neckera viticulosoides P. Beauv. (Palisot de Beauvois, 1805a).

Leptodontium quennoae C. Muell. (Muller, 1879a) fide Zander, 1972.

Leptodontium rhacomitroides Lor. et C. Muell. (Lorentz and Mueller in Mueller, 1879a).

Leptodontium racomitroides Lor. et C. Muell. (Lorentz and Mueller in Kindberg, 1889) err.

Distr. Southem South America (Mueller, 1879a; Paris, 1905a; Brotherus, 1924a; Kühnemann, 1938; Zander, 1972).

L. zygondontoides C. Muell. (Mueller, 1879a).

Distr. Southern South America (Mueller, 1879a; Paris, 1905a; Zander, 1972). 


\section{Leptohymenium}

L. squarrosum Hamp.

Erythrodontium squarrosum

\section{Leptopterigynandrum}

L. austro-alpinum C. Muell. (Mueller, 1879a).

Syn. Heterocladium austro-alpinum (C. Muell.) Broth. (Brotherus, 1907).

Pterigynandrum austro-alpinum C. Muell. (Mueller in Fleischer, 1912) nom. nud.

Distr. Southern South America (Mueller, 1897a; Paris, 1900a, 1905a; Brotherus, 1907, 1925; Fleischer, 1912, 1922; Kühnemann, 1938).

\section{Leptostomum}

L. bullockii (Thér.) Thér. (Thériot in Andrews, 1951 [1952]).

Syn. Leptodontium bullockii Thér. (Thériot, 1929b).

Distr. Southern South America (Theriot, 1929b, 1930; Andrews, 1951).

L. inclinans R. Brown (Brown, 1811).

Distr. Southern South America (Montagne, 1850).

L. menziesii R. Brown (Brown, 1811).

Syn. Gymnostomum menziesii (R. Brown) Hook. (Hooker, 1820).

Leptodontium menziesii R. Brown ex Thér. (R. Brown ex Thériot, 1929b) err.

Distr. Juan Fernandez (Brotherus, 1924b; Espinosa B., 1941; Bartram, 1959; Robinson, 1975).

Southern South America (Brown, 1811; Schwaegrichen, 1816, 1823; Hooker, 1820; Montagne, 1845b, 1850; Wilson and Hooker, 1847; Mueller, 1848a, 1885; Sullivant, 1859; Mitten, 1869; Jaeger, 1875; Bescherelle, 1889; Hariot, 1891; Neger, 1899; Cardot, 1900; 1901, 1908a, 1913a; Dusén, 1903a; Paris, 1905a; Spegazzini, 1922; Cardot and Brotherus, 1923; Herzog, 1923, 1938, 1954; Brotherus, 1924a; Thériot, 1929b; Roivainen, 1934; Kühnemann, 1938; Andrews, 1951; Bartram, 1952).

L. splachnoides Hook. et Arnott (Hooker and Arnott, 1832).

Syn. Leptodontium splachnoides (Hook. et Arnott) Thér. (Thériot, 1929a) err.

Leptostomum sulcinerve Kunz. (Kunze in Mueller, 1848a) nom. nud.

Distr. Southern South America (Hooker and Arnott, 1832; Schwaegrichen, 1842; Montagne, 1845b, 1850; Mueller, 1848a; Lorentz, 1866; Mitten, 1869; Jaeger, 1875; Paris. 1905a; Campo, 1921; Brotherus, 1924a; Thériot, 1929b, 1935a; Andrews, 1951; Herzog, 1954; Seki, 1974).

L. sulcinerve Kunz.

\section{Leptostomum splachnoides}

\section{Leptotheca}

L. gaudichaudii Schwaegr. (Schwaegrichen, 1824).

Syn. Bryum gaudichaudii (Schwaegr.) Spreng. (Sprengel, 1827).

Aulacomnium gaudichaudii (Schwaegr.) Mitt. (Mitten, 1856).

Brachymenium ovatum Hook. f. et Wils. (Hooker, J. D. and Wilson, 1844).

Hymenodon ovatus (Hook. f. et Wils.) C. Muell. (Mueller, 1851a).

Leptotheca spegazzinii C. Muell. (Mueller, 1885).

Distr. Juan Fernandez (Robinson, 1975).

Southern South America (Mueller, 1848a, 1885; Jaeger, 1875; Cardot, 1905a, 1908a;

Paris, 1905b; Cardot and Brotherus, 1923; Brotherus, 1924a; Kühnemann, 1938;

Herzog, 1939; Bartram, 1952; Seki, 1974).

Falkland Islands (Hooker, J. D. and Wilson, 1844; Wilson and Hooker, 1847; Mueller, 1851a; Mitten, 1869; Jaeger, 1875; Cardot, 1905a, 1908a; Paris, 1905a; Brotherus, 1924a; Kühnemann, 1938).

South Georgia (Clarke, 1973a).

L. spegazzinii C. Muell.

Leptotheca gaudichaudii

Leptotrichum hom. illeg.

L. affine C. Muell.

Ditrlchum difficile 
L. australe Mitt.

Ditrichum strictum

L. capillaceum (Hedw.) Mitt.

Distichium capillaceum

L. cylindricarpum C. Muell.

Ditrichum cylindricarpum

L. difficile (Dub.) C. Muell.

Ditrichum difficile

L. elongatum (Hook. f. et Wils.) Jaeg.

Ditrichum cylindricarpum

L. flexifolium Mitt.

Ditrichum difficile

L. glaciale C. Muell.

Sarconeurum glaciale

L. homomallum (Hedw.) Hamp.

Ditrichum heteromallum

L. hookeri C. Muell.

Ditrichum hookeri

L. hyalinum Mitt.

Ditrichum hyalinum

L. jamesonii Mitt.

Anisothecium vaginatum

L. kunzeanum C. Muell.

Microdus kunzeanus

L. laxifolium (Hook. f. et Wils.) C. Muell.

Ditrichum difficile

L. longisetum Lor.

Ditrichum longisetum

L. Iorifolium C. Muell. (Mueller, 1901a [1900]) nom. nud.

Syn. Leptotrichum sericeum Lor. (Lorentz in Mueller, 1901a [1900]) nom. nud.

Distr. Southern South America (Mueller, 1901a).

L. montagnei C. Muell.

Ditrichum conicum

L. praealtum Mitt.

L. rufescens Hamp.

L. sericeum Lor.

Ditrichum praealtum

Ditrichum rufescens

Leptotrichum lorifolium

\section{Lepyrodon}

L. glaucus (C. Muell.) Kindb.

Lepyrodon hexastichus

L. gunckelii Thér. (Thériot, 1934b [1935]).

Distr. Southem South America (Thériot, 1934b).

L. hexastichus (Mont.) Wijk et Marg. (van der Wijk and Margadant, 1960b).

Syn. Leucodon hexastichus Mont. (Montagne, 1845a).

Neckera implexa C. Muell. (Mueller, 1850) nom. illeg.

Leucodon implexus Kunz. ex Hook. f. (Kunze ex Hooker, 1867) nom. illeg.

Lepyrodon implexus Kindb. (Kindberg, 1888) nom. illeg.

Neckera glauca C. Muell. (Mueller, 1859).

Leucodon glaucus (C. Muell.) Jaeg. (Jaeger, 1877).

Lepyrodon glaucus (C. Muell.) Kindb. (Kindberg, 1888).

Leucodon kunzeanus C. Muell. (Mueller, 1844b [1845]).

Distr. Juan Fernandez (Brotherus, 1924b, 1925; Thériot, 1927b; Espinosa B., 1941; Bartram, 1959; Robinson, 1975).

Southern South America (Mueller, 1844b, 1850, 1859; Montagne, 1845a, 1850, 1856; Jaeger, 1877; Dusén, 1903a; Paris, 1905a; Cardot, 1908a; Fleischer, 1917; Herzog, 1923, 1938, 1939, 1940, 1954, 1957, 1960; Thériot, 1924; Brotherus, 1925; Reimers, 1926; Kühnemann, 1938; Bartram, 1952; Seki, 1974).

L. implexus Kindb.

Lepyrodon hexastichus

var. reptans Reim. (Reimers, 1926).

Distr. Southern South America (Reimers, 1926).

L. lagurus (Hook.) Mitt. (Mitten, 1869).

Syn. Leucodon lagurus Hook. (Hooker, 1819).

Hypnum lagurum Menz. (Menzies in Hooker, 1819) nom. nud.

Neckera lagura (Hook.) C. Muell. (Mueller, 1850).

Stereodon lagurus (Hook.) Mitt. (Mitten, 1860).

Hylocomium lagurus (Hook.) Kindb. (Kindberg, 1885). 
Distr. Southern South America (Hooker, 1819; Schwaegrichen, 1824; Bridel, 1827b;

Montagne, 1845b, 1850; Wilson and Hooker, 1847; Mueller, 1850, 1885, 1889;

Sullivant, 1859; Mitten, 1869; Jaeger, 1877; Bescherelle, 1889; Neger, 1899; Cardot, 1900, 1901, 1905a, 1908a, 1913a; Dusén, 1903a, 1905d (as Leptodon lagurus);

Paris, 1905a; Fleischer, 1917; Campo, 1921; Cardot and Brotherus, 1923; Herzog,

1923, 1939; Brotherus, 1925; Roivainen, 1934; Thériot, 1934b; Kühnemann, 1938; Bartram, 1952; Seki, 1974).

South Georgia (Cardot, 1906a, 1908a; Steere, 1961b).

var. laxior Card. et Broth. (Cardot and Brotherus, 1923).

Distr. Southern South America (Cardot and Brotherus, 1923).

L. parvulus Mitt. (Mitten, 1869).

Syn. Leucodon parvulus (Mitt.) Jaeg. (Jaeger, 1877).

Distr. Juan Fernandez (Mitten, 1885; Johow, 1896; Paris, 1905a; Brotherus, 1924b, 1925; Espinosa B., 1941; Bartram, 1959; Robinson, 1975). Southern South America (Mitten, 1869; Jaeger, 1877; Fleischer, 1917; Brotherus, 1925;
Herzog, 1939, 1954).

L. suborthostichus (C. Muell.) Hamp.

Lepyrodon tomentosus

L. tomentosus (Hook.) Mitt. (Mitten, 1869).

Syn. Leucodon tomentosus Hook. (Hooker, 1818).

Neckera tomentosa (Hook.) C. Muell. (Mueller, 1850).

Neckera suborthosticha C. Muell. (Mueller, 1850).

Leucodon suborthostichus (C. Muell.) De Not. (De Notaris, 1859).

Lepyrodon suborthostichus (C. Muell.) Hamp. (Hampe, 1862b).

Distr. Juan Fernandez (Brotherus, 1924, 1925; Bartram, 1959; Robinson, 1975).

Southern South America (Lorentz, 1866; Mitten, 1869; Jaeger, 1877; Kurtz, 1904; Paris, 1905a; Campo, 1921; Cardot and Brotherus, 1923; Brotherus, 1925; Roivainen, 1934; Thériot, 1935a; Hosseus, 1936, 1937; Herzog, 1938, 1957, 1960; Kühnemann,
1938).

var. minor Card. et Broth. (Cardot and Brotherus, 1923).

Distr. Southern South America (Cardot and Brotherus, 1923).

var. patagonicus Card. et Broth. (Cardot and Brotherus, 1923).

Distr. Southern South America (Cardot and Brotherus, 1923; Seki. 1974).

\section{Lescuraea}

L. chilensis Herz. (Herzog, 1954).

Distr. Southern South America (Herzog, 1954).

\section{Leskea}

L. albicans Hedw.

L. caespitosa Hedw.

L. concinna Hook.

L. cristata Hedw.

L. distans Mont.

L. fuegiana Besch.

L. gayana Mont.

L. glabella Hedw.

L. inclinata (Brid.) Web. et Mohr

L. julacea Schwaegr.

L. leucostega Brid.

L. microphylla (Hedw.) Sw.

L. mollis Hedw.

Cyclodictyon albicans

Sematophyllum caespitosum

Lopidium concinnum

Eriopus cristatus

Glyphothecium gracile

Hygroamblystegium fuegianum

Ptychomnion ptychocarpum

Homalia glabella

Bryum inclinatum

Myurella julacea

Stereophyllum leucostegum

Haplocladium microphyllum

Weymouthia mollis 
L. nitida Hook. f. et Wils.

Catagonium nitidum

L. ornithopodioides (Brid.) Brid.

Racopilum tomentosum

L. pellicula (C. Muell.) Broth. (Brotherus, 1907).

Syn. Anomodon pellicola C. Muell. (Mueller in Kindberg, 1891) nom. nud. Anomodon pellicula C. Muell. (Mueller, 1897a).

Distr. Southem South America (Mueller, 1897a; Paris, 1900a, 1903; Brotherus, 1907, 1918, 1925; Kühnemann, 1938).

L. pellucida Hook.

Dimerodontium pellucidum

L. pseudo-leskeoides Broth. (Brotherus in Kurtz, 1904) nom. nud.

Distr. Southern South America (Kurtz, 1904; Hosseus, 1936, 1937; Kühnemann, 1938).

L. remotifolia C. Muell.

Meteoriopsis remotifolia

L. rutilans Brid.

L. seminervis Kunz. ex Schwaegr.

L. varia Hedw. Vesicularia vesicularis Juratzkaea seminervis Amblystegium varium

\section{Leskeadelphus}

L. angustifolius Herz. (Herzog, 1952a).

Distr. Southern South America (Herzog, 1952a).

L. catenulatulus (C. Muell.) Herz. (Herzog, 1916a).

Syn. Pseudoleskea catenulatula C. Muell. (Mueller, 1897b).

Distr. Southern South America (Mueller, 1897a, b; Paris, 1905b; Brotherus, 1925; Kühnemann, 1938).

\section{Leucobryum}

L. argentinicum C. Muell. (Mueller, 1879a).

Distr. Southern South America (Mueller, 1879a; Paris, 1905a; Kühnemann, 1938).

L. crispum C. Muell. (Mueller, 1848a).

Distr. Southem South America (Hosseus, 1940).

L. giganteum C. Muell. (Mueller, 1848a).

Syn. Leucobryum longifolium Hamp. (Hampe, 1847) hom. illeg.

Distr. Southem South America (Jaeger, 1872, 1873).

L. longifolium Hamp.

Leucobryum giganteum

L. sordidum Aongstr. (Ångström, 1876a).

Distr. Southern South America (Hosseus, 1940).

\section{Leucodon}

L. coronatus (Mont) Mitt.

L. curtipendulus (Hedw.) T. Jens.

Forsstroemia coronata

L. felipponei Thér. (Thériot, 1939 [1941]).

Antitrichia curtipendula

Distr. Southern South America (Thériot, 1939).

L. glaucus (C. Muell.) Jaeg.

Lepyrodon hexastichus

L. gracilis Hamp.

L. hexastichus Mont.

L. implexus Kunz. ex Hook. f.

L. julaceus (Hedw.) Sull. (Sullivant, 1846).

Syn. Pterigynandrum julaceum Hedw. (Hedwig, 1801). 
Maschalocarpus julaceus (Hedw.) Spreng. (Sprengel, 1827).

Grimmia julacea (Hedw.) Web. et Mohr (Weber and Mohr, 1803).

Pterogonium julaceum (Hedw.) Schwaegr. (Schwaegrichen, 1811).

Hypnum julaceum (Hedw.) Schleich. (Schleicher, 1821) hom. illeg.

Fissidens julaceus (Hedw.) Lindb. (Lindberg, 1883).

Leucodontiella julacea (Hedw.) Nog. (Noguchi, 1947).

Distr. Southern South America (Hornschuch, 1820b; Bridel, 1827b; Montagne, 1850).

L. kunzeanus C. Muell.

L. lagurus Hook.

L. parvulus (Mitt.) Jaeg.

L. suborthostichus (C. Muell.) De Not.

L. tomentosus Hook.

\section{Leucodontella}

L. julacea (Hedw.) Nog.

\section{Leucoloma}

L. alboalare Dus.

L. australe (Besch.) Broth.

L. billardieri (Brid.) Broth.

L. capillare Dus.

L. capillifolium Broth.

L. dusenii Broth.

L. fuegianum Dus.

var. laxum Dus.

L. grandialare Dus.

L. hariotii (C. Muell.) Broth.

L. imponens (Mont.) Dus.
Lepyrodon hexastichus

Lepyrodon lagurus

Lepyrodon parvulus

Lepyrodon tomentosus

Lepyrodon tomentosus

Leucodon julaceus

L. longifolium (Brid.) Wijk et Marg. (van der Wijk and Margadant, 1960b).

Syn. Trichostomum longifolium Brid. (Bridel, 1806).

Didymodon longifolius (Brid.) Hook. f. et Wils. hom. illeg.

Distr. Juan Fernandez (Montagne, 1835, 1850; Johow, 1896).

Southern South America (Wilson and Hooker, 1847).

L. menziesii (Hook. f. et Wils.) Broth.

Dicranoloma hariotii

Dicranoloma australe

Dicranoloma billardieri

Dicranoloma capillare

Dicranoloma menziesii

Dicranoloma dusenii

Dicranoloma hariotii

Dicranoloma hariotii

Dicranoloma robustum

Dicranoloma hariotii

Dicranoloma imponens

L. muelleri Dus.

Dicranoloma menziesii

var. strictifolium Dus.

Dicranoloma muelleri

L. nigricaule (Aongstr.) Broth.

var. flexuosulum Dus.

var. gracile Dus.

Dicranoloma robustum var. strictifolium
Dicranoloma nigricaule

Dicranoloma nigricaule var. flexuosulum

Dicranoloma capillare

L. perremotifolium Dus.

Dicranoloma perremotifolium

var. fragile Dus.

Dicranoloma perremotifolium var. fragile

L. peruncinatum Dus.

Dicranoloma nigricaule

var. gracile Dus.

Dicranoloma capillare

L. pungens (Hook. f. et Wils.) Broth.

Dicranoloma robustum

L. robustum (Hook. f. et Wils.) Broth.

Dicranoloma robustum

var. flexuosum Dus.

Dicranoloma australe

var. lagunicola Dus.

Dicranoloma imponens

L. setosum (Hook. f. et Wils.) Broth.

Dicranoloma robustum var. setosum 
L. suberectum (Hamp.) Broth. Dicranoloma menziesii

L. subnigricaule Dus. (Dusén in Paris, 1905a) nom. nud.

Distr. Southern South America (Paris, 1905a).

L. syrrhodictyon (C. Muell.) Broth.

Dicranum syrrhopodontoides

\section{Leucomium}

L. crinitifolium (C. Muell.) Mitt. (Mitten, 1869).

Syn. Hypnum crinitifolium C. Muell. (Mueller, 1851a).

Distr. Southern South America (Mueller, 1851a; Mitten, 1869; Jaeger, 1878; Paris, 1905a; Brotherus, 1925).

Limbella nom. nud.

L. confluens (C. Muell.) C. Muell.

Sciaromium confluens

L. conspissata (Hook. f. et Wils.) C. Muell.

Sciaromium conspissatum

L. conspissatula C. Muell.

Sciaromium conspissatulum

L. drepanophyllopsis C. Muell.

L. krauseana C. Muell. Sciaromium drepanophyllopsis

L. negeri Broth. (Brotherus in Neger, 1899) nom. nud.

Sciaromium krauseanum

Distr. Southern South America (Neger, 1899).

L. pachyloma (Mont.) C. Muell.

Sciaromium pachyloma

L. pachylomata C. Muell.

L. platylomata C. Muell.

Sciaromium pachylomatum

Sciaromium platylomatum

\section{Limnobryum}

L. palustre (Hedw.) Rabenh.

Aulacomnium palustre

\section{Limprichtia}

L. revolvens (Sw.) Loesk.

Drepanocladus revolvens

\section{Lindigia}

L. Iorentzii C. Muell. (Mueller, 1879a).

Distr. Southern South America (Mueller, 1879a; Paris, 1905a; Brotherus, 1925; Kühnemann, 1938; Herzog, 1952a).

Lophiodon nom. rejic.

L. strictus Hook. f. et Wils.

Ditrichum strictum

\section{Lopidium}

L. concinnum (Hook.) Wils. (Wilson, 1854).

Syn. Leskea concinna Hook. (Hooker, 1818).

Hookeria concinna (Hook.) Hook. et Grev. (Hooker and Greville, 1825a).

Hypopterygium concinnum (Hook.) Brid. (Bridel, 1827b).

Lopidium pallens Hook. f. et Wils. (Hooker and Wilson in Wilson, 1854).

Hypopterygium pallens (Hook. f. et Wils.) Mitt. (Mitten, 1856).

Hypopterygium plumarium Mitt. (Mitten, 1869).

Lopidium plumarium (Mitt.) Hamp. (Hampe, 1879).

Hypopterygium pallens (Hook. f. et Wils.) Mitt. ssp. plumarium (Mitt.) Kindb.

(Kindberg, 1901).

Hypopterygium flexisetum Hamp. (Hampe in Lorentz, 1866) nom. nud.

Lopidium flexisetum Fleisch. (Fleischer, 1922) nom. nud.

Distr. Juan Fernandez (Skottsberg, 1914; Brotherus, 1924b. 1925; Espinosa B., 1941; Bartram, 1959; Matteri, 1973b; Robinson, 1975).

Southern South America (Montagne, 1850; Lorentz, 1866; Mitten, 1869; Jaeger, 1876; Neger, 1899; Kindberg, 1901; Paris, 1905a; Cardot, 1908a; Campo, 1915; Fleischer. 
1922; Cardot and Brotherus, 1923; Herzog, 1923, 1938, 1939, 1954, 1960;

Brotherus, 1925; Reimers, 1926; Thériot, 1934a; Kühnemann, 1938; Bartram, 1952;

Seki, 1974).

L. flexisetum Fleisch.

Lopidium concinnum

L. pallens Hook. f. et Wils.

Lopidium concinnum

L. plumarium (Mitt.) Hamp.

Lopidium concinnum

\section{Lorentziella}

L. gibertii C. Muell.

Lorentziella imbricata

L. glauca C. Muell. (Mueller, 1879a).

Distr. Southern South America (Mueller, 1879a; Jaeger and Sauerbeck, 1879b; Paris, 1905a;

Roth, 1911; Brotherus, 1924a; Kühnemann, 1938).

L. globiceps C. Muell.

Lorentziella imbricata

L. imbricata (Mitt.) Broth. (Brotherus, 1903).

Syn. Leptangium imbricatum Mitt. (Mitten, 1869).

Gigaspermum imbricatum (Mitt.) Kindb. (Kindberg, 1888).

Lorentziella globiceps C. Muell. (Mueller, 1879a).

Lorentziella paraguensis Besch. (Bescherelle, 1877).

Lorentziella gibertii C. Muell. (Mueller, 1888).

Distr. Southern South America (Mitten, 1869; Gibert, 1873; Jaeger and Sauerbeck, 1879b; Mueller, 1879a, 1888; Paris, 1896, 1904b, 1905a; Brothenus, 1903, 1924a; Kurtz. 1904; Roth, 1911, 1913; Felippone, 1912; Herter, 1928, 1933a, b, 1939a; Hosseus, 1935b, 1937, 1938a, d; Kühnemann, 1938).

L. paraguensis Besch.

Lorentziella imbricata

L. uruguensis C. Muell. (Mueller in Bescherelle, 1877).

Distr. Southern South America (Jaeger and Sauerbeck, 1879b: Paris, 1905a).

\section{Macrocoma}

M. filiforme (Hook. et Grev.) Grout

Macrocoma orthotrichoides

M. hymenostomum (Mont.) Grout (Grout, 1946).

Syn. Macromitrium hymenostomum Mont. (Montagne, 1845a).

Macromitrium hariotii Besch. ex C. Muell. (Bescherelle ex Mueller, 1885).

Distr. Juan Fernandez (Brotherus, 1924b, 1925).

Southern South America (Montagne, 1845a, 1850, 1856; Mueller, 1849, 1885; Mitten, 1869; Jaeger, 1874; Bescherelle, 1885a, 1889; Paris, 1905a; Cardot, 1908a; Cardot and Brotherus, 1923; Brotherus, 1925; Kühnemann, 1938).

M. orthotrichoides (Raddi) Wijk et Marg. (van der Wijk and Margadant, 1962).

Syn. Lasia orthotrichoides Raddi (Raddi, 1823).

Chaetephora orthotrichoides (Raddi) Brid. (Bridel, 1827b).

Orthotrichum filiforme Hook. et Grev. (Hooker and Greville, 1824a) nom. illeg.

Macromitrium filiforme Schwaegr. (Schwaegrichen, 1826) nom. illeg.

Leiotheca filiformis Brid. (Bridel, 1826) hom. illeg.

Macrocoma filiforme (Hook. et Grev.) Grout (Grout, 1944) nom. illeg.

Macromitrium quinquefarium Hornsch. (Hornschuch, 1840).

Distr. Southern South America (Schwaegrichen, 1826; Bridel, 1827a; Montagne, 1839c, 1850; Hornschuch, 1840; Mueller, 1849; Mitten, 1869; Paris, 1905a; Felippone, 1912;

Brotherus, 1925; Herter, 1933a; Kühnemann, 1938; Herzog, 1952a).

M. sullivantii (C. Muell.) Grout (Grout, 1944).

Syn. Macromitrium sullivanti C. Muell. (Mueller, 1862).

Macromitrium paraphysatum Mitt. (Mitten, 1869) fide Vitt, 1973.

Macromitrium paraphysatum Mitt. var. chilense Thér. (Thériot, 1921a) fide Vitt, 1973. 
Distr. Juan Fernandez (Robinson, 1975).

Southem South America (Mitten, 1869; Jaeger, 1874; Paris, 1905a; Thériot, 1921a; Brotherus, 1925).

\section{Macromitrium}

M. aciculare Brid.

see Macromitrium filicaule

M. anacamptophyllum C. Muell. (Mueller, 1879a).

Distr. Southern South America (Mueller, 1879a; Paris, 1905a; Kühnemann, 1938).

M. angulicaule C. Muell. (Mueller, 1897a).

Distr. Southern South America (Mueller, 1897a; Paris, 1900a, 1905a; Brotherus, 1925; Kühnemann, 1938).

M. apiculatum (Hook.) Schwaegr.

Groutiella apiculata

M. bifasciculare C. Muell. ex Dus. (Mueller ex Dusén, 1903a).

Distr. Southem South America (Dusén, 1903a; Kühnemann, 1938).

M. bifasciculatum C. Muell. (Mueller, 1898a).

Distr. Southem South America (Mueller, 1898a; Paris, 1900a, 1905a; Cardot, 1908a; Cardot and Brotherus, 1923; Brotherus, 1925).

M. campoanum Thér. (Thériot in Herzog, 1938).

Distr. Southem South America (Herzog, 1938).

M. coriaceum Bartr.

Macromitrium tenax

M. crassiusculum Lor. (Lorentz, 1866).

Distr. Southern South America (Lorentz, 1866; Jaeger, 1874; Paris, 1905a; Brotherus, 1925; Thériot, 1925a; Herzog, 1938, 1960).

M. didymodon Schwaegr.

Macromitrium richardii

M. fernandezianum Broth. (Brotherus, 1924b).

Distr. Juan Fernandez (Brotherus, 1924b. 1925; Espinosa B., 1941; Bartram, 19்59; Robinson, 1975).

M. filicaule C. Muell. (Mueller, 1849).

Distr. Juan Fernandez (? Montagne, 1835 (as M. aciculare Brid.)).

Southern South America (Paris, 1905a).

M. filiforme Schwaegr.

Macrocoma orthotrichoides

M. fimbriatum (P. Beauv.) Schwaeg. (Schwaegrichen, 1823).

Syn. Orthotrichum fimbriatum P. Beauv. (Palisot de Beauvois, 1805a).

Distr. Juan Fernandez (Montagne, 1850; Johow, 1896).

M. gracillimum (Besch.) Broth. (Brotherus, 1902).

Syn. Schlotheimia gracillima Besch. (Bescherelle, 1885a).

Distr. Southern South America (Bescherelle, 1885a, 1889; Brotherus, 1902, 1925; Paris, 1905b; Cardot, 1908a; Herzog, 1926, 1954; Kühnemann, 1938; Seki, 1974).

M. hariotii Besch. ex C. Muell.

Macrocoma hymenostomum

M. hymenostomum Mont. Macrocoma hymenostomum

M. jamesonii (Arnott) C. Muell.

Schlotheimia jamesonii

M. krausei Lor. (Lorentz, 1866).

Distr. Southern South America (Lorentz, 1866; Jaeger, 1874; Mueller, 1885; Cardot, 1908a; Brotherus, 1925; Herzog, 1938; Bartram, 1952). 
M. longipes (Hook.) Schwaegr. (Schwaegrichen, 1824).

Syn. Orthotrichum longipes Hook. (Hooker, 1818).

Distr. Southern South America (Wilson and Hooker, 1847).

M. longirostre (Hook.) Schwaegr. (Schwaegrichen, 1823).

Syn. Orthotrichum longirostre Hook. (Hooker, 1818).

Distr. Southern South America (Mitten, 1869; Paris, 1905a; Brotherus, 1925).

M. macrocomoides C. Muell. (Mueller, 1898a).

Distr. Southern South America (Mueller, 1898a; Paris, 1900a, 1905a; Cardot, 1908a; Brotherus, 1925; Kühnemann, 1938).

M. masafuerae Broth. (Brotherus, 1924b).

Distr. Juan Fernandez (Brotherus, 1924b, 1925; Bartram, 1959; Robinson, 1975).

M. microcarpum C. Muell. (Mueller, 1849).

Distr. Southern South America (Mueller, 1849; Montagne, 1850; Mitten, 1869; Jaeger, 1874;

Paris, 1905a; Brotherus, 1925; Thériot, 1935a; Herzog, 1939, 1960; Bartram, 1952).

M. microstomum (Hook. et Grev.) Schwaegr. (Schwaegrichen, 1827a).

Syn. Orthotrichum microstomum Hook. et Grev. (Hooker and Greville, 1824a).

Leiotheca microstoma (Hook. et Grev.) Brid. (Bridel, 1826).

Macromitrium saxatile Mitt. (Mitten, 1869) fide Robinson, 1975.

Distr. Juan Fernandez (Montagne, 1835; Mitten, 1869, 1885; Jaeger, 1874; Johow, 1896; Paris, 1905a; Brotherus, 1924b, 1925; Espinosa B., 1941; Bartram, 1959; Robinson, 1975).

M. ottonis (Schwaegr.) C. Muell.

Schlotheimia torquata

M. papillosum Thér. (Thériot in Herzog, 1938).

Distr. Southern South America (Herzog, 1938, 1954).

M. paraphysatum Mitt.

Macrocoma sullivantii

var. chilense Thér.

Macrocoma sullivantii

M. pertriste C. Muell. (Mueller, 1898a).

Distr. Southern South America (Mueller, 1898a; Paris, 1900a, 1905a; Thériot, 1921a, 1934b; Brotherus, 1925).

M. poeppigii Dub.

Zygodon pentastichus

M. pseudoserrulatum Bartr. (Bartram, 1965).

Distr. Southern South America (Bartram, 1965).

M. quinquefarium Hornsch.

Macrocoma orthotrichoides

M. richardii Schwaegr. (Schwaegrichen, 1826).

Syn. Macromitrium didymodon Schwaegr. (Schwaegrichen, 1827a).

Distr. Southern South America (Krieger, 1904).

M. saddleanum Besch. ex C. Muell. (Bescherelle ex Mueller, 1885).

Distr. Southern South America (Bescherelle, 1885a, 1889; Mueller, 1885; Paris, 1905a; Cardot, 1908a; Cardot and Brotherus, 1923; Brotherus, 1925; Herzog, 1938, 1954; Kühnemann, 1938).

M. saxatile Mitt.

Macromitrium microstomum

M. spurio-crispulum Dus. (Dusén, 1903a) nom. nud.

Distr. Southern South America (Dusén, 1903a; Paris, 1905a). 
M. subpiliferum Dus. (Dusén, 1903a) nom. nud.

Distr. Southem South America (Dusén, 1903a).

M. sullivantii C. Muell.

Macrocoma sullivantii

M. tenax C. Muell. (Mueller, 1884 [1883]).

Syn. Macromitrium coriaceum Bartr. (Bartram, 1946) fide Seki, 1974.

Distr. Southern South America (Mueller, 1884, 1885, 1889; Dusén, 1903a; Paris, 1905a; Cardot, 1908a; Cardot and Brotherus, 1923; Brotherus, 1925; Roivainen, 1934;

Kühnemann, 1938; Bartram, 1946; Seki, 1974).

var. theriotii Card. (Cardot, 1908a).

Syn. Macromitrium theriotii Card. (Cardot, 1908a) nom. inval.

Distr. Southem South America (Cardot, 1908a).

M. theriotii Card.

Macromitrium tenax var. theriotii

M. tomentosum Hornsch. Groutiella tomentosa

M. torquatum (Hedw.) C. Muell.

M. trichomitrium (Schwaegr.) C. Muell. Schlotheimia torquata Schlotheimia trichomitria

M. urceolatum (Hook.) Brid. (Bridel, 1826).

Syn. Orthotrichum urceolatum Hook. (Hooker, 1819). Leiotheca urceolata (Hook.) Brid. (Bridel, 1826).

Distr. Juan Fernandez (Montagne, 1835; Johow, 1896). Southern South America (Montagne, 1850).

\section{Macromitrium sp.}

Syn. Zygodon hatcheri Dus. (Dusén, 1903a).

Distr. Southem South America (Dusén, 1903a; Cardot, 1908a; Brotherus, 1925; Kühnemann, 1938).

Maschalocarpus nom. illeg.

M. julaceus (Hedw.) Spreng.

Leucodon julaceus

M. smithii (Hedw.) Spreng.

Leptodon smithii

\section{Meesia}

M. alpina Funck ex Bruch

M. austro-georgica C. Muell.

Meesia uliginosa var. alpina

Philonotis acicularis

M. hymenostoma Card. et Broth. (Cardot and Brotherus, 1923).

Distr. Southem South America (Cardot and Brotherus, 1923; Brotherus, 1924a; Kühnemann, 1938).

M. patagonica Dus. (Dusén, 1903a).

Distr. Southem South America (Dusén, 1903a; Cardot, 1908a; Brotherus, 1924a; Kabiersch, 1937; Kühnemann, 1938).

M. stygia (Sw.) Brid.

Cinclidium stygium

M. trichodes Spruc. var. alpina (Bruch) Braithw.

Meesia uliginosa var. alpina

M. uliginosa Hedw. var. alpina (Bruch) Hamp. (Hampe, 1837a).

Syn. Meesia alpina Funck ex Bruch (Funck ex Bruch, 1826).

Meesia trichodes Spruc. var. alpina (Bruch) Braithw. (Braithwaite, 1895).

Distr. Southem South America (Cardot and Brotherus, 1923; Kabiersch, 1937; Kühnemann, 1938).

M. vagans (Hook. f. et Wils.) C. Muell.

Philonotis vagans 


\section{Meteoriopsis}

M. ambigua (Hornsch.) Broth. (Brotherus, 1906).

Syn. Pilotrichum ambiguum Hornsch. (Hornschuch, 1840). Meteorium ambigum (Hornsch.) Mitt. (Mitten, 1869).

Distr. Southern South America (Hampe, 1879).

M. characea (C. Muell.) Broth. (Brotherus, 1906).

Syn. Neckera characea C. Muell. (Mueller, 1879a).

Pilotrichella characea (C. Muell.) Kindb. (Kindberg, 1888).

Distr. Southern South America (Mueller, 1879a; Paris, 1905b; Brotherus, 1906, 1925; Kühnemann, 1938).

var. nigrescens (C. Muell.) Wijk et Marg. (van der Wijk and Margadant, 1962).

Syn. Neckera characea C. Muell. var. nigrescens C. Muell. (Mueller, 1879a). Pilotrichella characea (C. Muell.) Kindb. var. nigrescens (C. Muell.) Par. (Paris, 1897).

Distr. Southern South America (Mueller, 1879a; Paris, 1897, 1905b).

var. tenuis (C. Muell.) Wijk et Marg. (van der Wijk and Margadant, 1962).

Syn. Neckera characea C. Muell. var. tenuis C. Muell. (Mueller, 1879a). Pilotrichella characea (C. Muell.) Kindb. var. tenuis (C. Muell.) Par. (Paris, 1897).

Distr. Southern South America (Mueller, 1879a; Paris, 1897, 1905b).

M. decurrens (Broth.) Broth. (Brotherus, 1906).

Syn. Meteorium decurrens Broth. (Brotherus, 1900).

Distr. Southern South America (Herzog, 1952a).

M. implanata (Mitt.) Broth. (Brotherus, 1906b).

Syn. Meteorium implanatum Mitt. (Mitten, 1869).

Distr. Southern South America (Herzog, 1952a).

M. onusta (Mitt.) Broth. (Brotherus, 1906b).

Syn. Meteorium onustum Spruc. ex Mitt. (Spruce ex Mitten, 1869).

Distr. Southern South America (Herzog, 1952a).

M. piligera (C. Muell.) Broth. (Brotherus, 1906b).

Syn. Meteorium piligerum C. Muell. (Mueller, 1901b).

Distr. Southern South America (Herzog, 1952a).

M. remotifolia (C. Muell.) Broth. (Brotherus, 1906b).

Syn. Leskea remotifolia C. Muell. (Mueller, 1847a [1846]).

Distr. Southern South America (Hosseus, 1940).

M. straminea (C. Muell.) Broth. ex Par. (Brotherus ex Paris, 1909).

Syn. Pilotrichum stramineum C. Muell. (Mueller, 1850). Meteorium stramineum (C. Muell.) Mitt. (Mitten, 1869).

Distr. Southern South America (Mueller, 1850; Mitten, 1869; Jaeger, 1877; Paris, 1905a; Brotherus, 1925).

\section{Meteorium}

M. ambiguum (Hornsch.) Mitt.

Meteoriopsis ambigua

M. ancistrodes (Mont.) Par.

Ancistrodes genuflexa

M. billardieri (Hamp.) Mitt.

Weynouthia cochlearifolia var. billardieri

M. decurrens Broth.

Meteoriopsis decurrens

M. excavatum Mitt. 
M. filipendulum Hook. f. et Wils.

M. flexicaule Wils.

M. genuflexum (C. Muell.) Mitt.

Ancistrodes genuflexa

M. illecebrinum (C. Muell.) Broth. (Brotherus, 1925).

Syn. Neckera illecebrina C. Muell. (Mueller, 1879a).

Pilotrichella illecebrina (C. Muell.) Kindb. (Kindberg, 1888).

Distr. Southern South America (Mueller, 1879a; Paris, 1905b; Brotherus, 1925; Kühnemann, 1938; Herzog, 1952a).

M. implanatum Mitt.

Meteoriopsis implanata

M. lorentzii C. Muell.

Squamidium lorentzii

M. medium (Aongstr.) Broth. (Brotherús, 1906b).

Syn. Papillaria media Aongstr. (Ångström, 1876a).

Distr. Southern South America (Herter, 1933a).

M. molle Wils.

Weymouthia mollis

M. onustum Spruc. ex Mitt.

Meteoriopsis onusta

M. piligerum C. Muell.

Meteoriopsis piligera

M. stramineum (C. Muell.) Mitt.

Meteoriopsis straminea

M. striatum Lindb. (Lindberg, 1864b [1865]).

Distr. Southern South America (Lindberg, 1864b; Paris. 1905a).

\section{Microdus}

M. euchlorus (Mont.) Besch. (Bescherelle in Paris, 1896).

Syn. Dicranum euchlorum Mont. (Montagne, 1845a).

Aongstroemia euchlora (Mont.) C. Muell. (Mueller, 1848a).

Dicranella euchlora (Mont.) Lor. (Lorentz, 1866).

Distr. Southern South America (Montagne, 1845a, 1850, 1856; Mueller, 1848a; Lorentz, 1866; Mitten, 1869; Jaeger, 1872; Paris, 1896, 1905a).

M. hilarianus (Mont.) Besch.

Dicranella hilariana

M. kunzeanus (C. Muell.) Broth. (Brotherus, 1924a).

Syn. Leptotrichum kunzeanum C. Muell. (Mueller, 1847c).

Seligeria kunzeana (C. Muell.) C. Muell. (Mueller, 1848a).

Dicranella kunzeana (C. Muell.) Mitt. (Mitten, 1869).

Seligeria kunzei Kindb. (Kindberg, 1888) nom. illeg.

Weisia tenuis Kunz. ex Mont. (Kunze ex Montagne, 1850) hom. illeg.

Microdus tenuis Par. (Paris, 1896) nom. illeg.

Distr. Southern South America (Mueller, 1847c, 1848a; Montagne, 1850; Mitten, 1869; Jaeger, 1873; Paris, 1896, 1905; Brotherus, 1924a).

M. tenuirostris (Schwaegr.) Besch.

Dicranella hilariana

M. tenuis Par.

Microdus kunzeanus

Micromitrium

M. tomentosum (Hornsch.) Par.

Groutiella tomentosa

\section{Microthamnium}

M. flavidum Aongstr.

Mittenothamnium simorhynchum

M. gracile (Hook. f. et Wils.) Jaeg.

Camptochaete gracilis

$M$. humile Besch.

Mittenothamnium humile

M. hylophilum C. Muell.

Mittenothamnium hylophilum

M. longo-reptans C. Muell. 
M. plinthophilum (C. Muell.) Par.

M. pseudo-elegans C. Muell.

M. pumilum (Hook. f. et Wils.) Jaeg.

M. sellovii (Hornsch.) Mitt.

M. tapes C. Muell.

M. thelistegum (C. Muell.) Mitt.

M. widgrenii Aongstr.
Mittenothamnium plinthophilum

Mittenothamnium pseudo-elegans Thamnobryum pumilum

Mittenothamnium sellovii

Mittenothamnium tapes

Mittenothamnium diminutivum

Mittenothamnium camptorryhnchium

\section{Mielichhoferia}

M. acuminata C. Muell. (Mueller, 1879a).

Distr. Southern South America (Mueller, 1879a; Paris, 1905a; Brotherus, 1924a; Kühnemann, 1938).

M. auriseta C. Muell. (Mueller, 1879a).

Distr. Southern South America (Mueller, 1879a; Paris, 1905a; Brotherus, 1924a; Kühnemann, 1938).

var. alpina C. Muell. (Mueller, 1879a).

Distr. Southern South America (Mueller, 1879a).

M. austro-georgica C. Muell. (Mueller, 1890a).

Distr. South Georgia (Mueller, 1890a; Paris, 1905a; Cardot, 1908a; Steere, 1961b; Clarke, 1973a).

M. brevicaulis Hornsch. (Hornschuch, 1840).

Distr. Southern South America (Hornschuch, 1840; Mueller, 1848a; Montagne, 1850: Mitten, 1869; Gibert, 1873; Jaeger, 1875; Krieger, 1904; Paris, 1905a; Brotherus, 1924a: Herter, 1933a).

M. chilensis (Mont.) Mitt. (Mitten, 1869).

Syn. Leptochlaena chilensis Mont. (Montagne, 1845a).

Distr. Southern South America (Montagne, 1845a, 1850, 1856; Mueller, 1848a; Mitter. 1869: Jaeger, 1875; Paris, 1905a; Brotherus, 1924a; Espinosa B., 1924).

M. coarctata C. Muell. (Mueller, 1879a).

Distr. Southern South America (Mueller, 1879a; Paris, 1905a; Brotherus, 1924a; Kühnemann. 1938)

M. cygnicolla Hamp.

Anomobryum cygnicollum

M. demissa C. Muell. (Mueller, 1858).

Distr. Southern South America (Mueller, 1858; Jaeger, 1875; Paris, 1905a; Brotherus. 1924a).

M. emergens (C. Muell.) Broth. (Brotherus, 1904).

Syn. Bryum emergens C. Muell. (Mueller, 1879a).

Webera emergens (C. Muell.) Kindb. (Kindberg, 1889).

Distr. Southern South America (Mueller, 1879a; Brotherus, 1904; Paris, 1905a; Kühnemann. 1938).

M. fulvonitens Card. et Broth. (Cardot and Brotherus, 1923).

Distr. Southern South America (Cardot and Brotherus, 1923; Brotherus, 1924a; Kühnemann. 19.38).

M. gayana Card.

Mielichhoferia pleurogyna

Ni. humipetens (C. Muell.) Par.

Haplodontium humipetens

M. immersidens C. Muell. (Mueller, 1879a).

Distr. Southern South America (Paris, 1905a; Brotherus, 1924a; Kühnemann, 1938). 
M. leptoclada C. Muell. (Mueller, 1879a).

Distr. Southern South America (Mueller, 1879a; Paris, 1905a; Brotherus, 1924a; Kühnemann, 1938).

M. longiseta C. Muell. (Mueller, 1848a).

Syn. Bryum nutans (Hedw.) Turn. var. minus Hook. (Hooker in Kunth, 1822).

Webera nutans Hedw. var. minor (Hook.) Brid. (Bridel, 1827a).

Distr. Juan Fernandez (Mitten, 1885; Johow, 1896; Paris, 1905a; Brotherus, 1924b; Robinson, 1975).

M. Iorentziana C. Muell. (Mueller, 1879a).

Syn. Mielichhoferia lorentzii C. Muell. ex Kindb. (Mueller ex Kindberg, 1889) nom. illeg.

Distr. Southern South America (Mueller, 1879a; Kurtz, 1904; Paris, 1905a; Brotherus, 1924a; Hosseus, 1936, 1937; Kühnemann, 1938).

M. Iorentzii C. Muell. ex Kindb.

Mielichhoferia lorentziana

M. manca (C. Muell.) Broth. (Brotherus, 1903).

Syn. Bryum mancum C. Muell. (Mueller, 1879a).

Webera manca (C. Muell.) Kindb. (Kindberg, 1889).

Distr. Southern South America (Mueller, 1879a; Brotherus, 1903, 1924a; Paris, 1906: Kühnemann, 1938).

M. micropoma C. Muell. (Mueller, 1879a).

Distr. Southern South America (Mueller, 1879a; Paris, 1905a; Brotherus, 1924a; Kühnemann, 1938).

var. brunnescens C. Muell. (Mueller, 1879a).

Distr. Southern South America (Mueller, 1879a).

M. multiflora Bartr. (Bartram, 1946).

Distr. Southern South America (Roivainen, 1934; Bartram, 1946).

M. ochracea C. Muell. (Mueller, 1879a).

Distr. Southern South America (Mueller, 1879a; Paris, 1905a; Brotherus, 1924a; Kühnemann, 1938).

M. pampae C. Muell. (Mueller, 1882).

Distr. Southern South America (Mueller, 1882; Paris, 1905a; Brotherus, 1924a; Kühnemann, 1938).

M. patagonica C. Muell. (Mueller, 1897a).

Distr. Southern South America (Mueller, 1897a; Paris, 1900a, 1905a).

M. pernana (C. Muell.) Par.

Haplodontium sanguinolentum

var. robustior (C. Muell.) Par.

Haplodontium pernanum var. robustior

M. pleurogena Mont. ex C. Muell.

Mielichhoferia pleurogyna

M. pleurogyna Mont. (Montagne, 1845a).

Syn. Mielichhoferia pleurogena Mont. ex C. Muell. (Montagne ex Mueller, 1848c) err.

Mielichhoferia gayana Card. (Cardot in Thériot, 1921b) nom. nud.

Distr. Southern South America (Montagne, 1845a, 1850, 1856; Mueller, 1848a, c; Lorentz, 1866; Mitten, 1869; Jaeger, 1875; Paris, 1905a; Thériot, 1921b, 1934b; Brotherus, 1924a; Looser, 1932; Bartram, 1952).

M. pohlioidea C. Muell. (Mueller, 1879a).

Distr. Southern South America (Mueller, 1879a; Kurtz, 1904; Paris, 1905a; Brotherus, 1924a; Hosseus, 1936, 1937, 1938c, d; Kühnemann, 1938). 
M. pulchra Broth. (Brotherus in Felippone, 1909).

Distr. Southern South America (Felippone, 1909; Brotherus, 1924a; Herter, 1933a; Herzog, 1952a).

M. sanguinolenta (C. Muell.) Par.

Haplodontium sanguinolentum

M. serridens C. Muell. (Mueller, 1879a).

Distr. Southern South America (Mueller, 1879a; Paris, 1905a; Brotherus, 1924a; Kühnemann, 1938).

M. skottsbergii Card. et Broth. (Cardot and Brotherus, 1923).

Distr. Southern South America (Cardot and Brotherus, 1923; Brotherus, 1924a; Kühnemann, 1938).

M. spegazzinii C. Muell. (Mueller, 1885).

Distr. Southern South America (Mueller, 1885; Paris, 1905a; Cardot, 1908a; Brotherus, 1924a; Kühnemann, 1938).

M. subpohlioidea Broth. (Brotherus, 1918).

Distr. Southern South America (Brotherus, 1918, 1924a; Hosseus, 1936, 1937; Kühnemann, 1938).

M. ulei C. Muell. (Mueller, 1898c).

Distr. Southern South America (Herter, 1943).

M. uruguensis Besch. (Bescherelle in Mueller, 1901a [1900]) nom. nud.

Distr. Southern South America (Mueller, 1901a).

\section{Mittenothamnium}

M. camptorrhynchium (Hamp.) Card. (Cardot, 1913b).

Syn. Hypnum camptorrhynchium Hamp. (Hampe, 1870).

Microthamnium widgrenii Aongstr. (Ångström, 1876a) nom. illeg.

Distr. Southern South America (Krieger, 1904).

M. diminutivum (Hamp.) Britt. (Britton, 1914).

Syn. Hypnum diminutivum Hamp. (Hampe, 1847).

Hypnum thelistegum C. Muell. (Mueller, 1851a).

Microthamnium thelistegum (C. Muell.) Mitt. (Mitten, 1869).

Distr. Southern South America (Hosseus, 1940; Herter, 1943; Herzog, 1952a).

M. humile (Besch.) Card. (Cardot, 1913b).

Syn. Microthamnium humile Besch. (Bescherelle, 1877).

Stereohypnum humile (Besch.) Herz. (Herzog, 1909).

Distr. Southern South America (Brotherus, 1918, 1925; Kühnemann, 1938).

M. hylophilum (C. Muell.) Card. (Cardot, 1913b).

Syn. Microthamnium hylophilum C. Muell. (Mueller, 1897a).

Distr. Southern South America (Mueller, 1897a; Paris, 1900a, 1905a; Brotherus, 1925; Kühnemann, 1938; Herzog, 1952a).

M. longoreptans (C. Muell.) Card. (Cardot, 1913b).

Syn. Microthamnium longo-reptans C. Muell. (Mueller, 1897a).

Distr. Southern South America (Mueller, 1897a; Paris, 1900a, 1905a; Brotherus, 1925; Kühnemann, 1938).

M. plinthophilum (C. Muell.) Card. (Cardot, 1913b).

Syn. Hypnum plinthophilum C. Muell. (Mueller, 1879a).

Microthamnium plinthophilum (C. Muell.) Par. (Paris, 1896). 
Distr. Southem South America (Mueller, 1879a; Paris, 1896, 1905a; Brotherus, 1925; Herter, 1933a; Kühnemann, 1938; Hosseus, 1939).

M. pseudo-elegans (C. Muell.) Card. (Cardot, 1913b).

Syn. Microthamnium pseudo-elegans C. Muell. (Mueller, 1897a).

Distr. Southem South America (Mueller, 1897a; Paris, 1900a, 1905a; Brotherus, 1925; Kühnemann, 1938).

M. sellovii (Hornsch.) Card. (Cardot, 1913b).

Syn. Hookeria sellovii Hornsch. (Hornschuch, 1840). Microthamnium sellovii (Hornsch.) Mitt. (Mitten, 1869).

Distr. Southem South America (Herter, 1928, 1933a, b).

M. simorhynchum (Hamp.) Card. (Cardot, 1913b).

Syn. Hypnum simorhynchum Hamp. (Hampe, 1870). Microthamnium flavidum Aongstr. (Ångström, 1876a) nom. illeg.

Distr. Southern South America (Krieger, 1904).

M. tapes (C. Muell.) Card. (Cardot, 1913b).

Syn. Microthamnium tapes C. Muell. (Mueller, 1897a). Stereohypnum tapes (C. Muell.) Fleisch. (Fleischer, 1908a).

Distr. Southern South America (Mueller, 1897a; Paris, 1905a; Brotherus, 1925; Kühnemann, 1938).

Mniadelphus nom. illeg.

M. apiculatus (Hook. f. et Wils.) Mitt.

Eriopus apiculatus

M. assimilis Broth. Distichophyllum assimile

M. cavifolius Card.

M. dicksonii (Hook. et Grev.) Hamp. Distichophyllum eremitae

M. eremitae Jaeg. Distichophyllum dicksonii

M. flaccidus (Hook. f. et Wils.) Hamp. Distichophyllum eremitae

$M$. hahnianus $C$. Muell. Distichophyllum flaccidum

M. hahnii C. Muell. Eriopus apiculatus

Eriopus apiculatus

M. krausei Lor. Distichophyllum kraussei

M. krausseiLor.

M. mollis C. Muell.

Distichophyllum kraussei

$\mathrm{M}$. nigricans $\mathrm{C}$. Muell.

Eriopus bescherellei

M. procumbens C. Muell.

Distichophyllum kraussei

M. rotundifolius (Hook. f. et Wils.) C. Muell.

Distichophyllum eremitae

Distichophyllum rotundifolium

\section{Mniobryum}

M. albicans (Wahlenb.) Limpr.

Pohlia wahlenbergii

M. alticaule (C. Muell.) Broth.

Pohlia wahlenbergii var. glacialis

var. crassinerve Broth. (Brotherus in Cardot and Brotherus, 1923).

Syn. Webera alticaulis (C. Muell.) Kindb. var. crassinervis Card. (Cardot in Cardot and Brotherus, 1923) nom. nud.

Distr. Southern South America (Cardot and Brotherus, 1923). Falkland Islands (Cardot and Brotherus, 1923).

var. robustius (C. Muell.) Par. (Paris, 1905a).

Syn. Bryum alticaule C. Muell. var. robustius C. Muell. (Mueller, 1889). Webera alticaulis (C. Muell.) Kindb. var. robustior (C. Muell.) Par. (Paris, 1898). 
Distr. Southern South America (Mueller, 1889; Paris, 1898, 1905a; Cardot, 1908a;

Kühnemann, 1938).

M. amplirete (C. Muell.) Broth.

Pohlia wahlenbergii var. glacialis

M. aspillacae Thèr. (Thériot, 1924).

Distr. Southern South America (Thériot, 1924; Brotherus, 1925).

M. austro-albicans (C. Muell.) Broth.

Pohlia wahlenbergii

M. philonoteum (C. Muell.) Broth. (Brotherus, 1903).

Syn. Bryum philonoteum C. Muell. (Mueller, 1885).

Webera philonotea (C. Muell.) Par. (Paris, 1906).

Webera philonotidea Par. (Paris, 1898) err.

Distr. Southern South America (Mueller, 1885; Paris, 1898, 1905a; Brotherus, 1903, 1924a;

Cardot, 1908a; Kühnemann, 1938; Herzog, 1954).

M. pulchellum (Hedw.) Loesk. (Loeske, 1910).

Syn. Bryum pulchellum Hedw. (Hedwig, 1801).

Distr. Southern South America (Hornschuch, 1820b; Montagne, 1850).

M. wahlenbergii (Web. et Mohr) Jenn.

Pohlia wahlenbergii

var. glaciale (Brid.) Wijket Marg.

Pohlia wahlenbergii var. glacialis

\section{Mnium}

M. affine Bland. ex Funck var. magellanicum Card. et Broth.

Plagiomnium rugicum

var. rugicum (Laur.) B.S.G.

Plagiomnium rugicum

M. albicans Wahlenb.

Pohlia wahlenbergii

M. aubertii Schwaegr.

Rhodobryum aubertii

$M$. beyrichianum Hornsch.

Rhodobryum beyrichianum

M. bimum Schreb.

Bryum pseudotriquetrum ssp. bimum

var. turbinatum (Hedw.) Wahlenb.

Bryum turbinatum

M. brevifolium (Broth.) C. Muell.

Rhizogonium brevifolium

M. caespiticium (Hedw.) With.

Bryum caespiticium

M. chloroloma C. Muell.

Mnium rostratum

M. cristatum (Hedw.) P. Beauv.

Eriopus cristatus

M. crudum Hedw.

Pohlia cruda

M. fontanum Hedw.

Philonotis fontana

var. magellanicum Brid.

M. lanatum P. Beauv.

M. lepto-limbatum C. Muell.

Philonotis fontana var. magellanica

M. lindigii Hamp.

Bryum argenteum var. lanatum

M. longicolle C. Muell. in Par.

Plagiomnium rugicum

Rhizogonium lindigii

M. longicolle C. Muell. (Mueller, 1901a [1900]) hom. illeg.

Rhizogonium longicolle

Distr. Southern South America (Mueller, 1901a).

M. longirostre Brid.

Mnium rostratum

M. palustre Hedw.

Aulacomnium palustre

M. polycarpum C. Muell.

Rhizogonium mnioides

M. pseudo-triquetrum Hedw.

Bryum pseudotriquetrum

M. punctulatum Brid.

Tortula robusta

M. reticulatum (Hook. f. et Wils.) C. Muell.

Philonotis vagans 
M. rigidum Hornsch.

M. roseum Hedw.

M. rostratum Schrad. (Schrader in Schrebius, 1802).

Syn. Hypnum rostratum (Schrad.) Web. et Mohr (Weber and Mohr, 1803).

Bryum rostratum (Schrad.) Sm. (Smith, 1804b).

Mnium longirostre Brid. (Bridel, 1803) nom. illeg.

Bryum longirostre Brid. (Bridel, 1819).

Mnium chloroloma C. Muell. (Mueller, 1882).

Distr. Southem South America Wilson and Hooker, 1847; Mueller, 1848a, 1882; Montagne, 1850; Gibert, 1873; Dusén, 1903a; Paris, 1905a; Cardot, 1908a; Brotherus, 1924a; Herter, 1933a, 1939a; Kühnemann, 1938; Hosseus, 1940; Herzog, 1952a).

M. rugicum Laur.

Plagiomnium rugicum

M. schleicheri (Cand.) Brid.

Bryum schleicheri

M. stygium (Sw.) B.S.G.

Cinclidium stygium

M. subbasilare (Hook.) C. Muell.

Goniobryum subbasilare

M. subpunctatum Card. et Broth.

Cinclidium stygium

M. truncorum Brid.

Bryum truncorum

M. turbinatum Hedw.

Bryum turbinatum

M. undulatum Hedw. (Hedwig, 1801).

Distr. Southem South America (Kühnemann, 1938).

\section{Molendoa}

M. excelsa (C. Muell.) Broth.

Molendoa sendtneriana

M. fuegiana Bartr. (Bartram, 1946).

Distr. Southern South America (Bartram, 1946).

M. sendtneriana (B.S.G.) Limpr. (Limpricht, 1886).

Syn. Anoectangium sendtnerianum B.S.G. (Bruch and others, 1846).

Zygodon excelsus C. Muell. (Mueller, 1879a) fide Zander, 1977.

Anoectangium excelsum (C. Muell.) Kindb. (Kindberg, 1889) fide Zander, 1977.

Molendoa excelsa (C. Muell.) Broth. (Brotherus, 1902) fide Zander, 1977.

Anoectangium lechlerianum Mitt. (Mitten, 1869) fide Zander, 1977.

Distr. Southern South America (Mueller, 1879a; Brotherus, 1902, 1924a; Paris, 1905a; Kühnemann, 1938; Bartram, 1965; Zander, 1977).

Mollia hom. illeg.

M. brachydontia (Bruch) Lindb.

Trichostomum brachydontium

M. humilis (Hedw.) Lindb.

Tortella humilis

M. tortuosa (Hedw.) Schrank ex Lindb.

Tortella tortuosa

\section{Monocranum}

M. dicranoides C. Muell. (Mueller, 1901a [1900]).

Distr. Southern South America (Mueller, 1901a).

Muelleriella hom. illeg.

M. crassifolia (Hook. f. et Wils.) Dus. (Dusén, 1905b).

Syn. Orthotrichum crassifolium Hook. f. et Wils. (Hooker, J. D. and Wilson, 1844).

Ulota crassifolia (Hook. f. et Wils.) Hook. f. (Hooker, 1867).

Muelleriella crassifolia (Hook. f. et Wils.) Dus. var. lingulata Dus. (Dusén, 1905b).

Distr. Southern South America Wilson and Hooker, 1847 (as vars 2 and 3): Mueller, 1849. 1885; Sullivant, 1859; Angström, 1872; Jaeger, 1874; Bescherelle. 1889; Dusén, 1905b; Paris, 1905b; Cardot, 1908a; Cardot and Brotherus, 1923: Brotherus, 1925; Vitt, 1976). 
Falkland Islands (Hooker, 1847 (as var. 1); Jaeger, 1874; Mueller, 1885; Bescherelle, 1889; Dusén, 1905b; Paris, 1905b; Cardot, 1908a; Cardot and Brotherus, 1923; Brotherus, 1925; Kühnemann, 1938; Vitt, 1976).

South Georgia (Cardot, 1906a, 1908a; Taylor, 1914; Cardot and Brotherus, 1923; Steere, 1961b; Vitt, 1976).

var. lingulata Dus.

Muelleriella crassifolia

\section{Muscoflorschuetzia}

M. pilmaiquen (Crosby) Crosby (Crosby, 1978).

Syn. Florschuetzia pilmaiquen Crosby (Crosby, 1977b) nom. illeg.

Distr. Southern South America (Crosby, 1977b).

\section{Myurella}

M. julacea (Schwaegr.) B.S.G. (Bruch and others, 1853).

Syn. Leskea julacea Schwaegr. (Schwaegrichen, 1804).

Distr. South Georgia (Newton, 1977a).

\section{Neckera}

N. amblyoglossa C. Muell. (Mueller, 1879a).

Distr. Southern South America (Mueller, 1879a; Paris, 1905a; Brotherus, 1925; Kühnemann, 1938).

N. appressa C. Muell.

Papillaria nigrescens

N. argentinica Lor. ex C. Muell. (Lorentz ex Mueller, 1879a).

Distr. Southern South America (Mueller, 1879a; Paris, 1905a; Brothenus, 1925; Williams, 1930; Kühnemann, 1938; Herzog, 1952a).

N. avellanedae C. Muell.

Orthostichopsis avellanedae

N. berteroana Schimp. ex Mitt. (Schimper ex Mitten, 1869).

Syn. Neckera berteroi Kindb. (Kindberg, 1888) nom. illeg.

Distr. Southern South America (Mitten, 1869; Jaeger, 1877; Paris, 1905a; Thériot, 1915; Brotherus, 1925).

N. berteroi Kindb.

Neckera berteroana

N. beyrichii Schwaegr.

Entodon beyrichii

N. billardieri Hamp.

N. characea C. Muell. Weymouthia cochlerifolia var. billardieri

var. nigrescens C. Muell.

Meteoriopsis characea

var. tenuis $\mathrm{C}$. Muell.

Meteoriopsis characea var. nigrescens

Meteoriopsis characea var. tenuis

N. chilensis Schimp. (Schimper, 1836).

Distr. Southern South America (Schimper, 1836; Montagne, 1850; Mueller, 1850; Mitten, 1869; Jaeger, 1877; Neger, 1899; Paris, 1905a; Cardot, 1908a; Campo, 1915; Thériot, 1915, 1917b; Costes, 1921; Herzog, 1923, 1938, 1940, 1954, 1957; Brotherus, 1924c, 1925; Reimers, 1926; Kühnemann, 1938; Espinosa B., 1941; Skottsberg, 1950; Seki, 1974).

N. coronata (Mont.) C. Muell.

Forsstroemia coronata

N. cumingii $\mathrm{C}$. Muell.

Weymouthia mollis

N. curtipendula Hedw.

Antitrichia curtipendula

N. cyathipoma C. Muell.

Pilotrichella cyathipoma

N. dillenii Lindb.

Homalia glabella

N. disticha Hedw.

Neckeropsis disticha

N. eriodon C. Muell. 
N. fasciculata (Hedw.) Arnott

Porothamnium fasciculatum

N. glabella (Hedw.) Web. et Mohr

Homalia glabella

N. glauca C. Muell.

Lepyrodon hexastichus

N. humboldtii (Hook.) C. Muell.

Rhacocarpus purpurascens

N. illecebrina C. Muell.

Meteorium illecebrinum

N. imberbis (Sm.) C. Muell.

Hedwigidium integrifolium

N. implexa C. Muell.

Lepyrodon hexastichus

N. jamesonii Tayl. (Taylor, 1846).

Distr. Southem South America (Mitten, 1869; Brotherus, 1925).

N. lagura (Hook.) C. Muell.

Lepyrodon lagurus

N. leucocaulon C. Muell.

Porothamnium leucocaulon

N. leucocolea Mitt.

Cryphidium leucocoleum

N. mollis (Hedw.) C. Muell.

Weymouthia mollis

N. patagonica Brid.

Homalia glabella

N. pennata Hedw. (Hedwig, 1801).

Distr. Southern South America (Montagne, 1850).

N. poeppigiana C. Muell.

Glyphothecium gracile

N. polytrichoides (Hedw.) Web. et Mohr

N. porteri Thér.

Lepidopilum polytrichoides

Neckera scabridens var. porteri

N. praelonga Lor. (Lorentz, 1866).

Distr. Southern South America (Lorentz, 1866; Paris, 1905a).

N. rotundata Broth. (Brotherus, 1924b).

Distr. Juan Fernandez (Brotherus, 1924b; Bartram, 1959; Robinson, 1975).

N. scabridens C. Muell. (Mueller, 1847C).

Distr. Southern South America (Mueller, 1847c, 1850; Sullivant, 1859; Lorentz, 1866; Mitten, 1869; Jaeger, 1877; Paris, 1905a; Cardot, 1908a; Herzog, 1923, 1954, 1960;

Brotherus, 1925; Reimers, 1926; Kühnemann, 1938; Bartram, 1952; Seki, 1974).

var. porteri Thér. (Thériot, 1915).

Syn. Neckera porteri Thér. (Thériot, 1915) nom. nud.

Distr. Southern South America (Thériot, 1915, 1917b; Costes, 1921).

N. scabriseta Schwaegr.

Lepidopilum scabrisetum

N. schnyderiC. Muell. Neckeropsis villae-ricae

N. smithii (Hedw.) C. Muell.

Leptodon smithii

N. suborthosticha C. Muell.

Lepyrodon tomentosus

N. tenella Schwaegr.

Cryphaea tenella

N. tomentosa (Hook.) C. Muell.

Lepyrodon tomentosus

N. trichoblepharis Schimp. (Schimper in Paris, 1896) nom. nud.

Distr. Southern South America (Paris, 1896, 1905a).

N. trichophora Schimp. (Schimper in Lorentz, 1866) nom. nud.

Distr. Southern South America (Lorentz, 1866; Jaeger, 1877; Paris, 1905a).

N. truncata C. Muell.

Entodon truncatus

N. turgidula C. Muell.

Squamidium turgidulum

var. tristis C. Muell.

Squamidium turgidulum var. triste 
N. undulata Hedw.

Neckeropsis undulata

N. uruguensis C. Muell. Neckeropsis villae-ricae

N. villae-ricae Besch. Neckeropsis villae-ricae

N. viticulosoides $\mathrm{P}$. Beauv. Leptodontium viticulosoides var. viticulosoides

\section{Neckeropsis}

N. disticha (Hedw.) Kindb. (Kindberg, 1894).

Syn. Neckera disticha Hedw. (Hedwig, 1801).

Distr. Southern South America (Paris, 1905a).

N. undulata (Hedw.) Reichdt. (Reichardt, 1870).

Syn. Neckera undulata Hedw. (Hedwig, 1801). Hypnum harrisonia Poir. (Poiret in Bridel, 1827b) nom. nud.

Distr. Southern South America (Bridel, 1801b, 1817, 1827b; Paris, 1905a; Hosseus, 1940; Herzog, 1952a).

N. villae-ricae (Besch.) Broth. ex Par. (Brotherus ex Paris, 1909).

Syn. Neckera villae-ricae Besch. (Bescherelle, 1877).

Neckera uruguensis C. Muell. (Mueller, 1879a).

Neckera schnyderi C. Muell. (Mueller, 1882).

Distr. Southern South America (Mueller, 1879a, 1882; Paris, 1905a; Brotherus, 1925; Herter, 1933a, 1939b; Kühnemann, 1938; Hosseus, 1940; Herzog, 1952a).

\section{Neobarbula}

N. magellanica Dus.

Tortula densifolia

Neuroloma hom. illeg.

N. fuegianum Card.

Andreaea fuegiana

Notarisia hom. illeg.

N. crispata (Hedw.) Mont.

Ptychomitrium crispatum

\section{Octoblepharum}

O. albidum Hedw. (Hedwig, 1801).

Syn. Bryum albidum (Hedw.) P. Beauv. (Palisot de Beauvois, 1805a).

Distr. Southern South America (Hornschuch, 1820b; Montagne, 1850; Mueller, 1879a; Kühnemann, 1938).

\section{Octodiceras}

O. berterii (Mont.) Jaeg.

Fissidens fontanus

O. dillenii (Pyl.) Brid.

Fissidens fontanus

O. fissidentoides Brid.

Fissidens semicompletus

O. fontanum (Pyl.) Lindb.

Fissidens fontanus

O. hedwigii Lindb.

Fissidens semicompletus

O. julianum (Cand.) Brid.

Fissidens fontanus

\section{Oedipodium}

O. griffithianum (Dicks.) Schwaegr. (Schwaegrichen, 1823).

Syn. Bryum griffithianum Dicks. (Dickson, 1801).

Gymnostomum griffithianum (Dicks.) Sm. (Smith, 1804b).

Hymenostomum griffithianum (Dicks.) Spreng. (Sprengel, 1827).

Distr. Southern South America (Matteri, 1979).

Falkland Islands (Cardot and Brotherus, 1923; Kühnemann, 1938). 


\section{Oligotrichum}

O. antarcticum (C. Muell.) Kindb.

O. atrichopsis C. Muell. (Mueller, 1901a [1900]).

Distr. Southem South America (Mueller, 1901a).

O. austro-aligerum G. L. Smith (Smith, 1969b).

Syn. O. magellanicum Card. et Broth. (Cardot and Brotherus, 1923) hom. illeg.

Distr. Southern South America (Cardot and Brotherus, 1923; Kühnemann, 1938; Herzog, 1954; Seki, 1974).

O. canaliculatum (Hook. et Arnott) Mitt. (Mitten, 1869).

Syn. Polytrichum canaliculatum Hook. et Arnott (Hooker and Arnott, 1832).

Catharinea canaliculata (Hook. et Arnott) C. Muell. (Mueller, 1848a).

Pogonatum liliputanum Thér. (Thériot, 1917b).

Distr. Juan Fernandez (Bartram, 1959; Robinson, 1975).

Southem South America (Hooker and Arnott, 1832: Schwaegrichen, 1842; Mueller, 1848a; Montagne, 1850; Mitten, 1869; Jaeger, 1875; Krieger, 1904; Paris, 1905a; Thériot, 1915, 1917b, 1925a, 1935a; Costes, 1921: Herzog, 1923, 1938, 1939, 1954, 1960; Brotherus, 1924c, 1925; Reimers, 1926; Espinosa B., 1941; Mullen and Frye, 1947; Bartram, 1952; Seki, 1974).

var. majus (Lor.) Par. (Paris, 1896).

Syn. Catharinea canaliculata (Hook. et Arnott) C. Muell. var. major Lor. (Lorentz, 1866).

Distr. Southem South America (Lorentz, 1866; Paris, 1896, 1905a).

var. minus Broth. (Brotherus, 1924b).

Syn. Oligotrichum subcanaliculatum Dus. (Dusén in Mullen and Frye, 1947) nom. nud.

Distr. Juan Fernandez (Brotherus, 1924b, 1925; Mullen and Frye, 1947; Bartram, 1959). Southem South America (Herzog, 1938, 1939; Mullen and Frye, 1947).

var. molinae (Mont.) Thér. (Thériot, 1924).

Syn. Polytrichum molinae Mont. (Montagne, 1845a).

Catharinea molinae (Mont.) C. Muell. (Mueller, 1848a).

Oligotrichum molinae (Mont.) Lor. (Lorentz, 1866).

Distr. Southern South America (Montagne, 1845a, 1850, 1856; Mueller, 1848a; Lorentz, 1866; Jaeger, 1875; Paris, 1905a; Thériot, 1924).

O. compressum (Hook. f. et Wils.) Kindb.

Atrichopsis compressa

O. crispatissimum C. Muell. (Mueller, 1901a [1900]).

Distr. Southern South America (Mueller, 1901a).

O. dendroides (Hedw.) Kindb.

O. gymnostomulum (C. Muell.) Kindb.

Dendroligotrichum dendroides

O. horridum (Mitt.) Kindb.

Psilopilum gymnostomulum

O. laevifolium Thér. (Thériot, 1924).

Polytrichadelphus magellanicus

Distr. Southern South America (Thériot, 1924; Brotherus, 1925; Herzog, 1938).

O. magellanicum (Hedw.) Kindb.

O. magellanicum Card. et Broth.

O. molinae (Mont.) Lor.

Polytrichadelphus magellanicus

O. obtusifolium Thèr. (Thériot, 1934b [1935]).

Oligotrichum austro-aligerum

Oligotrichum canaliculatum var. molinae

Distr. Southern South America (Thériot, 1934b).

O. riedelianum (Mont.) Mitt. (Mitten, 1869).

Syn. Polytrichum riedelianum Mont. (Montagne, 1841).

Distr. Southern South America (Krieger, 1904) 
O. rigidum (Lor.) Broth. (Brotherus, 1905).

Syn. Atrichum rigidum Lor. (Lorentz, 1864).

Catharinea rigida (Lor.) C. Muell. (Mueller, 1901a [1900]).

Distr. Southern South America (Lorentz, 1864, 1866; Jaeger, 1875; Mueller, 1901a; Paris, 1904a; Brotherus, 1905, 1925; Mullen and Frye, 1947).

O. squamosum (Hook. f. et Wils.) Kindb.

Dendroligotrichum squamosum

O. subcanaliculatum Dus.

Oligotrichum canaliculatum var. minus

O. tapes (C. Muell.) Kindb.

Psilopilum tapes

O. umbrosum (Mitt.) Kindb.

Polytrichadelphus umbrosus

\section{Oncophorus}

O. billardieri (Brid.) Brid.

Dicranoloma billardieri

O. cyathicarpus (Mont.) Mitt. Amphidium tortuosum

O. dichotomus (P. Beauv.) Brid.

Dicranoloma billardieri

O. fuegianus Card. (Cardot, 1900).

Syn. Cynodontium fuegianum (Card.) Card. (Cardot, 1901).

Dichodontium brotheri Dix. (Dixon in Paris, 1904b) nom. nud.

Distr. Juan Fernandez (Brotherus, 1924b; Bartram, 1959; Robinson, 1975).

Southern South America (Cardot, 1900, 1901, 1905a, 1908a; Paris, 1904b, 1905a;

Brotherus, 1924a; Kühnemann, 1938).

O. luteo-virens Bartr. (Bartram in Roivainen and Bartram, 1937).

Distr. Southern South America (Roivainen and Bartran, 1937).

O. starkei (Web. et Mohr) Brid.

Kiaeria starkei

O. striatus (Schrad.) Lindb.

Rhabdoweisia fugax

\section{Oreoweisia}

O. auridens (C. Muell.) Kindb.

Oreoweisia bogotensis

var. robusta (C. Muell.) Par. (Paris, 1896).

Syn. Weisia auridens C. Muell. var. robusta C. Muell. (Mueller, 1882).

Distr. Southern South America (Mueller, 1882; Paris, 1896, 1905a).

O. bogotensis (Hamp.) Mitt. (Mitten, 1869).

Syn. Weisia bogotensis Hamp. (Hampe, 1863).

Weisia auridens C. Muell. (Mueller, 1882).

Oreoweisia auridens (C. Muell.) Kindb. (Kindberg, 1889).

Distr. Southern South America (Mueller, 1882; Paris, 1905a; Brotherus, 1924a; Hosseus, 1938c, d; Kühnemann, 1938).

O. brevifolia Broth. (Brotherus, 1918).

Distr. Southern South America (Brotherus, 1918, 1924a; Kühnemann, 1938).

O. chilensis (C. Muell.) Kindb. (Kindberg, 1888).

Syn. Weisia chilensis Hamp. ex C. Muell. (Hampe ex Mueller, 1862).

Distr. Southern South America (Mueller, 1862; Jaeger, 1870; Paris, 1905a; Brotherus, 1924a).

O. patagonica Dus. (Dusén in Paris, 1905a) nom. nud.

Distr. Southern South America (Paris, 1905a).

\section{Orthodontium}

O. australe Hook. f. et Wils.

var. brevisetum Card. et Broth.

var. sinuosifolium Card. et Broth. 
O. gracile Schwaegr. exB.S.G. (Schwaegrichen ex Bruch and others, 1844).

Syn. Orthodontium tenellum Mitt. (Mitten, 1869).

Stableria tenella (Mitt.) Broth. (Brotherus, 1903).

Orthodontium laxirete Dus. (Dusén in Paris, 1905a) nom. nud.

Distr. Juan Fernandez (Bartram, 1959; Robinson, 1975).

Southem South America (Paris, 1905a; Thériot, 1923; Meijer, 1952; Herzog, 1954).

O. humile (Mont.) Mitt.

Pohlia humilis

O. laxirete Dus.

Orthodontium gracile

O. lineare Schwaegr. (Schwaegrichen, 1827a).

Syn. Apalodium linare (Schwaegr.) Mitt. (Mitten, 1885) hom. illeg.

Orthodontium australe Hook. f. et Wils. (Hooker, J. D. and Wilson, 1844).

Apalodium australe (Hook. f: et Wils.) Mitt. (Mitten, 1869).

Orthodontium australe Hook. f. et Wils. var. brevisetum Card. et Broth. (Cardot and Brotherus, 1923).

Orthodontium australe Hook. f. et Wils. var. sinuosifolium Card. et Broth. (Cardot and Brotherus, 1923).

Distr. Southern South America (Hooker, J. D. and Wilson, 1844; Wilson and Hooker, 1847; Mueller, 1848a; Mitten, 1869; Jaeger, 1875; Paris, 1905a; Cardot, 1908a; Cardot and Brotherus, 1923; Brotherus, 1924a; Kühnemann, 1938; Meijer, 1952).

Falkland Islands (Hooker J. D. and Wilson, 1844; Wilson and Hooker, 1847; Mueller, 1848a; Jaeger, 1875; Paris, 1905a; Cardot, 1908a; Cardot and Brotherus, 1923; Brothenus, 1924a; Kühnemann, 1938; Meijer, 1952).

O. pellucens (Hook.) B.S.G. (Bruch and others, 1844).

Syn. Bryum pellucens Hook. (Hooker, 1836).

Apalodium pellucens (Hook.) Mitt. (Mitten, 1869).

Apalodium confine (Hamp.) Mitt. (Mitten, 1885).

Distr. Southem South.America (Herzog, 1954).

O. tenellum Mitt.

Orthodontium gracile

O. tenue C. Muell.

Pohlia humilis

Orthopyxis nom. rejic.

O. palustris (Hedw.) P. Beauv.

Aulacomnium palustre

Orthorrhynchium

O. chilense Williams

Catagonium politum

\section{Orthostichidium}

O. excavatum (Mitt.) Broth.

Orthostichidium pentagonum

O. pentagonum (Hamp. et Lor.) C. Muell. (Mueller, 1897c).

Syn. Pilotrichum pentagonum Hamp. et Lor. (Hampe and Lorentz in Lorentz, 1868). Meteorium excavatum Mitt. (Mitten, 1869).

Orthostichidium excavatum (Mitt.) Broth. (Brotherus, 1906b).

Distr. Southern South America (Hosseus, 1940; Herzog, 1952a).

\section{Orthostichopsis}

O. avellanedae (C. Muell.) Broth. (Brotherus, 1906b).

Syn. Neckera avellanedae C. Muell. (Muëller, 1879a).

Pilotrichella avellanedae (C. Muell.) Kindb. (Kindberg, 1891).

Distr. Southern South America (Mueller, 1879a; Paris, 1905b; Brotherus, 1906b, 1918, 1925; Kühnemann, 1938).

\section{Orthotheca}

O. berteroana (Brid.) Brid. 
Orthotheciella nom. nud.

O. austro-chilense C. Muell.

Hygroamblystegium chilense

\section{Orthothecium}

O. filum (C. Muell.) Kindb.

Hygroamblystegium filum

O. vittatum Card. ex Syd.

Orthotrichum vittatum

\section{Orthotrichum}

O. affine Schrad. ex Brid. var. rupestre Brid.

Orthotrichum rupestre

O. anaglyptodon Card. et Broth. (Cardot and Brotherus, 1923).

Distr. Southern South America (Cardot and Brotherus, 1923; Kühnemann, 1938).

O. anderssonii C. Muell. ex Aongstr.

Orthotrichum leiothecium

O. angustissimum Dus. (Dusén, 1903a) nom. nud.

Distr. Southern South America (Dusén, 1903a).

O. antarcticum Card. (Cardot, 1900).

Distr. Antarctic, peninsula region (Cardot, 1900, 1901; Paris, 1905a; Cardot, 1908a; Brotherus, 1925; Steere, 1961a).

O. apiculatum Hook.

Groutiella apiculata

O. argentinicum Herz. (Herzog, 1957).

Distr. Southern South America (Herzog, 1957; Seki, 1974).

O. aristoblepharum Herz. (Herzog, 1954).

Distr. Southern South America (Herzog, 1954).

O. assimile C. Muell. (Mueller, 1849).

Distr. Southern South America (Mueller, 1849; Montagne, 1850; Mitten, 1869; Jaeger, 1874; Paris, 1905a; Thériot, 1921a, b, 1928; Brotherus, 1925; Herzog, 1954).

O. aurantiorum C. Muell. (Mueller, 1882).

Distr. Southern South America (Mueller, 1882; Paris, 1905a; Brotherus, 1925; Kühnemann, 1938).

O. bellum C. Muell. (Mueller, 1879a).

Distr. Southern South America (Mueller, 1879a; Paris, 1905a; Brotherus, 1925; Kühnemann, 1938).

O. bicolor Thér. (Thériot, 1921a).

Distr. Southern South America (Thériot, 1921a, 1928, 1935a; Brotherus, 1925).

O. brevicolle Mitt.

Ulota rufula

O. carinatum (Mitt.) Mitt.

Ulota carinata

O. chilense Mitt.

Ulota rufula

O. compactum Dus. (Dusén, 1903a).

Distr. Southern South America (Dusén, 1903a; Cardot, 1908a; Brotherus, 1925; Kühnemann, 1938; Herzog, 1960).

O. conceptionense Thér. (Thériot, 1926).

Distr. Southern South America (Thériot, 1926).

$O$. crassifolium Hook. f. et Wils.

Muelleriella crassifolia

O. crenato-erosa C. Muell. (Mueller, 1885).

Syn. Ulota crenato-erosa (C. Muell.) Kindb. (Kindberg, 1888). 
Distr. Southem South America (Mueller, 1885; Paris, 1905a; Cardot, 1908a; Brotherus, 1925; Kühnemann, 1938).

O. crispatum (Hedw.) Hook. et Grev.

Ptychomitrium crispatum

O. cupulatum Hoffm. ex Brid. (Hoffmann ex Bridel, 1801).

Distr. Southem South America (Montagne, 1850).

O. darwinii (Mitt.) Mitt.

Ulota fuegiana ssp. darwinii

O. diaphanum Schrad. ex Brid. var. brevipilum Warnst. (Warnstorf, 1904).

Distr. Southem South America (Warnstorf, 1904).

O. elegantulum Schimp. ex Mitt. (Schimper ex Mitten, 1869).

Distr. Southem South America (Lechler, 1857; Mitten, 1869; Jaeger, 1874; Mueller, 1885; Bescherelle, 1889; Cardot, 1905a (as Ulota magellanica fide Cardot, 1908a), 1908a; Paris, 1905a; Cardot and Brotherus, 1923; Brotherus, 1925; Kühnemann, 1938; Bartram, 1952; Bizot, 1967; Seki, 1974).

O. eremitense (Mitt.) Mitt.

Ulota glabella

O. erpodiaceum C. Muell. (Mueller, 1879a).

Distr. Southem South America (Mueller, 1879a; Kurtz, 1904; Paris, 1905a; Brotherus, 1925;

Hosseus, $1935 b$ and 1937 (both as O. expodiaceum); Kühnemann, 1938).

O. filiforme Hook. et Grev.

Macrocoma orthotrichoides

O. fimbriatum P. Beauv.

Macromitrium fimbriatum

O. fuegianum (Mitt.) Mitt.

Ulota fuegiana

O. fulvellum Mitt.

Ulota luteola

O. germanum Mont.

Ulota germana

O. glabellum (Mitt.) Mitt.

Ulota glabella

O. gymnomitrium (C. Muell.) Broth. (Brotherus, 1902).

Syn. Ulota gymnomitria C. Muell. (Mueller, 1897a).

Distr. Southern South America (Mueller, 1897a; Brotherus, 1902, 1925; Paris, 1905a; Cardot, 1908a; Kühnemann, 1938).

O. herteri Herz. (Herzog in Herter, 1937).

Distr. Southern South America (Herter, 1933a, 1937).

O. hymenomitrium Dus. (Dusén, 1903a) nom. nud.

Distr. Southern South America (Dusén, 1903a).

O. incanum C. Muell. (Mueller, 1885).

Syn. Ulota incana (C. Muell.) Kindb. (Kindberg, 1888).

Distr. South South America (Mueller, 1885; Paris, 1905a; Cardot, 1908a; Brotherus, 1925;
Kühnemann, 1938).

O. inclinatum C. Muell. (Mueller, 1885).

Syn. Ulota inclinata (C. Muell.) Kindb. (Kindberg, 1888).

Distr. Southern South America (Mueller, 1885; Bescherelle, 1885a; Paris, 1905a; Cardot,
1908a; Brotherus, 1925; Kühnemann, 1938).

O. jamesonii Arnott

Schlotheimia jamesonii

O. johnstonii Bartr. (Bartram, 1942).

Distr. Southern South America (Bartram, 1942, 1943).

O. jutlandicum Brid. 
O. lebrunii Besch. (Bescherelle, 1885a).

Distr. Southern South America (Bescherelle, 1885a; Paris, 1905a; Brotherus, 1925; Kühnemann, 1938).

O. leiothecium C. Muell. (Mueller, 1862).

Syn. Ulota leiothecia (C. Muell.) Jaeg. (Jaeger, 1874).

Orthotrichum anderssonii C. Muell. ex Aongstr. (Mueller ex Ångström, 1872).

Ulota anderssonii (Aongstr.) Jaeg. (Jaeger and Sauerbeck, 1879b).

Distr. Southern South America (Mueller, 1862, 1885; Ångström, 1872, 1876b; Jaeger, 1874; Bescherelle, 1885a; Paris, 1905a; Cardot, 1908a; Brotherus, 1925; Kühnemann, 1938).

O. ligulatum Dus. (Dusén, 1903a) nom. nud.

Distr. Southern South America (Dusén, 1903a).

O. lobbianum (Mitt.) Mitt.

Ulota lobbiana

O. longipes Hook.

Macromitrium longipes

O. longirostre Hook. Macromitrium longirostre

O. Iorentzii C. Muell. (Mueller, 1879a).

Distr. Southern South America (Mueller, 1879a; Paris, 1905a; Brotherus, 1925; Herzog, 1938; Kühnemann, 1938).

O. luteolum Hook. f. et Wils.

Ulota luteola, U. fuegiana and U. glabella

O. macloskii Dus. (Dusén, 1903a).

Distr. Southern South America (Dusén, 1903a; Cardot, 1908a; Brotherus, 1925; Kühnemann, 1938).

O. macrocalycinum (Mitt.) Mitt.

Ulota macrocalycina

O magellanicum Mont.

Ulota magellanica

O. malacothecium C. Muell. (Mueller, 1882).

Distr. Southern South America (Mueller, 1882; Paris, 1905a; Brotherus, 1925; Kühnemann, 1938).

O. marginatum Aongstr.

Ulota macrocalycina

O. microstomum Hook. et Grev. Macromitrium microstomum

O. nigritellum Dus. (Dusén, 1903a) nom. nud.

Distr. Southern South America (Dusén, 1903a; Paris, 1905a; Seki, 1974).

O. nutans C. Muell. (Mueller, 1879a).

Distr. Southern South America (Mueller, 1879a; Kurtz, 1904; Paris, 1905a; Brotherus, 1925; Hosseus, 1935b, 1937; Kühnemann, 1938).

O. paleomitrium Dus. (Dusén, 1903a) nom. nud.

Distr. Southern South America (Dusén, 1903a; Paris, 1905a; Herzog, 1957).

O. perpusillum Dus. (Dusén, 1903a) nom. nud.

Distr. Southern South America (Dusén, 1903a; Paris, 1905a).

O. phyllanthum (Brid.) Steud.

Ulota.phyllantha

O. podocarpi C. Muell. (Mueller, 1879a).

Distr. Southern South America (Mueller, 1879a; Paris, 1905a; Brotherus, 1925; Kühnemann, 1938).

O. polytrichoides (Hedw.) Brid.

Lepidopilum polytrichoides

O. pygmaeothecium C. Muell.

Ulota pygmaeothecia

O. quenoae C. Muell. (Mueller, 1882).

Distr. Southern South America (Mueller, 1882; Paris, 1905a; Brotherus, 1925; Hosseus, 1938a, d: Kühnemann, 1938). 
O. rufulum Mitt.

O. rupestre Schleich. ex Schwaegr. (Schleicher ex Schwaegrichen, 1816).

Syn. Dorcadion rupestre (Schwaegr.) Lindb. (Lindberg, 1879).

Orthotrichium affine Schrad. ex Brid. var. rupestre Brid. (Bridel, 1812) nom. nud.

Distr. Southern South America (Dusén, 1903a; Cardot, 1908a; Costes, 1921; Kühnemann, 1938).

O. rupicola C. Muell. (Mueller, 1884 [1883]).

Distr. Antarctic, peninsula region (Cardot, 1900, 1901, 1908a; Paris, 1905a; Steere, 1961a).

O. schnyderi C. Muell. (Mueller, 1882).

Distr. Southem South America (Mueller, 1882; Paris, 1905a; Brotherus, 1925; Kühnemann, 1938).

O. smithii (Hedw.) Brid.

Leptodon smithii

O. subassimile Dus. (Dusén, 1903a) nom. nud.

Distr. Southem South America (Dusén, 1903a).

O. subrupestre Thér. (Thériot, 1923b).

Distr. Southem South America (Theriot, 1921a (as O. rupestre), 1923b; Brotherus, 1925).

O. torquatum (Hedw.) Arnott

Schlotheimia torquata

O. truncato-dentatum C. Muell. (Mueller, 1882).

Distr. Southem South America (Mueller, 1882; Brotherus, 1925; Kühnemann, 1938).

O. ulotoides Herz. (Herzog, 1923).

Distr. Southern South America (Herzog, 1923).

O. urceolatum Hook.

Macromitrium urceolatum

O. verrucosum C. Muell. (Mueller, 1879a).

Distr. Southem South America (Mueller, 1879a; Paris, 1905a; Brotherus, 1925; Kühnemann, 1938).

O. vittatum Card. (Cardot, 1905a) hom. illeg.

Syn. Orthothecium vittatum Card. ex Syd. (Cardot ex Sydow, 1910) nom. inval.

Distr. Southern South America (Cardot, 1905a, 1908a; Brotherus, 1925; Kühnemann, 1938).

\section{Oxyrrhynchium}

O. altisetum (C. Muell.) Broth. (Brotherus, 1909).

Syn. Rhynchostegium altisetum C. Muell. (Mueller, 1897a).

Hypnum altisetum (C. Muell.) Broth. (Brotherus, 1900).

Distr. Southern South America (Mueller, 1897a; Paris, 1905b; Brotherus, 1909, 1925; Kühnemann, 1938).

O. clinocarpum (Tayl.) Broth. (Brotherus, 1909).

Syn. Hypnum clinocarpum Tayl. (Taylor, 1848a).

Brachythecium clinocarpum (Tayl.) Jaeg. (Jaeger, 1878).

Hypnum remotifolium Grev. (Greville, 1826).

Eurhynchium remotifolium (Grev.) Jaeg. (Jaeger, 1878).

Thuidium remotifolium (Grev.) Ren. et Card. (Renauld and Cardot, 1893b).

Oxyrrhynchium remotifolium (Grev.) Steere (Steere, 1948) hom. illeg.

Distr. Southem South America (Herzog, 1952a).

O. corralense (Lor.) Fleisch. ex Reim. (Fleischer ex Reimers, 1926).

Syn. Hypnum corralense Lor. (Lorentz, 1866).

Eurhynchium corralense (Lor.) Jaeg. (Jaeger, 1878). 
Eurhynchium confusum Thér. (Thériot, 1918).

Rhynchostegium subsquarrosum Herz. (Herzog, 1923).

Eurhynchium acanthophyllum (Mont.) Dus. ex Thér. (Dusén ex Thériot, 1934b). nom. inval. fide Thériot, 1934a.

Distr. Southern South America (Lorentz, 1866; Jaeger, 1878; Paris, 1904b; Thériot, 1918, 1934b; Campo, 1921; Herzog, 1923, 1938; Brotherus, 1924c, 1925; Reimers, 1926; Herter, 1933a, 1939b; Espinosa B., 1941; Skottsberg, 1950).

O. fuegianum (Card.) Herz. (Herzog, 1939).

Syn. Eurhynchium fuegianum Card. (Cardot, 1905a).

Rhynchostegiella fuegiana (Card.) Broth. (Brotherus, 1909).

Eurhynchiella fuegiana (Card.) Fleisch. (Fleischer, 1923).

Distr. Southern South America (Cardot, 1905a, 1908a; Cardot and Brotherus, 1923; Fleischer, 1923; Brotherus, 1925; Herzog, 1938, 1939, 1954; Kühnemann, 1938; Seki, 1974).

O. remotifolium (Grev.) Steere

Oxymhynchium clinocarpum

Pancovia hom. illeg.

P. denticulata (Hedw.) Kickx

Plagiothecium denticulatum

P. rutabula (Hedw.) Kickx

Brachythecium rutabulum

Papillaria

P. appressa (C. Muell.) Aongstr.

Papillaria nigrescens

P. cardotii Broth.

Floribundaria cardotii

P. filipendula (Hook. f. et Wils.) Jaeg.

Papillaria flexicaulis

P. flexicaulis (Wils.) Jaeg. (Jaeger, 1877).

Syn. Meteorium flexicaule Wils. (Wilson, 1854).

Trachypus flexicaulis (Wils.) Mitt. (Mitten, 1860).

Meteorium filipendulum Hook. f. et Wils. (Hooker and Wilson in Wilson, 1859).

Papillaria filipendula (Hook. f. et Wils.) Jaeg. (Jaeger, 1877).

Pilotrichella filipendula (Hook. f. et Wils.) Hamp. (Hampe, 1880b).

Distr. Juan Fernandez (Bartram, 1959; Robinson, 1975).

Southern South America (Paris, 1905a; Brotherus, 1925; Reimers, 1926; herzog, 1938, 1940).

P. laeviuscula Broth.

Papillaria tijucae

P. media Aongstr.

Meteorium medium

P. nigrescens (Hedw.) Jaeg. (Jaeger, 1877).

Syn. Hypnum nigrescens Hedw. (Hedwig, 1801).

Neckera appressa C. Muell. (Mueller, 1850).

Papillaria appressa (C. Muell.) Aongstr. (Ångström, 1876a).

Distr. Southern South America (Herzog, 1952a, b).

P. pilifolia C. Muell. (Mueller, 1901b).

Distr. Southern South America (Herzog, 1952a).

P. tijucae C. Muell. (Mueller, 1901b).

Syn. Papillaria laeviuscula Broth. (Brotherus in Ule, 1899) nom. nud.

Distr. Southern South America (Herzog, 1952a).

$P$. usneoides Broth.

Floribundaria usneoides

Pentastichella nom. nud.

P. aurea Dus.

Stenomitrium aureum

P. bartramioides Dus.

Zygodon bartramioides

$P$. jaffuelii Thér.

Zygodon porteri

P. pentasticha (Mont.) C. Muell.

Zygodon pentastichus

var. strictifolia Thér. 
P. robustula Dus.

Zygodon bartramioides

\section{Perisiphorus}

P. ciliatus (Hedw.) P. Beauv.

Hedwigia ciliata

\section{Peromnion}

P. magellanicum Sull.

Brachymenium indicum

Pharomitrium nom. rejic.

P. subsessile.(Brid.) Schimp.

Pterygoneurum subsessile

\section{Phasconica}

P. Iorentzii C. Muell. (Mueller, 1882).

Distr. Southem South America (Mueller, 1882; Paris, 1905a; Brotherus, 1924a; Hilpert, 1933; Kühnemann, 1938; Hosseus, 1939).

\section{Phascum}

P. brevipes (Hook.) Schwaegr.

Bruchia brevipes

P. calodictyon C. Muell. (Mueller, 1888).

Distr. Southem South America (Gibert, 1873 (as P. turgidum ?); Mueller, 1888; Paris, 1905a; Roth, 1911; Felippone, 1912; Brotherus, 1924a; Herter, 1928, 1933a; Kühnemann, 1938).

P. fruchartii C. Muell.

Astomum fruchartii

P. lamprocarpum C. Muell.

P. lamprothecium C. Muell.

$P$. nervosum Hook. Tetrapterum lamprothecium

var. robinsonii Mont. Pleuridium nervosum

P. recurvirostre C. Muell.

P. robinsonii Mont. ex C. Muell.

\section{Philibertiella}

P. ditrichoidea Card. (Cardot, 1914b).

Distr. Southem South America (Cardot, 1914b; Cardot and Brotherus, 1923; Brotherus, 1924a; Herzog, 1938, 1957).

P. nitens Herz. (Herzog, 1960 [1961]).

Distr. Southem South America (Herzog, 1960).

\section{Philonotis}

P. acicularis (C. Muell.) Kindb. (Kindberg, 1891).

Syn. Bartramia acicularis C. Muell. (Mueller, 1890a).

Philonotis varians Card. (Cardot, 1906a) fide Clarke, $1973 a$.

Philonotis elegantissima Broth. ex Dism. (Brotherus ex Dismier, 1910) hom. illeg.

Meesia austro-georgica C. Muell. (Mueller, 1890a) fide Clarke, 1973a.

Distr. Southem South America (Dismier, 1910; Thériot, 1924; Matteri, 1968).

South Georgia (Mueller, 1890a; Paris, 1905a; Cardot 1906a, 1908a; Dismier, 1910; Cardot and Brotherus, 1923; Brotherus, 1924a; Dixon, 1932; Kabiersch, 1937; Steere, 1961b; Matteri, 1968; Clarke, 1973a; Bell, 1977b).

P. acutissima (C. Muell.) Kindb. (Kindberg, 1889).

Syn. Bartramia acutissima C. Muell. (Mueller, 1882).

Distr. Southern South America (Mueller, 1882; Paris, 1905a; Brotherus. 1924a; Hosseus, 1938c, d, 1939; Kühnemann, 1938; Herzog, 1952a) 
P. balansae Besch. ex Kindb.

Philonotis curvata

$P$. balansaena Besch.

Philonotis curvata

P. bartramii Matteri (Matteri, 1973c).

Syn. Philonotis luteola Bartr. (Bartram, 1946) hom. illeg.

Distr. Southern South America (Bartram, 1946; Matteri, 1973c).

P. brevifolia Herz.

Philonotis krausei

P. buenos-ayrensis (C. Muell.) Par.

Philonotis curvata

P. crenatula (C. Muell.) Kindb. (Kindberg, 1889).

Syn. Bartramia crenatula C. Muell. (Mueller, 1882).

Distr. Southern South America (Mueller, 1882; Paris, 1905a; Brotherus, 1924a; Hosseus, $1935 a, b, 1937$; Kühnemann, 1938).

P. curvata (Hamp.) Jaeg. (Jaeger, 1875).

Syn. Bartramia curvata Hamp. (Hampe, 1862a).

Philonotis balansaena Besch. (Bescherelle, 1877).

Philonotis balansae Besch. ex Kindb. (Bescherelle ex Kindberg, 1888) nom. illeg.

Philonotula buenos-ayrensis C. Muell. (Mueller, 1897a).

Philonotis buenos-ayrensis (C. Muell.) Par. (Paris, 1900a).

Philonotula bonariensis C. Muell. (Mueller in Kuntze, 1898) err.

Distr. Southern South America (Mueller, 1897a; Kuntze, 1898; Paris, 1900a, 1905a; Krieger, 1904; Felippone, 1909; Dismier, 1910; Brotherus, 1924a; Herter, 1933a).

P. defecta (C. Muell.) Kindb.

Philonotis glaucescens

P. dimorpha Schimp. Philonotis vagans

P. elegantissima Broth. ex Dism.

Philonotis acicularis

P. elegantula (Tayl.) Jaeg.

Philonotis rufiffora

P. elongatula (C. Muell.) Kindb. (Kindberg, 1889).

Syn. Bartramia elongatula C. Muell. (Mueller, 1882).

Philonotis subelegantula Par. (Paris, 1897) nom. illeg.

Bartramia elegantula Par. (Paris, 1905a) nom. inval.

Distr. Southern South America (Mueller, 1882; Paris, 1897, 1900a, 1905a; Brotherus, 1924a; Hosseus, 1938c, d, 1939; Kühnemann, 1938).

P. esquelensis Matt. (Matteri, 1968).

Distr. Southern South America (Matteri, 1968).

P. exigua (Sull.) Broth.

Philonotis scabrifolia

P. flexipes (C. Muell.) Par. (Paris, 1900a).

Syn. Philonotula flexipes C. Muell. (Mueller, 1897a).

Bartramia flexipes (C. Muell.) C. Muell. (Mueller, 1901a [1900]).

Distr. Southern South America (Mueller, 1897a; Paris, 1900a, 1905a; Felippone, 1912;

Brotherus, 1924a; Herter, 1933a, 1943).

P. fontana (Hedw.) Brid. (Bridel, 1827b).

Syn. Mnium fontanum Hedw. (Hedwig, 1801).

Bryum fontanum (Hedw.) Gaertn., Meyer et Scherb. (Gärtner and others, 1802).

Bartramia fontana (Hedw.) Turn. (Turner, 1804a).

Distr. Southern South America (Mueller, 1848a; Montagne, 1850).

var. magellanica (Brid.) Brid. (Bridel, 1827b).

Syn. Mnium fontanum Hedw. var. magellanicum Brid. (Bridel, 1803).

Bartramia fontana (Hedw.) Turn. var. magellanica (Brid.) P. Beauv. (Palisot de Beauvois, 1805a).

Distr. Southern South America (Bridel, 1803, 1827b). 
P. fontanoides (Gill. et Grev.) Jaeg. (Jaeger, 1875).

Syn. Bartramia fontanoides Gill. et Grev. (Gillies and Greville in Greville, 1830).

Distr. Southem South America (Greville, 1830; Mitten, 1869; Jaeger, 1875; Paris, 1905a; Brotherus, 1924a; Kühnemann, 1938).

P. glabrata Broth. (Brotherus, 1924b).

Distr. Juan Femandez (Brotherus, 1924b; Bartram, 1959; Robinson. 1975).

P. glaucescens (Hornsch.) Broth. (Brotherus, 1895).

Syn. Bartramia glaucescens Hornsch. (Hornschuch, 1840).
Philonotis muhlenbergii (Schwaegr.) Brid. var. tenella Brid. (Bridel, 1827b).

Bartramia tenella (Brid.) C. Muell. (Mueller, 1849) nom. illeg.

Philonotis sphaerocarpa (H. (Jaeger, 1875).

Philonotis sphaerocarpa (Hedw.) Brid. 1875).
Bartramia defecta C. Muell. (Mueller, 1882).

Bartramidula defecta (C. Muell.) Broth (Kindberg, 1889).

Philonotis weberoides Dus. (Dusén Distr. Southem South America (Mueller, 1882; Brotherus, 1904, 1924a; Paris, 1905a; Dismier,
1910; Hosseus, 1938b, c, d, 1939; Kü Piovano, 1953;
Piovano, 1954). Piovano, 1954).

P. gourdonii Card. (Cardot, 1911b).

Distr. Antarctic, peninsula region (Cardot, 1911b, d, 1913a: Brotherus. 1924a; Steere, 1961a;

P. inflexa (C. Muell.) Broth.

Pohlia inflexa

P. kerguelensis $\mathrm{Par}$.

Breutelia integrifolia

P. krauseana Broth.

Philonotis krausei

var. longinervis Broth. (Brotherus, 1924b).

Distr. Juan Femandez (Brotherus, 1924b)

P. krausei (C. Muell.) Broth. (Brotherus, 1904).

Syn. Bartramia krausei C. Muell. (Mueller, 1874).
Glyphocarpus krausei (C. Muell.) Jaeg

Bartramidula krausei (C. Muell.)) Jaeg. (Jaeger, 1875).

Philonotis krauseana Broth. (Brotherus, (Paris, 1894).

Distr. Juan Fernandez (Brotherus, 1924 $\mathrm{b}$; 1954 ) fide Seki, 1974 .

Southem South America (Mueller, 1874 ;ram, 1959; Matteri, 1968 ; $189.1904 a$; Brotherus,

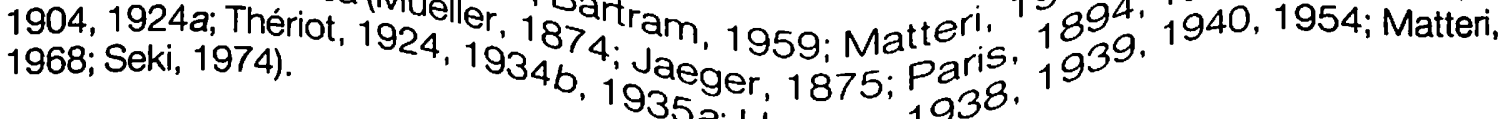

P. litorea Card. et Broth. (Cardot and Brother

Distr. Juan Fernandez (Skottsberg, 1914).
Falkland Islands (Cardot and Brotherus).

P. luteola Bartr.

P. macropyxis Dus. (Dusén in Paris, 1905

Distr. Southern South America (Paris, 1905 . $n_{u_{d}}$.

P. muhlenbergii (Schwaegr.) Brid. var. tene/la ${ }$ ).
P. nigricans Herz. (Herzog, 1916b).

philonotis bartramil

Distr. Southern South America (Herzo

P. nigroflava C. Muell. (Mueller, 1897 a). ${ }^{1}{ }^{1} 6_{b}$ ) Distr. Southern South America (Mueller,
and Brotherus, 1923; Brotheru, 1897 a. 1968). 
P. parallela Dus. (Dusèn, 1903a).

Distr. Southern South America (Dusén, 1903a; Paris, 1905a; Cardot, 1908a; Dismier, 1910; Cardot and Brotherus, 1923; Brotherus, 1924a; Herzog, 1957; Kühnemann, 1938; Matteri, 1968; Seki, 1974).

P. platensis Par. (Paris, 1900a).

Syn. Philonotula secunda C. Muell. (Mueller, 1897a).

Distr. Southern South America (Mueller, 1897a; Paris, 1900a, 1905a; Brothenus, 1924a; Kühnemann, 1938).

P. pomangium (C. Muell.) Kindb. (Kindberg, 1889).

Syn. Bartramia pomangium C. Muell. (Mueller, 1882).

Bartramia declivium C. Muell. (Mueller, 1882) nom. nud.

Distr. Southern South America (Mueller, 1882; Paris, 1905a; Brothenus, 1924a; Kühnemann, 1938).

P. riparia Dus. (Dusén in Paris, 1905a) nom. nud.

Distr. Southern South America (Paris, 1905a; Dismier, 1910).

P. rufiflora (Hornsch.) Reichdt. (Reichardt, 1870).

Syn. Bartramia rufiflora Hornsch. (Hornschuch, 1840).

Bartramia elegantula Tayl. (Taylor, 1847).

Philonotis elegantula (Tayl.) Jaeg. (Jaeger, 1875).

Distr. Southern South America (Taylor, 1847; Sullivant, 1859; Jaeger, 1875).

P. scabrifolia (Hook. f. et Wils.) Braithw. (Braithwaite, 1893).

Syn. Hypnum scabrifolium Hook. f. et Wils. (Hooker, J. D. and Wilson, 1844).

Bartramia scabrifolia (Hook. f. et Wils.) C. Muell. (Mueller, 1901a[1900]).

Bartramia pusilla Sull. (Sullivant, 1850) hom. illeg.

Bartramia exigua Sull. (Sullivant, 1859).

Glyphocarpus exiguus (Sull.) Jaeg. (Jaeger, 1875).

Bartramidula exigua (Sull.) Par. (Paris, 1894).

Philonotis exigua (Sull.) Broth. (Brotherus, 1904).

Bartramia willii C. Muell. (Mueller, 1890a).

Philonotis willii (C. Muell.) Broth. (Brotherus, 1904).

Bartramia ventanae C. Muell. (Mueller, 1897a).

Philonotis ventanae (C. Muell.) Par. (Paris, 1900a).

Distr. Juan Fernandez (Skottsberg, 1914; Brotherus, 1924b; Espinosa B., 1941; Bartram, 1959; Robinson, 1975).

Southern South America (Sullivant, 1850, 1859; Mueller, 1851a, 1885, 1897a, 1901a; Jaeger, 1875; Paris, 1894, 1900a, 1904a, 1905a; Neger, 1899; Dusén, 1903a; Brotherus, 1904, 1924a; Cardot, 1905a, 1908a, 1913a; Dismier, 1910; Cardot and Brotherus, 1923; Herzog, 1923, 1939, 1954, 1957; Roivainen, 1934; Hosseus, 1938c, d, 1939; Kühnemann, 1938; Bartram, 1952; Matteri, 1968; Seki, 1974).

Falkland Islands (Cardot, 1905a, 1908a; Brotherus, 1924a; Kühnemann, 1938).

South Georgia (Mueller, 1890a; Brotherus, 1904, 1924a; Paris, 1904a; Cardot, 1906a, 1908a; Dismier, 1910; Steere, 1961b; Matteri, 1968; Clarke, 1973a).

P. simplicissima (C. Muell.) Kindb. (Kindberg, 1889).

Syn. Bartramia simplicissima C. Muell. (Mueller, 1882).

Distr. Southern South America (Mueller, 1882; Paris, 1905a; Dismier, 1910; Kühnemann, 1938).

P. sphaerocarpa (Hedw.) Brid. ssp. tenella (Brid.) Dism.

Philonotis glaucescens

P. striata (C. Muell.) Kindb. (Kindberg, 1889).

Syn. Bartramia striata C. Muell. (Mueller, 1882).

Distr. Southern South America (Mueller, 1882; Paris, 1905a; Dismier, 1910; Brotherus, 1924a; Kühnemann, 1938). 
P. strictiuscula (C. Muell.) Par. (Paris, 1900a).

Syn. Philonotula strictiuscula C. Muell. (Mueller, 1897a).

Bartramia strictiuscula (C. Muell.) C. Muell. (Mueller, 1901a [1900]).

Distr. Southern South America (Mueller, 1897a, 1901a; Paris. 1900a; Brotherus, 1924a; Herter, 1933a).

P. subelegantula Par.

Philonotis elongatula

P. tenella (Brid.) Jaeg.

Philonotis glaucescens

P. trichophylla Herz. (Herzog, 1954) hom. illeg.

Distr. Southem South America (Herzog, 1954).

P. vagans (Hook. f. et Wils.) Mitt. (Mitten, 1860).

Syn. Bryum vagans Hook. f. et Wils. (Hooker, J. D. and Wilson, 1844).

Meesia vagans (Hook. f. et Wils.) C. Muell. (Mueller, 1848a).

Bartramia vagans (Hook. f. et Wils.) Mitt. (Mitten, 1869).

Philonotis vagans (Hook. f. et Wils.) Mitt. var. inundata Card. (Cardot, 1906a) fide

Clarke, 1973a.

Hypnum reticulatum Hook. f. et Wils. (Hooker, J. D. and Wilson, 1844) hom. illeg.

Goniobryum reticulatum Lindb. (Lindberg, $1868 b$ ).

Rhizogonium reticulatum (Hook. f. et Wils.) Mitt. (Mitten, 1869) hom. illeg.

Mnium reticulatum (Hook. f. et Wils.) C. Muell. (Mueller, 1885).

Philonotis dimorpha Schimp. (Schimper in Mitten, 1860) nom. nud.

Bartramia dimorpha Schimp. (Schimper in Mueller, 1901a (1900]) nom. nud.

Distr. Juan Fernandez (Espinosa B., 1941; Matteri, 1968; Robinson, 1975).

Southem South America (Hooker, J. D. and Wilson, 1844; Wilson and Hooker, 1847; Mueller, 1848a, 1885; Sullivant, 1859; Mitten, 1860, 1869; Ångström, 1872; Jaeger, 1875; Bescherelle, 1889; Neger, 1899; Dusén, 1903a, 1905d; Paris, 1904b, 1905a; Cardot, 1905a, 1908a, 1913a; Dismier, 1910; Cardot and Brotherus, 1923; Brotherus, 1924a; Roivainen, 1934; Thériot, 1934b; Kühnemann, 1938; Herzog, 1939, 1954; Bartram. 1952; Matteri, 1968; Seki, 1974).

South Georgia (Cardot, 1906a, 1908a; Dismier, 1910; Cardot and Brotherus, 1923;

Dixon, 1935; Steere, 1961b; Matteri, 1968; Clarke, 1973a).

var. evanidinervis Broth. (Brotherus, 1924b).

Distr. Juan Fernandez (Brotherus, 1924b; Espinosa B., 1941; Bartram, 1959).

var. inundata Card.

Philonotis vagans

$P$. varians Card.

Philonotis acicularis

P. ventanae (C. Muell.) Par.

Philonotis scabrifolia

$P$. weberoides Dus.

Philonotis glaucescens

P. willii (C. Muell.) Broth.

Philonotis scabrifolia

\section{Philonotula}

P. bonariensis C. Muell.

Philonotis curvata

P. buenos-ayrensis C. Muell.

Philonotis curvata

$P$. flexipes C. Muell.

Philonotis flexipes

$P$. secunda C. Muell.

Philonotis platensis

P. strictiuscula C. Muell.

Philonotis strictiuscula

Photinophyllum hom. illeg.

$P$. subbasilare (Hook.) Mitt.

Goniobryum subbasilare

\section{Phyllogonium}

P. callichroum Mont.

\section{Physcomitrium}

P. acutifolium Broth. (Brotherus, 1900).

Distr. Southern South America (Herter, 1933a) 
P. argentinicum Par. (Paris, 1897).

Syn. Physcomitrium pusillum C. Muell. (Mueller, 1882) hom. illeg.

Physcomitrium subpusillum C. Muell. (Mueller in Kindberg, 1889) nom. nud.

Distr. Southern South America (Mueller, 1882; Paris, 1897, 1905a; Brotherus, 1924a; Kühnemann, 1938).

P. badium Broth. (Brotherus, 1900).

Distr. Southern South America (Herter, 1933a; Herzog, 1954).

P. bonplandii (Hook.) De Not.

Funaria bonplandii

P. chlorodictyon C. Muell. (Mueller, 1879a).

Distr. Southern South America (Mueller, 1879a; Paris, 1905a; Brotherus, 1924a; Kühnemann, 1938).

P. cryptotheca C. Muell. (Mueller, 1901a [1900]) nom. nud.

Distr. Southern South America (Mueller, 1901a).

P. cupulare C. Muell. (Mueller, 1879a).

Distr. Southern South America (Mueller, 1879a; Paris, 1905a; Brotherus, 1924a; Kühnemann, 1938; Herter, 1939b).

var. latifolium Broth. (Brotherus, 1918).

Distr. Southern South America (Brotherus, 1918; Kühnemann, 1938).

P. densirete Besch. (Bescherelle in Mueller, 1901a [1900]) nom. nud.

Distr. Southern South America (Mueller, 1901a).

P. excurrens Broth. (Brotherus in Krieger, 1904) nom. nud.

Distr. Southern South America (Krieger, 1904).

P. felipponei Thér. (Thériot, 1939 [1941]).

Distr. Southern South America (Herter, 1939b; Thériot, 1939).

P. germanillae C. Muell. (Mueller, 1879a).

Distr. Southern South America (Mueller, 1879a; Kurtz, 1904; Paris, 1905a; Brotherus, 1924a; Hosseus, 1935a; Kühnemann, 1938; Herter, 1939b).

P. gibertii Mitt. (Mitten, 1885).

Distr. Southern South America (Mitten, 1885).

P. herteri Thér. (Thériot, 1939 [1941]).

Distr. Southern South America (Herter, 1939b; Thériot, 1939).

P. integrifolium C. Muell. (Mueller, 1901a [1900]) nom. nud.

Distr. Southern South America (Mueller, 1901a).

P. lorentzii C. Muell. (Mueller, 1879a).

Distr. Southern South America (Mueller, 1879a; Krieger, 1904; Kurtz, 1904; Paris, 1905a; Brotherus, 1918, 1924a; Herter, 1933a; Thériot, 1934b; Hosseus, 1935b, 1937. 1938a, c, d; Kühnemann, 1938).

P. macroglobum C. Muell. (Mueller, 1901a [1900]) nom. nud.

Distr. Southern South America (Mueller, 1901a).

P. mahui H. Robinson (Robinson, 1970b).

Distr. Southern South America (Robinson, 1970b).

P. orbignyanum (Mont.) Mont. (Montagne, 1839c).

Syn. Gymnostomum orbignyanum Mont. (Montagne, 1838). 
Distr. Southem South America (Montagne, 1838, 1856; Paris, 1905a; Brotherus, 1924a; Kühnemann, 1938).

P. pusillum C. Muell.

Physcomitrium argentinicum

P. pyriforme (Hedw.) Hamp. (Hampe, 1837b).

Syn. Gymnostomum pyriforme Hedw. (Hedwig, 1801).

Distr. Southern South America (Montagne, 1850).

P. roseae Williams (Williams, 1915).

Distr. Southem South America (Williams, 1915; Brotherus, 1924a).

P. serrifolium C. Muell. (Mueller, 1879a).

Distr. Southem South America (Mueller, 1879a; Kurtz, 1904; Paris, 1905a; Brotherus, 1924a; Hosseus, 1935b, 1937, 1938a, c, d; Kühnemann, 1938).

P. spegazzinii C. Muell. (Mueller, 1901a [1900]) nom. nud.

Distr. Southem South America (Mueller, 1901a).

P. subpusillum C. Muell.

Physcomitrium argentinicum

P. thieleanum Hamp. (Hampe, 1844).

Syn. Physcomitrium thielei Hamp. ex Kindb. (Hampe ex Kindberg, 1888) nom. illeg.

Distr. Southem South America (Hampe, 1844; Mueller, 1848a; Mitten, 1869; Gibert, 1873; Jaeger, 1874; Herter, 1928, 1933a; Herzog, 1952a).

P. thielei Hamp. ex Kindb.

Physcomitrium thieleanum

P. umbonatum Mitt. (Mitten, 1869).

Distr. Southern South America (Felippone, 1909; Herter, 1933a).

\section{Pilopogon}

P. gracilis (Hook.) Brid. (Bridel, 1826).

Syn. Didymodon gracilis Hook. (Hooker, 1818).

Thysanomitrion gracile (Hook.) Arnott (Arnott, 1827).

Weisia gracilis (Hook.) Spreng. (Sprengel, 1827).

Dicranum strictum Schwaegr. (Schwaegrichen in Gaudichaud, 1826 [1828]) hom.

illeg.
Didymodon gracilis Hook. var. robustum Hook. (Hooker in Gaudichaud, 1826 [1828]) nom. nud.

Thysanomitrion gracile (Hook.) Arnott var. robustum Arnott (Arnott, 1827).

Distr. Falkland Islands (Dumont D'Unille, 1825; Gaudichaud, 1825, 1826; Bridel, 1827b).

$P$. leptodus (Mont.) Broth.

Campylopus clavatus

P. richardii (Brid.) Broth.

Campylopus richardii

P. schilleri Herz. et Thér. (Herzog and Thériot in Herzog and others, 1933).

Distr. Southern South America (Herzog and others, 1933).

\section{Pilotrichella}

P. avellanedae (C. Muell.) Kindb.

Orthostichopsis avellanedae

P. billardieri (Hamp.) Jaeg.

Weymouthia cochlearifolia var. billardieri

P. characea (C. Muell.) Kindb.

Meteoriopsis characea

var. nigrescens (C. Muell.) Par.

Meteoriopsis characea var. nigrescens

var. tenuis (C. Muell.) Par.

Meteoriopsis characea var. tenuis

$P$. cumingii (C. Muell.) Lor.

Weymouthia mollis

P. cyathipoma (C. Muell.) Kindb. (Kindberg, 1888).

Syn. Neckera cyathipoma C. Muell. (Mueller, 1879a). 
Distr. Southern South America (Mueller, 1879a; Paris, 1905b; Brothenus, 1925; Kühnemann, 1938).

P. filipendula (Hook. f. et Wils.) Hamp.

Papillaria flexicaulis

$P$. illecebrina (C. Muell.) Kindb.

Meteorium illecebrinum

$P$. Kraussei Lor.

P. mollis (Hedw.) Jaeg.

Weymouthia cochlearifolia var. billardieri

Weymouthia mollis

P. turgidula (C. Muell.) Par.

var. tristis (C. Muell.) Par.

Squamidium turgidulum

Squamidium turgidulum var. triste

\section{Pilotrichum}

$P$. ambiguum Hornsch.

$P$. ancistrodes (Mont.) Lor.

P. ciliatum (Hedw.) C. Muell.

P. consimile (Mont.) C. Muell.

$P$. flagelliferum Brid.

$P$. genuflexum C. Muell.

$P$. gorveanum (Mont.) C. Muell.

P. patagonicum (Brid.) P. Beauv.

$P$. pentagonum Hamp. et Lor.

$P$. polytrichoides (Hedw.) Brid.

P. scabrisetum (Schwaegr.) Brid.

Meteoriopsis ambigua

P. smithii (Dicks.) P. Beauv.

$P$. stramineum C. Muell.

Ancistrodes genuffexa

Hedwigia ciliata

Cryphaea tenella

Pseudocryphaea flagellifera

Ancistrodes genufiexa

Dendrocryphaea gorveana

Homalia glabella

Orthostichidium pentagonum

Lepidopilum polytrichoides

Lepidopilum scabrisetum

Leptodon smithii

Meteoriopsis straminea

P. tenellum (Schwaegr.) C. Muell.

P. thieleanum (C. Muell.) Hamp.

Cryphaea tenella

Pinnatella thieleana

\section{Pinnatella}

P. callicostelloides (Thér.) Broth. (Brotherus, 1925).

Syn. Porotrichum callicostelloides Broth. ex Thér. (Brotherus ex Thériot, 1917b).

Distr. Southern South America (Paris, 1905b; Thériot, 1917b. 1926; Costes, 1921; Brotherus, 1925; Espinosa B., 1941; Herzog, 1954).

var. distichum Thér. (Thériot, 1924).

Distr. Southern South America (Thériot, 1924).

P. macrosticta Broth. (Brotherus, 1924b).

Distr. Juan Fernandez (Brotherus, 1924b, 1925; Robinson, 1975).

P. pinnatelloides (C. Muell.) Broth. ex Par.

Pinnatella thieleana

P. thieleana (C. Muell.) Broth. ex Par. (Brotherus ex Paris, 1909).

Syn. Hypnum thieleanum C. Muell. (Mueller, 1851a).

Porotrichum thieleanum (C. Muell.) Mitt. (Mitten, 1869).

Pilotrichum thieleanum (C. Muell.) Hamp. (Hampe, 1879).

Porotrichum thielei Aongstr. (Ångström, 1876a) hom. illeg.

Thamnium thielei Kindb. (Kindberg, 1902) nom. illeg.

Porotrichum pinnatelloides C. Muell. (Mueller, 1879a).

Pinnatella pinnatelloides (C. Muell.) Broth. ex Par. (Brotherus ex Paris, 1909).

Distr. Southern South America (Hampe, 1879; Mueller, 1879a; Kindberg, 1902; Paris, 1905b;

Brotherus, 1925; Kühnemann, 1938).

\section{Plagiobryum}

P. lamprocarpum (C. Muell.) Par. 


\section{Plagiomnium}

P. rugicum (Laur.) Koponen (Koponen, 1968).

Syn. Mnium rugicum Laur. (Laurer, 1827).

Mnium affine Bland. ex Funck var. rugicum (Laur.) B.S.G. (Bruch and others, 1838).

Mnium affine Bland. ex Funck var. magellanicum Card. et Broth. (Cardot and Brotherus, 1923).

Mnium lepto-limbatum C. Muell. (Mueller, 1897a) fide Koponen, 1971.

Distr. Southem South America (Mueller, 1897a; Paris, 1900a, 1905a; Cardot, 1908a; Cardot and Brotherus, 1923; Kühnemann, 1938; Koponen, 1971).

\section{Plagiopus}

P. ithyphyllus (Brid.) Mach.

Bartramia ithyphylla

P. pomiformis (Hedw.) Mach.

Bartramia pomiformis

P. strictus (Brid.) Mach.

Bartramia stricta

\section{Plagiothecium}

P. bellirete C. Muell. (Mueller, 1897a).

Distr. Southem South America (Mueller, 1897a; Paris, 1900a, 1905b; Brotherus, 1925; Kühnemann, 1938).

P. cacti (C. Muell.) Kindb.

Isopterygium cacti

P. denticulatum (Hedw.) B.S.G. (Bruch and others, 1851).

Syn. Hypnum denticulatum Hedw. (Hedwig, 1801).

Stereodon denticulatus (Hedw.) Mitt. (Mitten, 1863).

Pancovia denticulata (Hedw.) Kickx (Kickx, 1867).

Distr. Southem South America (Wilson and Hooker, 1847; Mueller, 1851a, 1885; Sullivant, 1859; Cardot, 1908a, 1913a; Roivainen, 1934).

var. obtusifolium (Turn.) Moore (Moore, 1873).

Syn. Hypnum denticulatum Hedw. var. obtusifolium Turn. (Turner, 1804a).

Hypnum obtusifolium (Turn.) Brid. (Bridel, 1812).

Plagiothecium obtusifolium (Turn.) Amann (Amann, 1928) nom. illeg.

Hypnum donnianum Sm. (Smith, 1804b).

Plagiothecium donnianum (Sm.) Mitt. (Mitten, 1869).

Distr. Southern South America (Mitten, 1869; Jaeger, 1878; Paris, 1905b).

P. donnianum (Sm.) Mitt.

Plagiothecium denticulatum var. obtusifolium

P. elegans (Brid.) Sull. var. nanum (Jur.) Mol.

Isopterygium elegans var. nanum

P. fuegianum (Besch.) Dus.

Isopterygium fuegianum

P. georgico-antarcticum (C. Muell.) Kindb. (Kindberg, 1891).

Syn. Hypnum georgico-antarcticum C. Muell. (Mueller, 1890a).

Distr. South Georgia (Mueller, 1890a; Paris, 1905b; Cardot, 1908a; Brotherus, 1925; Steere, 1961b).

P. letoplumosum Dus.

P. leucophyllum Hamp.

Isopterygium leptop/umosum Isopterygium leucophyllum

P. lucidulum (Hook. f. et Wils.) Mitt. (Mitten, 1869).

Syn. Hypnum lucidulum Hook. f. et Wils. (Hooker and Wilson in Wilson and Hooker, 1847).

Distr. Southern South America (Wilson and Hooker, 1847; Mueller, 1851a, 1885; Mitten, 1869; Jaeger, 1878; Paris, 1905b; Kühnemann, 1938).

Falkland Islands (Paris, 1905b; Kuhnemann, 1938).

P. Iucidum (Hook. f. et Wils.) Par. (Paris, 1896).

Syn. Hypnum lucidum Hook. f. et Wils. (Hooker, J. D. and Wilson, 1844).

Amblystegium lucidum (Hook. f. et Wils.) Jaeg. (Jaeger in Jaeger and Sauerbeck, 1879a). 
Distr. Southern South America (Hooker. J. D. and Wilson, 1844; Mueller, 1851a; Jaeger and Sauerbeck, 1879a; Paris, 1896, 1905b; Cardot, 1908a; Cardot and Brothenus, 1923; Brotherus, 1925; Kühnemann, 1938).

Falkland Islands (Cardot, 1908a; Brotherus, 1925; Kühnemann, 1938).

P. magellanicum Schimp.

Acrocladium auriculatum

P. magellanicum Par.

Isopterygium fuegianum

P. nanum Jur.

Isopterygium elegans var. nanum

P. nitidum (Hook. f. et Wils.) Bartr.

Catagonium nitidum

P. obtusifolium (Turn.) Amann

Plagiothecium denticulatum var. obtusifolium

P. orthocarpum Mitt. (Mitten, 1869).

Syn. Isothecium orthocarpum Brid. (Bridel, 1827b) nom. nud.

Hypnum orthocarpon Hornsch. ex C. Muell. (Hornschuch ex Mueller, 1851a) hom. illeg.

Distr. Southern South America (Hornschuch, 1820b; Bridel, 1827b; Mueller, 1851a; Mitten, 1869; Jaeger, 1878; Paris, 1905b).

P. ovatifolium Card. (Cardot, 1905a).

Distr. Southern South America (Cardot, 1905a, 1908a; Cardot and Brotherus, 1923; Brotherus, 1925; Kühnemann, 1938; Seki, 1974).

P. roseanum B.S.G. var. falklandicum Card. et Broth. (Cardot and Brotherus, 1923).

Distr. Falkland Islands (Cardot and Brotherus, 1923; Brotherus, 1925; Kühnemann, 1938).

P. schimperi Jur. et Mild. var. nanum (Jur.) Walth. et Mol. Isopterygium elegans var. nanum

P. simonovii Savicz. et Smirn. (Savich-Ljubitskaya and Smirnova, 1964a).

Distr. Antarctic, continental region (Savich-Ljubitskaya and Smirnova, 1964a; Greene, 1968a; Savich-Ljubitskaya, 1978).

P. subsimplex (Hedw.) Besch.

Sematophyllum subsimplex

\section{Platyhypnidium}

P. aquaticum (Jaeg.) Fleisch. (Fleischer, 1923).

Syn. Rhynchostegium aquaticum Jaeg. (Jaeger, 1878).

Distr. Southern South America (Hosseus, 1938c, 1939).

P. riparioides (Hedw.) Dix. (Dixon, 1933 [1934]).

Syn. Hypnum riparioides Hedw. (Hedwig, 1801).

Hypnum rusciforme Brid. (Bridel, 1801) nom. illeg.

Eurhynchium riparioides (Hedw.) Richs. (Richards, 1937).

Eurhynchium rusciforme Mild. (Milde, 1869) nom. illeg.

Distr. Southern South America (Bridel, 1827b).

Platylomidium nom. nud.

P. crispatula C. Muell.

Calyptopogon mnioides

\section{Platyneurum}

P. laticostatum (Card.) Broth. (Brotherus, 1924a).

Syn. Dicranum laticostatum Card. (Cardot, 1900).

Dicranum cirrhifolium Schimp. ex Dus. (Schimper ex Dusén, 1903a).

Distr. Southern South America (Mueller, 1885; Neger, 1899; Cardot, 1900, 1901, 1905a, 1908a; Dusén, 1903a, 1905a; Paris, 1904b; Cardot and Brotherus, 1923; Brotherus, 1924a; Roivainen and Bartram, 1937; Herzog, 1938, 1940, 1954, 1957; Kühnemann, 1938; Bartram, 1952; Bizot, 1967; Seki, 1974).

South Georgia (Cardot, 1906a, 1908a; Cardot and Brotherus, 1923; Brotherus, 1924a; Steere, 1961b; Bell, 1973b). 
Pleuridiopsis nom. nud.

P. amblyocalyx (C. Muell.) Par.

Astomiopsis amblyocalyx

P. subulata (C. Muell.) Par.

Astomiopsis subulata

\section{Pleuridium}

P. costesii Thér. (Thériot, 1921a).

Distr. Southem South America (Costes, 1921; Thériot, 1921a; Brotherus, 1924a).

P. macrothecium Dus. (Dusén, 1905a).

Distr. Southern South America (Dusén, 1905a; Roth, 1911; Campo, 1921; Thériot, 1921a; Brotherus, 1924a).

P. nervosum (Hook.) Mitt. (Mitten, 1856).

Syn. Phascum nervosum Hook. (Hooker, 1819).

Astomum nervosum (Hook.) C. Muell. (Mueller, 1847b).

Distr. Juan Fernandez (Mueller, 1856; Johow, 1896; Roth, 1911).

Southem South America (Mueller, 1847b, 1848a, 1856; Montagne, 1850; Roth, 1911).

P. robinsonii (Mont.) Mitt. (Mitten, 1869).

Syn. Phascum nervosum Hook. var. robinsonii Mont. (Montagne, 1835).

Phascum robinsonii Mont. ex C. Muell. (Montagne ex Mueller, 1848a) err.

Astomum robinsonii (Mont.) C. Muell. (Mueller, 1856).

Distr. Juan Fernandez (Montagne, 1835; Mueller, 1856; Mitten, 1869; Gibert, 1873; Jaeger, 1873; Paris, 1905b; Brotherus, 1924b; Robinson, 1975).

Southern South America (Montagne, 1835; Mueller, 1856: Jaeger, 1869, 1873; Mitten, 1869; Gibert, 1873; Dusén, 1905a; Paris, 1905b; Felippone, 1909; Thériot, 1921a, b; Brothenus, 1924a; Herter, 1928, 1933a, 1939a; Herzog, 1938, 1954; Bartram, 1952).

P. subnervosum (C. Muell.) Jaeg. (Jaeger in Paris, 1896).

Syn. Astomum subnervosum C. Muell. (Mueller, 1882).

Distr. Southern South America (Jaeger and Sauerbeck, 1879b; Mueller, 1882; Paris, 1896, 1905b; Roth, 1911; Brotherus, 1924a; Herter, 1933a; Kühnemann, 1938).

\section{Pleurochaete}

P. ecuadoriensis Broth. (Brotherus, 1920).

Distr. Southem South America (Hilpert, 1933).

\section{Pleurorthotrichum}

P. chilense Broth. (Brotherus, 1905c).

Distr. Southern South America (Brotherus, 1905c, 1924c, 1925; Espinosa B., 1941; Skottsberg, 1950; Herzog, 1954).

\section{Pleurozium}

P. schreberi (Brid.) Mitt. (Mitten, 1869).

Syn. Hypnum schreberiBrid. (Bridel, 1801b). Hylocomium schreberi (Brid.) De Not. (De Notaris, 1867). Hypnopsis schreberi (Brid.) Podp. (Podpěra, 1921). Entodon schreberi (Brid.) Moenk. (Mönkemeyer, 1927). Cuspidaria morenoi C. Muell. (Mueller, 1897a). Hypnum morenoi (C. Muell.) Par. (Paris, 1900a). Acrocladium morenoi (C. Muell.) Dus. (Dusén, 1903a). Hypnum parietinum Wahlenb. (Wahlenberg, 1812) hom. illeg.

Distr. Southern South America (Mueller, 1897a; Paris, 1900a, 1904C; Dusén, 1903a; Cardot, 1908a; Brotherus, 1924a, 1925).

\section{Pogonatum}


var. brevifolium (R. Brown) Brid.

Polytrichum alpinum

$P$. austro-georgicum (C. Muell.) Par.

Polytrichum alpinum

P. dendroides (Hedw.) Brid.

Dendroligotrichum dendroides

$P$. liliputanum Thér.

Oligotrichum canaliculatum

P. magellanicum (Hedw.) P. Beauv.

Polytrichadelphus magellanicus

P. oligodus (C. Muell.) Mitt. (Mitten, 1869).

Syn. Polytrichum oligodus Kunz. ex C. Muell. (Kunze ex Mueller, 1848a).

Cephalotrichum oligodus B.S.G. (Bruch and others, 1844) nom. nud.

Distr. Southern South America (Bruch and others, 1844; Mueller, 1848a; Montagne, 1850; Mitten. 1869; Jaeger, 1875; Paris, 1905b; Thériot, 1915, 1917b, 1921b; Campo, 1921; Costes, 1921; Brotherus, 1925; Bartram, 1952).

P. plurisetum (C. Muell.) Broth. (Brotherus, 1905a).

Syn. Polytrichum plurisetum C. Muell. (Mueller, 1879a).

Distr. Southern South America (Mueller, 1879a; Kurtz, 1904; Brotherus, 1905a, 1925;

Hosseus, 1936, 1937; Kühnemann, 1938).

var. brevipes (C. Muell.) O. Kühnemann (Kühnemann, 1938).

Syn. Polytrichum plurisetum C. Muell. var. brevipes C. Muell. (Mueller, 1879a). Pogonatum polycarpum Par. var. brevipes (C. Muell.) Par. (Paris, 1896).

Distr. Southern South America (Mueller, 1879a; Paris, 1896, 1905b; Kühnemann, 1938).

P. polycarpum Par. var. brevipes (C. Muell.) Par.

Pogonatum plurisetum var. brevipes

P. robustum (Lindb.) Jaeg.

Polytrichadelphus robustus

P. semi-angulatum Brid.

Polytrichadelphus semi-angulatus'

P. spurio-cirratum Broth. (Brotherus, 1910a).

Distr. Southern South America (Thériot, 1926).

\section{Pohlia}

P. albicans Lindb.

Pohlia wahlenbergii

P. calopyxis (C. Muell.) Broth. (Brotherus, 1903).

Syn. Bryum calopyxis C. Muell. (Mueller, 1882).

Webera calopyxis (C. Muell.) Kindb. (Kindberg, 1889).

Distr. Southern South America (Mueller, 1882; Brotherus, 1903, 1924a; Paris, 1906; Kühnemann, 1938).

P. clavata Schimp.

Bryum clavatum

P. commutata (Schimp.) Lindb.

Pohlia drummondii

P. cruda (Hedw.) Lindb. (Lindberg, 1879).

Syn. Mnium crudum Hedw. (Hedwig, 1801).

Hypnum crudum (Hedw.) Web. et Mohr (Weber and Mohr, 1803).

Bryum crudum (Hedw.) Turn. (Turner, 1804a).

Webera cruda (Hedw.) Fuernr. (Fürnrohr, 1829).

Lamprophyllum crudum (Hedw.) Lindb. (Lindberg, 1871).

Bryum synoico-crudum C. Muell. (Mueller, 1884 [1883]).

Webera synoico-cruda (C. Muell.) Kindb. (Kindberg, 1889).

Bryum viridatum C. Muell. (Mueller, 1890a).

Webera viridata (C. Muell.) Kindb. (Kindberg, 1891).

Distr. Southern South America (Mitten, 1869; Mueller, 1884, 1885, 1889; Neger, 1899;

Cardot, 1900, 1901, 1905a, 1908a, 1913a; Dusén, 1903a; Paris, 1906; Cardot and

Brotherus, 1923; Herzog, 1923, 1954, 1957; Brotherus, 1924a; Roivainen, 1934;

Kühnemann. 1938; Bartram, 1965; Seki, 1974).

South Georgia (Mueller, 1890a; Cardot, 1906a, 1908a; Paris, 1906; Dixon, 1935; Steere, $1961 b$; Clarke, 1973a). 
var. imbricata (Card.) Bartr. (Bartram, 1957).

Syn. Webera cruda (Hedw.) Fuernr. var. imbricata Card. (Cardot, 1900).

Distr. South Georgia (Clarke, 1973a).

Antarctic, peninsula region (Cardot, 1900, 1901, 1906b, 1907 C, 1908a, 1911b, d 1913a; Paris, 1906; Bartram, 1957; Steere, 1961 a; Greene and others, 1970; Robinson, 1972 (as P. cruda)).

P. cruegeri (Hamp.) Andrews

Bryum cruegeri

P. drummondii (C. Muell.) Andrews (Andrews, 1935).

Syn. Bryum drummondii C. Muell. (Mueller, 1862).

Webera drummondii (C. Muell.) Jaeg. (Jaeger, 1875).

Webera commutata Schimp. (Schimper, 1876).

Pohlia commutata (Schimp.) Lindb. (Lindberg, 1879).

Bryum commutatum (Schimp.) Boul. (Boulay, 1884) hom. illeg.

Distr. Southern South America (Cardot and Brotherus, 1923).

P. filiformis (Dicks.) Andrews

Anomobryum filiforme

P. gilliesii (Hook.) Mont.

Bryum gilliesii

P. humilis (Mont.) Broth. (Brotherus, 1903).

Syn. Bryum humile Mont. (Montagne, 1845a).

Orthodontium humile (Mont.) Mitt. (Mitten, 1869).

Webera humilis (Mont.) Broth. (Brotherus, 1924a).

Orthodontium tenue C. Muell. (Mueller, 1848a) nom. illeg.

Apalodium tenue Mitt. (Mitten, 1885) nom. illeg.

Distr. Southem South America (Montagne, 1845a, 1850, 1856; Mueller, 1848a; Mitten, 1869. 1885; Jaeger, 1875; Brotherus, 1903, 1924a; Paris, 1905a).

$P$. inclinata Sm. ex Brid.

Bryum inclinatum

P. inflexa (C. Muell.) Wijk et Marg. (van der Wijk and Margadant, 1965).

Syn. Bryum inflexum C. Muell. (Mueller, 1890a).

Webera inflexa (C. Muell.) Kindb. (Kindberg, 1891).

Philonotis inflexa (C. Muell.) Broth. (Brotherus, 1904).

Distr. South Georgia (Mueller, 1890a; Brotherus, 1904; Paris, 1905a; Cardot, 1906a, 1908a; Steere, 1961b; Clarke, 1973a, b).

P. Ionchochaete (Dus.) Broth. (Brotherus, 1909).

Syn. Webera lonchochaete Dus. (Dusén, 1903a).

Distr. Southem South America (Dusén, 1903a; Cardot, 1908a; Brotherus. 1909, 1924a; Kühnemann, 1938).

P. nevadensis (C. Muell.) Broth. (Brotherus, 1903).

Syn. Bryum nevadense C. Muell. (Mueller, 1879a).

Webera nevadensis (C. Muell.) Kindb. (Kindberg, 1889). Distr. Southern South America (Mueller, 1879a; Brotherus, 1903, 1924a; Paris, 1906;
Kühnemann, 1938).

P. nutans (Hedw.) Lindb. (Lindberg, 1879).

Syn. Webera nutans Hedw. (Hedwig, 1801).

Bryum nutans (Hedw.) Turn. (Turner, 1804a).

Lamprophyllum nutans (Hedw.) Lindb. (Lindberg, 1871).

Bryum sphagnadelphus C. Muell. (Mueller, 1885).

Webera sphagnadelphus (C. Muell.) Besch. (Bescherelle, 1889).

Bryum pulvinatum C. Muell. (Mueller, 1890a) hom. illeg. fide Clarke, 1973a.
Webera pulvinata Kindb. (Kindberg, 1891) fide Clarke, 1973a.

Pohlia pulvinata (Kindb.) Broth. (Brotherus, 1903) fide Clarke. $1973 a$.

Bryum caespitosum (Hopp. et Hornsch.) Brid. (Bridel, 1826). 
Distr. Southern South America Wilson and Hooker, 1847; Montagne, 1850; Sullivant, 1859; Mitten, 1869; Mueller, 1885; Bescherelle, 1889; Paris, 1906; Cardot, 1908a; Cardot and Brotherus, 1923; Brotherus, 1924a; Thériot, 1924; Kühnemann, 1938; Herzog, 1954: Seki. 1974).

Falkland Islands (Wilson and Hooker, 1847; Cardot and Brotherus, 1923).

South Georgia (Mueller, 1890a; Brotherus, 1903, 1924a; Cardot, 1906a, 1908a; Paris, 1906; Dixon, 1934; Steere, 1961b; Clarke, 1973a).

Antarctic, peninsula region (Cardot, 1901, 1907c, 1908a, 1911b, d, 1913a; Brotherus, 1924a; Bartram, 1957; Steere, 1961a; Robinson, 1972).

Antarctic, continental region (Savich-Ljubitskaya and Smimova, 1971; SavichLjubitskaya, 1978).

P. papillosa (Jaeg.) Broth. (Brotherus, 1903).

Syn. Bryum papillosum C. Muell. (Mueller, 1848a) hom. illeg.

Brachymenium papillosum Jaeg. (Jaeger, 1875).

Distr. Southern South America (Bartram, 1965).

P. platyphylla Schwaegr.

Bryum platyphyllum

P. pluriseta Herz. (Herzog, 1909).

Distr. Southern South America (Robinson, 1964).

P. polycarpa (Mitt.) Broth. (Brotherus, 1903).

Syn. Bryum polycarpum Mitt. (Mitten, 1869).

Webera polycarpa (Mitt.) Jaeg. (Jaeger, 1875).

Webera costesii Card. et Thér. (Cardot and Thériot in Thériot, 1917b).

Distr. Southern South America (Thériot, 1917b, 1924; Costes, 1921; Brotherus, 1924a).

P. pulvinata (Kindb.) Broth.

Pohlia nutans

P. racovitzae (Card.) Broth. (Brotherus, 1903).

Syn. Webera racovitzae Card. (Cardot, 1900).

Distr. South Georgia (Cardot, 1900).

Antarctic, peninsula region (Cardot, 1901, 1906a, b, 1907c, 1908a, 1911b, $c, d, 1912 b$, 1913a; Brotherus, 1903, 1924a; Wright, 1905 (as Bryum sp.); Paris, 1906; Dixon, 1935: Steere, 1961a).

var. laxiretis (Card.) Steere (Steere, 1961a).

Syn. Webera racovitzae Card. var. laxiretis Card. (Cardot, 1906b).

Distr. Antarctic, peninsula region (Cardot, 1906b, 1907c, 1908a; Horikawa and Ando, 1961 (as Bryum inconnexum); Steere, 1961a).

P. wahlenbergii (Web. et Mohr) Andrews (Andrews, 1935).

Syn. Hypnum wahlenbergii Web. et Mohr (Weber and Mohr, 1807).

Bryum wahlenbergii (Web. et Mohr) Schwaegr. (Schwaegrichen, 1816).

Webera wahlenbergii (Web. et Mohr) Fuernr. (Fürnrohr, 1829).

Mniobryum wahlenbergii (Web. et Mohr) Jenn. (Jennings, 1913).

Hypnum albicans Web. et Mohr (Weber and Mohr, 1803) nom. nud.

Mnium albicans Wahlenb. (Wahlenberg, 1812) nom. illeg.

Bryum albicans Roehl. (Röhling, 1813) hom. illeg.

Webera albicans Schimp. (Schimper, 1855 [1856]) nom. illeg.

Pohlia albicans Lindb. (Lindberg, 1879) nom. illeg.

Mniobryum albicans (Wahlenb.) Limpr. (Limpricht, 1892) nom. illeg.

Bryum austro-albicans C. Muell. (Mueller, 1884 [1883]).

Mniobryum austro-albicans (C. Muell.) Broth. (Brotherus, 1903).

Distr. Southern South America Wilson and Hooker, 1847; Mueller, 1848a; Paris, 1905a; Cardot and Brotherus, 1923; Brotherus, 1924a; Herzog, 1938, 1939, 1954, 1957; Hosseus, 1938c, d, 1939; Kühnemann, 1938; Bartram, 1952; Seki, 1974).

Falkland Islands Wilson and Hooker, 1847; Mueller, 1848a; Cardot, 1905a, 1908a; Paris, 1905a; Cardot and Brotherus, 1923; Brotherus, 1924a; Roivainen, 1934; Kühnemann, 1938).

South Georgia (Cardot and Brotherus, 1923; Steere, 1961b). 
var. glacialis (Brid.) E. F. Warburg (Warburg, 1962).

Syn. Mniobryum wahlenbergii (Web. et Mohr) Jenn. var. glaciale (Brid.) Wijk et Marg. (van der Wijk and Margadant, 1959).

Bryum glaciale Brid. (Bridel, 1827a).

Bryum alticaule C. Muell. (Mueller, 1884 [1883]) fide Clarke, 1973a.

Webera alticaulis (C. Muell.) Kindb. (Kindberg, 1889) fide Clarke, $1973 a$.

Mniobryum alticaule (C. Muell.) Broth. (Brotherus, 1903) fide Clarke, $1973 a$.

Bryum amplirete C. Muell. (Mueller, 1890a) fide Clarke, 1973a.

Webera ampliretis (C. Muell.) Kindb. (Kindberg, 1891) fide Clarke, 1973a.

Mniobryum amplirete (C. Muell.) Broth. (Brotherus, 1903) fide Clarke, 1973a.

Distr. Southem South America (Wilson and Hooker, 1847; Mueller, 1884, 1885, 1889;

Brotherus, 1903, 1924a; Cardot, 1905a, 1908a, 1913a; Paris, 1905a; Cardot and Brotherus, 1923; Herzog, 1923, 1954; Reimers, 1926; Kühnemann, 1938; Bartram, 1952).

Falkland Islands (Wilson and Hooker, 1847; Cardot and Brotherus, 1923; Brotherus, 1924a; Kühnemann, 1938).

South Georgia (Mueller, 1890a; Brotherus, 1903, 1924a; Paris, 1905a; Cardot, 1906a, 1908a; Dixon, 1932 (as Webera inflexa fide Dixon, 1934), 1934: Steere, 1961b; Clarke, 1973a; Bell, 1977b).

\section{Polytrichadelphus}

P. croceus Mitt.

P. dendroides (Hedw.) Mitt.

P. horridus Mitt.
Polytrichadelphus semi-angulatus

Dendroligotrichum dendroides

Polytrichadelphus magellanicus

P. magellanicus (Hedw.) Mitt. (Mitten, 1860).

Syn. Polytrichum magellanicum L. ex Hedw. (Hedwig, 1801).

Pogonatum magellanicum (Hedw.) P. Beauv. (Palisot de Beauvois, 1805a).

Catharinea magellanica (Hedw.) Brid. (Bridel, 1819).

Oligotrichum magellanicum (Hedw.) Kindb. (Kindberg, 1888).

Gymnopilum magellanicum (Hedw.) Schimp. (Schimper in Paris, 1895) nom. inval.

Polytrichadelphus horridus Mitt. (Mitten, 1869).

Oligotrichum horridum (Mitt.) Kindb. (Kindberg, 1888).

Distr. Juan Fernandez (Skottsberg, 1914: Brotherus, 1924b, 1925; Espinosa B., 1941; Bartram, 1959; Robinson, 1975).

Southern South America (Menzies, 1798; Hedwig, 1801; Bridel, 1803, 1806, 1827b; Schwaegrichen, 1816; Montagne, 1845b, 1850; Wilson and Hooker, 1847; Mueller, 1848a, 1885; Sullivant, 1859; Mitten, 1860, 1869; Duby, 1875a; Jaeger, 1875;

Bescherelle, 1889; Neger, 1899; Dusèn, 1903a; Cardot, 1905a, 1908a; Paris, 1905b; Rowlee, 1916; Cardot and Brotherus, 1923; Herzog, 1923. 1939, 1940, 1954; Brotherus, 1925; Reimers, 1926; Kühnemann, 1938; Seki, 1974).

Falkland Islands (Dumont D'Urville, 1825; Bridel, 1827b; Wilson and Hooker, 1847; Jaeger, 1875; Mueller, 1885; Bescherelle, 1889; Cardot, 1905a, 1908a; Paris, 1905b; Cardot and Brotherus, 1923; Brotherus, 1925; Kühnemann, 1938).

P. minimus Card.

Psilopilum minimum

P. robustus (Lindb.) Broth. (Brotherus, 1905a).

Syn. Polytrichum robustum Lindb. (Lindberg, 1868c).

Pogonatum robustum (Lindb.) Jaeg. (Jaeger, 1875) hom. illeg.

Distr. Southern South America (Lindberg, 1868c; Ångström, 1872; Jaeger, 1875; Brotherus, 1905a, 1925; Cardot, 1908a, 1913a; Cardot and Brotherus, 1923; Bartram, 1952).

P. semi-angulatus (Brid.) Mitt. (Mitten, 1869).

Syn. Pogonatum semi-angulatum Brid. (Bridel, 1827b).

Polytrichadelphus croceus Mitt. (Mitten, 1860).

Distr. Southern South America (Krieger, 1904).

P. squamosus (Hook. f. et Wils.) Mitt.

P. stricticaulis Dus. (Dusén, 1903a) nom. nud.

Distr. Southern South America (Dusén, 1903a; Paris, 1905b). 
P. umbrosus Mitt. (Mitten, 1869).

Syn. Oligotrichum umbrosum (Mitt.) Kindb. (Kindberg, 1888).

Distr. Southern South America (Mitten, 1869; Jaeger, 1875; Paris, 1905b; Brotherus, 1925).

\section{Polytrichastrum}

P. alpinum (Hedw.) G. L. Smith

Polytrichum alpinum

P. longisetum (Brid.) G. L. Smith

Polytrichum longisetum

\section{Polytrichum}

$P$. affine Funck

Polytrichum alpestre

P. alpestre Hopp. (Hoppe, 1801).

Syn. Polytrichum strictum Menz. ex Brid. (Menzies ex Bridel, 1801a).

Polytrichum strictum Menz. ex Brid. var. alpestre (Hopp.) Rabenh. (Rabenhorst, 1848).

Polytrichum affine Funck (Funck, 1802).

Polytrichum juniperinum Hedw. var. affine (Funck) Brid. (Bridel, 1806).

Polytrichum juniperinum Hedw. var. alpestre (Hopp.) Roehl. (Röhling, 1813).

Polytrichum juniperinum Hedw. var. strictum (Brid.) Roehl. (Röhling, 1813) nom. illeg.

Polytrichum juniperinum Hedw. ssp. strictum (Brid.) Nyl. et Sael. (Nylander and Saelan, 1859).

Polytrichum macroraphis C. Muell. (Mueller, 1890a) pro parte fide Greene, 1973.

Distr. Southern South America (Montagne, 1850; Mueller, 1885; Bescherelle, 1889; Dusén, 1903a, 1905d; Cardot, 1905a, 1908a; Paris, 1905b; Spegazzini, 1922; Cardot and Brotherus, 1923; Brotherus, 1925; Kühnemann, 1938; Martin, 1949).

Falkland Islands (Cardot and Brotherus, 1923; Kühnemann, 1938).

South Georgia (Mueller, 1890a; Paris, 1905b; Cardot, 1906a, 1908a; Cardot and Brotherus, 1923; Brotherus, 1925; Steere, 1961b; Greene, 1973; Bell, 1977b).

Antarctic, peninsula region (Cardot, 1900, 1901, 1906a, 1908a, 1911b, $d, 1913 a$; Paris, 1905 b; Brotherus, 1925; Steere, 1961a; Greene and others, 1970; Robinson, 1972).

P. alpinum Hedw. (Hedwig, 1801).

Syn. Pogonatum alpinum (Hedw.) Roehl. (Röhling, 1814).

Polytrichastrum alpinum (Hedw.) G. L. Smith (Smith, 1971).

Polytrichum austro-georgicum C. Muell. (Mueller, 1890a) fide Greene, 1973.

Pogonatum austro-georgicum (C. Muell.) Par. (Paris, 1898).

Polytrichum nano-cephalum C. Muell. (Mueller, 1890a).

Polytrichum plurirameum C. Muell. (Mueller, 1890a) fide Greene, 1973.

Polytrichum timmioides C. Muell. (Mueller, 1908a) fide Greene, 1973.

Pogonatum alpinum (Hedw.) Roehl. var. brevifolium (R. Brown) Brid. (Bridel, 1927b).

Polytrichum macroraphis C. Muell. (Mueller, 1890a) pro parte fide Greene, 1973.

Distr. Southern South America (Cardot, 1905a, 1908a; Thériot, 1935a; Kühnemann, 1938).

South Georgia (Mueller, 1890a; Paris, 1898, 1905b; Cardot, 1906a, 1908a; Cardot and Brotherus, 1923; Brotherus, 1925; Dixon, 1932; Steere, 1961b; Greene, 1973).

Antarctic, peninsula region (Cardot, 1900*, 1901*, 1906a*, $b, 1907 c, 1908 a^{*}, 1911 b^{*}$, $d^{*}, 1913 a^{*}$; Paris, 1905b*; Dixon, 1920, 1935; Brotherus, 1925*; Bartram, 1957*; Steere, 1961 $a^{*}$; Greene, 1968a; Greene and others, 1970; Robinson, 1972).

var. integrifolium Card. et Broth. (Cardot and Brotherus, 1923).

Distr. Southern South America (Cardot and Brotherus, 1923; Kühnemann, 1938).

P. antarcticum Card.

Polytrichum piliferum

P. antillarum Rich.

Polytrichum juniperinum

ssp. prionotum (C. Muell.) Walth.

ssp. secundulum (C. Muell.) Walth.

Polytrichum juniperinum var. tumescens

ssp. substrictum Walth.

Polytrichum juniperinum

$P$. australe Hook. f. et Wils.

Polytrichum juniperinum

Psilopilum australe

*and as Pogonatum alpinum (Hedw.) Roehl. var. brevifolium (R. Brown) Brid. fide Greene and others, 1970. 
P. austro-georgicum C. Muell.

Polytrichum alpinum

P. breve C. Muell.

Polytrichum juniperinum var. tumescens

$P$. canaliculatum Hook. et Arnott

Oligotrichum canaliculatum

P. compressum Hook. f. et Wils.

Atrichopsis compressa

P. dendroides Brid. ex Hedw.

Dendroligotrichum dendroides

P. elongatum P. Beauv. (Palisot de Beauvois, 1805a).

Distr. Southem South America (Palisot de Beauvois, 1805a; Bridel, 1806, 1827b; Schwaegrichen, 1816; Mueller, 1848a; Mitten, 1869; Jaeger, 1875; Paris, 1905b; Cardot, 1908a).

$P$. gracile Dicks.

Polytrichum longisetum

P. juniperifolium Schimp. ex Par.

Polytrichum juniperinum

$P$. juniperiforme Schimp. ex Mitt.

Polytrichum juniperinum

P. juniperinum Hedw. (Hedwig, 1801).

Syn. Polytrichum juniperinum Hedw. var. alpinum Schimp. (Schimper, 1860).

Polytrichum juniperinum Hedw. var. integrum C. Muell. (Mueller, 1848a).

Polytrichum antillarum Rich. (Richard in Bridel, 1827b) nom. illeg.

Polytrichum antillarum Rich. ssp. substrictum Walth. (Walther, 1934).

Polytrichum antillarum Rich. ssp. secundulum (C. Muell.) Walth. (Walther, 1934).

Polytrichum secundulum C. Muell. (Mueller, 1897b).

Polytrichum prionotrichum C. Muell. (Mueller, 1897a).

Polytrichum substrictum Hamp. (Hampe in Paris, 1897) nom. nud.

Polytrichum juniperiforme Schimp. ex Mitt. (Schimper ex Mitten, 1869).

Polytrichum juniperifolium Schimp. ex Par. (Schimper ex Paris, 1905b) nom. inval.

Distr. Southern South America (Mueller, 1848a, 1897a; Montagne, 1850; Lorentz, 1866; Mitten, 1869; Jaeger, 1875; Paris, 1897, 1905b; Dusén, 1903a, 1905d; Kurtz, 1904; Cardot, 1905a, 1908a; Thériot, 1921b, 1935a; Cardot and Brotherus, 1923; Espinosa B., 1924; Brotherus, 1925; Reimers, 1926; Roivainen, 1934; Walther, 1934; Hosseus, 1936, 1937, 1938c, d; Kühnemann, 1938; Herter, 1939b; Bartram, 1952; Bizot, 1967; Seki, 1974).

Falkland Islands (Kühnemann, 1938).

South Georgia (Cardot, 1906a, 1908a; Steere, 1961 b; Greene, 1973).

Antarctic, peninsula region (Greene, 1968a; Greene and others, 1970).

ssp. strictum (Brid.) Nyl. et Sael.

Polytrichum alpestre

var. affine (Funck) Brid.

Polytrichum alpestre

var. alpestre (Hopp.) Roehl.

Polytrichum alpestre

var. alpinum Schimp.

Polytrichum juniperinum

var. australe Walth. (Walther, 1934) hom. illeg.

Syn. Polytrichum spegazzinii C. Muell. (Mueller, 1885).

Distr. Southern South America (Mueller, 1885; Neger, 1899; Paris, 1905b; Cardot, 1908a;

Brotherus, 1925; Walther, 1934, 1940; Kühnemann, 1938; Herzog, 1939, 1957).

var. integrum C. Muell.

Polytrichum juniperinum

var. strictum (Brid.) Roehl.

Polytrichum alpestre

var. tumescens (C. Muell.) Herz. (Herzog, 1916a).

Syn. Polytrichum tumescens C. Muell. (Mueller, 1897a).

Polytrichum prionotum C. Muell. (Mueller, 1897a).

Polytrichum breve C. Muell. (Mueller, 1897a).
Polytrichum antillarum Rich. Ssp. prionotum (C. Muell.) Walth. (Walther, 1934).

Distr. Southern South America (Mueller, 1897a; Paris, 1905b; Brotherus, 1925; Walther. 1934).

P. Ionchopelma C. Muell. (Mueller, 1901a [1900]) nom. nud.

Distr. Southern South America (Mueller, 1901a). 
P. longisetum Sw. ex Brid. (Swartz ex Bridel, 1801).

Syn. Polytrichastrum longisetum (Brid.) G. L. Smith (Smith, 1971).

Polytrichum gracile Dicks. (Dickson in Menzies in Schrebius, 1802).

Distr. Southern South America (Herzog, 1939).

P. macroraphis C. Muell. Polytrichum alpestre pro parte and Polytrichum alpinum pro parte

P. magellanicum L. ex Hedw.

Polytrichadelphus magellanicus

P. molinae Mont.

Oligotrichum canaliculatum var. molinae

P. nano-cephalum C. Muell.

Polytrichum alpinum

P. noduliferum Dus.

Polytrichum piliferum

P. oligodus Kunz. ex C. Muell.

Pogonatum oligodus

P. patagonicum C. Muell.

Polytrichum piliferum

P. piliferum Hedw. (Hedwig, 1801).

Syn. Polytrichum pilifolium C. Muell. (Mueller, 1897a) hom. illeg.

Polytrichum patagonicum C. Muell. (Mueller, 1897a).

Polytrichum antarcticum Card. (Cardot, 1900) fide Greene and others, 1970.

Polytrichum subpiliferum Card. (Cardot, 1900).

Polytrichum trachynotum C. Muell. (Mueller, 1885).

Polytrichum noduliferum Dus. (Dusén in Paris, 1905b) nom. nud.

Distr. Juan Fernandez (Montagne, 1835; Paris, 1905b; Brotherus, 1925).

Southern South America (Mitten, 1869; Ángström, 1872; Mueller, 1885, 1897a; Cardot, 1900, 1901, 1905a, 1908a, 1913a; Dusén, 1903a; Kurtz, 1904; Paris, 1905b; Spegazzini, 1922; Brotherus, 1925; Reimers, 1926; Hosseus, 1936, 1937; Herzog, 1938, 1939, 1957; Kühnemann, 1938; Bartram, 1952; Seki, 1974).

Falkland Islands (Cardot, 1905a, 1908a; Paris, 1905b; Cardot and Brotherus, 1923; Brotherus, 1925; Kühnemann, 1938).

South Georgia (Cardot, 1906a, 1908a; Brotherus, 1925; Steere, 1961b; Greene, 1973).

Antarctic, peninsula region (Cardot, 1900, 1901, 1906a, b, 1907c, 1908a, 1911b, c, d, 1912b, 1913a; Wright, 1905; Paris, 1905b; Dixon, 1920, 1935; Brotherus, 1925; Bartram, 1957; Steere, 1961a; Greene and others, 1970; Robinson, 1972).

P. pilifolium C. Muell.

Polytrichum piliferum

P. plurirameum C. Muell.

Polytrichum alpinum

P. plurisetum C. Muell.

Pogonatum plurisetum

var. brevipes C. Muell.

Pogonatum plurisetum var. brevipes

P. prionotrichum C. Muell.

Polytrichum juniperinum

P. prionotum C. Muell.

Polytrichum juniperinum var. tumescens

P. pygmaeocaulon C. Muell. (Mueller, 1901a [1900]) nom. nud.

Distr. Southern South America (Mueller, 1901a).

$P$. riedelianum Mont.

Oligotrichum riedelianum

$P$. robustum Lindb.

Polytrichadelphus robustus

P. secundulum C. Muell.

Polytrichum juniperinum

P. spegazzinii C. Muell.

P. squamosum Hook. f. et Wils.

Polytrichum juniperinum var. australe

Dendroligotrichum squamosum

P. stolonigerum C. Muell. (Mueller, 1901a [1900]).

Distr. Southern South America (Mueller, 1901a).

P. strictum Menz. ex Brid.

Polytrichum alpestre

var. alpestre (Hopp.) Rabenh.

Polytrichum alpestre

$P$. subpiliferum Card.

Polytrichum piliferum

P. substrictum Hamp.

Polytrichum juniperinum

P. timmiaceum C. Muell. (Mueller, 1901a [1900]) nom. nud.

Distr. Southern South America (Mueller, 1901a). 
P. timmioides C. Muell.

$P$. trachynotum C. Muell.

P. trichodon Hook. f. et Wils.

Psilopilum trichodon

$P$. tumescens C. Muell.

var. pygmaeocaulon C. Muell. (Mueller, 1897a).

Distr. Southern South America (Mueller, 1897a; Paris, 1905b).

P. umbrosus Mitt. (Mitten in Brotherus, 1925).

Distr. Southern South America (Brotherus, 1925).

\section{Porothamnium}

P. arbusculans (C. Muell.) Fleisch. (Fleischer in Cardot and Brotherus, 1923).

Syn. Hypnum arbusculans C. Muell. (Mueller, 1874).

Thamnium arbusculans (C. Muell.) Jaeg. (Jaeger, 1877).

Thamnium arbusculans (C. Muell.) Jaeg. var. complanatum C. Muell. (Mueller in Kindberg, 1902) nom. nud.

Porothamnium arbusculans (C. Muell.) Fleisch. var. complanatum Card. (Cardot in Cardot and Brotherus, 1923).

Hypnum naumanii C. Muell. (Mueller, 1884 [1883]).

Hypnodendron naumanii (C. Muell.) Kindb. (Kindberg, 1891).

Thamnium naumanii (C. Muell.) Kindb. (Kindberg, 1902).

Thamnium decumbens Besch. (Bescherelle, 1885a).

Porothamnium decumbens (Besch.) Fleisch. (Fleischer, 1908b).

Porotrichum corralense Broth. (Brotherus in Kindberg, 1902) nom. nud.

Thamnium corralense Broth. (Brotherus in Reimers, 1926) nom. nud.

Distr. Juan Fernandez (Skottsberg, 1914; Brotherus, 1924b; Robinson, 1975).

Southem South America (Mueller, 1874, 1884, 1885, 1889; Jaeger, 1877; Bescherelle, 1885a, 1889; Neger, 1899; Kindberg, 1902; Paris, 1904b, 1905b; Cardot, 1908a; Fleischer, 1908b; Cardot and Brotherus, 1923; Herzog, 1923, 1938, 1939, 1960; Brotherus, 1925; Reimers, 1926; Gunckel, 1938; Kühnemann, 1938; Bizot, 1967; Seki, 1974).

var. complanatum Card.

Porothamnium arbusculans

P. decumbens (Besch.) Fleisch. Porothamnium arbusculans

P. explanatum (Mitt.) Fleisch. (Fleischer in Brotherus, 1925).

Syn. Porotrichum explanatum Mitt. (Mitten, 1869). Thamnium explanatum (Mitt.) Kindb. (Kindberg, 1902).

Distr. Southern South America (Kindberg, 1902).

P. fasciculatum (Hedw.) Fleisch. (Fleischer, 1908b).

Syn. Hypnum fasciculatum Hedw. (Hedwig, 1801).

Neckera fasciculata (Hedw.) Arnott (Arnott, 1827).

Porotrichum fasciculatum (Hedw.) Mitt. (Mitten, 1869).

Dendro-Hypnum fasciculatum (Hedw.) Hamp. (Hampe, 1870) nom. inval.

Thamnium fasciculatum (Hedw.) C. Muell. (Mueller, 1898b).

Distr. Juan Femandez (Brotherus, 1924b, 1925; Espinosa B., 1941; Bartram, 1959).

Southem South America (Montagne, 1850; Fleischer, 1908b).

P. leucocaulon (C. Muell.) Fleisch. (Fleischer in Brotherus, 1925).

Syn. Neckera leucocaulon C. Muell. (Mueller, 1847c).

Porotrichum leucocaulon (C. Muell.) Mitt. (Mitten, 1869).

Thamnium leucocaulon (C. Muell.) Kindb. (Kindberg, 1902).

Distr. Southern South America (Mueller, 1847 C; Montagne. 1850; Mitten, 1869; Jaeger, 1877; Kindberg, 1902; Paris, 1905b; Cardot, 1908a; Herzog, 1923, 1938, 1940, 1957;

Brotherus, 1925; Roivainen, 1934; Gunckel, 1938; Kühnemann, 1938; Bizot, 1967).

P. lorentzii (C. Muell.) Fleisch.

Porothamnium neckeraeforme 
P. mattogrossense (Broth.) Fleisch. (Fleischer in Brotherus, 1925).

Syn. Thamnium mattogrossense Broth. (Brotherus, 1900).

Distr. Southern South America (Brotherus, 1925; Kühnemann, 1938).

P. neckeraeforme (Hamp.) Fleisch. (Fleischer in Brotherus, 1925).

Syn. Hypnum neckeraeforme Hamp. (Hampe, 1866).

Porotrichum neckeraeforme (Hamp.) Mitt. (Mitten, 1869).

Thamnium neckeraeforme (Hamp.) Kindb. (Kindberg, 1902).

Porotrichum lorentzii C. Muell. (Mueller, 1879a).

Porothamnium lorentzii (C. Muell.) Fleisch. (Fleischer, 1908b).

Distr. Southern South America (Mueller, 1879a; Kindberg, 1902; Paris, 1905b; Fleischer, 1908b; Brotherus, 1925; Kühnemann, 1938).

P. panduraefolium (C. Muell.) Fleisch. (Fleischer, 1908b).

Syn. Hypnum panduraefolium C. Muell. (Mueller, 1855).

Porotrichum panduraefolium (C. Muell.) Mitt. (Mitten, 1869).

Thamnium panduraefolium (C. Muell.) Broth. (Brotherus, 1906b).

Porotrichum panduraeforme Kindb. (Kindberg, 1891) err.

Thamnium angustifolium Schimp. (Schimper in Lechler, 1857) nom. nud. fide Mitten, 1869.

Distr. Southern South America (Mueller, 1855; Lechler, 1857; Mitten, 1869; Jaeger, 1877;

Paris, 1905b; Brotherus, 1906b, 1925; Fleischer, 1908b; Campo, 1915, 1921; Thériot, 1918; Cardot and Brotherus, 1923; Herzog, 1923, 1938, 1954, 1960; Reimers, 1926;

Gunckel, 1938; Seki, 1974).

P. porrectulum (C. Muell.) Fleisch. (Fleischer in Brotherus, 1925).

Syn. Porotrichum porrectulum C. Muell. (Mueller, 1879a).

Thamnium porrectulum (C. Muell.) Broth. (Brotherus, 1906b).

Distr. Southern South America (Mueller, 1879a; Paris, 1905b; Brotherus, 1906b, 1925; Kühnemann, 1938).

P. valdiviae (C. Muell.) Fleisch. (Fleischer in Brotherus, 1925).

Syn. Hypnum valdiviae C. Muell. (Mueller, 1855).

Porotrichum valdiviae (C. Muell.) Mitt. (Mitten, 1869).

Thamnium valdiviae (C. Muell.) Kindb. (Kindberg, 1902).

Thamnium valdivicum C. Muell. (Mueller in Kindberg, 1902) nom. nud.

Hypnum neckera Schimp. (Schimper in Mueller, 1855) nom. nud.

Thamnium neckera Schimp. (Schimper in Jaeger, 1877) nom. nud.

Distr. Juan Fernandez (Robinson, 1975).

Southern South America (Mueller, 1855; Lorentz, 1866; Mitten, 1869; Jaeger, 1877;

Kindberg, 1902; Paris, 1905b; Cardot, 1908a; Thériot, 1918; Cardot and Brotherus, 1923; Brotherus, 1925; Reimers, 1926; Roivainen, 1934; Gunckel, 1938; Herzog,

1938, 1954, 1957, 1960; Kühnemann, 1938; Bartram, 1952: Bizot, 1967; Seki, 1974).

var. pallidum (Lor.) Wijk et Marg. (van der Wijk and Margadant, 1961).

Syn. Hypnum valdiviae C. Muell. var. pallidum Lor. (Lorentz, 1866).

Porotrichum valdiviae (C. Muell.) Mitt. var. pallidum (Lor.) Jaeg. (Jaeger, 1877).

Distr. Southern South America (Lorentz, 1866; Paris, 1900a, 1905b).

\section{Porotrichodendron}

P. porteri (Thér.) Broth. (Brotherus, 1925).

Syn. Porotrichum porteri Thér. (Thériot, 1917b).

Distr. Southern South America (Thériot, 1917b; Brotherus, 1925).

P. robustum Broth. (Brotherus in Herzog, 1916a).

Distr. Southern South America (Bartram, 1965).

\section{Porotrichum}

P. callicostelloides Broth. ex Thér. 
P. chilense Thér. (Thériot, 1924).

Distr. Southem South America (Thériot, 1918 (as Porothamnium valdiviae forma minor Thér.), 1924; Costes, 1921; Brotherus, 1925).

P. cochlearifolium (Schwaegr.) Mitt.

Weymouthia cochlearifolia

P. confertum Mitt.

P. corralense Broth.

Thamnobryumponfertum

P. crassinervium Mitt. ex Broth.

Porothamnium arbusculans

$P$. explanatum Mitt.

Thamnobryum rigidum

P. fasciculatum (Hedw.) Mitt.

Porothamnium explanatum

$P$. gracile (Hook. f. et Wils.) Mitt.

Porothamnium fasciculatum

$P$. latinerve Mitt.

Camptochaete gracilis

Thamnobryum rigidum

$P$. leucocaulon (C. Muell.) Mitt.

Porothamnium leucocaulon

P. Iorentzii C. Muell.

P. neckeraeforme (Hamp.) Mitt.

Porothamnium neckeraeforme

P. neckeroides (Hook.) Williams

Porothamnium neckeraeforme

Thamnium neckeroides

$P$. panduraeforme Kindb.

Porothamnium panduraefolium

P. panduraefolium (C. Muell.) Mitt.

Porothamnium panduraefolium

$P$. pinnatelloides $\mathrm{C}$. Muell.

Pinnatella thieleana

P. porrectulum C. Muell.

Porothamnium porrectulum

$P$. porteri Thér.

Porotrichodendron porteri

$P$. rigidum Mitt.

Thamnobryum rigidum

P. thieleanum (C. Muell.) Mitt.

Pinnatella thieleana

P. thieli Aongstr.

Pinnatella thieleana

P. valdiviae (C. Muell.) Mitt.

Porothamnium valdiviae

var. pallidum (Lor.) Jaeg.

Porothamnium valdiviae var. pallidum

Pottia

P. altipes Broth. (Brotherus, 1918).

Distr. Southern South America (Brotherus, 1918, 1924a; Kühnemann, 1938).

P. amblyophylla (Hook.) C. Muell.

Didymodon amblyophyllus

P. anderssonii Sauerb.

Pottia antarctica

P. antarctica Schimp. (Schimper in Jaeger, 1873) nom. nud.

Distr. Southern South America (Jaeger, 1873).

P. antarctica (Aongstr.) C. Muell. (Mueller, 1885).

Syn. Gymnostomum antarcticum Aongstr. (Ångström 1872).

Gymnostomum magellanicum Aongstr. ex Par. (Ångström ex Paris, 1896) nom. inval. Pottia magellanica Schimp. ex Par. (Schimper ex Paris, 1898) hom. illeg.

Pottia anderssonii Sauerb. (Sauerbeck in Jaeger and Sauerbeck, 1879b) nom. illeg.

Distr. Southern South America (Lechler, 1857; Ångström, 1872; Jaeger and Sauerbeck, 1879b; Mueller, 1885; Paris, 1898, 1905b; Dusén, 1906; Cardot, 1908a; Warnstorf, 1916; Kühnemann, 1938).

P. austro-georgica Card. (Cardot, 1906a).

Syn. P. austro-georgica Card. var. microphylla Card. et Broth. (Cardot and Brotherus, 1923) fide Matteri, $1977 a$.

Distr. Southern South America (Warnstorf, 1916)

South Georgia (Cardot, 1906a, 1908a; Cardot and Brotherus, 1923; Brotherus, 1924a; Steere, 1961b; Greene, 1968a; Matteri, 1977a)

Antarctic, peninsula region (Matteri, 1977b) 
var. microphylla Card. et Broth.

Pottia austro-georgica

P. brunnea (C. Muell.) Par.

Didymodon brunneus

P. cavifolia Ehrh. ex Fuernr.

Pterygoneurum ovatum

$P$. charcotii Card.

Pottia heimii

P. chubutensis Card. et Broth. (Cardot and Brotherus, 1923).

Distr. Southern South America (Cardot and Brotherus, 1923; Brotherus, 1924a; Kühnemann, 1938).

P. flavipes Mont. (Montagne, 1845a).

Syn. Pottia heimii (Hedw.) Hamp. var. flavipes (Mont.) Warnst. (Warnstorf, 1916).

Pottia flaviseta Mont. (Montagne, 1888) err.

Tortula flaviseta Mitt. (Mitten, 1869).

Distr. Southern South America (Montagne, 1845a, 1850, 1856; Mueller, 1849; Mitten, 1869; Reichardt, 1870; Paris, 1905b; Warnstorf, 1916; Brotherus, 1924a; Bartram, 1942, 1943).

P. flaviseta Mont.

Pottia flavipes

P. glauco-viridis (C. Muell.) Par.

Didymodon glauco-viridis

$P$. gracillima (C. Muell.) Par.

Trichostomum gracillimum

$P$. guessfeldtii Schlieph.

Pottia heimii var. guessfeldtii

P. gymna (C. Muell.) Par.

Didymodon gymnus

P. heimii (Hedw.) Hamp. (Hampe, 1837a).

Syn. Gymmnostomum heimii Hedw. (Hedwig, 1801).

Bryum heimii (Hedw.) Dicks. ex With. (Dickson ex Withering, 1801).

Desmatodon heimii (Hedw.) Mitt. (Mitten, 1864).

Tortula heimii (Hedw.) Mitt. (Mitten, 1869).

Didymodon heimii (Hedw.) Kindb. (Kindberg, 1897).

Pottia charcotii Card. (Cardot, 1911 d) fide Matteri, $1977 \mathrm{~b}$.

Pottia heimii (Hedw.) Hamp. var. charcotii (Card.) Savicz. et Smirn. (Savich-

Ljubitskaya and Smirnova, 1963a) fide Matteri, 1977 b.

Distr. Southern South America Wilson and Hooker, 1847; Mueller, 1849; Sullivant, 1859; Mitten, 1869; Jaeger, 1873; Dusén, 1907; Cardot, 1908a; Warnstorf, 1916; Cardot and Brotherus, 1923; Herzog, 1923; Brotherus, 1924a; Kühnemann, 1938).

Falkland Islands (Wilson and Hooker, 1847; Cardot, 1905a, 1908a).

Antarctic, peninsula region (Cardot, 1911b, d, 1913a; Brotherus, 1924a; Bartram, 1957; Steere, 1961a; Savich-Ljubitskaya and Smirnova, 1963a, 1965b; Robinson, 1972; Matteri, 1977b).

var. brevinervis Savicz. et Smirn. (Savich-Ljubitskaya and Smirnova, 1965b).

Distr. Antarctic, continental region (Savich-Ljubitskaya and Smirnova, 1965b; Greene, 1968a; Kuc, 1969; Savich-Ljubitskaya, 1978).

var. charcotii (Card.) Savicz. et Smirn.

Pottia heimii

var. eurystoma Card. et Broth. (Cardot and Brotherus, 1923).

Distr. Southern South America (Cardot and Brotherus, 1923; Kühnemann, 1938).

var. flavipes (Mont.) Warnst.

Pottia flavipes

var. guessfeldtii (Schlieph.) Warnst. (Warnstorf, 1916).

Syn. Pottia guessfeldtii Schlieph. (Schliephacke, 1884).

Stegonia guessfeldtii (Schlieph.) Hag. (Hagen in Hagen and Printz, 1929).

Distr. Southern South America (Schliephacke, 1884; Paris, 1905b; Warnstorf, 1916; Cardot and Brotherus, 1923; Brotherus, 1924a; Kühnemann, 1938).

var. magellanica Warnst. (Warnstorf, 1916).

Syn. Pottia magellanica Schimp. (Schimper in Mueller, 1885) nom. nud.

Pottia megapoda C. Muell. (Mueller, 1897a).

Pottia systyliopsis C. Muell. (Mueller, 1897a). 
Distr. Southern South America (Jaeger, 1873; Mueller, 1897a; Paris, 1905b; Cardot, 1908a; Warnstorf, 1916; Cardot and Brotherus, 1923; Brotherus, 1924a; Kühnemann, 1938).

var. maxima Card. (Cardot, 1905a).

Distr. Southern South America (Cardot, 1905a, 1908a; Cardot and Brotherus, 1923).

var. spegazzinii (C. Muell.) Warnst. (Warnstorf, 1916).

Syn. Pottia spegazzinii C. Muell. (Mueller, 1885).

Distr. Southern South America (Mueller, 1885; Dusén, 1903a, 1906; Paris, 1905b; Cardot, 1908a; Warnstorf, 1916; Brotherus, 1924a; Kühnemann, 1938).

var. thaxteri Card. et Thér. (Cardot and Thériot in Thériot, 1929c [1930]).

Distr. Southern South America (Thériot, 1929c).

P. imperfecta (C. Muell.) Par.

Barbula imperfecta

P. kunzeana C. Muell.

P. lamprocarpa (C. Muell.) Broth.

Hymenostylium kunzeanum

P. lamprothecium (C. Muell.) Broth.

Tetrapterum lamprocarpum

$P$. longirostris C. Muell.

Tetrapterum lamprothecium

P. lorentziana C. Muell.

Hymenostylium kunzeanum

$P$. lorentzii C. Muell.

Didymodon lorentzianus

Stegonia lorentzii

P. macrocarpa Schimp. (Schimper, 1836).

Syn. Pottia macropoda Schimp. ex Broth. (Schimper ex Brotherus, 1902) err. Tortula macrocarpa (Schimp.) Mitt. (Mitten, 1869).

Hyophila macrocarpa (Schimp.) Kindb. (Kindberg, 1888).

Distr. Southern South America (Schimper, 1836; Mueller, 1849; Montagne, 1850; Mitten, 1869; Jaeger, 1873; Brotherus, 1902; Paris, 1905b; Warnstorf, 1916).

P. macropoda Schimp. ex:Broth.

Pottia macrocarpa

P. magellanica Schimp.

Pottia heimii var. magellanica

P. magellanica Schimp. ex Par.

Pottia antarctica

P. megapoda C. Muell.

P. microthecia (C. Muell.) Par.

Pottia heimii var. magellanica

Didymodon microthecius

P. mucronata Warnst. (Warnstorf, 1916) nom. nud.

Distr. Southern South America (Warnstorf, 1916).

P. nuda (C. Muell.) Par.

Didymodon brunneus

$P$. orbignyana C. Muell.

P. ovata (Hedw.) Fuernr. ex Lindb.

Didymodon orbignyanus

Pterygoneurum ovatum

P. pellucida Card. (Cardot, 1911 d) nom. nud.

Distr. Southern South America (Cardot, 1911d).

$P$. physcomitrioides C. Muell.

Pottia truncata

P. poeppigiana (C. Muell.) C. Muell.

Trichostomopsis australasiae

P. recurvirostra Ehrh. ex Hedw.

Hymenostylium recurvirostre

P. spathulato-linearis (C. Muell.) Par.

Didymodon spathulato-linearis

$P$. spegazzinii C. Muell.

Pottia heimii var. spegazzinii

P. subsessilis (Brid.) B.S.G.

Pterygoneurum subsessile

P. systyliopsis C. Muell.

Pottia heimii var. magellanica

P. truncata (Hedw.) B.S.G. (Bruch and others, 1843).

Syn. Gymnostomum truncatum Hedw. (Hedwig, 1801).

Bryum truncatum (Hedw.) Brotero (Brotero, 1804).

Tortula truncata (Hedw.) Mitt. (Mitten in Godman, 1870). 
Bryum truncatulum L. ex With. (Withering, 1801) nom. illeg.

Pottia truncatula (With.) Bus. (Buse, 1858) hom. illeg.

Tortula truncatula (With.) Lindb. (Lindberg, 1879) nom. illeg.

Pottia physcomitrioides C. Muell. (Mueller, 1897a).

Distr. Southern South America (Mueller, 1897a; Krieger, 1904; Paris, 1905b; Felippone, 1909; Warnstorf, 1916; Brotherus, 1924a; Herter, 1933a; Kühnemann, 1938).

P. truncatula (With.) Bus.

Pottia truncata

\section{Pseudocrossidium}

P. chilense Williams (Williams, 1915).

Distr. Southern South America (Williams, 1915; Brotherus, 1924a; Hilpert, 1933).

P. leucocalyx (Mont.) Thér. (Thériot, 1923a [1921]).

Syn. Tortula leucocalyx Mont. (Montagne, 1838).

Barbula leucocalyx (Mont.) C. Muell. (Mueller, 1849).

Barbula pachyneura Dus. (Dusén, 1906).

Pseudocrossidium pachyneuron (Dus.) Thér. (Thériot, 1921a).

Distr. Southern South America (Montagne, 1838, 1839c, 1845a, 1850, 1856; Mueller, 1849; Mitten, 1869; Jaeger, 1873; Paris, 1904a; Dusén, 1906; Thériot, 1917b, 1921a, 1922. 1923a; Costes, 1921; Brotherus, 1924a, c; Espinosa B., 1941; Skottsberg, 1950).

$P$. pachyneuron (Dus.) Thér.

Pseudocrossidium leucocalyx

\section{Pseudocryphaea}

P. flagellifera (Brid.) Britt. (Britton in Brotherus, 1925).

Syn. Pilotrichum flagellifera Brid. (Bridel, 1827b).

Distr. Southern South America (Hosseus, 1940; Herzog, 1952a).

\section{Pseudodistichium}

P. atlanticum * Dix. (Dixon in Dixon and Fleischer, 1937).

Distr. Southern South America (Roivainen and Bartram, 1937; Robinson, 1964).

P. austro-georgicum Card. (Cardot, 1905b).

Distr. Southern South America (Roivainen and Bartram, 1937; Kühnemann, 1938).

Falkland Islands (Brotherus, 1924a; Kühnemann, 1938).

South Georgia (Cardot, 1905b, 1906a, 1908a; Cardot and Brotherus, 1923; Brotherus, 1924a; Steere, 1961b).

var. longifolium Broth. (Brotherus in Cardot and Brotherus, 1923).

Syn. Pseudodistichium falklandicum Card. (Cardot in Cardot and Brotherus, 1923) nom. nud.

Distr. Southern South America (Roivainen and Bartram, 1937; Kühnemann, 1938).

Falkland Islands (Cardot and Brotherus, 1923; Kühnemann, 1938).

P. falklandicum Card.

Pseudodistichium austro-georgicum var. longifolium

P. fuegianum Roiv. (Roivainen in Roivainen and Bartram, 1937).

Distr. Southern South America (Roivainen and Bartram, 1937; Kühnemann, 1938).

Antarctic, peninsular region (Robinson, 1972).

P. taitaoense* Froehl. (Froehlich, 1953).

Distr. Southern South America (Froehlich, 1953; Seki, 1974).

\section{Pseudoleskea}

$P$. antarctica Card.

Hygroamblystegium antarcticum

P. calochroa Card. Hygroamblystegium calochroum

$P$. catenulatula C. Muell.

Leskeadelphus catenulatulus

“According to Robinson (1972), both of these species are synonyms of Ditrichum conicum (Mont.) Mitt. 
P. filum (C. Muell.) Par.

Hygroamblystegium filum

$P$. fuegiana (Besch.) Broth. Hygroamblystegium fuegianum

var. gracilis Card. et Broth.

Hygroamblystegium fuegianum var. gracilis

var. skottsbergii Card.

$P$. laplatae C. Muell.

Hygroamblystegium fuegianum var. skottsbergii

P. Iurida Card.

Haplocladium microphyllum

P. microphylla (Hedw.) Sauerb.

Hygroamblystegium luridum

$P$. platyphylla Card.

P. pseudogracilis (C. Muell.) Par.

Haplocladium microphyllum

P. siambonica C. Muell.

Hygroamblystegium platyphyllum

Haplocladium microphyllum

P. sordidoviridis Card. et Broth.

Raviella siambonica

$P$. strictula Card.

$P$. uruguensis C. Muell.

Hygroamblystegium sordidoviride

Hygroamblystegium strictulum

Haplocladium microphyllum

\section{Pseudoscleropodium}

P. pseudopurum (C. Muell.) Broth. (Brotherus, 1925).

Syn. Cuspidaria pseudo-pura C. Muell. (Mueller, 1897a). Hypnum pseudo-purum (C. Muell.) Par. (Paris, 1900a).

Scleropodium pseudo-purum (C. Muell.) Broth. (Brotherus, 1908).

Distr. Southem South America (Mueller, 1897a; Paris, 1900a, 1905a; Brotherus, 1908, 1925; Kühnemann, 1938).

\section{Psilopilum}

P. angulatum Card. et Broth. (Cardot and Brotherus, 1923).

Distr. Southern South America (Cardot and Brotherus, 1923; Brotherus, 1925; Kühnemann. 1938; Seki, 1974).

P. antarcticum (C. Muell.) Par.

Psilopilum trichodon

var. densifolium Card. et Broth. (Cardot and Brotherus, 1923).

Distr. Southern South America (Cardot and Brotherus, 1923; Kühnemann, 1938).

Falkland Islands (Cardot and Brotherus, 1923).

P. australe (Hook. f. et Wils.) Mitt. (Mitten, 1860).

Syn. Polytrichum australe Hook. f. et Wils. (Hooker and Wilson in Wilson, 1854).

Distr. Southern South America (Wilson, 1854).

$P$. compressum (Hook. f. et Wils.) Mitt.

Atrichopsis compressa

P. cuspidatum Dus. (Dusén, 1905b). Distr. Southern South America (Dusén, 1905b; Cardot, 1908a; Brotherus, 1925; Kühnemann,
1938).

P. gymnostomulum (C. Muell.) Par. (Paris, 1898).

Syn. Catharinea gymnostomula C. Muell. (Mueller, 1879a). Oligotrichum gymnostomulum (C. Muell.) Kindb. (Kindberg, 1889).

Catharinea gymnostoma C. Muell. ex Par. (Mueller ex Paris, 1894) err.

Distr. Southern South America (Mueller, 1879a, 1885; Paris, 1898, 1905b; Brotherus, 1925; Hosseus, 1936, 1937; Kühnemann, 1938)

Falkland Islands (Mueller, 1885)

P. magellanicum Dus.

Psilopilum trichodon

P. minimum (Card.) G. L. Smith (Smith, 1969a).

Syn. Polytrichadelphus minimus Card. (Cardot, 1905a). Distr. Juan Fernandez (Brotherus, 1924b (as Psilopilum antarctıcum fide Smith, 1969a); Smith,
1969a). 
Southern South America (Cardot, 1905a, 1908a; Cardot and Brotherus, 1923 (as

Psilopilum compressum fide Smith, 1969a); Brotherus, 1925; Smith, 1969a).

P. subtapes Dus.

Psilopilum tapes var. apiculatum

P. tapes (C. Muell.) Par. (Paris, 1898).

Syn. Catharinea tapes C. Muell. (Mueller, 1890a).

Oligotrichum tapes (C. Muell.) Kindb. (Kindberg, 1891).

Distr. Southern South America (Cardot, 1913a; Brotherus, 1925).

South Georgia (Mueller, 1890a; Paris, 1898, 1905b; Cardot, 1908a; Brothenus, 1925;

Steere, 1961b; Greene, 1973).

var. apiculatum Card. (Cardot, 1908a).

Syn. Psilopilum subtapes Dus. (Dusén in Cardot, 1908a) nom. nud.

Distr. Southern South America (Cardot, 1908a; Cardot and Brotherus, 1923; Kühnemann, 1938).

P. trichodon (Hook. f. et Wils.) Mitt. (Mitten, 1869).

Syn. Polytrichum trichodon Hook. f. et Wils. (Hooker and Wilson in Wilson, 1847).

Catharinea antarctica C. Muell. (Mueller, 1884 [1883]).

Oligotrichum antarcticum (C. Muell.) Kindb. (Kindberg, 1889).

Psilopilum antarcticum (C. Muell.) Par. (Paris, 1898) fide Smith, 1971.

Psilopilum trichodon (Hook. f. et Wils.) Mitt. var. antarcticum Mitt. ex Par. (Mitten ex Paris, 1898) err.

Psilopilum magellanicum Dus. (Dusén, 1905b).

Distr. Southern South America (Cardot, 1905a, 1908a; Dusén, 1905b; Brothenus, 1925;

Kühnemann, 1938).

Falkland Islands (Cardot and Brotherus, 1923; Brotherus, 1925; Kühnemann, 1938).

South Georgia (Mueller, 1890a; Paris, 1898, 1905b; Cardot, 1906a, 1908a; Dixon, 1920; Brotherus, 1925; Steere, 1961b; Greene, 1973).

Antarctic, peninsula region (Greene, 1968a; Greene and others, 1970).

var. antarcticum Mitt.

Psilopilum trichodon

\section{Pterigynandrum}

P. austro-alpinum C. Muell.

$P$. bicolor Lindb. ex Aongstr.

$P$. julaceum Hedw.

P. longirostre Brid.

P. smithii (Hedw.) Lam. et Cand.

Leptopterigynandrum austro-alpinum

Enythrodontium squarrosum

Leucodon julaceus

Diplostichum longirostre

Leptodon smithii

\section{Pterobryon}

P. densum Hornsch. (Hornschuch, 1840).

Syn. Pterobryon densum Hornsch. var. condensatum Lor. (Lorentz in Arzeni, 1954) nom. nud.

Pterobryon lorentzii C. Muell. (Mueller, 1879a).

Distr. Southern South America (Mueller, 1879a; Paris, 1905b; Brotherus, 1925; Kühnemann, 1938).

var. condensatum Lor.

Pterobryon densum

$P$. lorentzii C. Muell.

Pterobryon densum

$P$. stolonaceum C. Muell.

Pterobryopsis stolonacea

\section{Pterobryopsis}

P. stolonacea (C. Muell.) Broth. (Brotherus, 1906).

Syn. Pterobryon stolonaceum C. Muell. (Mueller, 1879a).

Distr. Southern South America (Mueller, 1879a; Paris, 1905b; Brotherus, 1906, 1925; Herzog, 1952a). 
var. dilatata Herz. (Herzog, 1952a) nom. nud.

Distr. Southem South America (Herzog, 1952a).

\section{Pterogoniadelphus}

P. montevidensis (C. Muell.) Fleisch.

Felipponea montevidensis

\section{Pterogoniopsis}

P. cylindrica C. Muell. (Mueller, 1879a).

Distr. Southem South America (Mueller, 1879a; Paris, 1905b; Brotherus, 1925; Kühnemann, 1938).

\section{Pterogonium}

P. julaceum (Hedw.) Schwaegr.

Leucodon julaceus

\section{Pterygoneurum}

P. cavifolium Jur.

P. ovatum (Hedw.) Dix. (Dixon, 1933 [1934]).

Syn. Gymnostomum ovatum Hedw. (Hedwig, 1801).

Barbula ovata (Hedw.) Schimp. (Schimper in Lindberg, 1864a) nom. inval.

Pottia ovata (Hedw.) Fuernr. ex Lindb. (Fürnrohr ex Lindberg, 1864a) nom. inval.

Pottia cavifolia Ehrh. ex Fuernr. (Fürnrohr, 1829) hom. illeg.

Barbula cavifolia Schimp. (Schimper, 1860).

Desmatodon cavifolius Mitt. (Mitten, 1864) nom. illeg.

Pterygoneurum cavifolium Jur. (Juratzka, 1882) nom. illeg.

Pterygophyllum cavifolium Amann (Amann, 1916) err.

Tortula pusilla Mitt. (Mitten, 1869).

Distr. Southem South America (Dusén, 1906; Warnstorf, 1916).

$P$. roseae Williams

Aloina roseae

P. subsessile (Brid.) Jur. (Juratzka, 1882).

Syn. Gymnostomum subsessile Brid. (Bridel, 1806).

Schistidum subsessile (Brid.) Brid. (Bridel, 1819).

Anictangium subsessile (Brid.) Grev. et Arnottt (Greville and Arnott, 1824).

Anoectangium subsessile (Brid.) Spreng. (Sprengel, 1827).

Pottia subsessilis (Brid.) B.S.G. (Bruch and others, 1843).

Pharomitrium subsessile (Brid.) Schimp. (Schimper, 1860).

Tortula subsessilis (Brid.) Mitt. (Mitten, 1869).

Anoectangium acaule (Web. et Mohr) Roehl. (Röhling, 1809).

Distr. Southern South America (Mitten, 1869; Jaeger, 1873; Paris, 1905b).

Pterygophyllum hom. illeg.

$P$. anomalum (Schwaegr.) Mitt.

Achrophyllum anomalum

var. pallidum Card. et Broth. (Cardot and Brotherus, 1923).

Distr. Southem South America (Cardot and Brotherus, 1923; Welch, 1969; Seki, 1974).

$P$. apiculatum (Hook. f. et Wils.) Jaeg.

Eriopus apiculatus

$P$. arcuatum (Hedw.) Brid.

Hypnodendron arcuatum

P. cavifolium Amann

Pterygoneurum ovatum

P. chonoticum Mitt. (Mitten, 1885).

Distr. Southern South America (Mitten, 1885; Paris, 1905b: Cardot, 1908a: Brotherus, 1925; Welch, 1969).

P. crassirete Matteri (Matteri, 1972).

Syn. Hookeria obscura Mont. (Montagne, 1845a).

Pterygophyllum obscurum (Mont.) Mitt. (Mitten, 1869) hom. illeg. 
Distr. Juan Fernandez (Brotherus, 1924b).

Southern South America (Montagne, 1845a, 1850, 1856; Mueller, 1851a; Lorentz, 1866; Mitten, 1869; Jaeger, 1877; Neger, 1899; Paris, 1905b; Cardot, 1908a; Herzog, 1923, 1939. 1960; Brotherus, 1925; Reimers, 1926; Welch, 1969; Matteri, 1972; Seki, 1974).

Falkland Islands (Cardot, 1905a, 1908a; Kühnemann, 1938).

P. dentatum (Hook. f. et Wils.) Dix.

Achrophyllum dentatum

P. denticulatum Mitt. Achrophyllum dentatum

P. fragile Mitt. (Mitten, 1885).

Distr. Southern South America (Mitten, 1885; Paris, 1905b; Brotherus, 1925; Welch, 1969).

P. haesselianum Matteri (Matteri, 1972).

Distr. Southern South America (Matteri, 1972, 1975a; Seki, 1974).

P. lamellatum Dus. (Dusén, 1903a) nom. nud.

Syn. Hepaticina lamellata Fleisch. (Fleischer, 1922) nom. nud.

Distr. Southern South America (Dusén, 1903a; Fleischer, 1922).

P. magellanicum Besch. (Bescherelle, 1889).

Distr. Southern South America (Bescherelle, 1889; Hariot, 1891; Paris, 1905b; Cardot, 1908a; Brotherus, 1925; Kühnemann, 1938; Welch, 1969; Matteri, 1972, 1975a; Seki, 1974). Falkland Islands (Matteri, 1972).

var. oligodontium Matteri (Matteri, 1972).

Distr. Southern South America (Matteri, 1972, 1975a; Seki, 1974).

$P$. nigellum (Hook. f. et Wils.) Mitt.

Achrophyllum dentatum

P. obscurum (Mont.) Mitt. Pterygophyllum crassirete

P. peruncinatum Fleisch. (Fleischer, 1922) nom. nud.

Syn. Hepaticina peruncinata Dus. (Dusén in Fleischer, 1922) nom. nud.

Distr. Southern South America (Fleischer, 1922).

$P$. rigidum (Schwaegr.) Brid.

Lepidopilum polytrichoides

P. sublimbatum (C. Muell.) Mitt. Cyclodictyon sublimbatum

P. tenuinerve Broth. Achrophyllum tenuinerve

\section{Ptychomitrium}

P. aligrimmioides Broth. (Brotherus, 1918).

Distr. Southern South America (Brotherus, 1918, 1925; Kühnemann, 1938).

P. balansae Besch. (Bescherelle, 1877).

Syn. Glyphomitrium balansae (Besch.) Broth. (Brotherus, 1902).

Brachysteleum brevifolium C. Muell. (Mueller, 1879a).

Ptychomitrium brevifolium (C. Muell.) Kindb. (Kindberg, 1889).

Glyphomitrium brevifolium (C. Muell.) Broth. (Brotherus, 1902).

Brachysteleum uruguense C. Muell. (Mueller, 1882).

Ptychomitrium uruguense (C. Muell.) Kindb. (Kindberg, 1889).

Glyphomitrium uruguense (C. Muell.) Broth. (Brotherus, 1902).

Distr. Southern South America (Mueller, 1879a, 1882; Brotherus, 1902, 1918, 1925; Kurtz, 1904; Paris, 1905b; Williams, 1930; Herter, 1933a, 1943; Hosseus, 1935b, 1936, 1939; Kühnemann, 1938; Herzog, 1952a; Bizot and Piovano, 1953; Piovano, 1954).

P. brevifolium (C. Muell.) Kindb.

Ptychomitrium balansae

P. chimborazense (Mitt.) Jaeg. var. obtusifolium (Thér.) Broth. (Brotherus, 1925).

Syn. Brachysteleum chimborazense (Mitt.) Thér. var. obtusifolium Thér. (Thériot, 1923b).

Distr. Southern South America (Thériot, 1923b; Brotherus, 1925). 
P. crispatum (Hedw.) Jaeg. (Jaeger, 1874).

Syn. Encalypta crispata Hedw. (Hedwig, 1801).

Orthotrichum crispatum (Hedw.) Hook. et Grev. (Hooker and Greville, 1824a).

Ulota crispata (Hedw.) Sw. (Swartz in Hooker and Greville, 1824a) nom. inval.

Brachypodium crispatum (Hedw.) Brid. (Bridel, 1826).

Brachysteleum crispatum (Hedw.) Hornsch. (Hornschuch, 1840).

Notarisia crispata (Hedw.) Mont. (Montagne, 1840b).

Distr. Juan Fernandez (Montagne, 1850; Johow, 1896).

Southern South America (Hornschuch, 1840; Montagne, 1850).

$P$. cummingii Dub.

Ptychomitrium fernandesianum

P. deltorii (Thér.) Broth. (Brotherus, 1925).

Syn. Brachysteleum deltorii Thér. (Thériot, 1921a).

Distr. Southem South America (Thériot, 1921 a; Brotherus, 1925).

P. emersum (C. Muell.) Kindb. (Kindberg, 1889).

Syn. Brachysteleum emersum C. Muell. (Mueller, 1882).

Glyphomitrium emersum (C. Muell.) Broth. (Brotherus, 1902).

Distr. Southem South America (Mueller, 1882; Brotherus, 1902, 1918, 1925; Paris, 1905b; Kühnemann, 1938).

P. felipponei Thér. (Thériot in Felippone, 1929 [1930]).

Distr. Southern South America (Felippone, 1929; Herter, 1933a).

P. femandesianum (Mitt.) Jaeg. (Jaeger, 1874).

Syn. Glyphomitrium fernandesianum Mitt. (Mitten, 1860).

Ptychomitrium cummingii Dub. (Duby, 1880a).

Brachysteleum cummingii (Dub.) Hamp. (Hampe, 1880a).

Glyphomitrium cummingii (Dub.) Broth. (Brotherus, 1902).

Distr. Juan Fernandez (Mitten, 1860, 1885; Jaeger, 1874; Johow, 1896; Paris, 1905b;

Skottsberg, 1914; Brotherus, 1924b, 1925; Espinosa B., 1941; Robinson, 1975).

Southem South America (Duby, 1880a, b; Brotherus, 1902, 1925; Paris, 1905b; Thériot, 1935a).

var. majus Broth. (Brotherus, 1924b).

Distr. Juan Femandez (Brotherus, 1924b).

P. hieronymi Besch. (Bescherelle, 1891).

Syn. Glyphomitrium hieronymi (Besch.) Broth. (Brotherus, 1902).

Distr. Southem South America (Bescherelle, 1891; Brotherus, 1902, 1925; Paris, 1905b; Kühnemann, 1938).

P. ligulatum (Mitt.) Jaeg. (Jaeger, 1874).

Syn. Glyphomitrium ligulatum Mitt. (Mitten, 1869).

Brachysteleum ligulatum (Mitt.) C. Muell. (Mueller, 1885).

Distr. Southern South America (Mitten, 1869; Jaeger, 1874; Mueller, 1885; Paris, 1905b; Cardot, 1908a; Brotherus, 1925; Kühnemann, 1938).

P. sellowianum (C. Muell.) Jaeg. (Jaeger, 1874).

Syn. Brachysteleum sellowianum C. Muell. (Mueller, 1849).

Glyphomitrium sellowianum (C. Muell.) Mitt. (Mitten, 1869).

Brachysteleum sellowii Aongstr. (Ångström, 1876a) hom. illeg.

Ptychomitrium sellowii C. Muell. ex Kindb. (Mueller ex Kindberg, 1888) hom. illeg.

Distr. Southern South America (Mueller, 1849; Mitten, 1869; Gibert, 1873; Jaeger, 1874; Krieger, 1904; Paris, 1905b; Brotherus, 1925: Herter. 1933a)

var. uruguense Thér. (Thériot in Felippone, 1929 [1930]).

Distr. Southern South America (Felippone, 1929). 
P. sellowii C. Muell. ex Kindb.

P. uruguense (C. Muell.) Kindb.

P. vernicosum (C. Muell.) Kindb. (Kindberg, 1889).

Syn. Brachysteleum vernicosum C. Muell. (Mueller, 1882).

Glyphomitrium vernicosum (C. Muell.) Broth. (Brotherus, 1902).

Distr. Southern South America (Mueller, 1882; Brotherus, 1902, 1925; Paris, 1905b; Kühnemann, 1938).

\section{Ptychomnion}

P. aciculare (Brid.) Mitt. (Mitten, 1869).

Syn. Hypnum aciculare Brid. (Bridel, 1801b).

Stereodon acicularis (Brid.) Mitt. (Mitten, 1860).

Hypnum cucullifolium P. Beauv. (Palisot de Beauvois, 1805a).

Distr. Juan Fernandez (Montagne, 1835; Mitten, 1869, 1885; Johow, 1896; Paris, 1905b).

Southern South America (Palisot de Beauvois, 1805a; Bridel, 1817, 1819, 1827b; Wilson and Hooker, 1847; Montagne, 1850; Mueller, 1851a; Sullivant, 1859; Lorentz, 1866; Mitten, 1869; Ångström, 1872; Jaeger and Sauerbeck, 1879a; Eaton, 1892; Paris, 1905b; Cardot, 1908a; Kühnemann, 1938; Bartram, 1952; Kühnemann and Gonçalves, 1975).

P. cygnisetum (C. Muell.) Kindb. (Kindberg, 1888).

Syn. Hypnum cygnisetum C. Muell. (Mueller, 1885).

Ectropothecium cygnisetum C. Muell. (Mueller in Mitten, 1883) nom. nud.

Eurhynchium cygnisetum C. Muell. (Mueller in Jaeger, 1878) nom. nud.

Hylocomium splendidissimum De Not. (De Notaris in Bescherelle, 1889) nom. nud.

Distr. Juan Fernandez (Robinson, 1975).

Southern South America (Mueller, 1885; Bescherelle, 1889; Hariot, 1891; Neger, 1899; Paris, 1905b; Cardot, 1908a; Campo, 1915; Thériot, 1915; Cardot and Brotherus, 1923; Herzog, 1923, 1938, 1939, 1940, 1954, 1957, 1960; Brotherus, 1924c, 1925; Reimers, 1926; Kühnemann, 1938; Skottsberg, 1950; Robinson, 1970b; Seki, 1974; Kühnemann and Gonçalves, 1975).

var. chilense Broth. (Brotherus in Espinosa B., 1941) nom. nud.

Distr. Southern South America (Espinsoa B., 1941).

P. densifolium (Brid.) Jaeg. (Jaeger and Sauerbeck, 1879a).

Syn. Hypnum densifolium Brid. (Bridel, 1812).

Stereodon densifolius (Brid.) Mitt. (Mitten, 1860).

Distr. Southern South America (Cardot, 1905a, 1908a; Cardot and Brotherus, 1923;

Brotherus, 1925; Roivainen, 1934; Kühnemann, 1938; Robinson, 1970b; Seki, 1974; Kühnemann and Gonçalves, 1975).

var. gracile Card. et Broth. (Cardot and Brotherus, 1923).

Distr. Southern South America (Cardot and Brotherus, 1923; Kühnemann and Gonçalves, 1975).

P. falcatulum Broth. (Brotherus, 1924b).

Distr. Juan Fernandez (Brotherus, 1924b, 1925; Robinson, 1975; Kühnemann and Gonçalves, 1975).

var. gracilescens Broth. (Brotherus, 1924b).

Distr. Juan Fernandez (Brotherus, 1924b; Espinosa B., 1941; Kühnemann and Gonçalves, 1975).

P. fruticetorum C. Muell. (Mueller, 1898c).

Distr. Southern South America (Bizot, 1967).

P. horridum Card. et Broth. (Cardot and Brotherus, 1923).

Distr. Southern South America (Cardot and Brotherus, 1923; Kühnemann and Gonçalves, 1975). 
P. ptychocarpum (Schwaegr.) Mitt. (Mitten, 1869).

Syn. Hypnum ptychocarpum Schwaegr. (Schwaegrichen, 1844 [1845]).

Stereodon ptychocarpum (Schwaegr.) Mitt. (Mitten, 1860).

Leskea gayana Mont. (Montagne, 1845a).

Hypnum gayanum (Mont.) Lor. (Lorentz, 1866).

Distr. Juan Fernandez (Brotherus, 1924b, 1925; Espinosa B., 1941; Bartram, 1959; Robinson, 1975).

Southem South America (Mueller, 1844b, 1851a; Schwaegrichen, 1844; Montagne, 1845a, 1850, 1856; Lorentz, 1866; Mitten, 1869; Jaeger and Sauerbeck, 1879a; Bescherelle, 1889; Paris, 1905b; Cardot, 1908a; Thériot, 1918, 1935a; Campo, 1921; Cardot and Brotherus, 1923; Herzog, 1923, 1938, 1939, 1954, 1957, 1960; Brotherus, 1925; Reimers, 1926; Roivainen, 1934; Kühnemann, 1938; Bartram, 1952; Bizot, 1967; Robinson, 1970b; Seki, 1974; Kühnemann and Gonçalves, 1975).

P. subaciculare Besch. (Bescherelle, 1885a).

Distr. Juan Fernandez (Paris, 1905b; Brotherus, 1924b, 1925; Espinosa B., 1941; Bartram, 1959; Robinson, 1970b; Kühnemann and Gonçalves, 1975).

Southem South America (Bescherelle, 1885a, 1889; Paris, 1905b; Cardot, 1908a; Cardot and Brotherus, 1923; Brotherus, 1925; Kühnemann, 1938; Robinson, 1970b; Seki, 1974).

\section{Pulvinella}

P. albicans Broth. et Herz. (Brotherus and Herzog in Herzog, 1928).

Distr. Southem South America (Herzog, 1928).

\section{Racomitrium}

R. aciculare (Hedw.) Brid. var. aquaticum (Schrad.) Hueb.

Racomitrium aquaticum

R. andreaeoides Herz. (Herzog, 1954).

Distr. Southern South America (Herzog, 1954; Seki, 1974).

R. aquaticum (Schrad.) Brid. (Bridel, 1819).

Syn. Trichostomum aquaticum Brid. ex Schrad. (Bridel ex Schrader, 1803a).

Grimmia aquatica (Schrad.) C. Muell. (Mueller, 1849).

Trichostomum aciculare (Hedw.) P. Beauv. var. aquaticum (Schrad.) Web. et Mohr ex Brid. (Weber and Mohr ex Bridel, 1826) nom. inval.

Racomitrium aciculare (Hedw.) Brid. var. aquaticum (Schrad.) Hueb. (Hübener, 1833).

Trichostomum protensum Braun (Braun in Schultz, 1828).

Racomitrium protensum (Braun) Hueb. (Hübener, 1833).

Grimmia protensum (Braun) Mitt. (Mitten, 1879).

Distr. Southern South America (Paris, 1905b; Cardot, 1908a).

R. austro-canescens Dus. (Dusén, 1907).

Distr. Southern South America (Dusén, 1907; Cardot, 1908a; Brotherus, 1924a; Kühnemann, 1938).

R. austro-georgicum Par. (Paris, 1895).

Syn. Grimmia austro-patens C. Muell. (Mueller, 1890a).

Racomitrium substenocladum Card. (Cardot, 1911 d) fide Bell, 1973c.

Racomitrium skottsbergii Card. et Broth. (Cardot and Brotherus, 1923).

Grimmia austro-georgica C. Muell. ex Par. (Mueller ex Paris, 1905b) nom. inval.

Distr. Southern South America (Cardot and Brotherus, 1923; Brotherus . 1924a; Kühnemann, 1938; Roivainen, 1955a).

South Georgia (Mueller, 1890a; Paris, 1895, 1905b; Cardot, 1906a, 1908a; Cardot and Brotherus, 1923; Steere, 1961b; Bell, 1974).

Antarctic, peninsula region (Cardot, 1911b. d, 1913a; Cardot and Brotherus. 1923;

Brotherus, 1924a; Steere, 1961a; Bell, 1973c)

var. kranckii Roiv. (Roivainen, 1955a).

Distr. Southern South America (Roivainen, 1955a) 
R. bartramii (Roiv.) H. Robinson (Robinson, 1974).

Syn. Bucklandia bartramii Roiv. (Roivainen, 1955b).

Bucklandiella bartramii Roiv. (Roivainen, 1972).

Distr. Southern South America (Roivainen, 1955b, 1972; Robinson, 1974).

R. convolutum Mont.

Racomitrium crispulum

R. crispipilum (Tayl.) Jaeg. (Jaeger, 1874).

Syn. Trichostomum crispipilum Tayl. (Taylor, 1846).

Distr. Juan Fernandez (Robinson, 1975).

R. crispulum (Hook. f. et Wils.) Hook. f. et Wils. (Hooker and Wilson in Wilson, 1854).

Syn. Dryptodon crispulus Hook. f. et Wils. (Hooker, J. D. and Wilson, 1844).

Grimmia crispula (Hook. f. et Wils.) C. Muell. (Mueller, 1849) hom. illeg.

Racomitrium convolutum Mont. (Montagne, 1845a).

Grimmia convoluta (Mont.) C. Muell. (Mueller, 1851a).

Grimmia nigrita C. Muell. (Mueller, 1849) hom. illeg. fide Bell, 1974.

Racomitrium nigritum Jaeg. (Jaeger, 1874) fide Bell, 1974.

Distr. Juan Fernandez (Skottsberg, 1914; Brotherus, 1924b; Bartram, 1959; Robinson, 1975).

Southern South America (Montagne, 1845a, 1856; Hooker, 1847; Mueller, 1849, 1851a, 1885; Mitten, 1869; Jaeger, 1874; Paris, 1905b; Cardot, 1908a; Cardot and Brotherus, 1923; Brotherus, 1924a; Herzog, 1938, 1954; Kühnemann, 1938; Bartram, 1952; Roivainen, 1955a; Seki, 1974).

South Georgia (Cardot, 1906a, 1908a; Cardot and Brotherus, 1923; Brotherus, 1924a; Steere, 1961b; Bell, 1974).

Antarctic, peninsula region (Robinson, 1972; Bell, 1973c).

var. rupestre (Hook. f. et Wils.) Dix. (Dixon, 1926).

Syn. Dryptodon rupestris Hook. f. et Wils. (Hooker, J. D. and Wilson, 1844).

Grimmia rupestris (Hook. f. et Wils.) C. Muell. (Mueller, 1849).

Racomitrium rupestre (Hook. f. et Wils.) Hook. f. et Wils. (Hooker and Wilson in Wilson, 1854).

Distr. Southern South America (Hooker, J. D. and Wilson, 1844; Wilson and Hooker, 1847; Mueller, 1849, 1885; Sullivant, 1859; Mitten, 1869; Jaeger, 1874; Bescherelle, 1889; Eaton, 1892; Cardot, 1905a, 1908a; Paris, 1905b; Dusén, 1907; Cardot and Brotherus, 1923; Brotherus, 1924a; Thériot, 1925a; Roivainen, 1934, 1955a; Bartram, 1952; Seki, 1974).

South Georgia (Cardot, 1906a; Bell, 1974).

R. didymum (Mont.) Lor. (Lorentz, 1866).

Syn. Grimmia didyma Mont. (Montagne, 1845a).

Distr. Southern South America (Montagne, 1845a, 1850, 1856; Mueller, 1849; Lorentz, 1866; Mitten, 1869; Jaeger, 1874; Paris, 1905b; Brotherus, 1924a; Reimers, 1926; Thériot, 1935a; Herzog, 1938, 1939, 1954, 1960; Seki, 1974).

R. flavescens Card.

Racomitrium symphyodontum

R. flavo-pallidum Dus. (Dusén, 1903a) nom. nud.

Distr. Southern South America (Dusén, 1903a; Paris, 1905b).

R. geminatum Roiv. (Roivainen, 1955a).

Distr. Southern South America (Roivainen, 1955a).

R. geronticum C. Muell.

R. glaciale Kindb.

Racomitrium lanuginosum

Racomitrium lanuginosum

R. grimmioides Herz. (Herzog, 1957).

Distr. Southern South America (Herzog, 1957).

R. heterostichoides Card. (Cardot, 1905a).

Syn. Racomitrium horridum Dus. (Dusén in Roivainen, 1955a) nom. nud. 
Distr. Southern South America (Cardot, 1905a, 1908a; Dusén, 1907; Cardot and Brotherus, 1923; Brotherus, 1924a; Kühnemann, 1938; Roivainen, 1955a).

Falkland Islands (Cardot and Brotherus, 1923; Kühnemann, 1938).

South Georgia (Cardot, 1906a, 1908a; Cardot and Brotherus, 1923; Brotherus, 1924a; Steere, 1961b; Bell, 1974).

var. acutifolium Dus.

Racomitrium subulifolium

R. heterostichum (Hedw.) Brid. (Bridel, 1819).

Syn. Trichostomum heterostichum Hedw. (Hedwig, 1801).

Bryum heterostichum (Hedw.) Dicks. (Dickson, 1801).

Grimmia heterosticha (Hedw.) C. Muell. (Mueller, 1849).

Distr. Southem South America (Herzog, 1938).

Falkland Islands (Wilson and Hooker, 1847; Cardot, 1908a; Brotherus, 1924a; Kühnemann, 1938).

R. horridum Dus.

Racomitrium heterostichoides

R. hyalino-cuspidatum (C. Muell.) Kindb.

Grimmia hyalino-cuspidata

R. hypnoides Lindb.

Racomitrium lanuginosum

var. senile Lindb.

Racomitrium lanuginosum

R. integripilum Dus. (Dusén, 1907).

Distr. Southem South America (Dusén, 1907; Cardot, 1908a; Brotherus, 1924a; Kühnemann, 1938; Herzog, 1954).

R. laevigatum Jaeg. (Jaeger, 1874).

Syn. Grimmia laevigata Mitt. (Mitten, 1869) hom. illeg.

Distr. Juan Fernandez (Robinson, 1975).

Southem South America (Mitten, 1869; Jaeger, 1874; Dusén, 1903a, 1907; Paris, 1905b; Cardot, 1908a; Brotherus, 1924a; Kühnemann, 1938).

R. lamprocarpum (C. Muell.) Jaeg. (Jaeger, 1874).

Syn. Grimmia lamprocarpa C. Muell. (Mueller, 1849).

Distr. Falkland Islands (Wilson and Hooker, 1847 (as R. protensum var. fide Mueller, 1849); Mueller, 1849; Jaeger, 1874; Paris, 1905b; Cardot, 1908a; Cardot and Brotherus, 1923; Brotherus, 1924a; Kühnemann, 1938).

R. lanuginosum (Hedw.) Brid. (Bridel, 1819).

Syn. Trichostomum lanuginosum Hedw. (Hedwig, 1801).

Bryum lanuginosum (Hedw.) Brotero (Brotero, 1804).

Grimmia lanuginosa (Hedw.) C. Muell. (Mueller, 1849).

Bryum hypnoides L. ex With. (Linnaeus ex Withering, 1801) nom. illeg.

Trichostomum hypnoides Willd. exP. Beauv. (Willdenow ex Palisot de Beauvois, 1822) nom. illeg.

Racomitrium hypnoides Lindb. (Lindberg, 1866 [1867]) nom. illeg.

Racomitrium hypnoides Lindb. var. senile Lindb. (Lindberg, 1866 [1867]) nom. nud.

Racomitrium senile Schimp. (Schimper in Jaeger, 1874) nom. nud.

Racomitrium puccioanum De Not. (De Notaris, 1859).

Grimmia glacialis C. Muell. (Mueller, 1890a) hom. illeg.

Racomitrium glaciale Kindb. (Kindberg, 1891).

Racomitrium geronticum C. Muell. (Mueller, 1869).

Distr. Juan Fernandez (Brotherus, 1924b; Espinosa B., 1941; Bartram, 1959; Robinson, 1975).

Southern South America (Bridel, 1819; Montagne, 1845b. 1850; Wilson and Hooker, 1847; Sullivant, 1859; De Notaris, 1859; Lorentz, 1866; Mitten, 1869; Mueller, 1869. 1885; Jaeger, 1874; Bescherelle, 1889; Hariot. 1891: Neger, 1899; Cardot, 1900. 1901, 1905a, 1908a; Dusén, 1903a, 1907; Krieger, 1904: Paris, 1905b; Middleton, 1909; Thériot, 1921a; Cardot and Brotherus, 1923; Brotherus, 1924a; Reimers, 1926; Herzog, 1938, 1940, 1954, 1957, 1960; Kuhnemann. 1938; Bartram, 1952;

Roivainen, 1955a; Gunckel, 1971; Seki, 1974).

Falkland Islands (Dumont d'Unville, 1825; Gaudichaud. 1825. 1826; Wilson and Hooker, 1847; Paris, 1905b; Cardot, 1908a; Cardot and Brotherus. 1923: Kuhnemann. 1938). 
South Georgia (Mueller, 1890a; Paris, 1905b; Cardot, 1906a, 1908a; Dixon, 1920, 1932; Brotherus, 1924a; Steere, 1961b; Bell, 1974, 1977b).

Antarctic, peninsula region (Dixon, 1935; Greene, 1968a; ? error see Bell, 1973c).

R. limbatum Bartr.

Racomitrium subnigritum

R. looseri Thér. (Thériot, 1934b [1935]).

Distr. Southern South America (Thériot, 1934b).

R. loriforme Dus.

Racomitrium willii

R. nigritum Jaeg.

Racomitrium crispulum

R. pachydictyon Card. (Cardot, 1908a).

Syn. Racomitrium symphyodontum (C. Muell.) Par. var. muticum Card. (Cardot, 1905a).

Distr. Southern South America (Cardot, 1905a (and as R. flavescens pro parte and R. rupestre pro parte fide Cardot, 1908a), 1908a; Cardot and Brotherus, 1923; Brotherus, 1924a; Herzog, 1938; Kühnemann, 1938; Roivainen, 1955a; Seki, 1974).

South Georgia (Cardot, 1908a; Brotherus, 1924a; Steere, 1961b; Bell, 1974).

R. pachyneuron Herz. (Herzog, 1957).

Distr. Southern South America (Herzog, 1939, 1954, 1957, 1960; Seki, 1974).

R. patagonicum Par. (Paris, 1905b) nom. nud.

Syn. Racomitrium subnigritum Dus. (Dusén in Paris, 1905b) nom. nud.

Distr. Southern South America (Paris, 1905b).

R. plicatum Herz. (Herzog in Donat, 1936a).

Distr. Southern South America (Donat, 1936a; Seki, 1974).

R. protensum (Braun) Hueb.

Racomitrium aquaticum

R. ptychophyllum (Mitt.) Mitt. (Mitten in Hooker, 1867).

Syn. Grimmia ptychophylla Mitt. (Mitten in Lindsay, 1866).

Distr. Southern South America (Cardot and Brotherus, 1923; Kühnemann, 1938; Bartram. 1952).

Falkland Islands (Cardot and Brotherus, 1923; Kühnemann, 1938).

South Georgia (Cardot, 1906a, 1908a; Steere, 1961b; Bell, 1974).

R. puccioanum De Not.

R. rupestre (Hook. f. et Wils.) Hook. f. et.Wils.

R. scabrifolium Bartr.

R. senile Schimp.

R. skottsbergii Card. et Broth.

Racomitrium lanuginosum

R. stenocladoides Roiv. (Roivainen, 1955a).

Distr. Southern South America (Roivainen, 1955a).

R. stenocladum Dus. (Dusén, 1907).

Distr. Southern South America (Dusén, 1907; Cardot, 1908a; Brotherus, 1925; Herzog, 1938; Kühnemann, 1938; Seki, 1974).

var. obtusum Dus. (Dusén, 1907).

Distr. Southern South America (Dusén, 1907; Cardot, 1908a; Kühnemann, 1938).

R. striatipilum Card. (Cardot, 1905a).

Distr. Juan Fernandez (Brotherus, 1924b; Espinosa B., 1941).

Southern South America (Cardot, 1905a, 1908a; Dusén, 1907; Cardot and Brotherus. 1923; Brotherus, 1924a; Thériot, 1935a; Herzog, 1938; Kühnemann, 1938; Roivainen, 1955a; Seki, 1974).

Falkland Islands (Cardot and Brotherus, 1923).

South Georgia (Cardot, 1906a, 1908a; Dixon, 1934; Steere, 1961b; Bell, 1974). 
R. subcrispipilum (C. Muell.) Jaeg. (Jaeger, 1874).

Syn. Grimmia subcrispipila C. Muell. (Mueller, 1862).

Distr. Southern South America (Mueller, 1862; Paris, 1905b; Brotherus, 1924a).

R. sublamprocarpum (C. Muell.) Par. (Paris, 1898).

Syn. Grimmia sublamprocarpa C. Muell. (Mueller, 1885).

Distr. Southern South America (Mueller, 1885; Paris, 1898, 1905b).

R. subnigritum (C. Muell.) Par. (Paris, 1898).

Syn. Grimmia subnigrita C. Muell. (Mueller, 1885).

Racomitrium limbatum Bartr. (Bartram, 1946).

Distr. Juan Fernandez (Brotherus, 1924b; Bartram, 1959).

Southem South America (Mueller, 1885; Cardot, 1905a, 1908a; Paris, 1905b; Dusén, 1907; Cardot and Brotherus, 1923; Brotherus, 1924a; Kühnemann, 1938; Bartram, 1946, 1952; Herzog, 1954; Roivainen, 1955a; Seki, 1974).

Falkland Islands (Cardot and Brotherus, 1923; Kühnemann, 1938).

R. subnigritum Dus.

Racomitrium patagonicum

R. subrupestre Roiv. (Roivainen, 1955a).

Distr. Southern South America (Roivainen, 1955a).

R. substenocladum Card.

Racomitrium austro-georgicum

R. substriatipilum Roiv. (Roivainen, 1955a).

Distr. Southern South America (Roivainen, 1955a).

R. subulifolium Card. (Cardot, 1908a).

Syn. Racomitrium heterostichoides Card. var. acutifolium Dus. (Dusén, 1907).

Distr. Southern South America (Dusén, 1907; Cardot, 1908a; Cardot and Brotherus, 1923; Brotherus, 1924a; Kühnemann, 1938; Roivainen, 1955a; Herzog, 1957).

R. symphyodon Jaeg. (Jaeger, 1874).

Syn. Grimmia symphyodon Mitt. (Mitten, 1882) nom. illeg.

Distr. Southern South America (Jaeger, 1874).

R. symphyodontum (C. Muell.) Par. (Paris, 1897).

Syn. Grimmia symphyodonta C. Muell. (Mueller, 1849). Racomitrium flavescens Card. (Cardot, 1900).

Distr. Juan Fernandez (Brotherus, 1924b; Espinosa B., 1941).

Southern South America (Wilson and Hooker, 1847 (as R. fasciculare vars 2 and 3 fide Mueller 1849); Mueller, 1849, 1855; Cardot, 1900, 1905a pro parte, 1908a, 1910; Paris, 1905b; Dusén, 1907; Cardot and Brotherus, 1923; Brotherus, 1924a; Kühnemann, 1938; Herzog, 1954, 1957; Roivainen, 1955a; Seki, 1974).

Falkland Islands (Cardot, 1905a, 1908a; Paris, 1905b; Brotherus, 1924a; Kühnemann, 1938).

var. dusenii Card. (Cardot, 1908a) nom. nud.

Distr. Southern South America (Dusén, 1907 (as R. symphydontum var. muticum fide Cardot, 1908a); Cardot, 1908a).

var. muticum Card.

Racomitrium pachydictyon

R. willii (C. Muell.) Kindb. (Kindberg, 1891).

Syn. Grimmia willii C. Muell. (Mueller, 1890a).

Racomitrium loriforme Dus. (Dusén, 1907).

Racomitrium scabrifolium Bartr. (Bartram, 1946).

Distr. Juan Fernandez (Brotherus, 1924b; Espinosa B., 1941).

Southern South America (Paris, 1905b; Dusèn, 1907; Cardot. 1908a; Cardot and 
Brotherus, 1923; Brotherus, 1924a; Kühnemann, 1938; Bartram, 1946; Roivainen, 1955a; Seki, 1974).

South Georgia (Mueller, 1890a; Paris, 1905b; Cardot, 1906a, 1908a; Brotherus, 1924a; Steere, 1961b; Bell, 1974).

\section{Racopilum}

R. anomalum Schwaegr.

Achrophyllum anomalum

R. arcuatum (Hedw.) Jaeg. Hypnodendron arcuatum

R. chilense Dus. ex Herz. (Dusén ex Herzog, 1938).

Distr. Southern South America (Paris, 1905b; Brotherus, 1925; Herzog, 1938).

R. fernandezianum Card. (Cardot in Theiriot, 1921b).

Distr. Juan Fernandez (Skottsberg, 1914; Thériot, 1921b, 1927b; Brotherus, 1924b, 1925; Espinosa B., 1941; Bartram, 1959; Robinson, 1975).

Southern South America (Herzog, 1954).

R. leptocarpum Wils. (Wilson, 1854).

Distr. Juan Fernandez (Wilson, 1854).

R. ornithopodioides (Brid.) Lindb.

Racopilum tomentosum

R. tomentosum (Hedw.) Brid. (Bridel, 1827b).

Syn. Hypnum tomentosum Hedw. (Hedwig, 1801).

Hypopterygium tomentosum (Hedw.) C. Muell. (Mueller, 1850).

Hypnum ornithopodioides Brid. (Bridel, 1801b).

Leskea ornithopodioides (Brid.) Brid. (Bridel, 1812).

Racopilum ornithopodioides (Brid.) Lindb. (Lindberg, 1868d).

Distr. Juan Fernandez (Montagne, 1835; Mitten, 1885; Johow, 1896; Paris, 1905b;

Skottsberg, 1914; Robinson, 1975).

Southern South America (Bridel, 1801b, 1812, 1817, 1827b; Palisot de Beauvois, 1805a; Montagne, 1850; Mueller, 1879a; Krieger, 1904; Paris, 1905b; Brotherus, 1918; Kühnemann, 1938; Hosseus, 1940; Herzog, 1952a).

Rauia hom. illeg.

R. firmula (C. Muell.) Broth.

Rauiella firmula

R. microcalycina Broth. ex Par.

R. niveo-calycina (C. Muell.) Broth.

R. siambonica (C. Muell.) Broth.

Rauiella niveo-calycina

Rauiella niveo-calycina

Rauiella siambonica

\section{Rauiella}

R. firmula (C. Muell.) Wijk et Marg. (van der Wijk and Margadant, 1962).

Syn. Thuidium firmulum C. Muell. (Mueller, 1897a).

Rauia firmula (C. Muell.) Broth. (Brotherus, 1907).

Distr. Southern South America (Mueller, 1897a; Paris, 1906; Brotherus, 1907, 1925; Hosseus, 1938a, d, 1939; Kühnemann, 1938).

R. niveo-calycina (C. Muell.) Wijk et Marg. (van der Wijk and Margadant, 1962).

Syn. Thuidium niveo-calycinum C. Muell. (Mueller, 1897a).

Rauia niveo-calycina (C. Muell.) Broth. (Brotherus, 1907).

Rauia microcalycina Broth. ex Par. (Brotherus ex Paris, 1909) err.

Distr. Southern South America (Mueller, 1897a; Paris, 1906; Brotherus, 1907, 1925;

Kühnemann, 1938; Herzog, 1952a).

R. siambonica (C. Muell.) Wijk et Marg. (van der Wijk and Margadant, 1962).

Syn. Pseudoleskea siambonica C. Muell. (Mueller, 1897a).

Rauia siambonica (C. Muell.) Broth. (Brotherus, 1907).

Distr. Southern South America (Mueller, 1897a; Paris, 1905b; Brotherus, 1907, 1925; Kühnemann, 1938). 


\section{Renauldia}

R. chilensis Thér. (Thériot, 1926).

Distr. Southern South America (Thériot, 1926).

\section{Rhabdowesia}

s. crispata (With.) Lindb. (Lindberg, 1871).

Syn. Bryum crispatum Dicks. ex With. (Dickson ex Withering, 1801).

Distr. Juan Femandez (Robinson, 1975).

R. cyathicarpa (Mont.) Mitt.

Amphidium tortuosum

R. fugax (Hedw.) B.S.G. (Bruch and others, 1846).

Syn. Weisia fugax Hedw. (Hedwig, 1801).

Grimmia striata Schrad. (Schrader, 1803b) nom. illeg.

Oncophorus striatus (Schrad.) Lindb. (Lindberg, 1879).

Distr. Juan Fernandez (Mitten, 1885; Johow, 1896; Paris, 1905b; Lawton, 1961).

R. sphaerothecia (C. Muell.) Kindb.

Amphidium tortuosum

\section{Rhachithecium}

R. demissum (C. Muell.) Broth.

Rhachithecium perpusillum

R. perpusillum (Thwait. et Mitt.) Broth. (Brotherus, 1909).

Syn. Zygodon perpusillus Thwait. et Mitt. (Thwaites and Mitten in Mitten, 1873).

Hypnodon perpusillus (Thwait. et Mitt.) C. Muell. (Mueller, 1897a).

Hypnodon demissus C. Muell. (Mueller, 1897a).

Decodon demissus C. Muell. (Mueller, 1897a) nom. nud.

Rhachithecium demissum (C. Muell.) Broth. (Brotherus, 1909).

Distr. Southem South America (Mueller, 1897a; Paris, 1900a, 1904b; Brotherus, 1909, 1925; Kühnemann, 1938).

\section{Rhacocarpus}

R. australis (Hook. f. et Wils.) Par.

Rhacocarpus purpurascens

R. crasso-limbatus (C. Muell.) Par.

Rhacocarpus purpurascens

R. humboldtii (Hook.) Lindb.

Rhacocarpus purpurascens

var. argentinicus (C. Muell.) Par.

Rhacocarpus purpurascens var. argentinicus

R. orbiculatus (Mitt.) Par. (Paris, 1898).

Syn. Hedwigia orbiculata Mitt. (Mitten, 1869).

Harrisonia orbiculata (Mitt.) Jaeg. (Jaeger, 1876),

Distr. Southern South America (Mitten, 1869; Jaeger, 1876; Mueller, 1897d; Paris, 1898 , 1905b; Brotherus, 1925).

R. patagonicus Broth. (Brotherus in Dusén, 1905b).

Distr. Southern South America (Dusén, 1905b; Cardot, 1908a; Brotherus, 1925; Kühnemann, 1938).

R. purpurascens (Brid.) Par. (Paris, 1900a).

Syn. Hypnum purpurascens Brid. (Bridel, 1812).

Harrisonia purpurascens (Brid.) C. Muell. (Mueller, 1897d).

Anictangium humboldtii Hook. (Hooker, 1816).

Hedwigia humboldtii (Hook.) Hook. (Hooker, 1819).

Anoectangium humboldtii (Hook.) Brid. (Bridel, 1827b).

Harrisonia humboldtii (Hook.) Spreng. (Sprengel, 1827).

Neckera humboldtii (Hook.) C. Muell. (Mueller, 1850).

Rhacocarpus humboldtii (Hook.) Lindb. (Lindberg, 1862 [1863]).

Braunia humboldtii (Hook.) Hook. f. (Hooker, 1867).

Anoectangium humboldtii (Hook.) Brid. var. australis Hook. f. et Wils. (Hooker and Wilson in Wilson and Hooker, 1845). 
Hedwigia humboldtii (Hook.) Hook. var. australis (Hook. f. et Wils.) Wils. Wilson, 1854).

Harrisonia australis (Hook. f. et Wils.) Hamp. (Hampe, 1860).

Rhacocarpus australis (Hook. f. et Wils.) Par. (Paris, 1898).

Harrisonia crasso-limbata C. Muell. (Mueller, 1897d).

Rhacocarpus crasso-limbatus (C. Muell.) Par. (Paris, 1900a).

Distr. Juan Fernandez (Brotherus, 1924b, 1925; Robinson, 1975).

Southern South America (Wilson and Hooker, 1847; Mitten, 1869; Mueller, 1879a, 1885. 1897d; Paris, 1900a, 1905b; Cardot, 1908a; Cardot and Brotherus, 1923; Brotherus, 1925; Kühnemann, 1938; Seki, 1974).

Falkland Islands (Paris, 1900a, 1905b; Cardot, 1908a; Brotherus, 1925).

var. argentinicus (Brid.) Wijk et Marg. (van der Wijk and Margadant, 1961).

Syn. Harrisonia humboldtii (Hook.) Spreng. var. argentinica C. Muell. (Mueller, 1879a). Rhacocarpus humboldtii (Hook.) Lindb. var. argentinicus (C. Muell.) Par. (Paris, 1898).

Distr. Southern South America (Mueller, 1879a; Paris, 1898, 1905b; Kühnemann, 1938).

\section{Rhamphidium}

R. laetum (C. Muell.) Broth. (Brotherus, 1902).

Syn. Trichostomum laetum Kunz. ex C. Muell. (Kunze ex Mueller, 1849).

Tortula laeta (C. Muell.) Mitt. (Mitten, 1869).

Distr. Juan Fernandez (Montagne, 1850).

Southern South America (Mueller, 1849; Mitten, 1869; Jaeger, 1873; Brotherus, 1902, 1924a; Paris, 1905b).

\section{Rhaphidorrhynchium}

R. aberrans (Broth.) Broth.

\section{Sematophyllum aberrans}

R. amoenum (Hedw.) Fleisch. (Fleischer, 1923).

Syn. Hypnum amoenum Hedw. (Hedwig, 1801).

Isothecium amoenum (Hedw.) Brid. (Bridel, 1827b).

Sematophyllum amoenum (Hedw.) Mitt. (Mitten, 1869).

Rhaphidostegium amoenum (Hedw.) Jaeg. (Jaeger, 1878).

Rhaphidostegium amaenum (Hedw.) Par. (Paris, 1898) err.

Hypnum leptorhynchum Brid. (Bridel, 1812) hom. illeg.

Distr. Southern South America (Wilson and Hooker, 1847; Mueller, 1851a; Mitten, 1869; Jaeger, 1878; Paris, 1898, 1905b; Cardot, 1908a; Brotherus, 1925; Kühnemann, 1938).

Falkland Islands (Kühnemann, 1938).

R. berberidis (Dus.) Broth. (Brotherus, 1925).

Syn. Ectropothecium berberidis Dus. (Dusén, 1905b).

Rhaphidostegium berberidis (Dus.) Broth. (Brotherus, 1908).

Distr. Southern South America (Dusén, 1903a, 1905b; Brotherus, 1908, 1925; Cardot, 1008a; Seki, 1974).

R. brachypus (Hamp.) Broth. (Brotherus, 1925).

Syn. Hypnum brachypus Hamp. (Hampe, 1879).

Distr. Southern South America (Herzog, 1952a).

R. callidum (Mont.) Broth. (Brotherus, 1925).

Syn. Hypnum callidum Mont. (Montagne, 1845a).

Sematophyllum callidum (Mont.) Mitt. (Mitten, 1869).

Rhaphidostegium callidum (Mont.) Jaeg. (Jaeger, 1878).

Distr. Southern South America (Montagne, 1845a, 1850, 1856; Mueller, 1851a; Lorentz, 1866; Mitten, 1869; Jaeger, 1878; Paris, 1905b; Brotherus, 1908, 1924C; Cardot, 1908a;

Campo, 1915; Thériot, 1917 b, 1918; Costes, 1921; Cardot and Brotherus, 1923;

Herzog, 1923, 1938, 1939, 1954, 1957, 1960; Reimers, 1926; Kühnemann, 1938;

Espinosa B., 1941; Skottsberg, 1950; Bartram, 1952; Seki, 1974). 
R. dendroligotrichum (Dus.) Broth. (Brotherus, 1925).

Syn. Rhaphidostegium dendroligotrichum Dus. (Dusén, 1905b).

Rhaphidostegium polytrichadelphus Dus. (Dusén, 1903a) nom. nud.

Distr. Southern South America (Dusén, 1903a, 1905b; Paris, 1905b; Cardot, 1908a; Brotherus, 1925; Kühnemann, 1938; Herzog, 1954, 1960; Seki, 1974).

.R. leptophyllum (Mitt.) Broth. (Brotherus, 1925).

Syn. Sematophyllum leptophyllum Mitt. (Mitten, 1869). Rhaphidostegium leptophyllum (Mitt.) Jaeg. (Jaeger, 1878).

Distr. Southem South America (Mitten, 1869; Jaeger, 1878; Paris, 1905b; Brotherus, 1925).

R. masafuerae (Broth.) Broth.

Sematophyllum masafuerae

R. minutum (Broth.) Broth. (Brotherus, 1925).

Syn. Sematophyllum minutum Broth. (Brotherus, 1900).

Distr. Southem South America (Herzog, 1952a).

R. noduliferum (Mitt.) Broth. (Brotherus, 1925).

Syn. Sematophyllum noduliferum Mitt. (Mitten, 1869). Rhaphidostegium noduliferum (Mitt.) Jaeg (Jaeger, 1878).

Distr. Southem South America (Mitten, 1869; Jaeger, 1878; Paris, 1905b; Cardot, 1908a; Brotherus, 1925).

R. pallido-nitidum (C. Muell.) Broth. (Brotherus, 1925).

Syn. Cupressina pallido-nitida C. Muell. (Mueller, 1897a).

Ectropothecium pallido-nitidum (C. Muell.) Par. (Paris, 1900a).

Rhaphidostegium pallido-nitidum (C. Muell.) Broth. (Brotherus, 1908).

Distr. Southem South America (Mueller, 1897a; Paris, 1900a, 1904b; Brotherus, 1908, 1925; Kühnemann, 1938).

R. scorpiunus (Mont.) Broth. (Brotherus, 1925).

Syn. Hypnum scorpiurus Mont. (Montagne, 1845a).

Sematophyllum scorpiurus (Mont.) Mitt. (Mitten, 1869).

Rhaphidostegium scorpiurus (Mont.) Jaeg. (Jaeger, 1878).

Sematophyllum aureo-nitidum Bartr. (Bartram, 1946) fide Seki, 1974.

Distr. Southem South America (Montagne, 1845a, 1850, 1856; Mueller, 1851 a; Lorentz, 1866; Mitten, 1869; Jaeger, 1878; Paris, 1905b; Herzog, 1923, 1938, 1954, 1960; Brotherus, 1925; Bartram, 1946; Seki, 1974).

R. subsimplex (Hedw.) Broth.

Sematophyllum subsimplex

Rhaphidostegium hom. illeg.

R. aberrans Broth.

Sematophyllum aberrans

R. amaenum (Hedw.) Par.

R. amoenum (Hedw.) Jaeg.

Rhaphidorrhynchium amoenum

R. ampullatum Par.

Rhaphidorrhynchium amoenum

R. ampullulatum (C. Muell.) Broth.

Sematophyllum ampullulatum

R. aureo-viride (C. Muell.) Par.

Sematophyllum ampullulatum

R. berberidis (Dus.) Broth.

Sematophyllum aureo-viride

R. brachycladulum Broth.

Rhaphidorihynchium berberidis

R. bracteatum (C. Muell.) Kindb.

Sematophyllum brachycladulum

R. caespitosoides Broth.

Sematophyllum bracteatum

Sematophyllum aberrans

R. caespitosum (Hedw.) Besch.

Sematophyllum caespitosum

R. callidum (Mont.) Jaeg.

R. catilliforme (C. Muell.) Par.

Rhaphidorrhynchium callidum

Sematophyllum bracteatum 
R. cochleatulum (C. Muell.) Par. (Paris, 1898).

Syn. Aptychus cochleatulus C. Muell. (Mueller, 1897a).

Distr. Southern South America (Mueller, 1897a; Paris, 1898, 1905b).

R. condensatulum (C. Muell.) Par. (Paris, 1898).

Syn. Aptychus condensatulus C. Muell. (Mueller, 1897a).

Distr. Southern South America (Mueller, 1897a; Paris, 1898, 1905b).

R. dendroligotrichum Dus.

Rhaphidorrhynchium dendroligotrichum

R. diaphanodictyon (C. Muell.) Par. (Paris, 1898).

Syn. Aptychus diaphanodictus C. Muell. (Mueller, 1897a).

Distr. Southern South America (Mueller, 1897a; Paris, 1898, 1905b).

R. grandicellulosum (C. Muell.) Par.

Sematophyllum grandicellulosum

R. laxo-alare (C. Muell.) Par.

R. leptophyllum (Mitt.) Jaeg.

R. leucocytus (C. Muell.) Jaeg.

Entodon laxo-alaris

R. loxense (Hook.) Jaeg.

R. macrocytus (C. Muell.) Par.

R. masafuerae Broth.

Rhaphidornhynchium leptophyllum

R. micrangium (C. Muell.) Par.

R. microcarpoides (C. Muell.) Kindb.

Sematophyllum leucocytus

R. micropyxis (C. Muell.) Kindb.

Sematophyllum caespitosum

R. nano-cephalum (C. Muell.) Par.

Sematophyllum macrocytus

Sematophyllum masafuerae

Sematophyllum micrangium

Sematophyllum microcarpoides

Trichosteleum sp.

var. subglauculum (C. Muell.) Par.

Sematophyllum nanocephalum

R. nanum Kindb. (Kindberg, 1891) nom. nud.

Syn. Hypnum nanum C. Muell. (Mueller in Balansa and Savès, 1887) nom. nud.

Sematophyllum nanum Hert. (Herter, 1933a) nom. nud.

Distr. Southern South America (Felippone, 1912; Herter, 1933a).

R. noduliferum (Mitt.) Jaeg.

Rhaphidorthynchium noduliferum

R. pallens Dus.

Hypnum pallens

R. pallido-nitidum (C. Muell.) Broth.

Rhaphidorrhynchium pallido-nitidum

R. paraguariense C. Muell. ex Broth. (Mueller ex Brotherus, 1900).

Distr. Southern South America (Brotherus, 1918; Kühnemann, 1938).

R. patagonicum Broth. (Brotherus in Cardot and Brotherus, 1923).

Distr. Southern South America (Dusén, 1903a; Paris, 1905b; Cardot and Brotherus, 1923; Kühnemann, 1938; Seki, 1974).

R. polytrichadelphus Dus.

Rhaphidorrhynchium dendroligotrichum

R. prostratum Card. (Cardot in Skottsberg, 1914) nom. nud.

Distr. Juan Fernandez (Skottsberg, 1914).

R. purpureo-axis Dus. (Dusén in Paris, 1905b) nom. nud.

Distr. Southern South America (Paris, 1905b).

R. scorpiurus (Mont.) Jaeg.

R. secundifolium (C. Muell.) Jaeg.

R. serifolium (C. Muell.) Par.

R. stenopyxidium (C. Muell.) Kindb.

Rhaphidornhynchium scorpiurus

Drepanocladus secundifolius

Sematophyllum serifolium

Sematophyllum stenopyxidium 
R. strictipes Dus. (Dusén, 1903a) nom. nud.

Distr. Southem South America (Dusén, 1903a).

R. subsimplex (Hedw.) Besch.

Sematophylum subsimplex

R. temperatum (C. Muell.) Par.

Sematophyllum temperatum

$P$ cenerifolium (C. Muell.) Par.

R. tristifolium Dus. (Dusén, 1903a) nom. nud.

Distr. Southern South America (Dusén, 1903a).

\section{Rhaphidostichum}

R. gunckelii Thér. (Thériot, 1930).

Distr. Southem South America (Thẹriot, 1930).

\section{Rhexophyllum}

R. lacinatum Herz. (Herzog, 1916a).

Distr. Southem South America (Bartram, 1965).

\section{Rhizogonium}

R. brevifolium Broth. (Brotherus, 1891).

Syn. Mnium brevifolium (Broth.) C. Muell. (Mueller, 1901a [1900]).

Distr. Southem South America (Thériot, 1935a).

R. lindigii (Hamp.) Mitt. (Mitten, 1869).

Syn. Mnium lindigii Hamp. (Hampe, 1865).

Distr. Southern South America (Herzog, 1960).

R. longicolle C. Muell. (Mueller in Kindberg, 1891) nom. nud.

Syn. Mnium longicolle C. Muell. (Mueller in Paris, 1898) nom. nud.

Distr. Southem South America (Paris, 1898).

R. mnioides (Hook.) Wils. Wilson, 1854).

Syn. Hypnum mnioides Hook. (Hooker, 1820).

Mnium polycarpum C. Muell. (Mueller, 1848a) nom. illeg.

Rhizogonium polycarpum Schimp. (Schimper in Mueller, 1889) nom. illeg.

Aulacomnium chilense C. Muell. (Mueller, 1843b).

Distr, Juan Femandez (Brotherus, 1924b; Espinosa B., 1941; Robinson, 1975).

Southem South America (Hooker, 1820; Bridel, 1827b; Schwaegrichen, 1829; Mueller, 1843b, 1848a, 1885, 1889; Montagne, 1845b, 1850; Wilson and Hooker, 1847; Lorentz, 1866; Mitten, 1869; Ångström, 1872; Bescherelle, 1889; Neger, 1899; Dusén, 1903a; Cardot, 1905a, 1908a; Paris, 1905b; Thériot, 1915, 1918, 1934b; Campo, 1921: Cardot and Brotherus, 1923; Herzog, 1923, 1938, 1939, 1940, 1954. 1957, 1960; Brotherus, 1924a; Reimers, 1926; Roivainen, 1934; Kühnemann, 1938; Bartram, 1952; Bizot, 1967; Seki, 1974).

R. novae-hollandiae (Brid.) Brid. var. patagonicum Card. et Broth. (Cardot and Brotherus, 1923).

Distr. Juan Fernandez (Brotherus, 1924b; Espinosa B., 1941; Robinson, 1975).

Southern South America (Cardot and Brotherus, 1923).

R. polycarpum Schimp.

Rhizogonium mnioides

R. reticulatum (Hook. f. et Wils.) Mitt.

Philonotis vagans

R. spiniforme (Hedw.) Bruch (Bruch in Krauss, 1846).

Syn. Hypnum spiniforme Hedw. (Hedwig, 1801).

Distr. Southern South America (Herter, 1943)

R. spininervium (Hook.) Schimp.

R. subbasilare (Hook.) Schimp. 


\section{Rhodobryum}

R. andicola (Hook.) Par.

R. aubertii (Schwaegr.) Thér. (Thériot, 1922).

Syn. Mnium aubertii Schwaegr. (Schwaegrichen, 1816). Bryum aubertii (Schwaegr.) Brid. (Bridel, 1819).

Distr. Southern South America (Montagne, 1839c; Kühnemann, 1938 (as M. augertii)).

R. beyrichianum (Hornsch.) C. Muell. (Mueller in Hampe, 1874 [1875]).

Syn. Mnium beyrichianum Hornsch. (Hornschuch, 1840).

Bryum beyrichianum (Hornsch.) C. Muell. (Mueller, 1848a).

Bryum beyrichii Aongstr. (Ångström, 1876a) nom. illeg.

Bryum beyrichiae Hornsch. ex Kindb. (Hornschuch ex Kindberg, 1888) nom. illeg.

Distr. Southern South America (Mueller, 1848a; Montagne, 1850; Mitten, 1869; Paris, 1905b; Brotherus, 1924a; Reimers, 1926; Hosseus, 1940; Herter, 1943; Herzog, 1952a).

R. chilense Thér. (Thériot, 1924).

Distr. Southern South America (Thériot, 1924; Brotherus, 1925).

R. gracilescens C. Muell.

Bryum densifolium

R. hieronymi (C. Muell.) Par. (Paris, 1898).

Syn. Bryum hieronymi C. Muell. (Mueller, 1879a).

Distr. Southern South America (Mueller, 1879a; Paris, 1898, 1905b; Brotherus, 1924a; Kühnemann, 1938).

R. lechleri (C. Muell.) Par.

Bryum andicola

R. Iorentzianum (C. Muell.) Par. (Paris, 1898).

Syn. Bryum lorentzianum C. Muell. (Mueller, 1879a).

Distr. Southern South America (Mueller, 1879a; Paris, 1898, 1905b; Brotherus, 1924a; Hosseus, 1938b, c, d; Kühnemann, 1938).

R. platense (C. Muell.) Par. (Paris, 1898).

Syn. Bryum platense C. Muell. (Mueller, 1897a).

Distr. Southern South America (Mueller, 1897a; Paris, 1898, 1905b; Brotherus, 1924a; Kühnemann, 1938).

R. rigidum (Hornsch.) Par.

Bryum rigidum

R. roseolum (C. Muell.) Par.

Bryum roseolum

R. roseum (Hedw.) Limpr. (Limpricht, 1892).

Syn. Mnium roseum Hedw. (Hedwig, 1801).

Distr. Southern South America (Montagne, 1839C; Kühnemann. 1938).

R. subintegrum Broth. (Brotherus in Krieger, 1904) nom. nud.

Distr. Southern South America (Krieger, 1904).

\section{Rhyncho-hypnum}

R. leucostegum (Brid.) Hamp.

R. sellowii (Hornsch.) Hamp.

Stereophyllum leucostegum

Rhynchostegium sellowii

\section{Rhynchostegiella}

R. acanthophylla (Mont.) Broth.

Eurhynchiella acanthophylla

var. robusta Thér. (Thériot, 1917b).

Distr. Southern South America (Thériot, 1917b). 
R. chilensis Thér. (Thériot, 1934b [1935]).

Distr. Southem South America (Thériot, 1934b).

R. fuegiana (Card.) Broth.

Oxyrrhynchium fuegianum

R. leptoneura Card. (Cardot in Skottsberg, 1914) nom nud.

Distr. Juan Femandez (Skottsberg, 1914).

\section{Rhynchostegium}

R. acanthophyllum (Mont.) Jaeg.

R. altisetum C. Muell.

Eurhynchiella acanthophylla

R. aquaticum Jaeg. Oxyrrhynchium altisetum

R. berteroanum (Mont.) Jaeg. Platyhypnidium aquaticum

R. berteroi Kindb.

R. beskeanum (C. Muell.) Jaeg. (Jaeger, 1878).

Syn. Hypnum beskeanum C. Muell. (Mueller, 1857).

Distr. Southem South America (Herter, 1933a).

R. brevicuspis C. Muell. (Mueller, 1897a).

Distr. Southem South America (Mueller, 1897a; Paris, 1905b; Brotherus, 1925; Hosseus, 1938a, b, d; Kühnemann, 1938).

R. byssicladum Dus. (Dusén, 1903a) nom. nud.

Distr. Southem South America (Dusén, 1903a).

R. cacticola (C. Muell.) Par. (Paris, 1898).

Syn. Hypnum cacticola C. Muell. (Mueller, 1882).

Distr. Southem South America (Mueller, 1882; Paris, 1898, 1905b; Brotherus, 1925; Kühnemann, 1938).

R. campylocladulum C. Muell. (Mueller, 1897a).

Distr. Southem South America (Mueller, 1897a; Paris, 1905b; Brotherus, 1925; Hosseus, 1938a, b. d; Kühnemann, 1938).

R. capillirameum Dus. (Dusén in Paris, 1905b) nom. nud.

Distr. Southern South America (Paris, 1905b).

R. complanum (Mitt.) Jaeg. (Jaeger, 1878).

Syn. Hypnum complanum Mitt. (Mitten, 1869).

Distr. Juan Fernandez (Brotherus, 1924b, 1925; Espinosa B., 1941; Bartram, 1959; Robinson, 1975).

Southern South America (Mitten, 1869; Jaeger, 1878; Paris, 1905b; Brotherus, 1925; Herzog, 1954, 1960).

R. concavifolium Card. (Cardot in Skottsberg, 1914) nom. nud.

Distr. Juan Fernandez (Skottsberg, 1914).

R. confertum (Dicks.) B.S.G. (Bruch and others, 1852).

Syn. Hypnum confertum Dicks. (Dickson, 1801).

Distr. Southern South America (Montagne, 1850).

R. drepanocladioides (C. Muell.) Kindb. (Kindberg, 1891).

Syn. Hypnum drepanocladioides C. Muell. (Mueller, 1882).

Distr. Southern South America (Mueller, 1882; Paris, 1905b; Brotherus. 1925; Kühnemann. 1938). 
R. fissidens (C. Muell.) Kindb. (Kindberg, 1891).

Syn. Hypnum fissidens C. Muell. (Mueller, 1882).

Distr. Southern South America (Mueller, 1882; Paris, 1905b; Brotherus, 1925; Kühnemann, 1938).

R. glauco-virescens (C. Muell.) Kindb. (Kindberg, 1888).

Syn. Hypnum glauco-virescens C. Muell. (Mueller, 1879a).

Distr. Southern South America (Mueller, 1879a; Kurtz, 1904; Paris, 1905b; Hosseus, 1935b, 1937; Kühnemann, 1938).

R. globipyxis (C. Muell.) Kindb. (Kindberg, 1888).

Syn. Hypnum globipyxis C. Muell. (Mueller, 1879a).

Distr. Southern South America (Mueller, 1879a; Paris, 1905b; Brotherus, 1925; Kühnemann, 1938).

R. homaliocaulon (C. Muell.) Kindb. (Kindberg, 1888).

Syn. Hypnum homaliocaulon C. Muell. (Mueller, 1879a).

Distr. Southern South America (Mueller, 1879a; Kurtz, 1904; Paris, 1905b; Brotherus, 1925; Hosseus, 1935a, 1937; Kühnemann, 1938).

R. leptopteridium C. Muell. (Mueller, 1897a).

Distr. Southern South America (Mueller, 1897a; Paris, 1905b; Brotherus, 1925; Kühnemann, 1938).

R. leucodictyon C. Muell. (Mueller, 1897a).

Distr. Southern South America (Mueller, 1897a; Paris, 1905b; Brotherus, 1925; Kühnemann, 1938).

R. mollissimum Dus.

Brachythecium paradoxum

R. pampae (C. Muell.) Kindb. (Kindberg, 1888).

Syn. Hypnum pampae C. Muell. (Mueller, 1879a).

Distr. Southern South America (Mueller, 1879a; Kurtz, 1904; Paris, 1905b; Brotherus, 1925; Hosseus, 1935a, b, 1937; Kühnemann, 1938).

R. pendulum (Brid.) Jaeg.

Helicodontium pendulum

R. pinnicaule (C. Muell.) Kindb. (Kindberg, 1888).

Syn. Hypnum pinnicaule C. Muell. (Mueller, 1879a).

Distr. Southern South America (Mueller, 1879a; Kurtz, 1904; Paris, 1905b; Brothenus, 1925; Hosseus, 1935b; Kühnemann, 1938).

R. plagiotheciella C. Muell. (Mueller, 1897a).

Distr. Southern South America (Mueller, 1897a; Paris, 1905b; Brotherus, 1925; Kühnemann, 1938).

R. planirameum Card. (Cardot in Skottsberg, 1914) nom. nud.

Distr. Juan Fernandez (Skottsberg, 1914).

R. Sellowii (Hornsch.) Jaeg. (Jaeger, 1878).

Syn. Hypnum sellowii Hornsch. (Hornschuch, 1840).

Hypnum sellowianum C. Muell. (Mueller, 1847a [1846]) nom. illeg.

Rhyncho-hypnum sellowii (Hornsch.) Hamp. (Hampe, 1870) comb. inval.

Distr. Southern South America (Hornschuch, 1840; Mueller, 1851a; Mitten, 1869; Gibert, 1873; Jaeger, 1878; Paris, 1905b; Felippone, 1912; Brotherus, 1925; Herter, 1933a; Hosseus, 1938a, c, d; Kühnemann, 1938).

R. semi-tortulum Kindb. (Kindberg, 1891).

Syn. Hypnum semi-tortulum C. Muell. (Mueller, 1882) hom. illeg. 
Distr. Southem South America (Mueller, 1882; Paris, 1905b; Brotherus, 1925; Kühnemann, 1938).

R. serrulatum (Hedw.) Jaeg. (Jaeger, 1878).

Syn. Hypnum serrulatum Hedw. (Hedwig, 1801).

Distr. Juan Femandez (Montagne, 1835, 1839c; Johow, 1896).

Southem South America (Hornschuch, 1820b; Bridel, 1827 b; Montagne, 1850; Kühnemann, 1938).

R. striatellum Schimp. ex Par.

Rigodium toxarion

R. subsquarrosum Herz.

Oxyrrhynchium corralense

R. taphrophilum C. Muell. (Mueller, 1897a).

Distr. Southem South America (Mueller, 1897a; Paris, 1905b; Brotherus, 1925; Kühnemann, 1938).

R. tenuifolium (Hedw.) Reichdt. (Reichardt, 1870).

Syn. Hypnum tenuifolium Hedw. (Hedwig, 1801).

Isothecium tenuifolium (Hedw.) Brid. (Bridel, 1827b).

Amblystegiella tenuifolia (Hedw.) Fleisch. (Fleischer, 1920).

Helicodontium tenuifolium (Hedw.) C. Muell. (Mueller in Fleischer, 1920) nom. inval.

Distr. Juan Femandez (Mueller, 1885; Johow, 1896; Brotherus, 1924b; Herter, 1928, 1933b; Robinson, 1975).

Southem South America (Mitten, 1869; Gibert, 1873; Jaeger, 1878; Paris, 1905b; Felippone, 1912; Brotherus, 1925; Herter, 1933a, b; Kühnemann, 1938; Herzog, 1954).

var. ledifolium Jaeg. (Jaeger, 1878) nom. nud.

Distr. Southem South America (Jaeger, 1878).

var. minus Jaeg. (Jaeger, 1878) nom. nud.

Distr. Southem South America (Jaeger, 1878).

R. trieblingii C. Muell. (Mueller, 1897a).

Distr. Southern South America (Mueller, 1897a; Paris, 1905b; Brotherus, 1925).

\section{Rigodium}

R. acuminatum Card. (Cardot in Skottsberg, 1914) nom. nud.

Distr. Juan Fernandez (Skottsberg, 1914).

R. arborescens (C. Muell.) Broth. (Brotherus, 1909).

Syn. Hypnum arborescens C. Muell. (Mueller, 1858).

Rigodium lechleri Schimp. (Schimper in Lorentz,'1866) nom. illeg.

Heterocladium lechleri Schimp. (Schimper in Lorentz, 1866) nom. nud. pro parte.

Heterocladium prolixum Schimp. (Schimper in Lorentz, 1866) nom. nud. pro parte.

Distr. Juan Femandez (Espinosa B., 1941).

Southern South America (Lechler, 1857; Mueller, 1858; Lorentz, 1866; Jaeger, 1878; Neger, 1899; Paris, 1905b; Brotherus, 1909, 1925; Campo, 1915, 1921; Thériot, 1917b, 1918; Costes, 1921; Herzog, 1923, 1954, 1960; Roivainen, 1934; Seki, 1974).

R. argentinicum (C. Muell.) Kindb. (Kindberg, 1891).

Syn. Hypnum argentinicum C. Muell. (Mueller, 1882).

Distr. Southern South America (Mueller, 1882; Paris, 1905b; Brotherus, 1925; Kühnemann, 1938; Seki, 1974).

R. brachypodium (C. Muell.) Par. (Paris, 1898).

Syn. Hypnum brachypodium C. Muell. (Mueller, 1851a).

Hypnum brachypelma C. Muell. (Mueller, 1882) nom. inval.

Distr. Southern South America (Mueller, 1851a, 1882; Paris, 1898, 1905b; Brotherus, 1925; Bartram, 1952). 
R. carnosulum Dus. (Dusén, 1903a) nom. nud.

Distr. Southern South America (Dusén, 1903a; Paris, 1905b; Seki, 1974).

R. elegantulum Card.

Rigodium tamarix

var. fernandezianum Card. (Cardot in Skottsberg, 1914) nom. nud.

Distr. Southern South America (Skottsberg, 1914).

R. hylocomioides Card. et Broth. (Cardot and Brotherus, 1923).

Distr. Juan Fernandez (Brotherus, 1924b; Robinson, 1975).

Southern South America (Cardot and Brotherus, 1923; Brotherus, 1925; Thériot, 1926; Kühnemann, 1938; Herzog, 1939, 1940, 1960).

var. gracilius Card. et Broth. (Cardot and Brotherus, 1923).

Distr. Southern South America (Cardot and Brotherus, 1923).

R. implexum Kunz. ex Schwaegr. (Kunze ex Schwaegrichen, 1844 [1845]).

Syn. Hypnum implexum (Schwaegr.) C. Muell. (Mueller, 1844b [1845]) hom. illeg. Heterocladium implexum (Schwaegr.) Lor. (Lorentz, 1866).

Hypnum neei Mohr (Mohr in Mueller, 1851a) nom. nud.

Distr. Southern South America (Mueller, 1844b, 1851a; Schwaegrichen, 1844; Sullivant, 1859; Lorentz, 1866; Paris, 1905b; Campo, 1915 (as R. inflexum Kurz); Cardot and Brotherus, 1923; Herzog, 1923, 1938, 1939, 1940, 1954, 1960; Brotherus, 1925; Roivainen, 1934; Kühnemann, 1938; Bartram, 1952; Bizot, 1967; Seki, 1974).

R. lechleri Schimp.

R. looseri Thér.

R. nano-fasciculare C. Muell. ex Kindb.

Rigodium arborescens

Rigodium robustum

R. nano-fasciculatum C. Muell. ex Thér. Rigodium toxarion Rigodium toxarion

R. pendulum Herz. et Thér. (Herzog and Thériot in Herzog, 1939).

Distr. Southern South America (Herzog, 1939, 1954; Seki, 1974).

R. pseudo-thuidium Dus. (Dusén, 1905b).

Syn. Rigodium ptychomnioides Broth. (Brotherus, 1909) nom. nud.

Distr. Southern South America (Dusén, 1903a, 1905b; Paris, 1905b; Cardot, 1908a; Brotherus, 1925; Kühnemann, 1938; Herzog, 1954, 1960; Seki, 1974).

R. ptychomnioides Broth.

Rigodium pseudo-thuidium

R. robustum Broth. (Brotherus, 1924b).

Syn. Rigodium looseri Thér. (Thériot, 1927b) fide Robinson, 1975.

Distr. Juan Fernandez (Brotherus, $1924 b$ (and as R. arborescens fide Robinson, 1975); Thériot, 1927b; Bartram, 1959 (and as R. arborescens fide Robinson, 1975); Robinson, 1975).

R. tamarix C. Muell. (Mueller, 1897a).

Syn. Rigodium elegantulum Card. (Cardot in Skottsberg, 1914) nom. nud.

Distr. Juan Fernandez (Skottsberg, 1914; Thériot, 1921b).

Southern South America (Mueller, 1897a; Paris, 1905b; Cardot, 1908a; Cardot and Brotherus, 1923; Brotherus, 1925; Roivainen, 1934; Thériot, 1934b; Kühnemann, 1938; Seki, 1974).

R. toxarion (Schwaegr.) Jaeg. (Jaeger, 1878).

Syn. Hypnum toxarion Schwaegr. (Schwaegrichen, 1816).

Hypnum taxarion Schwaegr. ex Mont. (Schwaegrichen ex Montagne, 1835) err.

Eurhynchium striatellum Schimp. (Schimper in Mitten, 1869) nom. nud.

Rhynchostegium striatellum Schimp. ex Par. (Schimper ex Paris, 1898) nom. nud.

Heterocladium lechleri Schimp. (Schimper in Lorentz, 1866) nom. nud. pro parte.

Heterocladium prolixum Schimp. (Schimper in Lorentz, 1866) nom. nud. pro parte.

Rigodium nano-fasciculare C. Muell. ex Kindb. (Mueller ex Kindberg, 1891) err. 
Rigodium nano-fasciculatum C. Muell. ex Thèr. (Mueller ex Thériot, 1929d [1930]) fide Robinson, 1975.

Distr. Juan Femandez (Montagne, 1835; Mitten, 1885; Johow, 1896; Brotherus, 1924b, (and as $R$. arborescens fide Robinson, 1975); Espinosa B., 1941; Bartram, 1959; Robinson, 1975).

Southem South America (Montagne, 1850; Mitten, 1869; Jaeger, 1878; Bescherelle, 1889; Neger, 1899; Cardot, 1908a; Campo, 1915, 1921; Thériot, 1918, 1929d, 1934b; Brotherus, 1924c; Roivainen, 1934; Kühnemann, 1938; Herzog, 1939, 1954, 1960; Espinosa B., 1941; Skottsberg, 1950; Seki, 1974).

R. varium (Hedw.) Kindb.

Amblystegium varium

\section{Rudia}

R. clarazii (Dub.) Jaeg.

Helicodontium clarazii

\section{Sanionia}

S. orthothecioides (Lindb.) Loesk.

Drepanocladus uncinatus var. subjulaceus

S. symmetrica (Ren. et Card.) Wheld.

Drepanocladus uncinatus var. symmetricus

S. uncinata (Hedw.) Loesk.

Drepanocladus uncinatus

\section{Sarconeurum}

S. antarcticum Bryhn

Sarconeurum glaciale

S. glaciale (C. Muell.) Card. et Bryhn (Cardot and Bryhn in Cardot, 1907a).

Syn. Didymodon glacialis Hook. f. et Wils. (Hooker and Wilson in Wilson and Hooker, 1847) hom. illeg.

Leptotrichum glaciale C. Muell. (Mueller, 1851a).

Ditrichum glaciale (C. Muell.) O. Kuntze (Kuntze, 1891).

Sarconeurum antarcticum Bryhn (Bryhn, 1902).

Tortula saxicoḷa Dus. (Dusén, 1905b) hom. illeg. fide Greene, 1975.

Tortula lithophila Dus. (Dusén, 1906) fide Greene, 1975.

Tortula pygmaea Dus. (Dusén, 1907) fide Greene, 1975.

Distr. Southern South America (Dusén, 1905b, 1906, 1907; Cardot, 1908a; Cardot and Brotherus, 1923; Brotherus, 1924a; Kühnemann, 1938; Bizot, 1967; Seki, 1974; Greene, 1975).

Antarctic, peninsula region Wilson and Hooker, 1847; Mueller, 1851 a; Jaeger, 1873; Paris, 1904b; Cardot, 1908a; Steere, 1961a; Greene, 1967; Greene and others, 1970).

Antarctic, continental region (Bryhn, 1902; Cardot, 1907a, 1908a, 1910; Dixon and Watts, 1918; Hilpert, 1933; Bartram, 1938, 1957; Steere, 1961a; Savich-Ljubitskaya and Smirnova, 1962; Greene, 1967; Greene and others, 1970; Robinson, 1972; Savich-Ljubitskaya, 1978).

S. tortelloides* S. W. Greene (Greene in Greene and others, 1970).

Distr. Antarctic, peninsula region (Greene and others, 1970).

\section{Sauloma}

S. tenella (Hook. f. et Wils.) Mitt. (Mitten, 1860).

Syn. Hookeria tenella Hook. f. et Wils. (Hooker and Wilson in Wilson, 1854).

Distr. Southern South America (Bartram, 1946; Welch, 1969; Matteri, 1972, 1975a).

\section{Schimperobryum}

S. splendidissimum (Mont.) Marg. (Margadant, 1959).

Syn. Hookeria splendidissima Mont. (Montagne, 1835).

Cyathophorum splendidissimum (Mont.) Hamp. et Lor. (Hampe and Lorentz in Lorentz, 1866).

Lepidopilum splendidissimum (Mont.) Mitt. (Mitten, 1869).

Lamprophyllum splendidissimum (Mont.) Broth. (Brotherus, 1907).

Hookeria speciosissima Mont. ex Schwaegr. (Montagne ex Schwaegrichen, 1842) err.

'Transferred to Tortella by Robinson (1972). 
Distr. Juan Fernandez (Montagne, 1835, 1856; Schwaegrichen, 1842; Mueller, 1851a; Mitten, 1869, 1885; Jaeger, 1876; Johow, 1896; Skottsberg, 1914; Brotherus, 1924b, 1925; Thériot, 1927b; Espinosa B., 1941; Bartram, 1959; Matteri, 1972; Robinson, 1975).

Southern South America (Montagne, 1850; Mueller, 1851a; Lorentz, 1866; Mitten, 1869; Jaeger, 1876; Neger, 1899; Paris, 1904a; Brotherus, 1907, 1925; Cardot, 1908a; Campo, 1915; Cardot and Brotherus, 1923; Herzog, 1923, 1938, 1954; Thériot, 1924; Reimers, 1926; Kühnemann, 1938; Welch, 1969).

var. perdentatum Matteri (Matteri, 1972).

Distr. Juan Fernandez (Matteri, 1972).

Southern South America (Matteri, 1972; Seki, 1974).

\section{Schistidium}

S. alpicola (Hedw.) Limpr. (Limpricht, 1889).

Syn. Grimmia alpicola Sw. ex Hedw. (Hedwig, 1801).

Weisia alpicola (Hedw.) Poir. (Poiret, 1808) nom. inval.

Grimmia apocarpa Hedw. ssp. alpicola (Hedw.) C. Hartm. (Hartman in Lindberg, 1879).

Schistidium apocarpum (Hedw.) B.S.G. ssp. alpicola (Hedw.) Meyl. (Meylan, 1905).

Distr. Southern South America (Cardot and Brotherus, 1923; Kühnemann, 1938; Herzog, 1957).

var. rivulare (Brid.) Limpr.

Schistidium apocarpum

S. amblyophyllum (C. Muell.) Herz.

Grimmia amblyophylla

S. antarctici (Card.) Savicz. et Smirn. (Savich-Ljubitskaya and Smirnova, 1965a).

Syn. Grimmia antarctici Card. (Cardot, 1906a) fide Savich-Ljubitskaya and Smirnova, $1965 a$.

Grimmia antarctici Card. var. percompacta Bartr. (Bartram, 1938) fide Savich-

Ljubitskaya and Smirnova, 1965a.
Grimmia antarctici Card. var. pilifera Bartr. (Bartram, 1938) fide Savich-Ljubitskaya and Smirnova, 1965a.

Distr. Antarctic, peninsula region (Wilson and Hooker, 1847 (as Tortula gracilis see Cardot, 1908a, p. 241); Wright, 1905 (as G. amblyophylla fide Cardot, 1908a); Cardot, 1906a, 1907 c, 1908a, 1911b, c, d, 1912b, 1913a; Dixon, 1920; Brotherus, 1924a; Steere, 1961a; Greene, 1968a (as Tortula gracilis see Cardot, 1908a, p. 241); Robinson, 1972).

Antarctic, continental region (Bartram, 1938, 1957; Steere, 1961a; Savich-Ljubitskaya and Smirnova, 1965a; Robinson, 1972; Savich-Ljubitskaya, 1978).

S. apocarpum (Hedw.) B.S.G. (Bruch and others, 1845).

Syn. Grimmia apocarpa Hedw. (Hedwig, 1801).

Bryum apocarpum (Hedw.) L. ex With. (Withering, 1801).

Weisia apocarpa (Hedw.) Poir. (Poiret, 1808).

Grimmia rivularis Brid. (Bridel, 1801a).

Schistidium rivulare (Brid.) Podp. (Podpěra, 1911).

Grimmia apocarpa Hedw. var. rivularis (Brid.) Roehl. (Röhling, 1812).

Schistidium apocarpum (Hedw.) B.S.G. var. rivulare (Brid.) B.S.G. (Bruch and others, 1845).

Grimmia alpicola Sw. ex Hedw. var rivularis (Brid.) Wahlenb. (Wahlenberg, 1812).

Schistidium alpicola (Hedw.) Limpr. var. rivulare (Brid.) Limpr. (Limpricht, 1889).

Distr. Southern South America Wilson and Hooker, 1847; Mueller, 1849; 1885; Montagne, 1850; Mitten, 1869; Paris, 1904b; Cardot, 1905a, 1908a; Cardot and Brotherus, 1923; Brotherus, 1924a; Roivainen, 1934; Kühnemann, 1938).

Falkland Islands Wilson and Hooker, 1847; Cardot, 1905a, 1908a; Cardot and Brotherus, 1923; Brotherus, 1924a).

Antarctic, peninsula region (Wright, 1905; Cardot, 1908a, 1911c, 1912b; Brotherus, 1924a; Bartram, 1957; Steere, 1961a; Robinson, 1972).

ssp. alpicola (Hedw.) Meyl.

Schistidium alpicola

ssp. confertum (Funck) Loesk. (Loeske, 1913).

Syn. Grimmia conferta Funck (Funck, 1820). 
Schistidium confertum (Funck) B.S.G. (Bruch and others, 1845).

Grimmia apocarpa Hedw. var. conferta (Funck) Spreng. (Sprengel, 1827).

Grimmia apocarpa Hedw. ssp. conferta (Funck) Boul. (Boulay, 1884).

Distr. Southem South America (Cardot and Brotherus, 1923; Brotherus, 1924a; Kühnemann, 1938).

Falkland Islands (Cardot and Brotherus, 1923).

var. rivulare (Brid.) B.S.G.

Schistidium apocarpum

S. ciliatum (Hedw.) Brid. var. imberbe (Sm.) Hueb.

Hedwigidium integrifolium

S. confertum (Funck) B.S.G.

Schistidium apocarpum ssp. confertum

S. imberbe (Sm.) Nees et Hornsch.

Hedwigidium integrifolium

S. maritimum (Turn.) B.S.G. (Bruch and others 1845).

Syn. Grimmia maritima Turn. (Turner, 1804a).

Grimmia alpicola Sw. ex Hedw. var. maritima (Turn.) Wahlenb. (Wahlenberg, 1812).

Distr. Southem South America (Wilson and Hooker, 1847).

S. plagiopodium (Hedw.) Loesk.

Grimmia plagiopodia

S. praemorsum (C. Muell.) Herz.

Grimmia praemorsa

S. rivulare (Brid.) Podp.

Schistidium apocarpum

S. subsessile (Brid.) Brid.

Pterygoneurum subsessile

Schistophyllum hom. illeg.

S. acacioides (Schrad.) Lindb.

S. adianthoides (Hedw.) Lindb.

Fissidens osmundoides

S. asplenioides (Hedw.) Brid.

Fissidens adianthoides

S. bryoides (Hedw.) Lindb.

Fissidens asplenioides

Fissidens bryoides

S. julianum (Cand.) Lindb.

Fissidens fontanus

S. osmundoides (Hedw.) Lindb.

Fissidens osmundoides

var. microcarpum (B.S.G.) Kindb.

Fissidens osmundoides

S. semicompletum (Hedw.) Lindb.

Fissidens semicompletus

S. taxifolium (Hedw.) Lindb.

Fissidens taxifolius

\section{Schlotheimia}

S. aequifolia Dus. (Dusén in Naveau, 1928) nom. nud.

Distr. Southern South America (Naveau, 1928).

S. argentinica Lor. et C. Muell. (Lorentz and Mueller in Mueller, 1879a).

Distr. Southern South America (Mueller, 1879a; Paris, 1905b; Brotherus, 1925; Kühnemann, 1938).

S. asperrima Broth. (Brotherus, 1918).

Distr. Southern South America (Brotherus, 1918, 1925; Kühnemann, 1938).

S. gracillima Besch.

Macromitrium gracillimum

S. jamesonii (Arnott) Brid. (Bridel, 1826).

Syn. Orthotrichum jamesonii Arnott (Arnott, 1824b).

Macromitrium jamesonii (Arnott) C. Muell. (Mueller, 1845).

Distr. Southern South America (Hornschuch, 1840; Herter, 1933a).

S. linearifolia (C. Muell.) Wijk et Marg. (van der Wijk and Margadant, 1960b).

Syn. Macromitrium linearifolium C. Muell. (Mueller, 1845).

Schlotheimia longifolia Hornsch. (Hornschuch, 1840) hom. illeg.

Distr. Southern South America (Hornschuch, 1840; Mueller, 1849; Gibert, 1873; Paris, 1905b; Herter, 1933a). 
S. longifolia Hornsch.

S. nitida Schwaegr.

S. ottonis Schwaegr.

Schlotheimia torquata

S. striata Herz. (Herzog, 1952a).

Distr. Southern South America (Herzog, 1952a).

S. torquata (Hedw.) Brid. (Bridel, 1812).

Syn. Hypnum torquatum Hedw. (Hedwig, 1801).

Orthotrichum torquatum (Hedw.) Arnott (Arnott, 1827).

Macromitrium torquatum (Hedw.) C. Muell. (Mueller, 1845).

Schlotheimia nitida Schwaegr. (Schwaegrichen, 1826).

Schlotheimia ottonis Schwaegr. (Schwaegrichen, 1826).

Macromitrium ottonis (Schwaegr.) C. Muell. (Mueller, 1845).

Distr. Southern South America (Schwaegrichen, 1826; Bridel, 1827a; Hornschuch, 1840;

Mueller, 1849; Gibert, 1873; Jaeger, 1874; Herter, 1933a).

S. trichomitria Schwaegr. (Schwaegrichen, 1826).

Syn. Macromitrium trichomitrium (Schwaegr.) C. Muell. (Mueller, 1845).

Distr. Southern South America (Schwaegrichen, 1826, 1827a; Bridel, 1827a; Homschuch, 1840; Mueller, 1849; Gibert, 1873; Jaeger, 1874; Paris, 1905b; Felippone, 1912; Herter, 1933a).

\section{Sciaromium}

S. confluens (C. Muell.) Par. (Paris, 1898).

Syn. Hypnum confluens C. Muell. (Mueller, 1885).

Brachythecium confluens (C. Muell.) Kindb. (Kindberg, 1888).

Limbella confluens (C. Muell.) C. Muell. (Mueller, 1889) comb. inval.

Distr. Southern South America (Mueller, 1885, 1889; Paris, 1898, 1905b; Dusén, 1903a; Cardot, 1908a; Brotherus, 1925; Kühnemann, 1938; Seki, 1974).

S. conspissatulum Par. (Paris, 1898).

Syn. Limbella conspissatula C. Muell. (Mueller, 1897a) comb. inval.

Distr. Southern South America (Mueller, 1897a; Paris, 1898, 1905b; Brothenus, 1925; Kühnemann, 1938).

S. conspissatum (Hook. f. et Wils.) Mitt. (Mitten, 1869).

Syn. Hypnum conspissatum Hook. f. et Wils. (Hooker, J.D. and Wilson, 1844).

Limbella conspissata (Hook. f. et Wils.) C. Muell. (Mueller, 1889) comb. inval.

Distr. Southern South America (Mueller, 1851a, 1889; Sullivant, 1859; Lorentz, 1866; Jaeger and Sauerbeck, 1879a; Paris, 1905b; Cardot, 1908a; Thériot, 1915; Brotherus, 1925; Kühnemann, 1938; Herzog, 1939, 1954, 1960; Bartram, 1952; Seki, 1974).

Falkland Islands (Hooker, J. D. and Wilson, 1844; Wilson and Hooker, 1847; Mitten, 1869; Jaeger and Sauerbeck, 1879a; Paris, 1905b; Cardot, 1908a; Cardot and Brotherus, 1923; Brotherus, 1925; Kühnemann, 1938).

South Georgia (Cardot, 1906a, 1908a; Brotherus, 1925; Steere, 1961b).

var. maritimum (Card.) Herz.

Sciaromium obscurifolium

S. crassinervatum Mitt. (Mitten, 1869).

Distr. Southern South America (Herzog, 1939).

S. depastum Dus. (Dusén, 1903a).

Distr. Southern South America (Dusén, 1903a; Cardot, 1908a; Cardot and Brotherus, 1923; Brotherus, 1925; Kühnemann, 1938).

S. drepanophyllopsis Par. (Paris, 1898).

Syn. Limbella drepanophyllopsis C. Muell. (Mueller, 1897a) comb. inval. 
Distr. Southem South America (Mueller, 1897a; Paris, 1898, 1905b; Brotherus, 1925; Kühnemann, 1938).

S. flavidulum Dus. (Dusén, 1905b).

Distr. Southern South America (Dusén, 1905b; Cardot, 1908a; Brotherus, 1925).

S. gracile Dus. (Dusén, 1903a).

Distr. Southem South America (Dusén, 1903a; Cardot, 1908a; Brotherus, 1925; Kühnemann, 1938).

S. krauseanum Par. (Paris, 1898).

Syn. Limbella krauseana C. Muell. (Mueller, 1897a) comb. inval.

Distr. Southem South America (Mueller, 1897 a; Paris, 1898, 1905b; Cardot and Brotherus, 1923; Brothenus, 1925; Kühnemann, 1938).

S. maritimum Card.

Sciaromium obscurifolium

S. nigritum Dus. (Dusén, 1905b).

Distr. Southem South America (Dusén, 1905b; Cardot, 1908a; Brotherus, 1925).

S. obscurifolium Mitt. (Mitten, 1869).

Syn. Sciaromium maritimum Card. (Cardot, 1905a).

Sciaromium conspissatum (Hook. f. et Wils.) Mitt. var. maritimum (Card.) Herz. (Herzog, 1954).

Distr. Southem South America (Mitten, 1869; Jaeger and Sauerbeck, 1879a; Paris, 1905b; Cardot and Brotherus, 1923; Thériot, 1924, 1934b; Brotherus, 1925; Kühnemann, 1938; Herzog, 1954; Seki, 1974).

Falkland Islands (Cardot, 1905a, 1908a; Cardot and Brotherus, 1923; Brotherus, 1925; Kühnemann, 1938).

S. pachyloma (Mont.) Par. (Paris, 1898).

Syn. Gymnostomum pachyloma Mont. (Montagne, 1838).

Grimmia pachyloma (Mont.) Mitt. (Mitten, 1869).

Hypnum pachyloma (Mont.) C. Muell. (Mueller, 1889).

Limbella pachyloma (Mont.) C. Muell. (Mueller, 1897a) comb. inval.

Distr. Juan Fernandez (Brotherus, 1924b, 1925; Espinosa B., 1941; Bartram, 1959; Robinson, 1975).

Southem South America (Montagne, 1838, 1850; Mueller, 1851a, 1889, 1897a; Mitten, 1869; Jaeger, 1874; Paris, 1898, 1905b; Cardot and Brotherus, 1923; Espinosa B.. 1924; Brotherus, 1925; Reimers, 1926; Kühnemann, 1938; Bartram, 1952).

var. brevifolium Thér. (Thériot, 1924).

Distr. Southem South America (Costes, 1921 (as fo. brevifolium); Thériot, 1924).

var. gracilescens Herz. (Herzog, 1954).

Distr. Southem South America (Herzog, 1954).

S. pachylomatum Par. (Paris, 1898).

Syn. Limbella pachylomata C. Muell. (Mueller, 1897a) comb. inval.

Distr. Southem South America (Mueller, 1897a; Paris, 1898, 1905b; Kurtz, 1904; Brotherus, 1925; Kühnemann, 1938).

S. platylomatum Par. (Paris, 1898).

Syn. Limbella platylomata C. Muell. (Mueller, 1897a) comb. inval.

Distr. Southern South America (Mueller, 1897a; Paris, 1898, 1905b; Brotherus, 1925; Hosseus, 1936, 1937).

Sclerodontium nom. rejic.

S. pellucidum Schwaegr.

Dimerodontium pellucidum 


\section{Scleropodium}

S. pseudo-purum (C. Muell.) Broth.

\section{Scouleria}

S. patagonica (Mitt.) Jaeg. (Jaeger, 1876).

Syn. Grimmia patagonica Mitt. (Mitten, 1869).

Distr. Southern South America (Mitten, 1869; Jaeger, 1876; Paris, 1905b; Dusén, 1907; Cardot, 1908a; Brotherus, 1924a; Thériot, 1935a (as fo. squarrosa); Kühnemann, 1938; Bartram, 1952; Herzog, 1954, 1960; Seki, 1974; Matteri, 1979).

\section{Seligeria}

S. kunzeana (C. Muell.) C. Muell.

Microdus kunzeanus

S. kunzei Kindb.

Microdus kunzeanus

\section{Sematophyllum}

S. aberrans (Broth.) Bartr. (Bartram, 1959) fide Robinson, 1975.

Syn. Rhaphidostegium aberrans Broth. (Brotherus, 1924b).

Rhaphidorrhynchium aberrans (Broth.) Broth. (Brotherus, 1925).

Rhaphidostegium caespitosoides Broth. (Brotherus, 1924b) fide Robinson, 1975.

Sematophyllum caespitosoides (Broth.) Broth. (Brotherus, 1925).

Distr. Juan Fernandez (Brotherus, 1924b, 1925; Espinosa B., 1941; Bartram, 1959; Robinson, 1975).

S. amoenum (Hedw.) Mitt.

Rhaphidorrhynchium amoenum

S. ampullulatum (C. Muell.) Broth. (Brotherus, 1925).

Syn. Aptychus ampullulatus C. Muell. (Mueller, 1897a). Rhaphidostegium ampullulatum (C. Muell.) Broth. (Brotherus, 1908).

Aptychus ampullatus C. Muell. ex Par. (Mueller ex Paris, 1898) err.

Rhaphidostegium ampullatum Par. (Paris, 1898) hom. illeg.

Distr. Southern South America (Mueller, 1897a; Paris, 1898, 1905b; Brotherus, 1908, 1925; Kühnemann, 1938).

S. aquicola (C. Muell.) Kindb.

Bryum sp.

S. aureo-nitidium Bartr.

Rhaphidorrhynchium scorpiurus

S. aureo-viride (C. Muell.) Broth. (Brotherus, 1925).

Syn. Aptychus aureo-viridis C. Muell. (Mueller, 1897a). Rhaphidostegium aureo-viride (C. Muell.) Par. (Paris, 1898).

Distr. Southern South America (Mueller, 1897a; Paris, 1898, 1905b; Brotherus, 1925; Kühnemann, 1938).

S. brachycladulum (Broth.) Broth. (Brotherus, 1925).

Syn. Rhaphidostegium brachycladulum Broth. (Brotherus, 1924b).

Distr. Juan Fernandez (Brotherus, $1924 b$ (and as S. caespitosum fide Robinson, 1975), 1925; Bartram, 1959; Robinson, 1975).

S. bracteatum (C. Muell.) Broth. (Brotherus, 1925).

Syn. Hypnum bracteatum C. Muell. (Mueller, 1879a).

Rhaphidostegium bracteatum (C. Muell.) Kindb. (Kindberg, 1888).

Aptychus catilliformis C. Muell. (Mueller, 1897a).

Rhaphidostegium catilliforme (C. Muell.) Par. (Paris, 1898).

Sematophyllum catilliforme (C. Muell.) Broth. (Brotherus, 1925).

Distr. Southern South America (Mueller, 1879a, 1897a; Paris, 1898, 1900a, 1905b; Brotherus, 1925; Felippone, 1929; Herter, 1933a, 1943; Kühnemann, 1938; Hosseus, 1939). 
S. caespitosum (Hedw.) Mitt. (Mitten, 1869).

Syn. Leskea caespitosa Hedw. (Hedwig, 1801).

Rhapidostegium caespitosum (Hedw.) Besch. (Bescherelle, 1876).

Hypnum loxense Hook. (Hooker in Kunth, 1822).

Rhaphidostegium loxense (Hook.) Jaeg. (Jaeger, 1874).

Distr. Juan Femandez (Montagne, 1835).

Southem South America (Krieger, 1904; Hosseus, 1938c, d).

S. callidum (Mont.) Mitt.

S. catilliforme (C. Muell.) Broth.

Rhaphidorrhynchium callidum

Sematophyllum bracteatum

S. cirmifolium (Amott) Mitt. (Mitten, 1885).

Syn. Hookeria cirrhifolium Arnott (Arnott, 1827).

Hypnum cirrifolium (Arnott) Schwaegr. (Schwaegrichen in Gaudichaud, 1826 [1828]) hom. illeg.

Distr. Juan Fernandez (Montagne, 1835).

Falkland Islands (Gaudichaud, 1826).

S. crassiusculum (Brid.) Broth. (Brotherus, 1925).

Syn. Hypnum crassiusculum Brid. (Bridel, 1812).

Distr. Juan Femandez (Johow, 1896).

Southem South America (Montagne, 1850).

S. grandicellulosum (C. Muell.) Broth. (Brotherus, 1925).

Syn. Aptychus grandicellulosus C. Muell. (Mueller, 1897a).

Rhaphidostegium grandicellulosum (C. Muell.) Par. (Paris, 1898).

Distr. Southem South America (Mueller, 1897a; Paris, 1898, 1905b; Brotherus, 1925; Kühnemann, 1938).

S. kunkelii H. Robinson (Robinson, 1974).

Distr. Juan Fernandez (Robinson, 1974, 1975).

S. leptophyllum Mitt.

Rhaphidorrhynchium leptophyllum

S. leucocytus (C. Muell.) Sains. (Sainsbury, 1955).

Syn. Hypnum leucocytus C. Muell. (Mueller, 1851a).

Rhaphidostegium leucocytus (C. Muell.) Jaeg. (Jaeger, 1878).

Distr. Southern South America (Cardot, 1908a).

S. macrocytus (C. Muell.) Broth. (Brotherus, 1925).

Syn. Aptychus macrocytus C. Muell. (Mueller, 1897a).

Rhaphidostegium macrocytus (C. Muell.) Par. (Paris, 1898).

Distr. Southern South America (Mueller, 1897a; Paris, 1898, 1905b; Brotherus, 1925; Kühnemann, 1938).

S. masafuerae (Broth.) Bartr. (Bartram, 1959) fide Robinson, 1975.

Syn. Rhaphidostegium masafuerae Broth. (Brotherus, 1924b) fide Robinson, 1975. Raphidorrhynchium masafuerae (Broth.) Broth. (Brotherus, 1925).

Distr. Juan Fernandez (Brotherus, 1924b, 1925; Bartram, 1959; Robinson, 1975).

S. micrangium (C. Muell.) Broth. (Brotherus, 1925).

Syn. Aptychus micrangius C. Muell. (Mueller, 1897a).

Rhaphidostegium micrangium (C. Muell.) Par. (Paris, 1898).

Distr. Southern South America (Mueller, 1897a; Paris, 1905b; Brotherus, 1925; Kühnemann, 1938).

S. microcarpoides (C. Muell.) Broth. (Brotherus, 1925).

Syn. Hypnum microcarpoides C. Muell. (Mueller, 1879a).

Rhaphidostegium microcarpoides (C. Muell.) Kindb. (Kindberg, 1888). 
Distr. Southern South America (Mueller, 1879a; Paris, 1905b; Brotherus, 1925; Herter, 1933a; Kühnemann, 1938; Hosseus, 1939).

S. minutum Broth.

Rhaphidorrhynchium minutum

S. nanocephalum (C. Muell.) Broth. (Brotherus, 1925).

Syn. Aptychus nano-cephalus C. Muell. (Mueller, 1897a).

Rhaphidostegium nano-cephalum (C. Muell.) Par. (Paris, 1898).

Distr. Southern South America (Mueller, 1897a; Paris, 1898, 1905b; Brotherus, 1925; Kühnemann, 1938).

var. subglauculum (C. Muell.) Wijk et Marg. (van der Wijk and Margadant, 1959).

Syn. Aptychus nano-cephalus C. Muell. var. subglauculus C. Muell. (Mueller, 1897a).

Rhaphidostegium nano-cephalum (C. Muell.) Par. var. subglauculum (C. Muell.) Par. (Paris, 1898).

Distr. Southern South America (Mueller, 1897a; Paris, 1898, 1905b; Brotherus, 1925; Kühnemann, 1938).

S. noduliferum Mitt.

Rhaphidornynchium noduliferum

S. perichaetiale Thér. (Thériot in Felippone, 1929 [1930]).

Distr. Southern South America (Felippone, 1929; Herter, 1933a).

S. scorpiurus (Mont.) Mitt.

S. secundifolium (C. Muell.) Mitt.

Rhaphidorrhynchium scorpiurus

Drepanocladus secundifolius

S. serifolium (C. Muell.) Broth. (Brotherus, 1925).

Syn. Aptychus serifolius C. Muell. (Mueller, 1897a).

Rhaphidostegium serifolium (C. Muell.) Par. (Paris, 1898).

Distr. Southern South America (Mueller, 1897a; Paris, 1898, 1905b; Brotherus, 1925; Kühnemann, 1938).

S. stenopyxidium (C. Muell.) Broth. (Brotherus, 1925).

Syn. Hypnum stenopyxidium C. Muell. (Mueller, 1879a).

Rhaphidostegium stenopyxidium (C. Muell.) Kindb. (Kindberg, 1888).

Distr. Southern South America (Mueller, 1879a; Paris, 1905b; Brotherus, 1925; Herter, 1933a; Kühnemann, 1938; Hosseus, 1939).

S. subsimplex (Hedw.) Mitt. (Mitten, 1869).

Syn. Hypnum subsimplex Hedw. (Hedwig, 1801).

Isothecium subsimplex (Hedw.) Brid. (Bridel, 1827b).

Plagiothecium subsimplex (Hedw.) Besch. (Bescherelle, 1871-72 [1872]).

Rhaphidorrhynchium subsimplex (Hedw.) Broth. (Brotherus, 1925).

Rhaphidostegium subsimplex (Hedw.) Besch. (Bescherelle, 1876).

Hypnum simplex Brid. (Bridel, 1812) nom. illeg.

Distr. Southern South America (Bescherelle, 1889; Paris, 1905b; Cardot, 1908a).

S. temperatum (C. Muell.) Broth. (Brotherus, 1925).

Syn. Aptychus temperatus C. Muell. (Mueller, 1897a).

Rhaphidostegium temperatum (C. Muell.) Par. (Paris, 1898).

Distr. Southern South America (Mueller, 1897a; Paris, 1898, 1905b; Brotherus, 1925; Kühnemann, 1938).

S. tenerifolium (C. Muell.) Broth. (Brotherus, 1925).

Syn. Aptychus tenerifolius C. Muell. (Mueller, 1897a).

Rhaphidostegium tenerifolium (C. Muell.) Par. (Paris, 1898).

Distr. Southern South America (Mueller, 1897a; Paris, 1898, 1905b; Brotherus, 1925; Kühnemann, 1938).

S. tucumanense Bartr. (Bartram, 1965).

Distr. Southern South America (Bartram, 1965). 
Sigmatella hom. illeg.

S. microthamnioides C. Muell.

Taxithelium microthamnioides

Simophyllum nom. illeg.

S. viridulum Lindb.

Weisia controversa

Skitophyllum nom. illeg.

S. acacioides (Schrad.) Pyl.

Fissidens osmundoides

S. adianthoides (Hedw.) Pyl.

Fissidens adianthoides

S. asplenioides (Hedw.) Pyl.

Fissidens asplenioides

S. bryoides (Hedw.) Pyl.

Fissidens bryoides

S. dillenii Pyl.

Fissidens fontanus

S. fontanum Pyl.

Fissidens fontanus

S. julianum (Cand.) Brid.

Fissidens fontanus

S. osmundoides (Hedw.) Pyl.

Fissidens osmundoides

S. semi-completum (Hedw.) Pyl.

Fissidens semicompletus

S. taxifolium (Hedw.) Pyl.

Fissidens taxifolius

\section{Skottsbergia}

S. paradoxa Card. (Cardot, 1905b).

Distr. Southem South America (Roivainen and Bartram, 1937; Kühnemann, 1938).

South Georgia (Cardot, 1905a, 1906a, 1907b, 1908a; Brotherus, 1924a; Steere, 1961b).

Sphaerangium nom. illeg.

S. Iorentzii (C. Muell.) Par,

Astomum lorentzii

S. vesiculosum (C. Muell.) Kindb.

Acaulon vesiculosum

\section{Sphaerocephalus}

S. palustris (Hedw.) Lindb.

Aulacomnium palustre

\section{Sphagnum}

S. acutifolium Schrad. (Schrader, 1794).

Distr. Southern South Ȧmerica (Paris, 1905b (as forma diblastum (C. Muell.) Par.)).

Falkland Islands (Gaudichaud, 1825).

var. tenellum Schwaegr. (Schwaegrichen in Gaudichaud, 1826 [1828]) nom. nud.

Distr. Falkland Islands (Gaudichaud, 1826).

S. amblyphyllum (Russ.) Warnst. (Warnstorf, 1911).

Distr. Southern South America (Thériot, 1934b).

S. andinum Hamp. (Hampe, 1866).

Distr. Southem South America (Lorentz, 1866; Jaeger, 1870).

S. bicolor Besch. (Bescherelle, 1885a).

Distr. Southern South America (Mueller, 1885; Bescherelle, 1885a, 1889; Hariot, 1891).

S. capillaceum (Weiss) Schrank (Schrank, 1789).

Distr. Southern South America (Andrews, 1947).

S. chilense Lor. (Lorentz, 1866).

Distr. Southern South America (Lorentz, 1866).

S. cuspidatum Ehrh. ex Hoffm. (Ehrhardt ex Hoffmann, 1795 [1796]).

Distr. Southem South America Wilson and Hooker, 1847; Sullivant, 1859; Cardot, 1908a;

Warnstorf, 1911; Cardot and Brotherus, 1923; Thériot, 1934b; Andrews, 1949b).

Falkland Islands (Wilson and Hooker, 1847; Kühnemann, 1938). 
var. falcatulum (Besch.) Par. (Paris, 1897).

Distr. Southern South America (Paris, 1897, 1905b).

var. falcatum Russ. (Russow, 1867).

Distr. Southern South America (Dusén, 1903b; Paris, 1905b; Cardot, 1908a).

var. microporum (Warnst.) Par. (Paris, 1897).

Distr. Falkland Islands (Paris, 1897, 1905b).

var. miquelonense (Ren. et Card.) Ren. et Card. (Renauld and Cardot in Cardot, 1887).

Distr. Southern South America (Dusén, 1903b; Cardot, 1908a).

var. serratum (Aust.) Aust. (Austin, 1880).

Distr. Southern South America (Dusén, 1903b; Cardot 1908a).

var. submersum Schimp. (Schimper, 1858 [1857]).

Distr. Southern South America (Dusén, 1903b: Paris, 1905b).

S. cymbifolium (Ehrh.) Hedw. (Hedwig, 1782).

Distr. Southern South America (Sullivant, 1859; Mitten, 1869; Jaeger, 1870; Paris, 1905b; Spegazzini, 1922).

Falkland Islands (Bridel, 1827a; Wilson and Hooker, 1847).

var. condensatum Hook. f. (Hooker in Wilson and Hooker, 1847) hom. illeg.

Distr. Southern South America (Wilson and Hooker, 1847; Ångström. 1872; Paris, 1905b). var. magellanicum (Brid.) P. Beauv. (Palisot de Beauvois, 1805a).

Distr. Southern South America (Palisot de Beauvois, 1805a; Bridel, 1826).

var. paradisii Besch. (Bescherelle in Warnstorf, 1890a) nom. nud.

Distr. Southern South America (Warnstorf, 1890a).

S. diblastum C. Muell. (Mueller, 1887a).

Distr. Southern South America (Mueller, 1887a; Warnstorf, 1911; Brotherus, 1924a; Kühnemann, 1938).

S. dissimile Roiv. (Roivainen in Roivainen and Bartram, 1937).

Distr. Southern South America (Roivainen and Bartram, 1937).

S. dusenioides Roiv. (Roivainen in Roivainen and Bartram, 1937).

Distr. Southern South America (Roivainen and Bartram, 1937).

S. falcatulum Besch. (Bescherelle, 1885a).

Distr. Southern South America (Bescherelle, 1885a, 1889; Mueller, 1885; Hariot, 1891; Warnstorf, 1894, 1895, 1911; Cardot, 1905a, 1908a; Cardot and Brotherus, 1923; Brotherus, 1924a; Roivainen and Bartram, 1937; Kühnemann, 1938; Andrews, 1949b; Seki, 1974).

var. microporum Warnst. (Warnstorf, 1895).

Distr. Falkland Islands (Warnstorf, 1895, 1911; Cardot, 1908a; Kühnemann, 1938).

S. fimbriatum Wils. (Wilson in Wilson and Hooker, 1845).

Distr. Southern South America Milsson and Hooker, 1847; Sullivant, 1859; Mitten, 1869; Jaeger, 1870; Mueller, 18835; Cardot, 1900, 1901, 1905a, 1908a; Paris, 1905b; Warnstorf, 1911; Cardot and Brotherus, 1923; Brotherus, 1924a; Herzog, 1939; Andrews, 1947; Seki, 1974).

Falkland Islands (Wilson and Hooker, 1847; Jaeger, 1870; Mueller, 1885; Cardot, 1905a, 1908a; Paris, 1905b; Andrews, 1947).

South Georgia (Greene, 1964, 1968a).

var. molluscoides Card. (Cardot in Cardot and Brotherus, 1923).

Distr. Southern South America (Cardot and Brotherus, 1923; Kühnemann, 1938). 
var. robustum Braithw. ex Warnst. (Braithwaite ex Warnstorf, 1884a) nom. illeg.

Distr. Southem South America (Dusén, 1903a, $b$ (and as forma brachydasyclada and forma laxifolia), 1905d (as forma brachydasyclada); Paris, 1905b; Cardot, 1908a; Warnstorf, 1911; Cardot and Brotherus, 1923; Kühnemann, 1938; Herzog, 1939).

var. validus Card. (Cardot, 1884).

Distr. Southem South America (Warnstorf, 1911; Cardot and Brotherus, 1923; Brotherus, 1924a; Kühnemann, 1938).

Falkland Islands (Cardot, 1905a, 1908a; Cardot and Brotherus, 1923).

S. hahnianium C. Muell. (Mueller in Warnstorf, 1890a) nom. nud.

Distr. Southern South America (Warnstorf, 1890a).

S. imbricatum Hornsch. ex Russ. (Hornschuch ex Russow, 1867).

Distr. Southern South America (Wamstorf, 1911).

S. lechleri Warnst. (Warnstorf, 1907).

Distr. Southern South America (Warnstorf, 1907, 1911 (but see Andrews, 1947, p. 183).

S. magellanicum Brid. (Bridel, 1798).

Distr. Southem South America (Bridel, 1801 b, 1803, 1806; Roivainen and Bartram, 1937; Herzog, 1938, 1939; Kühnemann, 1938; Seki, 1974).

var. congestum (Schimp.) Roell (Röll, 1915).

Distr. Falkland Islands (Kühnemann, 1938).

var. versicolor (Warnst.) Wijk et Marg. (van der Wijk and Margadant, 1965).

Distr. Southem South America (Kühnemann, 1938).

S. medium Limpr. (Limpricht, 1881).

Distr. Southem South America Warnstorf, 1894, 1911 (as forma brachy-orthocladum Warnst.); Paris, 1905b; Cardot, 1908a; Brotherus, 1924a; Herter, 1933a, 1943; Martin, 1949).

var. congestum (Schimp.) Warnst. (Warnstorf, 1883).

Distr. Southern South America (Röll, 1886 (as forma bicolor Besch.); Cardot, 1905a, 1913a; Cardot and Brotherus, 1923).

Falkland Islands (Cardot and Brotherus, 1923).

var. fusco-rubellum Warnst. (Warnstorf in Dusén, 1903b) nom. nud.

Distr. Southem South America (Dusén, 1903a, b (as forma brachydasyclada); Kühnemann, 1938).

var. laeve (Russ.) Warnst. (Warnstorf, 1890b).

Distr. Southem South America Warnstorf, 1890b (as forma albescens Warnst.); Paris, 1905b).

var. pallido-carneum Warnst. (Warnstorf in Dusén, 1903b) nom. nud.

Distr. Southern South America (Dusén, 1903 b (as forma brachydasyclada).

var. purpurascens (Russ.) Warnst. (Warnstorf, 1884b).

Distr. Southern South America (Dusén, 1903b, 1905d (and as forma brachylomatoclada); Cardot and Brotherus, 1923; Kühnemann, 1938).

Falkland Islands (Cardot and Brotherus, 1923; Kühnemann, 1938).

var. roseum ((Roell) Warnst. (Warnstorf, 1888).

Distr. Southern South America (Cardot and Brotherus, 1923; Kühnemann, 1938).

var. violascens Warnst. (Warnstorf in Horrell, 1900).

Distr. Southerr, South America (Cardot and Brotherus, 1923; Kühnemann, 1938).

var. virescens Warnst. (Warnstorf, 1888).

Distr. Southern South America (Cardot and Brotherus, 1923).

S. nano-porosum Warnst. (Warnstorf, 1898).

Distr. Falkland Islands (Warnstorf, 1898, 1911; Cardot, 1905a, 1908a; Cardot and Brotherus, 1923; Brotherus, 1924a; Kühnemann, 1938). 
var. skottsbergii Card. (Cardot in Cardot and Brotherus, 1923).

Distr. Falkland Islands (Cardot and Brotherus, 1923; Kühnemann, 1938).

S. patagoniense Warnst. (Warnstorf, 1911).

Distr. Southern South America Warnstorf, 1911; Brotherus, 1924a; Herzog, 1939 (as S. patagonicum Warnst.)).

var. submersum Warnst. (Warnstorf, 1911).

Distr. Southern South America (Warnstorf, 1911).

S. personatum Roiv. (Roivainen in Roivainen and Bartram, 1937).

Distr. Southern South America (Roivainen and Bartram, 1937).

S. plumulosum Roell (Röll, 1886).

Distr. Southern South America (Warnstorf, 1911; Brotherus, 1924a; Kühnemann, 1938; Andrews, 1947).

S. pulchricoma C. Muell. (Mueller, 1848a).

Distr. Southern South America (Mitten, 1869; Jaeger, 1870; Herzog, 1938).

S. recurvum P. Beauv. (Palisot de Beauvois, 1805a).

Distr. Southern South America (Paris, 1905b; Cardot, 1908a; Andrews, 1949b).

S. rigescens Warnst. (Warnstorf, 1898).

Distr. Southern South America Warnstorf, 1898, 1911; Dusén, 1903a, b; Paris, 1905b; Cardot, 1908a; Brotherus, 1924a; Andrews, 1941).

S. schwabeanum Paul (Paul in Herzog, 1939).

Distr. Southern South America (Herzog, 1939).

S. squarrosum Crom. (Crome in Hoppe, 1801).

Distr. Southern South America (Hariot, 1891).

S. subfalcatulum Roiv. (Roivainen in Roivainen and Bartram, 1937).

Distr. Southern South America (Roivainen and Bartram, 1937; Kühnemann, 1938).

S. subnitens Russ. et Warnst. var. purpurascens Schlieph. ex C. Jens. (Schliephacke ex Jensen, 1890).

Distr. Southern South America (Dusén, 1903b; Paris, 1905b; Cardot, 1908a).

S. subrigidum Hamp. et Lor. (Hampe and Lorentz in Lorentz, 1868).

Distr. Southern South America (Paris, 1905b; Warnstorf, 1911; Brotherus, 1924a (but see Andrews, 1947)).

S. subserratum Roiv. (Roivainen in Roivainen and Bartram, 1937).

Distr. Southern South America (Roivainen and Bartram, 1937).

S. tenerum Sull. et Lesq. (Sullivant and Lesquereux, 1856).

Distr. Southern South America (Andrews, 1947).

S. teres (Schimp.) Aongstr. var. laxum Braithw. ex Warnst. (Braithwaite ex Warnstorf, 1881a).

Distr. Southern South America (Paris, 1905b).

S. torreyanum Sull. (Sullivant, 1849).

Distr. Southern South America (Warnstorf, 1911; Brotherus, 1924a).

S. trinitense C. Muell. (Mueller, 1848a).

Distr. Southern South America (Cardot and Brotherus, 1923).

Falkland Islands (Cardot and Brotherus, 1923; Kühnemann, 1938).

S. undulatum Warnst. (Warnstorf, 1894).

Distr. Southern South America (Warnstorf, 1894, 1895, 1911; Dusén, 1903b; Paris, 1905b; Cardot, 1908a; Brotherus, 1924a; Kühnemann, 1938). 


\section{Splachnobryum}

S. Iorentzii C. Muell. (Mueller, 1882).

Distr. Southern South America (Mueller, 1882; Paris, 1905b; Brotherus, 1924a; Hosseus, 1935b, 1937; Kühnemann, 1938).

S. valdiviae C. Muell. (Mueller, 1897c).

Distr. Southem South America (Mueller, 1897c; Paris, 1905b; Brotherus, 1924a).

\section{Splachnum}

S. magellanicum Brid.

Tayloria magellanica

S. mnioides Hedw.

Tetraplodon mnioides

S. octoblepharum Hook.

Tayloria octoblepharum

\section{Sporledera}

S. hampeana (C. Muell.) Mitt.

Bruchia hampeana

S. schwaegrichenii Hamp.

Bruchia hampeana

\section{Squamidium}

S. Iorentzii (C. Muell.) Broth. (Brotherus, 1906b).

Syn. Meteorium Iorentzii C. Muell. (Mueller, 1879a).

Distr. Southern South America (Mueller, 1879a; Paris, 1905a; Brotherus, 1906b, 1925; Kühnemann, 1938).

S. turgidulum (C. Muell.) Broth. (Brotherus, 1906b).

Syn. Neckera turgidula C. Muell. (Mueller, 1879a).

Pilotrichella turgidula (C. Muell.) Par. (Paris, 1897).

Distr. Southem South America (Mueller, 1879a; Paris, 1897, 1905b; Brotherus, 1906b, 1925; Kühnemann, 1938).

var. triste (C. Muell.) Wijk et Marg. (van der Wijk and Margadant, 1961).

Syn. Neckera turgidula C. Muell. var. tristis C. Muell. (Mueller, 1879a).

Pilotrichella turgidula (C. Muell.) Par. var. tristis (C. Muell.) Par. (Paris, 1897).

Distr. Southem South America (Mueller, 1879a; Paris, 1897, 1905b).

\section{Stableria}

S. tenella (Mitt.) Broth.

\section{Orthodontium gracile}

\section{Stegonia}

S. guessfeldtii (Schliep.) Hag.

Pottia heimii var. guessfeldtii

S. latifolia (Schwaegr.) Vent. ex Broth. (Brotherus, 1923).

Syn. Weisia latifolia Schwaegr. (Schwaegrichen, 1804).

Distr. Antarctic, peninsula region (Smith, 1972, p. 31).

S. lorentzii (C. Muell.) Hag. (Hagen in Hagen and Printz, 1929).

Syn. Pottia lorentzii C. Muell. (Mueller, 1879a).

Distr. Southern South America (Mueller, 1879a; Paris, 1905b; Warnstorf, 1916; Brotherus, 1924a; Kühnemann, 1938).

\section{Stenocarpidium}

S. leucodon C. Muell. (Mueller, 1897a).

Distr. Southem South America (Mueller, 1897a; Paris, 1905b; Brotherus, 1925; Hosseus, $1935 b, 1938 c, d$; Kühnemann, 1938).

\section{Stenomitrium}

S. aureum Par. (Paris, 1905b) nom. nud. 
Syn. Pentastichella aurea Dus. (Dusén, 1903a) nom. nud.

Distr. Southern South America (Dusén, 1903a; Paris, 1905b).

S. pentastichum (Mont.) Broth.

Zygodon pentastichus

S. robustulum Par.

Zygodon bartramioides

Stereodon nom. illeg.

S. acicularis (Brid.) Mitt.

Ptychomnion aciculare

S. auriculatus (Mont.) Mitt.

Acrocladium auriculatum

S. campoanus Thér.

Hypnum campoanum

S. cochlearifolius (Schwaegr.) Mitt.

S. cupressiformis (Hedw.) Brid. ex Mitt.

Weymouthia cochlearifolia

S. densifolius (Brid.) Mitt.

Hypnum cupressiforme

S. denticulatus (Hedw.) Mitt.

Ptychomnion densifolium

S. gracilis (Hook. f. et Wils.) Mitt.

Plagiothecium denticulatum

S. gracilis Dus.

Camptochaete gracilis

Hypnum gracile

S. lagurus (Hook.) Mitt.

Lepyrodon lagurus

S. lechleri (C. Muell.) Mitt.

Hypnum chrysogaster

S. mollis (Hedw.) Mitt.

Weymouthia mollis

S. nitidus (Hook. f. et Wils.) Mitt.

Catagonium nitidum

S. pallens Broth.

S. pseudo-pallens Dus. (Dusén in Naveau, 1928) nom. nud.

Hypnum skottsbergii

Distr. Southern South America (Naveau, 1928).

S. ptychocarpum (Schwaegr.) Mitt.

S. revolutus Mitt.

Ptychomnion ptychocarpum Hypnum revolutum

S. spegazzinii (C. Muell.) Broth. Hypnum spegazzinii

S. varius (Hedw.) Mitt.

Amblystegium varium

\section{Stereohypnum}

S. humile (Besch.) Herz.

S. tapes (C. Muell.) Fleisch.

\section{Mittenothamnium humile \\ Mittenothamnium tapes}

\section{Stereophyllum}

S. aptychopsis C. Muell. (Mueller, 1897a).

Distr. Southern South America (Mueller, 1897a; Paris, 1905b; Brotherus, 1925; Kühnemann, 1938; Herzog, 1952a).

S. argentinicum Broth. (Brotherus, 1918).

Distr. Southern South America (Brotherus, 1918, 1925; Kühnemann, 1938).

S. brevipes (C. Muell.) Mitt. (Mitten, 1869).

Syn. Hypnum brevipes C. Muell. (Mueller, 1851a).

Euglossophyllum brevipes (C. Muell.) Hamp. (Hampe, 1870) comb. inval.

Glossophyllum brevipes (C. Muell.) Hamp. (Hampe, 1879).

Distr. Southern South America (Brotherus, 1918; Kühnemann, 1938).

S. fuegianum Besch.

Brachythecium fuegianum

S. homalioides Besch. (Bescherelle, 1891).

Distr. Southern South America (Herzog, 1952a).

var. bellirete Herz. (Herzog, 1952a).

Distr. Southern South America (Herzog, 1952a). 
S. krausei (C. Muell.) Broth.

Juratzkaea krausei

S. lepidopiloides (C. Muell.) Wijk et Marg. (van der Wijk and Margadant, 1959).

Syn. Hypnum lepidopiloides C. Muell. (Mueller, 1879a).

Brachythecium lepidopiloides (C. Muell.) Kindb. (Kindberg, 1888).

Distr. Southem South America (Mueller, 1879a; Kurtz, 1904; Paris, 1904a; Hosseus, 1935a, 1937).

S. leucostegum (Brid.) Mitt. (Mitten, 1869).

Syn. Leskea leucostega Brid. (Bridel, 1827b).

Hypnum leucostegum (Brid.) C. Muell. (Mueller, 1851a).

Rhyncho-hypnum leucostegum (Brid.) Hamp. (Hampe, 1870) comb. inval.

Distr. Southem South America (Brotherus, 1918, 1925; Kühnemann, 1938; Herzog, 1952a).

S. seminerve (Schwaegr.) Mitt.

Juratzkaea seminenvis

\section{Streblotrichum}

S. gracile Par.

Tortella humilis

S. humile (Hedw.) P. Beauv.

Tortella humilis

S. piliferum (Hook.) Hilp.

Tortula pilifera

S. unguiculatum (Hedw.) Loesk.

Barbula unguiculata

\section{Streptocalypta}

S. fernandeziana Card. (Cardot in Skottsberg, 1914) nom. nud.

Distr. Southern South America (Skottsberg, 1914).

S. Iorentziana C. Muell. (Mueller, 1879a).

Syn. Streptocalypta:lorentzii C. Muell. ex Kindb. (Mueller ex Kindberg, 1889) nom. illeg.

Distr. Southern South America (Mueller, 1879a; Paris, 1905b; Brotherus, 1924a; Hilpert, 1933; Kühnemann, 1938).

S. Iorentzii C. Muell. ex Kindb.

Streptocalypta lorentziana

\section{Streptopogon}

S. crispatulus (C. Muell.) Par.

Calyptopogon mnioides

S. heterophyllus Herz. (Herzog, 1916a).

Distr. Southern South America (Herzog, 1952a).

S. mnioides (Schwaegr.) Mitt.

Calyptopogon mnioides

var. prostratus (Mont.) Jaeg.

Tortula prostrata

\section{Symblepharis}

S. pumila Mitt.

Holodontium pumilum

\section{Syntrichia}

S. anderssonii (Aongstr.) Herz.

Tortula anderssonii

S. apiculato-pilosa Dus.

Tortula apiculato-pilosa

S. arenae (Besch.) Herz.

Tortula arenae

S. brachychaete Dus.

Tortula brachychaete

S. brevicaulis Dus.

Tortula brevicaulis

S. costesii (Thér.) Herz.

Tortula costesii

S. crispatula C. Muell.

Calyptopogon mnioides

S. ericetorum (With.) Brid. 
S. flavido-pilosa Dus.

S. fuegiana Mitt.

S. hyperborea Brid.

S. intermedia Brid.

S. laevipila Brid.

S. lepto-syntrichia C. Muell.

S. levipila Brid. ex Moenk.

S. lingulaefolia (Card. et Broth.) Herz.

S. marginato-serrata Dus.

S. micro-runcinata Dus.

S. mniadelphus (C. Muell.) Herz. var. cochlearifolia Herz.

S. muelleri Bruch

S. muralis (Hedw.) Raab

S. papillosa (Wils.) Jur.

S. perichaetialis Herz. (Herzog, 1957) nom. nud.

Distr. Southern South America (Herzog, 1957).

S. perrubiginosa (Dus.) Par.

S. princeps (De Not.) Mitt.

S. prostrata (Mont.) Herz.

S. pseudo-robusta (Dus.) Herz.

S. robusta Dus.

S. robusta (Hook. et Grev.) Herz.

S. rubripungeus Broth. (Brotherus in Neger, 1899) nom. nud.

Distr. Southern South America (Neger, 1899).

S. runcinata C. Muell.

S. ruralis (Hedw.) Web. et Mohr var. laevipila (Brid.) Spreng.

S. scabrella (Dus.) Herz.

S. scabrinervis (C. Muell.) Herz.

S. serripungens (Lor. et C. Muell.) Herz.

S. squarripila (Thér.) Herz.
Tortula flavido-pilosa

Tortula fuegiana

Tortula mucronifolia var. arctica

Tortula intermedia

Tortula laevipila

Tortula lepto-syntrichia

Tortula laevipila

Tortula lingulaefolia

Tortula marginato-serrata

Leptodontium longicaule var. microruncinatum

Tortula mniadelphus

Tortula mniadelphus var. cochlearifolia

Tortula princeps

Tortula muralis

Tortula papillosa

Tortula anderssonii

Tortula princeps

Tortula prostrata

Tortula pseudo-robusta

Tortula pseudo-robusta

Tortula robusta

\section{Syrrhopodon}

S. argentinicus Lor. ex C. Muell. (Lorentz ex Mueller, 1879a).

Distr. Southern South America (Mueller, 1879a; Paris, 1905b; Brotherus, 1924a; Kühnemann, 1938).

S. berteroanus (Brid.) C. Muell.

Tortula robusta var. runcinata

Tortula ruralis

Tortula laevipila

Tortula scabrella

Tortula scabrinervis

Tortula serripungens

Tortula squarripila

S. berteroi Kindb.

Syrrhopodon incompletus var, berteroanus

S. decolorans C. Muell.

Syrrhopodon incompletus var. berteroanus

S. fasciculatus Hook. et Grev.

Syrrhopodon incompletus

Thyridium fasciculatum

S. incompletus Schwaegr. (Schwaegrichen, 1824).

Syn. Syrrhopodon decolorans C. Muell. (Mueller, 1897c).

Distr. Southern South America (Hosseus, 1940).

var. berteroanus (Brid.) Reese (Reese, 1977). 
Syn. Dicranum berteroanum Brid. (Bridel, 1826).

Orthotheca berteroana (Brid.) Brid. (Bridel, 1827a).

Syrrhopodon berteroanus (Brid.) C. Muell. (Mueller, 1849).

Syrrhopodon berteroi Kindb. (Kindberg, 1888) nom. illeg.

Syrrhopodon malouinensis C. Muell. (Mueller, 1849) nom. illeg.

Distr. Falkland Islands (Bridel, 1827a; Mueller, 1849; Jaeger, 1873; Paris, 1905b; Cardot, 1908a; Kühnemann, 1938).

S. malouinensis C. Muell.

Syrrhopodon incompletus var. berteroanus

S. reinwardtii Hornsch.

Zygodon reinwardtii

S. rigescens Schwaegr. (Schwaegrichen, 1827a).

Distr. Falkland islands (Schwaegrichen, 1827a; Mueller, 1849; Jaeger, 1873; Paris, 1905b; Cardot, 1908a; Kühnemann, 1938).

S. tortuosus Hornsch.

Amphidium tortuosum

Systegium hom. illeg.

S. fruchartii (C. Muell.) Kindb.

Astomum fruchartii

S. recurvirostre (C. Muell.) Kindb.

Tetrapterum recurvirostre

\section{Tamariscella}

T. pseudo-aequatorialis C. Muell.

Thuidium peruvianum

var. casuarina C. Muell.

Thuidium pseudo-aequatoriale var. casuarinum

\section{Taxicaulis}

T. adflatus C. Muell.

Isopterygium adflatum

T. byssobolax C. Muell.

Isopterygium byssobolax

T. pyrrhopus C. Muell.

Isopterygium pyrrhopus

T. saprophilus C. Muell.

Isopterygium saprophilum

\section{Taxithelium}

T. microthamnioides (C. Muell.) Par. (Paris, 1898).

Syn. Sigmatella microthamnioides C. Muell. (Mueller, 1897a).

Distr. Southern South America (Mueller, 1897a; Paris, 1898, 1905b).

\section{Tayloria}

T. dubyi Broth. (Brotherus, 1903).

Syn. Hymenocleiston magellanicum Dub. (Duby, 1875b).

Dissodon dubyanus Besch. (Bescherelle in Brotherus, 1924a) nom. nud.

Distr. Southern South America (Duby, 1875a, b; Jaeger and Sauerbeck, 1879b; Mueller, 1885; Bescherelle, 1885a, 1889; Brotherus, 1903, 1924a; Cardot, 1905a, 1908a, b; Paris, 1905b; Cardot and Brotherus, 1923; Kühnemann, 1938; Bartram, 1952; Seki, 1974).

var. edenensis (Besch.) Par. (Paris, 1905b).

Syn. Hymenocleiston magellanicum Dub. var. edenense Besch. (Bescherelle, 1885a).

Distr. Southern South America (Bescherelle, 1885a, 1889; Paris, 1905b; Cardot, 1908a).

T. magellanica (Brid.) Mitt. (Mitten, 1869).

Syn. Splachnum magellanicum Brid. (Bridel, 1802).

Eremodon magellanicus (Brid.) Brid. (Bridel, 1826).

Dissodon magellanicus (Brid.) Hamp. (Hampe in Mueller, 1851a).

Distr. Southern South America (Bridel, 1803, 1806, 1826; Schwaegrichen, 1811; Mueller, 1848a, 1851b, 1885; Montagne, 1850; Mitten, 1869; Ångström, 1872; Jaeger, 1874; Bescherelle, 1889; Cardot, 1900, 1901, 1908a; Paris, 1904b; Cardot and Brotherus, 1923; Brotherus, 1924a; Kühnemann, 1938; Seki, 1974).

var. gigantea Bartr. (Bartram, 1946).

Distr. Southern South America (Bartram, 1946). 
T. mirabilis (Card.) Broth. (Brotherus, 1903).

Syn. Dissodon mirabilis Card. (Cardot, 1900).

Distr. Southern South America (Cardot, 1900, 1901, 1908a; Brotherus, 1903, 1924a; Paris, 1904b; Cardot and Brotherus, 1923; Kühnemann, 1938; Bartram, 1952).

T. octoblepharum (Hook.) Mitt. (Mitten, 1883).

Syn. Splachnum octoblepharum Hook. (Hooker, 1819).

Dissodon plagiopus (Mont.) C. Muell. (Mueller, 1851a).

Distr. Southern South America (Ångström, 1872).

T. patagonica Dus. (Dusén in Paris, 1905b) nom. nud.

Distr. Southern South America (Paris, 1905b).

T. spathulata (Hook. f. et Wils.) C. Muell. (Mueller, 1848a).

Syn. Eremodon spathulatus Hook. f. et Wils. (Hooker and Wilson in Wilson, 1846).

Distr. Southern South America (Wilson, 1846; Paris, 1905b).

\section{Tetraplodon}

T. fuegianus Besch. (Bescherelle, 1885a).

Distr. Southern South America (Bescherelle, 1885a, 1889; Paris, 1905b; Cardot, 1908a; Brotherus, 1924a; Seki, 1974).

T. mnioides (Hedw.) B.S.G. (Bruch and others, 1844).

Syn. Splachnum mnioides Hedw. (Hedwig, 1801).

Distr. Southern South America (Robinson, 1964).

T. stenophysatus Herz. (Herzog, 1939).

Distr. Southern South America (Herzog, 1939).

\section{Tetrapterum}

T. lamprocarpum (C. Muell.) Broth. (Brotherus, 1924a).

Syn. Phascum lamprocarpum C. Muell. (Mueller, 1879a). Pottia lamprocarpa (C. Muell.) Broth. (Brotherus, 1902).

Distr. Southern South America (Mueller, 1879a; Brotherus, 1902, 1924a; Roth, 1911; Kühnemann, 1938).

T. lamprothecium (C. Muell.) Broth. (Brotherus, 1924a).

Syn. Phascum lamprothecium C. Muell. (Mueller, 1879a).

Pottia lamprothecium (C. Muell.) Broth. (Brotherus, 1902).

Distr. Southern South America (Mueller, 1879a; Brotherus, 1902, 1924a; Roth, 1911; Kühnemann, 1938).

T. recurvirostre (C. Muell.) Broth. (Brotherus, 1924a).

Syn. Phascum recurvirostre C. Muell. (Mueller, 1888). Astomium recurvirostre (C. Muell.) Roth (Roth, 1911). Systegium recurvirostre (C. Muell.) Kindb. (Kindberg, 1889). Astomum chilense Williams (Williams, 1915).

Distr. Southern South America (Williams, 1915; Thériot, 1921a).

\section{Thamnium}

T. angustifolium Schimp.

T. arbusculans (C. Muell.) Jaeg.

Porothamnium panduraefolium

var. complanatum C. Muell.

Porothamnium arbusculans

T. assimile Broth.

Porothamnium arbusculans

T. caroli Broth.

Thamnobryum rigidum

Thamnobryum carolii 
T. confertum (Mitt.) Par.

Thamnobryum confertum

T. corralense Broth.

Porothamnium arbusculans

$T$. crassinervium Broth.

Thamnobryum rigidum

T. decumbens Besch.

Porothamnium arbusculans

T. explanatum (Mitt.) Kindb.

Porothamnium explanatum

T. fasciculatum (Hedw.) C. Muell.

Porothamnium fasciculatum

$T$. ingae Broth.

Thamnobryum ingae

T. latinerve (Mitt.) Broth.

Thamnium rigidum

T. leucocaulon (C. Muell.) Kindb.

Porothamnium leucocaulon

T. mattogrossense Broth.

Porothamnium mattogrossense

T. naumannii (C. Muell.) Kindb.

Porothamnium arbusculans

T. neckera Schimp.

Porothamnium valdiviae

T. neckeraeforme (Hamp.) Kindb.

Porothamnium neckeraeforme

T. neckeroides (Hook.) Jaeg. (Jaeger, 1877).

Syn. Hypnum neckeroides Hook. (Hooker, 1818). Isothecium neckeroides (Hook.) Brid. (Bridel, 1827b).

Porotrichum neckeroides (Hook.) Williams (Williams, 1901).

Distr. Juan Fernandez (Johow, 1896).

Southem South America (Montagne, 1850; Paris, 1905b; Brotherus, 1925).

T. panduraefolium (C. Muell.) Broth.

Porothamnium panduraefolium

T. platycladum Card. (Cardot in Skottsberg, 1914) nom. nud.

Distr. Juan Fernandez (Skottsberg, 1914).

T. porrectulum (C. Muell.) Broth.

Porothamnium porrectulum

T. proboscideum Broth.

Thamnobryum proboscideum

T. pumilum (Hook. f. et Wils.) Kindb.

Thamnobryum pumilum

T. rigidum (Mitt.) Broth.

Thamnobryum rigidum

T. thielei Kindb.

Pinnatella thieleana

T. valdiviae (C. Muell.) Kindb.

Porothamnium valdiviae

T. valdivicum C. Muell.

Porothamnium valdiviae

\section{Thamnobryum}

T. carolii (Broth.) H. Robinson (Robinson, 1974).

Syn. Thamnium caroli Broth. (Brotherus, 1924b).

Distr. Juan Fernandez (Brotherus, 1924b, 1925; Espinosa B., 1941; Bartram, 1959; Robinson, 1974, 1975).

T. confertum (Mitt.) H. Robinson (Robinson, 1974).

Syn. Porotrichum confertum Mitt. (Mitten, 1885). Thamnium confertum (Mitt.) Par. (Paris, 1898).

Distr. Juan Fernandez (Mitten, 1885; Johow, 1896; Paris, 1898, 1905b; Brotherus, 1924b; Robinson, 1974, 1975).

T. ingae (Broth.) H. Robinson (Robinson, 1974).

Syn. Thamnium ingae Broth. (Brotherus, 1924b).

Distr. Juan Fernandez (Brotherus, 1924b, 1925; Thériot, 1927b; Espinosa B., 1941; Bartram, 1959; Robinson, 1974, 1975).

T. pumilum (Hook. f. et Wils.) Nieuwl. (Nieuwland, 1917).

Syn. Isothecium pumilum Hook. f. et Wils. (Hooker and Wilson in Wilson, 1859). 
Microthamnium pumilum (Hook. f. et Wils.) Jaeg. (Jaeger, 1878).

Thamnium pumilum (Hook. f. et Wils.) Kindb. (Kindberg, 1891).

Distr. Juan Fernandez (Kindberg, 1902).

T. proboscideum (Broth.) H. Robinson (Robinson, 1974).

Syn. Thamnium proboscideum Broth. (Brotherus, 1924b).

Distr. Juan Fernandez (Brotherus, 1924b, 1925; Bartram, 1959; Robinson, 1974, 1975).

T. rigidum (Mitt.) H. Robinson (Robinson, 1974).

Syn. Porotrichum rigidum Mitt. (Mitten, 1869).

Thamnium rigidum (Mitt.) Broth. (Brotherus, 1906).

Porotrichum latinerve Mitt. (Mitten, 1885).

Thamnium latinerve (Mitt.) Broth. (Brotherus, 1924b).

Thamnium crassinervium Broth. (Brotherus, 1906).

Porotrichum crassinervum Mitt. ex Broth. (Mitten ex Brotherus, 1924b) nom. inval.

Thamnium assimile Broth. (Brotherus, 1924b).

Distr. Juan Fernandez (Mitten, 1869, 1885; Jaeger, 1877; Johow, 1896; Brotherus, 1906, 1924b, 1925; Skottsberg, 1914; Thériot, 1921b; Espinosa B., 1941; Bartram, 1959; Robinson, 1974, 1975).

\section{Thuidiopsis}

T. chilensis (Mitt.) Broth. (Brotherus, 1925).

Syn. Thuidium chilense Mitt. (Mitten, 1869). Distr. Southern South America (Mitten, 1869; Jaeger, 1878; Paris, 1906; Herzog, 1923, 1954;
Brotherus, 1925).

T. corralensis (Broth.) Herz.

Thuidiopsis furfurosa

T. dusenii (Broth.) Broth. (Brotherus, 1925).

Syn. Thuidium dusenii Broth. (Brotherus in Cardot and Brotherus, 1923).

Distr. Southern South America (Cardot and Brotherus, 1923; Brotherus, 1925; Seki, 1974).

T. filaria (Mitt.) Broth.

Thuidiopsis furfurosa

T. furfurosa (Hook. f. et Wils.) Fleisch. (Fleischer, 1923).

Syn. Hypnum furfurosum Hook. f. et Wils. (Hooker and Wilson in Wilson, 1854).

Thuidium furfurosum (Hook. f. et Wils.) Reichdt. (Reichardt, 1870).

Thuidium filarium Mitt. (Mitten, 1869).

Cyrto-Hypnum filarium (Mitt.) Hamp. (Hampe, 1878).

Hypnum filarium (Mitt.) Hamp. (Hampe, 1879).

Thuidium filare Dus. (Dusén in Paris, 1906) nom. nud.

Thuidiopsis filaria (Mitt.) Broth. (Brotherus, 1925).

Hypnum unguiculatum Hook. f. et Wils. (Hooker and Wilson in Wilson, 1859).

Thuidium unguiculatum (Hook. f. et Wils.) Fleisch. (Fleischer, 1923).

Thuidiopsis unguiculata (Hook. f. et Wils.) Fleisch. (Fleischer, 1923).

Thuidium corralense Broth. (Brotherus in Cardot and Brotherus, 1923).

Thuidiopsis corralensis (Broth.) Herz. (Herzog, 1939).

Thuidium valdiviae Broth. (Brotherus, 1924b) fide Robinson, 1975.

Thuidiopsis valdiviae (Broth.) Broth. (Brotherus, 1925).

Distr. Juan Fernandez (Skottsberg, 1914 (as Thuidium valdiviense); Brotherus, 1924b, 1925; Bartram, 1959; Robinson, 1975).

Southern South America (Dusén, 1903a; Paris, 1906; Thériot, 1918; Cardot and Brotherus, 1923; Brotherus, 1925; Reimers, 1926; Dixon, 1929b; Herzog, 1938, 1939, 1954, 1960; Hosseus, 1940; Bartram, 1952; Bizot, 1967; Seki, 1974).

T. masafuerae (Broth.) Broth. (Brotherus, 1925).

Syn. Thuidium masafuerae Broth. (Brotherus, 1924b).

Distr. Juan Fernandez (Brotherus, 1924b, 1925; Espinosa B., 1941; Robinson, 1975).

T. unguiculata (Hook. f. et Wils.) Fleisch.

Thuidiopsis furfurosa

T. valdiviae (Broth.) Broth.

Thuidiopsis furfurosa 


\section{Thuidium}

T. amblyostomum (C. Muell.) Kindb. Haplocladium microphyllum var. attenuatifolium (C. Muell.) Par. Haplocladium microphyllum var. paludicola (C. Muell.) Par. Haplocladium microphyllum

T. austro-serpens (C. Muell.) Kindb.

Haplocladium microphyllum

T. brachypyxis C. Muell. (Mueller, 1897a).

Syn. Thuidium lorentzii Par. (Paris, 1898) nom. nud.

Distr. Southern South America (Mueller, 1897a; Paris, 1898, 1906; Brotherus, 1925; Kühnemann, 1938).

T. chacoanum C. Muell. (Mueller, 1897a).

Distr. Southem South America (Mueller, 1897a; Paris, 1906; Brotherus, 1925; Kühnemann, 1938).

T. chilense Mitt.

Thuidiopsis chilensis

T. condensatifolium Dus. (Dusén in Paris, 1906) nom. nud.

Distr. Southern South America (Paris, 1906).

T. corralense Broth.

Thuidiopsis furfurosa

T. cylindrella C. Muell. (Mueller, 1897a).

Distr. Southern South America (Mueller, 1897a; Paris, 1906; Kühnemann, 1938).

T. dusenii Broth.

Thuidiopsis dusenii

T. exasperatum Mitt. (Mitten, 1869).

Distr. Southern South America (Herzog, 1952a).

T. filare Dus.

Thuidiopsis furfurosa

T. filarium Mitt.

Thuidiopsis furfurosa

T. firmulum C. Muell.

Rauiella firmula

T. furfurosum (Hook. f. et Wils.) Reichdt.

Thuidiopsis furfurosa

T. inconspicuum Herz. (Herzog, 1923).

Distr. Southern South America (Herzog, 1923).

T. laterale Par.

T. laterculi (C. Muell.) Kindb.

Haplocladium microphyllum Haplocladium microphyllum

T. ligulifolium Herz. (Herzog, 1916b).

Distr. Southern South America (Herzog, 1916b).

T. lorentzii Par.

Thuidium brachypyxis

T. masafuerae Broth.

Thuidiopsis masafuerae

T. microphyllum (Hedw.) Jaeg.

T. niveo-calycinum C. Muell.

Rauiella niveo-calycina

T. occultirete C. Muell. (Mueller, 1897a).

Distr. Southern South America (Mueller, 1897a; Paris, 1906; Kühnemann, 1938).

T. persistens (C. Muell.) Kindb.

Haplocladium persistens

T. peruvianum Mitt. (Mitten, 1869).

Syn. Tamariscella pseudo-aequatorialis C. Muell. (Mueller, 1897a). Thuidium pseudo-aequatoriale (C. Muell.) Par. (Paris, 1898).

Distr. Southem South America (Mueller, 1897a; Paris, 1898, 1906; Kühnemann, 1938).

T. pseudo-aequatoriale (C. Muell.) Par. 
var. casuarinum (C. Muell.) Par. (Paris, 1898).

Syn. Tamariscella pseudo-aequatorialis C. Muell. var. casuarina C. Muell. (Mueller, 1897a).

Distr. Southern South America (Mueller, 1897a; Paris, 1898, 1906; Brotherus, 1925).

T. pseudo-gracile (C. Muell.) Par.

Haplocladium microphyllum

T. pseudo-recognitum (Hamp.) Kindb.

Thuidium urceolatum

T. pulvinatulum C. Muell. (Mueller, 1897a).

Distr. Southern South America (Mueller, 1897a; Paris, 1906; Brotherus, 1925; Herter, 1933a; Hosseus, 1939).

T. raphidostegum (C. Muell.) Mitt. (Mitten, 1869).

Syn. Hypnum raphidostegum C. Muell. (Mueller, 1851a).

Hypnum rhaphidostegum C. Muell. ex Par. (Mueller ex Paris, 1895) nom. inval.

Thuidium rhaphidostegium Mitt. ex Par. (Mitten ex Paris, 1898) nom. inval.

Distr. Southern South America (Mueller, 1851a; Mitten, 1869; Jaeger, 1878; Paris, 1898, 1906; Brotherus, 1925; Herzog, 1938).

T. recognitum (Hedw.) Lindb. var. delicatulum (Hedw.) Warnst. (Warnstorf, 1881b).

Syn. Hypnum delicatulum Hedw. (Hedwig, 1801).

Hypnum tamariscinum Hedw. var. delicatulum (Hedw.) Brid. (Bridel, 1812).

Distr. Southern South America (Montagne, 1850).

T. remotifolium (Grev.) Ren. et Card.

T. rhaphidostegium Mitt. ex Par.

Oxyrrhynchium clinocarpum

T. semi-lunare C. Muell.

Thuidium raphidostegum

T. tamariscinum (Hedw.) B.S.G. (Bruch and others, 1852).

Haplocladium microphyllum

Syn. Hypnum tamariscinum Hedw. (Hedwig, 1801).

Distr. Southern South America (Montagne, 1850).

T. torskii Kiaer ex C. Muell.

T. unguiculatum (Hook. f. et Wils.) Fleisch.

Haplocladium microphyllum

Thuidiopsis furfurosa

T. urceolatum Lor. (Lorentz, 1864).

Syn. Thuidium pseudo-recognitum (Hamp.) Kindb. (Kindberg, 1891).

Distr. Southern South America (Herzog, 1952a).

T. valdiviae Broth.

Thuidiopsis furfurosa

\section{Thyridium}

T. fasciculatum (Hook. et Grev.) Mitt. (Mitten, 1868).

Syn. Syrrhopodon fasciculatus Hook. et Grev. (Hooker and Greville, 1825b). Calymperes fasciculatum (Hook. et Grev.) Mitt. (Mitten, 1859) hom. illeg.

Distr. Southern South America (Mitten, 1859, 1869; Jaeger, 1873; Paris, 1905b).

\section{Thysanomitrion}

T. gracile (Hook.) Arnott

var. robustum Arnott

T. griseum Hornsch.

T. introflexum (Hedw.) Arnott

$T$. laetevirens Broth.

T. leptodus (Mont.) Broth.

T. richardii (Brid.) Schwaegr.

T. scabrisetum Hamp.

T. stenocarpum Hamp.
Pilopogon gracilis

Pilopogon gracilis

Campylopus griseus

Campylopus introflexus

Campylopus laetevirens

Campylopus clavatus

Campylopus richardii

Campylopus arctocarpus

Campylopus stenocarpus 


\section{Timmiella}

T. argentinica Broth. (Brotherus, 1918).

Distr. Southem South America (Brotherus, 1918, 1924a; Hosseus, 1936, 1937, 1938a, b, c, d, 1939; Thériot, 1936; Kühnemann, 1938).

T. umbrosa (C. Muell.) Broth. (Brotherus, 1902).

Syn. Trichostomum umbrosum C. Muell. (Mueller, 1879a).

Distr. Southem South America (Mueller, 1879a; Brotherus, 1902, 1924a; Kurtz, 1904; Paris, 1906; Williams, 1930; Hosseus, 1935a, b, 1937; Kühnemann, 1938).

\section{Tortella}

T. acaulon (C. Muell.) Broth. (Brotherus, 1902).

Syn. Trichostomum acaulon C. Muell. (Mueller, 1879a).

Distr. Southern South America (Mueller, 1879a; Brotherus, 1902, 1924a; Kurtz, 1904; Paris, 1906; Hosseus, 1935b, 1937; Kühnemann, 1938).

T. brachydontia (Bruch) C. Jens.

Trichostomum brachydontium

T. caespitosa (Schwaegr.) Limpr.

Tortella humilis

T. calycina (Schwaegr.) Dix. (Dixon, 1923).

Syn. Barbula calycina Schwaegr. (Schwaegrichen, 1823).

Tortula calycina (Schwaegr.) Hook. et Grev. (Hooker and Greville, 1824b).

Distr. Southern South America (Mitten, 1869; Jaeger, 1873; Paris, 1904a; Brotherus, 1925).

T. hosseusii Herz. (Herzog, 1938).

Distr. Southern South America (Herzog, 1938).

T. humilis (Hedw.) Jenn. (Jennings, 1913).

Syn. Barbula humilis Hedw. (Hedwig, 1801).

Tortula humilis (Hedw.) Turn. (Turner, 1804a).

Streblotrichum humile (Hedw.) P. Beauv. (Palisot de Beauvois, 1805a).

Mollia humilis (Hedw.) Lindb. (Lindberg, 1883).

Trichostomum humile (Hedw.) Mach. (Machado, 1919).

Barbula caespitosa Schwaegr. (Schwaegrichen, 1811).

Tortula caespitosa (Schwaegr.) Hook. et Grev. (Hooker and Greville, 1824b).

Tortella caespitosa (Schwaegr.) Limpr. (Limpricht, 1888).

Streblotrichum gracile Par. (Paris, 1905b) err.

Distr. Southern South America (Brotherus, 1918; Herter, 1933a, 1939b; Hosseus, 1938a, b, $d$, 1940; Kühnemann, 1938; Herzog, 1952a; Bizot and Piovano, 1953; Piovano, 1954).

T. perrufula (C. Muell.) Broth. (Brotherus, 1902).

Syn. Barbula perrufula C. Muell. (Mueller, 1897a).

Tortula perrufula (C. Muell.) Williams (Williams, 1930).

Distr. Southern South America (Mueller, 1897a; Paris, 1900a, 1906; Brotherus, 1902, 1924a; Herter, 1933a; Kühnemann, 1938).

T. pseudo-caespitosa (C. Muell.) Broth. (Brotherus, 1902).

Syn. Barbula pseudo-caespitosa C. Muell. (Mueller, 1879a).

Distr. Southern South America (Mueller, 1879a; Brotherus, 1902, 1924a; Kurtz, 1904; Paris, 1906; Hosseus, 1935b, 1937; Kühnemann, 1938).

var. brachybasis (C. Muell.) Par. (Paris, 1904a).

Syn. Barbula pseudo-caespitosa C. Muell. var. brachybasis C. Muell. (Mueller, 1879a).

Distr. Southern South America (Mueller, 1879a).

var. pungens (C. Muell.) Par. (Paris, 1904a).

Syn. Barbula pseudo-caespitosa C. Muell. var. pungens C. Muell. (Mueller, 1879a).

Distr. Southern South America (Mueller, 1879a). 
T. tortuosa (Hedw.) Limpr. (Limpricht, 1888).

Syn. Tortula tortuosa Hedw. (Hedwig, 1801).

Bryum tortuosum (Hedw.) L. ex With. (Linnaeus ex Withering, 1801).

Barbula tortuosa (Hedw.) Web. et Mohr (Weber and Mohr, 1803).

Mollia tortuosa (Hedw.) Schrank ex Lindb. (Schrank ex Lindberg, 1879).

Trichostomum tortuosum (Hedw.) Dix. (Dixon, 1896).

Distr. Southern South America (Cardot, 1905a, 1908a; Cardot and Brotherus, 1923;

Kühnemann, 1938; Bartram, 1952; Seki, 1974).

T. viridula C. Jens.

Weisia controversa

\section{Tortella sp.}

Syn. Trichostomum tortella C. Muell. (Mueller, 1879a).

Distr. Southern South America (Mueller, 1879a).

\section{Tortula}

T. aculeonervis (C. Muell.) Broth. (Brotherus, 1902).

Syn. Barbula aculeonervis C. Muell. (Mueller, 1879a).

Distr. Southern South America (Mueller, 1879a; Brotherus, 1902, 1924a; Kurtz, 1904; Paris, 1906; Hosseus, 1935b, 1937; Kühnemann, 1938).

T. aequatoriale Spruc.

T. aestiva (Hedw.) P. Beauv.

Trichostomum aequatoriale

T. amblyophylla (Hook.) Mitt.

Tortula muralis ssp. aestiva

T. amblyophylla (Mont.) Thér.

Didymodon amblyophyllus

Tortula platyphylla

T. amphidiifolia (C. Muell.) Broth. (Brotherus, 1902).

Syn. Barbula amphidiifolia C. Muell. (Mueller, 1879a).

Distr. Southern South America (Mueller, 1879a; Brotherus, 1902, 1924a; Kurtz, 1904; Paris, 1906; Hosseus, 1935b, 1937; Kühnemann, 1938).

T. anacamptophylla (C. Muell.) Broth.

Tortula lepto-syntrichia

T. anderssonii Aongstr. (Ångström, 1872).

Syn. Barbula anderssonii (Aongstr.) Jaeg. (Jaeger, 1873).

Syntrichia anderssonii (Aongstr.) Herz. (Herzog, 1954).

Barbula conotricha C. Muell. (Mueller, 1885).

Tortula conotricha (C. Muell.) Par. (Paris, 1906).

Barbula perrubiginosa Dus. (Dusén, 1903a).

Tortula perrubiginosa (Dus.) Par. (Paris, 1906).

Syntrichia perrubiginosa (Dus.) Par. (Paris, 1906) nom. nud.

Distr. Southern South America (Ångström, 1872; Jaeger, 1873; Mueller, 1885; Cardot, 1900. 1901, 1905a, 1908a; Dusén, 1903a, 1906; Paris, 1906; Cardot and Brotherus, 1923; Brotherus, 1924a; Roivainen, 1934; Kühnemann, 1938; Bartram, 1952; Herzog, 1954, 1957; Seki, 1974).

var. fagicola (C. Muell.) Card. (Cardot, 1908a).

Syn. Barbula conotricha C. Muell. var. fagicola C. Muell. (Mueller, 1885).

Tortula conotricha (C. Muell.) Par. var. fagicola (C. Muell.) Par. (Paris, 1906).

Distr. Southern South America (Mueller, 1885; Paris, 1906; Cardot, 1908a).

T. andicola Mont. (Montagne, 1838).

Distr. Southern South America (Bartram, 1965).

T. annulus (C. Muell.) Broth.

Tortula muralis

T. antarctica (Hamp.) Wils.

Tortula princeps

T. apiculato-pilosa Par. (Paris, 1906) nom. nud.

Syn. Syntrichia apiculato-pilosa Dus. (Dusén in Paris, 1906) nom. nud.

Distr. Southern South America (Paris, 1906). 
T. appressa Mitt. (Mitten, 1869).

Syn. Barbula appressa (Mitt.) Jaeg. (Jaeger, 1873).

Distr. Southern South America (Mitten, 1869; Jaeger, 1873; Paris, 1906).

T. arenae (Besch.) Broth. (Brotherus, 1902).

Syn. Barbula arenae Besch. (Bescherelle, 1885a).

Syntrichia arenae (Besch.) Herz. (Herzog, 1957).

Distr. Southern South America (Bescherelle, 1885a, 1889; Brotherus, 1902, 1924a; Paris, 1906; Cardot, 1908a; Herzog, 1923, 1939, 1957; Roivainen, 1934).

T. arvensis Mont.

Tortula ruralis

var. australis Mont.

Tortula flagellaris

T. atrata Thér. (Thériot, 1921a).

Distr. Southern South America (Thériot, 1921a; Brotherus, 1924a).

T. atro-virens (Sm.) Lindb.

Desmatodon convolutus

var. brevifolia Thér. (Thériot, 1921a).

Distr. Southern South America (Thériot, 1921a).

T. australasiae Hook. et Grev.

Trichostomopsis australasiae

T. berteroana (C. Muell.) Broth. ex Par.

Tortula muralis

T. berthoana Thér. (Thériot, 1926).

Distr. Southern South America (Thériot, 1926).

T. brachychaete Dus. (Dusén, 1905b).

Syn. Barbula brachychaete Dus. (Dusén, 1903a) nom. nud.

Syntrichia brachychaete Dus. (Dusén in Paris, 1906) nom. nud.

Distr. Southern South America (Dusén, 1903a, 1905b, 1906; Paris, 1906; Brotherus, 1924a; Kühnemann, 1938).

T. brachyclada Card. (Cardot, 1905a).

Distr. Southern South America (Cardot, 1905a, 1908a; Dusén, 1906; Brotherus, 1924a; Kühnemann, 1938).

Falkland Islands (Brotherus, 1924a; Kühnemann, 1938).

T. brachypelma Dus. (Dusén, 1905b).

Syn. Tortula pusilla Aongstr. (Ångström, 1872) hom. illeg.

Barbula pusilla Jaeg. (Jaeger, 1873).

Distr. Southern South America (Ångström, 1872; Jaeger, 1873; Mueller, 1885; Dusén, 1905b; Paris, 1906; Cardot, 1908a; Cardot and Brotherus, 1923; Brotherus, 1924a; Kühnemann, 1938; Bartram, 1952).

T. brevicaulis Par. (Paris, 1906) nom. nud.

Syn. Syntrichia brevicaulis Dus. (Dusén in Paris, 1906) nom. nud.

Distr. Southern South America (Paris, 1906).

T. breviseta Mont. (Montagne, 1845a).

Syn. Barbula breviseta (Mont.) C. Muell. (Mueller, 1849).

Distr. Southern South America (Montagne, 1845a, 1850, 1856; Mueller, 1849; Mitten, 1869; Jaeger, 1873; Paris, 1904a; Thériot, 1923a, b; Brotherus, 1924a; Herzog, 1938).

T. bullata Herz. (Herzog, 1923) hom. illeg.

Distr. Southern South America (Herzog, 1923).

T. caespitosa (Schwaegr.) Hook. et Grev.

Tortella humilis

T. calycina (Schwaegr.) Hook. et Grev.

Tortella calycina

T. calyculosa Mitt. 
T. campestris Dus. (Dusén, 1906).

Distr. Southern South America (Dusén, 1906; Cardot, 1908a; Thériot, 1921a; Cardot and Brotherus, 1923; Brotherus, 1924a; Kühnemann, 1938; Bartram, 1952; Seki, 1974).

T. carinata Gill. ex Grev. (Gillies ex Greville, 1830).

Distr. Southern South America (Greville, 1830).

T. characodonta (C. Muell.) Broth. (Brotherus, 1902).

Syn. Barbula characodonta C. Muell. (Mueller, 1882).

Distr. Southern South America (Mueller, 1882; Brotherus, 1902, 1918, 1924a; Paris, 1906; Kühnemann, 1938).

T. chilensis Mitt.

Trichostomum aequatoriale

T. chrysopila (C. Muell.) Par. (Paris, 1906).

Syn. Barbula chrysopila C. Muell. (Mueller, 1885).

Distr. Southern South America (Mueller, 1885; Paris, 1906; Cardot, 1908a).

T. chubutensis Dus. (Dusén, 1905b).

Distr. Southern South America (Dusén, 1905b, 1907; Brotherus, 1924a; Kühnemann, 1938).

T. chubutensis Broth. (Brotherus, 1931) hom. illeg.

Distr. Southern South America (Brotherus, 1931).

T. conferta Bartr. (Bartram, 1957).

Distr. Antarctic, peninsula region (Bartram, 1957; Steere, 1961a; Greene, 1967; Robinson, 1972 (as T. grossiretis)). Antarctic, continental region (Greene, 1967).

T. conotricha (C. Muell.) Par.

Tortula anderssonii

var. fagicola (C. Muell.) Par.

Tortula anderssonii var. fagicola

T. contorta (C. Muell.) Mont. (Montagne, 1850).

Syn. Barbula contorta Hamp. ex C. Muell. (Hampe ex Mueller, 1849).

Distr. Southern South America (Mueller, 1849; Montagne, 1850; Mitten, 1869; Jaeger, 1873; Paris, 1906; Brotherus, 1924a).

T. costesii Thér. (Thériot, 1921a).

Syn. Syntrichia costesii (Thér.) Herz. (Herzog, 1954).

Tortula curta Card. et Broth. (Cardot and Brotherus, 1923) hom. illeg.

Distr. Southern South America (Thériot, 1921a, b; Cardot and Brotherus, 1923; Brotherus, 1924a; Herzog, 1938, 1954; Kühnemann, 1938).

T. curta Card. et Broth. .

Tortula costesii

T. densifolia (Hook. f. et Wils.) Hook. f. et Wils. (Hooker and Wilson in Wilson and Hooker, 1847).

Syn. Barbula densifolia Hook. f. et Wils. (Hooker, J. D. and Wilson, 1844).

Neobarbula magellanica Dus. (Dusén, 1905b).

Distr. Southern South America (Mueller, 1849; Dusén, 1905b, 1906; Cardot, 1905a, 1908a; Cardot and Brotherus, 1923; Herzog, 1923; Kühnemann, 1938).

Falkland Islands (Hooker, J. D. and Wilson, 1844; Wilson and Hooker, 1847; Jaeger, 1873; Paris, 1906; Cardot, 1908a; Cardot and Brotherus, 1923; Brothenis, 1924a; Kühnemann, 1938).

T. epilosa Broth. ex Dus. (Brotherus ex Dusén, 1906).

Distr. Southern South America (Dusén, 1906; Brotherus, 1924a; Thériot, 1934b).

var. pilifera Thér. (Thériot, 1926).

Distr. Southern South America (Thériot, 1926). 
T. excelsa Card. (Cardot, 1906a).

Distr. Antarctic, peninsula region (Cardot, 1906a, 1908a; Brotherus, 1924a; Steere, 1961a; Robinson, 1972).

T. felipponei Thér. (Thériot in Felippone, 1929 [1930]).

Distr. Am. $t$ in Index muscorum, 5, 82, but not found in Felippone (1929).

T. ferruginea Bartr. (Bartram, 1965).

Distr. Southern South America (Bartram, 1965).

T. filaris (C. Muell.) Broth. (Brotherus, 1902).

Syn. Barbula filaris C. Muell. (Mueller, 1890a).

Distr. South Georgia (Mueller, 1890a; Brotherus, 1902, 1924a; Paris, 1906; Cardot, 1906a, 1908a; Steere, 1961b).

T. flagellaris (Schimp.) Mont. (Montagne, 1850).

Syn. Baarbula flagellaris Schimp. (Schimper, 1836).

Barbula perflaccida Broth. (Brotherus in Paris, 1906) nom. nud.

Tortula perflaccida Broth. ex Dus. (Brotherus ex Dusén, 1906).

Tortula ruralis (Hedw.) Gaertn., Meyer et Scherb. var. australis Mont. (Montagne, 1835).

Tortula arvensis Mont. var. australis Mont. (Montagne, 1850) err.

Distr. Juan Fernandez (Montagne, 1835; Johow, 1896; Brotherus, 1924b; Robinson, 1975).

Southern South America (Schimper, 1836; Mueller, 1849; Montagne, 1850; Mitten. 1869; Jaeger, 1873; Dusén, 1903a, 1906; Paris, 1904a, 1906; Cardot, 1908a; Campo, 1915; Thériot, 1917a, b, 1921a; Costes, 1921; Herzog, 1923; Brotherus, 1924a, c; Kühnemann, 1938; Espinosa B., 1941).

var. densiretis Thér. (Thériot, 1917a).

Distr. Southern South America (Thériot, 1917a).

T. flavido-pilosa Par. (Paris, 1906) nom. nud.

Syn. Barbula flavido-pilosa Dus. (Dusén, 1903a) nom. nud.

Syntrichia flavido-pilosa Dus. (Dusén in Paris, 1906) nom. nud.

Distr. Southern South America (Dusén, 1903a; Paris, 1906; Seki, 1974).

T. flavipes Broth. (Brotherus, 1924C) hom. illeg.

Distr. Southern South America (Brotherus, 1924c, 1925; Espinosa B., 1941; Skottsberg, 1950).

T. flaviseta Mitt.

Pottia flavipes

T. fontana (C. Muell.) Broth. (Brotherus, 1902).

Syn. Barbula fontana C. Muell. (Mueller, 1890a).

Distr. South Georgia (Mueller, 1890a; Brotherus, 1902, 1924a; Paris, 1906; Cardot, 1908a).

T. fragilis Tayl. (Taylor, 1847).

Distr. Southern South America (Hosseus, 1936, 1937, 1938a, d; Kühnemann, 1938).

T. fuegiana (Mitt.) Mitt. (Mitten, 1869).

Syn. Syntrichia fuegiana Mitt. (Mitten, 1860).

Barbula fuegiana (Mitt.) Jaeg. (Jaeger, 1873).

Barbula magellanica C. Muell. (Mueller, 1862).

Tortula magellanica (C. Muell.) Aongstr. (Ångström, 1876b) hom. illeg.

Barbula patagonica C. Muell. (Mueller, 1885) hom. illeg.

Barbula australis Par. (Paris, 1894) nom. nud.

Distr. Southern South America (Mitten, 1860, 1869; Mueller, 1862, 1885, 1889; Jaeger, 1873; Ångström, 1876b; Paris, 1894, 1906; Dusén, 1903a, 1906; Cardot, 1908a; Herzog. 1923, 1957; Brotherus, 1924a; Kühnemann, 1938).

Falkland Islands (Mitten, 1860, 1869; Jaeger, 1873; Paris, 1906; Cardot, 1908a: Brotherus, 1924a; Kühnemann, 1938). 
Antarctic, continental region (Giepp, 1902; Cardot, 1908a; Steere, 1961a).

T. fusca (C. Muell.) Mont.

Barbula fusca

$T$. fuscinervia Mitt.

Barbula fuscinervia

T. fusco-viridis Card. (Cardot, 1906a).

Distr. South Georgia (Cardot, 1906a, 1908a; Steere, 1961b).

T. geniculata Mont.

Barbula geniculata

T. glacialis (C. Muell.) Mont. (Montagne, 1850).

Syn. Barbula glacialis Kunz. ex C. Muell (Kunze ex Mueller, 1849).

Distr. Southern South America (Mueller, 1849; Montagne, 1850; Mitten, 1869; Jaeger, 1873;

Paris, 1906; Costes, 1921; Brotherus, 1924a).

T. graminicolor (C. Muell.) Mont.

T. gracilis (sensu Wilson and Hooker, 1847).

Barbula graminicolor

T. gromschii Thér. (Thériot, 1934b [1935]). see schistidium antarctici

Distr. Southern South America (Thériot, 1934b).

T. grossiretis Card. (Cardot, 1906a).

Distr. Southern South America (Cardot, 1900 and 1901 (as Barbula ruralis forma fide Cardot, 1908a), 1906a, 1908a; Thériot, 1918; Cardot and Brotherus, 1923; Brotherus, 1924a; Bartram, 1952).

South Georgia (Cardot, 1906a, 1908a; Brotherus, 1924a; Steere, 1961b).

Antarctic, peninsula region (Dixon, 1920; Steere, 1961a).

var. atrata Card. (Cardot, 1906a).

Distr. South Gerogia (Cardot, 1906a, 1908a; Steere, 1961b).

T. heimii (Hedw.) Mitt.

Pottia heimii

T. heteronema Card.

Tortula heteroneura

T. heteroneura Card. (Cardot, 1911d).

Syn. Tortula heteronema Card. (Cardot, 1911b) err.

Distr. Antarctic, peninsula region (Cardot, 1911b, d, 1913a; Brotherus, 1924a; Steere, 1961a).

T. humilis (Hedw.) Turn.

T. hyperborea (Brid.) Mont.

Tortella humilis

T. intermedia (Brid.) De Not. (De Notaris, 1838a).

Syn. Syntrichia intermedia Brid. (Bridel, 1826).

Tortula montana (Nees) Lindb. (Lindberg, 1879) hom. illeg.

Distr. Southern South America (Dusén, 1905d; Kühnemann, 1938).

T. inundata Mitt.

Didymodon inundatus

T. jaffuelii Thér. (Thériot, 1923b).

Distr. Southern South America (Thériot, 1923b; Brotherus, 1925).

T. kunzeana (C. Muell.) Mont. (Montagne, 1850).

Syn. Barbula kunzeana C. Muell. (Mueller, 1843a).

Barbula kunzei C. Muell. ex Kindb. (Mueller ex Kindberg, 1888) nom. illeg.

Distr. Scuthern South America (Mueller, 1843a, 1849; Montagne, 1850; Mitten, 1869; Jaeger, 1873; Paris, 1906; Williams, 1915; Costes, 1921; Thériot, 1921a; Brotherus, 1924a).

T. laeta (C. Muell.) Mitt.

T. laevigata Mitt.

Rhamphidium laetum

T. laevinervis Broth. ex Dus. (Brotherus ex Dusén, 1907).

Barbula laevigata 
Distr. Southern South America (Paris, 1906; Dusén, 1907; Brotherus, 1924a).

T. laevipila (Brid.) Schwaegr. (Schwaegrichen, 1823).

Syn. Syntrichia laevipila Brid. (Bridel, 1819).

Barbula laevipila (Brid.) Garov. (Garovaglio, 1840).

Tortula ruralis (Hedw.) Gaertn., Meyer et Scherb. var. laevipila (Brid.) Hook. et Grev. (Hooker and Greville, 1824b).

Syntrichia ruralis (Hedw.) Web. et Mohr var. laevipila (Brid.) Spreng. (Sprengel, 1827).

Syntrichia levipila Brid. ex Moenk. (Bridel ex Mönkemeyer, 1927) err.

Distr. Falkland Islands (Wilson and Hooker, 1847 (as vars 1 and 2); Kühnemann, 1938).

Antarctic, peninsula region (Wilson and Hooker, 1847 (as vars 3 and 4, see also Cardot, 1908a, p. 241); Greene, 1968a (as vars 3 and 4)).

T. lamprocalyx (C. Muell.) Mont.

Barbula lamprocalyx

T. lechleri (C. Muell.) Broth. (Brotherus, 1902).

Syn. Barbula lechleri C. Muell. (Mueller, 1859).

Distr. Southern South America (Mueller, 1859, 1885; Jaeger, 1873; Brotherus 1902, 1924a; Paris, 1906; Cardot, 1908a; Herzog, 1923).

T. lepto-syntrichia (C. Muell.) Broth. (Brotherus, 1902).

Syn. Barbula lepto-syntrichia C. Muell. (Mueller, 1890a).

Syntrichia lepto-syntrichia C. Muell. (Mueller, 1890a) nom. nud.

Barbula anacamptophylla C. Muell. (Mueller, 1890a).

Tortula anacamptophylla (C. Muell.) Broth. (Brotherus, 1902).

Distr. Southern South America (Cardot and Brotherus, 1923; Kühnemann, 1938).

South Georgia (Mueller, 1890a; Brotherus, 1902, 1924a; Cardot, 1906a, 1908a; Paris, 1906; Dixon, 1920, 1934; Steere, 1961b).

T. leucocalyx Mont.

Pseudocrossidium leucocalyx

T. lingulaefolia Card. et Broth. (Cardot and Brotherus, 1923).

Syn. Syntrichia lingulaefolia (Card. et Broth.) Herz. (Herzog, 1954) fide Seki, 1974.

Distr. Southern South America (Herzog, 1954; Seki, 1974).

South Georgia (Cardot and Brotherus, 1923; Brotherus, 1924a; Steere, 1961b).

T. lithophila Dus.

Sarconeurum glaciale

T. litorea Card. (Cardot in Cardot and Brotherus, 1923).

Distr. Falkland Islands (Cardot and Brotherus, 1923; Brotherus, 1924a; Kühnemann, 1938).

T. longipila Dus. (Dusén in Paris, 1906) nom. nud.

Distr. Southern South America (Paris, 1906).

T. lorentzii (C. Muell.) Broth. (Brotherus, 1902).

Syn. Barbula lorentzii C. Muell. (Mueller, 1879a).

Distr. Southern South America (Mueller, 1879a; Brotherus, 1902, 1924a; Paris, 1906; Hosseus, 1936, 1937, 1938a, b, d; Kühnemann, 1938).

T. macrocarpa (Schimp.) Mitt.

Pottia macrocarpa

T. magellanica Mont. (Montagne, 1850).

Syn. Barbula magellanica (Mont.) C. Muell. (Mueller, 1885) hom. illeg.

Distr. Southern South America (Montagne, 1850, 1856; Mueller, 1885; Paris, 1900a, 1906; Cardot, 1908a).

T. magellanica (C. Muell.) Aongstr.

Tortula fuegiana

T. marginato-serrata Par. (Paris, 1906) nom. nud.

Syn. Barbula marginato-serrata Dus. (Dusén, 1903a) nom. nud.

Syntrichia marginato-serrata Dus. (Dusén in Paris, 1906)nom. nud. 
Distr. Southern South America (Dusén, 1903a; Paris, 1906).

T. mendozensis Mitt.

Barbula mendozensis

T. micro-runcinata Par.

Leptodontium lingicaule var. microruncinatum

T. minutirosula (C. Muell.) Broth. (Brotherus, 1902).

Syn. Barbula minutirosula C. Muell. (Mueller, 1879a).

Distr. Southern South America (Mueller, 1879a; Brotherus, 1902, 1924a; Kurtz, 1904; Paris, 1906; Hosseus, 1935b, 1937; Kühnemann, 1938).

T. mniadelphus (C. Muell.) Broth. (Brotherus, 1901).

Syn. Barbula mniadelphus C. Muell. (Mueller, 1897b). Tortula uniadelphus C. Muell. (Mueller in Herzog, 1952c) err. Syntrichia mniadelphus (C. Muell.) Herz. (Herzog, 1954).

Distr. Southern South America (Thériot, 1923b).

var. cochlearifolia (Herz.) Seki (Seki, 1974).

Syn. Tortula unidelphus C. Muell. var. cochlearifolia Herz. (Herzog, 1952c) nom. nud. Syntrichia mniadelphus (C. Muell.) Herz. var. cochlearifolia Herz. (Herzog, 1954).

Distr. Southern South America (Herzog, 1952c, 1954; Seki, 1974).

T. mnioides (Schwaegr.) Mont.

Calyptopogon mnioides

T. monoica Card.

Tortula tenella

T. montana (Nees) Lindb.

Tortula intermedia

T. mucronifolia Schwaegr. var. arctica Hook. et Grev. (Hooker and Greville, 1824b).

Syn. Syntrichia hyperborea Brid. (Bridel, 1826).

Tortula hyperborea (Brid.) Mont. (Montagne, 1845b).

Barbula hyperborea Mont. ex Par. (Montagne ex Paris, 1894) err.

Distr. Southern South America (Montagne, 1839c, 1845b; Wilson and Hooker, 1847; Mueller, 1885; Kühnemann, 1938).

T. muelleri Hook. f. et Wils.

Tortula princeps

T. muralis Hedw. (Hedwig, 1801).

Syn. Bryum murale (Hedw.) L. ex With. (Linnaeus ex Withering, 1801).

Barbula muralis (Hedw.) Crom. (Crome, 1805).

Syntrichia muralis (Hedw.) Raab (Raab, 1819).

Barbula muralis (Hedw.) Crom. var. rupestris Schultz (Schultz, 1823).

Tortula muralis Hedw. var. rupestris Chev. (Chevallier, 1827).

Barbula muralis (Hedw.) Crom. var. australis Hamp. (Hampe in Mueller, 1862) nom. nud.

Barbula annulus C. Muell. (Mueller, 1882).

Tortula annulus (C. Muell.) Broth. (Brotherus ex Paris, 1906).

Barbula berteroana C. Muell. (Mueller, 1862).

Tortula berteroana (C. Muell.) Broth. ex Par. (Brotherus ex Paris, 1906) hom. illeg.

Barbula berteroi C. Muell. ex Kindb. (Mueller ex Kindberg, 1888) hom. illeg.

Barbula muricola C. Muell. (Mueller, 1857).

Tortula muricola (C. Muell.) Mitt. (Mitten, 1869).

Distr. Southern South America (Montagne, 1850; Mueller, 1862, 1879a, 1882; Mitten, 1869; Jaeger, 1873; Brotherus, 1900, 1924a, c; Krieger, 1904; Dusén, 1906; Paris, 1906; Williams, 1915; Costes, 1921; Thériot, 1921a, b; Herter, 1933a, 1939a, b; Roivainen, 1934; Herzog, 1938, 1939; Hosseus, 1938c, $d$, 1940; Espinosa B., 1941; Piovano, 1954).

var. aestiva Hedw. (Hedwig, 1801).

Syn. Tortula aestiva (Hedw.) P. Beauv. (Palisot de Beauvois, 1805a).

Barbula muralis (Hedw.) Crom. var. aestiva (Hedw.) Roehl. (Röhling, 1813).

Barbula aestiva (Hedw.) Schultz (Schultz, 1823).

Barbula muralis (Hedw.) Crom. ssp. aestiva (Hedw.) Kindb. (Kindberg, 1897).

Distr. Southern South America (Thériot, 1921a). 
var. longipila Dus. (Dusén, 1907).

Distr. Southern South America (Dusén, 1907; Kühnemann, 1938).

var. rupestris Chev.

Tortula muralis

T. muricola (C. Muell.) Mitt.

Tortula muralis

T. obscuriretis Thér. (Thériot, 1918).

Distr. Southern South America (Thériot, 1918; Campo, 1921; Brotherus, 1925).

T. occultilimbata Card.

Tortula platyphylla

T. papillosa Wils. (Wilson in Spruce, 1845).

Syn. Syntrichia papillosa (Wils.) Jur. (Juratzka, 1882).

Distr. Southern South America (Dusén, 1905b, 1906; Cardot, 1908a; Thériot, 1921a; Kühnemann, 1938; Herzog, 1954).

Falkland Islands (Paris, 1906; Cardot, 1908a; Brotherus, 1924a; Kühnemann, 1938).

var. chilensis Thèr. (Thériot, 1921a).

Syn. Tortula subpapillosa Card. et Broth. (Cardot and Brotherus, 1923).

Distr. Southern South America (Thériot, 1921a, 1930; Cardot and Brotherus, 1923; Brotherus, 1924a; Kühnemann, 1938).

T. patagonica Mitt.

Didymodon patagonicus

T. perangusta Dus. (Dusén, 1905d) nom. nud.

Distr. Southern South America (Dusén, 1905d; Kühnemann, 1938).

T. perarmata Broth. (Brotherus, 1924C).

Distr. Southem South America (Brotherus, 1924C, 1925; Espinosa B., 1941; Skottsberg, 1950).

T. percarnosa (C. Muell.) Broth. (Brotherus, 1902).

Syn. Barbula percarnosa C. Muell. (Mueller, 1879a).

Distr. Southern South America (Mueller, 1879a; Brotherus, 1902, 1924a; Paris, 1906; Kühnemann, 1938).

T. perflaccida Broth. ex Dus.

Tortula flagellaris

T. perpusilla (C. Muell.) Broth. (Brotherus, 1902).

Syn. Barbula perpusilla C. Muell. (Mueller, 1879a).

Distr. Southern South America (Mueller, 1879a; Brotherus, 1902, 1924a; Kurtz, 1904; Paris, 1906; Hosseus, 1935a; Kühnemann, 1938).

T. perrubiginosa (Dus.) Par.

Tortula anderssonii

T. perrufula (C. Muell.) Williams

Tortella perrufula

T. pilifera Hook. (Hooker, 1818).

Syn. Barbula pilifera (Hook.) Brid. (Bridel, 1826) hom. illeg.

Streblotrichum piliferum (Hook.) Hilp. (Hilpert, 1933).

Barbula crinita Schultz (Schultz, 1823) nom. illeg.

Barbula depressa Sull. (Sullivant, 1859).

Tortula subglacialis Thér. (Thériot, 1917b).

Distr. Juan Fernandez (Thériot, 1917b; Brotherus, 1924b).

Southern South America (Mueller, 1849; Montagne. 1850; Sullivant, 1859; Paris, 1904a; Thériot, 1917b, 1918, 1921a, b, 1923b, 1925b, 1928, 1934b; Campo, 1921; Costes, 1921; Cardot and Brotherus, 1923; Brotherus, 1924a; Reimers, 1926; Herzog, 1938, 1954, 1957, 1960; Bartram, 1952; Seki, 1974).

var. denticulata (Dus.) Wijk et Marg. (van der Wijk and Margadant, 1959).

Syn. Barbula flagellaris Schimp. var. denticulata Dus. (Dusén, 1906).

Barbula depressa Sull. var. denticulata (Dus.) Thér. (Thériot, 1917a).

Distr. Southern South America (Dusén, 1906; Cardot, 1908a; Thériot, 1917a; Campo, 1921; Kühnemann, 1938). 
var. gracilis (Dus.) Wijk et Marg. (van der Wijk and Margadant, 1959).

Syn. Barbula flagellaris Schimp. var. gracilis Dus. (Dusén, 1906).

Barbula depressa Sull. var. gracilis (Dus.) Thèr. (Thériot, 1917a).

Distr. Southern Southern South America (Dusén, 1906; Thériot, 1917a; Kühnemann, 1938).

var. oliviensis (Card.) Wijk et Marg. (van der Wijk and Margadant, 1959).

Syn. Barbula oliviensis Card. (Cardot, 1905a).

Barbula depressa Sull. var. Oliviensis (Card.) Thér. (Thériot, 1917a).

Distr. Southern South America (Cardot, 1905a, 1908a; Thériot, 1917a; Cardot and Brotherus, 1923; Kühnemann, 1938).

T. planicosta Herz. (Herzog, 1952b).

Distr. Southern South America (Herzog, 1952b).

T. platyphylla Mitt. (Mitten, 1869).

Syn. Desmatodon amblyophyllus Mont. (Montagne, 1845a).

Trichostomum amblyophyllum (Mont.) C. Muell. (Mueller, 1849).

Tortula amblyophylla (Mont.) Thér. (Thériot, 1923a) hom. illeg.

Tortula occultilimbata Card. (Cardot in Thériot, 1921b) nom. nud.

Distr. Southern South America (Montagne, 1845a, 1850, 1856; Mueller, 1849; Mitten, 1869; Jaeger, 1873; Paris, 1906; Thériot, 1921b, 1922, 1923a, 1934b; Brotherus, 1924a; Looser, 1932; Herzog, 1939, 1954).

T. podocarpi (C. Muell.) Broth. (Brotherus, 1902).

Syn. Barbula podocarpi C. Muell. (Mueller, 1879a).

Distr. Southern South America (Mueller, 1879a; Brotherus, 1902, 1924a; Paris, 1906; Kühnemann, 1938).

T. poeppigiana (C. Muell.) Mont.

Trichostomopsis australasiae

T. polycarpa Dus. (Dusén, 1906).

Distr. Southern South America (Dusén, 1906; Cardot, 1908a; Cardot and Brotherus, 1923; Brotherus, 1924a; Kühnemann, 1938; Seki, 1974).

T. princeps De Not. (De Notaris, 1838a).

Syn. Barbula principes (De Not.) C. Muell. (Mueller, 1849).

Syntrichia principes (De Not.) Mitt. (Mitten, 1859).

Barbula muelleri B.S.G. (Bruch and others, 1842) nom. illeg.

Syntrichia muelleri Bruch (Bruch in Bruch and others, 1842) nom. nud.

Tortula muelleri Hook. f. et Wils. (Hooker and Wilson in Wilson and Hooker, 1847) nom. illeg.

Barbula antarctica Hamp. (Hampe in Mueller, 1849).

Tortula antarctica (Hamp.) Wils. (Wilson, 1859).

Distr. Southern South America (Mitten, 1869; Jaeger, 1873; Paris, 1906; Brotherus, 1925; Seki, 1974).

Falkland Islands (Wilson and Hooker, 1847; Mueller, 1849; Jaeger, 1873; Paris, 1906; Cardot, 1908a; Cardot and Brotherus, 1923; Brotherus, 1925; Kühnemann, 1938).

T. prostrata Mont. (Montagne, 1845a).

Syn. Barbula prostrata (Mont.) Jaeg. (Jaeger, 1873).

Syntrichia prostrata (Mont.) Herz. (Herzog, 1960 [1961]).

Barbula mnioides Schwaegr. var. prostrata (Mont.) C. Muell. (Mueller, 1849).

Streptopogon mnioides (Schwaegr.) Mitt. var. prostrata (Mont.) Jaeg. (Jaeger, 1873).

Calyptopogon mnioides (Schwaegr.) Broth. var. prostratus (Mont.) Par. (Paris, 1904a).

Distr. Southern South America (Montagne, 1845a, 1850, 1856; Mueller, 1849; Mitten, 1869; Jaeger, 1873; Dusén, 1906; Paris, 1906; Thériot, 1915, 1928; Brotherus, 1924a; Herzog, 1938, 1960; Kühnemann, 1938; Bartram, 1952; Seki, 1974).

T. pseudo-latifolia Card. (Cardot, 1905a).

Distr. Southern South America (Cardot, 1905a, 1908a; Dusén, 1906; Brotherus, 1925;

Kuihnemann. 1938: Seki, 1974). 
T. pseudo-robusta Dus. (Dusén, 1906).

Syn. Syntrichia pseudo-robusta (Dus.) Herz. (Herzog, 1954). Syntrichia robusta Dus. (Dusén in Cardot, 1908a) nom. nud.

Barbula ventanae C. Muell. (Mueller in Thériot, 1932 [1933]) nom. nud.

Distr. Southem South America (Dusén, 1906; Cardot, 1908a; Thériot, 1921a, 1932; Cardot and Brotherus, 1923; Herzog, 1938, 1939, 1954; Kühnemann, 1938; Seki, 1974).

T. pulvinatula Dus. (Dusén, 1905b).

Distr. Southern South America (Dusén, 1905b, 1906; Brotherus, 1924a; Kühnemann, 1938).

T. punctulata (Brid.) Mitt.

Tortula robusta

T. purpureo-velutina Herz. (Herzog, 1954).

Distr. Southern South America (Herzog, 1954).

T. pusilla Mitt.

T. pusilla Aongstr.

Pterygoneurum ovatum

T. pygmaea Dus.

Tortula brachypelma

T. replicata (Tayl.) Wils.

Sarconeurum glaciale

T. rigidula (Hedw.) Lindb.

Barbula replicata

T. rivularis Dus. (Dusén, 1906).

Didymodon rigidulus

Distr. Southern South America (Dusén, 1906; Cardot, 1908a; Cardot and Brotherus, 1923; Brotherus, 1924a; Kühnemann, 1938).

T. robusta Hook. et Grev. (Hooker and Greville, 1824b).

Syn. Barbula robusta (Hook. et Grev.) Brid. (Bridel, 1827a).

Syntrichia robusta (Hook. et Grev.) Herz. (Herzog, 1954).

Barbula speciosa Hook. f. et Wils. (Hooker, J. D. and Wilson, 1844).

Mnium punctulatum Brid. (Bridel, 1827b).

Tortula punctulata (Brid.) Mitt. (Mitten, 1869).

Barbula punctulata (Brid.) Jaeg. (Jaeger, 1873).

Tortula serrulata Hook. et Grev. (Hooker and Greville, 1824b) fide Greene, 1964.

Barbula serrulata (Hook. et Grev.) Brid. (Bridel, 1827a) fide Greene, 1964.

Desmatodon serrulatus (Hook. et Grev.) Mitt. (Mitten, 1859) fide Greene, 1964.

Tortula rubra Mitt. (Mitten in Hooker, 1867) fide Greene, 1964.

Barbula rubra (Mitt.) Jaeg. (Jaeger, 1873) fide Greene, 1964.

Barbula dioica (R. Brown ter.) Par. (Paris, 1900a).

Desmatodon ruber (Mitt.) Hamp. (Hampe, 1880c).

Distr. Southern South America (Hooker and Greville, 1824b; Bridel, $1827 a$; Hooker, J. D. and Wilson, 1844; Wilson and Hooker, 1847; Mueller, 1849, 1885; Mitten, 1869; Jaeger, 1873; Bescherelle, 1889; Paris, 1900a, 1906; Cardot, 1905a, 1908a; Dusén, 1906;

Cardot and Brotherus, 1923; Brotherus, 1924a; Roivainen, 1934; Kühnemann, 1938; Herzog, 1939, 1940, 1954; Bartram, 1952; Seki, 1974).

Falkland Islands (Bridel, 1827a; Wilson and Hooker, 1847 (as T. robusta var. $\beta$ ); Mueller, 1849, 1885; Hooker, 1867; Mitten, 1869; Jaeger, 1873; Bescherelle, 1889; Paris, 1900a, 1906; Cardot, 1908a; Cardot and Brotherus, 1923; Brotherus, 1924a; Kühnemann, 1938).

South Georgia (Cardot, 1906a, 1908a; Dixon, 1920, 1932, 1934; Cardot and Brotherus, 1923; Brotherus, 1924a; Steere, 1961b; Greene, 1964).

var. laxa Bartr. (Bartram, 1946).

Distr. Southern South America (Roivainen, 1934; Bartram, 1946).

var. runcinata (C. Muell.) Broth. (Brotherus in Cardot and Brotherus, 1923).

Syn. Barbula runcinata C. Muell. (Mueller, 1890a).

Syntrichia runcinata C. Muell. (Mueller, 1890a) nom. nud.

Tortula runcinata (C. Muell.) Broth. (Brotherus, 1902).

Distr. Southern South America (Cardot and Brotherus, 1923: Kühnemann. 1938).

Falkland Islands (Cardot and Brotherus, 1923; Kühnemann, 1938).

South Georgia (Mueller, 1890a; Brotherus, 1902, 1924a; Paris, 1906). 
T. robustula Card. (Cardot, 1905a).

Distr. Southern South America (Cardot, 1905a, 1908a; Dusén, 1906; Brotherus, 1924a; Kühnemann, 1938).

Falkland Islands (Cardot, 1905a, 1908a; Cardot and Brotherus, 1923; Brotherus, 1924a; Kühnemann. 1938).

T. rubra Mitt.

Tortula robusta

T. runcinata (C. Muell.) Broth. Tortula robusta var. runcinata

T. ruralis (Hedw.) Gaertn., Meyer et Scherb. (Gärtner and others, 1802).

Syn. Barbula ruralis Hedw. (Hedwig, 1801).

Bryum rurale (Hedw.) L. ex With. (Linnaeus ex Withering, 1801).

Syntrichia ruralis (Hedw.) Web. et Mohr (Weber and Mohr, 1803).

Tortula arvensis Mont. (Montagne, 1850) err.

Distr. Southern South America (Jaeger, 1873; Dusèn, 1905a, 1906; Paris, 1906; Brothenus, 1924a: Roivainen, 1934; Kühnemann, 1938; Bartram, 1965).

var. australis Mont.

Tortula flagellaris

var. laevipila (Brid.) Hook. et Grev.

Tortula laevipila

T. santiagensis Broth. (Brotherus, 1924c).

Distr. Southern South America (Brotherus, 1924c; Espinosa B., 1941).

T. saxicola Card. (Cardot, 1905a).

Distr. Southern South America (Cardot, 1905a, 1908a; Kühnemann, 1938; Bizot, 1967; Seki, 1974).

T. saxicola Dus.

Sarconeurum glaciale

T. scabrella Dus. (Dusén, 1907).

Syn. Barbula scabrella Dus. (Dusén in Paris, 1906) nom. nud.

Syntrichia scabrella (Dus.) Herz. (Herzog, 1957).

Distr. Southern South America (Paris, 1906; Dusén, 1907; Thériot, 1921b, 1923b; Brotherus, 1924a; Herzog, 1957).

T. scabrinervis (C. Muell.) Mont. (Montagne, 1850).

Syn. Barbula scabrinervis C. Muell. (Mueller, 1849).

Syntrichia scabrinervis (C. Muell.) Herz. (Herzog, 1954).

Distr. Juan Fernandez (Brotherus, 1924b; Bartram, 1959; Robinson, 1975).

Southern South America (Mueller, 1849; Montagne, 1850; Mitten, 1869; Jaeger, 1873; Paris, 1906; Costes, 1921; Herzog, 1954).

T. schnyderi (C. Muell.) Broth. (Brotherus, 1902).

Syn. Barbula schnyderi C. Muell. (Mueller, 1882).

Distr. Southern South America (Mueller, 1882; Brotherus, 1902. 1924a; Dusén, 1906; Paris, 1906; Cardot, 1908a; Kühnemann, 1938).

T. serrata Dix. (Dixon, 1923).

Distr. South Gerogia (Greene, 1964, 1968a).

T. serripungens (Lor. et C. Muell.) Broth. (Brotherus, 1902).

Syn. Barbula serripungens Lor. et C. Muell. (Lorentz and Mueller in Mueller, 1879a). Syntrichia serripungens (Lor. et C. Muell.) Herz. (Herzog, 1954).

Distr. Southern South America (Mueller, 1879a; Brotherus, 1902, 1924a; Kurtz, 1904; Paris, 1906; Hosseus, 1935b, 1937, 1938b, c, d; Kühnemann. 1938; Herzog, 1952a, 1954).

var. exesa (C. Muell.) Herz. (Herzog, 1916a).

Syn. Barbula serripungens Lor. et C. Muell. var. exesa C. Muell. (Mueller, 1879a).

Distr. Southern South America (Mueller, 1879a; Kühnemann, 1938). 
T. serrulata Hook. et Grev.

T. sinuata Bartr. (Bartram, 1965).

Distr. Southern South America (Bartram, 1965).

T. socialis Dus. (Dusén, 1907).

Distr. Southem South America (Dusén, 1907; Brotherus, 1924a).

T. squarripila Thér. (Thériot, 1917b).

Syn. Syntrichia squarripila (Thér.) Herz. (Herzog, 1954).

Distr. Southem South America (Thériot, 1917b; Costes, 1921; Brotherus, 1924a; Bartram, 1952; Herzog, 1954, 1960).

T. stenophylla Card. et Broth. (Cardot and Brotherus, 1923) hom. illeg.

Distr. Southem South America (Cardot and Brotherus, 1923; Brotherus, 1924a; Thériot, 1935a; Kühnemann, 1938).

T. subenervis Dus. (Dusén in Paris, 1906) nom. nud.

Distr. Southem South America (Paris, 1906).

T. subglacialis Thér.

Tortula pilifera

T. subpapillosa Card. et Broth.

T. subsessilis (Brid.) Mitt.

Tortula papillosa var. chilensis Pterygoneurum subsessile

T. tenella Broth. (Brotherus, 1898).

Syn. Barbula tenella (Broth.) Par. (Paris, 1900a).

Tortula monoica Card. (Cardot, 1905a).

Distr. Falkland Islands (Cardot, 1905a, 1908a; Cardot and Brotherus, 1923; Brotherus, 1925; Kühnemann, 1938).

South Georgia (Cardot, 1906a, 1908a; Cardot and Brotherus, 1923; Brotherus, 1925; Dixon, 1934; Steere, 1961b).

Antarctic, peninsula region (Cardot, 1906a, 1908a; Brotherus, 1925; Steere, 1961a).

T. tortuosa Hedw.

Tortella tortuosa

T. truncata (Hedw.) Mitt.

Pottia truncata

T. truncatula (With.) Lindb.

Pottia truncata

T. umbrosa Dus. (Dusén, 1907).

Distr. Southem South America (Dusén, 1907; Brotherus, 1924a).

T. unguiculata (Hedw.) A. Roth. exP. Beauv.

Barbula unguiculata

T. uniadelphus C. Muell.

Tortula mniadelphus

var. cochlearifolia Herz.

Tortula mniadelphus var. cochlearifolia

T. vahliana (Schultz) Mont. (Montagne, 1850).

Syn. Barbula vahliana Schultz (Schultz, 1823).

Distr. Southern South America (Bruch and others, 1842; Montagne, 1850; Reichardt, 1870).

T. vinealis (Brid.) Spruc. var. flaccida (B.S.G.) Spruc.

Barbula vinealis var. flaccida

\section{Trachyloma}

T. subbasilare (Hook.) Mitt.

Goniobryum subbasilare

\section{Trachypus}

T. flexicaulis (Wils.) Mitt.

Papillaria flexicaulis

\section{Trematodon}

T. affinis B.S.G.

Trematodon reflexus

T. boryanus (Schwaegr.) Spreng. 
T. crispatissimus Hamp.

T. crispifolius Thèr. (Thèriot in Felippone, 1929 [1930]).

Distr. Southern South America (Felippone, 1929; Herter, 1933b).

T. felipponei Thèr. (Thériot in Felippone, 1929 [1930]).

Distr. Southern South America (Felippone, 1929; Herter, 1933b).

T. latifolius C. Muell. (Mueller, 1901a [1900]) nom. nud.

Distr. Southern South America (Mueller, 1901a).

T. longicollis Michx. var. crispatissimus Hornsch. (Hornschuch, 1840) hom. illeg.

Syn. Trematodon crispatissimus Hamp. (Hampe, 1879) hom. illeg.

Distr. Southern South America (Hornschuch, 1840; Gibert, 1873; Hampe, 1879; Paris, 1906; Roth, 1911; Herter, 1933a).

T. reflexus C. Muell. (Mueller, 1848a).

Syn. Trematodon affinis B.S.G. (Bruch and others, 1846) nom. nud.

Distr. Southern South America (Krieger, 1904; Bartram, 1965).

T. uruguensis Broth. (Brotherus in Felippone, 1909).

Distr. Southern South America (Felippone, 1909; Brotherus, 1924a; Herter, 1933a).

T. vaginatus C. Muell. (Mueller, 1857).

Distr. Southern South America (Krieger, 1904).

\section{Trichosteleum}

T. micropyxidium (C. Muell.) Broth. (Brotherus, 1908).

Syn. Aptychus micropyxis C. Muell. (Mueller, 1896b).

Distr. Southern South America (Hosseus, 1935b; Kühnemann, 1938).

\section{Trichosteleum sp.}

Syn. Hypnum micropyxis C. Muell. (Mueller, 1879a).

Rhapidostegium micropyxis (C. Muell.) Kindb. (Kindberg, 1888).

Distr. Southern South America (Mueller, 1879a; Kurtz, 1904; Paris, 1905b; Hosseus, 1937).

\section{Trichostomopsis}

T. australasiae* (Hook. et Grev.) H. Robinson (Robinson, 1970a).

Syn. Tortula australasiae Hook. et Grev. (Hooker and Greville, 1824b).

Barbula australasiae (Hook. et Grev.) Brid. (Bridel, 1827a).

Trichostomum australasiae (Hook. et Grev.) Jaeg. (Jaeger, 1873).

Asteriscium australasiae (Hook. et Grev.) Hilp. (Hilpert, 1933).

Barbula poeppigiana C. Muell. (Mueller, 1843a).

Pottia poeppigiana (C. Muell.) C. Muell. (Mueller, 1849).

Tortula poeppigiana (C. Muell.) Mont. (Montagne, 1850).

Weisia poeppigiana (C. Muell.) Mitt. (Mitten, 1869).

Hyophila poeppigiana (C. Muell.) Jaeg. (Jaeger, 1873).

Asteriscium poeppigianum (C. Muell.) Hilp. (Hilpert, 1933).

Hyophila poeppigii C. Muell. ex Kindb. (Mueller ex Kindberg, 1888) nom. illeg.

Didymodon subtophaceus Williams (Williams, 1903a) fide Robinson, 1970a.

Desmatodon subtophaceus (Williams) Williams (Williams, 1915) fide Robinson, 1970 .

Asteriscium subtophaceum (Williams) Hilp. (Hilpert, 1933).

Distr. Southern South America (Mueller, 1843a, 1849; Montagne, 1850; Mitten, 1869; Jaeger, 1873; Paris, 1904a, $b$; Dusén, 1906; Williams, 1915; Warnstorf, 1916; Thériot, 1917b, 1921 b, 1928; Costes, 1921; Brotherus, 1924a, C; Herzog, 1938, 1954, 1957;

Espinosa B., 1941; Skottsberg, 1950; Robinson, 1970a).

\footnotetext{
* = Didymodon australasii (Hook. et Grev.) Zander fide Zander, 1978.
} 
T. curvipes (C. Muell.) H. Robinson (Robinson, 1970a).

Syn. Barbula curvipes C. Muell. (Mueller, 1879a).

Asteriscium curvipes (C. Muell.) Hilp. (Hilpert, 1933).

Distr. Southern South America (Mueller, 1879a; Paris, 1904a; Brotherus, 1924a; Kühnemann, 1938; Robinson, 1970a).

T. umbrosat (C. Muell.) H. Robinson (Robinson, 1970a).

Syn. Barbula umbrosa C. Muell. (Mueller, 1879a).

Asteriscium umbrosum (C. Muell.) Herz. (Herzog, 1952a).

Asteriscium flavisetum Herz. (Herzog, 1952a).

Barbula flaviseta (Herz.) Wijk et Marg. (van der Wijk and Margadant, 1958).

Distr. Southern South America (Mueller, 1879a; Kurtz, 1904; Paris, 1904a; Brotherus, 1924a; Hosseus, 1935a, b, 1937; Kühnemann, 1938; Herzog, 1952a; Robinson, 1970a).

\section{Trichostomum}

T. acaulon C. Muell.

Tortella acaulon

T. aciculare (Hedw.) P. Beauv. var. aquaticum (Schrad.) Web. et Mohr ex Brid.

Racomitrium aquaticum

T. aequatoriale Spruc. ex Dix. (Spruce ex Dixon, 1924a).

Syn. Tortula aequatoriale Spruc. (Spruce in Dixon, 1924a) nom. nud.

Tortula chilensis Mitt. (Mitten, 1869 non Trichostomum chilense Mont.).

Distr. Southern South America (Mitten, 1869).

T. affine (C. Muell.) Mont.

Ditrichum difficile

T. amblyophyllum (Mont.) C. Muell.

Tortula platyphylla

T. aquaticum Brid. ex Schrad.

Racomitrium aquaticum

T. australasiae (Hook. et Grev.) Jaeg.

Trichostomopsis australasiae

T. australe Mitt.

Ditrichum strictum

T. brachydontium Bruch (Bruch in F. A. Mueller, 1829).

Syn. Didymodon brachydontius (Bruch) Wils. (Wilson in Hooker, 1833).

Mollia brachydontia (Bruch) Lindb. (Lindberg, 1879).

Tortella brachydontia (Bruch) C. Jens. (Jensen, 1923).

Distr. Juan Fernandez (Brotherus, 1924b; Espinosa B., 1941; Bartram, 1959; Robinson, 1975).

T. brunneum C. Muell.

Didymodon brunneus

T. calcareum (Nees et Hornsch.) Lindb.

Gymnostomum aeruginosum

T. campylocarpum C. Muell.

Bryoerthrophyllum campylocarpum

T. capillaceum (Hedw.) Sm.

Distichium capillaceum

T. chilense Mont.

Cheilothela chilensis

T. compactulum C. Muell. (Mueller, 1879a).

Distr. Southern South America (Mueller, 1879a; Kurtz, 1904; Paris, 1906; Brotherus, 1924a; Hosseus, 1935b, 1937, 1938c, d; Kühnemann, 1938).

T. convolutum Brid.

Desmatodon convolutus

T. crispipilum Tayl.

Racomitrium crispipilum

T. cylindricarpum (C. Muell.) Wils.

Ditrichum cylindricarpum

T. difficile Dub.

Ditrichum difficile

T. dusenii Par. (Paris, 1906) nom. nud.

Syn. Trichostomum glauco-viride Dus. (Dusén in Paris, 1906) nom. nud.

Distr. Southern South America (Paris, 1906).

$\dagger=$ Didymodon umbrosus (C. Muell.) Zander fide Zander, 1978 
T. elliotii Broth. ex Dus. (Brotherus ex Dusén, 1906).

Distr. Southern South America (Dusèn, 1906; Brothenus, 1924a; Herzog, 1954).

$T$. elongatum Hook. f. et Wils.

Ditrichum cylindricarpum

T. fernandezianum Card. (Cardot in Skottsberg, 1914) nom. nud.

Distr. Southern South America (Skottsberg, 1914).

T. glauco-viride C. Muell.

Didymodon glauco-viridis

T. glauco-viride Dus.

Trichostomum dusenii

T. gracillimum C. Muell. (Mueller, 1879a).

Syn. Anacalypta gracillima (C. Muell.) C. Muell. (Mueller, 1882) nom. inval. Pottia gracillima (C. Muell.) Par. (Paris, 1900a).

Distr. Southern South America (Mueller, 1879a; Paris, 1900a, 1906; Kurtz, 1904; Warnstorf, 1916; Brotherus, 1924a; Hosseus, 1935b, 1937; Kühnemann, 1938).

T. gymnum C. Muell.

Didymodon gymnus

T. heterostichum Hedw.

Racomitrium heterostichum

T. humile (Hedw.) Mach.

Tortella humilis

T. hypnoides Willd. ex P. Beauv.

Racomitrium lanuginosum

T. imperfectum C. Muell.

Barbula imperfecta

T. julaceum Dus. (Dusén in Paris, 1906) nom. nud.

Distr. Southern South America (Paris, 1906).

T. laetum Kunz. ex C. Muell.

Rhamphidium laetum

T. lambii Bartr. (Bartram, 1965).

Distr. Southern South America (Bartram, 1965).

T. lanuginosum Hedw.

$T$. laxifolium Hook. f. et Wils.

$T$. leptodum (Mont.) Mitt.

Racomitrium lanuginosum Ditrichum difficile Campylopus clavatus

T. ligulum C. Muell. (Mueller, 1901a [1900]) nom. nud.

Distr. Southern South America (Mueller, 1901a).

T. longifolium Brid.

Leucoloma longifolium

T. microthecium C. Muell. Didymodon microthecius

T. nudum C. Muell.

Didymodon brunneus

T. orthotrichoides C. Muell. Leptodontium orthotrichoides

T. plicatulum C. Muell. (Mueller, 1882).

Distr. Southern South America (Mueller, 1882; Paris, 1906; Kühnemann, 1938).

$T$. protensum Braun

Racomitrium aquaticum

T. pungens (Mitt.) Par. Leptodontium pungens

T. rigidulum (Hedw.) Turn.

Didymodon rigidulus

$T$. schimperi Mont.

Didymodon schimperi

$T$. spathulato-lineare C. Muell.

Didymodon spathulato-linearis

$T$. strictum Web. et Mohr

Ditrichum rufescens

T. tortella C. Muell. Tortella sp.

T. tortuosum (Hedw.) Dix.

Tortella tortuosa

T. tucumanense Bartr. (Bartram, 1965).

Distr. Southern South America (Bartram, 1965).

T. umbrosum C. Muell. 


\section{Triquetrella}

T. filicaulis Dus. (Dusén, 1906).

Distr. Southern South America (Dusèn, 1906; Brotherus, 1924a, c; Espinosa B., 1941).

T. patagonica C. Muell. (Mueller, 1897e).

Distr. Southern South America (Mueller, 1897e; Paris, 1900a, 1906: Thériot, 1917b; Costes, 1921; Brotherus, 1924a; Herzog, 1938; Kühnemann, 1938).

\section{Tristichiopsis}

T. mirabilis C. Muell.

Tristichium mirabile

\section{Tristichium}

T. Iorentzii C. Muell. (Mueller, 1879a).

Distr. Southem South America (Mueller, 1879a; Paris, 1906; Roth, 1911; Brotherus, 1924a; Kühnemann, 1938).

T. mirabile (C. Muell.) Herz. (Herzog, 1914).

Syn. Tristichiopsis mirabilis C. Muell. (Mueller, 1882).

Distr. Southem South America (Mueller, 1882; Paris, 1906; Brotherus, 1924a; Kühnemann. 1938).

Ulea hom. illeg.

U. nitida Thér. (Thériot in Felippone, 1929 [1930]).

Distr. Southern South America (Felippone, 1929; Herter, 1933a).

\section{Ulota}

U. anderssoni (Aongstr.) Jaeg.

Orthotrichum leiothecium

U. angustissima C. Muell. (Mueller, 1897a).

Distr. Southern South America (Mueller, 1897a; Paris, 1906; Brotherus, 1925).

U. aurantiaca Dus. in Malta (Dusén in Malta, 1927).

Distr. Southem South America (Malta, 1927).

U. brevicollis (Mitt.) Jaeg.

Ulota rufula

U. carinata Mitt. (Mitten, 1860).

Syn. Orthotricum carinatum (Mitt.) Mitt. (Mitten, 1869).

Distr. Southem South America (Mitten, 1860, 1869; Paris, 1906; Brotherus, 1925; Malta, 1927; Herzog, 1938, 1939).

$U$. chilensis (Mitt.) Jaeg.

Ulota rufula

U. crassifolia (Hook. f. et Wils.) Hook. f.

Muelleriella crassifolia

U. crenato-erosa (C. Muell.) Kindb.

Orthotrichum crenato-erosa

U. crispata (Hedw.) Sw.

Ptychomitrium crispatum

U. darwinii Mitt.

$U$. eremitensis Mitt.

Ulota fuegiana ssp. darwinii

Ulota glabella

U. fernandeziana Malta (Malta, 1927).

Distr. Juan Fernandez (Malta, 1927; Bartram, 1959; Robinson, 1975).

U. fuegiana Mitt. (Mitten, 1860).

Syn. Orthotrichum fuegianum (Mitt.) Mitt. (Mitten, 1869).

Orthotrichum luteolum Hook. f. et Wils. (Hooker and Wilson in Wilson and Hooker, 1847) pro parte. 
Distr. Southern South America Wilson and Hooker, 1847; Mitten, 1860, 1869; Ångström, 1872; Jaeger, 1874; Bescherelle, 1885a, 1889; Mueller, 1885; Cardot, 1900, 1901. 1905a, 1908a; Paris, 1906; Cardot and Brotherus, 1923; Brotherus, 1925; Malta, 1927; Kühnemann, 1938; Bartram, 1952; Herzog, 1957; Seki, 1974).

ssp. darwinii (Mitt.) Malta (Malta, 1927).

Syn. Ulota darwinii Mitt. (Mitten, 1860).

Orthotrichum darwinii (Mitt.) Mitt. (Mitten, 1869).

Distr. Southern South America (Mitten, 1860, 1869; Jaeger, 1874; Bescherelle, 1885a; Mueller, 1885; Paris, 1906; Cardot, 1908a; Brotherus, 1925; Malta, 1927; Kühnemann, 1938).

var. crispata Malta (Malta, 1927).

Distr. Southern South America (Malta, 1927; Bartram, 1952 (as var. crispa Malta); Herzog, 1954).

U. fulvella Mitt.

Ulota luteola

U. germana (Mont.) Mitt. (Mitten, 1860).

Syn. Orthotrichum germanum Mont. (Montagne, 1845a).

Distr. Southern South America (Montagne, 1845a, 1850, 1856; Mueller, 1849; Mitten, 1869; Jaeger, 1874; Paris, 1906; Cardot, 1908a; Herzog, 1923, 1954; Brothenus, 1925; Reimers, 1926; Malta, 1927; Thériot, 1935a; Kühnemann, 1938; Seki, 1974).

var. breviseta Malta (Malta, 1927).

Distr. Southern South America (Malta, 1927; Herzog, 1938; Seki, 1974).

U. glabella Mitt. (Mitten, 1860).

Syn. Orthotrichum glabellum (Mitt.) Mitt. (Mitten, 1869).

Orthotrichum luteolum Hook. f. et Wils. var. $\beta$ (Hooker and Wilson in Wilson and Hooker, 1847) pro parte.

Ulota eremitensis Mitt. (Mitten, 1860) fide Malta, 1927.

Orthotrichum eremitense Mitt. (Mitten, 1869) fide Malta, 1927.

Ulota hermitei Besch. (Bescherelle, 1889) nom. illeg.

Distr. Southern South America Milson and Hooker, 1847; Mitten, 1860, 1869; Jaeger, 1874; Bescherelle, 1885a, 1889; Mueller, 1885; Paris, 1906; Cardot, 1908a; Brotherus, 1925; Malta, 1927; Kühnemann, 1938; Seki, 1974).

U. gymnomitria C. Muell.

U. hamata Dus.

Orthotrichum gymnomitrium

$U$. hermitei Besch.

Ulota magellanica

U. immarginata Card.

U. incana (C. Muell.) Kindb.

Ulota glabella

Ulota pygmaeothecia

U. inclinata (C. Muell.) Kindb.

Orthotrichum incanum

Orthotrichum inclinatum

U. lativentrosa C. Muell. ex Malta (Mueller ex Malta, 1927).

Distr. Southern South America (Neger, 1899 (as Ulota latavenosa); Malta, 1927; Herzog. 1938).

U. leiothecia (C. Muell.) Jaeg.

Orthotrichum leiothecium

U. lobbiana Mitt. (Mitten, 1860).

Syn. Orthotrichum lobbianum (Mitt.) Mitt. (Mitten, 1869).

Ulota lobbii Mitt. ex Kindb. (Mitten ex Kindberg, 1888) nom. illeg.

Distr. Southern South America (Mitten, 1860, 1869; Jaeger, 1874; Paris, 1906; Cardot, 1908a; Brotherus, 1925; Malta, 1927).

U. lobbii Mitt. ex Kindb.

Ulota lobbiana

U. Iuteola (Hook. f. et Wils.) Wijk et Marg. (van der Wijk and Margadant, 1961).

Syn. Orthotrichum luteolum Hook. f. et Wils. (Hooker and Wilson in Wilson and Hooker, 1847) pro parte. 
Ulota fulvella Mitt. (Mitten, 1860) nom. illeg.

Orthotrichum fulvellum Mitt. (Mitten, 1869) nom. illeg.

Ulota savatieri Besch. (Bescherelle, 1885a).

Distr. Southem South America Wilson and Hooker, 1847; Sullivant, 1859; Mitten, 1860, 1869; Jaeger, 1874; Bescherelle, 1885a, 1889; Mueller, 1885; Eaton, 1892; Cardot, 1900. 1901, 1908a; Dusén, 1903a, 1905d; Paris, 1906; Cardot and Brotherus, 1923; Brotherus, 1925; Malta, 1927; Kühnemann, 1938; Herzog, 1957).

U. macrocalycina Mitt. (Mitten, 1860).

Syn. Orthotrichum macrocalycinum (Mitt.) Mitt. (Mitten, 1869). Orthotrichum marginatum Aongstr. (Ångström, 1872) fide Malta, 1927.

Ulota marginata (Aongstr.) Jaeg. (Jaeger in Jaeger and Sauerbeck, 1879b) fide Malta, 1927.

Ulota nothofagi Card. (Cardot, 1905a) fide Malta, 1927.

Distr. Southern South America (Mitten, 1860, 1869; Ångström, 1872, $1876 \mathrm{~b}$ (as $U$. fuegiana Mitt.); Jaeger, 1874; Bescherelle, 1885a; Mueller, 1885; Cardot, 1905a, 1908a; Paris, 1906; Brotherus, 1925; Malta, 1927; Kühnemann, 1938; Seki, 1974).

U. macrodontia Dus. ex Malta (Dusén ex Malta, 1927).

Distr. Southem South America (Dusén, 1903a; Malta, 1927; Herzog, 1954; Seki, 1974).

U. magellanica (Mont.) Jaeg. (Jaeger, 1874).

Syn. Orthotrichum magellanicum Mont. (Montagne, 1843).

Ulota hamata Dus. (Dusén, 1903a).

Distr. Southem South America (Montagne, 1843, 1845b, 1850, 1856; Mueller, 1849, 1885; Sullivant, 1859; Mitten, 1869; Angström, 1872; Jaeger, 1874; Bescherelle, 1885a; Dusén, 1903a, 1905d; Cardot, 1905a, 1908a; Paris, 1906; Brotherus, 1925; Malta, 1927; Herzog, 1938; Kühnemann, 1938; Bartram, 1952; Seki, 1974).

U. marginata (Aongstr.) Jaeg.

Ulota macrocalycina

U. nothofagi Card.

Ulota macrocalycina

U. persubulata Dus.

Ulota rufula

U. phyllantha Brid. (Bridel, 1819).

Syn. Orthotrichum phyllanthum (Brid.) Steud. (Steudel, 1824).

Weisia phyllantha (Brid.) Lindb. (Lindberg, 1879).

Orthotrichum jutlandicum Brid. (Bridel, 1826) nom. illeg.

Distr. Southern South America Wilson and Hooker, 1847 (as Orthotrichum crispum fide Malta, 1927); Sullivant, 1859; Mitten, 1869; Bescherelle, 1885a; Mueller, 1885; Paris, 1906; Cardot, 1908a; Cardot and Brotherus, 1923; Brotherus, 1925; Malta, 1927; Bartram, 1952).

U. pusilla Malta (Malta, 1927).

Distr. Southern South America (Malta, 1927).

U. pycnophylla Dus. ex Malta (Dusén ex Malta, 1927).

Distr. Southern South America (Malta, 1927).

U. pygmaeothecia (C. Muell.) Kindb. (Kindberg, 1888).

Syn. Orthotrichum pygmaeothecium C. Muell. (Mueller, 1885). Ulota immarginata Card. (Cardot, 1905a).

Distr. Southern South America (Bescherelle, 1885a; Mueller, 1885; Paris, 1905a; Cardot, 1905a, 1908a; Cardot and Brotherus, 1923; Malta, 1927; Kühnemann, 1938; Bartram, 1952; Seki, 1974).

U. rufula (Mitt.) Jaeg. (Jaeger, 1874).

Syn. Orthotrichum rufulum Mitt. (Mitten, 1869).

Orthotrichum brevicolle Mitt. (Mitten, 1869) fide Malta, 1927.

Ulota brevicollis (Mitt.) Jaeg. (Jaeger, 1874) fide Malta, 1927.

Orthotrichum chilense Mitt. (Mitten, 1869) fide Malta, 1927. 
Ulota chilensis (Mitt.) Jaeg. (Jaeger, 1874) fide Malta, 1927.

Ulota persubulata Dus. (Dusén, 1903a) nom. nud. fide Malta, 1927.

Distr. Juan Fernandez (Brotherus, 1924b; Bartram, 1959; Robinson, 1975).

Southern South America (Mitten, 1869; Jaeger, 1874; Dusén, 1903a; Paris, 1906; Herzog. 1923, 1938, 1954, 1957; Brotherus, 1925; Malta, 1927; Roivainen, 1934; Theriot, 1934b, 1935a; Seki, 1974).

var. fagiola Dus. ex Malta (Dusén ex Malta, 1927).

Distr. Southern South America (Malta, 1927).

var. patagonica Malta (Malta, 1927).

Distr. Southern South America (Malta, 1927; Seki, 1974).

U. savatieri Besch.

Ulota luteola

U. ventricosa (C. Muell.) Malta (Malta, 1927).

Syn. Zygodon ventricosus C. Muell. (Mueller, 1844b [1845]).

Distr. Southern South America (Mueller, i844b, 1849; Montagne, 1850; Mitten, 1869; Jaeger, 1874; Paris, 1906; Brotherus, 1925; Malta, 1927).

\section{Valdiviella}

V. pauperculata Thér. et Herz. (Thériot and Herzog in Herzog, 1928).

Distr. Southern South America (Herzog, 1928).

\section{Verrucidens}

V. intermedius Dix. (Dixon, 1920).

Syn. Hymenoloma intermedium (Dix.) Broth. (Brotherus, 1924a).

Distr. South Georgia (Dixon, 1920; Brotherus, 1924a; Steere, 1961b).

V. turpis (Card.) Card. (Cardot, 1908a).

Syn. Blindia turpis Card. (Cardot, 1905a).

Hymenoloma turpe (Card.) Card. et Broth. (Cardot and Brotherus, 1923).

Distr. Southern South America (Cardot, 1905a, 1908a; Cardot and Brotherus, 1923;

Brotherus, 1924a; Donat, 1936a; Kühnemann, 1938; Herzog, 1957; Seki, 1974).

\section{Vesicularia}

V. argentinica Broth. (Brotherus, 1918).

Distr. Southern South America (Brotherus, 1918, 1925; Kühnemann, 1938; Herzog, 1952a).

V. vesicularis (Schwaegr.) Broth. (Brotherus, 1908).

Syn. Hypnum vesciculare Schwaegr. (Schwaegrichen, 1827a).

Leskea rutilans Brid. (Bridel, 1827b).

Ectropothecium rutilans (Brid.) Mitt. (Mitten, 1869).

Distr. Southern South America (Krieger, 1904; Herzog, 1952a).

\section{Vesiculariopsis}

V. spirifolium (Dus.) Broth. (Brotherus, 1908).

Syn. Ectropothecium spirifolium Dus. (Dusén, 1905a).

Vesiculariopsis spiripes Broth. ex Par. (Brotherus ex Paris, 1909) err.

Achrohypnella subenervis Herz. (Herzog, 1954) fide Seki, 1974.

Distr. Southern South America (Dusén, 1903a, 1905b; Paris, 1904b; Brotherus, 1908, 1925; Cardot, 1080a; Kühnemann, 1938; Herzog, 1954; Matteri, 1972; Seki, 1974).

V. spiripes Broth. ex Par.

Vesiculariopsis spirifolium

Webera hom. illeg.

W. albicans Schimp.

Pohlia wahlenbergii

W. alticaulis (C. Muell.) Kindb. 
var. crassinervis Card.

var. robustior (C. Muell.) Par.

W. ampliretis (C. Muell.) Kindb.

W. antarctica (Hook. f. et Wils.) Jaeg.

W. calopyxis (C. Muell.) Kindb.

W. chilensis Par. (Paris, 1906) nom. nud.
Mniobryum alticaule var. crassinerve

Mniobryum alticaule var. robustius

Pohlia wahlenbergii var. glacialis

Bryum antarcticum

Pohlia calopyxis

Syn. Webera paucifolia Dus. (Dusén in Paris, 1906) nom. nud.

Distr. Southern South America (Paris, 1906).

W. commutata Schimp.

Pohlia drummondii

W. costesii Card. et Thér.

Pohlia polycarpa

W. cruda (Hedw.) Fuernr.

Pohlia cruda

var. imbricata Card.

W. drummondii (C. Muell.) Jaeg.

Pohlia cruda var. imbricata

W. emergens (C. Muell.) Kindb.

Pohlia drummondii

W. firmo-acuminata Dus. (Dusén in Paris, 1906) nom. nud.

Distr. Southern South America (Paris, 1906).

W. gerlachei Card.

Bryum gerlachei

W. hariotiana Besch.

W. humilis (Mont.) Broth.

Goniobryum subbasilare

Pohlia humilis

W. inflexa (C. Muell.) Kindb.

Pohlia inflexa

W. leptoclada Card. et Broth. (Cardot and Brotherus, 1923).

Distr. Falkland Islands (Cardot and Brotherus, 1923; Brotherus, 1924a; Kühnemann, 1938).

W. lonchochaete Dus.

Pohlia lonchochaete

W. looseri Thér. (Thériot, 1934b [1935]).

Distr. Southem South America (Looser, 1932; Thériot, 1934b).

W. magnifica Herz. (Herzog, 1954).

Distr. Southern South America (Herzog, 1954).

W. manca (C. Muell.) Kindb.

Mielichhoferia manca

W. meyeniana Hamp.

W. nevadensis (C. Muell.) Kindb.

Brachymenium meyenianum

W. nutans Hedw.

Pohlia nevadensis

var. minor (Hook.) Brid.

Pohlia nutans

W. paucifolia Dus.

W. philonotea (C. Muell.) Par.

W. philonotidea Par.

W. polycarpa (Mitt.) Jaeg.

W. pulvinata Kindb.

W. pyriformis Hedw.

W. racovitzae Card.

var. laxiretis Card.

W. sphagnadelphus (C. Muell.) Besch.

Mielichhoferia longiseta

Webera chilensis

Mniobryum philonoteum

Mniobryum philonoteum

Pohlia polycarpa

Pohlia nutans

Leptobryum pyriforme

Pohlia racovitzae

W. synoico-cruda (C. Muell.) Kindb.

Pohlia racovitzae var. laxiretis

Pohlia nutans

Pohlia cruda

W. timmiaecaulon Dus. (Dusén, 1903a) nom. nud.

Distr. Southem South America (Dusén, 1903a). 
W. viridata (C. Muell.) Kindb.

Pohlia cruda

W. wahlenbergii (Web. et Mohr) Fuernr.

Pohlia wahlenbergii

W. wilsonii (Mitt.) Jaeg.

Leptobryum wilsonii

\section{Weisa}

W. acuta Hedw.

Blindia acuta

W. alpicola (Hedw.) Poir.

Schistidium alpicola

$W$. amblyodon Brid.

$W$. antarctica (C. Muell.) Wils.

Weisia controversa var. amblyodon

W. apocarpa (Hedw.) Poir.

Dicranoweisia antarctica

$W$. argentinica $\mathrm{C}$. Muell.

Schistidium apocarpum

$W$. auridens C. Muell.

Hymenostomum argentinicum

var. robusta C. Muell.

W. balansaeana (Besch.) C. Muell.

W. bogotensis Hamp.

Oreoweisia bogotensis

W. calcarea (Nees et Hornsch.) C. Muell.

W. chilensis Hamp. ex C. Muell.

W. contecta Hook. f. et Wils.

Oreoweisia auridens var. robusta

Hymenostomum balansaeanum

Oreoweisia bogotensis

Gymnostomum aeruginosum

Oreoweisia chilensis

W. controversa Hedw. (Hedwig, 1801).

Syn. Grimmia controversa (Hedw.) Web. et Mohr (Weber and Mohr, 1803).

Bryum controversum (Hedw.) P. Beauv. (Palisot de Beauvois, 1805a).

Weisia viridula Hedw. ex Brid. (Hedwig ex Bridel, 1819) nom. illeg.

Simophyllum viridulum Lindb. (Lindberg, 1871) hom. illeg.

Tortella viridula C. Jens. (Jensen, 1923) nom. illeg.

Weisia flavipes Hook. f. et Wils. (Hooker and Wilson in Wilson, 1854).

Distr. Juan Fernandez (Bartram, 1959; Robinson, 1975).

Southern South America (Herter, 1933a; Piovano, 1954).

var. amblyodon (Brid.) Sendtn. (Sendtner, 1848).

Syn. Weisia amblyodon Brid. (Bridel, 1827a).

Weisia viridula Hedw. ex Brid. var. amblyodon (Brid.) B.S.G. (Bruch and others, 1846).

Distr. Southern South America (Herzog, 1954).

W. crispula sensu Wils. et Hook. (Wilson and Hooker, 1847). see Dicranoweisia antarctica

W. cryptodon Mont.

Camptodontium cryptodon

W. curvirostris C. Muell.

Hymenostylium recurvirostre

W. donniana (Sm.) Poir.

Grimmia donniana

W. felipponei Thér. (Thériot in Felippone, 1929 [1930]).

Distr. Southern South America (Felippone, 1929; Herter, 1933a).

$W$. flavipes Hook. f. et Wils.

Weisia controversa

W. fugax Hedw.

Rhabdoweisia fugax

W. gracilis (Hook.) Spreng.

Pilopogon gracilis

W. heteromalla Hedw.

Ditrichum heteromallum

W. kunzeana C. Muell.

Hymenostomum kunzeanum

W. latifolia Schwaegr.

Stegonia latifolia

W. longirostris Mitt.

Hymenostylium kunzeanum

W. patagonica Card. et Broth. (Cardot and Brotherus, 1923).

Distr. Southern South America (Cardot and Brotherus, 1923; Kühnemann, 1938). 
W. pentasticha (Brid.) Hedw.

W. perichaetialis Mont.

W. phyllantha (Brid.) Lindb.

W. plagiopodia (Hedw.) Poir.

W. poeppigiana (C. Muell.) Mitt.

W. recurvirostris Hedw.

W. recurvirostris (Hedw.) Dix.

W. richardii (Brid.) Schwaegr.

W. semi-involuta C. Muell.

W. sphaerothecia C. Muell.

W. tenuis Kunz. ex Mont.

$W$. verticillata Brid.

W. viridula Hedw. ex Brid.

var. amblyodon (Brid.) B.S.G.
Conostomum pentastichum

Eucamptodon perichaetialis

Ulota phyllantha

Grimmia plagiopodia

Trichostomopsis australasiae

Bryoerythrophyllum recurvirostre

Hymenostylium recurvirostre

Campylopus richardii

Hymenostomum semi-involutum

Amphidium tortuosum

Microdus kunzeanus

Eucladium verticillatum

Weisia controversa

Weisia controversa var. amblyodon

Weymouthia cochlearifolia var. billardieri

\section{Weymouthia}

W. billardieri (Hamp.) Broth.

W. cochlearifolia (Schwaegr.) Dix. (Dixon, 1927).

Syn. Hypnum cochlearifolium Schwaegr. (Schwaegrichen, 1816). Isothecium cochlearifolium (Schwaegr.) Mitt. (Mitten, 1856).

Stereodon cochlearifolius (Schwaegr.) Mitt. (Mitten, 1860).

Coelidium cochlearifolium (Schwaegr.) Reichdt. (Reichardt, 1870).

Porotrichum cochlearifolium (Schwaegr.) Mitt. (Mitten, 1883).

Lembophyllum cochlearifolium (Schwaegr.) Lindb. (Lindberg in Paris, 1896).

Distr. Southern South America (Montagne, 1850; Eaton, 1892; Paris, 1896, 1905a; Thériot, 1935a).

var. billardieri (Hamp.) Dix. (Dixon, 1927).

Syn. Neckera billardieri Hamp. (Hampe, 1860).

Pilotrichella billardieri (Hamp.) Jaeg. (Jaeger, 1877).

Meteorium billardieri (Hamp.) Mitt. (Mitten, 1882).

Weymouthia billardieri (Hamp.) Broth. (Brotherus, 1906b).

Pilotrichella kraussei Lor. (Lorentz, 1864).

Distr. Southern South America (Lorentz, 1864, 1866; Jaeger, 1877; Paris, 1905b; Brotherus, 1906b, 1925; Cardot, 1908a; Campo, 1915; Cardot and Brotherus, 1923; Herzog, 1923, 1938, 1939, 1940, 1954, 1960; Reimers, 1926; Bartram, 1952; Seki, 1974).

W. mollis (Hedw.) Broth. (Brotherus, 1906b).

Syn. Leskea mollis Hedw. (Hedwig, 1801).

Neckera mollis (Hedw.) C. Muell. (Mueller, 1850).

Meteorium molle Wils. (Wilson, 1854).

Isothecium molle (Hedw.) Mitt. (Mitten, 1856).

Stereodon mollis (Hedw.) Mitt. (Mitten, 1860) hom. illeg.

Pilotrichella mollis (Hedw.) Jaeg. (Jaeger, 1877).

Neckera cumingii C. Muell. (Mueller, 1850).

Pilotrichella cumingii (C. Muell.) Lor. (Lorentz in Jaeger, 1877).

Distr. Juan Fernandez (Montagne, 1835; Johow, 1896; Paris, 1905b; Skottsberg, 1914; Brotherus, 1924b; Thériot, 1927b; Espinosa B., 1941; Bartram, 1959; Robinson, 1975).

Southern South America (Montagne, 1850; Mueller, 1850, 1855; Lorentz, 1866; Mitten, 1869; Jaeger, 1877; Bescherelle, 1889; Neger, 1899; Paris, 1905b; Brotherus, 1906b, 1924c, 1925; Cardot, 1908a; Rowlee, 1916; Cardot and Brotherus, 1923; Herzog. 1923, 1938, 1954, 1960; Reimers, 1926; Roivainen, 1934; Espinosa B.. 1941; Skottsberg, 1950; Bartram, 1952; Bizot, 1967; Seki, 1974).

W. orbiculata Thér. 
Willia

W. austro-leucophaea (Besch.) Broth. (Brotherus, 1902).

Syn. Grimmia austro-leucophaea Besch. (Bescherelle, 1885a).

Guembelia austro-leucophaea C. Muell. (Mueller in Paris, 1895) nom. nud.

Willia grimmioides C. Muell. (Mueller, 1890a) fide Bell, 1974.

Willia senilis C. Muell. (Mueller, 1901a [1900]).

Grimmia senilis Besch. (Bescherelle in Mueller, 1901a [1900]) nom. nud.

Distr. Southern South America (Bescherelle, 1885a, 1889; Mueller, 1901a; Brotherus, 1902, 1924a; Paris, 1906; Cardot, 1908a).

South Georgia (Mueller, 1890a; Cardot, 1906a, 1908a; Paris, 1906; Brotherus, 1924a; Steere, 1961b; Bell, 1974).

W. grimmioides C. Muell.

Willia austro-leucophaea

W. senilis C. Muell.

Willia austro-leucophaea

\section{Wollyan}

W. pottiacea (Dus.) Herz.

Leptobryum pottiaceum

W. wilsonii (Mitt.) Herz.

Leptobryum wilsonii

Zieria hom. illeg.

Z. lamprocarpa (C. Muell.) Kindb.

Bryum lamprocarpum

\section{Zygodon}

Z. bartramioides Malta (Malta, 1924).

Syn. Pentastichella bartramioides Dus. (Dusén in Malta, 1924) nom. nud.

Zygodon cylindropyxis C. Muell. (Mueller in Malta, 1926) nom. nud.

Stenomitrium robustulum Par. (Paris, 1905b) nom. nud.

Pentastichella robustula Dus. (Dusén in Paris, 1905b) nom. nud.

Zygodon robustulus Broth. (Brotherus in Malta, 1926) nom. nud.

Distr. Southern South America (Neger, 1899; Paris, 1905b; Malta, 1924, 1926; Brotherus, 1925; Herzog, 1938, 1954; Seki, 1974).

Z. caildensis Aongstr.

Z. coarctatus Schimp.

Zygodon reinwardtii

Z. coralensis Lor. ex C. Muell.

Zygodon reinwardtii

Z. corralensis Lor. (Lorentz, 1866).

Zygodon corralensis

Syn. Ceratodon corralensis (Lor.) Jaeg. (Jaeger, 1873).

Zygodon coralensis Lor. ex C. Muell. (Lorentz ex Mueller, 1879a) err.

Distr. Juan Fernandez (Malta, 1926).

Southern South America (Lorentz, 1866; Jaeger, 1873, 1874; Paris, 1906; Malta, 1924 1926; Brotherus, 1925; Herzog, 1938, 1954; Seki, 1974).

Z. curvicaulis Dus.

Z. cyathicarpus Mont.

Zygodon intermedius

Z. cylindropyxis C. Muell.

Z. denticulatus Tayl.

$Z$. erythrocarpus C. Muell.

$Z$. excelsus C. Muell.

Z. gracillimus Dus. Amphidium tortuosum

$Z$. hatcheri Dus. Zygodon bartramioides Zygodon reinwardtii

Z. hyadesii Besch. var. inermis Dus. Zygodon reinwardtii var. hyadesii

Zygodon obtusifolius

Molendoa sendtneriana

Zygodon papillatus

Macromitrium sp.

Zygodon inermis

Z. hystrix Dus.

Zygodon pichinchensis 
Z. inermis Malta (Malta, 1926).

Syn. Zygodon hyadesii Besch. var. inermis Dus. (Dusén in Malta, 1926) nom. nud.

Distr. Southern South America (Malta, 1926; Herzog, 1940; Seki, 1974).

Z. intermedius B.S.G. (Bruch and others, 1838).

Syn. Zygodon kraussei Lor. (Lorentz, 1864).

Zygodon curvicaulis Dus. (Dusén, 1903a).

Zygodon pseudoventricosus C. Muell. (Mueller in Malta, 1926) nom. nud.

Distr. Juan Femandez (Malta, 1926; Thériot, 1927b; Bartram, 1959; Robinson, 1975).

Southern South America (Montagne, 1850; Lorentz, 1864, 1866; Jaeger, 1873, 1874;

Dusén, 1903a; Paris, 1906; Cardot, 1908a; Cardot and Brotherus, 1923; Brotherus, 1925; Malta, 1926; Kühnemann, 1938; Herzog, 1954; Seki, 1974).

Z. jaffuelii Thér.

Zygodon liebmannii

Z. kraussei Lor.

Zygodon intermedius

Z. liebmannii Schimp. (Schimper in Mueller, 1849).

Syn. Zygodon jaffuelii Thér. (Thériot, 1921a).

Distr. Southern South America (Costes, 1921; Thériot, 1921a; Malta, 1926; Herzog, 1938. 1954; Seki, 1974).

Z. linearis C. Muell.

Anoectangium lineare

Z. magellanicus Dus. ex Malta (Dusén ex Malta, 1926).

Distr. Southem South America (Brotherus, 1925; Malta, 1926; Seki, 1974).

Z. mandonianus Schimp. ex C. Muell.

Anoectangium lineare

Z. menziesii (Schwaegr.) Arnott (Arnott, 1827).

Syn. Codonoblepharon menziesii Schwaegr. (Schwaegrichen, 1824).

Distr. Juan Fernandez (Brotherus, 1924b; Robinson, 1975).

Southem South America (Mueller, 1844b, 1849; Montagne, 1850; Mitten, 1869; Jaeger, 1874; Paris, 1906; Brothenus, 1925; Malta, 1926; Herzog, 1954; Seki, 1974).

Z. obovalis Mitt. (Mitten, 1885).

Distr. Juan Fernandez (Mitten, 1885; Johow, 1896; Paris, 1900a, 1906; Brotherus, 1924b, 1925; Malta, 1926).

Z. obtusifolius Hook. (Hooker, 1819).

Syn. Zygodon erythrocarpus C. Muell. (Mueller, 1879a).

Zygodon rufulus Dus. (Dusén in Malta, 1926) nom. nud.

Distr. Southern South America (Mueller, 1879a; Paris, 1906; Malta, 1924, 1926; Brotherus, 1925; Kühnemann, 1938).

var. antarcticus Malta (Malta, 1924).

Distr. Southern South America (Malta, 1924, 1926).

Z. ochraceus C. Muell. (Mueller, 1879a).

Syn. Zygodon pygmaeus C. Muell. (Mueller, 1879a).

Distr. Southem South America (Mueller, 1879a; Paris, 1906; Brotherus, 1925; Malta, 1926; Kühnemann, 1938).

Z. orthotrichoides (C. Muell.) Broth.

Leptodontium orthotrichoides

Z. palmarum C. Muell.

Leptodontium orthotrichoides

Z. papillatus Mont. (Montagne, 1845a).

Syn. Codonoblepharon papillatum (Mont.) Jaeg. (Jaeger, 1874).

Zygodon papillosus Mont. ex Broth. (Montagne ex Brotherus, 1902) err.

Zygodon gracillimus Dus. (Dusén, 1903a) nom. nud. 
Distr. Southern South America (Montagne, 1845a, 1850, 1856; Mueller, 1849; Mitten, 1869; Jaeger, 1874; Brotherus, 1902, 1925; Dusén, 1903a; Paris, 1906; Malta, 1926; Herzog, 1960; Seki, 1974).

Z. papillosus Mont. ex Broth.

Zygodon papillatus

Z. pentastichus (Mont.) C. Muell. (Mueller, 1849).

Syn. Aulacomnium pentastichum Mont. (Montagne, 1845a).

Stenomitrium pentastichum (Mont.) Broth. (Brotherus, 1902).

Pentastichella pentasticha (Mont.) C. Muell. (Mueller in Thériot, 1918) comb. inval.

Zygodon uncinatus Mitt. (Mitten, 1869).

Codonoblepharon uncinatum (Mitt.) Jaeg. (Jaeger, 1874).

Macromitrium poeppigii Dub. (Duby, 1868).

Distr. Juan Fernandez (Skottsberg, 1914; Brotherus, 1924b; Malta, 1926; Espinosa B., 1941; Bartram, 1959; Robinson, 1975).

Southern South America (Montagne, 1845a, 1850, 1856; Mueller, 1849; Lorentz, 1866; Duby, 1868; Mitten, 1869; Jaeger, 1874; Neger, 1899; Brotherus, 1902, 1925; Paris, 1905a, b, 1906; Cardot, 1908a; Campo, 1915, 1921; Thériot, 1918; Cardot and Brotherus, 1923; Herzog, 1923, 1938, 1939, 1954, 1957, 1960; Malta, 1926;

Reimers, 1926; Seki, 1974).

var. crispatulus (Thèr.) Malta (Malta, 1926).

Syn. Zygodon porteri Thér. var. crispatulus Thér. (Thériot, 1926).

Distr. Southern South America (Malta, 1926; Thériot, 1926).

var. strictifolius Malta (Malta, 1926).

Syn. Pentastichella pentasticha (Mont.) C. Muell var. strictifolia Thér. (Thériot, 1924) comb. inval.

Distr. Southern South America (Thériot, 1924; Malta, 1926).

Z. perpusillus Thwait. et Mitt.

Rhachithecium perpusillum

Z. pichinchensis (Tayl.) Mitt. (Mitten, 1851).

Syn. Didymodon pichinchensis Tayl. (Taylor, 1848b).

Zygodon hystrix Dus. (Dusén in Paris, 1906) nom. nud.

Distr. Southern South America (Paris, 1906; Brotherus, 1925; Malta, 1926; Seki, 1974).

Z. podocarpi C. Muell. ex Malta (Mueller ex Malta, 1926).

Distr. Southern South Anerica (Malta, 1926).

Z. porteri Thér. (Thériot, 1926).

Syn. Pentastichella jaffuelii Thér. (Thériot, 1921a) comb. inval.

Distr. Southern South America. (Thériot, 1921a, 1926).

var. crispatulus Thèr.

Z. pseudoventricosus C. Muell.

Zygodon pentastichus var. crispatulus

$Z$. pygmaeus C. Muell.

Zygodon intermedius

Zygodon ochraceus

Z. reinwardtii (Hornsch.) Braun (Braun in Bruch and others, 1838).

Syn. Syrrhopodon reinwardtii Hornsch. (Hornschuch in Reinwardt and Hornschuch, 1826 [1829]). Zygodon denticulatus Tayl. (Taylor, 1847).

Zygodon subdenticulatus Hamp. (Hampe, 1865).

Zygodon caldensis Aongstr. (Ångström, 1876a).

Zygodon coarctatus Schimp. (Schimper in Paris, 1898) nom. nud.

Distr. Southern South America (Mitten, 1869; Jaeger, 1874; Paris, 1898, 1906; Brotherus, 1925; Malta, 1926; Bartram, 1952).

var. hyadesii (Besch.) Malta (Malta, 1926).

Syn. Zygodon hyadesii Besch. (Bescherelle, 1885a).

Distr. Southern South America (Bescherelle, 1885a, 1889; Paris, 1906; Cardot, 1908a; Cardot and Brotherus, 1923; Brotherus, 1925; Malta, 1926; Kühnemann, 1938; Seki, 1974). 
Z. robustulus Broth.

Z. rufulus Dus.

Z. subdenticulatus Hamp.

Zygodon reinwardtii

Z. tenerrimus (C. Muell.) C. Muell.

Gymnostomum tenerrimum

Z. theriotii Herz. (Herzog, 1938).

Distr. Southem South America (Herzog, 1938).

Z. tristis Herz. (Herzog, 1960 [1961]).

Distr. Southern South America (Herzog, 1960).

$Z$. uncinatus Mitt.

$Z$. ventricosus $\mathrm{C}$. Muell.

Zygodon pentastichus

Ulota ventricosa

\section{ACKNOWLEDGEMENTS}

IT is a pleasure to acknowledge the support given by Sir Vivian Fuchs, FRS, who was Director of the British Antarctic Survey when this work was started. I am also grateful to $\operatorname{Dr}$ M. W. Holdgate, $\mathrm{CB}$ and Mr J. N. R. Jeffers, respectively, the former and current Directors of the Institute of Terrestrial Ecology, who facilitated its continuation to completion.

I wish to thank Miss P. I. Edwards, a former Head of the Library in the Department of Botany, British Museum (Nat. Hist.), who most generously spent many hours helping in the search for and verification of references, and $\mathrm{Mr} \mathrm{A}$. Eddy, of the same institution, who gave valuable expert advice on the presentation of data in Sphagnum. The help of Professor F. A. Stafleu and DrW. D. Margadant, both of the University of Utrecht, is also gratefully acknowledged for assistance with dating queries. Staff in the Inter-Library Loans Service of the University of Birmingham (where the work was started), and of my present Institute, gave much essential assistance which facilitated my consultation of many works.

Of my colleagues, first Mrs J. Crawford and then Mrs A. Halcrow, gave continuous and unstinting support in the typing up of a voluminous manuscript, while Mr B. G. Bell is acknowledged for much helpful discussion throughout the work. A special word of thanks is reserved for $\mathrm{Dr} R$. J. Adie, who advised on the presentation of the text and spent countless hours seeing it through the various stages of publication, and for $\operatorname{Dr} \mathrm{S}$. W. Greene, who suggested the project, helped in the search for literature and advised on the solution of many problems, and whose constant encouragement ensured its completion and final publication. 


\section{REFERENCES}

For each reference the date following the author's name is the bibliographic or title-page date.

Where the date of issue, i.e. the taxonomically effective date, differs from the title-page date, the former is given in square brackets at the end of the reference. For further discussion see Introduction.

AMANN, J. 1916. Ėtude sur la flore bryologique du Valais, 1912-1914. Bull. Murithienne, Fasc. 39 (for 1914-15), 350-55.

AMANN, J. 1924. Le Bryum schleicheri Schwaegr. Revue bryol., 51, No. 2, 20-22.

AMANN, J. 1928. Nouvelles additions et rectifications à la flore des mousses de la Suisse. Mém. Soc. vaud. Sci. nat., Sèr. 5, Mèm. No. 16 , 2, No. 9 [Mém. No. 17, 3, No. 2], 25-64.

ANDO, H. 1972. Distribution and speciation in the genus Hypnum in the circum-Pacific region. $J$. Hattoribot. Lab., No. 35, 68-98.

ANDO, H. 1973. Studies on the genus Hypnum Hedw. II. J. Sci. Hiroshima Univ., Ser. B, Div. 2 (Botany). 14, No. 3, 165-207.

ANDO, H. 1976. Studies on the genus Hypnum Hedw. III. J. Sci. Hiroshima Univ., Ser. B, Div. 2 (Botany), 16, No. 1, 1-46. [Effective date 20 November 1977, according to Ando personal communication.]

ANDREWS, A. Le R. 1935. Bryaceae. (In Grout, A. J., ed. Moss flora of North America north of Mexico. Newfane, Vermont, A. J. Grout, 2, Pt. 3, 184210.)

ANDREWS, A. LE R. 1941. Notes on the Warnstorf Sphagnum herbarium. III. The subgenus Inophloea in South America. Bryologist, 44, No. 6, 155-59.

ANDREWS, A. LE R. 1947. Studies in the Warnstorf Sphagnum herbarium. IV. The group Acutifolia in South America. Bryologist, 50, No. 2, 181-86.

ANDREWS, A. LE R. 1949a. Taxonomic notes. VII. The genus Acrocladium. Bryologist, 52, No. 2, 72-77.

ANDREWS, A. LE R. 1949b. Studies in the Warnstorf Sphagnum herbarium. V. The group Cuspidata in South America. Bryologist, 52, No. 3, 124-30.

ANDREWS, A. LE R. 1951. Taxonomic notes. X. The family Leptostomaceae. Bryologist, 54, No. 4, 217-23. [Effective date 16 February 1952; see 55, No. 1, 84.]

ÅNGSTRÖM, J. 1872. Förteckning och beskrifning öfver mossor, samlade af Professor N.J. Andersson under Fregatten Eugenies verldsomsegling åren 1851-53. Öfvers. $K$. VetenskAkad. Förh., 29, No. 4, 3-29.

ÅNGSTRÖM, J. 1876a. Primae lineae muscorum cognoscendorum, qui ad Caldas Brasiliae sunt collecti. Öfvers. K. VetenskAkad. Förh., 33, No. 4, 3-50.

ÅNGSTRÖM, J. 1876b. Rättelser och tillägg till förteckning och beskrifning öfver mossor. samlade af Professor N. J. Andersson under Fregatten Eugenies verldsomsegling 1851-1853, i Öfversigt af Kongl. VetenskapsAkademians Förhandlingar 1872, No. 4. Öfvers. $K$. VetenskAkad. Förh., 33, No. 4, 50-52.

Arnell, H. W. and C. Jensen. 1910. Die Moose des Sarakgebietes, Abh. 2 und 3. Naturw. Unters. sarekgebirg, 3, Botanik, Lief. 3, 144-268.

ARNOTT, G. A. W. 1824a. Notice of a "Journal of a voyage from Rio de Janeiro to the coast of Peru, by Mr. William Jameson, surgeon, corresponding member of the Wernerian Natural History Society". Mem. Wernerian nat . Hist. Soc., 5, Pt. 1, 187-205.
ARNOT, [G.A.] W. 1824b. Notice sur quelques mousses de Rio-Janeiro. Mém. Soc. Hist. nat. Paris, 1, Pt. 2, 346-52.

ARNOT, G. A. W. 1827. Nouvelle disposition méthodique. Des espèces de mousses exactement connues, par M. G.-A. WalkerArnott, correspondent à Edimbourg; précédée d'un rapport fait à son sujet à la Société Linnéene par M. B. Kittel, d.m.; suivie de notes critiques et 'additions qui complètent ce travail et le mettent à la hauteur des connaissances actuelles. Mém. Soc. linn. Paris, 5, Pt. 2, 205-324.

Austin, C. F. 1877. Notes. Bot. Gaz., 2, No. 12, 142-43.

Austin, C. F. 1880. Bryological notes. Bull. Torrey bot. Club, 7, No. 2, 15.

BalANSA, B. andT. Savès. 1887. Musci exotici. Rev. bryol., 14, No. 4, 56-57.

BARTRAM, E. B. 1931. A review of the American species of Daltonia. Bull. Torrey bot. Club, 58, No. 1, 31-48.

BARTRAM, E. B. 1936. New mosses from Argentina. (In BAUER, E., ed. Musci europaei et americani exsiccati. Appendix ad seriem 46. Stadt Olbersdorf, Ludwig Richter, 1-7.)

BARTRAM, E. B. 1938. The Second Byrd Antarctic Expedition - botany. Ill. Mosses. Ann. Mo. bot. Gdn, 25, No. 2, 719-24.

BARTRAM, E. B. 1942. Additions to the moss flora of northern Chile. Revue bryol. lichen., N. S., 13, Fasc. 1, 130-34. [Issued as Travaux bryologiques dédiès à le Mémoire de PierreTranquille Husnot, Botaniste normand, Fondateur de la Revue Bryologique (1840-1929). Paris, Laboratoire de Cryptogamie, Muséum National d'Histoire Naturelle, 1942.]

BARTRAM, E. B. 1943. Additions to the moss flora of northern Chile. Farlowia, 1, No. 2, 191-94.

Bartram, E. B. 1946. New mosses from Tierra del Fuego. Farlowia, 2, No. 3, 309-19.

BARTRAM, E. B. 1952. Mosses of Chile and Argentina mainly collected by R. Santesson. Svensk bot. Tidskr., 46, Ht. 2, 242-53.

BARTRAM, E. B. 1957. Mosses from the United States Antarctic Service Expedition, 1940-41. Bryologist, 60, No. 2, 139-43.

BARTRAM, E. B. 1959. Mosses collected during Dr. and Mrs. C. Skottsberg's second expedition to the Juan Fernandez Islands, December 1954 to March 1955. Ark. Bot., Ser. And., 4, Häfte 1, 29-43.

BARTRAM, E. B. 1965. New and noteworthy mosses from northern Argentina. Revue bryol. lichen., N.S., 33 (for 1964-65), Fasc. 3-4, 323-27.

BAUER, E. 1931. Musci europaei et americani exsiccati. Schedae und Bemerkungen zur 43 serie, Nos. 2101-50. [Prague, E. Bauer.]

BAUER, E. 1934. Musci europaei et americani exsiccati. Schedae und Bemerkungen zur 45 serie. Nos. 2201-2250. Stadt Olbersdorf, Ludwig Richter.

BÉHERÉ, J. B. J. 1826. Muscologia Rothomagensis, ou tableau analytique des mousses découvertes jusqu'a ce jour aux environs de Rouen. Rouen, F. Baudry. 
BEL, B. G. 1973a. Notes on Antarctic bryophytes: I. New records from the Antarctic botanical zone. British Antarctic Survey Bulletin, No. 36, 131-32. [Effective date 21 June 1974.]

BEL, B. G. 1973b. A synoptic flora of South Georgian mosses: Il. Chorisodontium, Dicranoloma, Dicranum, Platyneurum and Conostomum. British Antarctic Survey Bulletin, No. 37, 33-52. [Effective date 15 August 1974.]

BEL, B. G. 1973c. Notes on Antarctic bryophytes: II. Records of Racomitria from the Antarctic botanical zone. British Antarctic Survey Bulletin, No. 37, 91-94. [Effective date 15 August 1974.]

BEL, B. G. 1974. A synoptic flora of South Georgian mosses: V. Willia and Racomitrium. British Antarctic Survey Bulletin, No. 38, 73-101.

BEL, B. G. 1976. Notes on Antarctic bryophytes: VI. The genus Dicranoweisia on Signy Island, South Orkney Islands. British Antarctic Survey Bulletin, No. 44, 97-100.

BELL, B. G. 1977a. Notes on Antarctic bryophytes: VIII. Two species of Campylopus originally described from South Georgia. British Antarctic Survey Bulletin, No. 46, 136-37.

BELL, B. G. 1977b. Notes on Antarctic bryophytes: IX. A previously unreported botanical collection made on South Georgia during the German International Polar-Year Expedition, 1882-83. British Antarctic Survey Bulletin, No. 46, 137-39.

BERTSCH, K. 1949. Moosflora. Stuttgart, Verlag Eugen Ulmer.

Bescherelle, E. 1871-72. Prodromus bryologiae mexicanae ou énumération des mousses du Mexique avec description des espèces nouvelles. Mém. Soc. natn. Sci. nat. math. Cherbourg, 16, [Sér. 2, 6], 145-256. [Effective date 1872, fide Crum, 1950.]

BesCheRELLE, E. 1876. Florule bryologique des Antilles Françaises. Annls Sci. nat., Sér. 6, 3, Botanique, 175-265.

BesCHERELIE, E. 1877. Note sur les mousses de Paraguay. Récoltées par M. Balansa de 1874 à 1877. Mém. Soc. natn. Sci. nat. math. Cherbourg, 21, [Sér. 3, 1], 257-72.

Bescherelle, E. 1885a. Mousses nouvelles de l'Amérique australe. Bull. Soc. bot. Fr., 32, LIV-LXIX

BesCherelle, E. 1885b. Liste des mousses du Paraguay distribuées en 1884 par M. Balansa. Revue bryol., 12, No. 2, 17-19.

Bescherelle, E. 1889. Mousses. (In Bescherelle, E. and C. MAssalongo. Muscinées. Mission Scientifique du Cap Horn 1882-83. Vol. 5. Botanique, 253-312.)

BESCHERELIE, E. 1891. Selectio novorum muscorum. J. Bot., Paris, 5, No. 9, 142-48; No. 15, 252-55; No. 20, 342-50.

Bizot, M. 1967. Quelques mousses africaines et americaines. Bull. Soc. bot. Fr., 114, No. 9, 423-27.

Bizot, M. andG. Piovano. 1953. Musci brasilienses. Dusenia, 5, Fasc. 5-6, 443-52.

BLANDOW, 0. 1809. Uebersicht der Mecklen-

burgischen Moose nach alphabetischer Ordnung entworfen. Neustrelitz, Kosten.

Boulay, J. N. 1884. Muscinées de la France. Part 1. Mousses. Paris, F. Savy.

BraithwaIte, R. 1893, The British moss flora. Vol. 2. London, R. Braithwaite, Pt. 15, 183-218.

BraithWAITE, R. 1895. The British moss flora. Vol. 2. London, R. Braithwaite, Pt. 16, 221-68.

BraithWAITE, R. 1898. The British moss flora. Vol. 3. London, R. Braithwaite, Pt. 18, 37-64.
BRIDEL, S. E. 1798. Muscologia recentiorum seu analysis, historia, et descriptio methodica omnium muscorum frondosorum, hucusque cognitorum, ad norman Hedwigii. Gothae, Carolum Guil. Ettinger; Paris, Barrois Junior, 2, Pt. 1, 1-222.

BRIDEL, S. E. 1801a. Animaduersiones in Muscologiae Recentiorum tomum secundum, ab ipso auctore propositae. J. Bot., Göttingen, 1 (for 1800), Stück 2, 268-99.

BRIDEL, S. E. 1801 b. Muscologia recentiorum seu analysis, historia et descriptio methodica omnium muscorum frondosorum hucusque cognitorum ad norman Hedwigii. Gothae, Carol Guil. Ettinger; Paris, Barrois Junior, 2, Pt. 2, 1-198.

BRIDEL, S. E. 1802. Fortsetzung der Nro. 13 abgebrochen Recension über: Sam. El. Bridel, Muscologia etc. Bot. Ztg Erlangen, 1, Ht. 3, No. 14, 209-18.

BRIDEL, S. E. 1803. Muscologia recentiorum seu analysis, historia et descriptio methodica omnium muscorum frondosorum hucusque cognitorum ad normam Hedwigii. Gothae, Carol Guil. Ettinger; Paris, Barrois Junior, 2, Pt. 3, 1-182.

BRIDEL, S. E. 1806. Muscologiae recentiorum. Supplementum seu species muscorum, Pars I. Gothae, Carol Guil. Ettingeriana.

BRIDEL, S. E. 1812. Muscologiae recentiorum. Supplementum seu species muscorum, Pars II. Gothae, Libraria Ettingeriana.

BRIDEL, S. E. 1817. Muscologiae recentiorum. Supplementum seu species muscorum, Pars III. Gothae, Libraria Ettingeriana.

BRIDEL, S. E. 1819. Muscologiae recentiorum supplementum Pars $\mathrm{N}$, seu mantissa generum specierumque muscorum frondosorum universa. (Methodus nova muscorum ad naturae normam melius instituta et muscologiae recentiorum accommodata). Gothae, A. Ukertum.

BRIDEL, S. E. 1826. Bryologia universa seu systematica ad novam methodum dispositio. historia et descripto omnium muscorum frondosorum hucusque cognitorum cum synonymia ex auctoribus probatissimis. Vol. I. Lipsiae, Joan. Ambros. Barth. (1-746.)

BRIDEL, S. E. 1827a. Bryologia universa seu systematica ad novam methodum dispositio, historia et descripto omnium muscorum frondosorum hucusque cognitorum cum synonymia ex auctoribus probatissimis, supplementum ad volumen primum. Lipsiae. Joan. Ambros. Barth. (747-856.)

BRIDEL, S. E. 1827 b. Bryologia universa seu systematica ad novam methodum dispositio, historia et descripto omnium muscorum frondosorum hucusque cognitorum cum synonymia ex auctoribus probatissimis. Vol. II. Lipsiae, Joan. Ambros. Barth. (1-848.)

Baitron, E. G. 1896. An enumeration of the plants collected by H. H. Rusby, in Bolivia, 1885-1886: II. Musci. Bull. Torrey bot. Club, 23, No. 12, 471-99.

Britton, E. G. 1913. Ditrichaceae. (In North America flora, Vol. 15, Pt. 1. New York, New York Botanical Garden, 55-67.)

BritTon, E. G. 1914. Notes on nomenclature - XII. Bryologist, 17, No. 1, 7-10.

Brotero, F. A. 1804. Musci. (In Flora Lusitanica, seu plantarum, quae in Lusitania vel sponte crescunt, vel frequentius coluntur, ex florum praesertim sexubus systematice distributarum, 
synopsis. Pars 2. Olisipone, Typographia Regıa, 403-25.)

Brotherus, V. F. 1891. Some new species of Australian mosses. Öfvers. finska VetenskSoc. Forh., 33 (for 1890-91), 89-110.

Brotherus, V. F. 1893. Contributions à la flore bryologique du Brésil. Acta Soc. Sci. fenn., 19, No. 5, 3-30. [Effective date 1891 according to Revue bryol., 18, No. 5, 80.]

Brotherus, V. F. 1895. Nouvelles contributions à la flora bryologique du Brésil. Bih. K. svenska VetenskAkad. Handl., 21, Afd. 3, No. 3, 1-76.

Brotherus, V. F. 1898. Some new species of Australian mosses. Öfvers. finska VetenskSoc. Forh., 40 (for 1897-98), 159-93.

BROTHERUS, V. F. 1900. Die Laubmoose der ersten regnellschen expedition. Bih. K. svenska VetenskAkad. Handl., 26, Afd. 3, No. 7, 1-65.

Brotherus, V. F. 1901. Musci (Laubmoose). II Unterklasse Andreaeales, Andreaeaceae; III Unterklasse Bryales: II. Spezieller Teil, Gruppe I. Acrocarpi. (In ENGLER, A. and K. PRANTL. Die natürlichen Pflanzenfamilien. Leipzig, Wilhelm Engelmann, Teil 1, Abt. 3, Hälfte 1, Lief. 207. 265-68, 277-88; Lief. 208, 289-336; Lief. 212, 337-84.)

Brotherus, V. F. 1902. Musci (Laubmoose). III Unterklasse Bryales: II. Spezieller Teil, Gruppe I. Acrocarpi. (In ENGLER, A. and K. PRANTL. Die natürlichen Pflanzenfamilien. Leipzig, Wilhelm Engelmann, Teil 1, Abt. 3, Hälfte 1, Lief. 214, 385-432; Lief. 215, 433-80.)

Brotherus, V. F. 1903. Musci (Laubmoose). III Unterklasse Bryales: II. Spezieller Teil, Gruppe I. Acrocarpi. (In ENGLER, A. and K. PRANTL. Die natürlichen Pflanzenfamilien. Leipzig, Wilhelm Engelmann, Teil 1, Abt. 3, Hälfte 1, Lief. 216, 481-528; Lief. 218, 529-76.)

Brotherus, V. F. 1904. Musci (Laubmoose). III Unterklasse Bryales: II. Spezieller Teil, Gruppe I. Acrocarpi. (In ENGLER, A. and K. PrantL. Die natürlichen Pflanzenfamilien. Leipzig, Wilhelm Engelmann, Teil 1, Abt. 3, Hälfte 1, Lief. 219, 577-624; Lief. 220, 625-72.)

Brotherus, V. F. 1905a. Musci (Laubmoose). III Unterklasse Bryales: II. Spezieller Teil, Gruppe I. Acrocarpi. (In ENGLer, A. and K. PrantL. Die natürlichen Pflanzenfamilien. Leipzig, Wilhelm Engelmann, Teil 1, Abt. 3, Hälfte 1, Lief. 222, 673-700.)

Brotherus, V. F. 1905b. Musci (Laubmoose). III Unterklasse Bryales: II. Spezieller Teil, Gruppe II. Pleurocarpi. (In ENGLER, A. andK. PrANTL. Die natürlichen Pflanzenfamilien. Leipzig, Wilhelm Engelmann, Teil 1, Abt. 3, Hälfte 2, Lief. 222 701-20; Lief. 223, 721-68.)

Brotherus, V. F. 1905c. Pleurorthotrichum, eine neue Laubmoosgattung aus Chile. Öfvers. finska VetenskSoc. Förh., 47, No. 15, 1-4.

Brotherus, V. F. 1906 a. Die Laubmoose der Deutschen Südpolar-Expedition 1901-1903. Dt. Südpol.-Exped., 8, Botanik, Ht. 1, 83-96.

Brotherus, V. F. 1906b. Musci (Laubmoose). III Unterklasse Bryales: II. Spezieller Teil, Gruppe II. Pleurocarpi. (In Engler, A. and K. PrantL. Die natürlichen Pflanzenfamilien. Leipzig, Wilhelm Engelmann, Teil 1, Abt. 3, Hälfte 2, Lief. 224. 769-816; Lief. 226, 817-64.)

Brotherus, V. F. 1907. Musci (Laubmoose). III Unterklasse Bryales: II. Spezieller Teil, Gruppe II. Pleurocarpi. (In ENGLER, A. and K. PRANTL. Die natürlichen Pflanzenfamilien. Leipzig, Wilhelm Engelmann, Teil 1, Abt. 3, Hälfte 2, Lief. 227/28,
865-960; Lief. 229, 961-1008.)

BROTHERUS, V. F. 1908. Musci (Laubmoose). III Unterklasse Bryales: II. Spezieller Teil, Gruppe II. Pleurocarpi. (In ENGLER, A. and K. PrantL. Die natürlichen Pflanzentamilien. Leipzig, Wilhelm Engelmann, Teil 1, Abt. 3, Hälfte 2, Lief. 231, 1009-56; Lief. 232/33, 1057-152.)

BrotHerus, V. F. 1909. Musci (Laubmoose). III Unterklasse Bryales: II. Spezieller Teil, Gruppe II. Pleurocarpi. (In Engler, A. and K. Prantr. Die natürlichen Pflanzenfamilien. Leipzig, Wilhelm Engelmann, Teil 1, Abt. 3, Hälfte 2, Lief. 234/35, 1153-246.)

Brotherus, V. F. 1910a. Contributions to the bryological flora of the Philippines, III. Philipp. J. Sci., Sect. C, Botany, 5, No. 2, 137-62.

BROTHERUS, V. F. 1910 b. Schedae ad bryothecam fennicam. N: ri 1-100. Helsingforsiae, J. Simelii.

BrotherUS, V. F. 1916. Descriptions of some new species of Australian, Tasmanian and New Zealand mosses. VI. Proc. Linn. Soc. N.S.W., 41, No. 163, 575-96.

BRotherus, V. F. 1918. Contributions à la flore bryologique de l'Argentine. Ark. Bot., 15, Häfte 1-2, 1-15.

Brotherus, V. F. 1920. Contributions à la flore bryologique de l'Ecuador. Revue bryol., 47, No. 1, 1-16.

Brotherus, V. F. 1923. Die Laubmoose Fennoskandias. Helsingfors, Akademische Buchhandlung; Berlin, R. Friedländer \& Sohn; London, Wheldon \& Wesley Ltd. [Societas pro Fauna et Flora Fennica, Vol. I.]

Brotherus, V. F. 1924a. Musci (Laubmoose). II Unterklasse Andreaeales, Andreaeaceae. III. Unterklasse Bryales: II. Spezieller Teil. (In Engler, A. and K. Prantr. Die natürlichen Pflanzenfamilien. Leipzig, Wilhelm Engelmann, Aufl. 2, 10, Hälfte 1, 129-31, 143-478.)

Brotherus, V. F. 1924b. The Musci of Juan Fernandez Islands. (In SKotTsBeRG, C. [J. F.], ed. The natural history of Juan Fernandez and Easter Island. Vol. 2, Botany, Pt. 3. Uppsala, Almquist and Wiksells, 409-48.)

Brotherus, V. F. 1924c. Musci nonnulli chilenses a C. Skottsberg anno 1917 lectae. Acta Horti gothoburg., 1, 189-95.

BRotheRUS, V. F. 1925. Musci (Laubmoose). III Unterklasse Bryales: II. Spezieller Teil. (In Engler, A. and K. Prantr. Die natürlichen Pflanzenfamilien. Leipzig, Wilhelm Engelmann, Aufl. 2, 11, Hälfte 2, 1-542.)

Brotherus, V. F. 1928. Musci. (In IRMischer, E., led.] Beiträge zur Kenntnis der Flora von Borneo. Mitt. Inst. allg. Bot., Hamb., 7, Ht. 2, 115-40.)

Brotherus, V.F. 1931. Neue exotische Laubmoose. Mitt. Inst. allg. Bot., Hamb., 8, Ht. 2 , $399-406$.

Brown, R. 1811. Some observations on the parts of frutification in mosses, with characters and descriptions of two new genera of that order. Trans. Linn. Soc. Lond., 10, Pt. 2, 312-24.

Brown, R. 1902. On the Musci of the calcareous districts of New Zealand, with descriptions of new species. Trans. Proc. N.Z. Inst., 35, 323-42. [Effective date July 1903.]

BruCH, [P.] 1826. Bryologische Beobachtungen. Flora, Jena, 9, No. 11, 161-66.

BruCH, P. 1832. Beschreibung einiger Laubmoose. Abh. bayer. Akad. Wiss. Math.-phys. KI., 1,
277-86.

BRUCH, P., Schimper, W. P. and W. T. GüMbel.* 1838. Bryologia europaea seu genera muscorum 
Europaeorum monographice illustrata. Stuttgart, Librariae E. Schweizerbart, Vol. 3, [Fasc. 4, 33-41.], Zygodon, 1-9. Vol. 4, [Fasc. 5, 165201.], Mnium, 1-37.

Bruch, P., Schimper, W. P. and W. T. GüMBel.* 1839. Bryologia europaea seu genera muscorum Europaeorum monographice illustrata. Stuttgart, Librariae E. Schweizerbart, Vol. 4, [Fasc. 6-9, 71-152.], Bryum, 1-82.

Bruch, P., Schimper, W. P. and W. T. GüMBel." 1841. Bryologia europaea seu genera muscorum Europaeorum monographice illustrata. Stuttgart, Librariae E. Schweizerbart, Vol. 3, [Fasc. 11, 259-68], Funaria, 1-10.

BRUCH, P., SCHIMPER, W. P. and W. T. GüMBEL.* 1842. Bryologia europaea seu genera muscorum Europaeorum monographice illustrata. Stuttgart, Librariae E. Schweizerbart, Vol. 2, [Fasc. 13-15, 63-108.], Barbula, 1-46. Vol. 4, [Fasc. 12, 3151.], Bartramia, 1-21.

BRUCH, P., SCHIMPER, W. P. and W. T. GüMBEL.* 1843. Bryologia europaea seu genera muscorum Europaeorum monographice illustrata. Stuttgart, Librariae E. Schweizerbart, Vol. 1, [Fasc. 17, 185-96.], Fissidens, 1-12. Vol. 2, [Fasc. 18-20, 29-41.], Pottia, 1-13.

Bruch, P., Schimper, W. P. and W. T. GüMbel.* 1844. Bryologia europaea seu genera muscorum Europaeorum monographice illustrata. Stuttgart, Librariae E. Schweizerbart, Vol. 3, [Fasc. 23-24, 288-90.], Tetraplodon, 1-8. Vol. 4, [Fasc. 21-22, 245-54.], Pogonatum, 1-10; [Fasc. 23-24, 6770.], Orthodontium, 1-4.

Bruch, P., SCHIMPER, W. P. and W. T. GüMBel." 1845. Bryologia europaea seu genera muscorum Europaeorum monographice illustrata. Stuttgart, Librariae E. Schweizerbart, Vol. 3, [Fasc. 25-28, 93-102.], Schistidium, 1-10.

BRUCH, P., SCHIMPER, W. P. and W. T. GüMBEL.* 1846. Bryologia europaea seu genera muscorum Europaeorum monographice illustrata. Stuttgart, Librariae E. Schweizerbart, Vol. 1, [Fasc. 29-30, 173-78.], Trematodon, 1-6; [Fasc. 33-36, 6171.], Weisia, 1-11; [Fasc. 33-36, 73-82.], Gymnostomum, 1-10; [Fasc. 33-36, 91-93.], Eucladium, 1-3; [Fasc. 33-36, 95-100.], Rhabdoweisia, 1-6. Vol. 2, [Fasc. 29-30, 13541.], Didymodon, 1-7; [Fasc. 29-30, 153-57.], Distichium, 1-5; [Fasc. 33-36, 17-20.], Blindia, 1-4. Vol. 3, [Fasc. 29-30, 155-58.], Hedwigidium, 1-4.

BruCH, P., SCHIMPER, W. P. and W. T. GüMBel.* 1850. Bryologia europaea seu genera muscorum Europaeorum monographice illustrata. Stuttgart, Librariae E. Schweizerbart, Vol. 5, [Fasc. 44-45, 31-36.], Cryphaea, 1-6; [Fasc. 44-45, 53-55.], Omalia, 1-3.

Bruch, P., SChimper, W. P. and W. T. Gümbel.* 1852. Bryologia europaea seu genera muscorum Europaeorum monographice illustrata. Stuttgart, Librariae E. Schweizerbart, Vol. 5, [Fasc. 49-51, 157-67.], Thuidium, 1-11; [Fasc. 49-51, 197208.], Rhynchostegium, 1-12.

BRUCH, P., SCHIMPER, W. P and W. T. GüMBEL.* 1852. Bryologia europaea seu genera muscorum Europaeorum monographice illustrata. Stuttgart, Librariae E. Schweizerbart, Vol. 5, [Fasc. 48, 179-95.], Plagiothecium, 1-17.

Bruch, P., Schimper, W. P, and W. T. Gümbel.*

- Publication dates, fascicule numbers and figures for continuous pagination within square brackets from Stafleu (1967, 57-63).
1853. Bryologia europaea seu genera muscorum Europaeorum monographice illustrata. Stuttgart, Librariae E Schweizerbart, Vol. 6, [Fasc. 52-54, 5-26.], Brachythecium, 1-22; [Fasc. 55-56, 4561.]. Amblystegium, 1-17.

Bruch, P., Schimper, W. P. and W. T. GüMbel.* 1854. Bryologia europaea seu genera muscorum Europaeorum monographice illustrata. Stuttgart, Librariae E. Schweizerbart, Vol. 6, [Fasc. 57-61. 77-130.], Hypnum, 1-54.

BRUCH, P SCHIMPER, W. P. and W. T. GüMBel.* 1855. Bryologia europaea seu genera muscorum Europaeorum monographice illustrata. Stuttgart, Librariae E. Schweizerbart, Vol. 6, [Fasc. 62-64, 131-56.], Andraea, 1-26.

Bruggeman-Nannenga, M. A. 1973. The section Pachylomidium (Genus Fissidens). I. The species of tropical and subtropical South America. Proc. K. ned. Akad. Wet., Series C. Biological and Medical Sciences, 76, No. 2, 172-89.

BryHn, N. 1902. Sarconeurum, genus muscorum novum. Sarconeurum antarcticum sp. nov. (In WILLE, N. Mittheilungen über einige von C. E. Borchgrevink auf dem antarctischen Festlande gesammelte Pflanzen. Nyt. Mag. Naturvid., 40, Ht. 3 and 4, 204-08.)

Buck, W. R. and L. R. LANDRUM. 1977. A new species of Astomiopsis (Ditrichaceae). Bryologist, 80, No. 2, 359-62.

BUSE, L. H. 1858. Musci Neerlandici specimena exsiccati. 'Neerlands mossoorten in getroogde exemplaren. Haarlem, Kruseman, Fasc. 3, No. 67.

Campo, J. A. 1915. Mousses chiliens determinés par $V$. F. Brotherus, et récoltés dans la province de Valdivia. Revta chil. Hist. nat... 19, Nos. 3-6, 74-75.

Campo, J. A. 1921. Pequeñ catalogo briologico de Mariluan. Revta chil. Hist. nat., 25, 507-10.

CARDOT, J. 1884. Notes sphagnologiques. Revue bryol., 11, No. 4, 54-56.

CARDot, J. 1887. Révision des Sphaignes de l'Amérique du Nord. Bull. Soc. r. Bot. Belg., 26, Pt. 1, 41-61.

CARDOT, J. 1900. Note préliminaire sur les mousses recueillies par l'Expédition Antarctique Belge. Revue bryol., 27, No. 3, 38-46.

CARDOT, J. 1901. Mousses et coup d'oeil sur la flore bryologique des Terres Magellaniques. Résult. Voyage S. Y. Belgica, 6, Botanique, 44 pp.

CARDOT, J. 1905a. Notice préliminaire sur les mousses recueillies par l'Expédition Antarctique Suédoise. I. Espèces de la région magellanique. Bull. Herb. Boissier, 2ème sér., 5, No. 11, 997-1011.

CARDOT, J. 1905b. Notice préliminaire sur les mousses recueillies par l'Expédition Antarctique Suédoise. Deux genres nouveaux de mousses acrocarpes. Revue bryol., 32, No. 3, 45-47.

CARDOT, J. 1905c. Mousses de l'île Formose. Beih. bot. Zbl., 19, Abt. 2, 85-148.

CARDOT, J. 1906a. Notice préliminaire sur les mousses recueillies par l'Expédition Antarctique Suédoise. Bull. Herb. Boissier, 2ème sér., 6, No. 1,1-17.

CARDOT, J. 1906b. Les mousses de l'Expédition Charcot. Revue bryol., 33, No. 3, 33-35.

CARDOT, J. 1907a. Musci. (In National Antarctic Expedition, 1901-1904. Natural History, Vol. III. Zoology and Botany. London, British Museum, 1-6.) 
CARDOT, J. 1907b. Une mousse a péristome asymétrique. Bull. Soc. Hist. nat. Ardennes, 14, 27-30.

CARdot, J. 1907c. Mousses. Paris, Masson et Cie. [Expédition Antarctique Françaises (1903-1905), Sciences naturelles: documents scientifiques, 1-9.]

CARDOT, J. 1908a. La flore bryologique des Terres Magellaniques, de la Géorgie du Sud et de l'Antarctide. Wiss. Ergebn. schwed. Südpolarexped., Bd. 4, Lief. 8, 298 pp.

CARDOT, J. 1908b. Sur le genre Hymenocleiston Duby. Annu. Conserv. Jard. bot. Genève, 11, and 12, 165-69.

CARDOT, J. 1910. Musci. Note sur les mousses rapportèes par l'Expédition du "Nimrod". (In British Antarctic Expedition, 1907-9. Reports on the Scientific Investigations. Biology. Vol. I, Pt. 4, 77-79.)

CARDOT, J. 1911a. Deux genres nouveaux de la région magellanique. Revue bryol., 38, No. 3, 49-52.

CARDOT, J. 1911 b. Sur les mousses rapportées par l'expédition antarctique du Pourquois-Pas? C. $r$. Ass. fr. Avanc. Sci., 153, No. 13, 599-603.

CARDOT, J. 1911 c. Les mousses de l'Expédition Nationale Antarctique Écossaise. Trans. $R$. Soc. Edinb., 48, Pt. 1, No. 3, 67-82.

CARDOT, J. 1911d. Note sur les mousses rapportées par la seconde Expédition Antarctique Française, sous le commandement du Dr. Jean Charcot. Revue bryol., 38, No. 6, 124-27.

CARDOt, J. 1912a. Atrichopsis Card., genre nouveau de la famille des Polytrichacées. Revue bryol., 39, No. 6, 95-96.

Cardot, J. 1912b. Les mousses de l'expédition national antarctique Écossaise. Rep. scient. Results Scott. natn. antarct. Exped., 3, Pt. 5 , 55-57.

CARDOT, J. 1913a. Mousses. Paris, Masson et Cie. [Deuxième Expédition Antarctique Française (1908-1910), Sciences naturelles: documents scientifiques, 1-30.]

CARDOT, J. 1913b. Quelques observations sur la nomenclature bryologique. Revue bryol., 40, No. 2, 17-22.

CARDOT, J. 1914a. Acrocladiopsis Card., genre nouveau de la tribu des Plagiothéciées. Revue bryol., 41, No. 1, 9.

CARDOT, J. 1914b. Philibertiella Card. Genre nouveau de la tribu des Ditrichées. Revue bryol., 41, No. 3, 37-38.

CARDOT, J. and V. F. BRotherus. 1923. Les mousses. K. svenska VetenskAkad. Handl., 63, No. 10, 1-73.

Chen, P-C. 1941. Studien über die ostasiatischen Arten der Pottiaceae. I. Hedwigia, 80, Ht. 1/2, 1-76; Ht. 3/4, 141-322.

Chevallier, F. F. 1827. Mousses. Musci. (In Flore générale des environs de Paris, selon la méthode naturelle. Description de toutes les plantes agames, cryptogames et phanérogams qui y croissent spontanément; leurs propriétés, leur usage dans la médicine, les arts et l'économie domestique; avec une classification naturelle des agames et des cryptogames, basée sur l'organisation de ce végétaux. Vol. 2. Paris, Chez Ferra Jeune, 26-99.)

ClARKE, G. C. S. 1973a. A synoptic flora of South Georgian mosses: III. Leptotheca, Philonotis, Mielichhoferia and Pohlia. British Antarctic Survey Bulletin, No. 37, 53-79. [Effective date 15
August 1974.]

CLARKE, G. C. S. 1973b. Notes on Antarctic bryophytes: Ill. The type specimen of Pohlia inflexa (C. Muell.) Wijk et Marg. British Antarctic Survey Bulletin, No. 37, 97-98. [Effective date 15 August 1974.]

ClifFord, H. T. 1955. On the distribution of Rhacomitrium crispulum (H. f. \&W.) H. f. \&W. Bryologist, 58, No. 4, 330-34.

ClifFord, H. T. 1957. New records for Antarctic mosses. Aust. J. Sci., 20, No. 4, 115.

CONARD, H. S. 1945. The Atracheata (Bryophyta) of lowa. Il. lowa mosses in print: A critique of the species reported for the state. Bryologist, 48, No. 3, $103-09$.

COSTES, N. 1921. Nomenclature de los principales musgos de la hoya de Marga-Marga (QuilpuéProvincia de Valparaiso). Revta chil. Hist. nat., 25, 130-32.

Crome, G. E. W. 1805. Sammlung deutscher Laubmoose. Nachlieferung I. Schwerin, J. W. Barensprungs Erben.

Crosby, M. R. 1975. Lectotypification of Schizomitrium B.S.G. (Musci, Hookeriaceae). Taxon, 24, Nos. 2/3, 353-55.

Crosby, M. R. 1976. The correct name for the moss Duseniella. Bryologist, 79, No. 2, 246-47.

Crosby, M. R. 1977a. Index muscorum supplementum: 1974-1975. Taxon, 26, Nos. 2-3, 285-307.

Crosby, M. R. 1977b. Florschuetzia, a new genus of Buxbaumiaceae (Musci) from southem Chile. Bryologist, 80, No. 1, 149-52.

Crosby, M. R. 1978. Muscoflorschuetzia (Musci), a new name for Florschuetzia Crosby, hom. illeg. Bryologist, 81, No. 2, 338.

Crum, H. A. 1950. Publication date of the prodromus bryologiae mexicanae. Bryologist, 53 , No. 1, 60-61.

Crum, H. A. 1952. Bibliographic notes on Palisot de Beauvois' prodrome. Bull. Torrey bot. Club, 79 , No. 5, 407-09.

Crum, H. A. 1957. A contribution to the moss flora of Ecuador. Svensk bot. Tidskr., 51, Ht. 1, 197-206.

CRUM, H. 1972. A taxonomic account of the Erpodiaceae. Nova Hedwigia, 23, Ht. 2-3, 20124. [Effective date 8 January 1973.]

Crum, H. A. andW. C. Steere. 1950. Additions to the moss flora of Panama. Bryologist, 53, No. 2, 139-52.

Crundwell, A. C. and E. NyHolm. 1963. Two new European species of Bryum. Bot. Notiser, 116, Fasc. 1, 94-98.

Crundwell, A. C. and E. NyHolm. 1964. The European species of the Bryum erythrocarpum complex. Trans. Br. bryol. Soc., 4, Pt. 4, 638-41.

Dalla Torre, K. W. and L. G. Sarnthein. 1904. Die Moose (Bryophyta) von Tirol, Vorarlberg und Liechtenstein. Flora der Gefürsteten Grafschaft Tirol, des Landes Vorarlberg und des Fürstenthumes Liechtenstein. Band. 5. Innsbruck, Verlag des Wagner'schen Universitäts-Buchhandlung.

DARBISHIRE, O. V. 1923. Cryptogams from the Antarctic. J. Bot., Lond., 61, No. 724, 105-07. DE CANDOLLE, M. 1815. Famille des mousses. (In Flore française, ou descriptions succinctes de toutes les plantes qui croissent naturellement en France, disposées selon une nouvelle méthode d'analyse, et précédées par un exposé des principes élémentaires de la botanique. Vol. 5 lou 
6). 3rd edition. Paris, Chez Desray, 204-36.)

DE LA PYLAIE, B. 1814. Suite de l'article sur la monographie des mousses rangées parmi les Hypnum de Linné, et que les auteurs modernes ou tantôt établies comme un genre particulier sous le nom de Fissidens, et tantôt raportées au genre Dicranum. J. Bot. Agric. Pharm. Med. Arts Paris, Sér. 2, 4, Nos. 4-6, 145-69. [Effective date 1815 , fide Sayre $(1959,47)$.]

DelGadILLO, C. 1973. A new species, nomenclatura changes, and generic limits in Aloina, Aloinella and Crossidium (Musci). Bryologist, 76, No. 2, 271-77.

Delgadillo, C. 1975. Taxonomic revision of Aloina, Aloinella and Crossidium (Musci). Bryologist, 78, No. 3, 245-303.

DE NotARIS, J. 1838a. Specimen de Tortulis italicis. Memorie Accad. Sci. Torino, 40, 281-325.

DE NOTARIS, J. 1838b. Syllabus muscorum in Italia et in insulis circumstantibus hucusque cognitorum. Taurini, J. de Notaris.

DE NotARIS, J. 1859. Musci Napoani sive muscorum ad flumen Napo in Columbia a clar. Osculati lectorum. Memorie Accad. Sci. Torino. Ser. 2, 18, 437-55.

DE NotARIS, G. 1867. Cronaca della Briologia Italiana. Part II. Comment. Soc. crittogam. Ital., 2 Fasc. 3, 269-312 [169-212].

DE NotARIS, G. 1869. Epilogo della briologia Italiana. Atti Univ. Genova, 1, 1-781.

Dickson, J. 1801. Fasciculus quartus plantarum crytogamicarum Britanniae. Londini, Auctor.

DISMIER, G. 1910. Revision des Philonotis de l'Amérique. Mém. Soc. bot. Fr., 2, No. 17, 1-37.

DixoN, H. N. 1896. The student's handbook of British mosses, with illustrations, and keys to the genera and species by H. G. Jameson. Eastbourne, $\vee T$. Sumfield.

Dixon, H. N. 1902. New varieties of British mosses. J. Bot., Lond., 40, No. 11, 374-80.

Dixon, H. N. 1912. On some mosses of New Zealand. J. Linn. Soc., Botany, 40, No. 277, 433-59.

DIXON, H. N. 1913. Studies in the bryology of New Zealand, with special reference to the herbarium of Robert Brown of Christchurch, New Zealand. Bull. N.Z. Inst., No. 3, Pt. 1, 3-29.

Dixon, H. N. 1914. Studies in the bryology of New Zealand, with special reference to the herbarium of Robert Brown of Christchurch, New Zealand. Bull. N.Z. Inst., No. 3, Pt. 2, 31-74.

DixON, H. N. 1920. Contributions to Antarctic bryology; mosses of Deception Island. Bryologist, 23, No. 5, 65-71.

Dixon, H. N. 1921. Miscellanea bryologica - VII. J. Bot., Lond., 59, No. 701, 132-39.

DixON, H. N. 1923. Studies in the bryology of New Zealand with special reference to the herbarium of Robert Brown, of Christchurch, New Zealand. Bull. N.Z. Inst., No. 3, Pt. 3, 75-152.

DixoN, H. N. 1924a. Miscellanea bryologica - IX. J Bot., Lond., 62, No. 740, 228-36.

Dixon, H. N. 1924b. The student's handbook of British mosses, with illustrations, and keys to the genera and species by H. G. Jameson. 3rd edition. Eastbourne, Sumfield and Day Ltd.

DixoN, H. N. 1926. Studies in the bryology of New Zealand, with special reference to the herbarium of Robert Brown, of Christchurch, New Zealand. Bull. N.Z. Inst., No. 3, Pt. 4, 153-238.

DixoN, H. N. 1927. Studies in the bryology of New Zealand, with special reference to the herbarium of Robert Brown, of Christchurch, New Zealand.
Bull. N.Z. Inst., No. 3, Pt. 5, 239-98.

DixoN, H. N. 1929a. Studies in the bryology of New Zealand, with special reference to the herbarium of Robert Brown of Christchurch, New Zealand. Bull. N.Z. Inst., No. 3, Pt. 6, 299-372.

Dixon, H. N. 1929b. Critical mosses. Revue Bryol., N.S., 2, Fasc. 1, 21-29.

DixoN, H. N. 1932. Some mosses from South Georgia. K. norske Vidensk. Selsk. Forh., 4, No. 47, 179-81.

DixoN, H. N. 1933. The nomenclature of the Species muscorum. Revue bryol. lichen., N.S., 6 Fasc. 1-4, 93-115. [Effective date 30 April 1934.]

Dixon, H. N. 1934. More mosses from South Georgia. K. norske Vidensk. Selsk. Forh., 7, No. 1,1-3.

DixON, H. N. 1935. The mosses. (In MARR, J. W. S. The South Orkney Islands. 'Discovery' Rep., 10, 381-82.)

Dixon, H. N. and M. Fleischer. 1937. Bryophyta nova (26-29). Annls bryol., 9 (for 1936), 139-41.

DIxON, H. N. and W. W. WaTts. 1918. Mosses. Scient. Rep. Australas. Antarct. Exped., Ser. C (Zoology and Botany), Vol. 7, Pt. 1, 5-7.

DONAT, A. 1936a. Resultados fitogeográficos de la expedición patagónica "Gaea" 1933. I. Algunas briófitas neuvas de la Cordillera Central

Patagónica. Revta sudam. Bot., 3, No. 3, 61-68.

DONAT, A. 1936b. Bericht über die

pflanzengeographischen Ergebnisse der Inlandeisexpedition der Argentinischen Geographischen Gesellschaft "Gaea" 1933. Ber. dt. bot. Ges., 44, Ht. 1, 27-46.

Dozr, F. and J. H. MolkenBoer. 1845. Musci frondosi inediti archipelagi indici, sive descriptio et adumbratio muscorum frondosorum in insulis Java, Borneo, Sumatra, Celebes, Amboina, nec non in Japonia nuper detectorum minusve cognitorum. Lugduni-Batavorum, H.W. Hazenberg and Soc., Fasc. 1, 1-24.

Du BuYsson, R. 1883. Essai analytique du genre Amblystegium. Mém. Soc. natn. Agric. Sci. Angers, N.S., 25, 161-79.

DuBY, J. E. 1868. Choix de cryptogames exotiques nouvelles ou mal connues. Mém. Soc. Phys. Hist. nat. Genève, 19, Pt. 2, 291-304.

DuBY, J. E. 1870. Choix de cryptogames exotiques nouvelles ou mal connues. Mém. Soc. Phys. Hist. nat. Genève, 20, Pt. 2, 351-64.

Duby, J.E. 1875a. Choix de mousses exotiques nouvelles ou mal connues. Mém. Soc. Phys. Hist. nat. Genève, 24, Pt. 1, 361-74.

DuBY, J. E. 1875b. Diagnoses muscorum quorum descriptiones et icones Soc. Phys. et Hist. nat. Genevensi primo Aprili communicavit. Flora, Jena, 58, (N.R., 33), No. 18, 282-85.

DuBY, J. E. 1880a. Choix de mousses exotiques nouvelles ou mal connues. Mém. Soc. Phys. Hist. nat. Genève, 27, Pt. 1, 1-10.

DuBY, J.E. $1880 b$. Aliquot diagnoses muscorum novorum aut non cognitorum. Flora, Jena, 63. (N.R., 38), No. 11, 168-74.

Dumont-D'URVILLE, M. J. 1825. Flore des Malouines. Mém. Soc. linn. Paris, 4 (for 1826), $\mathrm{Pt}$. 2, 573-621.

DuséN, P. 1903a. Patagonian and Fuegian mosses. Rep. Princeton Univ. Exped. Patagonia, 8, Botany, No. 1, Pt. 3, 63-126.

Dusén, P. 1903b. Beiträge zur Bryologie der Magellansländer, von Westpatagonien und Südchile. Teil I. Ark. Bot., 1, Häfte 1-3, 441-65.

Dusén, P. 1905a. Beiträge zur Bryologie der Magellansländer, von Westpatagonien und 
Südchile. Teil II. Ark. Bot., 4, Häfte 1-3, 1-45.

DusÉn, P. 1905b. Musci nonnulli novi e Fuegia et Patagonia reportati. Bot. Notiser, No. 7, 299310.

DusÉN, P. 1905c. Beiträge zur Bryologie der Magellansländer, von Westpatagonien und Südchile. Teil III. Ark. Bot., 4, Häfte 4, 1-24.

DusÉN, P. 1905D. Mousses et hépatiques. (In Autran, E. Énumèration des plantes récoltées par Miles Stuart Pennington pendant son premier voyage à la Terre de Feu en 1903. Revta Univ. B. Aires, 4, 297-98.)

DusÉN, P. 1906. Beiträge zur Bryologie der Magellansländer, von Westpatagonien und Südchile. Teil IV. Ark. Bot., 6, Häfte 1-2, 1-40.

DusÉN, P. 1907. Beiträge zur Bryologie der Magellansländer von Westpatagonien und Südchile. Teil V. Ark. Bot., 6, Häfte 3-4, 1-32.

EATON, D. C. 1892. List of mosses from Fuegia and Patagonia. (In List of plants collected by the U.S.S. Albatross in 1887-'91 along the western coast of America. Contr. U.S. natn. Herb., 1, No. 5, 135-42.)

EsPinOSA B., M. R. 1924. Enumeración de plantas colectadas en "Los Bronces". Revta chil. Hist. nat., 28, 88-97.

ESPINOSA, B., M.R. 1941. Lista sisemática de musgos y liquenes chilenos, continentales e insulares envidos por el Dr. C. Skottsberg. Boln Mus. nac. Hist. nat. Chile, 19, 113-32.

FAmiller, I. 1913. Die Laubmoose Bayerns. Eine Zusammenstellung der bisher bekannt gewordenen Standortsangaben. Denkschr. $K$. bayer. bot. Ges. Regensb., 12, (N.F., 6), 1-174.

FELIPPONE, F. 1909. Contribution à la flore bryologique de l'Uruguay, 1er fascicule. Buenos Aires, Jean-A. Alsina.

FELIPPONE, F. 1912. Contribution à la flore bryologique de l'Uruguay, 2 ème fascicule. Montévidéo, Ateliers Typographiques "Gimenez".

FELIPPONE, F. 1917. Contributrion à la flore bryologique de l'Uruguay, 3 ème fascicule. Montévidéo, Mosca Hnos.

FELIPPONE, F. 1928. Contribución à la flora briológica del Uruguay. Revta chil. Hist. nat., 32, 83-88. [Effective date February 1929.]

FELIPPONE, F. 1929. Contribution à la flore bryologique de l'Uruguay. Revue bryol., N.S., 2, Fasc. 3-4, 210-25. [Effective date 31 March 1930 , fide title page.]

FELIPPONE, F. 1930. Contribution à la flore bryologique de l'Uruguay. Corrigenda. Revue bryol., N.S., 3, Fasc. 1-2, 98.

FIEDLER, C. 1844. Synopsis der Laubmoose Mecklenburg's. Schwerin, C. Kürschner.

FILSON, R. B. 1966. The lichens and mosses of Mac.Robertson Land. A.N.A.R.E. Rep., Ser. B2, (Botany), No. 82, 169 pp.

Fleischer, M. 1904. Die Musci der Flora von Buitenzorg (zugleich Laubmoosflora von Java). Enthaltend alle aus Java bekannt gewordenen Sphagnales und Bryales, nebst kritschen Bemerkungen über vieler Archipelarten, sowie indischer unde australischer Arten. Leiden, E. J. Brill, Bd. 1, 1-379.

Fleischer, M. 1906. Der Musci der Flora von Buitenzorg. Zugleich Laubmoosflora von Java mit Berücksichtigung aller Familien und Gattungen der gesamten Laubmooswelt. Enthaltend alle aus Java bekannt gewordenen
Sphagnales und Bryales nebst kritischen Bemerkungen über viele Archipelarten, sowie indische unde australische Arten. Leiden, E. J. Brill, Bd. 3, Bogen 42, 645-60. [Effective date 1908, fide Stafleu and Cowan $(1976,844)$.]

FLEISCHER, M. 1908a. Grundlagen zu einer Monographie der Gattung Stereohypnum (Hpe.). Hedwigia, 47, Ht. 5/6, 271-99.

FLEISCHER, M. 1908b. Die Musci der Flora von Buitenzorg. Zugleich Laubmoosflora von Java mit Berücksichtigung aller Familien und Gattungen der gesamten Laubmooswelt. Enthaltend alle aus Java bekannt gewordenen Sphagnales und Bryales nebst kritschen Bemerkungen über viele Archipelarten, sowie indische unde australische Arten. Leiden, E. J. Brill, Bd. 3, Bogen 59, 917-32.

FLEISCHER, M. 1914. Kritische Revision von Carl Müllerschen Laubmoosgattungen. I. Hedwigia, 55, Ht. 3, 280-85.

FleISCHER, M. 1917. Kritische Revision von Carl Müllerschen Laubmoosgattungen. Il. Hedwigia, 59, Ht. 5, 212-19.

FleisCHER, M. 1920. Kritische Revision von Carl Müllerschen Laubmoosgattungen. III. Hedwigia, 61, Ht. 6, 402-08.

FLEISCHER, M. 1922. Kritische Revision der Carl Müllerschen Laubmoosgattungen. IV. Hedwigia, 63, Ht. 3/4, 209-16.

FLEISCHER, M. 1923. Die Musci der Flora von Buitenzorg. Zugleich Laubmoosflora von Javamit Berücksichtigung aller Familien und Gattungen der gesamten Laubmooswelt. Enthaltend alle aus Java bekanntgewordenen Sphagnales und Bryales nebst kritschen Bemerkungen über viele Archipelarten, sowie indische unde australische Arten. Leiden, E. J. Brill, Bd. 4, 1105-729.

FLOWERS, S. 1935. Bartramiaceae. (In GROUT, A. J. ed. Moss flora of North America north of Mexico. Newfane, Vermont, A. J. Grout, 2, Pt. 3, 152-83.)

FLOWERS, S. 1952. Monograph of the genus Anacolia. Bull. Torrey bot. Club, 79, No. 2, 161-85.

FraHM, J. P. 1974. Zur Unterscheidung und Verbreitung von Campylopus introflexus (Hedw.) Brid. und C. polytrichoides De Not. Revue bryol. lichen., N.S., 40, Fasc. 1, 33-44.

Frahim, J. P. 1975a. Conspectus der mittel- und südamerikanischen Campylopus - Arten (Dicranaceae). Bryophytorum Bibliotheca, 5, 1143.

FraHM, J. P. 1975b: Taxonomische Notizen zur Gattung Campylopus. Revue bryol. lichen., N.S. 41, Fasc. 3, 321-32.

FraHM, J. P.2 1976a. Zur Campylopus - Flora von Chile. Herzogia, 4, Ht. 1-2, 141-60.

FraHM, J. P. 1976b. Taxonomische Notizen zur Gattung Campylopus II. Revue bryol. lichen., N.S., 42, Fasc. 1, 603-16.

FriEs, E. M. 1825. Musci. (In Stirpes agri Femsionensis, quarum indicem cons. ampliss. phil. ord. Lundens. pp. J. G. Arrhenius, Vermelandus, in acad. Carol. D. XI Junii MDCCCXXV. Continuatio I. Londoni Gothorum Officina Berlingiana, 24-30.)

FroEHLICH, J. 1953. Zwei neue Laubmoose aus Süd-Chile und Feuerland. Ann/n naturh. Mus. Wien, 59 (for 1952-53), 117-18.

Frye, T. C. and M. W. Duckering. 1948. Atrichum polycarpum. Bryologist, 51, No. 3, 170-74.

FunCK, H. C. 1802. Botanische Notizen. Bot. Ztg, Erlangen, 1, Ht. 2, No. 8, 120-24.

FunCK, H. C. 1820. Deutschlands Moose. Ein 
Taschenherbarium zum Gebraüch auf botanischen Excursionen. Baiereuth, F. C. Bimer.

FürNROHR, A. E. 1827. Versuch einer nähern Beleuchtung der Disposition methodique des espèces de mousses par G. A. Walker-Arnott, $M$. Membre des Sociétés royale et Wernerienne d'Edinbourg etc., Paris, 1825 (4.72 seiten). Flora, Jena, 10, (Bd. 2, Beil. 1), 22-86.

FürnROHR, A. E. 1829. Sam. El. a Bridel-Brideri, a consil secret legation Saxo-Gothanae etc. Bryologia universa seu systematica ad novam methodum dispositio, historia et descriptio omnium muscorum hucusque cognitorum cum synonymia ex auctoribus probatissimis. Accedunt tabulae aeneae tredecim. Lipsiae sumtibus Joan. Ambros. Barth. 8 max. Vol. primum 1926, XLVl et 856 pag. Vol. secundum 1827, 848 pag. Flora, Jena, 12, (Bd. 2, Ergänzungsblätter), $1-60$.

Garovaglio, S. 1840. Bryologia austriaca excursoria ta tamquam clavis analytica ad omnes in imperio austriaco hucusque inventos muscos facile et tuto determinandos. Vindobonae, Friderici Volke.

GÄrTnER, G., Meyer, B. and J. Scherbius. 1802. Ordnung Musci, Moose. (In Oekonomischtechnische Flora der Wetterau. Band 3, Abt. 2. Frankfurt am Main, Philipp Heinrich Guilhauman, 68-139.)

GaUdichaUd, [M.] C. 1825. Rapport sur la flore des îles Malouines. Annls sci. nat., Botanique, 5 , 89-110.

Gaudichaud, M. C. 1826. Botanique, Musci, Jussieu. (In FreYcinet, M. L. DE. Voyage autour du monde, entrepris par ordre du roi, sous le ministère et conformément aux instructions des. exc. M. le vicomte du Bouchage, secrétaire d'état au département de la marine, exécuté sur les corvettes de S. M. I'Uranie et la Physicienne, pendant les années 1817, 1818, 1819 et 1820. Paris, Chez Pillet Aîné, Pt. 6, 221-30.) [Effective date February 1828 , fide Stafleu and Cowan, 1976, 922.]

GeNTH, F. F. 1835. Flora des Herzoghtums Nassau und der obern, so wie der untern Rheingegenden von speier beis Cöln Von F. F. Genth. I. Theil Cryptogamie. I. Abtheilung. Farnkränter, Lebermoose, Moose und Flechten. Im Verlage von Florian Kupferberg. Mainz. 1836. 8. LitBer. Flora allg. bot. Zeit., 5, No. 12, 177-91.

GepP, A. 1902. Cryptogamia: Musci. (In Report on the collections of natural history made in Antarctic regions during the voyage of the "Southern Cross". Vol. 21. London, The Trustees, British Museum (Nat. Hist.), 319.)

GIACOMINI, V. 1955. Sull'autonomia specifica e sul ciclo di forme di Campylopus polytrichoides De Not. Atti Ist. bot. Univ. Lab. crittogam. Pavia, Ser. 5, 13, Fasc. 1, 45-83.

Gibert, E. 1873. Musci. (In Enumeratio plantarum sponte nascentum agro Montevidensi cum synonimis selectis. Montevideo, La Asociación Rural del Uruguay, 129-32.)

Godman, F. Du C. 1870. Natural history of the Azores or western islands. London, John von Voorst.

Gradstein, S. R. and H. J. M. Sipman. 1978. Taxonomy and world distribution of Campylopus introflexus and $C$. pilifer ( $-C$. polytrichoides): a new synthesis. Bryologist, 81, No. 1, 114-21.

Grassi, M. M. 1975a. Notas de clase Bryophyta. I. Parte general. Fundación Miguel Lillo.
Miscelánea, No. 55, 1-38.

Grassi, M. M. 1975b. Notas de clase Bryophyta. (Atlas). Fundación Miguel Lillo. Miscelánea, No. 55, 1-57.

Grassı, M. M. 1976. Notas de clase Bryophyta. II. Parte especial. Fundación Miguel Lillo. Miscelánea, No. 55, 1-320.

Gray, S. F. 1821. A natural arrangement of British plants, according to their relations to each other as pointed out by Jussieu, de Candolle, Brown, etc. including those cultivated for use; with an introduction to botany, in which the terms newly introduced are explained; illustrated by figures. Vol. 1. London, Baldwin, Cradock and Joy.

Greene, D. M. 1972. The herbarium of the British Antarctic Survey. Britsh Antarctic Survey Bulletin, No. 31, 107-09.

Greene, S. W. 1964. Problems and progress in Antarctic bryology. (In Carrick, R., Holdgate, M. and J. Prévost, ed. Biologie antarctique. Paris, Hermann, 173-79.)

GREeNE, S. W. 1967. Bryophyte distribution. (In BUSHNeL, V., ed. Terrestrial life in Antarctica. Antarct. Map Folio Ser., Folio 5, 11-13.)

Greene, S. W. 1968a. Studies in Antarctic bryology: I. A basic check list for mosses. Revue bryol. lichen., N.S., 36, Fasc. 1-2, 132-38.

GReENE, S. W. 1968b. Studies in Antarctic bryology: II. Andreaea, Neuroloma. Revue bryol. lichen., N.S., 36, Fasc. 1-2, 139-46.

Greene, S. W. 1972. A new combination in Andreaea. British Antarctic Survey Bulletin, No. 31, 109. [Effective date 17 May 1973.]

GREENE, S. W. 1973. A synoptic flora of South Georgian mosses: I. Dendroligotrichum, Polytrichum and Psilopilum. British Antarctic Survey Bulletin, No. 36, 1-32. [Effective date 21 June 1974.]

Greene, S. W. 1975. The Antarctic moss Sarconeurum glaciale (C. Muell.) Card. et Bryhn in southern South America. British Antarctic Survey Bulletin, Nos. 41 and 42, 187-91. [Effective date 4 February 1976.]

Greene, S. W., Greene, D. M., Brown, P. D. and J. M. PACEY. 1970. Antarctic moss flora: I. The genera Andreaea, Pohlia, Polytrichum, Psilopilum and Sarconeurum. British Antarctic Survey Scientific Reports, No. 64, 118 pp.

GREVILLE, R. K. 1824. Flora Edinensis: or a description of plants growing near Edinburgh, arranged according to the Linnean system. With a concise introduction to the material orders of the class Cryptogamia, and illustrative plates. Edinburgh, William Blackwood; London, T. Cadell.

GreviLLe, R. K. 1826. Descriptions of two new species of Musci, belonging to the genera Neckera and Hypnum. Mem. Wernerian nat. Hist. Soc., 5, Pt. 2 (for 1824-25), 481-84.

GREVILLE, R. K. 1830. Descriptions of some new mosses discovered in South America by Dr. Gillies; with a plate. Edinb. J. nat. geogr. Sci., 2, $1-4$.

Greville, R. K. and G. A. W. Arnott. 1824. IV. Tentamen methodi muscorum; or a new arrangement of the genera of mosses, with characters, and observations on their distribution, history and structure. Mem. Wernerian nat. Hist. Soc., 5, Pt. 1 (for 1823-24), 42-89.

Grout, A. J. 1929. Check list of the pleurocarpous mosses of North America, north of Mexico. New Brighton, New York, A. J. Grout. [The only copy seen lacked a date, so that given in Index 
muscorum, 2, p. 172, under Drepanocladus uncinatus var. symmetricus has been accepted but not verified.]

Grout, A. J. 1939. Pottieae with Aloina by E. J. Craig; Tortula by W. C. Steere; Pottia and Pterygoneurum by W. T. Wareham. (In Grout, A. J., ed. Moss flora of North America north of Mexico, Newfane, Vermont, A. J. Grout, 1, Pt. 4, 193-253.)

Grout, A. J. 1944. Preliminary synopsis of the North American Macromitriae. Bryologist, 47, No. 1, 1-22.

Grout, A. J. 1946. North American flora. New York, New York Botanical Garden, 15A, Pt. 1, 1-62.

GunCKel, H. 1938. Los Porothamnium de la flora Corraleña. Revta chil. Hist. nat., 42, 118-22. [Effective date 1939.]

GUNCKEL, H. 1964. Un interesante musgo de la Selva Valdiviana: Eriodon conostomus Montagne. Revta univ., Santiago, 49 [An. Acad. chil. Cienc. nat., No. 27], 67-70. [Effective date 1965.]

GUNCKEL, H. 1971. Las primeras plantas herborizades en Chile en 1690. An. Inst. Patagonia, 2, Nos. 1-2, 134-41.

Hagen, I. 1915. Forarbejder til en Norsk Løvmosflora. XX. Dicranaceae. K. norske Vidensk. Selsk. Skr., (for 1914), Nr. 1, 1-192.

Hagen, I. and H. Printz. 1929. Forabeider til en Norsk Løvmosflora. XXI. Pottiaceae. K. norske Vidensk. Selsk. Skr., (for 1928), Nr. 3, 1-96.

HAMPE, E. 1837a. Musci frondosi Germaniae ad methodum naturalem dispositi. Flora, Jena, 20, No. 18, 273-87.

HAMPE, E. 1837 b. Prodromus florae Hercyniae. Linnaea, Halle, 11, Ht. 1, 17-105.

HAMPE, E. 1837c. Weberae novae chilensis descriptio. Linnaea, Halle, 11, Ht. 3, 278.

HAMPE, E. 1839. Relation über die von dem Reisenden C. Beyrich auf seiner letzten Reise in Nordamerika gesammelten Laubmoose. Linnaea, Halle, 13, Ht. 1, 39-48.

HAMPE, E. 1844. Icones muscorum novorum vel minus cognitorum. Decades 1-3. Bonnae, Henry et Cohen.

HAMPE, E. 1846. Ueber Hyophila javanica Brid. Bot. $Z$ tg, 4, No. 15, 266-69.

HAMPE, E. 1847. Ein Referat über die Columbischen Moose, welche von Herrn Moritz gesammelt wurden. Linnaea, Halle, 20, [Beitr. PfIKunde, 4], Ht. 1, 65-98.

HAMPE, E. 1860. Muscorum frondosorum florae Australasiae auctore Dr. F. Müller mox edendae species novas. Linnaea, Halle, 30, [Beitr. PflKunde, 14], Ht. 5, 623-40; Ht. 6, 641-46.

HAMPE, E. 1862a. Species novas muscorum ab Dr. A. Alexandro Lindigio in Nova-Granada mensibus Julio et Augusto a 1861 collectas. Linnaea, Halle, 31, [Beitr. Pflkunde, 15], Ht. 5, 518-32.

HAMPE, E. 1862b. Beitrag zu einer Moosflora von Neu-Granada. Flora, Jena, 45, (N.R., 20), No. 29, 449-58.

HAMPE, E. 1863. Species novas muscorum ab Alexandro Lindigio in Nova-Granada collectas. Linnaea, Halle, 32, [Beitr. PfIKunde, 16], Ht. 1, 127-28; Ht. 2, $129-64$.

HaMPe, E. 1865. Musci. (In Triana, J. and J. E. PLANCHON. Prodromus florae Novo-Granatensis ou énumération des plantes de la NouvelleGrenade avec description des espèces nouvelles. Annls Sci. nat., Sér. 5, 4, Botanique, 324-78.)
Hampe, E. 1866. Musci. (In Triana, J. and J. E. PlanCHON. Prodromus florae Novo-Granatensis ou énumération des plantes de la NouvelleGrenade avec description des espèces nouvelles. Annls Sci. nat., Sér. 5, 5, Botanique, 300-42.)

HAMPE, E. 1867a. Bryologische Mittheilungen aus dem Herbarium. Flora, Jena, 50, (N.R., 25), No. 5 , 65-80.

HAMPE, E. 1867b. Ditrichum Timm, statt Leptotrichum Hpe. Flora, Jena, 50, (N.R., 25), No. 12, 181-82.

Hampe, E. 1870. Musci frondosi. (In Warming, E., ed. Symbolae ad floram Brasiliae centralis cognoscendam, Particula VIII. Vidensk. Meddr dansk naturh. Foren., Ser. 3, Nos. 18-20, 26796.)

HAMPE, E. 1872. Musci frondosi a clar. Dr. A. Glaziou in vicinia urbis Rio de Janeiro lecti. (In WARMING, E., ed. Symbolae ad florem Brasiliae centralis cognoscendam. Pt. 10. Vidensk. Meddr dansk naturh. Foren., Ser. 3, 4, Nr. 1-5, 36-59.)

HAMPE, E. 1874. Musci frondosi a clar. Dr. A. Glaziou in vicinia urbis Rio de Janeiro lecti. (In WARMING, E., ed. Symbolae ad floram Brasiliae centralis cognoscendam. Particula 19. Vidensk. Meddr dansk naturh. Foren., Ser. 3, Nos. 9-11, 129-78.) [Effective date 1875, according to entry for Rhodobryum gracilescens in Index muscorum, 4, p. 148.]

HAMPE, E. 1878. Musci frondosi, a clar. Dr. A. Glaziou in vicinia urbis Rio Janeiro lecti (continuatio). (In WARMING, E., ed. Symbolae ad floram Brasiliae centralis cognoscendam, Particula XXIV. Vidensk, Meddr dansk naturh. Foren., Ser. 3 (for 1877-78), 251-74.)

HAMPE, E. 1879. Enumeratio muscorum frondosorum Brasiliae centralis, praecipue provinciarum Rio de Janeiro et S. Paulo, adhuc cognitorum. Vidensk. Meddr dansk naturh. Foren., Ser. 4 (for 1879-80), 73-164.

HAMPE, E. 1880a. Choix de mousses exotiques, nouvelles ou mal connues, par J. E. Duby, ancien pasteur, Docteur de Sciences communiq. le 5 Febr. 1880. Flora, Jena, 63, (N.R., 38), No. 21, 332-36.

HAMPE, E. 1880b. Supplementum ad volumen undecimum fragmentorum phytographie Australiae, indices plantarum acotyledonearum complectens. III. Musci frondosi Australiae continentalis, praesertim e baronis de Mueller collectionibus. (In MUELLE, F. Fragmenta phytographie Australiae, Vol. XI. Melbourne Joannis Ferres, 45-52.)

[HAMPE, E.] 1880c. Additmenta pro supplemento voluminis undecimi. (In MUELLER, F. Fragmenta phytographie Australiae, Vol. XI. Melbourne Joannis Ferres, 105-21.) [Unsigned and presumed to be by Hampe who worked up the mosses.]

Hampe, E. and A. Geheeb. 1881. Additmenta ad "Enumerationem muscorum hactenus in provinciis Brasiliensibus Rio de Janeiro et Sao Paulo detectorum". Flora, Jena, 64, (N.R., 39), No. 26, 401-16.

HARIOT, P. 1891. Contribution à la flore cryptogamique de la Terre de Feu. Bull. Soc. bot Fr., 38, [Sér. 2, 13], 416-22.

HARTMAN, C. J. 1838. Musci. (In Handboki Skandinaviens Flora, innefattande Sveriges och Norriges vexter, till och med Mossorona; med ett utkast till botanologien eller vextlären i allmänhet Vol. 2. 3rd edition. Stockholm, Zacharias 
Haeggström, 260-334.)

Hartman, C. J. 1849. Musci. (In Handboki Skandinaviens Flora, innefattande Sveriges och Norriges vexter till och med Mossorna; ordnade eften Prof. Fries' system. 5th edition. Stockholm, Zacharias Haeggström, 319-431.)

HASSKARL, J. K. 1851. Allgemeines Sach- und Namen-Register zu den ersten XXV Jahrgängen der Flora oder allgemeinen botanischen Zeitung von 1818-1842. Regensburg, G. Joseph Manz. [Note this is general index to Flora, Vols 1-25, 1818-42.]

HeDWIG, D. J. 1782. Fundamentum historiae naturalis muscorum frondosorum concernens eorum flores, fructus, seminalem propagationem adiecta generum dispositione methodica, iconibus illustratis. Part 2. Lipsiae, Siegfried Lebrecht Crusium.

HEDWIG, J. 1801. Species muscorum frondosorum. Descriptae et tabulis aeneis LXXVII coloratis illustratae. Opus posthumum editum a Friderico Schwaegrichen. Lipsiae, Joannis Ambrosii Barthii; Parisiis, Amand Koenig. [Reprinted: Species muscorum frondosorum. (In Cramer, $J$. and H. K. SwaNn, ed. Historiae naturalis classica, 16, 1960. Weinheim/Bergstr., H. R. Engelmann (J. Cramer); Codicote/Herts., Wheldon \& Wesley, Ltd.; New York, Hafner Publishing Co.)]

HERTER, [W.] G. 1927-28. Index montevidensis, plantae avasculares. (In HeRTER, [W.] G., ed. Estudios botánicos en la región Uruguaya. No. 1. Montevideo, Asociación Rural del Uruguay, 36 pp.)

HeRTER, [W.] G. 1928. Las plantas uruguayas de Ernesto Gibert. Nomenclator Gibertianus. (In HerteR, [W.] G., ed. Estudios botánicos en la región Uruguaya. No. V. Montevideo, Universitatis rei Publicae, $60 \mathrm{pp}$.)

HERTER, [W.] G. 1933a. Florula uruguayensis, plantae avasculares. (In HERTER, [W.] G., ed. Ostenia, coleccion de trabajos botánicos dedicados a Don Cornelio Osten en ocasion del LXX aniversario de su nacimiento. Estudios botánicos en la región Uruguaya No. XIII. Montevideo, [W. G. Herter], 7-84.) [Re-issued without change of pagination in separate covers entitled Florula uruguayensis, plantae avasculares. Estudios botánicos en la región Uruguaya No. III. Montevideo, W. G. Herter.]

HerTER, [W.] G. 1.933b. Las plantas uruguayas de Ernesto Gibert. Nomenclator Gibertianus 2. (In Herter, [W.] G., ed. Ostenia, coleccion de trabajos botánicos dedicados a Don Cornelio Osten en ocasion del LXX aniversario de su nacimiento. Estudios botánicos en la región Uruguaya No. XIII. Montevideo, [W. G. Herter], 153-71.) [Re-issued without change of pagination in separate covers entitled $L a s$ plantas uruguayas de Emesto Gibert, segunda parte. Nomenclator Gibertianus II. Estudios botánicos en la región Uruguaya No. Va.]

Herter, W. G. 1937. Plantae uruguayenses novae vel criticae I. Revta sudam. Bot., 5, Nos. 1/2, 13-36. [Part of Estudios botánicos en le región Uruguaya No. XVI, fide Herter, 1953.]

Herter, W. G. 1939a. Zur kenntnis der Flora von Osturuguay. Beih. bot. Zbl., 59, , Abt. B, Ht. 1, 245-86.

Herter, W. G. 1939b. Plantae uruguayenses novae vel criticae II. Revta sudam. Bot., 6, Nos. 3/4, 69-107. [Effective date September 1941. Part of Estudios botánicos en la región Uruguaya No. XVI, fide Herter, 1953.]
Herter, W. G. 1943. Plantae uruguayenses novae vel criticae IV. Revta sudam. Bot., 7, Nos. 6/8, 171-260. [Part of Estudios botánicos en la región Uruguaya No. XVI, fide Herter, 1953.]

Herter, [W.] G. 1953. Conspecus estudios botánicos en la región Uruguaya. Revta sudam. Bot., 10, No. 3, 99-100.

Herzog, T. 1909. Beiträge zur Laubmoosflora von Bolivia. Beih. bot. Zbl., 26 (for 1909-10), Abt. 2, Ht. 1, 45-102.

Herzog, T. 1911. Bemerkungen zu der neuren Laubmoos-Gattung Wollyna. Beih. bot. Zbl., 28, Abt. 2, Ht. 2, 268-71.

Herzog, T. 1914. Zwei kleistokarpe Moose der bolivianischen Hochcordillere. Flora, Jena, N.F., 7, Ht. 3, 317-26.

Herzog, T. 1916a. Die Bryophyten meiner zweiten Reise durch Bolivia. Lief. 1. Biblthca bot., Ht. 87 , 1-168.

Herzog, T. 1916b. Neue Laubmoose aus Ostasien und Südamerika. Hedwigia, 57, Ht. 3, 233-50.

Herzog, T. 1920. Die Bryophyten meiner zweiten Reise durch Bolivia Nachtrag. Biblthca bot., Ht. 88, 1-31.

Herzog, T. 1923. Beiträge zur Bryophytenflora von Chiles. Hedwigia, 64, Ht. 1/2, 1-18.

Herzog, T. 1926. Beiträge zur Moosflora Westpatagoniens. Hedwigia, 66, Ht. 2, 79-92.

HERZOG, T. 1927. Zwei Bryophytensammlungen aus Südamerika. Hedwigia, 67, Ht. 6, 249-68.

HeRzog, T. 1928. Drei neue Laubmoosgattungen. Revue bryol., N.S., 1, Nos. 2-3, 98-108.

Herzog, T. 1937. Neue bryophyten von Ruwenzori und aus dem patagonischen Inlandeisgebiet. Reprium nov. spec. Regni veg., 41, Nos. 14-25, 285-92.

Herzog, T. 1938. Contribución al conocimiento de la flora Briofita del sur de Chile. a. Parte sistemática. Archos Esc. Farm. Córdoba, No. 7 (Sección científica), 3-56.

HerzoG, T. 1939. I. Verzeichnis der gesammelten Bryophyten. (In Herzog, T., SchwaBe, G. H. and E. SCHWABE. Zur Bryophytenflora südchiles. Beih. bot. Zbl., 60, Abt. B, Ht. 1/2, 1-51.)

Herzog, T. 1940. Die Moose der Expedition Ljungner nach Patagonien, 1932/34. Ark. Bot., 29A, Häfte 4, 1-17.

Herzog, T. 1952a. Beiträge zur Kenntnis der argentinischen Bryophytenflora. Reprium nov. spec. Regni veg., 55, Ht. 1, 1-27.

HERZOG, T. 1952b. Miscellanae bryologica. III. Memo. Soc. Fauna Flora fenn., 27 (for 1950-51), 92-110. [Effective date 1952, according to footnotes on p. 80,96 and 112.]

HERZOG, T. 1952c. Eine neue Lebermoosgattung aus Westpatagonien, Chondrophyllum Herz. Revue bryol. lichen., N.S., 21, Fasc. 1-2, 46-49.

Herzog, T. 1954. Zur Bryophytenflora chiles. Revue bryol. lichen., N.S., 23, Fasc. 1-2, 27-99.

HERZOG, T. 1957. Eine Briophytensammlung aus dem argentinis Nationalpark Nahuelhuapi. Darwiniana, 11, No. 2, 207-22.

Herzog, T. 1960. Weitere Beiträge zur Bryophytenflora von Chile. Revue bryol. lichen., N.S., 29, Fasc. 3-4, 183-206. [Effective date March 1961, fide Lamy, 1978.]

HERZOG, T., THÉRIOT, I., BUCH, H., SCHIfFNER, V. and F. VERDOORN. 1933. Bryophyta nova (6-16). Annls bryol., 6, 124-35.

HILPERT, F. 1933. Studien zur Systematik der Trichostomaceen. Beih. bot. Zbl., 50, Abt. 2, Ht. 2, 585-640; Ht. 3, 641-706.

HoffMan, G. F. 1795. Deutschlands Flora oder 
botanisches Taschenbuch. Theil 2. Cryptogamie. Erlangen, Johann Jacob Palm. [Effective date 1796, fide Stafleu and Cowan, 1979, 240.]

HOLZINGER, J. M. 1911. Reviews of current literature. [Cardot, J. 1907. Musci. (In National Antarctic Expedition, 1901-1904. Natural History, Vol. IV, Zoology and Botany. London, British Museum, 1-6).] Bryologist, 14, No. 1, 11-12.

HOOKER, J. D. 1867. Order IV, Musci. (In Handbook of the New Zealand flora: a systematic description of the native plants of New Zealand and the Chatham, Kermadec's, Lord Auckland's, Campbell's, and Macquarrie's Islands. London, Reeve \& Co., Pt. 2, 393-497.)

HOOKER, J. D. and W. WiLson. 1844. Musci Antarctici, being characters with brief descriptions of the new species of mosses discovered during the voyage of H.M. Discovery Ships, Erebus and Terror, in the southern circumpolar regions, together with those of Tasmania and New Zealand. Lond. J. Bot., 3, 533-56.

HOOKER, W. J. 1816. Plantae cryptogamicae, quas in plaga orbis novi aequinoctiali collegerunt Alexander de Humboldt et Amat. Bonpland, adjectis tabulis species quasdam novas minusve cognitas exhibentibus necnon Alexandri de Humboldt notationibus quibusdam plantarum geographiam spectantibus. London, W. J. Hooker.

HOOKER, W. J. 1818. Musci exotici; containing figures and descriptions of new or little known foreign mosses and other cryptogamic subjects. Vol. 1. London, Longman, Hurst, Rees, Orme and Brown.

HOOKER, W. J. 1819. Musci exotici; containing figures and descriptions of new or little known foreign mosses and other cryptogamic subjects. Vol. 2. London, Longman, Hurst, Rees, Orme and Brown.

HOOKER, W. J. 1820. Musci exotici; containing figures and descriptions of new or little known foreign mosses and other cryptogamic subjects. Vol. 2. London, Longman, Hurst, Rees, Orme and Brown.

HOOKER, W. J. 1829. Bryum gilliesii. Botanical miscellany containing figures and descriptions of such plants as recommend themselves by their novelty, rarity or history or by the uses to which they are applied in the arts, in medicine, and in domestic economy together with occasional botanical notices and information. London, John Murray, 1 (for 1830), Pt. 1, 3-4, pl. 2.

HOOKER, W. J. 1830. Gymnostomum amblyophyllum. Botanical miscellany containing figures and descriptions of such plants as recommend themselves by their novelty, rarity or history or by the uses to which they are applied in the arts, in medicine, and in domestic economy together with occasional botanical notices and information. London, John Murray, 1 (for 1830), Pt. 3, 352-53, pl. 75.

HOOKER, W. J. 1833. Cryptogamia: Musci. (In SMITH, J. E. The English flora, Vol. 5 (or Vol. 2 of Dr. Hooker's British flora), Part 1, comprising the mosses, Hepaticae, lichens, Characeae and Algae. London, Longman, Rees, Orme, Brown, Green and Longman, 1-96.)

HOOKER, W. J. 1836. Bryum (Pohlia) pellucens. Hooker's Icon. PI., 1. Tab. 34.

HOOKER, W. J. 1837. Dicranum jamesoni. Hooker's Icon. Pl., 2, Tab. 179.
HOOKER, W. J. 1839. Andreaea subulata Harv. mst. Hooker's Icon. Pl., 3, Tab. 201.

HOOKER, W. J. 1840. Bruchia brevipes Harv. Hooker's Icon. Pl., 3, Tab. 231.

HOOKER, W. J. and G. A. W. ARNOTt. 1832. Order LXXIV. Musci. (In The botany of Captain Beechey's voyage comprising an account of the plants collected by Messrs Lay and Collie and other officers of the expedition, during the voyage to the Pacific and Bering's Strait, performed in His Majesty's ship Blossom, under the command of Captain F. W. Beechey, R. N., F. R., \& A. S., in the years 1825, 26, 27 and 28. London, Henry G. Bohn, Pt. 2, 53-54.)

HOOKER, W. J. and R. K. GREVLLE. 1824a. Sketch of the charcters of the species of mosses, belonging to the genera Orthotrichum (including Schlotheimia, Micromitrion and Ulota), Glyphomitrion, and Zygodon. Edinb. J. Sci., 1, No. 1, 110-33.

HoOKER, W. J. and R. K. GREvie. 1824b. On the genus Tortula of the order Musci. Edinb. J. Sci., 1, No. 2, 287-302.

HOOKER, W. J. andR. K. GReVILE. 1825a. On the genus Hookeria of Smith, of the order Musci. Edinb. J. Sci., 2, No. 2, 221-36.

HOOKER, W. J. and R. K. GReVLuE. 1825b. On the genus Calymperes of Swartz and Symhopodon of Schwaegrichen of the order Musci. Edinb. J. Sci., 3, No. 2, 218-27.

HOOKER, W. J. and T. TAYLOR. 1818. Muscologia Britannica; containing the mosses of Great Britain and Ireland systematically arranged and described; with plates illustrative of the characters of the genera and species. London, Longman, Hurst, Rees, Orme and Brown.

HOOKER, W. J. and W. WiLSON. 1844. Enumeration of the mosses and Hepaticae, collected in Brazil by George Gardner, Esq. Lond. J. Bot., 3, 149-67.

HOPPE, D. H. 1801. Nachtrag zu Abhandlung über die in Deutschland nachsenden Arten des Widerthons (Polytrichum). Bot. Taschenb., 1801, 196-99.

HoRIKAWA, Y. 1961. Mosses from the Ongul Islands and its neighbouring areas (preliminary report). Antarctic Rec., 11, No. 1, 876-78.

HoRIKAWA, Y. and H. ANDo. 1961. Mosses of the Ongul Islands collected during the 1957-1960 Japanese Antarctic Research Expedition. Hikobia. 2, No. 3, 160-78.

HORKAWA, Y. and H. ANDO. 1963. A review of the Antarctic species of Ceratodon described by Cardot. Hikobia, 3, No. 4, 275-80.

HoRIKAWA, Y. and $H$. ANDO. 1967. The mosses of the Ongul Islands and adjoining coastal areas of the Antarctic continent. JARE Sci. Rep., Special issue, No. 1, 245-52.

HORNSCHUCH, [C.] F. 1820a. Musci frondosi exotici herbarii Willdenowiani tum capensis A. B. Bergio lecti tum alii quidam ex australasiae alliisque orbis terraquei plagis $\mathrm{AL}$. a Chamissone relati. Descripsit et iconibus novarum maximeque memorabilium specierum illustravit. (In NEES AB EsEnBECK, C. G., ed. Horae physicae Berolinenses collectae ex symbolis virorum doctorum. Bonnae, Adolphi Marcus, 57-68.)

HORNSCHUCH, [C. F.] 1820b. Ueber die von Chamisso und Bergius gesammelten Moose. Flora, Jena, 3, No. 33, 511-22.

HORNSCHUCH, C. F. 1840. Musci. (In de MartuUs, C. F. P., ed. Flora Brasiliensis, enumeratio plantarum in Brasilia hactenus detectarum quas 
suis aliorumque botanicorum studiis descriptas et methodo naturali digestas partim icone illustratas. Vol. I. Monachii, Lipsiae apud Frid. Fleischner, Pt. 2, 2-100.)

HORNSCHUCH, [C.] F. 1841. Muscorum frondosorum novorum, quos in Africa Australiori collegerunt Ecklon, drège mundt et maire, descriptiones. Linnaea, Halle, 15, Ht. 1, 113-57.

Horrell, E. C. 1900. The European Sphagnaceae (after Warnstorf). J. Bot., Lond., 38, No. 12, 469-80.

Hosseus, C. C. 1934. Bemerkungen zum Vorkommen einiger Südamerikanischer Fabroniaceen. Reprium nov. spec. Regni veg., 35, Nos. 8-17, 174-76.

Hosseus, C. C. 1935a. Beiträge zur Kenntnis der Laubmoose der Provinz Cordoba (Argentinien). I. Reprium nov. spec. Regni veg., 38, Nos. 6-12, 131-36.

Hosseus, C. C. 1935b. Beiträge zur Kenntnis der Laubmoose der Provinz Cordoba (Argentinien). II. Reprium nov. spec. Regni veg., 38, Nos. 13-25, 209-18.

Hosseus, C. C. 1936. Beiträge zur Kenntnis der Laubmoose der Provinz Cordoba (Argentinien). III. Reprium nov. spec. Regni veg., 40, Nos. $1-10,135-48$.

Hosseus, C. C. 1937. Musgos de la República Argentina. Contribución al conocimiento de los Musgos de la Provincia de Córdoba (Argentina). I. Archos Esc. Farm. Córdoba, No. 5 (Sección científica), 1-42.

Hosseus, C. C. 1938a. Beiträge zur Kenntnis der Laubmoose der Provinz Cordoba (Argentinien). IV. Reprium nov. spec. Regni veg., 44, Nos. 5-10, 136-44.

Hosseus, C. C. 1938b. Beiträgè zur Kenntnis der Laubmoose der Provinz Cordoba (Argentinien). V. Reprium nov. spec. Regni veg., 44, Nos. 11-20, 241-43.

Hosseus, C. C. 1938 c. Beiträge zur Kenntnis der Laubmoose der Provinz Cordoba (Argentinien). VI. Reprium nov. spec. Regni veg., 45, Nos. 1-7, 20-43.

Hosseus, C. C. 1938d. Musgos de la República Argentina. Contribución al conocimiento de los Musgos de la Provincia de Córdoba (Argentina). II. Archos Esc. Farm. Córdoba, No. 8 (Sección científica), 1-58.

Hosseus, C. C. 1939. La flore briológica Argentina. Contribucion a su conocimiento. Physis, $B$. Aires, 15, No. 47, 1-12.

Hosseus, C. C. 1940. Musgos del Territoria de Misiones, neuvos para la Argentina. Cordoba, Imprenta de la Universidad.

HübeneR, J. W. P. 1833. Muscologia Germanica, oder Beschreibung der deutschen Laubmoose. Leipzig, Friedrich Hofmeister.

HULT, R. 1886. Mossfloran i trakterna mellan Aavasaksa och Pallastunturit. Acta Soc. Fauna Flora fenn., 3, No. 1, 1-111.

Husnot, T. 1888. Muscologia gallica. Descriptions et figures des mousses de France et des contrées voisines. Orne, T. Husnot, A. Cahan, par Athis; Paris, F. Savy. Pt. 1, Livr. 7, 193-224.

HusNot, T. 1894. Muscologia gallica. Descriptions et figures des mousses de France et des contrées voisines. Orne, T. Husnot, A. Cahan, par Athis, Pt. 2, Livr. 12, 349-80.

JAEGER, A. 1869. Uebersicht über die cleistocarpischen Moose. Ber. Tät. St Gall. naturw. Ges., 1868-69, 62-125.
JAEGER, A. 1870. Adumbratio florae muscorum totius orbis terrarum. Ber. Tät. St Gall. naturw. ges., 1869-70, 245-99.

JAEGER, A. 1872. Genera et species muscorum systematicae disposita seu adumbratio florae muscorum totius orbis terrarum. Ber. Tät. St Gall. naturw. Ges., 1870-71, 357-451.

JAEGER, A. 1873. Genera et species muscorum systematicae disposita seu adumbratio florae muscorum totius orbis terrarum. Ber. Tät. St Gall. naturw. Ges., 1871-72, 309-490.

JAEGER, A. 1874. Genera et species muscorum systematicae disposita seu adumbratio florae muscorum totius orbis terrarum. Ber. Tät. St Gall. naturw. Ges., 1872-73, 61-236.

JAEGER, A. 1875. Genera et species muscorum systematicae disposita seu adumbratio florae muscorum totius orbis terrarum. Ber. Tät. St Gall. naturw. Ges., 1873-74, 53-278.

JAEGER, A. 1876. Genera et species muscorum systematicae disposita seu adumbratio florae muscorum totius orbis terrarum. Ber. Tät. St Gall. naturw. Ges., 1874-75, 85-188.

JAEGER, A. 1877. Genera et species muscorum systematicae disposita seu adumbratio florae muscorum totius orbis terrarum. Ber. Tät. St Gall. naturw. Ges., 1875-76, 201-371.

JAEGER, A. 1878. Genera et species muscorum systematicae disposita seu adumbratio florae muscorum totius orbis terrarum. Ber. Tät. St Gall. naturw. Ges., 1876-77, 211-454.

JAEGER, A. and F. SAUERBECK. 1879a. Genera et species muscorum systematicae disposita seu adumbratio florae muscorum totius orbis terrarum. Ber. Tät. St Gall. natunw. Ges., 187778, 257-361.

JaEger, A. and F. Sauerbeck. $1879 b$. Supplementum ad adumbrationem muscorum et conspectus systematis. Ber. Tät. St Gall. naturw. Ges., 1877-78, 362-514.

JENNINGS, O. E. 1913. A manual of the mosses of western Pennsylvania. Pittsburgh, O. E. Jennings.

JENSEN, C. 1890. De danske Sphagnum-arten. (In Festskrift, udgivet af den Botaniske Forening i Kjøbenhavn $i$ anledning af dens halvhundredarrsfest, den 12, April 1890. Kjøbenhavn, Hoffensberg \& Trap's Etabl., 52-116.)

JENSEN, C. 1897. Mosser fra Øst-Grønland. Meddr Gronland, 15, Afd. 7 (for 1898), 365-443.

JENSEN, C. 1923. Danmarks Mosser eller Beskrivelse af de i Danmark med faerøerne fundne Bryofyter II. Andreaeales og Bryales. København, Kristiania, Glydendalske Boghandel, Nordisk Forlag.

JENSEN, C. 1939. Skandinaviens Bladmossflora. København, Ejnar Munksgaard.

JENSEN, T. 1856. Bryologica Danica eller de Danske bladmosser. Kjøbenhavn, C. G. Iversens Boghandel Theiles Bogtrykkeri.

JoHow, F. 1896. Cryptogamae - Bryophyta. (In Estudios sobre la flore de las Juan Fernandez. Santiago de Chile, Imprenta Cervantes, 174-84.)

[JURATZKA, J.] 1861. Verine, Gesellschaften, Anstalten. Öst. bot. Z., 11, No. 12, 409-13. [Article signed J. J. Ascribed to J. Juratzka by Index muscorum, 3, p. 80 under Hypnum heufleri.]

JURATZKA, J. 1882. Die Laubmossflora von Oesterreich-Ungarn. Wien, W. Braumüller.

KabiersCH, W. 1937. Studien über die ostasiatischen Arten einiger Laubmoosfamilien: 
II. Rhizogoniaceae, Bartramiaceae,

Aulacomniaceae, Meeseaceae. Hedwigia, 77, Ht. 2/3, 71-136,

KarCzmarz, K. 1966. Taxonomic studies on the genus Acrocladium Mitt. Nova Hedwigia, 11, Ht. 1-4, 499-505.

KıCKx, J. 1867. Flore cryptogamique des Flandres. Vol. 1. Gand, Librairie Générale de H. Hoste; Paris, Librairie de J. B. Baillière et Fils.

KINDBERG, N. C. 1883. Die arten der Laubmoose (Bryineae) Schwedens und Norwegens. Bih. K. svenska VetenskAkad. Handl., 7, No. 9, 1-167.

KINDBERG, N. C. 1885. Table analytique des mousses pleurocarpes européennes. Revue bryol., 12, No. 2, 24-31.

KINDBERG, N. C. 1888. Enumeratio bryinearum exoticarum quam alphabetice disposuit. Linkoeping, Officina Corresp. Ostrogoth.

KINDBERG, N. C. 1889. Enumeratio bryinearum exoticarum quam alphabetice disposuit. Supplementum primum, circiter 1000 acrocarpas complectens. Linkoeping, Officina Corresp. Ostrogoth.

KINDBERG, N. C. 1891. Enumeratio bryinearum exoticarum quam alphabetice disposuit. Supplementum secundum, circiter 970 species complectens. Linkoeping, Officina Corresp. Ostrogoth.

KINDBERG, N. C. 1894. Check-list of European and North American mosses (Bryineae). (Concluded.) Can. Rec. Sci., 6, No. 2, 72-76.

KINDBERG, N. C. 1897. European and North American Bryineae (mosses) Parts I \& II. Linköping, Lithografiska Aktiebolag.

KINDBERG, N. C. 1901. Grundzüge einer Monographie über die Laubmoos-Familie Hypopterygiaceae. Hedwigia, 40, Ht. 5, 275303.

KINDBERG, N. C. 1902. Grundzüge einer Monographie der Laubmoos-Gattung Thamnium. Hedwigia, 41, Ht. 4, 203-24; Ht. 5, 225-68.

KLINGgRÄFF, H. E. M. vON. 1858. Die höheren Cryptogamen Preussens. Ein Beitrag zur Flora der Provinz. Königsberg, Verlag von Wilhelm Koch.

KOPONEN, T. 1968. Generic revision of Mniaceae Mitt. (Bryophyta). Ann. bot. fenn., 5, No. 2, 11.7-51.

KOPONEN, T. 1969. The taxonomic status and typification of Mnium subpunctatum. Bryologist, 72, No. 1, 61-62.

KOPONEN, T. 1971. A monograph of Plagiomnium sect. Rosulata (Mniaceae). Ann. bot. fenn., 8, No. 4, 305-67.

Krauss, F. 1846. Pflanzen des Cap- und NatalLandes. Flora, Jena, 29, (N.R., 4), No. 9, 129-38.

KRIEGER, W. 1904. Ein Beitrag zur Kenntnis der Moosflora von Uruguay. Hedwigia, 43, Ht. 5 , 349-50.

Kuc, M. 1969. Some mosses from an Antarctic oasis. Revue bryol. lichen., N.S., 36, Fasc. 3-4, 655-72.

KüHNEMANN, O. 1938. Catalogo de los musgos argentinos. Lilloa, 2, 37-183.

KüHNEMANN, O. 1944. Generos de briofitas de los alrededores de Buenos Aires. Lilloa, 10, 5-232.

KüHNEMANN, O. and M. F. GoNÇALVES. 1975. Monografia preliminar para la flora criptogámica fueguina; Los musgos de la familia Ptychomniaceae (Bryophyta). Darwiniana, 19. Nos. 2-4, 583-617.

KüHNEMANN, O. and M. F. GonçALVEs. 1976. Sobre
Cryphaeophilum molle (Dus.) Fleisch. Meteoriaceae-Briofitas. Boln Soc. argent. Bot., 17, Nos. 3-4, 247-51.

KuNTH, C. S. 1822. Musci, Juss. (In Synopsis plantarum, quasin itinere ad plagam aequinoctialem Orbis Novi, collegerunt $A$, de Humboldt et A. Bonpland. Vol. 1. Paris, F. G. Levrault atque Argentorati, 46-65.)

KUNTZE, O. 1891. Cryptogamae cellulares Musci. (In Revisio generum plantarum vascularium omnium ataque cellularium multarum secundum leges nomenclaturae internationales, cum enumeratione plantarum exoticarum in itinere mundi collectarum. Pt. 2. Leipzig, Arthur Felix London, Dulau \& Co.; Milano, U. Hoepli; New York, Gust E. Stechert; Paris, Charles Klincksieck, 829-40.)

KUNTZE, O. 1898. Cryptogamae cellulares Musci. (In Revisio generum plantarum vascularium omnium atque cellularium multarum secundum leges nomenclaturae intemationales, cum enumeratione plantarum exoticarum in itinere mundi collectarum. Pt. 3. Leipzig, Arthur Felix; London, Dulau \& Co.; Melbourne, George Robertson \& Co.; Milano, U. Hoepli; New York, Gust E. Stechert; Paris, Charles Klincksieck, 382-83.)

KURTZ, F. 1904. Flora. (In Río, M. E. andL. ACHÁVAL. Geografia de la Provincia de Córdoba. Vol. I. Buenos Aires, Compañia Sud-Americana de Billets de Banco, 270-343.)

LAMARK, M. DE and [A.P.] DE CANDOLLE. 1805. Mousses. Musci. (In Flore française, ou descriptions succinctes de toutes les plantes qui croissent naturellement en France, disposées selon une nouvelle méthode d'analyse, et précédées par un exposé des principes élémentaires de la botanique. Triosième édition. Paris, Chez Desray, 438-545.)

LAMY, D. 1978. Dates de parution des volumes de la 2e. série de la Revue bryologique et lichénologique. Revue bryol. lichen., N.S., 44, Fasc. 2, 203-04.

LANGE, J. and C. JENSEN. 1887. Muscineae, Cryptogamae cellulares. (In LANGE, J. Conspectus florae Groenlandicae. Part 2. Meddr Gronland, 1880-87, Ht. 3, Afdel. 2, 309-446.)

LAURER, [J.F.] 1827. Beiträge zur kryptogamischen Flora der Insel Rügen. Flora, Jena, 10, No. 19, 289-99.

LAWTON, E. 1961. A revision of the genus Rhabdoweisia. Bryologist, 64, Nos. 2-3, 140-56.

LECHLER, W. 1857. Berberides americae australis, accedit enumeratio plantarum, quas in america australi auctor detexit. Stuttgart, $\mathrm{E}$. Schweizerbart, 49-59.

LIMPRICHT, K. G. 1881. Zur Systematik der Torfmoose. Bot. Zbl., 7, No. 36, 311-19.

LIMPRICHT, K. G. 1886. Die Laubmoose Deutschlands, Oesterreichs und der Schweiz. (In RABENHORST, L., ed. Kryptogamen-Flora von Deutschland, Oesterreich und der Schweiz, Auflage 2, Bd. 4. Leipzig, Eduard Kummer, Abt. 1, Lief. 4, 193-256.)

LIMPRICHT, K. G. 1888. Die Laubmoose Deutschlands, Oesterreichs und der Schweiz. (In RABENHORST, L., ed. Kryptogamen-Flora von Deutschland, Oesterreich und der Schweiz, Auflage 2, Bd. 4. Leipzig, Eduard Kummer, Abt. 1, Lief, 10, 577-640.)

LIMPRICHT, K.G. 1889. Die Laubmoose

Deutschlands, Oesterreichs und der Schweiz. (In 
RABENHORST, L., ed. Kryptogamen-Flora von Deutschland, Oesterreich und der Schweiz, Auflage 2, Bd. 4. Leipzig, Eduard Kummer, Abt. 1, Lief, 12, 705-68.)

LMPRICHT, K. G. 1892. Die Laubmoose

Deutschlands, Oesterreichs und der Schweiz. (In

RABENHORST, L., ed. Kryptogamen-Flora von

Deutschland, Oesterreich und der Schweiz,

Auflage 2, Bd. 4. Leipzig, Eduard Kummer, Abt.

2, Lief. 18, 257-320; Lief. 20, 385-448.)

LINDBERG, S. O. 1861. Om en ny art af slägtet

Hypnum. Öfvers. K. VetenskAkad. Förh., 18, No. 8, 371-75.

LINDBERG, S. O. 1862. Om ett nytt slägte Epipterygium, bland bladmossoma. Öfvers, $K$. VetenskAkad. Förh., 19, No. 10, 599-609. [Effective date 1863.]

LINDBERG, S. O. 1863. Bidrag till mossornas synonymi. Ö́vers. K. VetenskAkad. Förh., 20, No. 7, 383-418.

LINDBERG, S. O. 1864a. De Tortulis et ceteris Trichostomeis europaeis. Öfvers. $K$. VetenskAkad. Förh., 21, No. 4, 213-54.

LINDBERG, S. O. 1864b. Uppställing af familjen Funariaceae. Öfvers. K. VetenskAkad. Förh., 21, No. 10, 589-608. [Effective date 15 December 1865.]

LINDBERG, S. O. 1866. Förteckning öfver mossor, insamlade under de svenska expeditinerna till Spitsbergen 1858 och 1861. Öfvers. K. VetenskAkad. Förh., 23, No. 10, 535-61. [Effective date 1867, according to Index muscorum, 3, 170.]

LINDBERG, S. O. 1868a. Animadversiones de Hypno elegante Hook. et speciebus europaeis Plagiothecii. Notis. Sällsk. Faun. Fl. fenn. Förh., 9, [N.S., 6], 19-38.

LINDBERG, S. O. 1868b. Observationes de Mniaceis europaeis. Notis. Sällsk. Faun. Fl. fenn. Förh., 9, [N.S., 6], 39-88.

LINDBERG, S. O. 1868c. Observationes de formis praesertim europaeis Polytrichoidearum (Bryacearum nematodontearum). Notis. Sällsk. Faun. FI. fenn. Förh., 9, [N.S., 6], 89-158.

LINDBERG, S. O. 1868d. Musci novi scandinavici. Notis. Sällsk. Faun. Fl. fenn. Förh., 9, [N.S., 6], 253-99.

LINDBERG, S. O. 1871. Revisio critica iconum in opere Flora Danica muscos illustrantium. Acta Soc. Sci. fenn., 10 (for 1875), 3-118.

LINDBERG, S. O. 1872. Contributio ad floram cryptogamam Asiae boreali-orientalis. Acta Soc. Sci. fenn., 10 (for 1875), 221-80.

LNDBERG, S. O. 1879. Musci Scandinavici in systemate novo naturali dispositi. Upsaliae, Officina lesaiae Edquist.

LINDBERG, S. O. 1883. Kritisk granskning af mossorna uti Dillenii historia muscorum. Program. Helsingfors, J. C. Frenckell and Son.

LINDBERG, S. O. and H. W. ARNELL. 1890. Musci Asiae borealis. Beschreibung der von den Schwedischen Expeditionen nach Sibirien in den Jahren 1875 und 1876 gesammelten Moose mit Berücksichtigung aller Früheren bryologischen angaben für das Russische Nord-Asien. Zweiter Theil. Laubmoose. K. svenska VetenskAkad. Handl., 23, No. 10, 163 pp.

LINDSAY, W. L. 1866. On new or rare cryptogams from Otago, New Zealand. Trans. Proc. bot. Soc. Edinb., 8, 280-84.

LOESKE, L. 1903. Mossflora des Harzes. Hilfbuch für die bryologische Forschung im Harze und dessen Umgebund mit Verbreitungsangaben und Bestimmungstabellen. Leipzig, Gebrüder
Borntraeger.

LOESKE, L. 1907. Drepanocladus, eine biologische Mischgattung. Hedwigia, 46 (for 1906-07), Ht. 5, 300-21.

LOESEKE, L. 1910. Studien zur vergleichenden Morphologie und phylogenetischen Systematik der Laubmoose. Berlin, Max Lande.

LOESKE, L. 1911. Zur Moosflora von Füssen und Hohenschwangau. Hedwigia, 50, Ht. 5, 210-48.

LOESKE, L. 1913. Die Laubmoose Europas: I. Grimmiaceae. Berlin, Schöneberg.

LoNGton, R. E. and M. W. Holdgate. 1979. The South Sandwich Islands: IV. Botany. British Antarctic Survey Scientific Reports, No 94, 55 pp.

LOOSER, G. 1932. Excursión botánica y zoológica a la alta Cordillera de las Condes. An. Univ. Chile, Ser. 3, 2, No. 3, 275-301.

LOOSER, G. 1952. El Botanico frances Thèriot y su labor acerca de los musgos Chilenos. Revta univ., Santiago, 37, No. 1, [An. Acad. chil. Cienc. nat., No. 17], 221-43.

LORENTZ, P. G. 1864. III. Pugillus specierum novarurn e exoticarum. (In LORENTZ, P. G. Moosstudien. Leipzig, Wilhelm Engelmann, 15571.)

LORENTZ, P. G. 1866. Musci frondosi in Chile prope Valdiviam et prope Corral lecti per Dr. Krause. Bot. Ztg, 24, No. 24, 185-89.

LORENTZ, P. G. 1868. Musci frondosi a clarissimo $H$. Krause in Ecuador, Prov. Loja collecti. Bot. Ztg, 26, No. 47, 793-800; No. 48, 809-22.

MaCHADO, A. 1919. Catálogo descritivo de Briologia Portugësa. Lisboa, Imprensa de Manuel Lucas Torres. [Title page lacks date, so that given in index muscorum, 5, p. 144, under Trichostomum humile has been accepted but not verified.]

MACOUN, J. and N. C. KINDBERG. 1892. Catalogue of Canadian plants. Part VI. Musci. Montreal, William Foster Brown \& Co.

MAKU, M. 1979. Familias y géneros de musgos Chilenos. Bryologist, 82, No. 4, 513-24.

MALTA, N. 1924. Studien über die Laubmoosgattung Zygodon Hook. et Tayl. (11 and 12). Latv. Üniv. Rak., 10, 303-35.

MALTA, N. 1926. Die Gattung Zygodon Hook. et Tayl. Eine monographische studie. Latv. Üniv. bot. dárza Darbi, No. 1, 185 pp.

MALTA, N. 1927. Die Ulota-Arten Süd-Amerikas. Acta Horti bot. Univ. latv., 2s, Nos. 2/3, 165-208.

MARGADANT, W. D. 1959. Typification and conservation of generic names in Musci. Acta Bot. Neerl., 8, No. 3, 271-76.

MARTIN, R. R. 1949. Consideraciones generales sobre la flora actual de los turberos de Tierra del Fuego. Revta argent. Agron., 16, No. 3, 149-55.

MATHIEU, C. 1853. Mousses. (In Flore générale de Belgique, contenant la description de toutes les plantes qui croissent dans ce pays. Ouvrage publié sous le patronage de sa Majesté Le Roi des Belges. Tome II. Cryptogamie. Bruxelles, Leipzig, Gand, C. Muquardt, 20-73.)

MatTERI, C. M. 1968. Las especies de "Philonotis (Bartramiaceae)" del sur de Argentina. Revta Mus. argent. Cienc. nat. Bernardino Rivadavia, Ciencias botánica, 3, No. 4, 185-234.

MATTERI, C. M. 1972. Las Hookeriaceae (Musci) Andino-Patagonicas II. Revta Mus. argent. Cienc. nat. Bernardino Rivadavia, Ciencias botánica, 4, No. 2, 243-80.

MATTERI, C. M. 1973a. El genero Breutelia (Bartramiaceae, Musci) en la region AndinoPatagonica. Revta Mus. argent. Cienc. nat. 
Bernardino Rivadavia, Ciencias botánica, 4, No. 4, 321-60.

MATTERI, C. M. 1973b. Revision de las

Hypopterygiaceae (Musci)

Austrosudamericanas. Boln Soc. argent. Bot., 25, Nos. 2-3, 229-50.

MATTERI, C. M. 1973c. Notas briologicas (Musci) I. An. Inst. Patagonia, 4, Nos. 1-3, 273.

Matteri, C. M. 1973d. Notas briologicas (Musci) II. An. Inst. Patagonia, 4, Nos. 1-3, 274.

MATTERI, C. M. 1975a. Hookeriales. (In GuARrera, S. A., GAMUNDI DE AMOs, I. and D. Rabinovich DE HALPERIN, ed. Flora criptogámica de Tierre del Fuego. Buenos Aires, Fundación para la Educación, la Cienca y la Culutra, 14, Fasc. 9, 7-60.)

MatTERI, C. M. 1975b. Las Hookeriaceae (Musci) Andino-Patagonicas, I. Nova Hedwigia, 26, No. 4, 649-724. [Effective date 1976, personal communication from C. M. Matteri.]

MATTERI, C. M. 1977a. A synoptic flora of South Georgian mosses: VII. Pottia. British Antarctic Survey Bulletin, No. 46, 23-28.

MATTERI, C. M. 1977 b. Notes on Antarctic bryophytes: $X$. The genus Pottia from the Antarctic botanical zone. British Antarctic Survey Bulletin, No., 46, 140-43.

MATTERI, C. M. 1979. Notas briológicas (Musci) IV y V. Bryologist, 82, No. 1, 99-102.

MEIJER, W. 1952. The genus Orthodontium. Acta bot. neerl., 1, No. 1, 3-80.

MENZIES, A. 1798. A new arrangement of the genus Polytrichum, with some emendations. Trans. Linn. Soc. Lond., 4, 63-84.

MEYLAN, C. 1905. Catalogue des mousses du Jura. Bull. Soc. vaud. Sci. nat., 5 ème sér., 41, No. 152 41-96; No. 153, 97-172.

MidDleton, R. M. 1909. The first Fuegian collection J. Bot., Lond., 47, No. 6, 207-12.

MiLDE, J. 1864a. Ein Sommer in Süd-Tirol. Bot. Ztg. 22, Nos. 17-19 (Beilag), 1-22.

MILDE, J. 1864b. Zur Cryptogamen-flora Süd-Tirols. Bot. Ztg, 22, Nos. 43 and 44 (Beilag), 1-18.

MILDE, J. 1869. Bryologia Silesia. Laubmoose Flora von Nord-und Mittle-Deutschland, unter besonderer Berücksichtigung Schlesiens und mit Hinzunahme der Floren von Jütland, Holland, der Rheinpfalz, von Baden, Franken, Böhmen, Mähren und der Umgegend von München. Leipzig, Arthur Felix.

MITTEN, W. 1851. Catalogue of cryptogamic plants collected by Professor $W$. Jameson in the vicinity of Quito. Hooker's J. Bot. \& Kew Gdns Miscell., 3, 49-57; 351-61.

MitTEN, W. 1856. A list of the Musci and Hepaticae collected in Victoria, Australia, by Dr. F. Mueller. Hooker's J. Bot. \& Kew Gdns Miscell., 8, 257-66.

MITTEN, W. 1859. Musci Indiae Orientalis; an enumeration of the mosses of the East Indies. J. Proc. Linn. Soc., Supplement to Botany, 1, [supplement to Vol. III of main series], 1-171.

MitTEN, W. 1860. Descriptions of some new species of Musci from New Zealand and other parts of the Southern Hemisphere, together with an enumeration of the species collected in Tasmania by William Archer, Esq.; arranged upon the plan proposed in the 'Musci Indiae Orientalis'. J. Linn. Soc., Botany, 4, 64-100. [Read 17 March 1859.]

MITTEN, W. 1863. On the Musci and Hepaticae from the Cameroons Mountain and from the River Niger. J. Proc. Linn. Soc., Botany, 7 (for 1864), $147-69$.
MitTEN, W. 1864: The "Bryologia" of the survey of the 49th parallel of latitude. J. Linn. Soc., Botany, 8 (for 1865), 12-55.

MITTEN, W. 1868. A list of Musci collected by the Rev. Thomas Powell in the Samoa or Navigator's Islands. J. Linn. Soc., Botany, 10 (for 1869), 166-95.

MITTEN, W. 1869. Musci austro-americani. J. Linn. Soc., Botany, 12, 1-659.

MiTTEN, W. 1873. New species of Musci collected in Ceylon by Dr Thwaites. J. Linn. Soc., Botany, 13, 293-326.

MiTTEN, W. 1875. Musci (mosses). (In Mews, J. C. St. Helena; physical, historical, and topographical description of the island, including its geology, fauna, flora and meteorology. London, L. Reeve \& Co.,357-66.)

MitTEN, W. 1876. The Musci and Hepaticae collected by H.N. Moseley, M.A., naturalist to H.M.S. 'Challenger'. J. Linn. Soc., Botany, 15, No. 82, 59-73.

MITTEN, W. 1879. Musci. (In An account of the petrological, botanical and zoological collections made in Kerguelen's Land and Rodriguez during the Transit of Venus Expeditions, carried out by order of Her Majesty's Goverment in the years 1874-75. Phil. Trans. R. Soc., 168 (extra volume), 24-39, 388-96.)

MITTEN, W. 1883. Australian mosses. Trans. R. Soc. Vict., 19, 49-96.

MitTEN, W. 1885. Musci. (In HemsLeY, W. B. Report on the botany of Juan Fernandez, the southeastern Moluccas, and the Admiralty Islands. Report on the Scientific Results of the Voyage of H.M.S Challenger during the years 1873-76, under the command of Captain George S. Nares, R.N., F.R.S., and the late Captain Frank Tourle Thomson, R.N. Botany, Vol. 1, Pt. 3, 78-83; 258-62.)

MitTEN, W. 1886. The mosses and Hepaticae collected in Central Africa by the late Right Rev. James Hannington, Bishop of Mombasa, F.L.S., F.G.S., \&c., with some others, including those gathered by Mr. H. H. Johnson on Kilimanjaro. J. Linn. Soc., Botany, 22, No. 146, 298-329.

MoHR, D. M. H. 1806. Observations on Orthotrichum and Neckera, together with some other genera of mosses. Ann. Bot., Lond., 2, No. 6, 532-47.

MOLENDO, I. 1875. Bayerns Laubmoose. Vorläufige Uebersicht mit besonderer Rücksicht auf Niederbayern. Ber. naturw. Ver. Passau, 187174, 1-278.

MöLLER, H. 1907. Ett par upplysningar angående den snart utkommande förteckningen öfver Skandinaviens mossor. Bot. Notiser for 1907, 141-45.

MÖNKEMEYER, W. 1911. Untersuchungen über Cratoneura und Hygroamblystegia. Hedwigia, 50, (for 1910-11), Ht. 5/6, 263-78.

MÖNKEMEYER, W. 1927. Die Laubmoose Europas. (In RABENHORST, L., ed. Kryptogamenflora von Deutschland, Österreich und der Schweiz, Bd. 4, Ergänzungsband. Leipzig, Akademische Verlagsgesellschaft, m.b.H., 960 pp.)

MONTAGNE, C. 1835. Prodromus florae fernandesianae. Pars prima, sistens enumerationem plantarum cellularium quas in Insulá Juan Fernandez à Cl. Bertero collectas describi edique curavit. Ann/s Sci. nat., Sér. 2, 4, Botanique, 86-99.

MONTAGNE, C. 1837. Monographie du genre Conomitrium, de la famille des mousses. Annls 
Sci. nat., Sér. 2, 8, Botanique, 239-52.

MONTAGNE, C. 1838. Centurie de plantes cellulaires exotiques nouvelles, Musci. Ann/s Sci. nat., Sér. 2, 9, Botanique, 38-57.

MONTAGNE, C. 1839a. Cryptogamae brasilienses seu plantae cellulares quas in itinere per Brasiliam à celeb. Auguste de Saint-Hilaire collectas recensuit observationibusque nonnullis illustravit. Annls Sci. nat., Sér. 2, 12, Botanique, 42-55.

MONTAGNe, C. $1839 b$. Sertum patagonicum. Cryptogames de la Patagonie. (In D'ORBIGNY, A. Voyage dans l'Amérique méridionale (le Brésil, la République orientale de l'Uruguay, la République Argentine, la Patagonie, la République du Chili, la République de Bolivia, la République du Pérou), éxecuté pendant les années 1826, 1827, 1828, $1829,1830,1831,1832$ et 1833. Paris, Chez P. Bertrand; Strasbourg, Chez Ve. Levrault, Vol. 7, Botanique, Cryptogamie, Pt. 1, 1-19.) [Effective dates p. 1-16, 1839 (?); p. 17-19, 1840, fide Stafleu, 1967, 326.]

MONTAGNE, C. $1839 c$. Florula boliviensis. Cryptogams de la Bolivia recueillies par Alcide d'Orbigny. (In D'Orbigny, A. Voyage dans l'Amérique méridionale (le Brésil, la République orientale de l'Uruguay, la République Argentine, la Patagonie, la République du Chili, la République de Bolivia, la République du Pérou), exécuté pendant les années 1826, 1827, 1828, 1829, 1830, 1831, 1832 et 1833. Paris, Chez P. Bertrand; Strasbourg, Chez V ${ }^{\circledR}$. Levrault, Vol. 7 , Botanique, Cryptogamie, Pt. 2, 1-119.) [Effective date p. 1-104, 1839; p. 105-19, 1840, fide Stafleu, 1967, 326.]

Montagne, C. 1840a. Seconde centurie de plantes cellulaires exotiques nouvelles. Décades VI, VII e: VIII. Annls Sci. nat., Sér 2, 14, Botanique, 321-50.

Montagne, C. 1840 b. Plantes cellulaires. D. C. Muscinae. (In BARKER-WEBB, P. andS. BERTHELot, Histoire naturelle des illes Canaries, Vol. 3, Pt. 2. Phytographia Canariensis, Sectio Ultima. Paris, Béthune, 1-67.)

Montagne, C. 1841. Seconde centurie de plantes cellulaires exotiques nouvelles. Décade $X(1)$ Cryptogamarum Brasiliensium continuatio. Annals Sci. nat., Sér. 2, 16, Botanique, 266-82.

Montagne, C. 1842. Botanique - plantes cellulaires: Musci. (In SAGRA, R. DE LA. Historie physique, politique et naturelle de l'ile de Cuba. Paris, Arthus Bertrand, 493-533.) .

MontAGNe, C. 1843. Quatrième centurie de plantes cellulaires exotiques nouvelles, Musci. Annls Sci. nat., Sér. 2, 19, Botanique, 238-43.

MontAGNe, C. 1845a. Cinquième centurie de plantes cellulaires exotiques nouvelles. Décades I à VI. Annls Sci. nat., Sér. 3, 4, Botanique, 86-123.

Montagne, C. 1845b. Plantes cellulaires. (In HOMBRON, M. and C. JACQUINOT. Voyage au Pole Sud et dans l'Océanie sur les corvettes l'Astrolabe et la Zélée, exécuté par ordre du roi pendant les années 1837-1838-1839-1840, sous le commandement de M. J. DumontD'Urville, Capitaine de vaisseau. Botanique, Vol. 1, Paris, Gide et Cie, 281-335.)

Montagne, C. 1846. Cryptogames cellulaires, Musci. (In Gaudichaud, M. 1844-46. Voyage autour du monde exécuté pendant les années 1836 et 1837 sur la corvette La Bonite commandée par $M$. Vaillant capitaine de Vaisseau publié par ordre du rois sous les auspices du département de la marine.
Botanique, Vol. 1, Paris, Arthus Bertrand, 267314.) [Date fide Staflleu, 1967, 166.]

Montagne, C. 1850 . Flora chilena, plantas cellulaires. I. Musgos. (In GAY, C. Historia fisica y politica de Chile. Vol. 7, Botanica. Paris, C. Gay; Chile, Museo de Historia natural de Santiago, 5-202.)

Montagne, C. Xs 1856. Classis Muscinae. (IN SYLLOGE GENERUM SPECIERUMOUE CRTTOGAMARUM. ParisiIs, J-B. BaIlliére, LoNdiNI, H. Baillière, 1-95.)

Montagne, C. 1888. Pottia flaviseta. (In JaCkson, D. $B$., ed General index to the Journal (Botany, Vols. $(-X X)$ and Proceedings, Nov. 1838 to June 1886, of the Linnean Society of London, 328.)

MoORE, D. 1873. A synopsis of the mosses of Ireland. Proc. R. Ir. Acad., Ser. 2, 1, Science (for 1872-73), No. 8, 329-474.

MoRITZI, A. 1846. Systematisches Verzeichniss der von $H$. Zollinger in den Jahren 1842-1844 auf Java gesammelten Pflanzen, nebst einer kurzen Beschreibung der neuen Gattungen und Arten. Solothurn, A. Moritzi. [Date fide Stafleu, 1967. 329.]

MUELLER, C. 1843a. De muscis frondosis nonnullis novis vel minus cognitis. Linnaea, Halle, 17. [Beitr. PflKunde, 1], Ht. 5, 581-601.

MUELLER, C. 1843b. Aulacomnion chilense nov. sp. Bot. Ztg, 1, No. 38, 649-56.

MUELLER, C. 1844a. Relation über die von Gardner in Brasilien gesammelten Laubmoose. Bot. Ztg, 2, No. 42, 726-28.

MUELLER, C. 1844b. Beiträge zu einer Flora der Aequinoctial-Gegenden. Laubmoose. Linnaea, Halle, 18, [Beitr. PflKunde, 12], Ht. 6, 667-709. [Effective date 1845, according to note on $p$. 508.]

MUelefe, C. 1845. Synopsis Macromitriorum hactenus cognitorum. Bot. Ztg, 3, No. 33, 53945.

MUeller, C. 1847a. Beiträge zu einer Flora der Aequinoctial-Gegenden der neuen Welt. Columbische Laubmoose. Linnaea, Halle, 19, [Beitr. PflKunde, 3], Ht. 2, 193-220. [Effective date February 1846, fide Van Steenis-Kruseman, M. J. and W. T. Stearn, 1954, p. cxcviii.]

MUELLER, C. $1847 b$. Systema muscorum ordinis "Cleistocarpi" Brid. Bot. Ztg, 5, No. 7, 97-102.

MUELLER, C. 1847c. De muscis nonnullis novis vel minus cognitis exoticis. Bot. Ztg, 5, No. 46, 801-06; No. 47, 825-30.

MUELLER, C. 1848a. Synopsis muscorum frondosorum omnium hucusque cognitorum. Vol. I. Berlin, Alb. Foerstner. [Reprinted 1973: Synopsis muscorum frondosorum omnium hucusque cognitorum, with a supplement consisting of the articles published in the Botanische Zeitung, Vols. 6-9: Vol. I. Musci vegetationis acrocarpicae. Amsterdam, A. Asher \& Co. B. V., Fasc. 1, 1-160; Fasc. 2, 161-320; Fasc. 3, 321-480.]

Mueller, C. $1848 b$. Plantae Kegelianae surinamenses. Musci frondosi. Linnaea, Halle, 21, [Beitr. PfIKunde, 5], Ht. 2, 181-200.

MUELLER, C. 1848 c. Ueber die Laubmoose der, von den Herren Funck and Schlium in Columbien veranstalteten, Käuflichen Sammlung des Herren J. Linden in Luxemburg. Bot. Ztg, 6, No. 44, 761-68.

MUELLER, C. 1849. Synopsis muscorum frondosorum omnium hucusque cognitorum. Vol. I. Berlin, Alb. Foerstner, [Reprinted 1973 Synopsis muscorum frondosorum omnium hucusque cognitorum, with a supplement 
consisting of the articles published in the Botanische Zeitung, Vols. 6-9: Vol. I. Musci vegetationis acrocarpicae. Amsterdam, A. Asher \& Co. B. V., Fasc. 4, 481-640; Fasc. 5, 641-812.]

MUeller, C. 1850. Synopsis muscorum frondosorum omnium hucusque cognitorum. Vol. II. Berlin, Alb. Foerstner. [Reprinted 1973: Synopsis muscorum frondosorum omnium hucusque cognitorum, with a supplement consisting of the articles published in the Botanische Zeitung, Vols. 6-9: Vol. II. Musci vegetationis pleurocarpicae cum appendice. Amsterdam, A. Asher \& Co. B. V., Fasc. 6, 1-160.]

MUelleR, C. 1851a. Synopsis muscorum frondosorum omnium hucusque cognitorum. Vol. II. Berlin, Alb. Foerstner. [Reprinted 1973: Synopsis muscorum frondosorum omnium hucusque cognitorum, with a supplement consisting of the articles published in the Botanische Zeitung, Vols. 6-9. Vol. II. Musci vegetationis pleurocarpicae cum appendice. Amsterdam, A. Asher \& Co. B. V., Fasc. 7 and 8 , 161-510; Fasc. 9 and 10, 511-722.]

Mueller, C. 1851 b. Die, von Samuel Mossman im Jahre 1850 in Van Dieman's Land, Neuseeland und Neuholland gemachte Laubmoossammlung bestimmt. Bot. Ztg, 9, No. 31, 545-52; No. 32, 561-57.

MUELLER, C. 1855. De muscis novis, incomplete descriptis, neglectis criticisve. Bot. Ztg, 13, No. 44, 761-69; No. 45, 782-89.

MuelleR, C. 1856. Symbolae ad synopsin muscorum. Bot. Ztg, 14, No. 24, 415-21; No. 26, 455-59.

Mueller, C. 1857. Beiträge zu einer Flora der Kryptogamen Brasiliens, insbesondere der Insel Santa Catharina. Bot. Ztg, 15, No. 23, 378-87.

MuelleR, C. 1858. Additamenta nova ad synopsin muscorum. Bot. Ztg, 16, No. 22, 154-56; No. 24 , 169-72.

MuELLER, C. 1859. Supplementum novum ad synopsin muscorum. Bot. Ztg, 17, No. 22, 197-98; No. 23, 205-07; No. 24, 214-15; No. 25 219-21; No. 26, 229-31; No. 28, 246-48.

Mueller, C. 1862. Additamenta ad synopsin muscorum nova. Bot. Ztg, 20, No. 40, 327-29; No. 41, 337-39; No. 42, 348-50; No. 43, 361-62. No. 44, 373-74.

MUELleR, C. 1864. Manipulus muscorum novorum. Bot. Ztg, 22, No. 45, 339-42; No. 49, 373.

Mueller, C. 1869. Mittheilungen über Rhacomitrium lanuginosum und die verwandten Arten. Verh. zool. -bot. Ges. Wien, 19, Ht. 1, 223-24.

MUELLER, C. 1872. Musci Australici praesertim Brisbanici novi. Linnaea, Berlin, 37 (for 1871-73), [Beitr. PfIKunde, N.F., 3], Ht. 2, 143-82.

MUELLER, C. 1874. Novitates bryothecae müllerianae: II. Musci Novo-Granatenses. Wallisiani adjectis nonnullis aliis muscis novis andinis vel tropico-Americanis vel australasiacis. Linnaea, Berlin, 38, [Beitr. PfIKunde, N.F., 4], Ht. 5, 572-620.

MuELLER, C. 1875. Musci Schweinfurthiani in itineribus duobus in Africam Centralem per annos 1868-1871 collecti, determinati ex expositi. Linnaea, Berlin, 39, [Beitr. PfIKunde, N.F., 5], Ht. 4-6, 325-474.

MUeller, C. 1879a. Prodromus bryologiae Argentinicae. I. Linnaea, Berlin, 42 (for 1878-79), [Beitr. PfIKunde, N.F., 8], Ht. 3-5, 217-460.

Mueller, C. $1879 b$. Musci Fendleriani Venezuelenses. Linnaea, Berlin, 42, [Beitr.
PfIKunde., N.F., 8], Ht. 5-7, 461-502.

MUELLER, C. 1882. Prodromus bryologiae Argentinicae, II. Linnaea, Berlin, 43 (for 1880-82), [Beitr. PflKunde, N.F., 9], Ht. 5 and 6, 341-486.

MuelleR, C. 1884. Die auf der Expedition SMS "Gazelle" von Dr. Naumann gesammelten Laubmoose. Bot. Jb., 5, Ht. 1, 76-88. [Effective date 31 December 1883.]

MUELLER, C. 1885. Bryologia fuegiana. Flora, Jena, 68, Nos. 21-23, 391-429.

MUELLER, C. 1887a. Sphagnorum novorum descriptio. Flora, Jena, 70, Nos. 26 and 27, 403-22.

MUelleR, C. $1887 \mathrm{~b}$. Erpodiaceae quatuor novae. Flora, Jena, 70, No. 28, 446-50.

Mueller, C. 1888. Musci cleistocarpici novi. Flora, Jena, 71, No. 1, 1-13.

MUelleR, C. 1889. Laubmoose (Musci frondosi). Die Forschungsreise S.M.S. "Gazelle", Theil IV, Botanik. Berlin, Ernst Siegfried Mittler und Sohn.

MUELLER, C. 1890a. Bryologia austro-georgiae. (In NeUMAYER, G. Die Intemationale Polarforschung 1882-83. Die Deutschen Expeditionen und ihre Ergebnisse. Berlin, A. Asher and Co., Bd. 2, 279-322.)

MUELLER, C. 1890b. Die Moose von vier Kilimandscharo Expeditionen. Flora, Jena, 73, [N.R., 48], No. 5, 465-99.

MUELLER, C. 1896a. Bryologia provinciae Schen-si Sinensis. Nuovo G. bot. ital., N.S., 3, Fasc. 1, 89-129.

MuelleR, C. $1896 b$. Musci nonnulli novi Guianae Anglicae prope Georgetown ad cataractas "Marshall Falls" fluvii Mazarunii a Cl. J. Quelch collecti. Malpighia, 10, Fasc. 11-12, 512-20.

Mueller, C. 1897a. Prodromus bryologiae Argentinicae atque regionum vicinarum. III. Hedwigia, 36, Ht. 2, 84-128; Ht. 3, 129-44.

Mueller, C. $1897 \mathrm{~b}$. Prodromus bryologiae Bolivianae. Nuovo G. bot. ital., N.S., 4, Fasc. 1, 5-50; Fasc. 2, 113-72.

MUELLER, C. 1897 c. Bryologia Guatemalensis ex collectionibus domin Bernoulli \& Cario (18661878). V. Türckeim et aliorum. Bull. Herb. Boissier, 5, No. 3, 171-220.

MUELLER, C. $1897 d$. Synopsis generis Harrisonia. Öst. bot. Z., 47, No. 11, 387-98; No. 12, 417-20.

MUELLER, C. 1897 e. Triquetrella genus muscorum novum conditum et descriptum. Öst. bot. Z., 47, No. 12, 420-24.

MUELLER, C. 1898a. Symbolae ad bryologiam Australiae. II. Hedwigia, 37, Ht. 2, 76-112; Ht. 3, 113-44; Ht. 4, 145-71.

MUELLER, C. 1898 b. Analecta bryographica Antillarum. Hedwigia, 37, Ht. 4, 219-24; Ht. 5,

Mueller, C. 1898c. Bryologia serrae itatiaiae (Minas Geraës Brasiliae) adjectis nonnullis speciebus affinibus regionum vicinarum. Bull. Herb. Boissier, 6, No. 1, 18-48; No. 2, 89-126.

MUELLER, C. 1900 . Symbolae ad bryologiam Brasiliae et regionum vicinarium. Hedwigia, 39, Ht. 5, 235-89.

MUELleR, C. 1901a. Genera muscorum frondosorum, classes Schistocarporum, Cleistocarporum, Stegocarporum complectentia, exceptis Orthotrichaceis et Pleurocarpis. Leipzig, Eduard Kummer. [Effective date 1900, fide Index muscorum, 1, p. 56 under Andreaea liliputana.]

MUELLER, C. $1901 \mathrm{~b}$. Symbolae ad bryologiam Brasiliae et regionum vicinarum. Hedwigia, 40, Ht. 1, 55-80; Ht. 2, 81-99.

Mueller, C. and V. F. Brotherus. 1900. Ergebnisse 
einer Reise nach dem Pacific. (H. Schauimland, 1896-97.) Musci Schauinslandiani. Ein Beitrag zur Kenntnis der Moosflora der Pacifischen Inseln. Abh. naturw. Ver. Bremen, 16, Ht. 3, 493-512.

Mueller, C. and E. HAMPE. 1855. Musci frondosi Australasiae ab Drc. Ferd. Müller lecti. Linnaea, 26 (for 1853), Ht. 4, 489-505.

Muelier, F. A. 1829. Erestes Verzeichnis sardinischer Laubmoose, wie auch derjenigen welche von meinem Freunde Herm Fleischer bei Smyma aufgefunden worden sind, nebst Beschreibungen und Abbildungen einiger neuen Arten. Flora, Jena, 12, No. 25, 385-400.

MULLen, D. andT. C. Frye. 1947. Middle and South American species of Oligotrichum. Bryologist, 50 , No. 1, 67-79.

Naveau, R. 1928. Mousses critiques. Revue bryol., N.S., 1, No. 1, 38-40.

Nees von EsenbeCK, C. G., HornsChuch, F. and J. STURM. 1823. Bryologia Germanica, oder Beschreibung der in Deutschland und in der Schweiz wachsenden Laubmoose, Vol. 1. Nürnberg, J. Sturm.

NEGER, F. W. 1899. Informe sobre las observaciones botánicas efectuadas en la Cordillera de Villarrica en el verano 1896-97. An. Univ. Chile, 103, No. 6, 903-67.

NewTON, M. E. 1974a. A synoptic flora of South Georgian mosses: IV. Bartramia and Breutelia. British Antarctic Survey Bulletin, No. 38, 59-71.

NewTON, M. E. 1974b. Taxonomic notes on Cheilothela (Lindb.) Broth. and Dicranella (C. Muell.) Schimp. J. Bryol., 8, Pt. 2, 265-68.

NEWTON, M. E. 1974c. Notes on Antarctic bryophytes: IV. Encalypta Hedw. British Antarctic Survey Bulletin, No. 39, 1-6.

NEWTON, M. E. 1974d. Notes on Antarctic bryophytes: V. Brachythecium majusculum. M. E. Newton, sp. nov. British Antarctic Survey Bulletin, No. 39, 45-48.

NEWTON, M. E. 1977a. A synoptic flora of South Georgian mosses: VI. Cheilothela, Dicranella, Distichium, Myurella, and Catagonium. British Antarctic Survey Bulletin, No. 46, 1-21.

NeWTON, M. E. 1977b. Notes on Antarctic bryophytes: VII. The occurrence of Distichium B.S.G. and Dicranella (C. Muell.) Schimp. in the Antarctic botanical zone. British Antarctic Survey Bulletin, No. 46, 131-35.

NEWTON, M. E. 1979a. A taxonomic assessment of Brachythecium on South Georgia. British Antarctic Survey Bulletin, No. 48, 119-32.

NEWTON, M. E. 1979b. A synoptic flora of South Georgian mosses: VIII. Calliergon and Brachythecium. British Antarctic Survey Bulletin, No. 48, 133-57.

NieuWLAND, J. A. 1917. Critical notes on new and old genera of plants. X. Am. Midl. Nat., 5, No. 2, 50-52.

Noguchl, A. 1947. A review of the Leucodontinae and Neckerinae of Japan, Loo Choo and of Formosa I. J. Hattori bot. Lab., No. 2, 27-79.

NyLANDER, W. andT. SaELAN. 1859. Herbarium musei fennici: förteckning öfver Finska musei växtsamling, utgifven af sällskapet pro fauna et flora fennica. Helsingfors, Finska Litteratursällakapets Tryckeri.

OCHI, H. 1967. Notes on moss flora. V. Hikobia, 5 , Nos. 1-2, 14-38.

OCHI, H. 1969. Notes on moss flora. VI. Hikobia, 5 , Nos. 3-4, 153-71.
OcH, H. 1970. A revision of the sub-family Bryoideae in Australia, Tasmania, New Zealand and the adjacent islands. J. Fac. Educ. Tottori Univ., Natural Science, 21, No. 1, 7-64.

$\mathrm{OCHI}, \mathrm{H} .1972$. A revision of African Bryoideae, Musci (first part). J. Fac. Educ. Tottori Univ., Natural Science, 23, No. 1, 1-126.

$\mathrm{OCH}, \mathrm{H} .1976$. On the taxonomic status of Bryum inconnexum Card. in Antarctica. Misc. bryol. lichen., 7, No. 6, 116-17.

$\mathrm{OCH}, \mathrm{H} .1977$. Central and South American bryaceous mosses, new to science or of geographical significance. J. Fac. Educ. Tottori Univ., Natural Science, 27, No. 2, 33-41.

$\mathrm{OCHI}, \mathrm{H}$. 1979. A taxonomic review of the genus Bryum, Musci, in Antarctica. (In MATSUDA, T. and T. HoshIAl, ed. Proceedings of the Symposium on Terrestrial Ecosystem in the Syowa Station Area. Mem. natn. Inst. Polar Res. Tokyo, Special Issue, No. 11, 70-80.)

Pausot de Beauvois, A. M. F. J. 1805a. Prodrome des cinquième et sixième familles de l'Aethéogamie, les mousses, les lycopodes. Paris, l'Auteur et Fournier fils. [For combinations effective in 1804 see Crum, 1952.]

Pausot dE BeAuvoIs, A. M. F. J. 1805b. Bartrame. (In Dictionnaire des Sciences Naturelles, Vol. 4. Strasburg and Paris, F. G. Levrault, 87-88.) [Effective date 8 January 1806, fide Sayre, 1959, 70.]

Palisot de Beauvois, [A. M. F. J.] 1822. Muscologie, outraité sur les mousses. Mém. Soc. linn. Paris, 1, Pt. 2, 388-472.

PARIS, E. G. 1894. Index bryologicus sive enumeratio muscorum hucusque cognitorum adjunctis synonymia distributioneque geographica locupletissimis. Pt. 1. Pariis, Paul Klincksieck, 1-324. [Date of issue fide Paris, 1900b.]

PARIS, E. G. 1895. Index bryologicus sive enumeratio muscorum hucusque cognitorum adjunctis synonymia distributioneque geographica locupletissimis. Pt. 2. Pariis, Paul Klincksieck, 325-644. [Date of issue fide Paris, 1900b.]

PARIS, E. G. 1896. Index bryologicus sive enumeratio muscorum hucusque cognitorum adjunctis synonymia distributioneque geographica locupletissimis. Pt. 3. Pariis, Paul Klincksieck, 645-964. [Date of issue fide Paris, 1900b.]

PARIS, E. G. 1897. Index bryologicus sive enumeratio muscorum hucusque cognitorum adjunctis synonymia distributioneque geographica locupletissimis. Pariis, Paul Klincksieck, Pt. 4, 965-1284. [Date of issue fide Paris, 1900b.]

PARIS, E. G. 1898. Index bryologicus sive enumeratio muscorum hucusque cognitorum adjunctis synonymia distributioneque geographica locupletissimis. Pariis, Paul Klincksieck, Pt. 5, 1285-380. [Date of issue fide Paris, 1900b.]

PARIS, E. G. 1900a. Index bryologicus. Supplement. Genève et Bale, Georg and Cie.

PARIS, E. G. 1900b. Musci Novo-Zelandici Browniana. Revue bryol., 27, No. 4, 49-51.

PARIS, E. G. 1903. Index bryologicus sive enumeratio muscorum ad diem ultimam anni 1900 cognitorum adjunctis synonymia distributioneque geographica locupletissimis. 2nd edition. Paris, Librarie Scientifique A. Hermann, 1, Fasc. 1, 1-64. 
PARIS, E. G. 1904a. Index bryologicus sive enumeratio muscorum ad diem ultimam anni 1900 cognitorum adjunctis synonymia distributioneque geographica locupletissimis. 2nd edition. Paris, Librarie Scientifique A. Hermann, 1, Fasc. 2-6, 65-384.

PARIS, E. G. 1904b. Index bryologicus sive enumeratio muscorum ad diem ultimam anni 1900 cognitorum adjunctis synonymia distributioneque geographica locupletissimis. 2nd edition. Paris, Librarie Scientifique A. Hermann, 2, Fasc. 1-6 [7-12], 1-375.

PARIS, E. G. 1904c. Index bryologicus sive enumeratio muscorum ad diem ultimam anni 1900 cognitorum adjunctis synonymia distributioneque geographica locupletissimis. 2nd edition. Paris, Librarie Scientifique A. Hermann, 3, Fasc. 13, 1-72.

Paris, E. G. 1905a. Index bryologicus sive enumeratio muscorum ad diem ultimam anni 1900 cognitorum adjunctis synonymia distributioneque geographica locupletissimis. 2nd edition. Paris, Librarie Scientifique A. Hermann, 3, Fasc. 14-18, 73-400.

PARIS, E. G. $1905 \mathrm{~b}$. Index bryologicus sive enumeratio muscorum ad diem ultimam anni 1900 cognitorum adjunctis synonymia distributioneque geographica locupletissimis. 2nd edition. Paris, Librarie Scientifique A. Hermann, 4, Fasc. 19-24, 1-368.

PARIS, E. G. 1906. Index bryologicus sive enumeratio muscorum ad diem ultimam anni 1900 cognitorum adjunctis synonymia distributioneque geographica locupletissimis. 2nd edition. Paris, Librarie Scientifique A. Hermann, 5, Fasc. 25-27, 1-160.

PARIS, E. G. 1909. Collatio nominum brotherianorum et indicis bryologici. Paris, Libraria J.-B. Baillière et filii.

Piovano, G. 1954. Alcuni muschi nuovi per l'Argentina. Nuovo G. bot. ital., N.S., 61, Fasc. 2-3, 425-28. [Effective date 10 June 1955.]

PODPĚra, J. 1911. Ein Beitrag zu der Kryptogamenflora der bulgarischen Hochgebirge. Beih. bot. Zbl., 28, Abt. 2, Ht. 2, 173-224.

PODPĚrA, J. 1921. Ad Bryophytorum Cisuralensium cognitionem additementum. Spisy vydáv. prĭir. Fak. Masaryk. Univ., Čis. 5, 1-42.

POIRET, J. L., ed. 1808. Encyclopédie méthodique botanique, par M. Lamarck, de l'Institut National de France. Vol. 8. Paris, H. Agasse.

Porter, C. E. 1900. Los musgos colectados por la espedición Antártica Belga en el estrecho de Magallanes i Tierra del Fuego estractados de la "Revue Bryologique". Revta chil. Hist. nat., 4, No. 7, 102-06.

RAAB [W.] 1819. Excursion in die Gegend von Muggendorf von Herrn Präsidenten und Pofessor Nees von Esenbeck und seinen Zuhörern aus Erlangen. Flora, Jena, 2, No. 19, 289-304.

RABENHORST, [G.] L. 1848. Leber-, Laubmoose und Farrn. (In RABENHORST, L. Deutschlands Kryptogamen-Flora. Bd. 2. Leipzig, Eduard Kummer, Abt. 3, 1-352.)

RABENHORST, [G.] L. 1863. Kryptogamen-flora, Sachsen, der Ober-Lausik, Thüringen und Nordböhmen mit Berüchsichtigung der benachbarten Länder. Abt. 1. Leipzig, Eduard Kummer.

RADDI, G. 1823. Crittogame brasiliane. Memorie Mat. Fis. Soc. ital. Sci., 19, Fasc. 1, 27-57.
RAYNER, G. W. 1940. MacRobertson Land and Kemp Land, 1936. Discovery' Rep., 19, 165-79.

REESE, W. R. 1977. The genus Symhopodon in the Americas. I. The elimbate species. Bryologist, 80, No. 1, 2-31.

REICHARDT, H. W. 1868. Diagnosen der neuen Arten von Laubmoosen welche die Novara-Expedition mitbrachte. Verh. zool.-bot. Ges. Wien, 18, Ht. 1 and 2, 193-98.

Reichardt, H. W. 1870. Fungi, Hepaticae et Musci frondosi. (In Grunow, A., KrempelHuber, J., Reichardt, H. W., Mettenius, G. and J. Milde. Reise de österreichischen Fregatte Novara um die Erde in den Jahren 1857, 1858, 1859 unter den Befehlen des Commodore B. von Wüllerstorfurbair. Botanischer theil, Bd. 1. Wien, Kaiserlich-Königlichen Hof- und Staatsdruckerei, 166-96.)

REID, C. and H. N. RidLeY. 1888. Fossil Arctic plants from the lacustrine deposit at Hoxne, in Suffolk. Geol. Mag., N.S., 5, Dec. 3, No. 10, 441-44.

Reimers, H. 1926. Beiträge zur Bryophytenflora Südamerikas. I. Die von Pater A. Hollermayer in Chile 1918/1923 gesammelten Leber- und Laubmoose. Hedwigia, 66, Ht. 1, 27-75.

ReIMERS, H. 1936. Über die Laubmoosgattungen Bryoporteria Thér., Camptodontium Dus., Hymenoloma Dus., Verrucidens Card. und ihre systematische Stellung. Notizbl. bot. Gart. Mus. Berl., 13, No. 116, 36-52.

Reimers, H. 1937. Die europäischen Haplocladium-Arten mit besonderer Berücksichtigung ihrer aussereuropäischen Verbreitung und ihrer Verwandtschaft. Hedwigia, 76 (for 1936-37), Ht. 5/6, 191-298.

REINWARDT, [G. C. C.] and [C. F.] HornsChuCh. 1826. Musci frondosi javanici reddidi coniunctis studiis et opera. Nova Acta physico-med. Acad. Caesar. Leop. Carol., 14, Pt. 2 (for 1829), 697-732.

RelHaN, R. 1802. Cryptogamia, Musci. (In Flora Cantabrigiensis exhibens plantas agri Cantabrigiensis indigenas, secundum systema sexuale digestas. Ed altera [2nd edition]. Cantabrigiae, J. Burges, 412-41.)

RENAULD, F. 1901. Nouvelle classification des Leucoloma. Revue bryol., 28, No. 4, 66-70.

Renauld, F. 1909. Essai sur les Leucoloma et supplément au prodrome de la flore bryologique de Madagascar des Mascareignes et des comores publiés par ordre de S.A.S. Le Prince Albert $1^{\text {o4 }}$. Monaco, Institut de France.

Renauld, F. and J. Cardot. 1888. Notice sur quelques mousses de l'Amérique du Nord. Revue bryol., 15, No. 5, 69-72.

Renauld, F. and J. CARDOt. 1889. Mousses nouvelles de l'Amérique du Nord. Mém. Soc. r. bot. Belg., 28, Pt. 1, 121-34.

RENAULD, F. and J. CARDOT. 1892. Musci. (In Durand, T. and H. PITTIER, ed. Primitiae florae Costaricensis, Fasc. 2, Bull. Soc. r. Bot. Belg., 31, Pt. 1 (Mémoires), 143-74.) [Effective date 1893, fide Index muscorum, 1, 6, under Acidodontium megalocarpum.]

Renauld, F. and J. Cardot. 1893a. Pleurocarpae, Hypnaceae. (In Nordamerikanische Laubmoose Torfmoose und Lebermoose gesammelt von Dr. Julius Röll in Darmstadt. Hedwigia, 32, Ht. 5, 334-71.)

RenAULD, F. and J. CARDOT. 1893b. Musci Americae Septentrionalis, ex operibus nouvissimis recensiti et methodici dispositi (suite). Revue bryol., 20, Nos. 1-2, 1-32.

Richards, P. W. 1937. A collection of bryophytes 
from the Azores. Annls bryol., 9 (for 1936), 131-38.

Richards, P. W. and E. C. Wallace. 1950. An annotated list of British mosses. Trans. Br. bryol. Soc., 1, Pt. 4, i-xxxi.

RoBINSON, H. [E.] 1964. Five bryophytes of interest from Chile. Bryologist, 67, No. 1, 53-55.

RoBinson, H. [E.] 1970a. A revision of the genus Trichostomopsis. Phytologia, 20, No. 3, 184-91.

Robinson, H. [E.] 1970b. Notes on the moss genera, Camptochaete, Physcomitrium and Ptychomnion, in Chile. Phytologia, 20, No. 6, 329-31.

ROBINSON, H. E. 1972. Observations on the origin and taxonomy of the Antarctic moss flora. (In LLANO, G. A., ed. Antarctic terrestrial biology. Washington, D.C., American Geophysical Union, 163-77.) [Antarctic Research Series, Vol. 20.]

RoBinson, H. [E.] 1974. Notes on the mosses of Juan Femandez and southern South America. Phytologia, 29, No. 2, 116-19.

RoBinson, H. [E.] 1975. The mosses of Juan Femandez Islands. Smithson. Contrib. Bot., No. $27,88 \mathrm{pp}$.

RöHLNG, I. C. 1809. Kritische Bemerkungen über meine Laubmoose. Ein Beitrag zur richtigen Bestimmung der Moosarten. Annaln wetterauisch. Ges., 1. Ht. 2, 178-201.

RöHLING, I. C. 1812. Kritische Bemerkungen über meine Laubmoose. Annln wetterauisch. Ges., 3 , Ht. 1, 79-122.

RöHLING, I. C. 1813. Moose (In Deutschlands Flora. Ein botanisches Taschenbuch. 2nd edition. Vol. 3. Kryptogamische Gewächse. Frankfurt am Main, Friedrich Wilmans, Abt. 1, 32-134.)

RöHLING, I. C. 1814. Kritische Bemerkungen über meine Laubmoose. Annin wetterauisch. Ges., 3, Ht. 2, 193-235.

RoIvainen, H. 1934. Musci nonnulli ex Chile austrocentrali. Suomal. eläin-ja kasvit. Seur. van. kasvit. Julk., 5, No. 10, 30-36.

RoIvainen, H. 1954. Studien über die Moose Feuerlands. Suomal. eläin-ja kasvit. Seur. van. kasvit. Julk., 28, No. 2, 1-205.

RoIvaINen, H. 1955a. Contribution to the Fuegian species of the genus Rhacomitrium Brid. Suomal. eläin-ja kasvit. Seur. van. Tiedon., 9, No. 2, 85-98.

ROIVAINEN, H. 1955b. Bucklandia bartramii, a new genus and species of leaf moss from Tierra del Fuego. Suomal. eläin-ja kasvit. Seur. van. Tiedon., 9, No. 2, 98-100.

RoIvAINEN, H. 1972. Bucklandiella Roiv., nomen novum (Musci). Ann. bot. fenn., 9, No. 2, 116.

RoIvainen, H. and E. B. BARTRAM. 1937. Bryological investigations in Tierra del Fuego. I. Sphagnaceae - Dicranaceae. Suomal. eläin-ja kasvit. Seur. van. kasvit. Julk., 9, No. 2, 1-58.

RöLL, [J.] 1886. Zur Systematik der Torfmoose. Flora, Jena. 69, No. 6, 89-94; No. 15, 227-42; Nos. 27-28, 419-27.

RöLL, J. 1915. Die Thüringer Torfmoose und Laubmoose. Hedwigia, 56, Ht. 1-2, 1-176.

Roth, A. W. 1802. Neue Beyträge zur Botanik. Theil 1. Frankfurth am Mayn, Friedrich Wilmans.

Roth, G. 1904. Die europäischen Laubmoose, Bd. II. Leipzig, Wilhelm Engelmann, Lief. 10, 513640.

RoTH, G. 1908. Übersicht über die europäischen Drepanocladus, einschliesslich der neueren Formen. Hedwigia, 48, Ht. 3, 152-77.

RoTH, G. 1910. Die aussereuropäischen Laubmoose. Bd. I. Dresden, C. Heinrich, Lief. 1, $1-96$
Rотн, G. 1911. Die aussereuropäischen Laubmoose. Bd. I. Dresden, C. Heinrich, Lief. 2, 97-192; Lief. 3, 193-272; Lief. 4, 273-331.

Rотн, G. 1913. Nachtrag I zu Band I der aussereuropäischen Laubmoose von 1910-11. Hedwigia, 53, Ht. 2, 81-98.

Rотн, G. 1914. Nachtrag II zu Band I der aussereuropäischen Laubmoose von 1910-11. Hedwigia, 55, Ht. 6, 267-74.

ROWLEE, W. W. 1916. Plants from southern Patagonia collected by Charles Wellington Furlong. Bull. Torrey bot. Club, 43, No. 6 , 305-21.

Russow, E. 1867. Beiträge zur Kenntniss der Torfmoose. Arch. Naturk. Liv- Est- u. Kurlands, Ser. 2, Biologische Naturkunde, 7, Lief. 1, 83162.

SaINSBURY, G. O. K. 1931. The moss Dicranoloma setosum (H. f. \& W.) Par. Bryologist, 34, No. 5, 72-74. [Effective date 8 February 1932.]

SAINSBURY, G. O. K. 1952. Critical New Zealand mosses. Revue bryol. lichen., N.S., 21, Fasc. 3-4, 213-25.

SAINSBURY, G. O. K. 1955. A handbook of the New Zealand mosses. Bull. R. Soc. N.Z.., No. 5, 490 pp.

SALMON, E. S. 1903. Bryological notes. (30) Calyptopogon mnioides (Schwaegr.) Broth. J. Bot., Lond., 41, No. 1, 1-8.

SANIONE, C. 1883. Additamentum secundum in harpidiorum cognitionem. Bot. Zbl., 13, No. 13, 425-40.

SAVICH-LJUBITSKAYA, L. I. 1978. Listostebel'nye mkhi vostochnoi Antarktidy [Leafy mosses of eastern Antarctic region]. Bot. Zh. SSSR, 63, No. 2, 272-90.

SaVich-LJUBitskaya, L. I. andZ. N. Smirnova. 1959. Novyi vid roda Bryum Hedw. iz oazisa Bangera [A new species of the genus Bryum Hedw. from Bunger's Oasis]. Inf. Byull. sov. antarkt. Eksped. No. 7, 34-39. [English translation: Vol. 1, 1964, 308-13.]

SAVICH-LJUBitskaya, L. I. and Z. N. Smirnova. 1960. Novaya raznovidnost Bryum korotkevicziae Sav.-Ljub. et Z. Smirn. [New variety of Bryum korotkevicziae Sav.-Ljub. et Z. Smirn.]. Inf. Byull. sov. antarkt. Eksped., No. 17, 25-27. [English translation: Vol. 2, 1964, 206-08.]

SAVICH-LJUBITSKAYA, L. I. and Z. N. SMIRNOVA. 1962 Endem Antarktidy - mokh Sarconeurum glaciale (Hook. fil. et Wils.) Card. et Bryhn [The endemic moss of the Antarctic continent Sarconeurum glaciale (Hook. fil. et Wils.) Card. et Bryhn]. (In Rezul'taty biologicheskekh issledovonii Sovetskoy Antarkticheskoi Ekspeditsii, 1955-58 [Biological Results of Soviet Antarctic Expedition, 1955-58]. Moscow and Leningrad, Akademii Nauk SSSR, 1, 295309.)

SAVICH-LJUBITSKAYA, L. I. and Z. N. SMIRNOVA. 1963a. O predstavitelyakh roda Pottia Fuernr. v Antarktide. De specibus Pottiae Fuernr. in Antarctida inventis. (In Botanicheskie materialy Otdela sporovyleh rastenii. Akademiya Nauk SSSR Botanicheskii Institut im V.L. Komarova, 16, 188-95.)

SAVICH-LuUBITSKaya, L. I. andZ. N. SMIRNOVA. 1963b. K biologii i geografii Bryoerythrophyllum recurvirostre (Hedw.) Chen - novogo vida dlya brioflory Antarktidy [A contribution to the biology and geography of Bryoerythrophyllum recurvirostre (Hedw.) Chen - a new species in the bryoflora of Antarctica]. Bot. Zh. SSSR, 48, 
No. 3, 350-61.

SAVICH-LuUBITSKAYA, L. I. andZ. N. SMIRNOVA. 1964a. Glubokovodnyi predstavitel' roda Plagiothecium Br. et Sch. v Antarktide [A deepwater member of the genus Plagiothecium $\mathrm{Br}$. et Sch. in Antarctica]. Inf. Byull. sov. antarkt. Eksped., No. 49, 33-39. [English translation: Vol. 5, No. 4, 1965, 240-43.]

SAVICH-LUUBITSKAYA, L. I. and Z. N. SMiRNOVA. 1964b. Zametka o Bryum argenteum Hedw. iz Antarktidy. Notula de Bryo argenteno Hedw. ex Antarctida. Nov. sist. Nizshikh Rast., (for 1964), 292-301.

SAVICH-LJUBITSKAYA, L. I. andZ. N. SMIRNOVA. 1965a. O Grimmia antarctici Card. iz Vostochnoy Antarktidy. De Grimmia antarctici Card. ex Antarctide Orientalis. Nov. sist. Nizshikh Rast., (for 1965), 251-58.

SAVICH-LJUBITSKAYA, L. I. andZ. N. Smirnova. 1965b. Dopolnienia k state o predstavitelyakh roda Pottia Fuernr. v Antarktide. De specibus Pottiae Fuernr. in Antarctide inventis supplementa. Nov. sist. Nizshikh Rast., (for 1965), 158-59.

SAVICH-LJUBITSKAYA, L. I. andZ. N. Smirnova. 1969. Grimmia doniana Sm. iz Vostochnoy Antarktidy. Grimmia doniana Sm. ex Antarctida orentali. Nov. sist. Nizshikh Rast., 6, 249-53.

SAVICH-LJUBITSKAYA, L. I. and Z. N. SMIRNOVA. 1970. Grimmia plagiopoda Hedw. iz Vostochnoy Antarktidy [Grimmia plagiopoda Hedw. from East Antarctica]. Inf. Byull. sov. antarkt. Eksped., No. 80, 94-97. [English translation: Vol. 8, No. 2, 1972, 97-98.]

SAVICH-LJUBITSKAYA, L. I. andZ. N. SmiRnova. 1971. Mokh Pohlia nutans (Hedw.) Lindb. v Vostochnoy Antarktide [Moss Pohlia nutans (Hedw.) Lindb. in East Antarctica]. Inf. Byull. sov. antarkt. Eksped., No. 82, 72-76. [English translation: Vol. 8, No. 4, 1973, 222-24.]

SAVICH-LJUBITSKAYA, L. I. andZ. N. SMIRNOVA. 1972. Bryum algens Card.- - naibolee pasprostranennyi mokh Vostochnoy Antarktidy. Bryum algens Card. is the most common moss in East Antarctica. (In Trudy Sovetskoy Antarkticheskoy Ekspeditsii. Nauchnye rezul'taty rabot Sovetskikh Antarkticheskikh Ekspeditsii, 60 328-45.)

SAYRE, G. 1959. Dates of publications describing Musci 1801-1821. Troy, New York, G. Sayre.

SAYRE, G. 1969. Cryptogamae exsiccatae-an annotated bibliography of published exsiccatae of Algae, Lichenes, Hepaticae and Musci. Introduction, I. General cryptogams, II. Algae, III. Lichenes. Mem. N.Y. bot. Gdn, 19, No. 1, 1-174.

SAYRE, G. 1971. Cryptogamae exsiccatae-an annotated bibliography of published exsiccatae of Algae, Lichenes, Hepaticae and Musci. IV. Brypophyta. Mem. N.Y. bot. Gdn, 19, No. 2, 175-276.

SAYRE, G. 1975. Cryptogamae exsiccatae-an annotated bibliography of published exsiccatae of Algae, Lichenes, Hepaticae and Musci. V. Unpublished exsiccatae. Mem. N.Y. bot. Gdn, 19. No. 3, 277-423.

SCHIMPER, W. P. 1836. Muscorum chilensium species novas descripsit. Annls Sci. nat., Sér. 2, 6. Botanique, 145-49.

SCHIMPER, W. P. 1838. Ueber Octodiceras julianum. Flora, Jena, 21 . No 17, 267-72.

SCHIMPER, W. P. 1855. Corollarium bryologiae europaeae, conspectum diagnosticum familiarum, generum et specierum, adnotationes novas atque emendationes complectens. Stuttgartiae, E. Schweizerbart. [Effective date 1856, according to Stafleu and Cowan (1976, 379).]

SCHIMPER, W. P. 1858. Mémoire pour servir à I'histoire naturelle des Sphaignes (Sphagnum L.). Mém. prés. div. Sav. Acad. Sci. Inst. Fr., Science mathematiques et physiques, 15, 1-97. [Effective date 1857, according to Index muscorum, 4, 63, under Sphagnum acutifolium var. purpureum. According to the author catalogue of the British Museum (Nat. Hist.) preprints were circulated in 1857.]

SCHIMPER, W. P. 1860. Synopsis muscorum europaeorum praemissa introductione de elementis bryologicis tractante. Stuttgartiae, E. Schweizerbart.

SCHIMPER, W. P. 1876. Synopsis muscorum europaeorum praemissa introductione de elementis bryologicis tractante. Vol. II. Specierum descriptio. Editio secunda. Stuttgartiae, E. Schweizerbart (E. Koch).

SCHLEICHER, J. C. 1821. Catalogus hucusque absolutus omnium plantarum in Helvetia cis et transalpina sponte nascentum. 4th edition. Camberii, Gorrin et Routin.

SCHLIEPHACKE, K. 1884. Pottia güssfeldtii, ein neues Laubmoos. Ber. dt. bot. Ges., 2, Ht. 10, 461-62. [Effective date 20 January 1885.]

SCHRADER, H. A. 1794. Spicilegium florae germanicae. Pars. I. Hannoverae, Ritscher.

Schrader, H. A. 1803a. Plantae cryptogamicae nouae, rariores aut minus cognitae. J. Bot., Göttingen, 1 (for 1801), Stück 1, 66-80.

SCHRADER, M. 1803b. [Review of:] Muscologia recentiorum seu analysis, historia et descriptio methodica omnium muscorum hucusque cognitorum ad Norman Hedwigii. A. Sam. E. Bridel Gothae et Parisiis T. I. (xxiv und 179s) 4. 1797; Tom. II Pars I ( $x$ und 222s) cum tab. aen sex 1798; Pars 2 (xiv und 182s) cum tab. aen sex 1801. J. Bot., Göttingen, 1 (for 1801), Stück 1, 188-205.

SchranK, F. von PAULA. 1789. Baiersche Flora. Vol. II. München, Strobl.

Schrealus, J. 1802. Recensionen. Leipzig bei Barth: Jo. Hedwig, Prof. Botan. in acad. Lips. Species muscorum frondosorum descriptae et tabulis aeneis LXXVII coloratis illustratae. Opus posthumum editum a Fr. Schwägrichen, 1801. 352 S. in 4. Bot. Ztg Erlangen, 1, Ht. 1, No. 5 , 65-80.

ScHULTZ, C. F. 1823. Recensio generum Barbulae et Syntrichiae. Nova Acta physico-med. Acad. Caesar. Leop. Carol., 11, Pt. 1, 191-232.

ScHULTZ, C. F. 1828. Observationes bryologicae. (In [HORNSCHUCH, C. F.] Sylloge plantarum novarum itemque minus cognitarum a praestantissimus botanicis adhuc viventibus collecta et a societate regia botanica ratisbonensi edita, tomus secundus. Ratisbonae, C. E. Brenck, 119-54, 177-217.) [Editorship ascribed to Hornschuch following Stafleu, 1967, 220.]

SChultze-Motel, W. 1970. Monographie de Laubmoosgattung Andreaea. I. Die costaten arten. Wildenowia, 6, No. 1, 25-110.

SCHWAEGRICHEN, A. D. 1804. Musci novi detecti et descripti. (In SCHULTES, J. A. Reise auf dem Glockner, Theil 2. Wien, J. V. Degen, Appendix, 362-66.)

SchWAEGRichen, F. 1811. Joannis Hedwig. Species muscorum frondosorum. Descriptae et tabulis aeneis coloratis illustratae. Opus postumum. 
Supplementum primum, Sectio primum. Lipsiae, Joannis Ambrosii Barth; Parisiis, Treuttel et Würtz.

SchWAEGRICHEN, F. 1816, Joannis Hedwig. Species muscorum frondosorum. Descriptae et tabulis aeneis coloratis illustratae. Opus postumum. Supplementum primum, Sectio posterior [=Section 2]. Lipsiae, Joannis Ambrosii Barth; Parisiis, Treuttel et Würtz.

SchWAEGRICHEN, F. 1823. Joannis Hedwig. Species muscorum frondosorum. Descriptae et tabulis aeneis coloratis illustratae. Opus postumum. Supplementum secundum, Sectio prior. Lipsiae, Joannis Ambrosii Barth; Parisiis, Truettel et Würtz; Londini, Treuttel et Würtz, Treuttel filium et Richter.

SCHWAEgriCHEN, F. 1824. Joannis Hedwig. Species muscorum frondosorum. Descriptae et tabulis aeneis coloratis illustratae. Opus postumum. Supplementum secundum, Sectio posterior. Lipsiae, Joannis Ambrosii Barth; Parisiis, Treuttel et Würtz; Londini, Treuttel et Würtz, Treuttel filium et Richter.

SCHWAEGRICHEN, F. 1826. Joannis Hedwig. Species muscorum frondosorum. Descriptae et tabulis aeneis coloratis illustratae. Opus postumum. Supplementum secundum, Volumen secundum, Sectio prior. Lipsiae, Joannis Ambrosii Barth; Parisiis, Treuttel et Würtz; Londini, Treuttel et Würtz, Treuttel filium et Richter.

SCHWAEgRICHEN, F. 1827a. Joannis Hedwig. Species muscorum frondosorum. Descriptae et tabulis aeneis coloratis illustratae. Opus postumum. Supplementum secundum, Volumen secundum, Sectio secundae. Lipsiae, Joannis Ambrosii Barth; Parisiis, Treuttel et Würtz; Londini, Treuttel et Würtz, Treuttel filium et Richter.

Schwaegrichen, F. 1827b. Joannis Hedwig. Species muscorum frondosorum. Descriptae et tabulis aeneis coloratis illustratae. Opus postumum. Supplementum tertium, Volumen primum, Sectio prima. Lipsiae, Joannis Ambrosii Barth; Parisiis, Treuttel et Würtz; Londini, Treuttel et Würtz, Treuttel filium et Richter.

SchWAEgrichen, F. 1828. Joannis Hedwig. Species muscorum frondosorum. Descriptae et tabulis aeneis coloratis illustratae. Opus postumum. Supplementum tertium, Volumen primum, Sectio secunda. Lipsiae, Joannis Ambrosii Barth; Parisiis, Treuttel et Würtz; Londini, Treuttel et Würtz, Treuttel filium et Richter.

SchWAEgrichen, F. 1829. Joannis Hedwig. Species muscorum frondosorum. Descriptae et tabulis aeneis coloratis illustratae. Opus postumum. Supplementum tertium, Volumen secundum, Sectio prima. Lipsiae, Joannis Ambrosii Barth; Parisiis, Treuttel et Würtz; Londini, Treuttel et Würtz, Treuttel filium et Richter.

SCHWAEGRICHEN, F. 1830. Joannis Hedwig. Species muscorum frondosorum. Descriptae et tabulis aeneis coloratis illustratae. Opus postumum. Supplementum tertium, Volumen secundum, Sectio secunda. Lipsiae, Joannis Ambrosii Barth; Parisiis, Treuttel et Würtz; Londini, Treuttel et Würtz, Treuttel filium et Richter.

Schwaegrichen, F. 1842. Joannis Hedwig. Species muscorum frondosorum. Descriptae et tabulis aeneis coloratis illustratae. Opus postumum. Supplementum quartum, Sectio prima. Lipsiae, Joannis Ambrosii Barth; Parisiis, Treuttel et Würtz; Londini, Longman, Brown, Rees and Longmans.
Schwaegrichen, C. F. 1844. Ueber einige Moose. Linnaea, Halle, 18, [Beitr. PfIKunde, 2], Ht. 5 , 557-62. [Effective data 1845, according to note on p. 508.]

SeEmann, B. C. 1873. Flora vitiensis: a description of the plants of the Viti or Fiji Islands, with an account of their history, uses and properties. London, L. Reeve and Co., Pt. 10, 325-434. [Dates of whole work 1865-73.]

SEKI, T. 1974. A moss flora of Provincia de Aisén, Chile. Results of the second scientific expedition to Patagonia by Hokkaido and Hiroshima Universities, 1967. J. Sci. Hiroshima Univ., Ser. B, Div. 2 (Botany), 15, No. 1, 9-101. [Effective date 31 March 1976; personal communication from author.]

SENDTNER, O. 1848. Beobachtungen über die klimatische Verbreitung der Laubmoose durch das österreichische Küstenland und Dalmatien. Flora, Jena, 31, (N.R., 6), No. 14, $229-40$.

SkotTsberG, C. [J.F.] 1914. Botanische Ergebnisse der Schwedischen Expedition nach Patagonien und dem Feuerlande 1907-1909. IV. Studien über die Vegetation der Juan Fernandez-Inseln. K. svenska VetenskAkad. Handl., 51, No. 9, $1-73$.

SKotTSBERG, C. [J.F.] 1950. Apuntes sobre la flore y vegetación de Frai Jorge (Coquimbo, Chile). Acta Horti gothoburg., 18 (for 1948), 91-184.

$S_{M I T H}$ G. L. 1969a. On Atrichopsis, with notes on some austral Psilopilum species (Polytrichaceae). Bull. Torrey bot. Club, 96, No. 1,60-69.

SMITH, G. L. 1969b. New combinations in Polytrichaceae. Phytologia, 18, No. 7, 403.

SMITH, G. L. 1971. A conspectus of the genera of Polytrichaceae. Mem. N.Y. bot. Gdn, 21, No. 3 , 1-83.

SMITH, J. E. 1803. Trichostomum capillaceum. (In English botany; or coloured figures of British plants, with their essential characters, synonyms, and places of growth: to which will be added occasional remarks. The figures by James Sowerby. London, J. E. Smith, 16, Tab. 1152.)

SMITH, J. E. 1804a. Grimmia donniana. (In English botany; or coloured figures of British plants, with their essential characters, synonyms, and places of growth: to which will be added occasional remarks. The figures by James Sowerby. London, J. E. Smith, 18, Tab. 1259).

SMITH, J. E. 1804b. Flora britannica, Vol. 3. London, J. Taylor.

SMITH, J. E. 1807. Grimmia pulvinata. (In English botany; or coloured figures of British plants, with their essential characters, synonyms, and places of growth: to which will be added occasional remarks. The figures by James Sowerby. London, J. E. Smith, 24, Tab. 1728.)

SMITH, J. E. 1809. Grimmia atro-virens. (In English botany; or coloured figures of British plants, with their essential characters, synonyms, and places of growth: to which will be added occasional remarks. The figures by James Sowerby. London, J. E. Smith, 28, Tab. 2015.)

SMITH, J. E. 1811. Gymnostomum imberbe. (In English botany; or coloured figures of British plants, with their essential characters, synonyms, and places of growth: to which will be added occasional remarks. The figures by James Sowerby. London, J. E. Smith, 32, Tab. 2237.)

SMITH, R. I. L. 1972. Vegetation of the South Orkney Islands with particular reference to Signy Island. British Antarctic Survey Scientific Reports, No. $68,124 \mathrm{pp}$. 
SPEGAZZINI, C. 1922. Resultados de la primera expedición a Tierra del Fuego (1921) envida par la Fácultad de Ciencias Exactas, Fisicas y Naturales de la Universidad Nacional de Buenos Aires. Cryptogamae nonnullae fuegianae. An. Soc. cient. argent., 94, Entr. 1-2, 59-85.

SPRENGEL, C. 1827. Cryptogamia. Sect. II. Musci frondosi et Hepatici. (In LiNNAEI, C. Systema vegetabilum. Editio decima sexta. Vol. IV. Pars 1. Gottingae, Librariae Dieterichianae, 135-236.)

SPRUCE, R. 1845. On several mosses, new to the British flora. Lond. J. Bot., 4, 169-95.

SPRUCE, R. 1847. Musci pyrenaici, quos in Pyrenaeis centralibus occidentalibusque nec non in agro syrtico, AD MDCCCXLV-VI, Fasc. 1, Nos. 1-160. Londoni.

Spruce, R. 1849. The Musci and Hepaticae of the Pyrenees. Ann. Mag. nat. Hist., Ser. 2, 3, No. 16, 269-93.

SPRUCE, R. 1867. Catalogus muscorum fere omnium quos in terris Amazonicis et Andinis per annos 1849-1860. Londini, E. Newman.

StAfleu, F. A. 1967. Taxonomic literature. A selective guide to botanical publications with dates, commentaries and types. Regnum veg., 52, $556 \mathrm{pp}$.

STAFLEU, F. A., ED. 1978. International code of botanical nomenclature, adopted by the twelfth International Botanical Congress, Leningrad, July 1975. Regnum veg., 97, 457 pp.

Stafleu, F. A. and R. S. Cowan. 1976. Taxonomic literature, a selective guide to botanical publications and collections with dates, commentaries and types. Edition 2. Vol. 1, A-G. Regnum veg., 94, 1136 pp.

Stafleu, F. A. and R. S. Cowan. 1979. Taxonomic literature, a selective guide to botanical publications and collections with dates, commentaries and types. Edition 2. Vol. 2, H-Le. Regnum veg., 98, 991 pp.

Stafleu, F. A. and R. S. Cowan. 1983. Taxonomic literature, a selective guide to botanical publications and collections with dates. commentaries and types. Edition 2. Vol. 4. Regnum veg., 110, $1214 \mathrm{pp}$.

STEERE, W. C. 1948. Contribution to the bryogeography of Ecuador. 1. A review of the species of musci previously reported. Bryologist 51, No. 3, 65-167.

STEERE, $W$. C. 1961a. A preliminary review of the bryophytes of Antarctica. (In Science in Antarctica. Part I. The life sciences in Antarctica. Washington, D.C., National Academy of Sciences-National Research Council, 20-33.) [National Academy of Sciences-National Research Council Publication 839.]

StEere, W. C. 1961b. The bryophytes of South Georgia. (In Science in Antarctica. Part I. The life sciences in Antarctica. Washington, D.C., National Academy of Sciences-National Research Council, 34-48.) [National Academy of Sciences-National Research Council Publication 839.]

STEUDEL, E. [G.] 1824. Nomenclator botanicus enumerans ordine alphabetico nomina atque synonyma tum generica tum specifica et a Linnaeo et recentioribus de re botanica scriptoribus plantis cryptogamica imposita. Part 2. Stuttgardtiae et Tubingae, J. G. Cottae.

StuRM, J. 1813. Deutschlands Flora in Abbildungen nach der Natur mit Beschreibungen. Abt. II. Cryptogamia. Ht. 14. Nürnberg, J. Sturm.

Sullivant, W. S. 1846. Musci Alleghanienses, sive enumeratio muscorum atque hepaticarum quos in itinere a Marylandia usque ad Georgiam per tractus montium. Columbus, Ohio, W. S. Sullivant.

SULUVANT, W. S. 1849. Contributions to the bryology and hepaticology of North America. Pt. II. Mem. Am. Acad. Arts Sci., N.S. 4, Pt. 1, 169-76.

SULLIVANT, W.S. 1850. Notices of several new species of mosses and Hepaticae from Tierra del Fuego. Hooker's J. Bot. \& Kew Gdns Miscell., 2, 315-18.

SULLIVANT, W. S. 1857. Notices of new species of mosses, from the Pacific islands. Proc. Am. Acad. Arts Sci., 3 (for 1852-57), 73-81. [Effective date 1854, according to entry under Cryphaea cuspidata in Index muscorum, 1, 514.]

SULLIVANT, W. S. 1859. Musci. (In United States Exploring Expedition during the years 1838, $1839,1840,1841,1842$ under the command of Charles Wilkes, U.S.N. Vol. XVII. Botany. Philadelphia, C. Sherman and Son, 3-32.)

SULIVANT, W. S. and [C.] L. LESOUEREUX. 1856. Musci boreali-Americani quorum specimina exsiccata. Columbi Ohioensium, Auctorum.

SUUUVANT, W. S. and L. LesQuereux, ed. 1865. Musci boreali-Americani quorum specimina exsiccata. Editio secunda. Columbi Ohioensium, W. S. Sullivant and L. Lesquereux.

[SWARTZ, O,] 1801. Dispositio systematica muscorum frondosorum Sueciae, adjectis descriptionibus et iconibus novarum specienum. 12 m. pp. 112. Erlangae, apud Jo. Jac. Palm. 1799. Mon. Rev. Lond., Ser. 2, 34, Appendix, 535-38.

SWARTZ, O. 1803. Cinclidium, eine neue Moosgattung. J. Bot., Göttingen, 1 (for 1801), Stück 1, 25-31.

SWARTZ, O. 1806a. Conostomum, eine neue Moosgattung. Neues J. Bot., 1, Stück 3, 24-36.

SWARTZ, O. 1806b. Cryptogamia. In Flora Indiae occidentalis aucta atque illustrata sive descriptiones plantarum in Prodromo recensitarum. Vol. 3. Erlangen, Jo. Jacobi Palmii. $1569-940$.

SYDOW, P. 1910. Moose. Just's bot. Jber., 36 (for 1908), Abt. 1, 30-90.

TAYLOR, N. 1914. Plants collected on the South Georgian Expedition. (In MURPHY, R. C. A report on the South Georgia Expedition. Sci. Bull. Brooklyn Inst., 2, No. 4, 60-63.)

TAYLOR, T. 1846. The distinctive characters of some new species of Musci, collected by Professor William Jameson, in the vicinity of Quito, and by Mr. James Drummond at Swan River. Lond. J. Bot., 5, 41-67.

TAYLOR, T. 1847. Description of new Musci and Hepaticae collected by Prof. W. Jameson on Pichincha, near Quito by Thomas Taylor M.D. Lond. J. Bot., 6, 328-42.

TAYLOR, T. 1848a. On some new Musci, collected by Professor $W$. Jameson on Pichincha. Lond. Bot., 7, 187-99.

TAYLOR, T.1848b. On the specific characters of certain new cryptogamic plants, lately received from, and collected by, Professor William Jameson, on Pichincha, near Quito. Lond. J. Bot., 7, 278-85.

THÉRIOT, I. 1911. Breutelia (Eubreutelia) subplicata Broth. sp. nov. ined. Revta chil. Hist. nat., 15, No. 1, 38-39.

THÉRIOT, I. 1915. Sur quelques mousses recueillies au Chili par le Prof.. Carlos E. Porter. Revta chil. Hist. nat., 19, Nos. 1-2, 30-37. 
THÉRIOT, I. 1916. Une rectification. Bryologist, 19, No. $1,10$.

THÉRIOT, I. 1917a. Note sur une mousse du Chili, Barbula flagellaris Sch. Recl Publs Soc. Havr. Etud. Diverses,84, 55-62.

THÉRIOT, I. 1917b. Contribution à la flore bryologique du Chili, II. Revta chil. Hist. nat., 21, Nos. 1-2, 6-37.

THÉRIOT, I. 1918. Contribution à la flore bryologique du Chili, III. Revta chil. Hist. nat., 22, Nos. 2-3, 79-94.

THÉRIOT, I. 1919. Notes bryologiques, [l et II]. Bull. Soc. bot. Genève, 2ème sér., 11, Nos. 1-4, 24-29.

THÉRIOT, I. 1921a. Contribution à la flore bryologique du Chili, IV. Revta chil. Hist. nat., 25, 289-312.

THÉRIOT, I. 1921b. Courte historie de rescapées. Recl Publs Soc. Havr. Étud. Diverses, 88, 29-36.

THÉRIOT, I. 1922. Deuxième contribution à la flore bryologique de Madagascar. Recl Publs Soc. Havr. Etud. Diverses, 89, 111-32.

THÉRIOT, I. 1923a. Notes bryologiques [III, IV et V]. Bull. Soc. bot. Genève, 2ème sér., 14, Nos. 1-10 (for 1922), 44-49. [Effective date 1919, according to Index muscorum, 5, 72, Tortula amblyophylla yet on p. 44 of article "Communique en séance du 12 mars 1919. Par suite d'une regrettable omission, les présentes notes n'ont pas été publiées dans le vol. XII du Bulletin, auquel elles étaint destinées: toutefois le tirage à part a été distribué par l'auteur dès 1921 [Réd.]".]

THÉRIOT, I. 1923b. Contribution à la flore bryologique du Chili, V. Revta chil. Hist. nat., 27, 9-15.

THÉRIOT, I. 1924. Contribution à la flore bryologique du Chili, VI. Revta chil. Hist. nat., 28, 129-39.

THÉRIOT, I. 1925a. Contribution à la flore bryologique du Chili, VII. Revta chil. Hist. nat., 29, 287-92.

THÉRIOT, I. 1925b. Notes bryologiques, [VI et VII]. Bull. Soc. bot. Genève, 2ème sér., 17, 252-57. [Effective date 10 April 1926.]

THÉRIOT, I. 1926. Contribution à la flore bryologique du Chili, VIII. Revta chil. Hist. nat., 30, 341-61.

THÉRIOT, I. 1927a. Le genre Funaria au Chili. Revta chil. Hist. nat., 31, 30-37.

THÉRIOT, I. $1927 \mathrm{~b}$. Mousses récoltées dans l'île Más a Tierra (Juan Fernández) en 1927, por M. Gualterio Looser. Revta chil. Hist. nat., 31, 256-59.

THÉRIOT, I. 1928. Contribution à la flore bryologique du Chili, IX. Revta chil. Hist. nat., 32, 252-55. [Effective date February 1929.]

THÉRIOT, I. 1929a. La genre Leptodontium au Chili. Revta chil. Hist. nat., 33, 515-17.

THÉRIOT, I. 1929b. Mousses du Chili austral récoltées par le Prof. Rol. Thaxter, de Cambridge, Mass. Revue bryol., N.S., 2, Fasc. 3-4, 165-66. [31 March 1930, fide title page.]

THÉRIOT, I. 1929c. Mousses critiques. Revue bryol., N.S., 2, Fasc. 3-4, 231-33. [31 March 1930, fide title page.]

THÉRIOT, I. 1930. Contribution à la flore bryologique du Chili, X. Revta chil. Hist. nat., 34, 258-62.

THÉRIOT, I. 1931. Liste et correction des fautes orthographiques ou autres erreurs contenues dans la 2e édition des Musci de Brotherus, in Engler-Prantl, Die natürlichen Pflanzenfamilien. Revue bryol., N.S., 4, Fasc. 4, 170-85. [Effective date September 1932.]

THÉRIOT, I. 1932. Barbula ventanica ou Barbula ventanae? Revue bryol. lichen., N.S., 5, Fasc. 2-3, 140. [Effective date 10 April 1933.]
THÉRIOT, I. 1933. Bryoporteria nouveau genre de la famille Seligeriaceae. Revta chil. Hist. nat., 37, 23-25.

THÉRIOT, I. 1934a. Quelques mousses du Chili austral récoltées a freire par $\mathrm{Mr}$. Raul Gillet $\mathrm{L}$., en fevrier 1934. Revta chil. Hist. nat., 38, 83-85.

THÉRIOT, I. 1934b. Contribucion à la flore bryologique du Chili, 11e article. Revue bryol. lichen., N.S., 7, Fasc. 3-4, 167-183. [Effective date 10 April 1935.]

THÉRIOT, I. 1935a. Contribuction à la flore bryologique du Chili, XII. Revta chil. Hist. nat., 39, 16-21.

THÉRIOT, I. 1935b. Quelques nouveautés pour la flore bryologique du Sud-Amérique. Ann/s Cryptog. exot., 8, Fasc. 3-4, 135-37. [Effective date November 1936.]

THÉRIOT, I. 1936. Quatre formes nouvelles de mousses de l'Argentine. (In BAUER, E., ed. Musci europaei et americani exsiccati. Schedae und Bemerkungen zur 46, Ser. 1936. [Stadt Olbersdorf, Ludwig Richter].)

THÉRIOT, I. 1939. Bryales uruguayenses. Revta sudam. Bot., 6, Nos. 3-4, 108-09. [Effective date September 1941.]

TIXIER, P. 1977. Clastobryoidées et taxa apparentés. Revue bryol. lichen., 43, Fasc. 4, $397-464$

Touw, A. 1971. A taxonomic revision of the Hypnodendraceae (Musci). Blumea, 19, No. 2. 211-354.

TURNER, D. 1804a. Muscologiae hibernicae spicilegium. London, J. White.

TURNER, D. 1804b. Remarks upon the Dillenian Herbarium. Trans. Linn. Soc. Lond., 7, 101-15.

TURNER, D. 1805. Remarks upon some parts of the Hedwigian system of mosses, with a monograph of the genus Bartramia. Ann. Bot., Lond., 1, No. 3, 517-30.

ULE, E. 1899. Bryotheca brasiliensis, herausgegeben von Dr. C. H. Brotherus in Helsingfors, bestimmt von Carl Müller, C. H. Brotherus und C. Warnstorf. Hedwigia, Beibl., 38, No. 1, 57-59.

VAN DEN Bosch, R. B. and C. M. van deR SANDE LACOSTE. 1860. Bryologia javanica seu descriptio muscorum frondosorum Archipelagi indici iconibus illustrata. Vol. 1. Lugduni-Batavorum, E. J. Brill, Fasc. 24, 141-48.

VAN DER WiJk, R. and W. D. Margadant. 1958. NeW combinations in mosses. I. Taxon, 7, No. 9, 287-90.

VAN DER WIJK, R. andW. D. MARGADANT. 1959. NeW combinations in mosses. II. Taxon, 8, No. 2 , 70-75.

VAN DER WIJK, R. and W. D. MARgadant, 1960A. NeW COMBINATIONS IN MOSSES. IV. TAXON, 9, No. 2, 50-52.

VAN DER WIJK, R. and W. D. MARGADANT. $1960 b$. New combinations in mosses. V. Taxon, 9, No. 6, 189-91.

VAN DER WIJK, R. and W. D. MARgadant, 1961. NeW combinations in mosses. VI. Taxon, 10, No. 1 , 24-26.

VAN DER WiJk, R. and W. D. MARgadant. 1962. NeW combinations in mosses. VII. Taxon, 11, No. 7 , 221-23.

VAN DER WIJK, R. and W. D. MARgadant. 1965. NeW combinations in mosses. VIII. Taxon, 14, No. 6, 196-98.

VAN DER WiJK, R., Margadant, W. D. and P. A. FLORSCHÜTZ. 1959. Index muscorum. Vol. 1 (A- 
C). Regnum veg., 17, $548 \mathrm{pp}$.

VAN DER WIJK, R., MARgadant, W. D. and P. A. FLORSCHÜTZ. 1962. Index muscorum. Vol. 2 (DHypno). Regnum veg., 26, 535 pp.

VAN DER WIJK, R., Margadant, W. D. and P. A.

FLORSCHÜTZ. 1964. Index muscorum. Vol. 3

(Hypnum-O). Regnum veg., 33, 529 pp.

VAN DER WIJK, R., MARgadant, W. D. and P. A.

FLORSCHÜTZ. 1967. Index muscorum. Vol. 4 (P-

S). Regnum veg., 48, $604 \mathrm{pp}$.

VAN DER WIJK, R., MARGADANT, W. D. and P. A.

FLORSCHÜTZ. 1969. Index muscorum. Vol. 5 (T-Z,

Appendix). Regnum veg., 65, 922 pp.

van Steenis-Kruseman, M. J. and W. T. Stearn. 1954. Dates of publication. (In VAN STEENISKruSEMAN, C. G. G. J., ed. Flora malesiana, being an illustrated systematic account of Malaysian flora including keys for determination, diagnostic descriptions, references to the literature, synonymy, and distribution, and notes on the ecology of its wild and commonly cultivated plants. Djakarta, Noordhoff-Kolff N. V. Ser. 1, 4, No. 5, CLXIII-CCXIX.)

VeNTURI, G. 1872. Muschi raccolti dal Signor Odoardo Beccari nella Terra dei Bogos in Abissinia. Nuovo G. bot. ital., 4, Fasc. 1, 7-22.

VENTURI, G. and A. BotTINI. 1884. Enumerazione critica del Muschi Italiani. Atti Soc. crittogam. Ital., Ser. 2, 3, Dispensa 3a, 153-227.

VERDOORN, F. 1938. Hepaticae selectae et criticae Series IX (1936) et Series X (1937) and Musci selecti et critici Series III (1936) et Series IV (1937). Annis bryol., 10 (for 1937), 124-30.

VITT, D. H. 1973. A revisionary study of the genus Macrocoma. Revue bryol. lichen., N.S., 39, Fasc. 2, 205-20.

VITT, D. H. 1976. A monograph of the genus Muelleriella Dusén. J. Hattori bot. Lab., No. 40 , 91-113.

VITT, D. H. and M. R. Crosby. 1972. Achrophyllum - a new name for a genus of mosses. Bryologist, 75, No. 2, 174-75.

Wahlenberg, G. 1812. Musci. (In F̈lora Lapponica exhibens plantas geographicae et botanice consideratas, in Lapponiis Suecicis scilicet Umensi, Pitensi, Lulensi, Tornensi et Kemensi nec non Lapponiis Norvegicis scilicet Nordlandia et Finmarkia utraque indigenas, et itineribus annorum 1800, 1802, 1807 et 1810 denuo investigatas. Berolini, Taberna libraria scholae realis, 300-400.)

WAHLENBERG, G. 1826. Musci. (In Flora Suecica enumerans plantas sueciae indigenas cum synopsi classium ordinumque, characteribus generum, differentiis specierum, synonymis citationibusque selectis, locis regionibusque natalibus, descriptionibus habitualibus nomina incolarum et qualitates plantarum illustrantibus. Post Linnaeum edita. Part 2. Upsaliae, Palmblad \& Co., 697-795.)

WALTHER, A. and L. MOLENDO. 1868. Die Laubmoose Oberfrankens. Beiträge zur Pflanzengeographie und Systematik und zur Theorie vom Ursprunge der Arten. Leipzig. Wilhelm Engelmann.

WALTHER, K. 1934. Untersuchungen über die Variabilität innerhalb des Formenkreises von Polytrichum juniperinum Willd. Annls bryol., 7. 121-56.

WALTHER, K. 1940. Revision des Verwandtschaftskreises von Polytrichum piliferum Schreb. Reprium nov. Spec. Regni veg.,
48, Nos. 12-20, 251-55.

WARBURG, E. F. 1962. New combinations in British mosses. Trans. Br. bryol. Soc., 4, Pt. 2, 247-48.

WARNSTORF, C. 1881a. Die europäischen Torfmoose. Eine Kritik und Beschreibung derselben. Berlin, Theobald Grieben.

WARNSTORF, C. $1881 \mathrm{~b}$. Thuidium delicatulum (Hedw.) Lindberg, in Steiermark und wahrscheinlich auch in Deutschland verbreitet. Bot. Zbl., 5, No. 6, 183-85.

WARNSTORF, C. 1883. Die Torfmoose des v. Flotow'schen Herbarium in königl bot. Museum in Berlin. Flora, Jena, 66, (N.R., 41), No. 24, 371-80.

WARNSTORF, C. 1884a. Neue europäische Sphagnumformen. Hedwigia, 23, Ht. 8, 116-25.

WARNSTORF, C. 1884b. Spagnologische Rückbliche, (Schluss). Verzeichniss der mir ois jetzt aus Europa bekannt gewordenen Sphagnumformen. Flora, Jena, 67, (N.R., 42), No. 31, 597-611.

WARNSTORF, C. 1888. Europaeische Torfmoose Serie 1. Flora v. Deutschland (Brandenburg). Nearuppin, Waldsumpf bei Stendenitz.

WARNSTORF, C. 1890a. Beiträge zur Kenntniss exotischer Sphagna. Hedwigia, 29, Ht. 4, 179 211; Ht. 5, 213-58.

WARNSTORF, C. $1890 \mathrm{~b}$. Contributions to the knowledge of North American Sphagna. IV. Bot. Gaz., 15, No. 10, 242-55.

WARNSTORF, C. 1894. Characteristik und Uebersicht der nord-, mittel- und südamerikanischen Torfmoose nach dem heutigen Standpunkte der Sphagnologie (1893). Hedwigia, 33, Ht. 6, 307-37.

WARNSTORF, C. 1895. Beiträge zur Kenntnis exotisher Sphagna. Allg. bot. Z., 1, No. 10 , 187-89.

WARNSTORF, C. 1898. Beiträge zur Kenntniss exotischer und europäischer Torfmoose. Bot. Zbl., 76, No. 12, 385-90; No. 13, 417-23.

WARNSTORF, C. 1903. Die europäischen Harpidien Beih. bot. Zbl., 13, 388-430.

WARNSTORF, C. 1904. Laubmoose. (In Kryptogamenflora der Mark Brandenburg und angrenzender Gebiete herausgegeben von dem Botanischen Verein der Provinz Brandenburg. $B d$. 2. Leipzig, Gebrüder Borntraeger, Ht. 1, 1-240; Ht. 2, 241-432.)

WARNSTORF, C. 1906. Laubmoose. (In Kryptogamenflora der Mark Brandenburg und angrenzender Gebiete herausgegeben von dem Botanischen Verein der Provinz Brandenburg. Bd. 2. Leipzig, Gebrüder Borntraeger, Ht. 5, 833-1160.)

WARNSTORF, C. 1907. Neue europäische und ausereuropäische Torfmoose. Hedwigia, 47, Ht. $1 / 2,76-112$.

WARNSTORF, C. 1911. Sphagnales-Sphagnaceae (Sphagnologia universalis). (In ENGLER, A. Das Pflanzenreich, Regni vegetabilis conspectus im Auftrage der Königl. preuss. Akademie der Wissenschaften, Ht. 51. Leipzig, Wilhelm Engelmann.)

WARNSTORF, C. 1915. Bryophyta nova europaea et exotica. Ill. Musci. Hedwigia, 57, Ht. 1, 78-80; Ht.
2/3, 81-131.

WARNSTORF, C. 1916. Pottia-Studien als Vorarbeiten zu einer Monographie des Genus "Pottia Ehrh." sens. str. Hedwigia, 58, Ht. 1/2, 35-80; Ht. 3/4, 81-152.

WATTS, W. W. and T. WHITELEgGE. 1902. Census muscorum australiensium. A classified catalogue 
of the frondose mosses of Australia and Tasmania, collated from available publications and herbaria records. Part 1, Proc. Linn. Soc. N.S.W., Ser. 2, 27, Pt. 3, No. 107, Supplement, 1-90.

[WEBER, F. and D. M. H. MoHR]. 1803. Index musei plantarum cryptogamarum. Kiloniae, 1-6. [Authorship of this work, seen in the Linnean Society of London, follows the ascription by Sayre, 1959, 17.]

WEBER, F. and D. M. H. Mohr. 1807. Botanisches Taschenbuch auf das Jahr 1807. Deutschlands kryptogamische Gewächse. Abt. 1. Filices, Musci frondosi et Hepatici. Kiel, Akademischen Buchhandlung.

WEIMARCK, H. 1937. Förteckning över Skandinaviens Växter utgiven av Lunds Botaniska Förening. Pt. 2. Mossor. Lund, Carl Bloms.

WELCH, W. 1969. Hookeriaceae species and distribution in South America. Proc. Indiana Acad. Sci., 78 (for 1968), 396-405.

WHELDON, J. A. 1921. Key to the Harpidoid Hypna. Naturalist, Hull, (for 1921), No. 774, [No. 548 of current series], 245-48.

WILLAMS, R. S. 1901. Contributions to the botany of the Yukon Territory. 2. An enumeration of the mosses collected. Bull. N.Y. bot. Gdn, 2, No. 6 , 105-48.

WIWAMS, R. S. 1903a. Bolivian mosses. Part I. Bull. N.Y. bot. Gdn, 3, No. 9, 104-34.

WIWAMS, R. S. 1903b. Additional mosses of the Upper Yukon River. Bryologist, 6, No. 4, 61-62.

WIШAMS, R. S. 1915. Mosses from the west coast of South America. Bull. Torrey bot. Club, 42, No. 7, 393-404.

WILLAams, R. S. 1916. Peruvián mosses. Bull. Torrey bot. Club, 43, No. 6, 323-34.

WILIAMs, R. S. 1924. Orthormynchium chilense sp. nov. Bryologist, 27, No. 6, 87-88. [Effective date 15 January 1925, fide title page of Vol. 28, No. 1.]

WI and Argentina by J. N. Rose in 1915. J. Wash. Acad. Sci., 20, No. 19, 465-75.

WILSON, W. 1844. Grimmia orbicularis. (In HOOKER, W. J. Supplement to the English botany of the late Sir J. E. Smith and Mr. Sowerby. London, J. Sowerby, J. W. Salter and Mrs. Sowerby, Vol. 4, Tab. 2888.)

WILSON, W. 1846. Remarks on the new species of Musci from Quito and Swan River, indicated by Dr. Taylor in the London Journal of Botany, Vol. V. p. 41. Lond. J. Bot., 5, 447-55.

WILSON, W. 1847. Brief characters of some new mosses, collected in New Granada by Mr. W. Purdie. Lond. J. Bot., 6, 289-92.

WILSON, W. 1848. Brief characters of Aulacopilum, a new genus of mosses, from New Zealand. Lond. J. Bot., 7, 90-91.

WILSON, W. 1854. Musci, Juss. (In HOOKER, J. D. The botany of the Antarctic voyage of H.M. Discovery ships Erebus and Terror in the years 1839-1843, under the command of Captain Sir James Clark Ross, Kt., R.N., F.R.S., \& L.S. etc. Vol. II. Flora Novae-Zelandiae. Pt. 2. Flowerless plants. London, Lovell Reeve, No. 5, 57-80; No. 6, 81-125.)

WILSON, W. 1855. Bryologia Britannica; containing the mosses of Great Britain and Ireland systematically arranged and described according to the method of Bruch and Schimper, with illustrative plates. London, Longman, Brown, Green and Longmans.
WILSON, W. 1859. Musci, Juss. (In HOOKER, J. D. The botany of the Antarctic voyage of H.M. Discovery ships Erebus and Terror, in the years 1839-1843, under the command of Captain Sir James Clark Ross, Kt., R.N., F.R.S. \& L.S., etc. Vol. III. Flora Tasmaniae. Pt. 2. Monocotyledones and Acotyledones. London, Lovell Reeve, No. 8, 161-221.)

WILSON, W. and J. D. HOOKER. 1845. Musci. (In HOOKER, J. D. The botany of the Antarctic voyage of H.M. Discovery ships Erebus and Terror in the years 1839-1843, under the command of Captain Sir James Clark Ross, Kt., R.N., F.R.S., \&c. Vol. I. Flora Antarctica. Pt. I. Botany of Lord Auckland's group and Campbell's Island. London, Reeve Brothers. [Reprinted 1963, Weinheim, J. Cramer], No. 8, 117-43.)

WILSON, W. and J. D. HOOKER. 1847. Musci. (In HOOKER, J. D. The botany of the Antarctic voyage of H.M. Discovery ships Erebus and Terror, in the years 1839-43, under the command of Captain Sir James Clark Ross, Kt., R.N., F.R.S., \&c. Vol. I. Flora Antarctica. Pt. 2. Botany of Fuegia, The Falklands, Kerguelen's Land, etc. London, Reeve Brothers. [Reprinted 1963. Weinheim, J. Cramer], No. 22, 395-96; No. 23, 397-423.)

WITHERING, W. 1801. A systematic arrangement of British plants; with an easy introduction to the study of botany. Vol. III. 4th edition. London, T. Cadell, Jun. and W. Davies, G. and J. Robinson, Wynne and Scholey, J. Walker, J. Cuthell, and James Wallis.

WRIGHT, C. H. 1905. The mosses of the South Orkneys. (In Brown, R. N. R., WRIGHT, C. H. and O. V. DARBISHIRE. The botany of the South Orkneys. Trans. Proc. bot. Soc. Edinb., 23, Pt. 1, 103-04.)

ZANDER, R. H. 1972. Revision of the genus Leptodontium (Musci) in the New World. Bryologist, 75, No. 3, 213-80.

ZANDER, R. H. 1977. The tribe Pleuroweisiae (Pottiaceae, Musci) in middle America. Bryologist, 80, No. 2, 233-69.

ZANDER, R. H. 1978. New combinations in Didymodon (Musci) and a key to the taxa in North America north of Mexico. Phytologia, 41, No. 1, 11-32.

ZetTERSTEDT, J. E. 1876. Musci et Hepaticae Finmarkiae circa sinum altensem crescentes. $K$. svenska VetenskAkad. Handl., 13, No. 13, 42 pp. 